
The University of Toronto

Chemical Library

Presented

to

The University of Thy onto Library

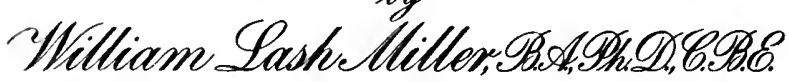

Professor Emeritus of STryssical Ch chemistry

for

A Departmental Libranytobleunder the control is the Professor. of

Chemistry according to the conditions setoutina letter from the Librarian of the University dated March 21.:"

1938. 


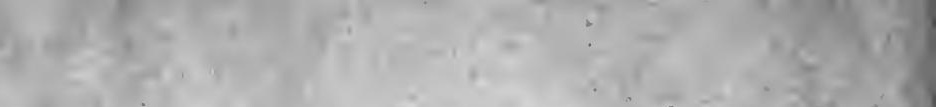

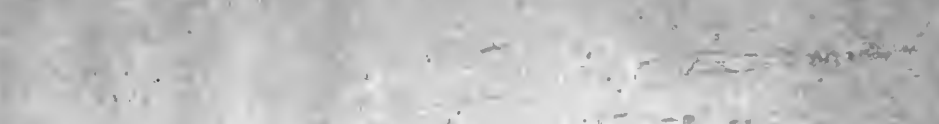

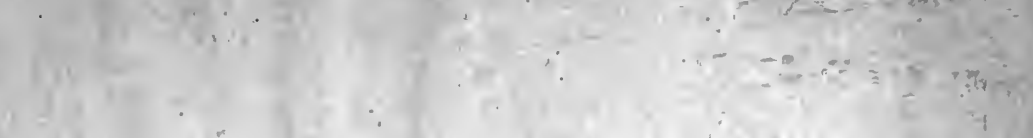
(19.2. . $\quad \therefore$ $+$ $-$

. $\quad 5=5$

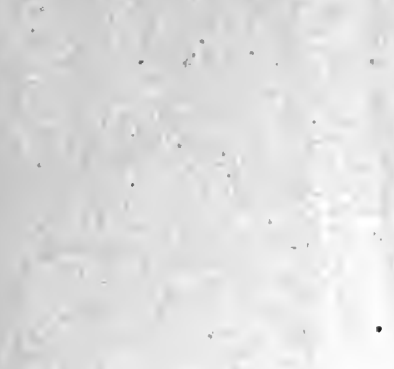
1 -1
$-\quad \because$ 

Digitized by the Internet Archive in 2007 with funding from Microsoft Corporation 


\section{A N U A L \\ oF \\ C H E M I S T R Y. \\ 1}

A GUIDE TO LECTURES AND LABORATORY-WORK FOR BEGINNERS

IN CHEMISTRY A TEXT-BOOK SPECIALLY ADAPTED

FOR STUDENTS OF PHARMACY AND MEDICINE.

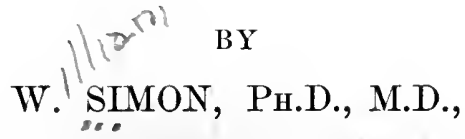

PROFESSOR OF CHEMISTRY AND TOXICOLOGY IN THE COLLEgE OF PHYSICIANS AND SURGENS; PROFESSOR OF CHEMISTRY AND ANALYTICAL CHEMISTRY IN THE MARYLAND COLLEGE OF PHARMACY, BALTMORE, MD.

WITH SIXTEEN ILLUSTRATIONS ON WOOD.
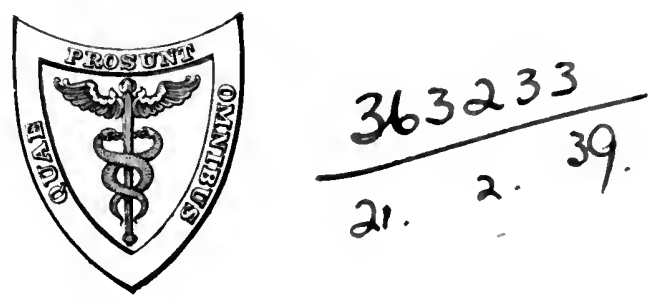

P H I L A DE L PH I A :

HENRY C. LEA'S SON \& CO.

1884 . 


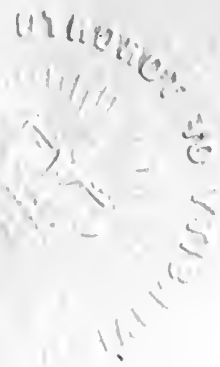

Entered according to the Act of Congress, in the year 1884, by HENRY C. LEA'S SON \& CO.,

in the Office of the Librarian of Congress. All rights reserved. 


\section{P R E F A C E.}

THIs work the author has intended, not only as a guide to those attending a general course of lectures on Chemistry, but especially for the use of pharmaceutical and medical students, whose wants it is believed it will fully supply, especially when supplemented by the practical manipulations in physiological chemistry at the laboratories of medical schools wherein micro-chemical and spectroscopic operations are fully taught, and in colleges of pharmacy where are exemplified to the student the practical chemical manipulations pertaining to the manufacture of officinal preparations.

The material has been divided into seven parts. In the first the fundamental properties of matter are briefly and so far considered as their knowledge is absolutely necessary for an understanding of chemical phenomena.

The second part treats of those principles of chemistry which are the foundation of our science, and enters briefly into a discussion of our theoretical views regarding the atomic constitution of matter. Though the author prefers to present these theories to his classes at the proper times during the course of lectures, he did not deem it desirable to have them scattered throughout the work, choosing rather to present them compactly in such a form that the student may be able to study them, after having acquired some knowledge of chemical phenomena.

The third and fourth parts are devoted to the consideration of the non-metallic and metallic elements and their compounds. The old classification of metals and non-metals, organic and inorganic com- 
pounds, has been adhered to for the reason that the author believes it to be the best adapted for purposes of instructing beginners in chemistry. All our classifications of either natural objects or phenomena are imperfect, because nature does not draw those distinct lines of demarcation which we adopt as necessary for our studies. The most simple and natural classification is, therefore, always to be preferred, even if, as in the above case, the student might derive from it the impression that matter was thus separated into distinct groups.

In considering the various elements and their compounds, much stress has been laid upon their occurrence in nature, for an understanding of chemical phenomena as they take place in the animated and inanimated world, depends largely on a knowledge of these facts.

Of elements those only are considered having either intrinsically or in combination a practical interest, or which take an active part in the various chemical changes in nature.

For the special benefit of pharmaceutical and medical students all chemicals mentioned in the United States Pharmacopœia are included, and when of sufficient interest are fully considered.

The fifth part is devoted to qualitative analysis and will serve the student as a guide in his laboratory work.

The sixth treats of organic chemistry. It was with much hesitation that the author has so briefly considered this portion of his subject, but he felt that a more extended consideration of this highly developed branch of chemical science in a volume of so condensed a character would unduly curtail attention to matters equally important.

The seventh and last part, giving some of the more important facts of physiological chemistry, has been added for the benefit of the medical student, but it is hoped that it may also serve as a guide to others who extend their studies to this very interesting part of the science.

The decimal system has been strictly adhered to in all weights and measures; degrees of temperature are expressed in the same system, except where otherwise stated.

Having frequently noticed how difficult it is for beginners to become familiar with the variously shaded colors of chemicals and 
their reactions, it was the author's desire to illustrate the work with a number of plates presenting the colors of those most important. Chromo-lithography having proved futile, this object has been in a manner accomplished by supplying a portion of the edition with plates made with the actual substance when possessing stability of color, otherwise the tints have been painted by the hand of her whose name is affixed to the plates, and to whose delicate appreciation of color is due the accuracy of the production.

W. S.

Baltimore, September, 1884. 



\section{O N TENTS.}

I.

FUNDAMENTAL PROPERTIES OF MATTER. RESULTS OF THE ATTRACTION BETWEEN MASSES, SURFACES, AND MOLECULES.

1. Extension or flgure.

PAGE

Matter-State of -aggregation-Solids-Cohesion-Crystallized, amorphous, polymorphous, isomorphous substances_Liquids-Gases

-Law of Mariotte .

2. Divisibility.

Mechanical comminution-Action of heat on matter-Molecular theory-Law of A vogadro-Motion of molecules, heat-Latent, free, specific heat-Melting, boiling, distillation, sublimation-Thermometer .

3. Gravitation.

Action of gravitation-Weight, specific weight-HydrometersWeight of gases-Barometer-Changes in the atmospheric pressure -Influence of pressure on state of aggregation . . . .

4. Porosity.

Nature of porosity-Surface, surface-action-Adhesion-Capillary attraction-Absorption-Diffusion-Dialysis

I I.

PRINCIPLES OF CHEMISTRY. RESULTS OF THE ATTRACTION BETWEEN ATOMS.

\section{Chemical divisibility.}

Decomposition by heat-Elements-Compound substancesChemical affinity-Atoms-Chemistry-Atomic and molecular weight-Chemical symbols and formula

6. Laws of chemical combination.

Law of the constancy of composition-Law of multiple proportions-Law of chemical combination by volume-Law of equivalents-Quantivalence 
7. Determination of atomic weights.

PAGE

Determination of atomic weights by chemical decomposition, by means of specific weights of gases or vapors, by means of specific heat .

8. Decomposition of compounds. Groups of compounds.

Decomposition by heat, light, and electricity-Mutual action of substances upon each other-Analysis and synthesis-Acid, basic, and neutral substances-Salts-Residues, radicles . . . .

\section{General remarks regarding elements.}

Relative importance of different elements-Classification of elements-Metals and non-metals-Natural groups of elements-Physical properties of elements-Allotropic modifications-Relationship between elements and the compounds formed by their union-Nomenclature-How to study chemistry

\section{I I.}

\section{NON-METALS AND THEIR COMBINATIONS.}

Symbols, atomic weights, and derivation of names-Occurrence in nature-Time of discovery . . . . . . . . . .

10. Oxygen.

History-Occurrence in nature-Preparation-Physical and chemical properties-Combustion-Ozone . . . . . .

\section{Hydrogen.}

History-Occurrence in nature-Preparation-Properties-Water -Mineral waters-Drinking water-Distilled water-Hydrogen dioxide

\section{Nitrogen.}

Occurrence in nature-Preparation-Properties-Atmospheric air -Ammonia-Compounds of nitrogen and oxygen-Nitric acid, tests for it

13. Carbon.

Occurrence in nature-Properties-Diamond-Graphite-Tests for carbon-Carbon dioxide-Carbonic acid-Tests for carbonic acid -Carbon monoxide-Compounds of carbon and hydrogen-Flame -Silicium-Boron-Tests for silicic and boracic acids . . .

\section{ulphur.}

Occurrence in nature-Properties-Sublimed, washed, and precipitated sulphur-Sulphur dioxide-Sulphurous acid; tests for it -Sulphur trioxide-Sulphuric acid, its manufacture and properties -Tests for sulphates-Sulpho-acids-Hyposulphurous acid-Hydrosulphuric acid; tests for it 
15. Phosphorus.

PAGE

Occurrence in nature-Manufacture, properties, and modifications-Poisonous properties and detection in cases of poisoningOxides of phosphorus-Phosphorous acid ; tests for it-Metaphosphoric, pyrophosphoric, orthophosphoric acid; tests for themHypophosphorous acid; tests for it-Phosphoretted hydrogen .

$92-99$

16. Chlorine.

Haloids or halogens-Preparation and properties of chlorineChlorine water-Hydrochloric acid; tests for it-Nitrohydrochloric acid-Compounds of chlorine with oxygen-Hypochlorous acid-Chloric acid; tests for it

17. Bromine. Iodine. Fluorine.

Bromine-Hydrobromic acid-Tests for bromides-IodineHydriodic acid-Tests for iodine and iodides-Fluorine-Hydrofluoric acid

$105-108$

\section{V.}

\section{METALS AND THEIR COMBINATIONS.}

18. General remarks regarding metals.

Derivation of names, symbols, and atomic weights-Meltingpoints, specific gravities, time of discovery,-valence, occurrence in nature, classification, and general properties of metals .

19. Potassium.

General remarks regarding the alkali-metals-Occurrence in nature-Potassjum hydrate, carbonate, bicarbonate, chlorate, sulphate, sulphite, hypophosphite, iodide, bromide-analytical reactions

$115-120$

20. Sodium.

Occurrence in nature-Sodium chloride, carbonate, bicarbonate, sulphate, sulphite, hyposulphite, phosphate, nitrate-Analy tical reactions-Lithium

\section{Ammonium.}

General remarks-Ammonium chloride, carbonate, sulphate, nitrate, phosphate, iodide, bromide, and sulphide-Analytical reactions .

22. Magnesium.

General remarks-Occurrence in nature-Metallic magnesium -Magnesium carbonate, oxide, sulphate, sulphite-Analytical reactions

23. Calcium.

General remarks regarding alkaline earths-Occurrence in nature-Calcium oxide, hydrate, carbonate, sulphate, phosphate, acid phosphate, and hypophosphite-Bone-black and bone-ashChlorinated lime-Sulphurated lime-Analytical reactions for calcium-Barium and strontium; their salts and analytical reactions 
24. Aluminium.

PAGE

Occurrence in .nature-Metallic aluminium-Alum $\rightarrow$ Aluminium hydrate, oxide, sulphate, and chloride-Clay-Glass-Ultramarine-Analytical reactions . . . . . . .

25. Iron.

General remarks regarding the metals of the iron group-Occurrence in nature-Manufacture of iron-Properties-Reduced iron-Ferrous and ferric oxides and chlorides-Dialyzed ironFerrous iodide, bromide, sulphide, and sulphate-Ferric súlphate and nitrate-Ferrous carbonate, phosphate, and hypophosphiteScale compounds of iron-Analytical reactions . . . .

$143-152$

26. Manganese. Chromium. Cobalt. Nickel.

Manganese; its oxides and sulphate-Potassium permanganateManganese reactions-Chromium-Potassium dichromate-Chromium trioxide-Chromic oxide and hydrate-Reactions for chromium compounds-Cobalt and nickel

$153-159$

27. Zinc.

Occurrence in nature-Metallic zinc-Zinc oxide, chloride, bromide, iodide, carbonate, sulphate, and phosphide-Analytical reactions-Antidotes-Cadmium .

$160-163$

28. Lead. Copper. Bismuth.

General remarks regarding the metals of the lead group-Lead - Lead oxides, nitrate, carbonate, iodide-Poisonous properties of lead-Antidotes-Lead reactions-Copper-Cupric and cuprous oxide-Cupric sulphate and carbonate-Ammonio-copper compounds-Poisonous properties and antidotes-Copper reactionsBismuth-Bismuthyl nitrate and carbonate-Bismuth reactions .

$163-171$

29. Silver. Mercury.

Silver-Silver nitrate, oxide, iodide-Antidotes-Silver reactions-Mercury-Mercurous and mercuric oxides, chlorides, iodides, sulphates, nitrates, sulphides-Ammoniated mercuryAntidotes-Mercury reactions . . . . . . .

30. Arsenic.

General remarks regarding the metals of the arsenic groupArsenious and arsenic oxides and acids-Sodium arseniate-Arseniuretted hydrogen-Sulphides of arsenic-Arsenious iodideAnalytical reactions-Preparatory treatment of organic matter for arsenic analysis-Antidotes

$182-192$

31. Antimony. Tin. Gold. Platinum. Molybdenum.

- Antimony-Trisulphide, oxysulphide, and pentasulphide of antimony-Antimonious chloride and oxide-Antidotes-Antimony reactions-Tin-Stannous and stannic chloride-Tin reactions-Gold-Platinum-Molybdenum 
V.

\section{ANALYTICAL CHEMISTRY.}

32. Introductory remarks and preliminary examination.

PAGE

General remarks-Apparatus needed for qualitative analysisReagents needed-General mode of proceeding in qualitative analysis-Use of reagents-Preliminary examination-Physical properties-Action on litmus-Heating on platinum-foil-Heating on charcoal alone and mixed with sodium carbonate-Flame-tests -Colored borax-beads_Liquefaction of solid substances-Table I : Preliminary examination

\section{Separation of metals in different groups.}

General remarks-Group-reagents-Acidifying the solutionAddition of hydrosulphuric acid-Separation of the metals of the arsenic group from those of the lead group-Addition of ammonium sulphide and ammonium carbonate-Table II: Separation of metals in different groups

34. Separation of the metals of each group.

Table III: Treatment of the precipitate formed by hydrochloric acid-Treatment of the precipitate formed by hydrosulphuric acid-Table IV : Treatment of that portion of the hydrosulphuric acid precipitate which is insoluble in ammonium sulphide-Table V: Treatment of that portion of the hydrosulphuric acid precipitate which is soluble in ammonium sulphide-Table VI : Treatment of the precipitate formed by ammonium hydrate and sulphide-Table VII : Treatment of the precipitate formed by ammonium carbonate-Table VIII : Detection of the alkalies and of magnesia .

\section{Detection of acids.}

General remarks-Detection of acids by means of the action of strong sulphuric acid-Table IX : Preliminary examination for acids-Detection of acids by means of reagents added to their neutral or acid solution-Table $\mathbf{X}$ : Detection of the more important acids by means of reagents added to the solution-Table XI : Systematically arranged table, showing the solubility and insolubility of inorganic salts and oxides

36. Detection of impurities in offlcinal inorganic chemical preparations.

General remarks-Examination of sulphuric, sulphurous, nitric, phosphoric, and hydrochloric acids-Examination of the compounds of potassium, sodium, ammonium, calcium, magnesium, aluminium, iron, zinc, manganese, chromium, lead, copper, bismuth, silver, mercury, arsenic, and antimony 


\section{VI. \\ CONSIDERATION OF CARBON COMPOUNDS, OR ORGANIC CHEMISTR Y.}

37. Introductory remarks. Elementary analysis.

PAGE

Definition of organic chemistry-Elements entering into organic compounds-General properties of organic compoundsDifference in the analysis of organic and inorganic substancesQualitative analysis of organic substances-Ultimate or elementary analysis-Determination of carbon, hydrogen, oxygen, nitrogen, sulphur, and phosphorus-Determination of atomic composition from results obtained by elementary analysis-Empirical and molecular formulas-Rational, constitutional, structural, or graphic formulas . . . . . . . . . .

38. Constitution, decomposition, and classiflcation of organic compounds.

Radicles or residues-Chains-Homologous series-TypesSubstitution-Derivatives-Isomerism-Metamerism-Polymerism-Various modes of decomposition-Action of heat upon organic substances-Dry or destructive distillation-Action of oxygen upon organic substances-Combustion-Decay-Fermentation and putrefaction-Action of chlorine, bromine, nitric acid, alkalies, dehydrating and reducing agents upon organic substances-Classification of organic compounds . . . .

39. Hydrocarbons.

Occurrence in nature-Formation of hydrocarbons-Properties -Paraffine or methane series-Coal, coal-oil, petroleum-Illuminating gas-Coal-tar-Olefines-Benzene series or aromatic hydrocarbons-Volatile or essential oils_Camphors-Resins

40. Alcohols.

Constitution of alcohols-Occurrence in nature-Formation and properties of alcohols-Monatomic normal alcohols-Methyl alcohol-Ethyl alcohol-Alcoholic liquors-Wines, beer, spirits -Amyl alcohol-Glycerine-Phenoles

41. Aldehydes. Haloid derivatives.

Aldehydes-Trichloraldehyde-Chloral hydrate-ChloroformIodoform

42. Monobasic fatty acids.

General constitution of organic acids-Occurrence in natureFormation of acids_Properties-Fatty acids-Acetic acid-Vinegar-Reactions for acetates-Acetate of potassium, sodium, zinc, iron, lead, and copper-Valerianic acid and its salts-Oleic acid .

43. Dibasic and tribasic organic acids.

Oxalic acid, oxalates, and analytical reactions-Tartaric acid; analytical reactions-Potassium tartrate-Potassium-sodium tartrate-Antimony-potassium tartrate-Action of certain organic acids upon certain metallic oxides--Scale compounds-Citric acid ; analytical reactions-Citrates-Lactic acid. 
44. Aromatic acids and some of their derivatives.

PAGE

Constitution of aromatic compounds-Carbolic acid; tests for it-Creasote-Su'phocarbolic acid-Picric acid-Benzoic acid-

Oil of bitter almond-Salicylic acid-Gallic acid-Tannic acid . $295-301$

45. Ethers.

Constitution-Formation of ethers-Occurrence in natureGeneral properties-Ethyl ether-Acetic ether-Ethyl nitrite-Amyl nitrite-Fats and fat oils-Soap.

$302-309$

46. Carbohydrates.

Constitution-Properties-Occurrence in nature-Groups of carbohydrates-Grape-sugar; tests for it-Fruit-sugar-InositeCane-sugar-Milk-sugar-Starch-Dextrine-Gums-CelluloseNitro-cellulose-Glycogen-Glucosides-Santonin

47. Amines and amides. Organic bases. Alkaloids.

Forms of nitrogen in organic compounds-Amines-AmidesFormation of amines and amides-Aniline-Occurrence in nature -Alkaloids; their general properties-General mode of obtaining alkaloids-Antidotes-Detection of alkaloids in cases of poisoning-Important alkaloids-Coniine-Nicotine-Opium-Morphine, its salts and reactions-Codeine-Narcotine and narceineMeconic acid-Cinchona alkaloids-Quinine; its salts and reactions-Cinchonine-Strychnine and its reactions-BrucineAtropine; its reactions-Veratrine; its reactions-Caffeine.

48. Cyanogen and its compounds.

Occurrence in nature-Constitution of cyanogen compoundsDicyanogen-Hydrocyanic acid-Potassium cyanide-Silver cyanide-Mercuric cyanide-Reactions for cyanides-AntidotesCyanic acid-Sulphocyanic acid-Metallocyanides--Potassium ferrocyanide-Reactions for ferrocyanides-Potassium ferricyanide -Nitrocyanmethane

49. Albuminous substances or proteids.

Occurrence in nature-General properties-A nalytical reactions -Classification-Serum-albumin-Egg-albumin-Vegetable albumin- Blood-fibrin- Muscle-fibrin-Vegetable fibrin-Milkcasein-Legumin-Peptone-Hrmoglobin-Animal cryptolitesPepsin

\section{VII.}

\section{PHYSIOLOGICAL CHEMISTRY.}

\section{Chemical changes in plants and animals.}

General remarks-Difference between vegetable and animal life -Formation of organic substances by the plant-Decomposition of vegetable matter in the animal system-Animal food-Nutrition of animals, digestion-Waste products of animal lifeChemical changes after death 
51. Animal fluids and tissues.

PAGE

Constituents of the animal body-Blood-Examination of blood stains-Chyle-Lymph-Saliva-Gastric juice-Bile-Biliary pigments-Biliary acids; tests for them-CholesterinLecithin-Pancreatic juice-Feces-Bone-Teeth-Hair, nails, etc.-Mucus-Muscles-Brain . . . . . . .

52. Milk.

Properties and composition-Changes in milk-Butter-Cheese -Adulteration of milk-Testing milk-Lactometer, creamometer, lactoscope-Analysis of milk . . . . . .

53. Urine and its normal constituents.

Secretion of urine-General properties-Composition-Urea; its properties and determination-Uric acid; tests for it-Hippuric acid; tests for it

54. Examination of normal and abnormal urine.

Points to be considered in the analysis of urine-Color-Odor -Reaction-Specific gravity-Determination of total solids, and of total organic and inorganic constituents-Detection and estimation of albumin-Detection and estimation of sugar-Detection of bile-Urinary deposits-Urinary calculi .

\section{A P P E N D I X.}

Table of weights and measures

Table of elements. 


\section{LIST OF ILLUSTRATIONS.}

FIG.

1. Structure of matter . + . . . . . . . . . . 21

2. Structure of matter . . . . . . . . . . . . . . 22

3. Thermometric scales $\quad . \quad$. $\quad . \quad$. . . . . . . . 25

4. Dialyzer . . . . . . . . . . . . . 34

5. Apparatus for the decomposition of mercuric oxide _ . . . $\quad$. 36

6. Structure of flame

7. Detection of arsenic . . . . . . . . . . . . . . 188

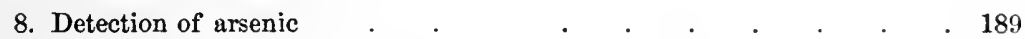

9. Detection of arsenic . . . . . . . . . . . . 190

10. Detection of arsenic . . . . . . . . . . . . . . 191

11. Apparatus for qualitative analysis . . . . . . . . 200

12. Apparatus for qualitative analysis . . . . . . . . . 200

13. A pparatus for qualitative analysis . . . . . . . . . 200

14. Apparatus for qualitative analysis . . . . . . . . . 201

15. A pparatus for qualitative analysis . . . . . . . . . 201

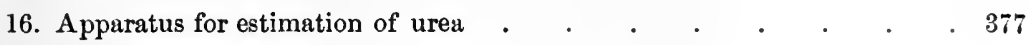

\section{A B B R E V I A T I O N S.}

C.C. = Cubic centimeter.

B.P. = Boiling-point.

F.P. = Fusing-point.

Spec. gr. = Specific gravity.

U. S. P. = United States Pharmacopœia. 



\title{
PRACTICAL CHEMISTRY,
}

\section{PHARMACEUTICAL AND MEDICAL.}

\author{
I. \\ INTRODUCTION.
}

FUNDAMENTAL PROPERTIES OF MATTER. RESULTS OF THE ATTRACTION BETWEEN MASSES, SURFACES, AND MOLECULES.

As the science of chemistry has for its object the study of the nature of all substances or of all varieties of matter, it is necessary first to consider some of the properties which belong to every kind of matter and are known as essential or fundamental properties. The fundamental properties of matter having a special interest for those studying chemistry are: extension, divisibility, porosity, and indestructibility.

\section{EXTENSION OR FIGURE.}

Matter is anything occupying space, and this property is known as extension. All bodies, without exception, fill a certain amount of space. That portion of matter lying within the surrounding surface of a body is called its mass. We distinguish three different conditions of matter, namely: Solids, Liquids, and Gases. These conditions of matter are known as the three states of aggregation, and we will now consider the peculiarities of matter when existing in either of these states. 
Solid state. Solids are distinguished by a self-subsistent figure. A solid substance forms for itself, as it were, a casing in which its smallest particles are enclosed. The question arises, by what means are these particles connected, how are they kept together? No other answer can be given, than that the particles themselves attract each other to such an extent that force is necessary to separate them. We see, consequently, that some form of attraction or attractive power is acting between the particles of a solid mass, and we call this kind of attraction cohesion, to distinguish it from other forms of attraction.

The external appearance or the figure of a solid body is various. It may be an irregular or a natural regular figure. Of these two forms, only the latter is here of interest, as it includes all the different crystallized substances.

Crystals are solid substances, bounded by plane surfaces sym- . metrically arranged according to fixed laws.

The first condition essential to the formation of crystals is the possibility of free motion of the smallest particles of the matter to be crystallized; in that case only will they be able to attract each other in such a way as to assume a regular shape or form crystals. Particles of a solid mass can move freely only after they have been transferred to the liquid or gaseous state. There are two different methods of liquefaction, viz., application of heat (melting), or solution in some suitable agent (dissolving). In the liquid condition thus produced, the smallest particles can follow their own attraction and unite to form crystals on removal of the cause of liquefaction (heat or solvent).

Not all matter can form crystals; some substances never have been obtained in a crystallized state, and these are known as amorphous bodies (starch, gum, glue).

Some substances capable of erystallization may also be obtained in an amorphous state (carbon, sulphur). Other substances are capable of assuming different crystalline shapes under different conditions. Thus sulphur when liquefied by heat, assumes, on cooling, a shape different from the sulphur crystallized from a solution. One and the same substance under the same conditions always assumes the same shape. Substances

1 It will be shown later that all matter consists of smallest particles. 
capable of assuming two or more different forms of crystals are said to be polymorphous. When substances of different kinds crystallize in exactly the same form we call them isomorphous (sulphate of magnesia and sulphate of zinc).

Liquid state. The characteristic features of liquids are, that they have no self-subsistent figure; that they consequently require some vessel to hold them; and that they present a horizontal surface. Whilst in a solid substance the smallest particles are held together by cohesion to such an extent that they cannot change their relative position without force, in a liquid this cohesion acts with much less energy and permits of a comparatively free motion of the particles. That cohesion is not altogether suspended in a liquid is shown by the formation of drops or round globules, which of course consist of a large number of smallest particles. If there were no cohesion at all between these particles of a liquid, drops could not be formed.

Gaseous state. Matter in the gaseous state has absolutely no self-subsistent figure. Gases fill any vessel or room èntirely; the smallest particles show the highest degree of mobility and move freely in every direction. Cohesion is entirely suspended in gases. The most characteristic feature of gases is their great elasticity or tension. Elasticity is that property of matter, by virtue of which it requires force to change its bulk or shape, and a continued application of that force to maintain the change, as it returns to its former bulk or shape when the force is removed. In gases this elasticity is so complete, that a law has been established in relation to it. This law is known as the Law of Mariotte (though really discovered by Boyle, of England, in 1661), and may be expressed thus: The volume of a gas is inversely as the pressure; the density and elastic force are directly as the pressure and inversely as the volume. For instance: If a vessel contains one cubic foot of a'gas under a pressure of -ten pounds, the volume will be reduced to one-half, one-tenth, or one-hundredth of one cubic foot, if the pressure be increased to 20,100 , or 1000 pounds respectively. On the contrary, the gas will expand to 2, 10 , or 100 cubic feet, if the pressure is reduced to 5,1 , or one-tenth pound respectively. Vapors, produced by evaporation of liquids or solids, have the same properties as gases. 


\section{Questions.}

1. What is matter, and what is mass?

2. Mention the principal fundamental properties of matter.

3. Mention the three states of aggregation.

4. Describe the characteristic properties of matter in the solid, liquid, and gaseous state.

5. What is cohesion?

6. Give a definition of a crystallized substance.

7. Under what circumstances will matter crystallize?

8. State the difference between amorphous, polymorphous, and isomorphous substances.

9 . What is meant by elasticity of gases?

10. State the Law of Mariotte.

\section{DIVISIBILITY.}

Mechanical comminution. All matter admits of being subdivided into smaller particles, and this property is called divisibility. The processes by which we accomplish the comminution of a solid substance may be of a mechanical nature, such as cutting, crushing, grinding, but besides these modes of subdivision we have another agent by which matter may be divided into smaller particles, and this agent is heat.

Action of heat on matter. Let us take a piece of ice and convert it, by means of mortar and pestle, into a very fine powder. When the smallest particle of this tinely powdered ice is placed under the microscope and heat applied we will observe that it turns liquid, thus proving that it was capable of further subdivision, that it consisted of smaller particles, which have now by the action of heat become movable. By further applying heat to the liquid particle of water we may convert it into a gas or vapor, which will escape into the air, or which we may collect into an empty flask. The flask will be filled completely by this water-gas (or steam) obtained by vaporizing that minute particle of ice-dust. This fact demonstrates that mechanical comminution does not carry us beyond a certain degree of subdivision of matter. That is to say, the smallest fragment of the finest powder still consists of a very large number of much smaller particles. To the smallest particles which compose matter, the name molecules has been given. 
Molecular theory. The expression molecule, is derived from the Latin word molecula-little mass, and means the smallest particle of matter that can exist by itself, or into which matter is capable of being subdivided by physical actions. To explain more fully what is meant by the expression, molecule, we will return to the conversion of water into steam.

When water boils at the ordinary atmospheric pressure it expands about 1800 times, or one cubic inch of water yields about 1800 cubic inches, equal to about one cubic foot of steam. In explaining this fact we have either to assume, that the water, as well as the steam, is homogeneous matter (Fig. 1), or that the

FIG. 1.
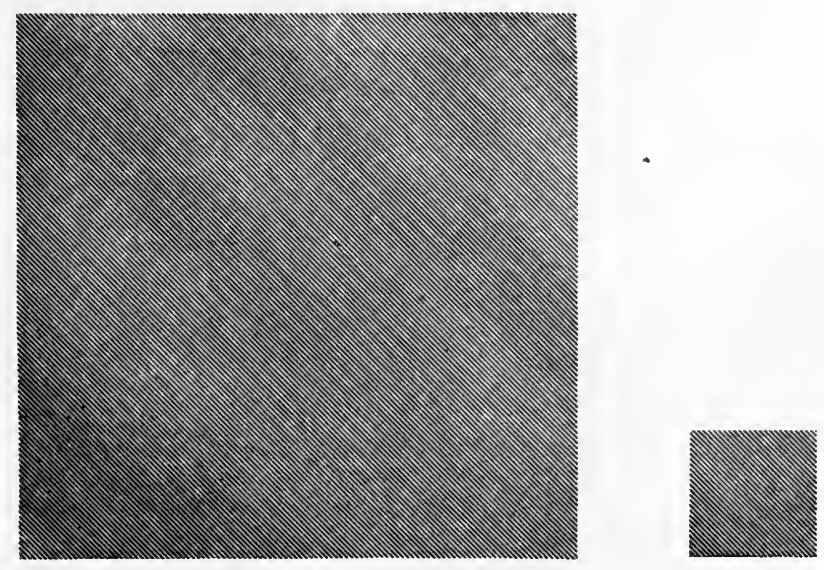

water consisted of small particles of a given size, which now exist in the steam again as such, with the only difference that they are more widely separated from each other (Fig. 2).

Of the many proofs which we have of the fact that the latter assumption is correct, I will mention but one, viz., that the quantities of vapor formed by volatile liquids at any certain temperature above the boiling-point in close vessels of the same size are the same, no matter whether the vessel was entirely empty or contains the vapors of one, two, or more other substances. For instance: If we place one cubic inch of water into a flask holding one cubic foot, from which flask the air has been previously removed, and then heat the flask to the boilingpoint, the cubic inch of water will evaporate, filling the vessel 
with steam. Upon now introducing into the flask a second and third liquid-for instance, alcohol and ether, we find that of each of these liquids exactly the same quantity will evaporate which would have evaporated if these liquids had been introduced into

FIG. 2.

the empty flask. This fact will undoubtedly prove that there nuust be small particles of steam which are not in close contact, that there are spaces between these particles which may be occupied by the particles of a second, third, or more substances. And to these particles of matter we give the name molecules, and the spaces between them we call intermolecular spaces.

We have thus demonstrated the correctness of the so-called molecular theory, but the proof given is but one of many. Of these molecules (though individually by far too small to make any impression whatever upon our senses), our conception is so perfect, that we even have formed an idea of the actual size of these minute particles of matter. Very good reasons lead us to believe that the diameter of a molecule is equal to about 500000000 of one inch, and that one cubic inch of a gas under ordinary conditions contains about one hundred thousand million million millions of molecules.

These figures at first glance appear to be beyond the limit of human conception, but in order to give some idea of the size of these molecules it may be mentioned that if a space of water as large as a pea were to be magnified to the size of our earth, 
each molecule being magnified in the same proportion, these molecules would represent balls of about two inches in diameter.

Whilst molecules consequently are exceedingly small particles, yet they are not entirely immeasurable; they are, as Sir W. Thomson says, pieces of matter of measurable dimensions, with shape, motion, and laws of action, intelligible subjects of scientific investigation.

Before leaving the molecular theory, I will mention the Law of Avogadro, which may be stated as follows: All gases or vapors, without exception, contain, in the same volume, the same number of molecules, provided temperature and pressure are the same. Or, in other words: Equal volumes of different gases contain, under equal circumstances, the same number of molecules. The correctness of this law may be mathematically proved by the law of Mariotte, but many other facts and considerations lead to the same assumption. We shall learn, hereafter, that the law of Avogadro is one of the greatest importance for the science of chemistry.

Motion of molecules. Heat. If we place over a gas-flame a vessel containing a lump of ice of the temperature of $0^{\circ} \mathrm{C}$., or $32^{\circ} \mathrm{F}$., the ice gradually melts and becomes converted into water; but if we measure with a thermometer the temperature of the water at the moment when the last particle of ice is melted, we still find it at the freezing-point or at $32^{\circ} \mathrm{F}$. From the position of the vessel over the flame, as well as from the fact that the ice has been liquefied, we know that the vessel and its contents have absorbed heat. Yet vessel and water show the same temperature as before. If the heat of the flame is allowed to continue its action on the ice-cold water, the thermometer will soon indicate a rapid absorption of heat until it reaches $100^{\circ} \mathrm{C}$. or $212^{\circ} \mathrm{F}$. Here the water begins to boil and escapes in the form of steam, but the temperature again remains stationary until the last particle of water has disappeared.

There must be, consequently, some relation between the state of aggregation of a substance and that agent which we call heat. It was the heat which liquefied the ice, it was the heat which converted the liquid water into steam or gaseous water. Yet the water, having absorbed considerable heat during the process of melting, shows a temperature of $0^{\circ}$, and the steam, 
having also absorbed large quantities of heat, shows $100^{\circ}$, the temperature of boiling water. A certain amount of heat has consequently been lost or at least hidden. What has become of it?

According to our present theory, heat is caused by the motion of molecules. All molecules of any substance are in a constant vibratory motion, and the velocity of this motion determines the degree of what we call heat.

An increase of heat is equal to an increase of the vibratory motion of the molecules and a decrease in temperature is caused by slower motion. The transfer of heat is a transfer of the motion of some particles to other particles.

One of the effects of increased heat is in nearly all cases an increase in volume, or, in other words, all substances expand when heated, and contract on cooling.

Another effect of the application of heat is, as we have just learned, the conversion of solids into liquids, and of liquids into gases. We also noticed the apparent loss of heat during this conversion, and can easily account for it now by saying, that a certain amount of vibratory motion, or a certain velocity of the molecules, is required to convert solids into liquids and liquids into gases. The molecules of steam vibrate with a much greater velocity than those of water of the same temperature, and the molecules of water move with greater velocity than those of ice of the same temperature. In other words, the different states of aggregation depend on the rapidity of the motion of molecules and the heat which is necessary to convert solids into liquids and liquids into gases, and which is not indicated by the thermometer, is called latent heat.

This latent heat may again be converted into free heat (heat capable of being indicated by a thermometer) by reconverting the gas into a liquid, or this latter into a solid. In both cases a liberation of heat, which is a transfer of the motion of the molecules upon the surroundings, will be noticed.

Increase of volume by heat. The increase of volume by heat is not alike for all matter. Gases expand more than liquids, liquids more than solids, and of the latter the metals more than most other solid substances. Whilst the expansion of any two or more different solids or liquids is not alike, gases show a fixed regularity in this respect, namely, all gases, without ex- 
ception, expand or contract alike, when the temperature is raised or lowered an equal number of degrees.

Melting and boiling. The temperature at which a solid substance is converted into a liquid and this into a gas, is of a certain fixed degree or point for every substance, and the temperatures at which this conversion takes place are known as melting- (fusing-) and boiling-points.

Not all matter is capable of existing in the three states of aggregation, like water. Thus we know carbon in the solid state only.

Other substances, again, may assume two, but not the third state. Some substances pass from the solid directly into the gaseous state (ammonic chloride, calomel), and the process of converting a solid into a gas directly, and this back again into a solid, is called sublimation.

Distillation is the conversion of a liquid into a gas, and the recondensation of the gas into a liquid.

Thermometers are instruments indicating different temperatures. Use is made in their construction of the change in volume of different substances by the action of heat. The most common thermometer is the mercury thermometer. This instrument may be easily constructed by filling with mercury a glass tube, having a bulb at the lower end, and placing it into boiling water. The point to which the mercury rises is marked B. P. (boiling-point), and the tube sealed by fusion of the glass. It is then placed in melting ice, and the point to which the mercury sinks is

Fig. 3.

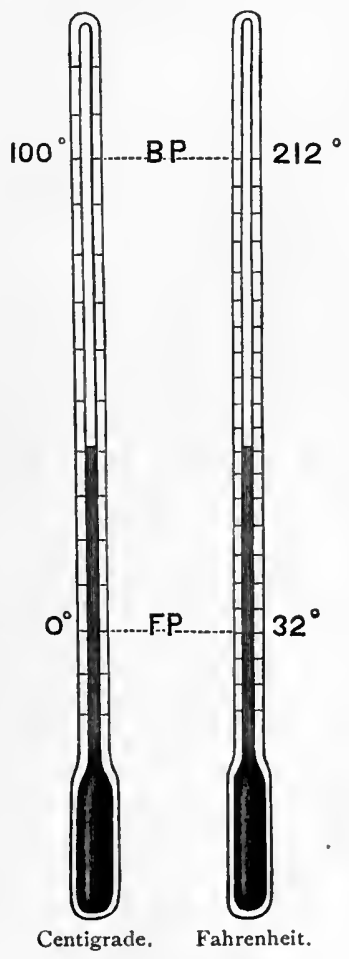

Thermometric scales. marked F. P. (freezing-point). The distance between the boiling- and freezing-points is then divided into 100 degrees in the so-called centigrade thermometer, or into 180 degrees in the 
Fahrenheit thermometer. The inventor of the latter instrument, Fahrenheit, commenced counting not from the freezingpoint, but $32^{\circ}$ below it, which causes the freezing-point to be at $32^{\circ}$, the boiling-point at $180^{\circ}$ above it, or at $212^{\circ}$. (Fig. 3.)

Specific heat. Equal weights of different substances require different quantities of heat to raise them to the same temperature. For instance: The same quantity of heat which is sufficient to raise one pound of water from $60^{\circ}$ to $70^{\circ}$, will raise the temperature of one pound of olive oil from $60^{\circ}$ to $80^{\circ}$, or two pounds of olive oil from $60^{\circ}$ to $70^{\circ}$. Olive oil consequently requires only one-half of the heat necessary to raise an equal weight of water the same number of degrees. Specific heat is therefore the heat required to raise a certain weight of a substance a certain number of degrees, compared with the heat required to raise an equal weight of water the same number of degrees.

The heat required to raise one pound of water one degree centigrade, is usually taken as the unit of comparison. On thus comparing olive oil, we find its specific heat to be $\frac{1}{2}$. If we say the specific heat of mercury is $\frac{1}{32}$, we indicate that equal quantities of heat will be required to raise one pound of water or 32 pounds of mercury one degree centigrade, or that the heat which raises one pound of water one degree will raise one pound of mercury $32^{\circ}$.

\section{Questions.}

11. What two kinds of divisibility of matter do we distinguish, and by what actions are they accomplished?

12. Explain the term molecule.

13. Mention one of the facts which prove that a gas consists of particles with intervais between them.

14. State the Law of Avogadro.

15. Mention the effects produced by increased velocity of the molecules of a mass.

16. Give an explanation of the expressions-latent heat, free heat, and specific heat.

17. Explain the construction of a mercury thermometer.

18. How many degrees of Fahrenheit are equal to $50^{\circ} \mathrm{C}$.?

19. How many degrees of centigrade are equal to $167^{\circ} \mathrm{F}$.?

20. What is distillation, and what is sublimation? 


\section{GRAVITATION.}

Action of gravitation. All masses attract each other and this attraction is known as gravitation. The action of gravitation between the thousands of heavenly bodies moving in the universe is to be considered by astronomy, but some of the phenomena caused by the mutual attraction of the substances composing the earth are of importance for our present considerations.

Such phenomena caused by gravitation are the falling of substances, the flowing of rivers, the resistance which a substance offers on being moved, lifted, or carried. A body thrown up into the air or deprived of its support will fall back upon the earth. In this case the attraction of the earth for the substance has caused its fall. It might appear that in this case the attraction was not mutual, but exerted by the earth only; it has been proved, however, by most exact experiments, that there is also an attraction of the falling substance for the earth, but the amount or the extent of this attraction is in proportion to the mass of the bodies, and consequently too insignificant in the above case to be noticed.

Weight of a substance is an expression used to indicate the amount of attraction which the earth exerts for that substance. Hereagain the attraction of the substance for the earth is not taken into consideration. All our weighing is a comparison with, or measurement by some standard weight, such as pound, ounce, gramme.

Specific weight or specific gravity denotes the weight of a body, as compared with the weight of an equal bulk or equal volume of another substance, which is taken as a standard or unit. The word density is frequently used for specific weight, as density means comparative mass. By the density of a body is consequently meant its mass (or quantity of matter) compared with the mass of an equal volume of some body arbitrarily chosen as a standard. The standard or unit adopted for all solids and liquids is water at the temperature of $60^{\circ} \mathrm{F} .=15.5^{\circ} \mathrm{C}$.

Specific weight is generally expressed in numbers, which denote how many times the weight of an equal bulk of water is 
contained in the weight of the substance in question. If we say that mercury has a specific gravity or density of 13.6 , or that alcohol has a specific gravity of 0.79 , we mean that equal volumes of water, mercury, and alcohol represent weights in the proportion of $1,13.6$, and 0.79 , or 100,1360 , and 79 .

The standards or units chosen for comparing the specific gravity of gases are either atmospheric air or hydrogen.

In order to obtain the specific gravity of any liquid, it is only necessary to weigh equal volumes of water and the liquid to be examined, and then to divide the weight of the liquid by the weight of the water.

A second method by which the specific gravity of liquids may be determined is by means of the instruments known as hydrometers, or, if made for some special purposes, as alcoholometers, urinometers, alkalimeters, lactometers, ete.

Hydrometers are instruments usually made of glass tubes, having a weight at the lower end, to maintain them in an upright position in the fluid to be tested as to specific gravity, and a stem above, bcaring a scale. The principle upon which their construction depends is the fact, that a solid substance, when placed in a liquid heavier than itself, will displace a volume of this liquid equal to the whole weight of the displacing substance. The hydrometer will consequently sink lower in liquids of lower specific gravity than in heavier ones, as the instrument has to displace a larger bulk of liquid in the lighter than in the heavier liquid in order to displace its own weight.

Weight of gases. We have so far considered the gravity of solids and liquids only, and the next question will be: Do gases also possess weight, are they also attracted by the earth? The fact that a gas, when generated or liberated, expands in every direction, might indicate that the molecules of a gas have no weight, are not attracted by the earth. A few simple experiments will, however, show that gases, like all other substances, have weight. Thus a flask from which the atmospheric air contained in it has been removed will weigh less than the same flask when filled with atmospheric air or with any other gas.

Barometer. A second method, by which the fact that atmospheric air possesses weight may be demonstrated, is by means 
of the barometer. The atmosphere is that ocean of gas which encircles the earth with a layer some 50 or 100 miles in thickness, exerting a considerable pressure upon all substances by its weight. The instruments used for measuring that pressure are known as barometers, and the most common form of these is the mercury barometer. It may be constructed by filling with mercury a glass tube closed at one end, and about three feet long, and then inverting it in a vessel containing mercury, when it will be found that the mercury no longer fills the tube to the top, but only to a height of about 30 inches, leaving a vacuum above. The column of mercury is maintained at this height by the pressure of the atmosphere upon the surface of the mercury in the vessel; a column of mercury about 30 inches high must consequently exert a pressure equal to the pressure of a column of the atmosphere of the same diameter as that of the mercury column.

As the weight of a column of mercury, having a base of one square inch and a height of about 30 inches, is equal to about 15 pounds, a column of atmosphere having also a base of one square inch must also weigh 15 pounds. In other words, the atmospheric pressure is equal to about $1 b$ pounds to the square inch, or about one ton to the square foot. This enormous pressure is borne without inconvenience by the animal frame in consequence of the perfect uniformity of the pressure in every direction.

A barometer may be constructed of other liquids than mercury, but as the height of the column must always bear an inverse proportion to the density of the liquid used, the length of the tube required must be greater for lighter liquids. As water is 13.6 times lighter than mercury, the height of a water column to balance the atmospheric pressure is 13.6 times 30 inches, or about 34 feet, which would therefore be the height of the column of water required.

Changes in the atmospheric pressure. The height of the mercury column in a barometer is not the same at all times, but varies within certain limits. These variations are due to a number of causes disturbing the density of the atmosphere, and are chiefly atmospheric currents, temperature, and the amount of moisture contained in the atmosphere. 
As the density of the atmosphere diminishes gradually from the level of the sea upwards, the height of the mercury column will be lower in localities situated at an elevation. This diminution of pressure is so regular that the barometer is used for estimating elevations.

Influence of pressure on state of aggregation. We have seen that the volume of a substance, and, more especially, of a gas, depends upon pressure and temperature, an increase of pressure and decrease of temperature causing the volume to become smaller. We also learned that liquids may be converted into gases, and that this conversion takes place at a certain fixed temperature called the boiling-point. This point, however, changes with the pressure. An increased pressure will raise, a decreased pressure will lower, the boiling-point.

Thus water boils at the normal pressure of one atmosphere at $100^{\circ}$, but it will boil at a lower temperature on mountains in consequence of the diminished atmospheric pressure. If the pressure be increased, as, for instance, in steam-boilers, the boiling-point will be raised. , Thus the boiling-point of water under a pressure of 2 atmospheres is at $122^{\circ}$, of 5 atmospheres at $153^{\circ}$, of 10 atmospheres at $180^{\circ}$.

\section{Questions.}

21. What is gravitation?

22. Mention some phenomena caused by gravitation.

23. Give a definition of weight.

24. What is specific weight?

25. Name the substances adopted as standards for the determination of specific gravities of solids, liquids, and gases.

26. What is the use made of hydrometers, and on what principle is their construction based?

27. Explain construction and use of the mercury barometer.

28. Mention some of the causes which have an influence upon the height of the mercury column in the barometer.

29. What is the atmospheric pressure upon a surface of five square feet?

30. State the relation between boiling-point, temperature, and pressure. 


\section{POROSITY.}

Nature of porosity. We have seen that the molecules of any substance are not in absolute contact, but that there are spaces between them which we call intermolecular spaces, and the property of matter to have spaces between the particles composing it is known as porosity.

In the case of solids, these spaces or pores are sometimes of considerable size, visible even to the naked eye, as, for instance, in charcoal, whilst in most cases these spaces cannot be discovered, even by the microscope. That even apparently very dense substances are porous, can be demonstrated by the fact that liquids may be pressed through metallic disks of considerable thickness, or that gases may be caused to pass through plates of metal or stone, and finally also by the fact that substances suffer expansion or contraction in consequence of increased or diminished heat, or in consequence of mechanical pressure.

Surface. In every-day life the expression surface refers to that part of a substance which is open to our senses, visible and measurable; but from a more scientitic point of view, we have also to take into consideration those surfaces which, in consequence of their porosity, extend to the interior of matter and are invisible to our eyes and absolutely immeasurable by instruments.

Surface-action. Attraction acts differently under different conditions, and accordingly we assign different names to it. We call it cohesion when it acts between molecules, gravitation when acting between masses, and surface-action or surfaceattraction when the attraction is exerted either by the visible surface or by that surface which pervades the whole interior of matter. The phenomena caused by this surface-action are extremely manifold, and some are of sufficient interest to be taken into consideration.

Adhesion. Most solid substances, when immersed in water, alcohol, or many other liquids, become moist; immersed in mercury, they remain dry. We explain this fact by saying that the 
surfaces of most solid substances exert an attraction for the particles of such liquids as water and alcohol to such an extent that these particles adhere to the surface of the solids. Such an attraction, however, does not manifest itself for the particles of mercury. This form of surface-attraction by which liquids are caused to adhere to solids is called adhesion.

This adhesion may also be noticed between two plates of even surface. A drop of water pressed between these plates will cause them to adhere to each other. The application and use of glue and mucilage depend on this kind of surface-action.

Capillary attraction. Whilst it is the general rule, that liquids in a vessel present a horizontal surface, this rule does not hold good near the sides of the vessel. When the liquids wet the vessel, as in the case of water in a glass vessel, the surface is somewhat concave in consequence of the attraction of the glass surface for the particles of water; on the contrary, when the liquids do not wet the vessel, as in the case of mercury in a glass vessel, the surface is somewhat convex. The smaller the diameter of the ressel holding the liquids, the more concave or convex will the surface be. If a narrow tube is placed in a liquid, this surface-action will be more striking, and it will be found that a liquid wetting the tube will not only have a completely concave surface, but the level of the liquid stands perceptibly higher in the tube than the level of the liquid outside. Substances not wetting the tube will show the reverse action, namely, the surface inside of the tube will be convex, and will be below the level of the liquid outside.

The attraction of the surface of tubes for liquids, manifesting itself in the concave shape of the surface and in the elevation of the liquid near the tube, is known as capillary attraction. Capillary elevations and depressions depend upon the diameter of the tube, temperature, and the nature of the liquid. The narrower the tube, the higher the elevation or the lower the depression; both are diminished by increased temperature. Capillary elevations and depressions, all other circumstances being equal, are inversely proportional to the diameters of the tubes.

Surface-attraction of solids for gases. Any dry solid substance, carefully weighed, will, after having been exposed to a higher 
temperature, show a decrease in weight whilst yet warm. Upon cooling, the original weight will be restored. This fact cannot be explained otherwise than that some substance or substances must have been expelled by heat, and that this substance or these substances are reabsorbed on cooling.

This is actually the case, and the substances expelled and reabsorbed are the gaseous constituents of the atmospheric air, chiefly the aqueous vapor.

Every solid substance upon our earth condenses upon its surface more or less of the gaseous constituents of the atmosphere. This condensation takes place upon the outer as well as upon the inner surface. The amount of gas absorbed depends upon the nature of the gas as well as upon the nature of the absorbing solid. Some of the so-called porous substances, such as charcoal, generally condense or absorb larger quantities than solids of a more dense and compact structure. Heat, as stated above, counteracts this absorbing power.

Surface-attraction of solids for liquids or for solids held in solution. When a mixture of different liquids, or a mixture of different solids dissolved in a liquid, is brought in contact with or filtered through a porous solid substance, such as charcoal or boneblack, it will be found that the surface of the solid substance retains a certain amount of the liquids or of the solids held in solution, and that it retains more of one kind than of another.

It is this peculiarity of surface-attraction which is made use of in purifying drinking water by allowing it to pass through charcoal. Bone-black is similarly used for decolorizing sugarsyrup and other liquids.

Absorbing power of liquids. In a similar manner as in the case of solids, liquids also exert an attraction for gases. When a gas is condensed within the pores or upon the surface of a solid, or when it is taken up and condensed by a liquid, we eall this process absorption. This absorbing power of different liquids for different gases varies greatly; it is facilitated by low temperature and high pressure, and counteracted by high temperature and removal of pressure. Thus: One volume of water absorbs at ordinary temperature and pressure about 0.03 volume 
of oxygen, 1 volume of carbon dioxide, 30 volumes of sulphur dioxide, and 800 volumes of ammonia.

Diffusion. When a cylindrical glass vessel has been partially filled with water, and alcohol, which is specifically lighter than water, is poured upon it, care being taken to prevent a mixing of the two liquids, so as to form two distinct layers, it will be found that after a certain lapse of time the two liquids have mixed with each other, particles of water having entered the alcohol and particles of alcohol the water, until a uniform mixture of the two liquids has taken place. Upon repeating the experiment with a layer of water over a column of a solution of common salt, it will again be found that the two liquids gradually enter one into the other until a uniform salt solution has been formed.

In a similar manner, two or more gases introduced into a vessel or a room will readily mix with each other. This gradual passage of one liquid into another, of a dissolved substance into another liquid, or of one gas into another gas, is called diffusion.

Endosmosis. Dialysis. This diffusion takes place also when two liquids are separated by some porqus diaphragm, such as bladder or parchment paper, and it is then called endosmosis or dialysis.

The apparatus used for dialysis is called a dialyzer (Fig. 4), and

FIG. 4.

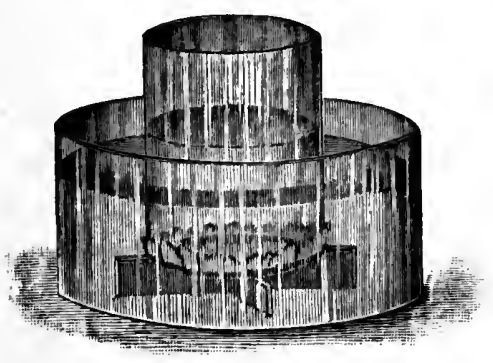

Dialyzer. usually consists of a glass cylinder, open at one end and closed at the other by the membrane to be used as the separating medium. This vessel is then placed into another, and the two liquids are introduced into the two vessels. If the inner vessel be filled with a salt solution and the outer one with pure water, it will be found that part of the salt solution passes throngh the membrane into the water, whilst at the same time water passes over to the salt solution.

On subjecting different substances to this process of dialysis, it has been found that some substances pass through the mem- 
brane with much greater facility or in larger quantities than others, and that some do nof pass through at all. As a general rule, crystallizable substances pass through more freely than amorphous substances. Those substances which do not pass through membranes in the process of dialysis are known as collö̈ds, those which diffuse rapidly crystalloids.

Capillary attraction, or, more generally speaking, surfaceattraction, is undoubtedly to some extent the cause of the phenomena of endosmosis, the surface of the diaphragm exercising an attraction upon the liquids.

Diffusion of gases. A diffusion similar to that of liquids takes place also when two different gases are separated from each other by some porous substance, such as burned clay, gypsum, and others.

It has been found that specifically lighter gases diffuse with greater rapidity than the heavier ones. The quantities of two different gases which diffuse into one another in a given time, are inversely as the square roots of their specific gravities. Oxygen is sixteen times as heavy as hydrogen; when the two gases diffuse, it will be found that four times as much hydrogen has penetrated into the oxygen as of the latter gas into the hydrogen.

\section{Questions.}

31. What is porosity?

32. What two meanings may be assigned to the word surface?

33. Mention some phenomena caused by surface-action.

34. Explain the term adhesion.

35. Under what circumstances can capillary attraction be noticed, and how does it manifest itself?

36. Give an explanation of the word absorption, and mention some instances of the absorption of gases by solids or liquids.

37. What do we understand by diffusion of gases or liquids?

38. Define the word endosmosis.

39. Which substances are most apt to dialyze, and which have no such tendency?

40. State the law regarding diffusion of gases through porous substances. 


\section{II. \\ PRINCIPLES OF CHENISTRY.}

RESULTS OF THE ATTRACTION BETIVEEN ATOUS.

\section{万. CHEMICAL DIVISIBILITY.}

Decomposition by heat. The results of the action of heat upon matter have been stated to be: Increased velocity of the motion of molecules, increase in volume of the substance heated, and in many cases a conversion of solids into liquids and of these into gases. Besides these results, another effect may be frequently noticed which has not yet been mentioned.

To illustrate this action of heat, we will select the red oxide of inercury, a solid substance which is insoluble in water, almost tasteless, and of a brick-red color. When this oxide of mercury is placed in a glass tube and heated, it will be found to disappear

Fig. 5.

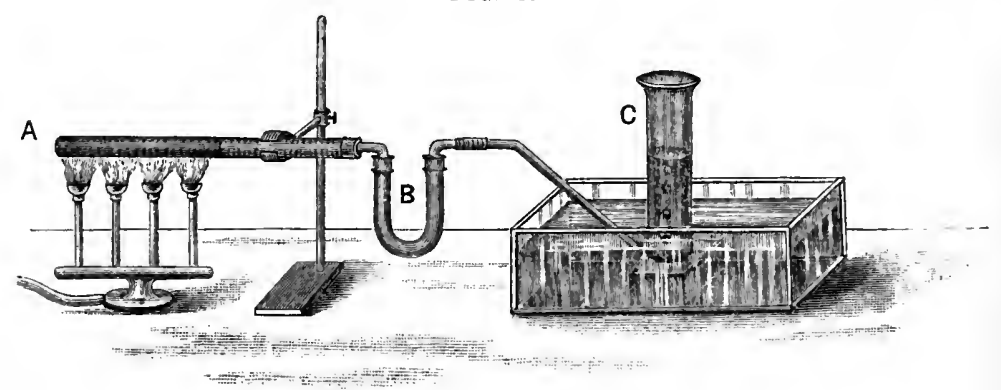

Decomposition of mercuric oxide in $\mathrm{A}$; collection of mercury in $\mathrm{B}$, and of oxygen in $\mathrm{C}$.

gradually, and we might assume that it has been converted into a.gas from which, upon cooling it, the red oxide of mercury would be re-obtained. If the apparatus for heating the oxide of mercury is so constructed that the escaping gases may be col- 
lected and cooled, we shall not find the red oxide in our receiver, but in its place a colorless gas, whilst at the same time globules of metallic mercury are found to be deposited in the cooler parts of the apparatus (Fig. 5).

The action of heat has consequently in this case produced an effect entirely different from the effects spoken of heretofore. There is no doubt that the first action of the heat upon the oxide of mercury is an increased velocity of the motion of its molecules and simultaneously an increase of its volume, but afterwards a decomposition of the oxide takes place, and two substances different from the oxide are liberated.

One of these substances is a silvery-white, heavy, liquid metal, the mercury; the other substance is a colorless, odorless gas, which supports eombustion much more freely than atmospherie air, and is known as oxygen.

Elements. We have thus succeeded in proving that red oxide of mercury may be converted or decomposed by the mere action of heat into mercury and oxygen. It is but natural to inquire whether it would be possible further to subdivide the mercury or the oxygen again into two or more new substances of different properties. To this question, which has been experimentally propounded to Nature over and over again, we have but one answer, viz., oxygen and mercury are substances incapable of decomposition by any means as yet at our disposal. They resist the powerful influences of electricity and heat, even when raised to the highest attainable degrees of intensity, and they issue unchanged from every variety of reaction hitherto devised with the view of resolving them into simpler forms of matter.

We are, therefore, justified in regarding oxygen and mercury as non-decomposable or simple substances, in contradistinction to compound or decomposable substances, such as the red oxide of mercury.

All substances which cannot by any known means be resolved into simpler forms of matter, are called elements; all substances which may, by one process or another, be subdivided or decomposed in such a manner that new substances with new properties are formed, are called compound substances or compounds.

While the number of known compounds exeeeds many 
thousands, the number of elements is comparatively small, but sixty-six of these simple substances being known to exist on our earth. And yet this small number of elements, by combining with each other in many different proportions, form all that boundless variety of matter which we see in nature.

Chemical affinity. There must be some cause which enables or even forces the different elements to unite with each otber so as to form compound bodies. There must be, for instance, a cause which enables oxygen and mercury to combine and form a red powder.

This cause is to be found in the existence of another form of the general attraction, which causes the smallest particles of different elements to unite to form new substances with new properties. This kind of attractive power is called chemical force, affinity, or chemism, and bodies possessing this capacity of uniting with each other are said to have an aftinity for each other.

There is a great difference between chemical attraction and the various forms of attraction spoken of heretofore. Cohesion simply holds together the molecules of the same substance, adhesion acts between the molecules of solid and liquid substances, gravitation acts between masses. But all these forces do not change the nature, the external and internal properties of matter; this is done when chemical force or affinity is operating, when a chemical change takes place.

For instance: In a piece of yellow sulphur the molecules are held together by cohesion, and we can counteract this cohesion by mechanical subdivision, converting it into a fine powder; or by the application of heat we can further subdivide the sulphur, melt, and finally volatilize it; or we can throw a piece of sulphur into the air, when it will fall back upon the earth in consequence of gravitation; or we can dip it into water, when it becomes moist in consequence of surface-action. Yet in all these cases sulphur remains sulphur.

It is entirely different when sulphur enters into chemical combination exerting chemical attraction, for instance, when it burns, this means, when it combines with the oxygen of the atmospheric air. In this case a new substance, a disagreeably smelling gas, a compound of oxygen and sulphur, is formed.

It is consequently a complete change in the properties of 
matter which follows the action of true chemical attraction; we might define affinity to be a force by which elements unite and new substances are generated.

Atoms. Molecules, as stated heretofore, are the smallest particles of matter which can exist. All matter consists of molecules, consequently the red oxide of mercury must also consist of molecules.

By heating the oxide of mercury, oxygen and mercury are obtained, each of which also must consist of molecules. As the oxide of mercury consists of molecules, and as these molecules are neither pure oxygen nor pure mercury, we must come to the conclusion that a molecule of the oxide of mercury is composed of a small particle of oxygen and a small particle of mercury. We consequently learn that a molecule of a compound substance is composed of yet smaller particles of elements, and these smallest particles of elements capable of entering into combination are called atoms, whilst molecules are the smallest particles of matter which are capable of existing in a free state.

Chemistry is the science of affinity, and affinity is the attraction acting between atoms and causing them to unite and form molecules. As every chemical change is due to the motion of atoms, chemistry may also be defined as the science of the motion of atoms taking place in consequence of chemical affinity.

Atomic weight. All matter possesses weight; this is true of a mass as well as any part of it, and must consequently be also true of the atoms and molecules of which matter consists. It is, of course, impossible to weigh a single atom or a single molecule, yet science has formed an opinion in regard to the relative weights of these minute particles. The experiment referred to above may be so conducted as to ascertain the weight of the products of decomposition (viz., the oxygen and the mercury) of a given, previously weighed quantity of oxide of mercury. In doing this, it will be invariably found that every 13.5 parts by weight of the oxide of mercury yield upon heating 12.5 parts by weight of mercury and 1 part of oxygen, that we have consequently in 13.5 pounds of oxide 12.5 pounds of mercury and 1 pound of oxygen.

If we assume that a molecule of the oxide is composed of one 
atom of mercury and one atom of oxygen, we are justified in saying that a mercury atom is 12.5 times heavier than an oxygen atom.

In a manner similar to this, the weights of the atoms of all different elements have been compared with each other, and the element having the lightest atom has been selected as the unit of comparison. The element having the lightest atom is hydrogen, and we say the atomic weight of hydrogen is 1 , and compare with this weight the weights of all other elements. In doing this, we find that the atom of oxygen weighs sixteen times as much as the atom of hydrogen, and we consequently say the atomic weight of oxygen is $\mathbf{1 6 .}$.

We have learned before, from the decomposition of the red oxide of mercury, that the mercury atom is 12.5 times as heavy as that of oxygen. As the atomic weight of this element is 16, the atomic weight of mercury must be 12.5 times 16 , or 200 .

Whilst atomic weight is the weight of the atom of any element as compared to the weight of an atom of hydrogen, molecular weight is the combined weight of the atoms forming the molecule. Thus the molecular weight of oxide of mercury is $200+16=216$.

Chemical symbols. For reasons to be better understood hereafter, chemists designate each element by a symbol, and the first or the first two letters of the Latin name of the element have generally been selected. Thus, the symbol of hydrogen is $\mathrm{H}$, of oxygen $\mathrm{O}$, of mercury $\mathrm{Hg}$ (from hydrargyrum), of sulphur $\mathrm{S}$, etc. These symbols designate, moreover, not only the elements, but one atom of these elements. For instance: $\mathrm{O}$ not only signifies oxygen, but one atom or 16 parts by weight of oxygen; and $\mathrm{Hg}$, one atom or 200 parts by weight of mercury.

Chemical formulas. In a similar manner as atoms of elements are represented by symbols, the molecules of a compound substance are designated, and such a representation of a compound substance by symbols is called its formula. Thus, $\mathrm{HgO}$ is the formula of the red oxide of mercury, and it tells at once that it is a substance composed of one atom or 200 parts by weight of mercury, and one atom or 16 parts by weight of oxygen.

In the molecule of a compound body there must be at least 
two atoms, each one of a different element, but there may be in a molecule of a compound more than two atoms belonging to two or more elements.

For instance: The composition of water is $\mathrm{H}_{2} \mathrm{O}$; this means, a molecule of water contains 2 atoms of hydrogen and 1 atom of oxygen. When there is more than one atom of an element in a molecule the number of these atoms is designated by placing the figure on the right of the symbol and a little below it, as in $\mathrm{H}_{2} \mathrm{O}$, whilst $2 \mathrm{HO}$ or $2(\mathrm{HO})$ would designate 2 molecules of a substance containing one atom of hydrogen and one atom of oxygen.

\section{Questrons.}

41. How does heat act upon red oxide of mercury?

42. State the difference between mechanical and chemical divisibility.

43. Define the terms element and compound.

44. How many elements and how many compound substances are known?

45. What is chemical affinity, and how does it differ from other forces?

46. What is an atom and how does it differ from a molecule?

47. What is chemistry?

48. Give a definition of atomic weight and of molecular weight?

49. The atom of which element has been selected as the unit for comparison of atomic weights?

50. Give an explanation of chenical symbols and formulas.

\section{LAIVS OF CHEMICAL COMBINATION.}

Law of the constancy of composition. This law was the first ever recognized in chemical science; it was discovered towards the close of the last century, and may be stated thus: $A$ definite compound always contains the same elements in the same proportion; or, in other words: All chemical compoumds are definite in their nature and in their composition.

To make this law perfectly understood, the difference between a mechanical mixture and a chemical compound must be pointed out. Two powders, for instance sugar and starch, may be mixed together very intimately in a mortar, so that it scems impossible for the eye to discover more than one body. But in looking at this powder by the aid of a microscope, the particles 
of sugar as well as those of starch may be easily distinguished. The mixture thus produced is a mechanical mixture of molecule clusters.

It is somewhat different when two substances, for instance two metals, are fused together, or when two gases or two liquids (oxygen and nitrogen, water and alcohol) are mixed together, or when finally a solid is dissolved in a liquid (sugar in water). In these instances no separate particles can be discovered even by the microscope. The mistures thus produced are mixtures of molecules. Such mixtures always exhibit properties intermediate between those of their constituents and in regular gradation according to the quantity of each one present. The proportions in which substances may thus be mixed are variable.

In a true chemical compound the proportions of the constituent elements admit of no variation whatever; it is not formed by the mixing of molecules, but by the combination of atoms into molecules; the properties of a compound thus formed usually differ very widely from those of the combining elements.

Law of multiple proportions. If two elements, $A$ and $B$, are capable of uniting in several proportions, the quantities of $B$ which combine with a fixed quantity of $A$ bear a simple ratio to each other. Thus $A$ may combine with $B$, or $A$ with $2 \mathrm{~B}$, or $\mathrm{A}$ with $3 \mathrm{~B}$, etc.

This law was discovered at the beginning of the present century, when it was found that the ratio of carbon to hydrogen in olefiant gas is as 6 to 1 , in marsh gas as 6 to 2 , and that the ratio of carbon to oxygen in carbon monoxide is as 6 to 8 , in carbon dioxide as 6 to 16 .

These and similar instances led to the discovery of the law of multiple proportions, and it was this law which led Dalton, in 1804 , to the adoption of the atomic theory. In thinking and reasoning about this law, he could find no other explanation than that there must be small particles of definite weight which combine with each other, and to these small particles he gave the name atoms.

The law of chemical combination by volume may be stated as follows: "When two or more gaseous constituents combine chemically to form a gaseous compound, the volumes of the individual constituents bear a simple relation to the volume of the product." For instance: 
1 volume of hydrogen combines with 1 volume of chlorine, forming 2 volumes of hydrochloric acid gas; 2 volumes of hydrogen combine with 1 volume of oxygen, forming 2 volumes of water-vapor; 3 volumes of hydrogen combine with 1 volume of nitrogen, forming 2 volumes of ammonia.

If the different combining volumes of the gases mentioned are weighed, it will be found that there exists a simple relation between these volumes and the atomic or molecular weights of the elements.

For instance: Equal volumes of hydrogen and chlorine combine, and the weights of these volumes are as $1: 35.5$, which numbers also represent the atomic weights of the two elements. Two volumes of hydrogen combine with one volume of oxygen and the weights of the volumes are as $1: 8$ or $2: 16$, the latter being the atomic weight of oxygen.

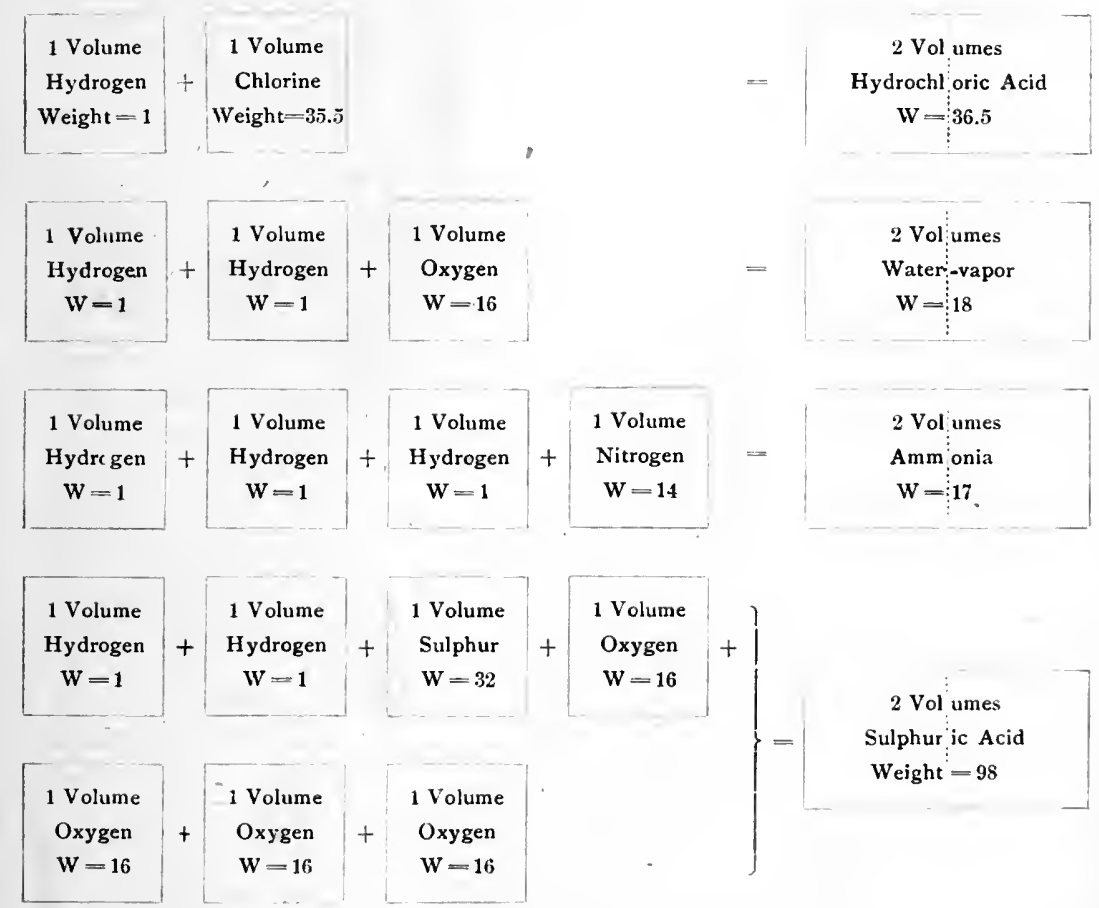

The above table shows the simple relation which exists between combining volumes, atomic and molecular weights, and that such a relation exists is not surprising, if we remember the 
law of Avogadro, which has been stated heretofore, and says that all gases under equal conditions contain the same number of molecules.

The weighing of equal volumes of gases is consequently identical with the weighing of equal numbers of molecules. The molecular weight of a substance can therefore be found by weighing this substance in the gaseous state and comparing with it the weight of an equal volume of another gas, the molecular weight of which is known. The gas usually adopted for this comparison is hydrogen.

If, for instance, we weigh equal volumes of hydrogen, chlorine, oxygen, hydrochloric acid, and steam, we find weights in the proportion of $2,71,32,36.5$ and 18 . These numbers at the same time express the molecular weights of these substances, but the numbers also show that atomic and molecular weights of elements are not identical, but that the latter weight is twice that of the atomic weight, or that the molecules of elements consist of two atoms. ${ }^{1}$

Law of equivalents. Quantivalence. When one element replaces another element in a compound, the quantities of the two elements are said to be equivalent to each other, and according to the law of equivalents the replacement of elements one by another always takes place in definite proportions. Formerly it was believed that all atoms were equivalent amongst each other, and, accordingly, atomic weights were frequently designated as equivalent weights.

This view, however, is not correct, as it is found that frequently one atom of one element displaces two or more atoms of another element. This fact, as well as other considerations, has led to the assumption of the quantivalence of atoms. This property will be best understood by selecting a few compounds of different elements with hydrogen, for consideration.

\begin{tabular}{llll}
\multicolumn{1}{c}{ I. } & II. & III. & IV. \\
$\mathrm{HCl}$ & $\mathrm{H}_{2} \mathrm{O}$ & $\mathrm{H}_{3} \mathrm{~N}$ & $\mathrm{H}_{4} \mathrm{C}$ \\
$\mathrm{HBr}$ & $\mathrm{H}_{2} \mathrm{~S}$ & $\mathrm{H}_{3} \mathrm{As}$ & $\mathrm{H}_{4} \mathrm{Si}$ \\
HI & $\mathrm{H}_{2} \mathrm{Se}$ & $\mathrm{H}_{3} \mathrm{P}$ &
\end{tabular}

I A few exceptions to this general rule will be mentioned at the proper places. 
We see here, that $\mathrm{Cl}, \mathrm{Br}$, and $\mathrm{I}$ combine with $\mathrm{H}$ in the proportion of atom for atom; $\mathrm{O}, \mathrm{S}$, Se combine with $\mathrm{H}$ in the proportion of 2 atoms of hydrogen for 1 atom of the other element; $\mathrm{N}, \mathrm{As}, \mathrm{P}$ combine with $3 ; \mathrm{C}$ and $\mathrm{S}$ with 4 atoms of hydrogen.

It has been, moreover, found that the compounds mentioned in column $\mathrm{I}$. are the only ones which can possibly be formed by the union of the elements $\mathrm{Cl}, \mathrm{Br}$, and $\mathrm{I}$ with $\mathrm{H}$. They invariably combine in this proportion only. Other elements show a similar behavior. For instance, the metal sodium combines with chlorine or bromine in one proportion only, forming the compounds $\mathrm{NaCl}$ or $\mathrm{NaBr}$.

Looking at columns II., III., and IV., we see that the elements mentioned there combine with 2, 3, and 4 atoms of hydrogen, respectively. It is evident, therefore, that there must be some peculiarity in the power of attraction of different elements towards other elements, and to this property of the atoms of elements of holding in combination one, two, three, four, or more atoms of other elements the name atomicity, quantivalence, or simply valence, has been given.

According to this theory of the valence of atoms, we distinguish univalent, bivalent, trivalent, quadrivalent, etc., elements. All elements which combine with hydrogen in the proportion of one atom to one atom are univalent, as, for instance, $\mathrm{Cl}, \mathrm{Br}, \mathrm{I}, \mathrm{Fl}$, and all elements which combine with these in but one proportion, that is, atom with atom, bear the same valence or are also univalent, as, for instance, $\mathrm{Na}, \mathrm{K}, \mathrm{Ag}$, etc.

Those elements which combine with hydrogen or other univalent elements in the proportion of one atom to two atoms are bivalent, such as $\mathrm{O}, \mathrm{S}$, Se.

Trivalent and quadrivalent elements are those the atoms of which combine with 3 or 4 atoms of hydrogen, respectively. Figuratively speaking, we may say that the atoms of univalent elements have but one, those of bivalent elements two, of trivalent elements three, of quadrivalent elements four points of attraction, by means of which they may attach themselves to other atoms.

To indicate the valence of the elements, dots or numbers are frequently placed above the chemical symbols, thus $\mathrm{H}^{\mathrm{i}}, \mathrm{O}^{\mathrm{ii}}, \mathrm{N}^{\mathrm{iii}}$, $\mathrm{C}^{\text {iiii }}$ or $\mathrm{C}^{\mathrm{iv}}$. 
Whilst the valence of some elements is invariably the same under all circumstances, other elements show a different valence (this means a different combining power for other atoms) under different conditions. For instance: Phosphorus combines both with 3 and 5 atoms of chlorine, forming the compounds $\mathrm{PCl}_{3}$ and $\mathrm{PCl}_{5}$. As chlorine is a univalent element, we have to assume that phosphorus has in one case 3 , in another case 5 points of attraction. Many similar instances are known, and will be spoken of later.

The doctrine of the valence of atoms has modified our views of the equivalence of atoms. We say the atoms of univalent, bivalent, or trivalent elements are equivalent among each other; but two atoms of a univalent element are equivalent to one atom of a bivalent element, or two atoms of a bivalent element to one atom of a quadrivalent element, etc.

After having explained this valence of atoms, it may now be better understood why the atoms do not exist as such in a free and uncombined state in an element, but combine with each other to form molecules. The atoms having the tendency of combining with, or attaching themselves to atoms, are bound to exert that attraction, and if they are not combined with atoms of other elements, they combine with each other. For instance : Oxygen gas is not a mass of oxygen atoms, but of oxygen molecules, each molecule being formed by the union of two atoms.

\section{Questions.}

51. State the law of the constancy of composition.

52. What is the difference between a mixture and a compound?

53. Mention some instances of the production of molecular mixtures.

54. State the law of multiple proportions.

55. What considerations led Dalton to the adoption of the atomic theory?

56. What regularity regarding volume is noticed when gases combine chemically?

57. To what was the term equivalent quantities applied formerly, and what is to be understood by it to-day?

58. Explain the term quantivalence or atomicity.

59. Mention some univalent, bivalent, trivalent, and quadrivalent elements. 
60. Suppose a certain volume of hydrogen to weigh 20 grains, how much will an equal volume of oxygen and how much an equal volume of hydrochloric acid gas weigh, provided pressure and temperature be the same?

\section{DETERMINATION OF ATOMIC WEIGHTS.}

Determination of atomic weights by chemical decomposition. The great difficulties originally encountered in the determination of atomic weights cannot be described here, and the present methods only, of which there are principally three, will be considered. These methods depend either on chemical action or physical properties.

One of the chemical methods used for the determination of atomic weights has been stated before in describing the decomposition of the red oxide of mercury by heat. The principle of this method is the determination of the proportions by weight in which the element, the atomic weight of which is unknown, combines with an element the atomic weight of which is known. For instance: If in decomposing a substance we find it to contain in 72 parts by weight 16 parts by weight of oxygen and 56 parts by weight of another element, we have a right to assume the atonic weight of this second element to be 56, provided, however, that the compound is actually formed by the union of one atom of oxygen and one atom of the other element. These 56 parts by weight might, however, represent 2 or 3 or more atoms. If 56 represented 2 atoms, the atomic weight would be but 28 ; if 4 atoms, 14 .

As this mode of determination gives no clew to the number of atoms present in the molecule, the results obtained are liable to be incorrect. In fact, the atomic weights of a number of elements had originally been determined incorrectly by using the above or similar methods, and many of these old atomic weights had to be corrected (generally doubled) in order to obtain the correct numbers.

Thus, in examining water, it was found that it contains 8 parts by weight of oxygen to every 1 part of hydrogen, and the conclusion was drawn that the atomic weight of oxygen was 8 , the molecule of water being formed by the union of one atom of hydrogen and one atom of oxygen. It will be demonstrated 
below why we assume, to-day, that the atomic weight of oxygen is 16 , and the molecule of water is composed of 2 atoms of hydrogen and 1 of oxygen.

Another chemical method of determining atomic weights is the replacement of hydrogen atoms in a known substance by the element the atomic weight of which is to be determined. For instance: Hydrochloric acid is composed of one atom of chlorine weighing 35.5, and one atom of hydrogen weighing 1 , the molecular weight of hydrochloric acid being 36.5. If in this hydrochloric acid the hydrogen is replaced by some other element, for instance by sodium, we are enabled to determine the atomic weight of sodium by weighing its quantity and that of the liberated hydrogen. Suppose by the action of 36.5 grains of hydrochloric acid on sodium 1 grain of hydrogen be replaced by 23 grains of sodium, we would say that the atomic weight of sodium is equal to 23 .

The same difticulty exists in this mode of determination of atomic weights which was alluded to above, viz., not knowing whether it was actually one atom of sodium that displaced the one part of hydrogen, a doubt is left whether the determination is absolutely correct.

Determination of atomic weights by means of specific weights of gases or vapors. It has been stated before that equal volumes of gases contain, under like conditions, the same number of molecules (no matter how small or large the number of atoms within the molecules may be), and that the molecules of elements contain (in most cases) two atoms. These facts give in themselves the necessary data for the determination of atomic weights.

For instance: If a certain volume of hydrogen is found to weigh 2 grains, and an equal volume of some other gaseous element is found to weigh 71 grains, then the atomic weight of the latter element must be 35.5 , because 2 and 71 represent the relative weights of the molecules of the two elements. Each molecule being composed of 2 atoms, these molecular weights have to be divided by 2 in order to find the atomic weights, which are, consequently, 1 and 35.5 respectively.

In comparing oxygen by this method with hydrogen, it is found that equal volumes of these gases weigh 32 and 2 respec- 
tively, that the atomic weight of oxygen is consequently 16 , and not 8 , as determined by chemical methods.

This mode of determining atomic weights may be applied to all elements which are gases or which may be converted into them. There are, however, elements which cannot be volatilized, and in this case it becomes necessary to determine the specific gravity of some gaseous compound of the element. The element carbon itself has never been volatilized, but we know many of its volatile compounds, which may be used in the determination of its atomic weight.

Determination of atomic weights by specific heat. Specific heat has been stated to be the quantity of heat required to raise the temperature of a given weight of any substance a given number of degrees, as compared with the quantity of heat required to raise the temperature of the same weight of water the same number of degrees.

In comparing atomic weights with the numbers expressing the specific heats, it is found that the higher the atomic weight the lower the specific heat, and the lower the atomic weight the higher the specitic heat. This simple relation may thus be expressed: Atomic weights are inversely proportional to the specific heats; or, the product of the atomic weight multiplied by the specific heat is a constant quantity for the elements examined.

$\begin{array}{lccc}\begin{array}{l}\text { Elements. } \\ \text { Lithium, }\end{array} & \begin{array}{c}\text { Specific heats. } \\ \text { (Water=1.) }\end{array} & \text { Atomic weights. } & \begin{array}{c}\text { Product of specific } \\ \text { heat } \times \text { atomic weight. }\end{array} \\ \text { Sodium, } & 0.9408 & 7 & 6.59 \\ \text { Sulphur, } & 0.2934 & 23 & 6.75 \\ \text { Zinc, } & 0.2026 & 32 & 6.48 \\ \text { Bromine (solid), } & 0.0956 & 65.2 & 6.24 \\ \text { Silver, } & 0.0843 & 80 & 6.75 \\ \text { Bismuth, } & 0.0308 & 108 & 6.16 \\ & & 210 & 6.48\end{array}$

An examination of this table will show this relation between atomic weight and specific heat, and also that the product of atomic weight multiplied by specific heat is equal to about 6.5 . The variations noticed in this constant quantity of about 6.5 may be due to errors made in the determinations of the specific 
heats, and subsequent determinations may cause a more absolute agreement.

However, the agreement is sufficiently close to justify the deduction of a law which says: The atoms of all elements have exactly the same capacity for heat. This law was first recognized by Dulong and Petit in 1819, and is simply a generalization of the facts stated.

To show more clearly what is meant by saying that all atoms have the same capacity for heat, we will select three elements to illustrate this law.

If we take of lithium 7 grams, of sulphur 32 grams, of silver 108 grams, we have of course in these quantities equal numbers of atoms, because 7, 32, and 108 represent the atomic weights of these elements. If we expose these stated quautities of the three elements to the same action of heat, we shall find that the temperature increases equally for all three substances; that is to say: the same time will be required to raise 7 grans of lithium $1^{\circ}$, which is necessary to raise either 32 grams of sulphur or 108 grams of silver $1^{\circ}$.

The quantity of heat necessary to raise the atom of any element a certain number of degrees is, consequently, the same. As heat is the consequence of motion, the result of the facts stated may also be expressed by saying: It requires the same energy to cause different atoms to vibrate with such a velocity as to acquire the same temperature, no matter whether these atoms be light or heavy.

It is evident that these facts give us new means of determining atomic weights by simply dividing 6.5 by the specific heat of the element. The specific heat of sulphur, for instance, has been found to be 0.2026. 6.5 divided by this number is 31.6 , or nearly 32 . Originally the atomic weight of sulphur had been determined by chemical methods to be 16 , but its specific heat, as well as other properties, has shown this number to be but one-half of the weight, 32, now adopted.

It may be mentioned that elements possess essentially the same specific heat, whether they exist in a free state or are in combination; this fact will, in many cases, be of use in the determination of atomic weights. 


\section{Questions.}

61. What are the three principal methods used for the determination of atomic weights?

62. Why are chemical means not always sufficient to determine atomic weights?

63. How can the specific gravity of elements in the gaseous state be used for the determination of atomic weight?

64. Describe a method of the determination of atomic weight by chemical means.

65. State one of the reasons why the atomic weight of oxygen has been changed from 8 to 16 .

66. What relation exists between atomic weight and specific heat?

67. State the Law of Dulong and Petit.

68. Suppose the specific heat of an element to be 0.1138 , what will its atomic weight be?

69. Suppose the specific gravity of an elementary gas to be 14, what will its atomic weight be?

70. Suppose 216 grams of an element replace 2 grams of hydrogen in 73 grams of $\mathbf{H C i}$, what will the atomic weight of the element be?

\section{DECOMPOSITION OF COMPOUNDS. GROUPS OF COMPOUNDS.}

Action of heat upon compounds. All phenomena taking place in nature are, without exception, due to motion. Chemistry considers the motion of atoms, without which no chemical change takes place. The causes for chemical changes are either physical actions (heat, electricity), or the decomposing influence of one substance upon another substance in consequence of greater affinity between them under given conditions.

The decomposing action of heat upon compounds has been mentioned before in connection with the decomposition of red oxide of mercury into mercury and oxygen. Similarly to this process, many other compound substances are decomposed by heat either into elements, or, more frequently, into simpler forms of combination. This means that the molecule of a substance containing, for instance, 10 atoms, is split up into 2, 3, or more molecules, each one containing a portion of the 10 atoms. 
For instance: A piece of marble, which is carbonate of calcium, or $\mathrm{CaCO}_{3}$, is decomposed by heat into oxide of calcium, $\mathrm{CaO}$, and carbon dioxide, $\mathrm{CO}_{2}$.

The amount of heat required for decomposition differs widely according to the nature of the substance. Some substances can only be produced at a temperature below the freezing-point of water, a higher temperature causing their decomposition; other substances will be decomposed at temperatures between the freezing- and boiling-points; others again, and to these belong the majority of compounds, may be raised to red or white heat before decomposition sets in ; and still another number of compounds have never yet been decomposed by heat. Theoretically, however, we assume that all compounds may be decomposed by heat, should it be possible to raise it to a sufficiently high degree.

Decomposition by electricity. Similarly to heat, electricity also decomposes many substances, provided they are in a liquid or gaseous state. These decompositions are usually accomplished by allowing an electric current to pass through the liquid, or electric sparks to pass through the gas. Thus hydrochloric acid, $\mathrm{HCl}$, may be decomposed into hydrogen and chlorine. There is a certain relation between electrical and chemical action, as the amount of electricity which for instance sets free 35.5 grams of chlorine, will also set free 80 grams of bromine or 127 grams of iodine. The figures $35.5,80$, and 127 represent the atomic weights of these elements.

Decomposition by light. Another cause of decomposition is, in many cases, the action of light. The art of photography is based upon this kind of decomposition. Many substances, easily affected by light, have to be kept in the dark to prevent them from being decomposed.

The phenomena of heat, light, and electricity resemble each other in so far as they are phenomena of motion. Heat is the consequence of the motion of material particles (molecules); light is the consequence of the vibratory motion of the hypothetical medium rether; electricity is the consequence of the motion of an assumed electric fluid.

These motions, in being transferred to atoms, have, as shown 
above, frequently the tendency of splitting up the molecules of compound substances.

Mutual action of substances upon each other. As a general rule, it may be said that no chemical action takes place between two substances, both of which are in the solid state, because the molecules do not come in sufficiently close contact to exchange their atoms. The free motion of the molecules in liquid or gaseous substances facilitates such a close contact, and consequently chemical action. It is often sufficient to have but one of the acting substances in the gaseous or liquid state, whilst the second one is a solid. (By converting two solids into extremely fine powder and mixing them together thoroughly, chemical combination may follow, provided the affinity between them be sufficiently strong.)

The action of substances upon each other may be represented by the following equations, in which the letters stand for elements or groups of elements:

1. $\mathrm{A}+\mathrm{B}=\mathrm{AB}$.

2. $\mathrm{AB}+\mathrm{C}=\mathrm{AC}+\mathrm{B}$.

3. $\mathrm{AB}+\mathrm{CD}=\mathrm{AC}+\mathrm{BD}$.

4. $\mathrm{AB}+2 \mathrm{C}=\mathrm{AC}+\mathrm{BC}$.

As instances illustrating the above, may be mentioned the tollowing chemical reactions:

1.

$$
\underset{\text { Hydrogen. }}{\mathrm{H}}+\underset{\text { Chlorine. }}{\mathrm{Cl}}=\underset{\substack{\text { Hydrochloric } \\ \text { acid. }}}{\mathrm{HCl}}
$$

The formula here given for the formation of hydrochloric acid is not entirely correct, because the action between hydrogen and chlorine does not take place between free atoms, but between the molecules of the two atoms, each molecule containing two atoms. The more correct way of writing the formula would therefore be:

Or

$$
\mathrm{HH}+\mathrm{ClC}=2 \mathrm{HCl},
$$

$$
2 \mathrm{H}+2 \mathrm{Cl}=2 \mathrm{HCl} \text {. }
$$

2. Hydrochloric acid and sodium form chloride of sodium and hydrogen :

$$
\mathrm{HCl}+\mathrm{Na}=\mathrm{NaCl}+\mathrm{H} .
$$


The formula more correctly written would be:

$$
2 \mathrm{HCl}+2 \mathrm{Na}=2 \mathrm{NaCl}+2 \mathrm{H} .
$$

3.

$$
\underset{\substack{\text { Hydrochloric } \\ \text { acid. }}}{\mathrm{HCl}}+\underset{\substack{\text { Nitrate of } \\ \text { silver. }}}{\mathrm{AgNO}_{3}}=\underset{\substack{\text { Chloride of } \\ \text { silver. }}}{\mathrm{AgCl}}+\underset{\text { Nitric acid. }}{\mathrm{HNO}_{3} .}
$$

This forni of decomposition, known as double decomposition, is one of the most common kinds of chemical changes met with in chemical operations.

4.

$$
\underset{\substack{\text { Hydrosulphuric } \\ \text { acid. }}}{\mathrm{H}_{2} \mathrm{~S}}+\underset{\text { Oxygen. }}{3 \mathrm{O}}=\underset{\text { Water. }}{\mathrm{H}_{2} \mathrm{O}}+\underset{\substack{\text { Sulphur } \\ \text { dioxide. }}}{\mathrm{SO}_{2}}
$$

All the decompositions mentioned above are caused by the affinity which the atoms of one substance have for atoms of another substance. For instance: The decomposition of the hydrochloric acid by sodium must be explained by saying, that sodium has a greater affinity for chlorine than hydrogen, as the latter is expelled by the sodium.

No general rule can, however, be given for the amount of affinity with which the atoms of different elements attract each other, because this attraction differs under different conditions. For instance: Water passed in the form of steam over red-hot iron is decomposed; oxide of iron and free hydrogen being formed :

$$
\mathrm{Fe}+\mathrm{H}_{2} \mathrm{O}=\mathrm{FeO}+2 \mathrm{H} .
$$

This decomposition would indicate that the attraction between iron and oxygen is greater than between hydrogen and oxygen. But in passing free hydrogen over heated oxide of iron the reverse action takes place, water and free iron being formed:

$$
\mathrm{FeO}+2 \mathrm{H}=\mathrm{Fe}+\mathrm{H}_{2} \mathrm{O} \text {. }
$$

This reaction would indicate that the affinity between oxygen and hydrogen is greater than that between oxygen and iron. Many similar instances are known and will be spoken of later.

Chemical reactions. This expression is used for any chemical change, brought about with the intention of studying the nature of a substance. The expression reagent is applied to those substances used for bringing about such changes. 
Analysis and synthesis. These expressions refer to two method of research in chemistry, accomplished by two kinds of reactions, analytical and synthetical.

Analysis is that mode of research by which compound substances are broken up into their elements or into simpler forms of combination, and analytical reactions are all chemical processes by which the nature of an element, or of a group of elements, may be recognized.

Synthesis is that method of research by which elements are made to unite to produce compound substances.

Analytical and synthetical methods, or reactions, frequently blend into one another. This means: A reaction made with the intention of recognizing a substance may at the same time produce some compound of interest from a synthetical point of view.

Acids. The many compounds formed by the union of elements are so various in their nature, that no system of classification proposed up to the present time can be called perfect. There are, however, a few groups or classes of compounds, the properties of which are so well marked, that a substance belonging to either of them may easily be recognized. These groups are the acids, bases, and neutral substances.

Acids are characterized by the following properties:

1. They have (when soluble in water) an acid or sour taste.

2. They change the color of many organic substances, for instance of litmus, from blue to red.

3. They contain hydrogen, which can be replaced by metals, the compound thus formed being a salt.

According to the number of hydrogen atoms replaceable by netals, we distinguish monobasic, bibasic, and tribasic acids. Hydrochloric acid, $\mathrm{HCl}$, is a monobasic, sulphuric acid, $\mathrm{H}_{2} \mathrm{SO}_{4}$, is a bibasic, phosphoric acid, $\mathrm{H}_{3} \mathrm{PO}_{4}$, is a tribasic acid.

Bases or basic substances show properties which are opposite to those of acids. These properties are:

1. They have (when soluble in water) the taste of lye, or an alkaline taste.

2. They restore the color of organic substances when previously changed by acids, for instance of litmus, from red to blue. 
3. When acted upon by acids, they form salts. For instance: Potassium hydrate is a base; when brought in contact with hydrochloric acid it forms the salt potassium chloride and water,

$$
\mathrm{KHO}+\mathrm{HCl}=\mathrm{H}_{2} \mathrm{O}+\mathrm{KCl} \text {. }
$$

Neutral substances. All substances having neither acid nor basic properties are neutral. Water, for instance, is a neutral substance, having no acid or alkaline taste, and no action on red or blue litmus. Many neutral substances, to some extent even water, appear to possess the characteristic properties of both classes, acids and bases; of neither class, however, to a very great extent.

Salts. A salt is a compound formed by the union of an acid and a base (nsually with the simultaneous formation of water), or by the action of an acid on a metal (usually with the liberation of hydrogen).

According to the number of hydrogen atoms replaced in an acid, we distinguish neutral and acid salts. A neutral salt is one formed by replacement of all the replaceable hydrogen atoms of an acid. For instance: $\mathrm{KCl}, \mathrm{K}_{2} \mathrm{SO}_{4}, \mathrm{~K}_{3} \mathrm{PO}_{4}$. (As monobasic acids have but one atom of hydrogen which can be replaced, they form neutral salts only.)

Acid salts are acids in which only a portion of the replaceable hydrogen atoms has been replaced. For instance: $\mathrm{KHSO}_{4}$, $\mathrm{K}_{2} \mathrm{HPO}_{4}, \mathrm{KH}_{2} \mathrm{PO}_{4}$.

Double salts are salts formed by replacement of hydrogen in an acid by more than one metal. For instance: $\mathrm{KNaSO}_{4}$.

Residue, radical or compound radical, are expressions for unsaturated groups of atoms known to enter as a whole into different compounds, but having no separate existence. For instance: The bivalent oxygen combines with two atoms of the univalent hydrogen, forming the saturated compound $\mathrm{H}_{2} \mathrm{O}$, water. If we take from this $\mathrm{H}_{2} \mathrm{O}$ one atom of $\mathrm{H}$, there is left the group of atoms $\mathrm{HO}$, consisting of an atom of oxygen in which but one point of attraction is actually saturated, the second one not being provided for.

This group, $\mathrm{HO}$, is a residue or radical, and is known to enter into many compounds; it is, for instance, a constituent of 
all the different hydrates, such as potassium hydrate, KHO, calcium hydrate, $\mathrm{Ca} 2 \mathrm{HO}$, etc.

According to the number of points of attraction left unprovided for in a radical, we distinguish univalent, bivalent, trivalent, and quadrivalent radicals.

Carbon is a quadrivalent element forming with the univalent hydrogen the saturated compound $\mathrm{CH}_{4}$. By removal of one, two, or three hydrogen atoms the radicals $\mathrm{CH}_{3}{ }^{\prime}, \mathrm{CH}_{2}{ }^{\prime \prime}, \mathrm{CH}^{\prime \prime \prime}$, are formed.

\section{Questions.}

71. What physical actions have a tendency to decompose compound substances?

72. Explain the terms reaction and reagent.

73. Mention some instances of decomposition produced by the action of one substance upon another substance.

74. Why can no general rules be established in regard to the amount of attraction which different elements have for each other?

75. What is the difference between analytical and synthetical methods?

76. Define the terms acid, basic, and neutral substances. By what means can they be recognized?

77. Distinguish between mono-, bi-, and tri-basic acids.

78. What are salts and how are they formed?

79. Define neutral, acid, and double salts.

80. Explain the term radical or residue.

\section{GENERAL REMARKS REGARDING ELEMENTS.}

Relative importance of different elements. Of the total number of about sixty-six elements, but few comparatively (about onefourth) are of great and general importance for the earth, and the phenomena taking place upon it. These important elements form the greater part of the mass of the solid portion of the earth, of the water and atmosphere, and of all animal and vegetable matter.

Another number of elements are of less importance, either because they are not found in any large quantity, or do not take any active or essential part in the formation of organic 
matter; yet they are of interest and importance on account of being used in their elementary' state, or in the form of different compounds in every-day life for various purposes.

A third number of elements are found in such minute quantities in nature that they are almost exclusively of scientific interest. The existence of some elements, the discovery of which has been claimed, is even doubtful.

The elements enumerated in column I. are those of great and general interest; in II. those claiming our interest on account of the use made of them; in III. those having scientific interest only.

I.

Aluminium
Calcium
Carbon
Cblorine
Hydrogèn
Iron
Magnesium
Nitrogen
Oxygen
Phosphorus
Potassium
Silicon
Sodium
Sulphur

II.

Antimony

Arsenic

Barium

Bismuth

Boron

Bromine

Cadmium

Chromium

Cobalt

Copper

Fluorine

Gold

Iodine

Lithinm

Lead

Manganese

Mercury

Molybdenum

Nickel

Platinum

Silver

Strontium

Tin

Zine
III.

Beryllium (Glucinium)

Crsium

Cerium

Columbium (Niobium)

Didymium

Erbium

Gallium

Indium

Iridium -

Lanthanum

Osmium

Palladium

Rhodium

Rubidium

Ruthenium

Scandium

Selenium

Tantalum

Tellurium

Thallium

Thorium

Titanium

Tungsten

Uranium

Vanadium

Ytterbium

Yttrium

Zirconium

Classification of elements may be based upon either physical or chemical properties, or upon a consideration of both. A very natural classification of all elements is the one dividing them into the two groups of metals and non-metals. 
Metals are all elements which have that peculiar lustre kuown as metallic lustre; which are good conductors of heat and electricity; which, in combination with oxygen, form compounds generally showing basic properties; and which are capable of replacing hydrogen in acids, thus forming salts.

Non-metals or metalloids are all elements not having the abovementioned properties. Their oxides in combination with water generally have acid properties. In all other respects, the chemical and physical properties of non-metals differ widely. Their number amounts to 14 , the other 52 elements being metals.

Natural groups of elements. Besides classifying all elements into metals and non-metals, certain members of both classes exhibit so much resemblance in their properties, that many of them have been arranged into natural groups. The members of such a natural group frequently show some connection between atomic weights and properties.

$\begin{array}{llll}\text { Chlorine, 35.5 } & \text { Sulphur, 32 } & \text { Lithium, } 7 & \text { Calcium, 40 } \\ \text { Iodine, 127 } & \text { Tellurium, 128 } & \text { Potassium, 39 } & \text { Barium, 137 } \\ \text { Bromine, 80 } & \text { Selenium, 79.5 } & \text { Sodium, 23 } & \text { Strontium, 87 }\end{array}$

Each three elements mentioned in the above four columns resemble each other in many respects, forming a natural group. The relation between the atomic weights will hardly be suspected by looking at the figures, but will be noticed at once by adding together the atomic weights of the first and last elements and dividing this sum by 2 , when the atomic weights (very nearly, at least) of the middle members of the series are obtained. Thus:

$$
\begin{array}{rlrl}
\frac{35.5+127}{2} & =81.25 ; & \frac{32+128}{2}=80 ; \\
\frac{7+39}{2}=23 ; & \frac{40+137}{2}=88.5 .
\end{array}
$$

This relationship between atomic weights and propertics has been used for arranging all elements systematically in such a manner that the existing connection is clearly pointed out. For several reasons, however, these systems are not altogether perfect, and need therefore not be further considered in this book. 
Physical properties of elements. Most elements are, at the ordinary temperature, solid substances, two are liquids (bromine and mercury), five are gases (oxygen, hydrogen, nitrogen, chlorine, and fluorine). Most of the solid elements may be collrerted into liquids and gases by the action of heat. Some solid elements, however, have so far resisted all attempts to change their state of aggregation.

Most, if not all, of the solid elements may be obtained in the crystallized state; a few are amorphous and crystallized, or polymorphous. The physical properties of many elements in these different states differ widely. For instance: Carbon is known crystallized as diamond and graphite, or amorphous as charcoal. The property of elements to assume such different conditions is called allotropic modification.

Some of the gaseous elements are also capable of existing in allotropic modifications. For instance: Oxygen is known as such and as ozone, the latter differing from the common oxygen both in its physical and chemical properties. The explanation given for this surprising fact, that one and the same element has different properties in certain modifications is, that either the molecules or the atoms within the molecules are arranged differently. Ozone, for instance, has three atoms of oxygen in the molecule, while the common oxygen molecule contains but two atoms.

Most of the elements are tasteless and odorless; a few, however, have a distinct odor and taste, as, for instance, iodine and brumine.

Relationship between elements and the compounds formed by their union. The properties of the compounds formed by the combination of elements are so various that it is next to impossible to give any general rule by which they might be indicated. It may be said, however, that nearly all of the gaseous compounds contain at least one gaseous element, and that solid elements, when combining with each other, generally form solid substances, rarely liquids, and never compounds showing the gaseous state at the ordinary temperature.

Nomenclature. The chemical nomenclature of compound substances has undergone considerable changes within the last 
twenty years. These changes were made in conformity with our present or modern views of the constitution of compounds, but many years may yet pass before a uniform system of nomenclature will be generally adopted.

When two elements combine in one proportion only, little difficulty is experienced in the formation of a name, as, for instance, in iodide of potassium or potassium iodide, KI, chloride of sodium or sodium chloride, $\mathrm{NaCl}$.

When two elements combine in more than one proportion, the syllables mono, di, tri, tetra, and penta are frequently used to designate the relative quantity of the elements. For instance: Carbou monoxide, $\mathrm{CO}$, carbon dioxide, $\mathrm{CO}_{2}$, phosphorus trichloride, $\mathrm{PCl}_{3}$, phosphorus pentachloride, $\mathrm{PCl}_{5}$.

In many cases the syllables ous and $i c$ are used to distinguish the proportions in which two elements combine; the syllable ous being used for the simpler or lower, the syllable ic for the more complex or higher form of combination. For instance: Phosphorous chloride, $\mathrm{PCl}_{3}$, and phosphoric chloride, $\mathrm{PCl}_{5}$; ferrous oxide, $\mathrm{FeO}$, ferric oxide, $\mathrm{Fe}_{2} \mathrm{O}_{3}$.

The syllables mono and sesqui are also occasionally used to mark this difference, as, for instance, monoxide of iron, $\mathrm{FeO}$, sesquioxide of iron, $\mathrm{Fe}_{2} \mathrm{O}_{3}$.

When two oxides of the same element ending in ous and ic form acids (by entering in combination with water), the same syllables are used to distinguish these acids: Phosphorous oxide, $\mathrm{P}_{2} \mathrm{O}_{3}$, forms phosphorous acid; phosphoric oxide, $\mathrm{P}_{2} \mathrm{O}_{5}$, forms phosphoric acid.

The salts formed by these acids are distinguished by using the syllables ite and ate. Phosphite of sodium is derived from phosphorous acid, phosphate of sodium from phosphoric acid. Sulphites and sulphates are derived from sulphurous and sulphuric acid, respectively.

According to the new nomenclature, the name of the metal precedes that of the acid or acid radical in an acid. For instance, sodic phosphite or sodium phosphite, instead of phosphite of sodium; potassic sulphate or potassium sulphate, instead of sulphate of potassium. The acids themselves are looked upon as hydrogen salts, and are named accordingly: hydrogen nitrate for nitric acid, hydrogen chloride for hydrochloric acid, etc. 
When the number of elements and the number of atoms increase in the molecule, the names become in most cases more complicated. The rules applied to the formation of such complicated names will be spoken of later.

How to study chemistry. In studying chemistry, the student is advised to impress upon his memory five points regarding every important element or compound. These points are:

1. Occurrence in nature. (Whether in free or combined state; whether in the air, water, or solid part of our earth.)

2. Mode of preparation by artificial means.

3. Physical properties. (State of aggregation and influence of heat upon it; color, odor, taste, solubility, etc.)

4. Chemical properties. (Atomic and molecular weight; valence; amount of attraction towards other elements or compounds; acid, alkaline, or neutral reaction; reactions by which it may be recognized and distinguished from other substances.)

5. Application and use made of it in every-day life, in the arts, manufactures, or medicine.

Of the most important elements and compounds, the history of their discovery, and, occasionally, some special points of interest, should also be noticed.

All students having the facilities for working in a chemical laboratory are strongly advised to make all those experiments and reactions which will be mentioned in connection with the different substances to be considered in this book.

\section{Questions.}

81. Why are not all the elements of equal importance?

82. State the physical and chemical properties of metals.

83. $\mathrm{H}_{\circlearrowleft \mathrm{w}}$ are metals distinguished from non-metals ?

84. What relation often exists between the atomic weights of elements belonging to the same group?

85. Explain the term allotropic modification.

86. Mention some elements capable of existing in allotropic modifications.

87. What relation exists between the properties of elements and the properties of the compounds formed by their union? 
88. In which cases are the syllables mono-, di-, tri-, tetra-, and pentaused in chenical nomenclature?

89. What use is made of the syllables ous and $i c$, ite and ate, in distinguishing compounds from each other?

90. Which five points should be specially considered in studying the nature of elements or compounds? 


\section{III.}

\section{NON-METALS AND THEIR COMBINATIONS.}

The total number of the non-metals is fourteen; two of them, selenium and tellurium, are of so little importance that they need not be considered in this book.

\section{Symbols, atomic weights, and derivation of names.}

Boron, $\quad$ B $=11$. From borax, the substance from which boron was first obtained.

Bromine, $\quad \mathrm{Br}=79.8$. From the Greek $\beta \rho \tilde{\omega} \mu o s$ (bromos), stench, in allusion to the intolerable odor.

Carbon, $\quad \mathrm{C}=12$. From the Latin carbo, coal, which is chiefly carbon.

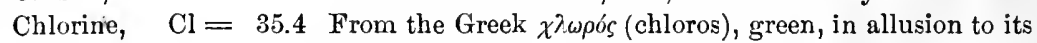
green color.

Fluorine, $\quad \mathrm{Fl}=19$. From fluorspar, the mineral fluoride of calcium, used as flux (Fluo, to flow).

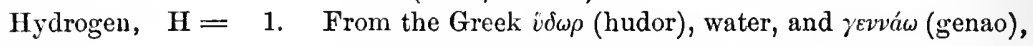
to generate.

Iodine, $\quad I=126.6$. From the Greek iov (ion), violet, referring to the color of its vapors.

Nitrogen, $\quad \mathrm{N}=14$. From the Greek vitpov (nitron), nitre, and $\gamma \varepsilon v v a ́ \omega$ (genao), to generate.

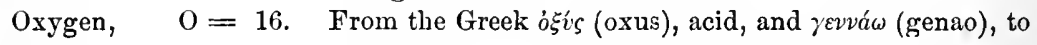
generate.

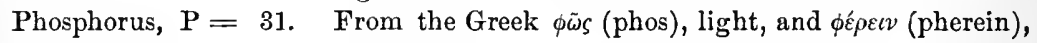
to bear.

Silicium, $\quad \mathrm{Si}=28$. From the Latin silex, flint, or silica, the oxide of silicium.

Sulphur, $\quad \mathrm{S}=32$. From $s a l$, salt, and $\pi \tilde{v} \rho$ (pur), fire, referring to the combustible properties of sulphur. 


\section{State of aggregation.}

Under ordinary conditions the non-metals show the following states:

Gases.

$\begin{aligned} & \text { Hydrogen, } \\ & \text { Oxygen, } \\ & \text { Nitrogen, }\end{aligned}$
Chlorine,
Fluorine,
$\begin{gathered}\text { Easily liquefied. } \\ \text { converted into } \\ \text { liquids. }\end{gathered}$

Liquids. Bromine, $63^{\circ}$

Solids.

$\begin{array}{lrr} & \text { F. P. } & \text { B. P. } \\ \text { Phosphorus, } & 44^{\circ} & 280^{\circ} \\ \text { Iodine, } & 107 & 175 \\ \text { Sulphur, } & 111 & 400 \\ \text { Carbon, } & & \\ \text { Boron, } \\ \begin{array}{l}\text { Silicon, } \\ \text { Silufusible. }\end{array}\end{array}$

\section{Occurrence in nature.}

a. In a free or combined state.

Carbon in coal, organic matter, carbon dioxide, carbonates.

Nitrogen in air, ammonia, nitrates, organic matter.

Oxygen in air, water, organic matter, most minerals.

Sulphur chiefly as sulphates and sulphides.

\section{b. In combination only.}

Boron in boric acid and borax.

Bromine in saline springs and sea-water as bromide of magnesium, etc.

Chlorine as chloride of sodium in sea-water, etc.

Fluorine as fluoride of calcium, fluorspar.

Hydrogen in water and organic matter.

Iodine as iodides in sea-water.

Phosphorus as phosphate of calcium, iron, etc., in bones.

Silicon as silicic acid or silica, and in silicates.

\section{Time of discovery.}

Sulphur Long known in the elementary state; recognized as elements in Carbon $\}$ the latter part of the eighteenth century.

Phosphorus, 1669, by Brandt, of Germany.

Chlorine, 1770, by Scheele, of Sweden.

Nitrogen, 1772, by Rutherford, of England.

Oxygen, 1774, by Priestley, of England, and Scheele, of Sweden.

Hydrogen, 1781, by Cavendish, of England.

Boron, 1808, by Gay-Lussac, of France.

Fluorine, 1810, by Ampère, of France.

Iodine, 1812, by Courtois, of France.

Silicon, 1823, by Berzelius, of Sweden. 


\section{Valence.}

Univalent.

Hydrogen.

Chlorine.

Bromine.

Iodine.

Fluorine.

\section{Trivalent.}

Nitrogen.

Boron.

Phosphorus.
Quadrivalent.

Carbon.

Silicon.

\section{OXYGEN.}

$$
\mathrm{O}^{\mathrm{i}}=16 .
$$

History. Oxygen was discovered in the year 1774 by Priestley, in England, and Scheele, in Sweden, independently of each other; its true nature was soon afterwards recognized by Lavoisier, of France, who gave it the name oxygen, from the two

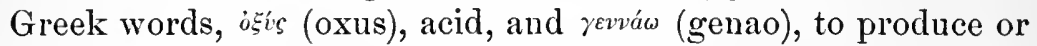
generate. Oxygen means, consequently, geperator of acids.

0ccurrence in nature. There is no other element on our earth present in so large a quantity as oxygen. It has been calculated that not less than about one-third of the total weight of our earth is made up of oxygen; it is found in a free or uncombined state in the atmosphere, of which it forms about onefifth of the weight. Water contains eight-ninths of its weight of oxygen, and most of the rocks and different mineral constituents of our earth contain oxygen in quantities varying from 30 to 50 per cent.; finally, it is found as one of the common constituents of most animal and vegetable matter.

Preparation. The oxides of the so-called noble metals (gold, silver, mercury, platinum) are easily decomposed by heat into the metal and oxygen:

$$
\begin{aligned}
\mathrm{HgO} & =\mathrm{Hg}+\mathrm{O} \\
\mathrm{Ag}_{2} \mathrm{O} & =2 \mathrm{Ag}+\mathrm{O} .
\end{aligned}
$$

A more economical method of obtaining oxygen is the decomposition of potassium chlorate into potassium chloride and oxygen by application of heat.

$$
\mathrm{KClO}_{3}=\mathrm{KCl}+3 \mathrm{O} .
$$

If the potassium chlorate is mixed with about ten per cent. of manganese dioxide and this mixture heated, the liberation of 
oxygen takes place with greater facility and at a lower temperature than by heating potassium chlorate by itself.

Apparently, the manganese dioxide takes no active part in the decomposition; as its total amount is found in an unaltered condition after all potassium chlorate has been decomposed by heat. A satisfactory explanation regarding this action of manganese dioxide is yet wanting.

A third method is to heat to redness, in an iron vessel, the manganese dioxide, which then suffers a partial decomposition :

$$
3 \mathrm{MnO}_{2}=\mathrm{Mn}_{3} \mathrm{O}_{4}+2 \mathrm{O} \text {. }
$$

In this case but one-third of the total amount of oxygen present is liberated, while two-thirds remain in combination with the manganese.

Other methods of obtaining oxygen are decomposition of water by electricity, heating of chromates, nitrates, barium dioxide, sulphuric acid, and other substances, which evolve a portion of the oxygen present in the molecules.

The quantity of oxygen liberated from a given quantity of a substance may easily be calculated from the atomic and molecular weights of the substances entering into decomposition. For instance : 100 pounds of oxygen may be obtained from how many pounds of potassium chlorate, or from how many pounds of manganese dioxide?

The molecular weight of potassium chlorate is found by adding up the weights of 1 atom of potassium $=39+1$ atom of chlorine $=35.5+3$ atoms of oxygen $=48$; total $=122.5$. Every 122.5 parts by weight of potassium chlorate liberate the weight of 3 atoms or 48 parts by weight of oxygen. If 48 are obtained from 122.5, 100 are obtained from 255.2.

$$
48: 122.5:: \begin{array}{rl}
100 & : x \\
x & x=255.2 .
\end{array}
$$

In a similar manner, it will be found that 815.6 pounds of manganese dioxide are necessary to produce 100 pounds of oxygen. $\mathrm{MnO}_{2}=55+32=87 . \quad 3 \quad \mathrm{MnO}_{2}=3 \times 87=261$. Every 261 parts furnish $2 \times 16=32$ parts of oxygen.

$$
\begin{aligned}
& 32: 261:: 100: x \\
& x=815.6 \text {. }
\end{aligned}
$$

Physical properties. Oxygen is a colorless, inodorous, tasteless gas; up to a few years ago it had been looked upon as a perma- 
nent or stable gas, as all attempts to liquefy or solidify it had failed. Lately, however, these efforts have been successful, and oxygen has been converted (though in very small quantities) into a liquid by the application of intense cold and an enormous pressure.

Oxygen is but sparingly soluble in water (about 3 volumes in 100 at common temperature). A litre of oxygen at the standard temperature and pressure weighs 1.43 gram.

Chemical properties. The principal feature of oxygen is its great affinity for almost all other elements, both metals and nonmetals; with nearly all these elements it combines in a direct manner. The more important elements with which oxygen does not combine directly are: $\mathrm{Cl}, \mathrm{Br}, \mathrm{I}, \mathrm{Fl}, \mathrm{Au}, \mathrm{Ag}$, and $\mathrm{Pt}$, but even with these it combines indirectly, excepting $\mathrm{Fl}$.

The act of combination between other substances and oxygen is called oxidation, and the products formed, oxides. Whenever the heat generated by oxidation (or by any other chemical action) is sufficiently high to cause the emission of light, the process is called combustion. Oxygen is the chief supporter of all the ordinary phenomena of combustion. Substances which burn in atmospheric air burn with greater facility in pure oxygen. This property is taken advantage of to recoguize and distinguish oxygen from most other gases. Processes of oxidation evolving no light are called slow combustion. An instance of slow combustion is the combustion of the different organic substances in the living animal; the oxygen being supplied during the process of respiration.

It is not absolutely necessary for a process of oxidation that free oxygen be present, as many substances contain oxygen in such a form of combination that they part with it easily when brought in contact with substances having a greater aftinity for it. Such substances are called oxidizing agents, as, for instance, nitric acid, potassium chlorate, potassium permanganate, etc.

Ozone is an allotropic modification of oxygen, which is formed when non-luminous electric discharges pass through atmospheric air or through oxygen; when phosphorus, partially covered with water, is exposed to air, and also during a number of chemical decompositions. Ozone differs from ordinary oxygen 
by a peculiar odor, by being an even stronger oxidizing agent than common oxygen, by liberating iodine from potassic iodide, etc. This latter action may be used for demonstrating the presence of ozone by suspending in the gas paper moistened with a solution of potassium iodide and starch. The iodine, liberated by the ozone, forms, with starch, a dark blue compound. Theoretically, we assume that ozone contains three, common oxygen but two, atoms in the molecule.

\section{Questions.}

91. By whom and at what time was oxygen discovered?

92. How is oxygen found in nature?

93. Mention three processes by which oxygen may be obtained?

94. How much oxygen may be obtained from 490 grains of potassium chlorate?

95. State the physical and chemical properties of oxygen.

96. What is combustion, and how does it differ from slow combustion?

97. Mention some oxidizing agents.

98. What is ozone, and how does it differ from common oxygen?

99. Under what circumstances is ozene formed?

100. State the molecular weight of oxygen and of ozone.

\section{HYDROGEN.}

$$
\mathrm{H}^{\mathrm{i}}=1 \text {. }
$$

History. Hydrogen was obtained by Paracelsus in the 16th century; its elementary nature was recognized by Cavendish, in 1781. The name is derived from $v \delta \omega \rho$ (hudor), water, and

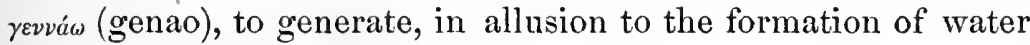
by the combustion of hydrogen.

0ccurrence in nature. Hydrogen is found chiefly in the form of water, and enters into the composition of most animal and vegetable substances.

Preparation. Hydrogen may be obtained by passing an electric current through water, by which it is decomposed into its elements :

$$
\mathrm{H}_{2} \mathrm{O}=2 \mathrm{H}+\mathrm{O} \text {. }
$$


A second process is the decomposition of water by metals. Some metals, such as potassium and sodium, decompose water at the ordinary temperature, whilst others, iron for instance, decompose it at a red heat:

$$
\begin{aligned}
\mathrm{K}+\mathrm{H}_{2} \mathrm{O} & =\mathrm{KHO}+\mathrm{H} \\
\mathrm{Fe}+\mathrm{H}_{2} \mathrm{O} & =\mathrm{FeO}+2 \mathrm{H} .
\end{aligned}
$$

A very convenient way of liberating hydrogen is the decomposition of dilute hydrochloric or sulphuric acid by zinc or iron :

$$
\begin{aligned}
& \mathrm{Zn}+2 \mathrm{HCl}=\underset{\substack{\mathrm{Zinc} \\
\text { chloride. }}}{\mathrm{ZnCl}_{2}}+2 \mathrm{H} ; \\
& \mathrm{Fe}+\mathrm{H}_{2} \mathrm{SO}_{4}=\underset{\substack{\text { FeSrous } \\
\text { sulphate. }}}{\mathrm{FeSO}_{4}}+2 \mathrm{H} .
\end{aligned}
$$

Whenever hydrogen is generated, care should be taken to expel all atmospheric air from the ressel in which the generation takes place, before the hydrogen is ignited, as otherwise an explosion may result.

Properties. Hydrogen is a colorless, inodorous, tasteless gas; it is the lightest of all known substances, having a specific gravity of 0.0692 as compared with atmospheric air $=1$.

In its chemical properties, hydrogen resernbles the metals more than the non-metals; it easily burns in atmospheric air, or in pure oxygen, with a non-luminous, colorless flame, producing during this process of combustion a higher temperature than can be obtained by the combustion of an equal weight of any other substance :

$$
\mathrm{H}_{2}+\mathrm{O}=\mathrm{H}_{2} \mathrm{O} \text {. }
$$

The formation of water by the combustion of hydrogen distinguishes it from other gases.

Two volumes of hydrogen combine with one volume of oxygen, forming two volumes of gaseous water.

Water, $\mathrm{H}_{2} \mathbf{O}=18$. Water is not found in nature in an absolutely pure state. The purest natural water is rain-water, collected after the air has been purified from dust, etc., by previous rain. Comparatively pure water may be obtained by melting ice, since, when water containing impurities is partially frozen, these are mostly left in the uncongealed water. 
The waters of springs, wells, rivers, etc., differ widely from each other; they all contain more or less of substances dissolved by the water in its course through the atmosphere or through the soil and rocks. The constituents thus absorbed by the water, are either solids or gases.

Solids generally found in natural waters, are common salt (sodiurn chloride), gypsum (calcium sulphate), and carbonate of lime (calcium carbonate); frequently found are chlorides and sulphates of potassium and magnesium, traces of silica and salts of iron. The gases absorbed by the water are chiefly constituents of the atmospheric air, oxygen, nitrogen, and carbon dioxide. One hundred volumes of water contain about two volumes of nitrogen, one volume of oxygen, and one volume of carbon dioxide.

Mineral waters are spring waters, containing one or more substances in such quantities that they impart to the water a peculiar taste and generally a decided medicinal action. According to the predominating constituents we distinguish bitter waters, containing larger quantities of magnesium salts; iron waters, containing carbonate of iron; hepatic waters, containing sulphuretted hydrogen; acidulous waters, containing larger quantities of carbonic acid, etc.

Drinking water. A good drinking water should neither be an absolutely pure water, nor a water containing too much of foreign matter. Water containing from 1 to 3 parts of total solids (chiefly carbonate of lime and common salt) in 10,000 parts of water and about 1 volume of carbon dioxide in 100 rolumes of water, may be said to be a good drinking water. There are, however, good drinking waters which contain more of total solids than the amount mentioned above.

Most objectionable in drinking water are organic substances, especially when derived from animal matter and in a state of desomposition. The presence of organic matter in water may be demonstrated by evaporating about one litre of it in a small porcelain or platinum dish over a steam bath. The residue left represents the total solids, and is generally of a white color. If this residue, upon being further heated over a flame, turns black (by separation of carbon), the presence of organic matter is indicated. 
Another method to prove the presence of organic matter is the addition of a solution of potassium permanganate. On heating $100 \mathrm{cc}$. of water, acidulated with $10 \mathrm{cc}$. of diluted sulphuric acid to the boiling-point, and adding enough of a dilute solution of potassium permanganate ( 1 in 1000) to impart to the liquid a decided rose-red tint, this tint should not be entirely destroyed by boiling for five minutes, as, otherwise, organic or other oxidizable matter is present.

Distilled water, Aqua destillata. The process for obtaining pure water is distillation in a suitable apparatus. From $\mathbf{1 0 0 0}$ parts of water used for distillation, the first 50 parts distilling over should not be used, as they contain the gaseous constituents. The solids contained in the water are left in the undistilled portion.

Properties of water. Water is a colorless, inodorous, tasteless liquid. It is perfectly neutral, yet it has a tendency to combine both with acid and basic substances. These compounds are usually called hydrates. Water is the most common solvent, both in nature and in artificial processes. As a general rule, solids are dissolved more quickly and in larger quantities by hot water than by cold, but to this there are many exceptions. For instance: Common salt is nearly as soluble in cold as in hot water; sulphate of sodium is most soluble in water of $33^{\circ}$, and some calcium salts are less soluble in hot than in cold water.

Many salts combine with water in crystallizing; crystallized sulphate of sodium, for instance, contains more than half its weight of water. This water is called water of crystallization, and is generally expelled at a temperature of $100^{\circ} \mathrm{C}$.

Hydrogen dioxide, $\mathrm{H}_{2} \mathrm{O}_{2}$. This compound may be obtained by the action of carbonic acid on barium dioxide suspended in water, when carbonate of barium and hydrogen dioxide are formed :

$$
\mathrm{BaO}_{2}+\mathrm{H}_{2} \mathrm{O}+\mathrm{CO}_{2}=\mathrm{BaCO}_{3}+\mathrm{H}_{2} \mathrm{O}_{2} \text {. }
$$

The liquid, separated by decantation from the insoluble carbonate, may be concentrated under the receiver of an air-pump and is, when thus obtained, a colorless liquid of a specific 
gravity 1.45, possessing remarkable bleaching properties. By higher temperatures, as well as by the action of many substances, it is readily decomposed into water and oxygen.

\section{Questions.}

101. Mention two processes by which hydrogen may be obtained.

102. Show by symbols the decomposition of water by potassium and sulphuric acid by iron.

103. State the chemical and physical properties of hydrogen.

104. How many pounds of zinc would be required to liberate 100 pounds of hydrogen?

105. State the composition of water in parts by weight and volume.

106. Mention the most common solid and gaseous constituents of natural waters.

107. How does a mineral water differ from other waters? Mention some different kinds of mineral waters and their chief constituents.

108. What substances are most objectionable in drinking water, and how can they be recognized?

109. What are the characteristics of a good drinking water?

110. What are the purest natural waters, and by what process may chemically pure water be obtained?

\section{NITROGEN.}

$$
\mathrm{N}_{\mathrm{iii}}=14 \text {. }
$$

0ccurrence in nature. By far the larger quantity of nitrogen is found in the atmosphere in a free state. Compounds containing nitrogen are chiefly the nitrates, ammonia, and many organic substances.

Preparation. Nitrogen is usually obtained from atmospheric air by the removal of oxygen. This may be accomplished by burning a piece of phosphorus in a confined portion of air, when phosphoric oxide, a white solid substance, is formed, whilst nitrogen is left in an almost pure state.

Properties. Nitrogen is a colorless, inodorous, tasteless gas. It is neither, like oxygen, a supporter of combustion, nor, like hydrogen, a combustible substance; in fact, nitrogen is distinguished by having very little affinity for any other element, 
and it scarcely enters directly into combination with any substance. Nitrogen is not poisonous, yet not being a supporter of combustion it cannot sustain animal life. Nitrogen is a trivalent element, combining with three atoms of hydrogen.

Atmospheric air is a mixture of about four-fifths of nitrogen and one-fifth of oxygen, with small quantities of aqueous vapor, carbon dioxide, and ammonia, containing frequently also traces of nitric acid and carburetted hydrogen, occasionally sulphuretted hydrogen and sulphur dioxide. Besides these gases there are frequently suspended in the air solid particles of dust and very minute cells of either animal or vegetable origin.

100 volumes of atmospheric air contain of

\begin{tabular}{|c|c|c|}
\hline Oxygen, & 20.61 & volumes. \\
\hline Nitrogen, & 77.95 & "6 \\
\hline Carbon dioxide, & 0.04 & 46 \\
\hline Aqueous vapor, & 1.40 & ، \\
\hline $\left.\begin{array}{l}\text { Ammonia, } \\
\text { Nitric aeid, }\end{array}\right\}$ & & \\
\hline
\end{tabular}

An analysis of air may be made by the following method: A graduated glass tube, containing a measured volume of air, is placed with the open end downward into a dish containing mercury. A small piece of phosphorus is then introduced and allowed to remain in contact with the air for several hours, when it gradually combines with the oxygen. The remaining volume of air is chiefly nitrogen, the loss in volume represents oxygen.

For the determination of carbon dioxide and water, a measured volume of air is passed through two U-shaped glass tubes. One of these tubes has previously been filled with pieces of calcium chloride, the other tube with pieces of potassium hydrate, and both tubes have been weighed separately. In passing the measured air through these tubes the first one will retain all the moisture, the second one all the carbon dioxide; the increase in weight of the tubes at the end of the operation will give the amounts of the two constituents.

That oxygen is found in the atmosphere in a free state is explained by the fact that all elements having affinity for oxygen hagve entered into combination with it, whilst the excess is left uncombined. Nitrogen is found uncombined, because it has so little affinity for other elements. 
Ammonia, $\mathbf{N H}_{3}=17$. This compound is constantly formed in nature by the decomposition of organic (chiefly animal) matter, such as meat, urine, blood, etc. It is also obtained during the process of destructive distillation, which is the heating of non-volatile organic substances in closed vessels. The manufacture of illuminating gas is such a process of destructive distillation; coal is heated in retorts, and the larger amount of nitrogen contained in the coal is converted and liberated as ammonia gas, which is condensed by passing the gases through water.

Another method of obtaining ammonia is the decomposition of ammonium salts by the oxides or hydrates of sodium, potassium, or calcium. Usually ammonium chloride is mixed with calcium hydrate and heated, when calcium chloride, water, and ammonia are formed:

$$
2\left(\mathrm{NH}_{4} \mathrm{Cl}\right)+\mathrm{Ca} 2 \mathrm{HO}=\mathrm{CaCl}_{2}+2 \mathrm{H}_{2} \mathrm{O}+2 \mathrm{NH}_{3} .
$$

Ammonia is a colorless gas, of a very pungent odor, an alkaline taste, and a strongly alkaline reaction. In pure oxygen it burns, forming water and free nitrogen.

By the mere application of a pressure of seven atmospheres or by intense cold $\left(-40^{\circ}\right)$, ammonia may be converted into a liquid, which at $-80^{\circ}$ forms a solid crystalline mass. Water dissolves about 700 times its volume of ammonia, forming ammonium hydrate:

$$
\mathrm{NH}_{3}+\mathrm{H}_{2} \mathrm{O}=\mathrm{NH}_{4} \mathrm{HO} .
$$

Water of ammonia, Aqua ammoniæ (Spirit of hartshorn). This is a solution of ammonia gas in water or hydrate of ammonium in water. The common water of ammonia contains 10 per cent. by weight of ammonia, and has a specific gravity of 0.959 ; the stronger water of ammonia contains 28 per cent., and has a specitic gravity of 0.900 . Ammonia water has the abovementioned odor, taste, and reaction like the gas itself.

Compounds of nitrogen and oxygen. Five distinct compounds of nitrogen and oxygen are known. They are named and constituted as follows: 
Nitrogen monoxide, $\mathrm{N}_{2} \mathrm{O}$,

$$
\text { " dioxide, } \mathrm{N}_{2} \mathrm{O}_{2}=2(\mathrm{NO}) \text {, }
$$

" trioxide, $\mathrm{N}_{2} \mathrm{O}_{3}$,

"tetroxide, $\mathrm{N}_{2} \mathrm{O}_{4}=2\left(\mathrm{NO}_{2}\right)$,

" pentoxide, $\mathrm{N}_{2} \mathrm{O}_{5}$,

\begin{tabular}{cccc}
\multicolumn{5}{c}{ Composition. } \\
By weight. & \multicolumn{2}{c}{ By volume. } \\
N & o & v & 0 \\
28 & 16 & 2 & 1 \\
28 & 32 & 2 & 2 \\
28 & 48 & 2 & 3 \\
28 & 64 & 2 & 4 \\
28 & 80 & 2 & 5
\end{tabular}

The first, third, and fifth of these compounds are capable of combining with water to form acids, known as hyponitrous, nitrous, and nitric acid, respectively.

Nitrogen monoxide, $\mathbf{N}_{2} \mathbf{O}=44$. (Sometimes called nitrous oxide; also, laughing gas). This compound may be easily obtained by heating ammonium nitrate in a flask at a temperature not exceeding $250^{\circ}$, when the salt is decomposed into nitrogen monoxide and water :

$$
\mathrm{NH}_{4} \mathrm{NO}_{3}=2 \mathrm{H}_{2} \mathrm{O}+\mathrm{N}_{2} \mathrm{O} \text {. }
$$

Nitrogen monoxide is a colorless, almost inodorous gas, of distinctly sweet taste. It supports combustion almost as energetically as oxygen, but differs from this element by its solubility in cold water, which absorbs nearly its own volume. The most characteristic feature of this gas is the intoxicating power it exerts upon the animal system. It is frequently used as an anæsthetic in dental surgery.

Nitrogen dioxide, N0 $=30$. This is a colorless gas which is generally formed when nitric acid acts upon metals or upon substances which deoxidize it. It is capable of combining directly with one more atom of oxygen, thereby forming $\mathrm{NO}_{2}$, nitrogen tetroxide, which is a gas of a deep red color. Nitrogen trioxide is of no practical interest.

Nitric acid, Acidum nitricum, $\mathrm{HNO}_{3}=63$ (Hydric nitrate). Nitrogen pentoxide is of scientific interest only. When brought in contact with water it readily combines with it, forming nitric acid :

$$
\mathrm{N}_{2} \mathrm{O}_{5}+\mathrm{H}_{2} \mathrm{O}=2 \mathrm{HNO}_{3}
$$


The usual method for obtaining nitric acid is the decomposition of sodium nitrate by sulphuric acid:

$$
\begin{aligned}
& \mathrm{NaNO}_{3}+\mathrm{H}_{2} \mathrm{SO}_{4}=\mathrm{HNO}_{3}+\mathrm{NaHSO}_{4} ; \\
& \text { Bisulphate } \\
& 2 \mathrm{NaNO}_{3}+\mathrm{H}_{2} \mathrm{SO}_{4}=2 \mathrm{HNO}_{3}+\underset{\substack{\text { Neutral sul- } \\
\mathrm{Na}_{2} \mathrm{SO}_{4}}}{\text { seutratef }}
\end{aligned}
$$

Or

The operation is accomplished by mixing the two substances in a retort and heating, when nitric acid distils over and is collected in a suitable receiver, whilst sulphate of sodium remains in the retort.

The acid thus obtained is an almost colorless, fuming, corrosive liquid, of a peculiar, somewhat suffocating odor, and a strongly acid reaction.

Common nitric acid of a specific gravity 1.42, is composed of 69.4 per cent. of $\mathrm{HNO}_{3}$ and 30.6 per cent. of water. The diluted nitric acid of the U. S. P. is made by mixing one part of the common acid with six parts of water.

Nitric acid is completely volatilized by heat: it stains animal matter distinctly yellow; it is a monobasic acid forming salts called nitrates. These salts are all soluble in water, for which reason nitric acid cannot be precipitated by any reagent. Nitric acid is a strong oxidizing agent; this means it is capable of giving off part of its oxygen to substances having aftinity for it.

\section{Tests for nitric acid or nitrates.}

1. Nitric acid when heated with copper filings, or nitrates when heated with copper filings and sulphuric acid, evolve red fumes of nitrogen tetroxide.

2. The solution of a nitrate, to which a few small pieces of ferrous sulphate have been added, will show a reddish-purple or black coloration upon pouring a few drops of strong sulphuric acid down the side of the test-tube, so that it may form a layer at the bottom of the tube.

3. Solution of indigo is decolorized by nitric acid.

As an antidote in cases of poisoning by nitric acid a solution of carbonate of sodium, or a mixture of magnesia and water, may be administered with the view of neutralizing the acid. 


\section{QuEsTIONS.}

111. State the physical and chemical properties of nitrogen.

112. Mention the principal constituents of atmospheric air and the quantity in which they are present.

113. By what processes can the four chief constituents of atmospheric air be determined?

114. Mention some decompositions by which ammonia is generated.

115. Explain the process of making water of ammonia.

116. State the physical and chemical properties of ammonia gas and ammonia water.

117. How is nitrogen monoxide obtained, and what are its properties?

118. Describe the process for making nitric acid, and give symbols for decomposition.

119. How does nitric acid act on animal matter, and what are its properties generally?

120. Give tests and antidote for nitric acid.

\section{CARBON.}

$$
\mathrm{C}^{\mathrm{iv}}=12 .
$$

0ccurrence in nature. Carbon is a constituent of all organic matter. In a pure state it is found crystallized as diamond and graphite, amorphous in a more or less pure condition in the various kinds of coal, charcoal, boneblack, lampblack, etc. As carbon dioxide carbon is found in the air, as carbonic acid in water, as carbonates (marble, limestone, etc.) in the solid portion of our earth.

Properties. The three different allotropic modifications of carbon differ widely from each other in their physical properties.

Diamond is the purest form of carbon; it crystallizes in regular octahedrons, cubes, or in some figures geometrically connected with these. Diamond is the hardest substance known; it is infusible, but burns when heated intensely, forming carbon dioxide.

Graphite, plumbago, or black-lead, is carbon crystallized in short six-sided prisms; it is a somewhat rare, dark gray mineral, chiefly used for lead-pencils.

Amorphous carbon is a soft, black, solid substance. 
Neither form of carbon is fusible, volatile, or soluble in any of the common solvents.

Carbon is a quadrivalent element; it has little affinity for metals, but combines with many of the non-metals, chiefly with oxygen, hydrogen, and nitrogen, forming the organic substances.

\section{Tests for carbon.}

1. Most non-volatile (organic) substances containing carbon, blacken when heated on platinum foil.

2. The product of combustion of carbon (or of combustible matter containing it), $\mathrm{CO}_{2}$, renders lime-water turbid.

Carbon dioxide, $\mathrm{CO}_{2}=44$. (Formerly named carbonic acid, or anhydrous carbonic acid.) This compound is always formed during the combustion of carbon or of organic matter; also during the decay (slow combustion), fermentation, and putrefaction (processes of decomposition) of organic matter; it is constantly produced in the animal system, exhaled from the lungs, and given off through the skin. Many spring waters contain considerable quantities of the gas, one single spring in Nauheim, Germany, liberating as much as 3000 pounds of carbon dioxide a day.

Many carbonates are decomposed by heating into oxides of the metals and carbon dioxide.

Lime-burning is such a process of decomposition :

$$
\underset{\substack{\text { Calcium } \\ \text { carbonate. }}}{\mathrm{CaCO}_{3}}=\underset{\substack{\text { Calcium } \\ \text { oxide }}}{\mathrm{CaO}}+\mathrm{CO}_{2}
$$

Another method for the generation of carbon dioxide is the decomposition of any carbonate by an acid:

$$
\underset{\substack{\text { Calcium } \\ \text { carbonate. }}}{\mathrm{CaCO}_{3}}+\underset{\substack{\text { Hydrochloric } \\ \text { acd. }}}{2 \mathrm{HCl}}=\underset{\substack{\text { Calceum } \\ \text { chloride. }}}{\mathrm{CaCl}_{2}}+\mathrm{H}_{2} \mathrm{O}+\mathrm{CO}_{2} .
$$

Carbou dioxide is a colorless, odorless gas, having a faint acid taste. By a pressure of 38 atmospheres, at a temperature of $0^{\circ} \mathrm{C}$., carbon dioxide is converted into a colorless liquid, which by intense cold $\left(-79^{\circ}\right)$ may be converted into a white, solid, crystalline, snow-like substance. The specific gravity of carbon dioxide is 1.524; it is consequently about one-half heavier than atmospheric air. 
Cold water absorbs at the ordinary pressure about its own volume of carbon dioxide, and much larger quantities under an increased pressure (soda water).

Carbon dioxide is not combustible, and not a supporter of combustion; on the contrary, it has a decided tendency to extinguish flames, air containing one-fourth of its volume of carbon dioxide being unable to support the combustion of a candle. Whilst not poisonous when taken into the stomach, carbon dioxide acts as a direct poison when inhaled.

Common atmospheric air contains about 4 volumes of carbon dioxide in 10,000 of air, or 0.04 per cent. In the process of respiration this air is inhaled, and a portion of the oxygen is absorbed in the lungs by the blood, which conveys it to the different portions of the animal body, and receives in exchange for the oxygen a quantity of carbon dioxide, produced by the union of a former supply of oxygen with the carbon of the different organs to which the blood is supplied.

The air issuing from the lungs contains this carbon dioxide, which amounts to about 4 volumes in 100 of exhaled air, which is 100 times more than contained in fresh air. It is not advisable to breathe for any length of time air containing more than 0.1 per cent. of carbon dioxide; in air containing 0.5 per cent. most persons are attacked by headache, still larger quantities produce insensibility, and air containing 8 per cent. of carbon dioxide causes death in a few minutes.

As exhaled air contains from 3.5 to 4 per cent. of carbon dioxide, it is entirely unfit to be breathed again. The total amount of carbon dioxide evolved by the lungs and skin of a grown person amounts to about 0.7 cubic foot per hour. Hence the necessity for a constant supply of fresh air by ventilation. This becomes the more necessary where an additional quantity of carbon dioxide is supplied by illuminating flames. A burning candle, for instance, produces nearly as much carbon dioxide as an adult man, and an ordinary gas flame as much as five persons.

Many processes by which carbon dioxide is constantly produced in nature have been mentioned above, and we might assume that the amount of 0.04 per cent. of carbon dioxide contained in the atmospheric air would gradually increase. This, however, is not the case, because the plants, and more especially 
all green parts of the plants, are capable of absorbing carbon dioxide from the air, whilst at the same time they liberate oxygen.

This process of vegetable respiration (if we may so call it), which takes place under the influence of sunlight, is, consequently, the reverse of that of animal respiration. The animal uses oxygen and liberates carbon dioxide; the plant consumes this carbon dioxide and liberates oxygen.

Carbon dioxide is an acid oxide, which combines with water, forming carbonic acid :

$$
\mathrm{CO}_{2}+\mathrm{H}_{2} \mathrm{O}=\mathrm{H}_{2} \mathrm{CO}_{3} \text {. }
$$

Carbonic acid, $\mathrm{H}_{2} \mathrm{CO}_{3}$, is not known in a pure state, but always diluted with much water, as in all the different natural waters. Carbonic acid is a bibasic, extremely weak acid, the salts of which are known as carbonates. Many of these carbonates (calcium carbonate, for instance) are abundantly found in nature.

Tests for carbon dioxide, carbonic acid, and carbonates.

1. Pass the gas through lime-water, which is rendered turbid by the formation of calcium carbonate:

$$
\mathrm{Ca} 2 \mathrm{HO}+\mathrm{CO}_{2}=\mathrm{CaCO}_{3}+\mathrm{H}_{2} \mathrm{O} .
$$

2. From carbonates, evolve the gas by the addition of some acid, and examine it by the same method.

3. The soluble carbonates of potassium and sodium give precipitates with the solutions of most metallic salts; for instance, with the chlorides of $\mathrm{Ba}, \mathrm{Ca}, \mathrm{Sr}, \mathrm{Mg}, \mathrm{F} e, \mathrm{Zn}, \mathrm{Cu}$, ete.

Carbon monoxide, Carbonic oxide, $\mathbf{C O}=28$. Carbon monoxide is a colorless, odorless, tasteless, neutral gas, almost insoluble in water; it burns with a pale-blue flame, forming carbon dioxide; it is very poisonous, when inhaled, by forming with the coloring matter of the blood a compound which prevents the absorption of oxygen. Carbon monoxide is formed when earbon dioxide is passed over red-hot coal :

$$
\mathrm{CO}_{2}+\mathrm{C}=2 \mathrm{CO} .
$$

The conditions necessary for the formation of carbon monoxide are, consequently, present in any stove or furnace where 
coal burns with an insufficient supply of air. The carbon dloxide formed in the lower parts of the furnace is decomposed by the coal above. The blue flames frequently playing over a coal fire are burning carbon monoxide. This gas is also formed by the decomposition of oxalic acid (and many other organic substances) by sulphuric acid:

$$
\underset{\substack{\text { Oxalic } \\ \text { acid. }}}{\mathrm{H}_{2} \mathrm{C}_{2} \mathrm{O}_{4}}+\underset{\substack{\text { Sulphuric } \\ \text { acdd }}}{\mathrm{H}_{2} \mathrm{SO}_{4}}=\mathrm{H}_{2} \mathrm{SO}_{4} \cdot \mathrm{H}_{2} \mathrm{O}+\mathrm{CO}_{2}+\mathrm{CO} .
$$

Compounds of carbon and hydrogen. There are no other two elements which are capable of forming so large a number of different combinations as carbon and hydrogen. Several hundred of these hydro-carbons are known, and their consideration belongs to the domain of organic chemistry.

Two of these hydro-carbons, however, may be briefly mentioned, as they are of importance in the consideration of common flames. These compounds are: methane (marsh-gas, firedamp), $\mathrm{CH}_{4}$; and ethene (olefiant gas), $\mathrm{C}_{2} \mathrm{H}_{4}$.

Both compounds are colorless, almost odorless gases, and both are products of the destructive distillation of organic substances. Destructive distillation is the heating of non-volatile organic substances in such a manner that the oxygen of the atmospheric air has no access, and to such an extent that the molecules of the organic matter are split up into simpler compounds. Among the gaseous products formed by this operation, more or less of the two hydro-carbons mentioned above is found.

Marsh-gas is also frequently formed by the decomposition of organic matter in the presence of moisture (leaves, etc., in swamps), and during the formation of coal in the interior of the earth; the gas there liberated giving rise to the explosions in coal mines.

Flame is a gas in the act of combustion. Of combustible gases, have been mentioned: hydrogen, carbon monoxide, marsh-gas, and olefiant gas. These four gases are actually those gases which are chiefly found in any of the common flames produced by the combustion of organic matter, such as paper, wood, oil, wax, or illuminating gas itself. 
These gases are generated by destructive distillation, the heat being either supplied by a separate process (manufacture of illuminating gas by heating wood or coal in retorts), or generated during the combustion itself.

In a burning candle, for instance, the fat is constantly decomposed by the heat of the flame itself, the generated gases burning continuously until all fat has been decomposed, and the products of decomposition have been burned up or converted into carbon dioxide and water.

An ordinary flame (Fig. 6) consists of three parts or cones. The inner or central portion is chiefly unburnt gas; the second is formed of partially burnt and burning gas; the outer cone, showing the highest temperature, but scarcely any light, is that part of the flame where complete combustion takes place.

The light of a flame is caused by solid particles of carbon heated to a white heat. The separation of carbon in the flame is explained by the fact that hydrogen has a greater affinity for oxygen than carbon; only a limited amount of oxygen can penetrate into the flame, and the hydrogen of the hydrocarbon will consume this oxygen, the carbon being liberated momentarily until it reaches the outer cone, where it finds sufticient oxygen with which to combine.

If a sufficient amount of air be previously mixed with the illuminating gas, as is done in the Bunsen FIG. 6.

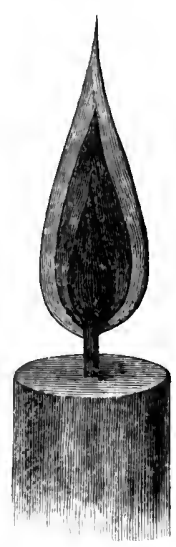

Structure of flame. burner, no separation of carbon takes place and no light, but a more intense heat is generated.

Silicium or Silicon, $\mathrm{Si}=\mathbf{2 8}$, and Boron, $\mathrm{B}=\mathbf{1 1}$, are elements which, in many respects, resemble carbon. Both, like carbon, are infusible, non-volatile, and insoluble in all common solvents. Like carbon, they are known in the amorphous state, and as crystals which resemble diamond or graphite. Silicium is, like carbon, quadrivalent; boron, however, is trivalent.

Silicium is found in nature very abundantly as silicium dioxide, or silica, $\mathrm{SiO}_{2}$ (rock-crystal, quartz, agate, sand), and in the form of silicates, which are silicic acid, $\mathrm{H}_{4} \mathrm{SiO}_{4}$, in which the hydrogen has been replaced by metals. Most of our common 
rocks, such as granite, porphyry, bașalt, feldspar, mica, etc., are such silicates, or a mixture of them. Small quartities of silica are found in spring waters, and also enter into vegetable and animal bodies.

Boron is found in but few localities, either as boric or boracic acid, $\mathrm{H}_{3} \mathrm{BO}_{3}$, or borate of sodium (borax). Formerly the total supply of boron was derived from Italy; lately large quantities of borax have been discovered in California.

Boracic acid is a white, solid substance, which is sparingly soluble in water, and has but weak acid properties.

\section{Tests for silicic and boracic acid or their salts.}

1. Silicic acid and most silicates are insoluble in water and acids. By fusion with a mixture of the carbonates of sodium and potassium, the silicate of these metals (known as solnble glass) is formed. By dissolving this salt in water, neutralizing the solution with hydrochloric acid, and supersaturating with ammonia, a portion of the silica separates as the gelatinous hydrate. Complete separation of the silica is accomplished by evaporation to dryness of the acidified solution.

2. Boracic acid and borates color the flame green, especially when moistened with sulphuric acid; their solution, when acidified by hydrochloric acid, turns turmeric paper brown (after being dried), and when neutral gives, with barium and silver salts, white precipitates of the borates of barium or silver.

\section{Questions.}

121. How is carbon found in nature?

122. State the physical and chemical properties of carbon in its three allotropic modifications.

123. Mention three different processes by which carbon dioxide is generated in nature, and some processes by which it is generated by artificial means.

124. State the physical and chemical properties of carbon dioxide.

125. Explain the process of respiration from a chemical point of view.

126. What is the percentage of carbon dioxide in the atmospheric air, and why does its amount not increase?

127. State the composition of carbonic acid and of a carbonate. How can they be recognized by analytical methods? 
128. Under what circumstances will carbon monoxide form, and how does it act when inhaled?

129. What is destructive distillation, and what gases are generally formed during that process?

130. Explain the structure and luminosity of flames.

\section{SULPHUR.$$
\mathrm{S}^{\mathrm{ii}}=32 \text {. }
$$

Occurrence in nature. Sulphur is found in the uncombined state in volcanic districts, the chief supply being derived from Sicily. In combination sulphur is widely diffused in the form of sulphates (gypsum, $\mathrm{CaSO}_{4} \cdot 2 \mathrm{H}_{2} \mathrm{O}$ ), and frequently as sulphides (iron pyrites, $\mathrm{FeS}_{2}$, galena, $\mathrm{PbS}$, cinnabar, $\mathrm{HgS}$, etc.). Sulphur also enters into organic compounds, during the decomposition of which sulphur is evolved as sulphuretted hydrogen, which gas is also a constituent of some waters.

Properties. Sulphur is a yellow, brittle, solid substance, having neither taste nor odor. It is insoluble in water and alcohol, soluble in carbon disulphide, oil of turpentine, and fat oils. Sulphur is polymorphous; it crystallizes, from a solution in bisulphide of carbon, in octahedrons with a rhombic base; when, however, liquefied by heat it crystallizes in six-sided prisms, and is obtained as a brown, amorphous substance by pouring melted sulphur into cold water.

Sulphur melts at $115^{\circ}$ to an amber-colored liquid, which is fluid as water; increasing the heat gradually, it becomes brown and thick, and at about $200^{\circ}$ it is so tenacious that it scarcely flows; when heated still further the sulphur again becomes thin and liquid, and, finally, boils at a temperature of about $400^{\circ}$.

In its chemical properties sulphur resembles oxygen, being like this element bivalent, and supporting, when in the form of vapor, the combustion of many substances, especially of metals. Many compounds of oxygen and sulphur show an analogous composition, as, for instance, $\mathrm{H}_{2} \mathrm{O}$ and $\mathrm{H}_{2} \mathrm{~S}, \mathrm{CO}_{2}$ and $\mathrm{CS}_{2}, \mathrm{CuO}$ and $\mathrm{CuS}$.

Crude sulphur is the sulphur obtained from the localities where it is found. It generally contains from 2 to 4 per cent. of 
earthy impurities. Melted sulphur poured into round moulds, is known as roll-sulphur.

Sublimed sulphur, Sulphur sublimatum (Flowers of sulphur). Obtained by heating sulphur in suitable vessels to the boilingpoint, and passing the vapor into large chambers, where it deposits in the form of a powder, composed of small crystals.

Washed sulphur, Sulphur lotum, is sublimed sulphur washed with a very dilute ammonia water, and then with pure water; the object of this treatment being to free the sulphur from all adhering sulphurous and sulphuric acid.

Precipitated sulphur, Sulphur præcipitatum (Milk of sulphur). Made by boiling hydrate of calcium with sulphur and water, filtering the solution, adding to it dilute hydrochloric acid until nearly neutral, washing and drying the precipitated sulphur.

By the action of sulphur on calcium hydrate are formed polysulphide of calcium, hyposulphite of calcium, and water :

$$
\underset{\substack{\text { Hydrate } \\
\text { of calcium. }}}{3(\mathrm{Ca} 2 \mathrm{HO}}+\underset{\text { Sulphur. }}{12 \mathrm{~S}}=\underset{\begin{array}{c}
\text { Polysulphide } \\
\text { of calcium. }
\end{array}}{2 \mathrm{CaS}_{5}}+\underset{\begin{array}{c}
\text { Hyposulphite } \\
\text { of calcium. }
\end{array}}{\mathrm{CaS}_{2} \mathrm{O}_{3}}+\underbrace{3 \mathrm{H}_{2} \mathrm{O} .}_{\text {Water. }}
$$

On adding hydrochloric acid to the solution, both substances are decomposed and sulphur is liberated:

$$
2 \mathrm{CaS}_{5}+\mathrm{CaS}_{2} \mathrm{O}_{3}+6 \mathrm{HCl}=3 \mathrm{CaCl}_{2}+3 \mathrm{H}_{2} \mathrm{O}+12 \mathrm{~S} \text {. }
$$

Precipitated sulphur differs from sublimed sulphur by being in a more finely divided state, and by having a much paler yellow, almost white color.

Sulphur dioxide, $\mathrm{SO}_{2}=64$. (Sulphurous anhydride, improperly also called sulphurous acid).

Two combinations of sulphur and oxygen are known; they are sulphur dioxide, $\mathrm{SO}_{2}$, and sulphur trioxide, $\mathrm{SO}_{3}$.

Sulphur dioxide is always formed when sulphur or substances containing it in a combustible form $\left(\mathrm{H}_{2} \mathrm{~S}, \mathrm{CS}_{2}\right.$, etc. $)$ burn in air. It is also formed by the action of strong sulphuric acid on many metals ( $\mathrm{Cu}, \mathrm{Hg}, \mathrm{Ag}$, etc.), or on charcoal:

$$
\begin{gathered}
\underset{\substack{\text { Sulphuric } \\
\text { acid. }}}{2 \mathrm{H}_{2} \mathrm{SO}_{4}}+\underset{\text { Copper. }}{\mathrm{Cu}}=\underset{\substack{\text { Cupric } \\
\text { sulphate. }}}{\mathrm{CuSO}_{4}}+\underset{\text { Water. }}{2 \mathrm{H}_{2} \mathrm{O}}+\underset{\substack{\text { Sulphur } \\
\text { dioxide. }}}{\mathrm{SO}_{2} .} \\
2 \mathrm{H}_{2} \mathrm{SO}_{4}+\mathrm{C}=\mathrm{CO}_{2}+2 \mathrm{H}_{2} \mathrm{O}+2 \mathrm{SO}_{2} .
\end{gathered}
$$


Sulphur dioxide is a colorless gas, having a suffocating, disagreeable odor; it liquefies at a temperature of $-10^{\circ}$, and solidifies at $-60^{\circ}$; it is very soluble in water, forming sulphurous acid; it is a strong deoxidizing, bleaching, and disinfecting agent; when inhaled in a pure state it is poisonous, when diluted with air it produces irritation of the air-passages and coughing.

Sulphurous acid, Acidum sulphurosum, $\mathrm{H}_{2} \mathrm{SO}_{3}=82$. Onc volume of cold water absorbs about 43 volumes of sulphur dioxide, or 100 parts by weight of water absorb 3.5 parts by weight of sulphur dioxide. To make the officinal acid, the gas is to be generated by heating charcoal and sulphuric acid in a flask, and passing the gas through a wash-bottle containing water, into distilled water for absorption.

Thus obtained, sulphurous acid is a colorless acid liquid, which has the odor as well as the disinfecting and bleaching properties of sulphur dioxide; it is completely volatilized by heat. Sulphurous acid is a bibasic acid, the salts of which are termed sulphites.

\section{Tests for sulphurous acid and sulphites.}

1. Sulphurous acid, or the gas liberated from sulphites, by the addition of sulphuric acid decolorizes an acidified solution of permanganate of potassium, in consequence of the deoxidation of the latter.

2. Similarly to the above, an acid solution of bichromate of potassium is turned green by conversion of chromic acid into chromic oxide.

3. When sulphurous acid or sulphites are added to diluted sulphuric acid and zinc (which evolve hydrogen), sulphuretted hydrogen gas is liberated:

$$
\mathrm{H}_{2} \mathrm{SO}_{3}+6 \mathrm{H}=\mathrm{H}_{2} \mathrm{~S}+3 \mathrm{H}_{2} \mathrm{O} .
$$

4. Chloride of barium added to solution of a sulphite produces a white precipitate of sulphite of barium, soluble in diluted hydrochloric acid; sulphate of barium is insoluble in hydrochloric acid:

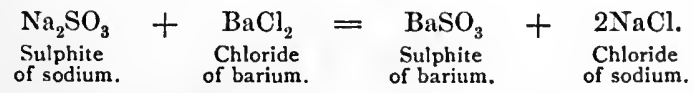


Sulphur trioxide, $\mathbf{S O}_{3}=\mathbf{8 0}$ (Anhydrous sulphuric acid). This is a white, silk-like, solid substance, having a powerful affinity for water; it may be obtained by the action of phosphoric oxide on strong sulphuric acid; it is merely of scientific interest.

Sulphuric acid, Acidum sulphuricum, $\mathrm{H}_{2} \mathrm{SO}_{4}=98$ (Oil of vitriol, Hydrogen sulphate). There is no other acid, and perhaps no other substance, manufactured by chemical action which is so largely used in chemical operations, and in the manufacture of so many of the most important articles, as sulphuric acid.

Sulphuric acid was accidentally discovered in the fifteenth century by Basile Valentine, a very laborious monk, who obtained it by heating ferrous sulphate (green vitriol) in a retort. To the liquid distilling over he gave the name oil of vitriol, in allusion to the thick or oily appearance, and the green vitriol from which it was obtained. The article of commerce called Nordhausen oil of vitriol, or fuming sulphuric acid, is yet made by this process of heating ferrous sulphate. The acid thus obtained is sulphuric acid, containing some sulphur trioxide $\left(\mathrm{H}_{2} \mathrm{SO}_{4} \cdot \mathrm{SO}_{3}\right)$.

Sulphuric acid is found in nature in combination with metals as sulphates. Thus calcium sulphate (gypsum), barium sulphate (heavy-spar), magnesium sulphate (Epsom salt), and others occur in nature.

Manufacture of sulphuric acid. Sulphuric acid is manufactured on a very large scale by passing into large leaden chambers simultaneously, the vapors of sulphur dioxide (obtained by burning sulphur or pyrites in furnaces), nitric acid, and steam, a supply of atmospheric air being also provided for. The oxygen of the nitric acid oxidizes the sulphur dioxide, which, at the same time, takes up water:

$$
\mathrm{SO}_{2}+\mathrm{O}+\mathrm{H}_{2} \mathrm{O}=\mathrm{H}_{2} \mathrm{SO}_{4} \text {. }
$$

Only a portion of the oxygen necessary for oxidation is derived from nitric acid directly; the larger quantity is obtained from the atmospheric air, the nitrogen dioxide serving as an agent for the transfer of the atmospheric oxygen.

By the action of nitric acid on sulphurous acid, are formed sulphuric acid, water, and nitrogen dioxide:

$$
3 \mathrm{H}_{2} \mathrm{SO}_{3}+2 \mathrm{HNO}_{3}=3 \mathrm{H}_{2} \mathrm{SO}_{4}+\mathrm{H}_{2} \mathrm{O}+2 \mathrm{NO} .
$$


Nitrogen dioxide is capable of readily absorbing oxygen from the atmospheric air, forming nitrogen tetroxide:

$$
\mathrm{NO}+\mathrm{O}=\mathrm{NO}_{2} \text {. }
$$

The nitrogen tetroxide again transfers one atom of its oxygen to the sulphurous acid, and so on indefinitely, as long as sulphurous acid and oxygen are present:

$$
\text { . } \mathrm{H}_{2} \mathrm{SO}_{3}+\mathrm{NO}_{2}=\mathrm{H}_{2} \mathrm{SO}_{4}+\mathrm{NO} \text {. }
$$

The liquid sulphuric acid thus formed in the lead-chamber, collects at the bottom of the chamber, whenee it is drawn off. In this state it is known as chamber acid (specific gravity 1.6), and is not pure, but contains an excess of water, and frequently either some sulphurous or nitric acid. By evaporationin shallow leaden pans it is further concentrated, until it shows a specific gravity of 1.72. When this point is reached the acid acts upon the lead, wherefore the further concentration is conducted in vessels of glass or platinum, until a specific gravity of 1.84 is obtained. This acid contains about 96 per cent. of sulphuric acid; the remaining 4 per cent. of water cannot be expelled by heat.

Properties of sulphuric acid. Pure acid has a specific gravity of 1.848; it is a colorless liquid, of oily consistence, boiling at $338^{\circ}$. It has a great tendency to combine with water, absorbing it readily from the atmospheric air. Upon mixing sulphuric acid and water, heat is generated in consequence of the combination taking place between the two substances. To the same tendency of sulphuric acid to combine with water, must be ascribed its property of destroying and blackening organic matter. Organic substances generally contain the elements carbon, hydrogen, and oxygen. Sulphuric acid added to such organic substances removes the elements hydrogen and oxygen (or at least a portion of them), combines them into water with which it unites, leaving behind compounds so rich in carbon that the black color predominates. It is due to this decomposing action of sulphuric acid upon organic matter that traces of the latter color sulphuric acid dark yellow, brown, and when present in larger quantities almost black. The poisonous, caustic properties are due to the same action. 
Sulphuric acid is a very strong, bibasic acid, which expels or displaces most other acids; its salts are known as sulphates.

The diluted sulphuric acid, Acidum sulphuricum dilutum, is a mixture of 1 part of acid and 9 parts of water.

\section{Tests for sulphuric acid and sulphates.}

1. Barium chloride produces a white precipitate of barium sulphate, insoluble in all acids:

$$
\mathrm{H}_{2} \mathrm{SO}_{4}+\mathrm{BaCl}_{2}=\mathrm{BaSO}_{4}+2 \mathrm{HCl} \text {. }
$$

2. Soluble lead salts (acetate of lead) produce a white precipitate of sulphate of lead.

Antidotes. Magnesia, carbonate of sodium, chalk, and soap, to neutralize the acid. Stomach pump must not be used.

Sulpho-acids. Whilst but two oxides of sulphur exist in the separate state, there are a large number of sulpho-acids known. They are:

$$
\begin{aligned}
& \text { Hydrosulphuric acid, } \mathrm{H}_{2} \mathrm{~S} \text {. } \\
& \mathrm{Hy} \text { drosulphurous acid, } \mathrm{H}_{2} \mathrm{SO}_{2} \text {. } \\
& \text { Sulphurous acid, } \mathrm{H}_{2} \mathrm{SO}_{3} \text {. } \\
& \text { Sulphuric acid, } \mathrm{H}_{2} \mathrm{SO}_{4} \text {. } \\
& \mathrm{H}_{y} \text { posulphurous acid, } \mathrm{H}_{2} \mathrm{~S}_{2} \mathrm{O}_{3} \text {. } \\
& \text { Hyposulphuric acid, } \mathrm{H}_{2} \mathrm{~S}_{2} \mathrm{O}_{6} \text {. } \\
& \text { Trithionic acid, } \mathrm{H}_{2} \mathrm{~S}_{3} \mathrm{O}_{6} \text {. } \\
& \text { Tetrathionic acid, } \mathrm{H}_{2} \mathrm{~S}_{4} \mathrm{O}_{7} \text {. } \\
& \text { Pentathionic acid, } \mathrm{H}_{2} \mathrm{~S}_{5} \mathrm{O}_{6} \text {. }
\end{aligned}
$$

The last four members of this series are known as polythionic acids; they are merely of scientific interest.

Hyposulphurous acid, $\mathrm{H}_{2} \mathrm{~S}_{2} \mathrm{O}_{3}$, is of interest in some of its salts, which are known as hyposulphites. The acid itself is not known in the separate state, because it decomposes into sulphur and sulphurous acid when attempts are made to liberate it from its salts.

Hydrosulphuric acid, $\mathrm{H}_{2} \mathbf{S}=34$ (Sulphuretted hydrogen, Hydrogen sulphide). This compound has been mentioned as being liberated by the decomposition of organic matter (putrefaction) 
and as a constituent of some spring waters. It is also formed during the destructive distillation of organic matter containing sulphur. The best mode of obtaining it is the decomposition of metallic sulphides by diluted sulphuric or hydrochloric acid. Ferrous sulphide is usually selected for decomposition :

$$
\mathrm{FeS}+\mathrm{H}_{2} \mathrm{SO}_{4}=\mathrm{FeSO}_{4}+\mathrm{H}_{2} \mathrm{~S} \text {. }
$$

Hydrosulphuric acid is a colorless gas, having an exceedingly offensive odor and a disgusting taste. Water absorbs about three volumes of the gas, and this solution is feebly acid. It is highly combustible in air, burning with a blue flame and forming sulphur dioxide and water. It is directly poisonous when inhaled, the sulphur entering into combination with the iron of the blood. Plenty of fresh air, or air containing a very little chlorine, should be used as antidotes.

Hydrosulphuric acid gas and its-solution in water are frequently used as reagents in analytical chemistry for precipitating and recognizing metals. This use depends on the property of the sulphur to combine with many metals to form insoluble compounds, the color of which is frequently very characteristic:

$$
\mathrm{CuSO}_{4}+\mathrm{H}_{2} \mathrm{~S}=\mathrm{CuS}+\mathrm{H}_{2} \mathrm{SO}_{4} .
$$

The salts of hydrosulphuric acid are known as sulphides.

\section{Tests for hydrosulphuric acid or sulphides.}

1. Hydrosulphuric acid or soluble sulphides when added to soluble salts of lead, copper, mercury, etc., give black precipitates of the sulphides of these metals.

2. From insoluble sulphides, liberate the gas by sulphuric or hydrochloric acid and test as above.

Bisulphide of carbon, Carbonei bisulphidum, $\mathrm{CS}_{2}=76$. This compound is obtained by passing vapors of sulphur over heated charcoal. It is a colorless, highly refractive, very volatile and inflammable, neutral liquid, having a characteristic odor and a sharp, aromatic taste. It boils at $46^{\circ}$; it is insoluble in water, soluble- in alcohol, ether, chloroform, fixed and volatile oils; for the two latter it is an excellent solvent. 


\section{Questions.}

131. How is sulphur found in nature?

132. Mention of sulphur : atomic weight, valence, color, odor, taste, solubility, behavior when heated, and allotropic modifications.

133. State the processes for obtaining sublimed, washed, and precipitated sulphur.

134. State composition and mode of preparing sulphur dioxide and sulphurous acid; what are they used for, and what are their properties?

135. Explain the process for the manufacture of sulphuric acid on a large scale.

136. Mention of sulphuric acid: color, specific gravity, its action on water and organic substances.

137. Give tests for sulphates and sulphites, sulphuric and sulphurous acid.

138. What is the difference between sulphates, sulphites, and sulphides?

139. How is hydrosulphuric acid formed in nature, and by what process is it obtained artificially? What are its properties, and what is it used for?

140. Mention antidotes in cases of poisoning by sulphuric and hydrosulphuric acid.

\section{PHOSPHORUS.$$
\mathbf{P}^{\mathrm{iii}}=31 \text {. }
$$

0ccurrence in nature. Phosphorus is found in nature chiefly in the form of phosphates of calcium (apatite, phosphorite), iron, and aluminium, and occurs diffused, though generally in small quantities, through all soils upon which plants will grow, as phosphorus is an essential constituent of the food of most plants. Through the plants it enters the animal system, where it is found either in organic compounds, or, - and this in, by far, the greater quantity,-as tricalcium phosphate principally in the bones, which contain about 60 per cent. of it. From the animal system it is chiefly eliminated by the urine.

Manufacture of phosphorus. Phosphorus was first made and discovered in 1669 by Brandt, of Hamburg, Germany, a bankrupt merchant, who obtained it in small quantities by distilling urine previously evaporated and mixed with sand. 
The manufacture of phosphorus to-day depends on the conversion of common tricalcium phosphate into acid phosphate of calciurn by the action of sulphuric acid:

$$
\underset{\substack{\text { Tricalcium } \\ \text { phosphate. }}}{\mathrm{Ca}_{3} 2 \mathrm{PO}_{4}}+\underset{\substack{\text { Sulphuric } \\ \text { acid. }}}{2 \mathrm{H}_{2} \mathrm{SO}_{4}}=\underset{\substack{\text { Acid phosphate } \\ \text { of calcium. }}}{\mathrm{CaH}_{4} 2 \mathrm{PO}_{4}}+\underset{\substack{\text { Calcium } \\ \text { sulphate. }}}{2 \mathrm{CaSO}_{4} .}
$$

The soluble acid phosphate of calcium is separated from the insoluble calcium sulphate, mixed with charcoal and sand, and evaporated to dryness; the mixture is then introduced into iron retorts and heated, when the decomposition takes place. By heating the aid phosphate of calcium, water is expelled and metaphosphate of calcium is formed:

$$
\mathrm{CaH}_{4} 2 \mathrm{PO}_{4}=\mathrm{Ca}_{2} \mathrm{PO}_{3}+2 \mathrm{H}_{2} \mathrm{O} .
$$

The action of charcoal and sand upon the metaphosphate of calcium at red heat, causes the formation of silicate of calcium and a deoxidation of the liberated phosphoric acid by the carbon:

$$
\cdot 2\left(\mathrm{Ca}_{2} \mathrm{PO}_{3}\right)+2 \mathrm{SiO}_{2}+10 \mathrm{C}=2 \mathrm{CaSiO}_{3}+10 \mathrm{CO}+4 \mathrm{P} .
$$

The phosphorus is liberated in the form of vapor, which is condensed by passing through water, carbonic oxide escaping at the same time.

Properties of phosphorus. When recently prepared, phosphorus is a colorless, translucent, solid substance, which has somewhat the appearance and consistency of bleached wax. In the course of time, and especially on exposure to light, it becomes by degrees less translucent, opaque, white, yellow, and finally yellowish-red. At the freezing-point phosphorus is brittle; as the temperature increases it becomes more and more soft, until it fuses at $44^{\circ}$ forming a yellowish fluid, which at $290^{\circ}$ (in the absence of oxygen) is converted into a colorless vapor.

The most characteristic features of phosphorus are its great affinity for oxygen, and its luminosity, visible in the dark, from which latter property its name, signifying bearer of light, has been derived. In consequence of its affinity for oxygen, phosphorus has to be kept under water, as it invariably takes fire when exposed to the air, the slow oxidation taking place 
upon the surface of the phosphorus soon raising it to $60^{\circ}$, at which temperature it ignites, burning with a bright white flame and giving off dense, white fumes of phosphoric oxide. The luminosity of phosphorus, due to this slow oxidation, is seen when a piece of it is exposed to the air, and whitish vapors are emitted which are luminous in the dark; at the same time an odor, resembling that of garlic, is noticed.

Phosphorus is insoluble in water, sparingly soluble in alcohol, ether, fatty and essential oils, very soluble in bisulphide of carbon, from which solution it separates in the form of crystals.

Phosphorus not only combines directly with oxygen, but also with chlorine, bromine, iodine, sulphur, and with many metals, the latter compounds being known as phosphides.

Phosphorus is trivalent in some compounds, as in $\mathrm{PCl}_{3}, \mathrm{P}_{2} \mathrm{O}_{3}$; quinquivalent in others, as in $\mathrm{PCl}_{5}, \mathrm{P}_{2} \mathrm{O}_{5}$.

The molecules of most elements contain two atoms; phosphorus is an exception to this rule, its molecule containing four atoms. The molecular weight of phosphorus is consequently $4 \times 31=124$.

Allotropic modifications. Several allotropic modifications of phosphorus are known, of which the red phosphorus (frequently called amorphous phosphorus) is the most important. This variety is obtained by exposing common phosphorus for about two days to a temperature of $240^{\circ}$ to $250^{\circ}$, in an atmosphere of carbon dioxide. Phosphorus is thereby gradually converted into a red powder, which differs widely from common phosphorus. It is not poisonous, not luminous, not soluble in the solvents above mentioned, not combustible before it has been heated to about $280^{\circ}$, when it is reconverted into common phosphorus.

Use of phosphorus. By far the largest quantity of all phosphorus (both common and red) is used for matches, which are made by dipping the wooden splints into some combustible substance, as melted sulphur or paraffin, and then into a paste, made by thoroughly mixing phosphorus with glue in which some oxidizing agent (potassium nitrate or chlorate) has been dissolved.

Pharmaceutical preparations containing phosphorus in the 
elementary state are phosphorated oil (oleum phosphoratum), and pills of phosphorus (pilulæ phosphori).

Phosphorus is also used for making phosphoric acid and other compounds.

Poisonous properties of phosphorus, and antidotes. Common phosphorus is extremely poisonous, two kinds of phosphoruspoisoning being distinguished. They are the acute form, consequent upon the ingestion of a poisonous dose, and the chronic form affecting the workmen employed in the manufacture of phosphorus or of lucifer matches.

There is no antidote to phosphorus which acts chemically. Oil of turpentine has been used successfully, though its action has not been sufficiently explained. Efforts should be made to eliminate the poison as rapidly as possible by means of a stomach-pump, emetics, or cathartics. Oil or fatty matter (milk) must not be given, as they act as solvents of the phosphorus, causing its more ready assimilation.

Detection of phosphorus in cases of poisoning. Use is made in detecting phosphorus (when in the elementary state) of its luminous properties. Organic matter (contents of stomach, food, etc.) containing phosphorus will often show this luminosity when agitated in the dark. If this process fails, in consequence of too small a quantity of the poison, a portion of the matter to be examined is rendered fluid by the addition of water, slightly acidulated with sulphuric acid, and placed in a flask, which is connected with a bent glass-tube leading to a Liebig's condenser. The apparatus is placed in the dark, and the flask is heated. If phosphorus be present, a luminous ring will be seen where the glass tube, leading from the flask, enters the condenser. The heat should be gradually raised to the boiling-point, the liquid kept boiling for some time, and the products of distillation collected in a glass vessel. Phosphorus volatilizes with the steam, and small globules of it may be found in the collected fluid. If, however, the quantity of phosphorus in the examined matter was very small, it may all have become oxidized during the distillation, and the fluid will then contain phosphorous acid, the tests for which will be stated below.

It should be mentioned that the luminosity of phosphorus 
vapors is diminished, or even prevented, by vapors of essential oils (oil of turpentine, for instance), ether, olefiant gas, and a few other substances.

0xides of phosphorus. Two oxides of phosphorus are known in the separate state. They are phosphorus trioxide or phosphorous oxide, $\mathrm{P}_{2} \mathrm{O}_{3}$, and phosphorus pentoxide or phosphoric oxide, $\mathrm{P}_{2} \mathrm{O}_{5}$. The first is obtained by slow oxidation of phosphorus, the second by burning phosphorus in dry air or oxygen. Both oxides are white solids, which combine readily with water, forming the corresponding acids.

Phosphorous acid, $\mathrm{H}_{3} \mathrm{PO}_{3}=\mathbf{8 2}$. This acid is obtained by dissolving phosphorous oxide in water:

$$
\mathrm{P}_{2} \mathrm{O}_{3}+3 \mathrm{H}_{2} \mathrm{O}=2 \mathrm{H}_{3} \mathrm{PO}_{3} .
$$

It is a colorless, acid liquid, which forms salts known as phosphites ; it is a strong deoxidizing agent, easily absorbing oxygen, forming phosphoric acid.

Tests for phosphorous acid.

1. Added to mercuric chloride, a white precipitate of mercurous chloride is formed.

2. Added to silver nitrate, a black precipitate of metallic silver is produced.

3. After being heated with nitric acid, it shows reactions of phosphoric acid.

Phosphoric acids. Phosphoric oxide is capable of combining chemically with one, two, or three molecules of water, forming thereby three different acids :

$$
\begin{array}{ll}
\mathrm{P}_{2} \mathrm{O}_{5}+\mathrm{H}_{2} \mathrm{O}=\mathrm{H}_{2} \mathrm{P}_{2} \mathrm{O}_{6}=2 \mathrm{HPO}_{3} \text { Metaphosphoric acid. } \\
\mathrm{P}_{2} \mathrm{O}_{5}+2 \mathrm{H}_{2} \mathrm{O}=\mathrm{H}_{4} \mathrm{P}_{2} \mathrm{O}_{7} & \text { Pyrophosphoric acid. } \\
\mathrm{P}_{2} \mathrm{O}_{5}+3 \mathrm{H}_{2} \mathrm{O}=\mathrm{H}_{6} \mathrm{P}_{2} \mathrm{O}_{8}=2 \mathrm{H}_{3} \mathrm{PO}_{4} \text { Orthophosphoric acid. }
\end{array}
$$

These three acids show different reactions, act differently upon the animal system, and form different salts.

Metaphosphoric acid, $\mathrm{HPO}_{3}=\mathbf{8 0}$ (Glacial phosphoric acid). This acid is always formed when phosphoric oxide is dissolved in water; gradually and more rapidly on heating with water it 
absorbs the latter, forming orthophosphoric acid; by evaporating the latter sufficiently, metaphosphoric acid is reformed.

Metaphosphoric acid is a monobasic acid which coagulates albumen (pyro- and ortho-phosphoric acids do not) and gives a white precipitate with ammonio-silver nitrate. It acts as a poison, whilst common phosphoric acid is comparatively harmless.

Pyrophosphoric acid, $\mathbf{H}_{4} \mathbf{P}_{2} \mathbf{0}_{7}=178$. This is a tetra-basic acid which gives a white precipitate with ammonio-silver nitrate, whilst orthophosphoric acid gives a yellow precipitate.

Phosphoric acid, Orthophosphoric acid, Acidum phosphoricum, $\mathbf{H}_{3} \mathbf{P O}_{4}=98$ (Trihydric phosphate, Common or tribasic phosphoric acid). Nearly all phosphates found in nature are orthophosphates.

Phosphoric acid may be made by burning phosphorus, dissolving the phosphoric oxide in water and boiling for a sufficient length of time to convert the meta- into ortho-phosphoric acid.

For medicinal purposes, phosphoric acid is made by gently heating pieces of phosphorus with diluted nitric acid, when the phosphorus is oxidized, red fumes of nitrogen tetroxide escaping. The liquid is evaporated until the excess of nitric acid has been expelled and enough of water added to obtain an acid which contains 50 per cent. of the pure acid, $\mathrm{H}_{3} \mathrm{PO}_{4}$.

Phosphoric acid is a colorless, odorless liquid, which, on heating, loses water, and is finally volatilized at a low red heat. It is a tribasic acid forming three series of salts, namely:

$\mathrm{Na}_{3} \mathrm{PO}_{4}=$ Trisodium phosphate.

$\mathrm{Na}_{2} \mathrm{HPO}_{4}=$ Disodium hydrogen phosphate.

$\mathrm{NaH}_{2} \mathrm{PO}_{4}=$ Dihydric sodium phosphate.

$\mathrm{H}_{3} \mathrm{PO}_{4}=$ Trihydric phosphate.

If the metal is bivalent, the formulas are thus :

$\mathrm{Ca}_{3} 2 \mathrm{PO}_{4}=$ Tricalcium phosphate.

$\mathrm{Ca}_{2} \mathrm{H}_{2} 2 \mathrm{PO}_{4}=$ Dicalcium orthophosphate.

$\mathrm{CaH}_{4} 2 \mathrm{PO}_{4}=$ Monocalcium orthophosphate.

\section{Tests for phosphoric acids and phosphates.}

1. Add to phosphoric acid, or to an aqueous solution of a phosphate, a mixture of magnesium sulphate, ammonium chloride, 
and ammonia water; a crystalline precipitate falls, which is dimagnesium ammonium phosphate:

$$
\begin{gathered}
\mathrm{H}_{3} \mathrm{PO}_{4}+\mathrm{MgSO}_{4}+3 \mathrm{NH}_{4} \mathrm{HO}=\mathrm{MgNH}_{4} \mathrm{PO}_{4}+\left(\mathrm{NH}_{4}\right)_{2} \mathrm{SO}_{4}+3 \mathrm{H}_{2} \mathrm{O} \\
\mathrm{Na}_{2} \mathrm{HPO}_{4}+\mathrm{MgSO}_{4}+\mathrm{NH}_{4} \mathrm{HO}=\mathrm{MgNH}_{4} \mathrm{PO}_{4}+\mathrm{Na}_{2} \mathrm{SO}_{4}+\mathrm{H}_{2} \mathrm{O} .
\end{gathered}
$$

2. Add to a neutral solution of a phosphate, silver nitrate: a yellow precipitate of phosphate of silver is produced, which is soluble both in ammonia and nitric acid:

$$
\mathrm{Na}_{3} \mathrm{PO}_{4}+3 \mathrm{AgNO} \mathrm{gg}_{3}=\mathrm{Ag}_{3} \mathrm{PO}_{4}+3 \mathrm{NaNO}_{3} .
$$

3. Add to phosphoric acid, or to a phosphate dissolved in nitric acid, an excess of a solution of ammonium molybdate in dilute nitric acid and apply heat: a yellow precipitate of phospho-molybdate of ammonium is produced. This test is by far the most delicate, and even traces of phosphoric acid may be recognized by it; moreover, it can be used in an acid solution, while the first two tests cannot.

4. Add to a neutral solution of a phosphate, calcium or barium chloride: a white precipitate of calcium or barium phosphate is produced, which is soluble in acids.

Hypophosphorous acid, $\mathrm{H}_{3} \mathrm{PO}_{2}$ or $\mathrm{HPH}_{2} \mathbf{O}_{2}$. This acid is of little interest in the free state, but many of its salts, named hypophosphites, are frequently used in medicine.

Although containing three atoms of hydrogen, hypophosphorous acid is a monobasic acid, only one of the hydrogen atoms being replaceable by metals.

\section{Tests for hypophosphites.}

1. Heated over a flame they burn with a phosphorescent light.

2. From solutions of mercuric chloride and silver nitrate they precipitate the metals in consequence of the deoxidizing action of hypophosphorous acid.

3. With zinc and diluted sulphuric acid they evolve hydrogen and phosphoretted hydrogen.

4. An acid solution of potassium permanganate is readily decolorized. 
Phosphoretted hydrogen. When phosphorus is heated with solutions of potassium or calcium hydrate, a number of products, chiefly hypophosphites, and phosphoretted hydrogen are formed. The latter compound has the composition $\mathrm{PH}_{3}$, and is a colorless, badly smelling, poisonous gas, which, when generated as directed above, is spontaneously inflammable. This lastnamed property is most likely due to the presence of small quantities of another compound of phosphorus and hydrogen which has the composition $\mathrm{P}_{4} \mathrm{H}_{2}$.

\section{Questions.}

141. In what forms of combination is phosphorus found in nature?

142. Give an outline of the process for manufacturing phosphorus.

143. What are the symbol, valence, atomic and molecular weight of phosphorus?

144. State chemical and physical properties both of common and red phosphorus.

145. By what methods may phosphorus be detected in cases of poisoning?

146. What two oxides of phosphorus are known; what is their composition, and what four acids do they form by combining with water?

147. State the officinal process for making phosphoric acid, and what are its properties?

148. By what tests may the three phosphoric acids be recognized and distinguished from phosphorous acid?

149. What is a phosphide, phosphite, phosphate, and hypophosphite?

150. What is glacial phosphoric acid, and in what respect does its action upon the animal system differ from the action of common phosphoric acid?

\section{CHLORINE.}

$$
\mathrm{Cl}^{1}=35.4 \text {. }
$$

Haloids or halogens. The four elements, fluorine, chlorine, bromine, and iodine, which form a natural group of elements, are known as haloids or halogens. The relation shown by the atomic weights of these four elements has been mentioned in connection with the consideration of natural groups of elements generally (see page 59). In many other respects a resemblance or relation can be discovered. For instance: All haloids are 
univalent elements, they combine with hydrogen, forming the acids $\mathrm{HFl}, \mathrm{HCl}, \mathrm{HBr}, \mathrm{HI}$; they combine directly with most metals, forming fluorides, chlorides, bromides, and iodides. The relative combining energy lessens as the atomic weight increases ; fluorine with the lowest atomic weight having the greatest, iodine with the highest atomic weight the smallest, affinity for other elements. The first two members of the group are gases, the third (bromine) is a liquid, the last (iodine) a solid at ordinary temperature. They all show a distinct color in the gaseous state, have a disagreeable odor, and possess disinfecting properties.

0ccurrence in nature. Chlorine is chiefly found as sodium chloride or common salt, $\mathrm{NaCl}$, either dissolved in water (small quantities in almost every spring water, larger quantities in some mineral waters, and the principal amount in sea-water), or as solid deposits in the interior of the earth.

Other chlorides, such as those of potassium, magnesium, calcium, are also found in nature. As common salt, chlorine enters the animal system, taking there an active part in many of the physiological and chemical changes.

Preparation of chlorine. Most methods of liberating chlorine depend on an oxidation of the hydrogen of hydrochloric acid by suitable oxidizing agents, the hydrogen being' converted into water, whilst chlorine is set free.

As oxidizing agents, may be used potassium chlorate, potassium bichromate, potassium permanganate, chromic acid, nitric acid, and many other substances.

The most common and cheapest mode of obtaining chlorine is to heat manganese dioxide, usually called black oxide of manganese, with hydrochloric acid, or a mixture of manganese dioxide and sodium chloride with sulphuric acid:

$$
\mathrm{MnO}_{2}+4 \mathrm{HCl}=\mathrm{MnCl}_{2}+2 \mathrm{H}_{2} \mathrm{O}+2 \mathrm{Cl} .
$$

Chlorine is also liberated by the action of acids on bleachingpowder, which is a mixture of calcium chloride and calcium hypochlorite :

$$
\mathrm{CaCl}_{2} \cdot \mathrm{Ca} 2 \mathrm{ClO}+2 \mathrm{H}_{2} \mathrm{SO}_{4}=2 \mathrm{CaSO}_{4}+2 \mathrm{H}_{2} \mathrm{O}+4 \mathrm{Cl} .
$$


Properties. Chlorine is a greenish-yellow gas, having a disagreeable taste and an extremely penetrating, suffocating odor, acting energetically upon the air-passages, producing violent coughing and inflammation. It is about two and a half times heavier than air, soluble in water, and convertible into a greenish-yellow liquid by a pressure of about four atmospheres.

Chemically, the properties of chlorine are well marked, and there are but few elements which have as strong an affinity for other elements as chlorine; it unites with all of them directly, except with oxygen, nitrogen, and carbon, but even with these it may be made to combine indirectly. The act of combination between chlorine and other elements is frequently attended by the evolution of so much heat that light is produced, or, in other words, combustion takes place. Thus hydrogen, phosphorus, and many metals burn easily in chlorine. The affinity between chlorine and hydrogen is very great, a mixture of the two gases being highly explosive. Such a mixture, kept in the dark, will not undergo chemical change, but when ignited, or when exposed to the direct sunlight, the combination occurs instantly with an explosion. The affinity of chlorine for hydrogen is also demonstrated by its property of decomposing water, ammonia, and many hydrocarbons (compounds of carbon with hydrogen), such as oil of turpentine, $\mathrm{C}_{10} \mathrm{H}_{16}$, and others :

$$
\begin{gathered}
\mathrm{H}_{2} \mathrm{O}+2 \mathrm{Cl}=2 \mathrm{HCl}+\mathrm{O} ; \\
\mathrm{NH}_{3}+3 \mathrm{Cl}=3 \mathrm{HCl}+\mathrm{N} \\
\mathrm{C}_{10} \mathrm{H}_{16}+16 \mathrm{Cl}=16 \mathrm{HCl}+10 \mathrm{C} .
\end{gathered}
$$

As shown by these formulas, hydrochloric acid is formed. whilst the other elements are set free.

Chlorine is a strong disinfecting, deodorizing, and bleaching agent; it acts as such either directly by combining with certain elements of the coloring or odoriferous matter, or, indirectly, by decomposing water with liberation of oxygen, which, in the nascent state (that is, at the moment of liberation), has a strong tendency to oxidize other substances.

Chlorine water, Aqua chlori, is water saturated with pure chlorine at a temperature of about $10^{\circ}$. One volume of water absorbs at that temperature about two volumes of chlorine, which is equal to 0.4 per cent. by weight. Chlorine water is a greenish-yellow 
liquid, having the odor and taste of chlorine. It must be kept in the dark, as otherwise decomposition takes place.

Hydrochloric acid, Acidum hydrochloricum, $\mathrm{HCl}=36.4$ (Chlorhydric acid, Muriatic acid, Hydrogen chloride). One volume of hydrogen combines with one volume of chlorine to form two volumes of hydrochloric acid.

Another method for obtaining it is the decomposition of chlorides by sulphuric acid:

Or

$$
\begin{aligned}
& \mathrm{NaCl}+\mathrm{H}_{2} \mathrm{SO}_{4}=\mathrm{HCl}+\mathrm{NaHSO}_{4} ; \\
& 2 \mathrm{NaCl}+\mathrm{H}_{2} \mathrm{SO}_{4}=2 \mathrm{HCl}+\mathrm{Na}_{2} \mathrm{SO}_{4} .
\end{aligned}
$$

Thus obtained, hydrochloric acid is a colorless gas, having a sharp, penetrating odor, and is very irritating when inhaled. It is not combustible, not a supporter of combustion, and has great affinity for water, which property is the cause of the formation of white clouds whenever the gas comes in contact with the vapors of water, or with moist air; the white clouds being formed of minute particles of the liquid hydrochloric acid.

Whilst hydrochloric acid is a gas, this name is also used for its solution in water, one volume of which at ordinary temperature takes up over 400 volumes of the gas.

The hydrochloric acid of the U. S. P. is the acid containing 31.9 per cent. of $\mathrm{HCl}$. It is a colorless, fuming liquid, having the odor of the gas, strong acid properties, and a specific gravity of 1.16. The officinal diluted hydrochloric acid is made by mixing 6 parts of the above acid with 13 parts of water.

The same antidotes may be used as for nitric acid.

\section{Tests for hydrochloric acid and chlorides.}

1. To hydrochloric acid, or to solution of chlorides, add silver nitrate; a white, curdy precipitate is produced, which is soluble in ammonium hydrate, but insoluble in nitric acid:

$$
\begin{aligned}
& \mathrm{AgNO}_{3}+\mathrm{NaCl}=\mathrm{NaNO}_{3}+\mathrm{AgCl} \\
& \mathrm{AgNO}_{3}+\mathrm{HCl}=\mathrm{HNO}_{3}+\mathrm{AgCl}
\end{aligned}
$$

2. Add solution of mercurous salt (mercurous nitrate): a 
white precipitate is produced, which blackens on the addition of ammonia :

$$
\mathrm{HgNO}{ }_{3}+\mathrm{NaCl}=\mathrm{NaNO}_{3}+\mathrm{HgCl} .
$$

3. Add solution of lead acetate: a white precipitate of lead chloride is formed, which is soluble in much water.

4. To a dry chloride add strong sulphuric acid and heat: vapors of hydrochloric acid gas are evolved, which may be recognized by the odor, or by their action on silver nitrate.

Aqua regia, Nitro-hydrochloric acid (Acidum nitro-hydrochloricum, Nitro-muriatic acid). Obtained by mixing 4 parts of nitric acid with 15 parts of hydrochloric acid.

The two acids act chemically upon each other, forming chloronitrous or chloronitric gas, chlorine and water:

$$
\begin{aligned}
& \mathrm{HNO}_{3}+3 \mathrm{HCl}=\mathrm{NOCl}+2 \mathrm{H}_{2} \mathrm{O}+2 \mathrm{Cl} ; \\
& \mathrm{HNO}_{3}+3 \mathrm{HCl}=\mathrm{NOCl}_{2}+2 \mathrm{H}_{2} \mathrm{O}+\mathrm{Cl} .
\end{aligned}
$$

The dissolving power of this acid upon gold and platinum depends on the action of the free chlorine and the action of the chloronitrous and chloronitric gas, both of which part easily with their chlorine.

Compounds of chlorine with oxygen. There is no method known to combine chlorine and oxygen directly, all the compounds formed by the union of these elements being obtained by indirect processes. The oxides of chlorine are the following:

Hypochlorous oxide, $\mathrm{Cl}_{2} \mathrm{O}$

Chlorous oxide, $\mathrm{Cl}_{2} \mathrm{O}_{3}$

$+\mathrm{H}_{2} \mathrm{O}=2 \mathrm{HClO}$.

$+\mathrm{H}_{2} \mathrm{O}=2 \mathrm{HClO}_{2}$.

Chlorous tetroxide, $\mathrm{Cl}_{2} \mathrm{O}_{4}$ does not combine with water to form an acid.

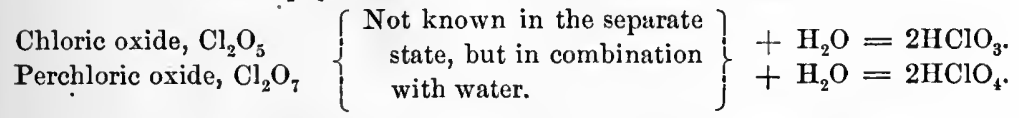

All these oxides, as well as the corresponding acids formed by their union with water, are distinguished by the great facility with which they decompose, frequently with violent explosion, for which reason many of their compounds are used in the manufacture of explosive mixtures. 


\section{Chlorine acids.}

$\begin{array}{ll}\text { Hydrochloric acid, } & \mathrm{HCl} . \\ \text { Hypochlorous acid, } & \mathrm{HClO} . \\ \text { Chlorous acid, } & \mathrm{HClO}_{2} \text {. } \\ \text { Chloric acid, } & \mathrm{HClO}_{3} \text {. } \\ \text { Perchloric acid, } & \mathrm{HClO}_{4} \text {. }\end{array}$

With the exception of hydrochloric acid, which has been considered, none of these five acids is of practical interest as such, but many of the salts of hypochlorous and chloric acid, known as hypochlorites and chlorates respectively, are of great and general importance.

Hypochlorous acid, HClO, may be obtained by the action of chlorine water on mercuric oxide, insoluble mercuric oxychloride being also formed:

$$
2 \mathrm{HgO}+4 \mathrm{Cl}+\mathrm{H}_{2} \mathrm{O}=\mathrm{Hg}_{2} \mathrm{OCl}_{2}+2 \mathrm{HClO} .
$$

Hypochlorous acid is a colorless, monobasic acid possessing strong bleaching properties.

Hypochlorites are formed by the action of chlorine on the hydrates of potassium, sodium, calcium, etc., at the ordinary temperature :

$$
2 \mathrm{NaHO}+2 \mathrm{Cl}=\mathrm{NaCl}+\mathrm{NaClO}+\mathrm{H}_{2} \mathrm{O} .
$$

Chloric acid, $\mathrm{HClO}_{3}$, may be obtained from potassium chlorate by the action of hydrofluosilicic acid; it is, however, an unstable substance which decomposes frequently with a violent explosion.

Chlorates are generally obtained by the action of chlorine on alkaline hydrates at a temperature of about $100^{\circ}$ :

$$
6 \mathrm{KHO}+6 \mathrm{Cl}=5 \mathrm{KCl}+\mathrm{KClO}_{3}+3 \mathrm{H}_{2} \mathrm{O} .
$$

Mixtures of hypochlorites and chlorides are converted into chlorates by boiling their solution :

$$
3 \mathrm{KCl}+3 \mathrm{KClO}=5 \mathrm{KCl}+\mathrm{KClO}_{3} .
$$

\section{Tests for chlorates and hypochlorites.}

1. Chlorates liberate oxygen when heated by themselves.

2. Chlorates liberate chlorous tetroxide, $\mathrm{Cl}_{2} \mathrm{O}_{4}$, a deep yellow explosive gas, on the addition of strong sulphuric acid. 
3. Chlorates deflagrate when sprinkled on red-hot charcoal.

4. Hypochlorites evolve a peculiarly smelling gas (hypochlorous acid) on the addition of acids, and are strong bleaching agents.

\section{Questions.}

151. State the names, and general physical and chemical properties of the four halogens.

152. How is chlorine found in nature, and why does it not occur in a free state?

153. State the general principie for liberating chlorine from hydrochloric acid, and explain the action of the latter on manganese dioxide.

154. Mention of chlorine: its atomic weight, molecular weight, valence, color, odor, action when inhaled, and solubility in water.

155. How does chlorine act chemically upon metals, hydrogen, phosphorus, water, ammonia, hydrocarbons, and coloring matters?

156. Mention two processes for making hydrochloric acid; state its composition, properties, and tests by which it may be recognized.

157. What is aqua regia?

158. State the composition of hypochlorous and chloric acid.

159. What is the difference in the action of chlorine upon a solution of potassium hydrate at ordinary temperature or at the boiling-point?

160. How many pounds of manganese dioxide, and how many of hydrochloric acid gas are required to liberate 142 pounds of chlorine?

\section{BROMINE-IODINE-FLUORINE.}

Bromine, Bromum, $\mathbf{B r}=80$. This element is found in seawater and many mineral waters, chiefly as magnesium bromide, which compound, however, represents in all these waters a comparatively small percentage of the total quantity of the different salts present. Most of these salts are separated from the water by evaporation and crystallization, and the remaining motherliquor, containing the magnesium bromide, is treated with chlorine which liberates bromine, the vapors of which are condensed in cooled receivers:

$$
\mathrm{MgBr}_{2}+2 \mathrm{Cl}=\mathrm{MrgCl}_{2}+2 \mathrm{Br} \text {. }
$$

Bromine is at common temperature a dark, reddish brown liquid, giving off brown fumes of an exceedingly suffocating and irritating odor; it is very volatile, and freezes at about 
$-24^{\circ}$; it is but sparingly soluble in water, more freely in alcohol, abundantly in ether and bisulphide of carbon; it is a strong disinfectant, and its aqueous solution is also a bleaching agent.

Hydrobromic acid, Acidum hydrobromicum, $\mathrm{HBr}=\mathbf{8 1}$, may be obtained by the formation of bromide of phosphorus, $\mathrm{PBr}_{5}$ (the two elements combine directly), and its decomposition by water:

$$
\mathrm{PBr}_{5}+4 \mathrm{H}_{2} \mathrm{O}=5 \mathrm{HBr}+\mathrm{H}_{3} \mathrm{PO}_{4} .
$$

Hydrobromic acid is, like hydrochloric acid, a colorless gas, of strong acid properties, easily soluble in water. A 10 per cent. solution is the officinal acid.

Hypobromic acid, $\mathrm{HBrO}$, Bromic acid, $\mathrm{HBrO}_{3}$, and their salts, the hypolromites and bromates, are analogous to the corresponding chlorine compounds.

\section{Tests for bromides.}

1. Silver nitrate produces in solutions of bromides a slightly yellowish-white precipitate of silver bromide, insoluble in nitric acid, sparingly soluble in ammonium hydrate.

2. Addition of chlorine water liberates bromine, which may be dissolved by shaking with chloroform or ether.

3. Mucilage of starch added to the liberated bromine is colored yellow.

4. Strong sulphuric acid added to a dry bromide liberates yellowish-red vapors of bromine.

Iodine, Iodum, $I=12 \%$. Iodine is found in nature in combination with sodium and potassium, in some spring waters and in sea-water, from which latter it is taken up by sea-plants and many aquatic animals. Iodine is chiefly derived from the vitrified ashes of sea-weeds, known as kelp. By washing this kelp with water, the soluble constituents are dissolved the larger quantities of sodium chloride, sodium and potassium carbonate are removed by evaporation and crystallization, and from the remaining mother-liquor iodine is obtained by treating the liquor with manganese dioxide and hydrochloric (or 
sulphuric) acid. The liberated iodine distils, and is collected in cooled receivers.

Iodine is a heavy, bluish-black, crystalline substance of a somewhat metallic lustre, a distinctive odor, a sharp and acrid taste, and a neutral reaction. It fuses at $114^{\circ}$, and boils at $180^{\circ}$, being converted into beautiful purple vapors, but it is also volatilized at ordinary temperature in small quantities. It is sparingly soluble in water, more soluble in water containing certain salts, for instance potassium iodide; it is solnble in 11 parts of alcohol (tincture of iodine), very soluble in ether, bisulphide of carbon, and chloroform. The solution of iodine in alcohol or ether has a brown, the solution in bisulphicle of carbon or in chloroform a violet color. Iodine stains the skin brown, and when taken internally acts as an irritant poison.

Hydriodic acid, Hydrogen iodide, HI. This is a colorless gas, readily soluble in water; the solution is unstable, being easily decomposed with liberation of iodine.

Whilst not of much importance itself, many of its salts, the iodides, are of great interest.

\section{Tests for iodine and iodides.}

1. Add to free iodine (or to an iodide, after it has been decomposed by a few drops of chlorine water) mucilage of starch: a dark blue color is produced, due to the formation of "blue iodized starch."

2. Liberate from solution of an iodide the iodine by chlorine water, and shake the solution with bisulphide of carbon or chloroform. After standing a few minutes the liquids form a layer of a beautiful violet color.

3. Add to solution of an iodide, solution of silver nitrate: a pale yellow precipitate of silver iodide, AgI, falls, which is insoluble in nitric acid, and sparingly soluble in dilute ammonium hydrate.

4. Add lead acetate to a neutral solution of an iodide: a yellow precipitate of lead iodide, $\mathrm{PbI}_{2}$, is produced.

5. Add mercuric chloride to a neutral solution of an iodide: a red precipitate of mercuric iodide, $\mathrm{HgI}_{2}$, is produced. 
Fluorine, $\mathbf{F l}=19$. This element is found in nature, chiefly as fluorspar, calcium fluoride, $\mathrm{CaFl}_{2}$. Fluorine itself is scarcely known in the elementary state, because it is very difficult to obtain any material (from which a vessel might be made) which is not chemically acted upon by fluorine. All metals, glass, organic substances, etc., which have been used as vessels for liberating fluorine were destroyed before satisfactory results were obtained. Most likely, fluorine is a pale green gas, of highly suffocating and poisonous properties.

Hydrofluoric acid, HFl (Hydrogen fuoride). A colorless gas, very irritating, soluble in water. It is obtained by the action of sulphuric acid on fluorspar:

$$
\mathrm{CaFl}_{2}+\mathrm{H}_{2} \mathrm{SO}_{4}=2 \mathrm{HFl}+\mathrm{CaSO}_{4} .
$$

Hydrofluoric acid, either in the gaseous state or its solution in water, is used for etching on glass. This property is due to the affinity which fluorine has for the constituents of glass, and which are dissolved by it.

\section{Questions.}

161. How is bromine found in nature?

162. State the physical and chemical properties of bromine.

163. What is hydrobromic acid, and how can it be made?

164. By what tests may bromine and bromides be recognized?

165. What is the chief source of iodine?

166. What are the chemical and physical properties of iodine?

167. What is tincture of iodine, what is its color, and how does it stain the skin?

168. Mention reactions by which iodine and iodides may be recognized.

169. By what element may bromine and iodine be liberated from their compounds?

170. How is hydrofluoric acid made, and what is it used for? 


\section{METALS AND THEIR COMBINATIONS.}

\section{GENERAL REMARKS REGARDING METALS.}

OF the total number of fifty-three metallic elements, only twenty-six are of sufficient general interest and importance to deserve consideration in this book.

\section{Derivation of names, symbols, and atomic weights.}

Aluminium, $\quad \mathrm{Al}=27 . \quad$ From alum, a salt containing it.

Antimony, $\quad \mathrm{Sb}=120$. From the Greek avti (anti), against, and moine, a (Stibium) French word for monk, from the fact that some monks were poisoned by compounds of antimony. Stibium, from the Greek ori $\beta$ (stibi), the name for the native sulphide of antimony.

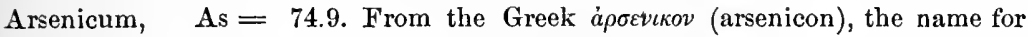
the native sulphide of arsenic.

Barium, $\quad \mathrm{Ba}=136.8$. From the Greek $\beta a \rho v s$ (barys), heavy, in allusion to the high specific gravity of barium sulphate, or heavy-spar.

Bismuth. $\quad \mathrm{Bi}=210$. From the German wismuth, an expression used Iong ago by the miners in allusion to the variegated tints of the metal when freshly broken.

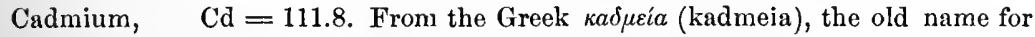
calamine (zinc carbonate), with which cadmium is frequently associated.

Calcium, $\quad \mathrm{Ca}=40 . \quad$ From the Latin calx, lime, the oxide of calcium.

Chromium, $\quad \mathrm{Cr}=52.4$. From the Greek $\chi \rho \tilde{\omega} \mu \alpha$ (chroma), color, in allusion to the beautiful colors of all its compounds.

Cobalt, $\quad$ Co $=$ 58.9. From the German Kobold, which means a demon, inhabiting the mines.

Copper, $\quad \mathrm{Cu}=63.2$. From the Latin cuprum, copper, and this from the Island Cyprus, where copper first was obtained by the ancients.

Gold, $\quad A u=196.2$. Gold means bright yellow in several old languages. 
Lead, $\quad \mathrm{Pb}=206.5$. Both words signify something heavy.

(Plumbum)

Lithium, $\quad \mathrm{Li}=7$. From the Greek $\lambda i \theta \varepsilon t o s$ (litheios), stony.

Magnesium, $M g=24$. From Magnesia, a town in Asia Minor, where magnesium carbonate was found as a mineral.

Manganese, $\mathrm{Mn}=54$. Probably from magnesium, with the compounds of which it was long confounded,

Mercury, $\quad \mathrm{Hg}=199.7$. From Mercury, the messenger of the Greek gods. (Hydrargyrum) Hydrargyrum means liquid silver.

Molybdenum, $\mathrm{Mo}_{\mathrm{o}}=$ 95.5. From the Greek $\mu 0 \lambda i \beta \delta$ s (molybdos), lead.

Nickel, $\quad \mathrm{Ni}=58$. From the old German word nickel, which means worthless.

Platinum, $\quad \mathrm{Pt}=$ 194.4. Platina is the diminutive of the Spanish word plata, silver.

Potassium, $\quad \mathrm{K}=9.3$ From pot-ash; potassium carbonate being the chief (Kalium) constituent of the lye of wood-ashes. Kali is the Arabic word for ashes.

Silver,

(Argentum)

Sodium,

$\mathrm{Na}=23$

(Natrium)

$\mathrm{Ag}=107.7$. Both words signify white.

Strontium,

old name for natural deposits of sodium carbonate.

$\mathrm{St}=$ 87.4. From Strontian, a village in Scotland, where strontium carbonate is found.

Tin,

(Stannum)

Zinc,

$\mathrm{Sn}=117.7$. Both words most likely signify stone.

$\mathrm{Zn}=64.9$. Most likely from the German zinn or tin, both metals 'having been confounded with each other.

\section{Melting-points of metals.}

\begin{tabular}{|c|c|c|c|}
\hline & & c. & F. \\
\hline Fusible below the & Mercury, & $-40^{\circ}$ & $-39^{\circ}$ \\
\hline boiling-point of & Potassium, & +62.5 & +144.5 \\
\hline water & Sodium, & 97 & 207.7 \\
\hline & Lithium, & 180 & 356 \\
\hline & Tin, & 228 & 443 \\
\hline & Bismuth, & 260 & 500 \\
\hline Fusible below & Cadmium, & 300 & 572 \\
\hline red heat & Lead, & 325 & 617 \\
\hline & Zinc, & 412 & 773 \\
\hline & Aluminium, & $425 ?$ & 796 \\
\hline & Antimony, & $450 ?$ & 842 \\
\hline Unknown & $\begin{array}{l}\text { Barium. } \\
\text { Calcium. } \\
\text { Strontium. } \\
\text { Arsenic. } \\
\text { Magnesium_red heat. }\end{array}$ & & \\
\hline
\end{tabular}




\begin{tabular}{|c|c|c|c|}
\hline & & c. & F. \\
\hline & Silver, & $1020^{\circ}$ & $1873^{\circ}$ \\
\hline & Copper, & 1100 & 2012 \\
\hline & Gold, & 1200 & 2192 \\
\hline & Cast-iron, & 1800 & 3272 \\
\hline $\begin{array}{l}\text { Infusible below a } \\
\text { red heat }\end{array}$ & $\begin{array}{l}\text { Pure iron, } \\
\text { Nickel, } \\
\text { Cobalt, } \\
\text { Manganese, }\end{array}$ & Highest heat of $f$ & \\
\hline & $\begin{array}{l}\text { Molybdenum, } \\
\text { Chromium, } \\
\text { Platinum, }\end{array}$ & $\begin{array}{l}\text { Agglomerate, but } \\
\text { Fusible in the ox }\end{array}$ & $\begin{array}{l}\text { ot melt in forge. } \\
\text { rogen blowpipe. }\end{array}$ \\
\hline
\end{tabular}

\section{Specific gravities of metals at $15.5^{\circ} \mathrm{C}$.}

$\begin{array}{lllr}\text { Lithium, } & 0.593 & \text { Manganese, } & 8.00 \\ \text { Potassium, } & 0.865 & \text { Cobalt, } & 8.54 \\ \text { Sodium, } & 0.972 & \text { Molybdenum, } & 8.63 \\ \text { Calcium, } & 1.57 & \text { Cadmium, } & 8.70 \\ \text { Magnesium, } & 1.75 & \text { Nickel, } & 8.80 \\ \text { Strontium, } & 2.54 & \text { Copper, } & 8.96 \\ \text { Aluminium, } & 2.60 & \text { Bismuth, } & 9.90 \\ \text { Barium, } & 4.00 & \text { Silver, } & 10.50 \\ \text { Arsenic, } & 5.88 & \text { Lead, } & 11.45 \\ \text { Antimony, } & 6.80 & \text { Mercury, } & 13.59 \\ \text { Zinc, } & 6.90 & \text { Gold, } & 19.50 \\ \text { Tin, } & 7.29 & \text { Platinum, } & 21.50 \\ \text { Iron, } & 7.79 & & \end{array}$

\section{Time of discovery of the metals.}

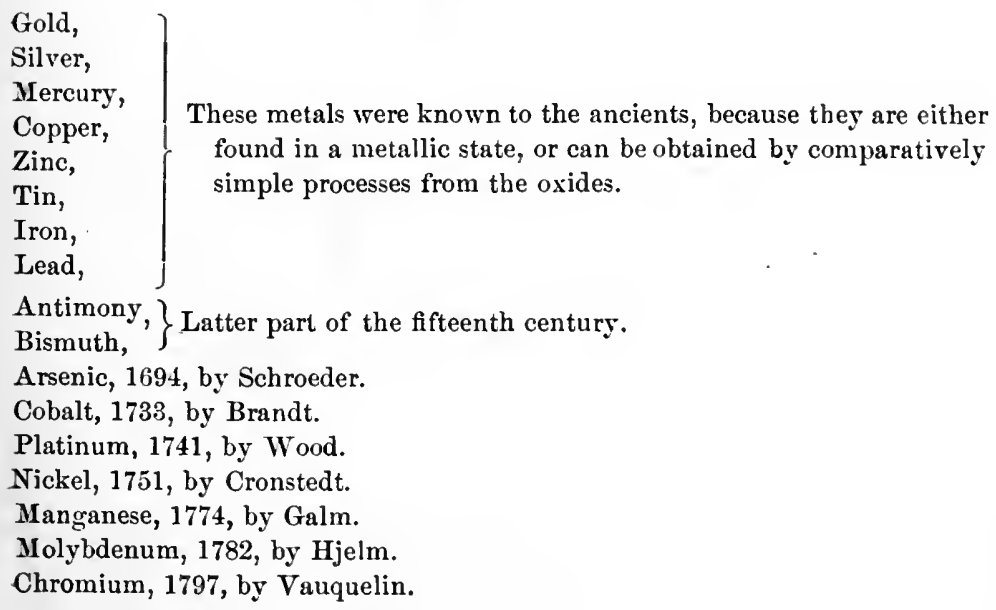


$\left.\left.\begin{array}{l}\begin{array}{l}\text { Potassium, } \\ \text { Sodium, } \\ \text { Barium, } \\ \text { Calcium, } \\ \text { Strontium, } \\ \text { Magnesium, }\end{array} \quad\{1807-1808\end{array}\right\} \begin{array}{c}\text { H. Davy discovered methods for the separation } \\ \text { of these metals from their oxides. }\end{array}\right\}$

Cadmium, 1817, by Stromeyer.

Lithium, 1817, by Arfvedson.

Aluminium, 1828, by Wöhler.

\section{Valence of metals.}

Univalent.

Lithium,

Potassium,

Sodium,

Silver.

Bivalent.

Barium,

Calcium,

Strontium,

Magnesium,

Cadmium,

Zine,

Copper,

Mercury.

Trivalent.

Bismuth,

Gold.
Bi- and trivalent.
Aluminium,
Chromium,
Cobalt,
Iron,
Manganese,
Nickel.

$B i$ - and quadrivalent.

Lead,

Platinum,

Tin.

Tri- and quinquivalent.

Antimony,

Arsenic.

Sixvalent.

Molybdenum.

\section{Occurrence in nature.}

a. In a free or combined state.

Gold,
Platinum, Almost exclusively in the metallic state.

$\left.\begin{array}{l}\text { Silver, } \\ \text { Mercury, }\end{array}\right\}$ As metals or sulphides.

Bismuth, generally metallic, also as oxide and sulphide.

Copper, rarely metallic; chiefly as sulphide, oxide, and carbonate. 


\section{b. In combination only.}

$\left.\begin{array}{l}\text { Potassium, } \\ \text { Sodium, } \\ \text { Lithium, }\end{array}\right\}$ Chiefly as chlorides or silicates.

Barium, as sulphate.

Calcium,

Strontium, As carbonates, sulphates, silicates.

Magnesium, j

Aluminium, in silicates.

Iron,

Zinc, As oxides, carbonates, sulphides

Cadmium,

Arsenic,

Antimony,

Lead,

Cobalt,

Nickel,

Molybdenum,

Chromium,

Manganese,
Tin,

\section{Classification of metals.}

\section{Light metals.}

Sp. gr. from 0.6 to 4 .

Sulphides soluble in water.
Heavy metals.

Sp. gr. from 6 to 21.5 .

Sulphides insoluble in water.

\section{Light metals.}

Earth metals.

$\mathrm{Al}$, and many rare metals.

Oxides insoluble.
Alkaline earth metals. $\mathrm{Ba}, \mathrm{Ca}, \mathrm{Sr},(\mathbf{M g})$. Oxides soluble;

Carbonates insoluble.
Alkali-metals. $\mathrm{K}, \mathrm{Na}, \mathrm{Li},\left(\mathrm{NH}_{4}\right)$. Oxides, carbonates, and most salts soluble.

\section{Heavy metals.}

Arsenic group.

Lead group.

Iron group.

$\mathrm{As}, \mathrm{Sb}, \mathrm{Sn}, \mathrm{Au}, \mathrm{Pt}, \mathrm{Mo}$. $\mathrm{Pb}, \mathrm{Cu}, \mathrm{Bi}, \mathrm{Ag}, \mathrm{Hg}, \mathrm{Cd}$,

Sulphidès insoluble in dilute aciḑs.

$\mathrm{Fe}, \mathrm{Co}, \mathrm{Ni}, \mathrm{Mn}, \mathrm{Zn}, \mathrm{Cr}$.

Sulphides soluble in dilute acids.

Sulphides soluble in am- Sulphides insoluble in monium sulphide. ammonium sulphide.

Properties of metals. All metals have a peculiar lustre known as metallic lustre, and all are good conductors of heat and electricity. The color of most metals is white, grayish- or 
bluish-white, or dark gray; a few metals show a distinct color, as, for instance, gold and calcium (yellow), copper (red).

At ordinary temperatures metals are solids with the exception of mercury, all are fusible, and some are so volatile that they may be distilled. Most, probably all, metals may be obtained in a crystallized condition.

The combinations of metals among themselves are called alloys, or, when mercury is one of the constituents, amalgams. These combinations, which may usually be obtained by fusing the metals together, must be looked upon as molecular mixtures, not as definite chemical compounds. All alloys still exhibit the metallic nature in their general physical characters. It is different, however, when metals combine with non-metals; in this case the metallic characters are almost invariably lost.

All metals combine with chlorine, fluorine, and oxygen; most metals also with sulphur, bromine, and iodine, forming the respective chlorides, fluorides, oxides, sulphides, bromides, and iodides.

Most metals may be obtained from their oxides by heating the same with charcoal, the carbon combining with the oxygen of the oxide, whilst the metal is liberated :

Or

$$
\begin{array}{r}
\mathrm{MO}+\mathrm{C}=\mathrm{CO}+\mathrm{M} ; \\
2 \mathrm{MO}+\mathrm{C}=\mathrm{CO}_{2}+2 \mathrm{M} .
\end{array}
$$

Questions.

171. How many metals are known, and about how many are of general interest?

172. Mention some metals showing very low and some showing very high fusing-points.

173. What range of specific gravities do we find among the metals?

174. Mention some univalent, some bivalent, and some metals showing a different valence under different conditions.

175. Mention some metals which are found in nature in an uncombined state; some which are found as oxides, sulphides, chlorides, and carbonates, respectively.

176. Into what two groups are the metals divided?

177. State the three groups of light metals.

178. What is a metal ?

179. What is an alloy, and what an amalgam?

180. By what process can most metals be obtained from their oxides? 


\section{POTASSIUM.}

$$
\boldsymbol{K}^{\mathbf{i}}=39 \text {. }
$$

General remarks regarding alkali-metals. The metals potassium, sodium, lithium (rubidium and cæsium) form the group of the alkali-metals, which, in many respects, show a great resemblance in chemical and physical properties. For reasons to be explained hereafter, the compound radical ammonium is usually classed among the alkali-metals.

The alkali-metals are all univalent; they decompose water at the ordinary temperature with liberation of hydrogen; they combine spontaneously with oxygen and chlorine; their oxides, hydrates, sulphates, nitrates, phosphates, carbonates, sulphides, chlorides, iodides, and nearly all of their other salts are soluble in water; all these compounds are white, solid substances, many of which are fusible at a red heat. Of all metals, those of the alkalies are the only ones forming hydrates and carbonates which are not decomposed by heat.

The metals themselves are of a silver-white color and extremely soft; on account of their tendency to combine with oxygen, they must be kept in a liquid not containing that element (coal-oil) or in an atmosphere of hydrogen.

The metals may be obtained by heating their carbonates with carbon in iron retorts, the escaping vapors being passed under coal-oil for condensation of the metal:

$$
\mathrm{K}_{2} \mathrm{CO}_{3}+2 \mathrm{C}=3 \mathrm{CO}+2 \mathrm{~K} \text {. }
$$

0ccurrence in nature. Potassium is chiefly found in nature as a double silicate of potassinm and aluminium (granitic rocks, feldspar, and other minerals) or as chloride and nitrate. By the gradual disintegration of the different granitic rocks containing silicate of potassium, this has entered into the soil, whence it is taken up by plants as one of the necessary constituents of their food.

In the plant the potassium enters largely into combination with organic acids (tartaric acid, citric acid, etc.), and when the plant is burned, ashes are left, containing the potassium now in the form of carbonate. By washing such ashes (chiefly woodashes) with water and filtering, the insoluble matter (carbonates, 
phosphates, and sulphates of calcium and magnesium, silica, etc.) is left behind, whilst a lye is obtained containing the soluble constituents, of which potassium carbonate is the principal one, chlorides and sulphates of potassium and sodium being also present in small quantities.

By evaporation of this lye to dryness an impure potassium carbonate is obtained, which is sold as crude potash.

Up to within twenty years ago the chief supply of potash was obtained by this process, and the trees of thousands of acres were burned with the view of obtaining potash. To-day this mode of manufacturing potash is very limited and is rapidly decreasing, as, fortunately, a new supply of soluble potassium salts has been discovered in the salt-mines of Stassfurt, Germany, where large quantities of potassium chloride (and some sulphate) are found, from which the carbonate and other salts are manufactured.

Potassium hydrate, Potassa, $\mathbf{K H O}=\mathbf{5 6}$ (Caustic potash), may be obtained by the action of the metal on water:

$$
\mathrm{K}+\mathrm{H}_{2} \mathrm{O}=\mathrm{H}+\mathrm{KHO} \text {. }
$$

The usual process for making potassium hydrate is to boil together a dilute solution of potassium carbonate or bicarbonate and calcium hydrate :

$$
\mathrm{K}_{2} \mathrm{CO}_{3}+\mathrm{Ca} 2 \mathrm{HO}=\mathrm{CaCO}_{3}+2 \mathrm{KHO} .
$$

The two metals exchange places, insoluble calcium carbonate is formed, which gradually subsides, whilst the liquid contains potassium hydrate. By evaporation to dryness the solid compound is obtained.

Potassium hydrate is a white, hard, solid substance, soluble in 0.5 part of water and 2 parts of alcohol; it fuses at a low red heat, forming an oily liquid, which may be poured into suitable moulds to form pencils; at a strong red heat it is slowly volatilized without decomposition; it is a very strong base, readily combining with all acids; when taken internally it acts as a powerful corrosive, and most likely also as a poison. Antidotes : dilute acids, vinegar, to form salts; or fat, oil, or milk, to form soap. 
Liquor potassa is a 5 per cent. solution of the potassium hydrate in water.

Potassium carbonate, $\mathrm{K}_{2} \mathrm{CO}_{3}=138$, Potassii carbonas, U. S. P., $\left(\mathrm{K}_{2} \mathrm{CO}_{3}\right)_{2} 3 \mathrm{H}_{2} \mathrm{O}=330$ (Carbonate of potassium), is obtained from ashes in an impure state as described above, or from the native chloride by the so-called Leblanc process, which will be described in connection with sodium carbonate. Crude potash when calcined in a furnace until white is known as pearlash.

Potassium carbonate is very deliquescent, is soluble in an equal weight of water, and has strong basic and alkaline properties.

Potassium bicarbonate, Potassii bicarbonas, $\mathrm{KHCO}_{3}=100(\mathrm{Bi}$ carbonate of potassium). Obtained by passing carbon dioxide through a strong solution of potassium carbonate, when the less soluble bicarbonate is formed and separates in crystals:

$$
\mathrm{K}_{2} \mathrm{CO}_{3}+\mathrm{H}_{2} \mathrm{O}+\mathrm{CO}_{2}=2 \mathrm{KHCO}_{3} \text {. }
$$

Potassium nitrate, Potassii nitras, $\mathrm{KNO}_{3}=101$ (Nitre, Saltpetre). Potassium and sodium nitrate are found as an incrustation upon and throughout the soil in certain dry and hot countries, as, for instance, in Peru, Chili, and India. The formation of these nitrates is to be explained by the absorption of ammonia (present in the atmosphere) by the soil, where it is gradually oxidized and converted into nitric acid, which then combines with the strongest base present in the soil. If this base be potash, potassium nitrate will be formed; if soda, sodium nitrate; if lime, calcium nitrate.

Upon the same principle is based the manufacture of nitre on a large scale, which is accomplished by mixing animal refuse matter with earth and lime, and placing the mixture in heaps under roof, to prevent lixiviation by rain. By the decomposition (putrefaction) of the animal matter, ammonia is formed, which, by oxidation, is converted into nitric acid, which then combines with the calcium of the lime, forming calcium nitrate. This is dissolved by water, and to the solution potassium carbonate (or chloride) is added, when calcium carbonate (or chloride) and potassium nitrate are formed :

$$
\mathrm{Ca}_{2} \mathrm{NO}_{3}+\mathrm{K}_{2} \mathrm{CO}_{3}=2 \mathrm{KNO}_{3}+\mathrm{CaCO}_{3} .
$$


Large quantities of potassium nitrate are also made by decomposing sodium nitrate (Chili saltpetre) by potassium chloride:

$$
\mathrm{NaNO}_{3}+\mathrm{KCl}=\mathrm{KNO}_{3}+\mathrm{NaCl} .
$$

Potassium nitrate crystallizes in six-sided prisms; it is soluble in about 5 parts of cold, and 0.5 part of boiling water. It has a cooling, saline, and pungent taste, and a neutral reaction. When heated with deoxidizing agents or combustible substances, these are readily oxidized.

It is this oxidizing power which is made use of in the manufacture of gunpowder - an intimate mixture of potassium nitrate, sulphur, and carbon. Upon heating or igniting the gunpowder, the sulphur and carbon are oxidized, a considerable quantity of various gases $\left(\mathrm{CO}, \mathrm{CO}_{2}, \mathrm{~N}, \mathrm{SO}_{2}\right.$, etc.) being formed, the sudden generation and expansion of which cause the explosion.

Potassium chlorate, Potassii chloras, $\mathrm{KClO}_{3}=122.4$ (Chlorate of potassium), may be obtained by the action of chlorine on a boiling solution of potassium hydrate :

$$
6 \mathrm{Cl}+6 \mathrm{KHO}=5 \mathrm{KCl}+\mathrm{KClO}_{3}+3 \mathrm{H}_{2} \mathrm{O} .
$$

A cheaper process for the manufacture of potassium chlorate is the action of chlorine upon a boiling solution of potassium carbonate, to which calcium hydrate has been added:

$$
\mathrm{K}_{2} \mathrm{CO}_{3}+6(\mathrm{Ca} 2 \mathrm{HO})+12 \mathrm{Cl}=2 \mathrm{KClO}_{3}+\mathrm{CaCO}_{3}+5 \mathrm{CaCl}_{2}+6 \mathrm{H}_{2} \mathrm{O} .
$$

Potassium chlorate crystallizes in plates of a pearly lustre; it is soluble in 17 parts of cold, and 2 parts of boiling water. It is even a stronger oxidizing agent than potassium nitrate, for which reason care must be taken in mixing it with organic or other deoxidizing agents, or with strong acids, which will liberate chloric acid. When heated by itself, it is decomposed into potassium chloride and oxygen.

Potassium sulphate, Potassii sulphas, $\mathrm{K}_{2} \mathrm{SO}_{4}=174$. Obtained by the decomposition of potassium chloride, nitrate, or carbonate, by sulphuric acid :

$$
\begin{gathered}
2 \mathrm{KCl}+\mathrm{H}_{2} \mathrm{SO}_{4}=2 \mathrm{HCl}+\mathrm{K}_{2} \mathrm{SO}_{4} ; \\
\mathrm{K}_{2} \mathrm{CO}_{3}+\mathrm{H}_{2} \mathrm{SO}_{4}=\mathrm{H}_{2} \mathrm{O}+\mathrm{CO}_{2}+\mathrm{K}_{2} \mathrm{SO}_{4} .
\end{gathered}
$$


Potassium sulphate exists in small quantities in plants, and in nearly all animal tissues and fluids, more abundantly in urine.

Potassium hydrogen sulphate, bisulphate or acid sulphate of potas sium, may be obtained by the action of one molecule of potassium chloride upon one molecule of sulphuric acid:

$$
\mathrm{KCl}+\dot{\mathrm{H}}_{2} \mathrm{SO}_{4}=\mathrm{HCl}+\mathrm{KHSO}_{4} \text {. }
$$

Potassium sulphite, Potassii sulphis, $\mathrm{K}_{2} \mathrm{SO}_{3} 2 \mathrm{H}_{2} \mathrm{O}=194$. Obtained by the decomposition of potassium carbonate by sulphurous acid :

$$
\mathrm{K}_{2} \mathrm{CO}_{3}+\mathrm{H}_{2} \mathrm{SO}_{3}=\mathrm{H}_{2} \mathrm{O}+\mathrm{CO}_{2}+\mathrm{K}_{2} \mathrm{SO}_{3} .
$$

Potassa sulphurata, U. S. P. (Sulphurated potassa, Sulphuret of potash). A mixture of potassium sulphide and hyposulphite. It is made by heating a mixture of sulphur and potassium carbonate in a covered crucible, and pouring the fused mass on a marble slab :

$$
3 \mathrm{~K}_{2} \mathrm{CO}_{3}+8 \mathrm{~S}=\mathrm{K}_{2} \mathrm{~S}_{2} \mathrm{O}_{3}+2 \mathrm{~K}_{2} \mathrm{~S}_{3}+3 \mathrm{CO}_{2} \text {. }
$$

The freshly prepared substance has a liver-brown color, turning gradually to. greenish-yellow; it is very apt to absorb oxygen, both the sulphide and hyposulphite becoming oxidized, and finally converted into sulphates.

Potassium hypophosphite, Potassii hypophosphis, $\mathrm{KH}_{2} \mathrm{PO}_{2}=104$, may be obtained by decomposing a solution of calcium hypophosphite by potassium carbonate:

$$
\mathrm{Ca} 2 \mathrm{PH}_{2} \mathrm{O}_{2}+\mathrm{K}_{2} \mathrm{CO}_{3}=2 \mathrm{NaPH}_{2} \mathrm{O}_{2}+\mathrm{CaCO}_{3} .
$$

Potassium iodide, Potassii iodidum, $\mathrm{KI}=\mathbf{1 6 5 . 6}$ (Iodide of potassium), is made by the addition of iodine to a solution of potassium hydrate until the dark brown color no longer disappears :

$$
6 \mathrm{KHO}+6 \mathrm{I}=5 \mathrm{KI}+\mathrm{KIO}_{3}+3 \mathrm{H}_{2} \mathrm{O} \text {. }
$$

'Iodide and iodate of potassium are formed, and may be separated by crystallization. A better method, however, is to boil the liquid containing both salts to dryness, and to heat the mass after having been mixed with some charcoal, in a crucible, when the iodate is converted into iodide:

$$
\mathrm{KIO}_{3}+3 \mathrm{C}=\mathrm{KI}+3 \mathrm{CO} .
$$


The cooled mass is dissolved in hot water, filtered, and crystallized, when potassium iodide is obtained in colorless, cubical crystals, which are very soluble in water.

Potassium bromide, Potassii bromidum, $\mathrm{KBr}=118.8$ (Bromide of potassium), may be obtained in a manner analogous to that given for potassium iodide, by the action of bromine upon potassium hydrate, etc.

Or it may be made by the decomposition of a solution of ferrous bromide by potassium carbonate:

$$
\mathrm{FeBr}_{2}+\mathrm{K}_{2} \mathrm{CO}_{3}=2 \mathrm{KBr}+\mathrm{FeCO}_{3} .
$$

Ferrous carbonate is precipitated, whilst potassium bromide remains in solution, from which it is obtained by crystallization.

Potassium salts of interest, which have not yet been mentioned, will be considered under the head of their respective acids. Some of these salts are the potassium chromate and permanganate, and the salts formed from organic acids, such as potassium tartrate, acetate, etc.

\section{Analytical reactions.}

1. To a solution of potassium chloride, or to any salt of potassium, after a few drops of hydrochloric acid have been mixed with it, add platinum chloride and some alcohol: a yellow crystalline precipitate falls, which is a double chloride of platinum and potassium, $\mathrm{PtCl}_{4} 2 \mathrm{KCl}$.

2. Add to a concentrated solution of a potassium salt strong solution of tartaric acid: a white precipitate of potassium acid tartrate is slowly formed.

3. Potassium compounds color the flame of a Bunsen-burner or of alcohol violet. (Presence of sodium interferes with this flame-test.)

4. All compounds of potassium are white (unless the acid has a coloring effect), soluble in water, and not volatile at a low red heat. 


\section{Questions.}

181. How is potassium found in nature, and from what sources is the chief supply of potassium salts obtained?

182. What color have the salts of the alkali-metals, and which are insoluble?

183. Mention two processes for making potassium hydrate, and what are its properties?

184. Show by symbols the conversion of carbonate into bicarbonate of potassium.

185. Explain the principle of the manufacture of potassium nitrate, and what is its action in gunpowder?

186. How is potassium chlorate made, and what are its properties?

187. Give the processes for manufacturing iodide and bromide of potassium, both in words and symbols.

188. State the composition of potassium sulphate and sulphite. How can they be obtained?

189. What is sulphuret of potash?

190. Mention tests for potassium compounds.

20. SODIUM.

$$
\mathrm{Na}^{1}=23 .
$$

Occurrence in nature. Sodium is found very widely diffused through all soils in small quantities. It occurs in large quantities in combination with chlorine, as rock-salt, or common salt, which forms considerable deposits in some regions, or is dissolved in spring waters, and is by them carried to the rivers, and finally to the ocean, which contains immense quantities of sodium chloride. It is also found as nitrate, silicate, etc.

Sodium and Sodium hydrate, Soda, $\mathbf{N a H O}=40$, may be obtained by the same processes mentioned for potassium and potassium hydrate.

Sodium chloride, Sodii chloridum, $\mathbf{N a C l}=\mathbf{5 8 . 4}$ (Chloride of sodium, Common salt). This is the most important of all sodium compounds, and is also the material from which the other compounds are directly or indirectly obtained. Common table-salt frequently contains small quantities of calcium and magnesium chloride, the presence of which causes an absorption of mois- 
ture, as these compounds are hygroscopic, whilst pure sodium chloride is not.

In the animal system, sodium chloride is found in all parts, it being of great importance by aiding the absorption of albuminoid substances and the phenomena of endosmosis; also by furnishing, through decomposition, the hydrochloric acid of the gastric juice.

Sodium chloride is soluble in about 2.8 parts of water, at all temperatures.

Sodium carbonate, Sodii carbonas, $\mathrm{Na}_{2} \mathrm{CO}_{3} \cdot 10 \mathrm{H}_{2} \mathrm{O}=286$ (Carbonate of sodium, Washing soda, Sal sodoe). This compound is, of all alkaline substances, the one which is manufactured in the largest quantities, being used in the manufacture of many highly important articles, as, for instance, soap, glass, etc.

Sodium carbonate is made according to Leblanc's process from the chloride by first converting it into sulphate (salt-cake) by the action of sulphuric acid:

$$
2 \mathrm{NaCl}+\mathrm{H}_{2} \mathrm{SO}_{4}=2 \mathrm{HCl}+\mathrm{Na}_{2} \mathrm{SO}_{4} .
$$

The escaping vapors of hydrochloric acid are absorbed by water, and this liquid acid is largely used in the manufacture of bleaching-powder. The sodium sulphate is mixed with coal and limestone (calcium carbonate) and the mixture heated in furnaces, when decomposition takes place, calcium sulphide, sodium carbonate, and carbonic oxide being formed:

$$
\mathrm{Na}_{2} \mathrm{SO}_{4}+4 \mathrm{C}+\mathrm{CaCO}_{3}=\mathrm{CaS}+\mathrm{Na}_{2} \mathrm{CO}_{3}+4 \mathrm{CO} .
$$

The resulting mass, known as black-ash, is washed with water, which dissolves the sodium carbonate, whilst calcium sulphide enters into combination with calcium oxide, thus forming an insoluble double compound of oxy-sulphide of calcium.

The liquid obtained by washing the black-ash, when evaporated to dryness, yields crude carbonate of sodium, or "soda-ash;" when this is dissolved and crystallized it takes up ten molecules of water, forming the ordinary "soda."

Sodium carbonate is also manufactured by a process which depends on the decornposition of sodium chloride by ammonium bicarbonate under pressure, when sodium bicarbonate and ammonium chloride are formed. 
Sodium carbonate has strong alkaline properties; it is soluble in 1.6 part of water at ordinary temperature, and in much less water at higher temperatures; the crystals lose water on exposure to the air, falling into a white powder; heat facilitates the expulsion of the water of crystallization, and is applied in making the dried sodium carbonate, Sodii carbonas exsiccata, of the U. S. P.

Sodium bicarbonate, Sodii bicarbonas, $\mathrm{NaHCO}_{3}=84$ (Bicarbonate of sodium). Made by passing carbon dioxide over sodium carbonate from which the larger portion of water of crystallization has been expelled:

$$
\mathrm{Na}_{2} \mathrm{CO}_{3}+\mathrm{H}_{2} \mathrm{O}+\mathrm{CO}_{2}=2 \mathrm{NaHCO}_{3} .
$$

It is a white powder, having a cooling, mildly saline taste, and a slightly acid reaction. Soluble in 12 parts of cold water and insoluble in alcohol. It is decomposed by hot water into sodium carbonate, water, and carbon dioxide.

Sodium sulphate, Sodii sulphas, $\mathrm{Na}_{2} \mathrm{SO}_{4} \cdot 10 \mathrm{H}_{2} \mathrm{O}=322$ (Sulphate of sodium, Glauber's salt). Made, as mentioned above, by the action of sulphuric acid on sodium chloride, dissolving the salt thus obtained in water and crystallizing. Large, colorless, transparent crystals, rapidly efflorescing on exposure to air. Soluble in 2.8 parts of water at $15^{\circ}$, in 0.25 part at $33^{\circ}$, and in 0.4 part of boiling water.

Sodium sulphite, Sodii sulphis, $\mathrm{Na}_{2} \mathrm{SO}_{3} \cdot 7 \mathrm{H}_{2} \mathrm{O}=252$. Made by passing sulphur dioxide into solution of sodium hydrate:

$$
2 \mathrm{NaHO}+\mathrm{SO}_{2}=\mathrm{Na}_{2} \mathrm{SO}_{3}+\mathrm{H}_{2} \mathrm{O} .
$$

Sodium hyposulphite, Sodii hyposulphis, $\mathrm{Na}_{2} \mathrm{~S}_{2} \mathrm{O}_{3} \cdot 5 \mathrm{H}_{2} \mathrm{O}=248$. Made by digesting a mixture of solution of sodium sulphite and powdered sulphur, when combination slowly takes place:

$$
\mathrm{Na}_{2} \mathrm{SO}_{3}+\mathrm{S}=\mathrm{Na}_{2} \mathrm{~S}_{2} \mathrm{O}_{3} .
$$

It is used, under the name of "hypo," in photography to dissolve chloride, bromide, or iodide of silver.

Disodium hydrogen phosphate, Sodii phosphas, $\mathrm{Na}_{2} \mathrm{HPO}_{4} \cdot 12 \mathrm{H}_{2} \mathrm{O}$ $=358$ (Phosphate of sodium), is made from calciuın phosphate 
by the action of sulphuric acid, which removes two-thirds of the calcium, forming calcium sulphate, while acid phosphate of calcium is formed and remains in solution:

$$
\mathrm{Ca}_{3} 2 \mathrm{PO}_{4}+2 \mathrm{H}_{2} \mathrm{SO}_{4}=2 \mathrm{CaSO}_{4}+\mathrm{CaH}_{4} 2 \mathrm{PO}_{4} .
$$

The solution is filtered and sodium carbonate added, when calcium phosphate is precipitated, phosphate of sodium, carbon dioxide, and water being formed:

$$
\mathrm{CaH}_{4} 2 \mathrm{PO}_{4}+\mathrm{Na}_{2} \mathrm{CO}_{3}=\mathrm{CaHPO}_{4}+\mathrm{H}_{2} \mathrm{O}+\mathrm{CO}_{2}+\mathrm{Na}_{2} \mathrm{HPO}_{4} .
$$

The filtered and evaporated solution yields crystals of phosphate of sodium, which have a slightly alkaline reaction.

Sodium nitrate, Sodii nitras, $\mathrm{NaNO}_{3}=85$ (Nitrate of sodium, Chili saltpetre, Cubic nitre). Found in nature, and is purified by crystallization.

Other sodium salts which are officinal are the sodium bisul phite, $\mathrm{NaHSO}_{3}$; borate (borax), $\mathrm{Na}_{2} \mathrm{~B}_{4} \mathrm{O}_{7} \cdot 10 \mathrm{H}_{2} \mathrm{O}$; bromide, $\mathrm{NaBr}$; iodide, $\mathrm{NaI}$; chlorate, $\mathrm{NaClO}_{3}$. The three latter salts may be obtained by processes analogous to those given for the corresponding potassium compounds.

\section{Tests for sodium.}

1. As all the salts of sodium are soluble in water, we cannot precipitate this metal in the form of a compound by any of the common reagents. (Potassium antimoniate precipitates neutral solution of sodium salts, but this test is not reliable.) .

2. The chief reaction for sodium is the flame-test, compounds of sodium imparting to a colorless flame an intensely yellow color. (The spectroscope shows a characteristic yellow line.)

3. Sodium compounds are white and not volatile at or below a red heat.

Lithium, $\mathrm{Li}=8$. Found in nature in combination with silicic acid in a few rare minerals or as chloride in some spring waters. Of inorganic salts, the bromide and carbonate of lithium are officinal. The hydrate, carbonate, and phosphate of lithium are much less soluble than the corresponding compounds of potassium and sodium. Iithium compounds color the flame beautifully crimson or carmine-red. 


\section{Questions.}

191. What is the composition of common salt; how is it found in nature, and what is it used for?

192. Describe Leblanc's process for manufacturing sodium carbonate on a large scale.

193. How much water is in 100 pounds of the crystallized sodium carbonate?

194. What is Glauber's salt, and how is it made?

195. State the composition of disodium hydrogen phosphate, and how is it prepared from calcium phosphate?

196. What difference exists between sodium carbonate and bicarbonate, both in regard to physical and chemical properties?

197. Give the composition of sodium hyposulphite, and what is it used for?

198. Which sodium salts are soluble, and which are insoluble?

199. How does sodium and how lithium color the flame?

200. Which lithium salts are officinal ?

\section{AMMONIUM.}

$\mathrm{NH}_{4} \mathrm{i}=18$.

General remarks. The salts of ammonium show so much resemblance, both in their physical and chemical properties, to those of the alkali-metals, that they may be most conveniently studied at this place.

The compound radical $\mathrm{NH}_{4}$ acts in these ammonium salts very much like one atom of an alkali-metal, and has, therefore, frequently been looked upon as a compound metal. The physical metallic properties (lustre, etc.) of ammonium cannot be fully demonstrated, as it is not capable of existing in a separate or free state. There is known, however, an alloy of ammonium and mercury, which may be obtained by dissolving potassium in mercury and adding to the potassium-amalgam thus formed, a strong solution of ammonium chloride, when potassium chloride and ammonium-amalgam are formed. The latter is a soft, spongy, metallic-looking substance, which readily decomposes into mercury, ammonia, and hydrogen:

$$
\begin{gathered}
\mathrm{HgK}+\mathrm{NH}_{4} \mathrm{Cl}=\mathrm{KCl}+\mathrm{NH}_{4} \mathrm{Hg} \\
\mathrm{NH}_{4} \mathrm{Hg}=\mathrm{NH}_{3}+\mathrm{H}+\mathrm{Hg}
\end{gathered}
$$


The source of all ammonium compounds is ammonia, $\mathrm{NH}_{3}$, or ammonium hydrate, $\mathrm{NH}_{4} \mathrm{HO}$, which have both been considered heretofore.

Ammonium chloride, Ammonii chloridum, $\mathrm{NH}_{4} \mathrm{Cl}=53.4$ (Chloride of ammonium, Sal-ammoniac). Obtained by saturating the "ammoniacal liquor" of the gas-works with hydrochloric acid, evaporating to dryness, and subliming the impure article for purification.

The pure ammonium chloride is either a white, crystalline powder, or presents itself in the form of long, fibrous crystals, which are tough and flexible; it has a cooling, saline taste; is soluble in 3 parts of cold, and 1.5 part of boiling water; and, like all ammonium compounds, is completely volatilized by heat.

Ammonium carbonate, Ammonii carbonas, $\mathrm{NH}_{4} \mathrm{HCO}_{3} \cdot \mathrm{NH}_{4} \mathrm{NH}_{2} \mathrm{CO}_{2}$ $=157$ (Carbonate of ammonium). The commercial ammonium carbonate is not the pure salt, but, as shown by the above formula, a combination of acid carbonate of ammonium with carbamate of ammonium. It is obtained by sublimation of a mixture of ammonium chloride and calcium carbonate, when calcium chloride is formed, ammonia gas and water escape, and ammonium carbonate condenses in the cooler part of the apparatus:

$$
2 \mathrm{CaCO}_{3}+4 \mathrm{NH}_{4} \mathrm{Cl}=\mathrm{NH}_{4} \mathrm{HCO}_{3} \cdot \mathrm{NH}_{4} \mathrm{NH}_{2} \mathrm{CO}_{2}+2 \mathrm{CaCl}_{2}+\mathrm{H}_{2} \mathrm{O}+\mathrm{NH}_{3} \text {. }
$$

Ammonium carbonate thus obtained forms white, translucent masses, losing both ammonia and carbon dioxide on exposure to the air, becoming opaque, and finally converted into a white powder of acid carbonate of ammonium:

$$
\mathrm{NH}_{4} \mathrm{HCO}_{3} \cdot \mathrm{NH}_{4} \mathrm{NH}_{2} \mathrm{CO}_{2}=\mathrm{NH}_{4} \mathrm{HCO}_{3}+2 \mathrm{NH}_{3}+\mathrm{CO}_{2} \text {. }
$$

When ammonium carbonate is dissolved in water, the carbamate unites with one molecule of water, forming neutral carbonate of ammonium :

$$
\mathrm{NH}_{4} \mathrm{NH}_{2} \mathrm{CO}_{2}+\mathrm{H}_{2} \mathrm{O}=\left(\mathrm{NH}_{4}\right)_{2} \mathrm{CO}_{3} .
$$

A solution of the common ammonium carbonate in water, is, consequently, a liquid containing both acid and neutral carbonate of ammonium; by the addition of some ammonia water the 
acid carbonate is also converted into the neutral salt. The solution thus obtained is frequently used as a reagent.

The aromatic spirit of ammonia (sal volatile) is a solution of neutral ammonium carbonate in diluted alcohol to which some essential oils have been added.

Ammonium sulphate, $\left(\mathrm{NH}_{4}\right)_{2} \mathrm{SO}_{4}$, Ammonium nitrate, $\mathrm{NH}_{4} \mathrm{NO}_{3}$, and Ammonium phosphate, $\left(\mathbf{N H}_{4}\right)_{2} \mathbf{H P O}_{4}$, may be obtained by the addition of the respective acids to ammonia water or ammonium carbonate :

$$
\begin{aligned}
\mathrm{H}_{2} \mathrm{SO}_{4}+2 \mathrm{NH}_{4} \mathrm{HO} & =\left(\mathrm{NH}_{4}\right)_{2} \mathrm{SO}_{4}+2 \mathrm{H}_{2} \mathrm{O} . \\
\mathrm{HNO}_{3}+\mathrm{NH}_{4} \mathrm{HO} & =\mathrm{NH}_{4} \mathrm{NO}_{3}+\mathrm{H}_{2} \mathrm{O} . \\
\mathrm{H}_{3} \mathrm{PO}_{4}+2 \mathrm{NH}_{4} \mathrm{HO} & =\left(\mathrm{NH}_{4}\right)_{2} \mathrm{HPO}_{4}+2 \mathrm{H}_{2} \mathrm{O} . \\
\mathrm{H}_{2} \mathrm{SO}_{4}+\left(\mathrm{NH}_{4}\right)_{2} \mathrm{CO}_{3} & =\left(\mathrm{NH}_{4}\right)_{2} \mathrm{SO}_{4}+\mathrm{H}_{2} \mathrm{O}+\mathrm{CO}_{2} .
\end{aligned}
$$

Ammonium iodide, Ammonii iodidum, $\mathrm{NH}_{4} \mathrm{I}$, and Ammonium bromide, Ammonii bromidum, $\mathrm{NH}_{4} \mathrm{Br}$, may be obtained by mixing strong solutions of potassium iodide (or bromide) and ammonium sulphate, and adding alcohol, which precipitates the potassium sulphate formed; by evaporation of the solution the ammonium iodide (or bromide) is obtained :

$$
\begin{aligned}
& 2 \mathrm{KI}+\left(\mathrm{NH}_{4}\right)_{2} \mathrm{SO}_{4}=2 \mathrm{NH}_{4} \mathrm{I}+\mathrm{K}_{2} \mathrm{SO}_{4} ; \\
& 2 \mathrm{KBr}+\left(\mathrm{NH}_{4}\right)_{2} \mathrm{SO}_{4}=2 \mathrm{NH}_{4} \mathrm{Br}+\mathrm{K}_{2} \mathrm{SO}_{4} .
\end{aligned}
$$

Another mode of preparing these compounds is the decomposition of ferrous bromide (or iodide) by ammonium hydrate:

$$
\mathrm{FeBr}_{2}+2 \mathrm{NH}_{4} \mathrm{HO}=2 \mathrm{NH}_{4} \mathrm{Br}+\mathrm{Fe} 2 \mathrm{HO} \text {. }
$$

Ammonium hydrogen sulphide, $\mathrm{NH}_{4} \mathrm{HS}$ (Ammonium hydrosulphide, Ammonium sulphydrate). Obtained by passing sulphuretted hydrogen gas through water of ammonia until this is saturated:

$$
\mathrm{H}_{2} \mathrm{~S}+\mathrm{NH}_{4} \mathrm{HO}=\mathrm{NH}_{4} \mathrm{HS}+\mathrm{H}_{2} \mathrm{O} .
$$

The solution thus obtained is, when recently prepared, a colorless liquid, having the odor both of ammonia and of sulphuretted hydrogen; when exposed to the air it soon assumes a yellow color. By the addition of ammonia water it is converted into ammonium sulphide, $\left(\mathrm{NH}_{4}\right)_{2} \mathrm{~S}$ :

$$
\mathrm{NH}_{4} \mathrm{HS}+\mathrm{NH}_{4} \mathrm{HO}=\left(\mathrm{NH}_{4}\right)_{2} \mathrm{~S}+\mathrm{H}_{2} \mathrm{O} .
$$


Both substances, the ammonium hydrogen sulphide and ammonium sulphide, are valuable reagents, frequently used for the precipitation of certain heavy metals, or for dissolving certain metallic sulphides.

\section{Analytical reactions.}

1. All compounds of ammonium are volatilized by heating upon platinum foil.

2. All compounds of ammonium evolve, when heated with the hydrates of calcium, potassium, or sodium, ammonia gas, which may be recognized by its odor, or by its action on paper moistened with solution of cupric sulphate, which is thereby colored dark blue.

3. Add to solution of ammonium salt some platinum chloride and a few drops of hydrochloric acid; a yellow precipitate of ammonium platinum chloride, $\left(\mathrm{NH}_{4} \mathrm{Cl}\right)_{2} \mathrm{PtCl}_{4}$, is produced.

4. Ammonium salts are colorless, and (almost all) soluble in water.

\section{Questions.}

201. What is ammonium, and why is it classed with the alkali-metals?

202. Is ammonium known the separate state?

203. What is ammonium-amalgam, how is it obtained, and what are its properties?

204. What is the source of ammonium compounds?

205. State the composition, mode of preparation, and properties of sal-ammoniac.

206. How is ammonium carbonate manufactured, and what difference exists between the solid article and its solution?

207. State the composition of ammonium sulphide and of ammonium hydrogen sulphide; how are they made, and what are they used for?

208. By what processes may ammonium sulphate, nitrate, and phosphate be obtained from ammonium hydrate or ammonium carbonate, and what chemical change takes place?

209. How does heat act upon ammonium compounds?

210. Give analytical reactions for ammonium salts. 


\section{MAGNESIUM.}

$$
M \mathrm{gi}^{\mathrm{il}}=24 \text {. }
$$

General remarks. Magnesium occupies a position intermediate between the metals of the alkalies and the alkaline earths, of which latter it was formerly classed as a member. To some extent it also resembles the heavy metal zinc, with which it has, in common, the volatility of the chloride, the solubility of its sulphate, and the isomorphism of several of its compounds with the analogously constituted compounds of zinc.

0ccurrence in nature. Magnesium is widely diffused in nature and several of its compounds are found in large quantities. It occurs as chloride and sulphate in many spring waters and in the salt-mines of Stassfurt; as carbonate in the mineral magnesite; as a double carbonate of magnesium and calcium in the mineral dolomite (magnesia-limestone), which forms entire mountains; as silicate of magnesium in the minerals serpentine, meerschaum, talc, asbestos, etc.

Metallic magnesium may be obtained by the decomposition of magnesium chloride by sodium:

$$
\mathrm{MgCl}_{2}+2 \mathrm{Na}=2 \mathrm{NaCl}+\mathrm{Mg} \text {. }
$$

Magnesium is an almost silver-white metal, losing its lustre rapidly in moist air by oxidation of the surface. It decomposes hot water with liberation of hydrogen :

$$
\mathrm{Mg}+2 \mathrm{H}_{2} \mathrm{O}=2 \mathrm{H}+\mathrm{Mg} 2 \mathrm{HO} .
$$

When heated to a red heat it burns with a brilliant bluishwhite light, forming magnesium oxide.

Magnesium carbonate, Magnesii carbonas, $4\left(\mathrm{MgCO}_{3}\right) \cdot \mathrm{Mg} 2 \mathrm{H0} \cdot 5 \mathrm{H}_{2} \mathrm{O}$ $=484$ (Carbonate of magnesium, Magnesia alba, Light magnesia). The neutral magnesium carbonate, $\mathrm{MgCO}_{3}$, is found in nature, but the officinal preparation is a mixture of carbonate, hydrate, and water. It is obtained by the addition of a solution of magnesium sulphate to solution of sodium carbonate and boiling, when the carbonate is precipitated, some carbon dioxide evolved, and sodium sulphate remains in solution:

$$
5 \mathrm{MgSO} \mathrm{gS}_{4}+6 \mathrm{Na}_{2} \mathrm{CO}_{3}+6 \mathrm{H}_{2} \mathrm{O}=\underset{9}{4}(\underset{\mathrm{MgCO}}{3}) \cdot \mathrm{Mg} 2 \mathrm{HO} .5 \mathrm{H}_{2} \mathrm{O}+5 \mathrm{Na}_{2} \mathrm{SO}_{4}+\mathrm{CO}_{2} .
$$


By tiltering, washing, and drying the precipitate, it is obtained in the form of a white, light powder; if, however, the above-mentioned solutions are mixed, evaporated to dryness, and the sodium sulphate removed by washing, the magnesium carbonate is left in a more dense condition, and is then known as heavy magnesia.

Large quantities of magnesium carbonate are also manufactured by treating dolomite (the double carbonate of magnesium and calcium) with water and carbon dioxide under pressure. Bicarbonate of magnesium is produced and dissolved in the carbonic acid. water, from which solution the carbonate is precipitated by heating.

Magnesium oxide, Magnesia, Mg0 $=\mathbf{4 0}$ (Calcined magnesia, Light magnesia), is obtained by heating light magnesium carbonate in a crucible to a full red heat, when all carbon dioxide and water are expelled:

$$
\left.4(\mathrm{MgCO})_{3}\right) . \mathrm{Mg} 2 \mathrm{HO} .5 \mathrm{H}_{2} \mathrm{O}=5 \mathrm{MgO}+4 \mathrm{CO}_{2}+6 \mathrm{H}_{2} \mathrm{O} \text {. }
$$

It is a very light, amorphous, white, almost tasteless powder, which absorbs gradually moisture and carbon dioxide from the air ; in contact with water it forms the hydrate $\mathrm{Mg} 2 \mathrm{HO}$, which is very sparingly soluble in water. Milk of magnesia is the hydrate suspended in water (1 part in about 15$)$.

The heavy magnesia of the U.S. P. differs from the common or light magnesia, not in its chemical composition, but merely in its physical condition, being a white, dense powder obtained by heating the heavy carbonate of magnesiunı.

Magnesium sulphate, Magnesii sulphas, $\mathrm{MgSO}_{4} \cdot \mathbf{7 H}_{2} \mathbf{O}=\mathbf{2 4 6}$ (Sulphate of magnesium, Epsom salt), is obtained either from spring waters or by decomposition of the native carbonate by sulphuric acid :

$$
\mathrm{MgCO}_{3}+\mathrm{H}_{2} \mathrm{SO}_{4}=\mathrm{MgSO}_{4}+\mathrm{CO}_{2}+\mathrm{H}_{2} \mathrm{O} .
$$

It forms colorless crystals, which have a cooling, saline, and bitter taste, a neutral reaction, and are easily soluble in water.

Magnesium sulphite, Magnesii sulphis, $\mathrm{MgSO}_{3} 6 \mathrm{H}_{2} \mathbf{O}=212$, may be obtained by adding sulphurous acid to magnesium carbonate :

$$
\mathrm{M} \mathrm{IgCO}_{3}+\mathrm{H}_{2} \mathrm{SO}_{3}=\mathrm{MgSO}_{3}+\mathrm{CO}_{2}+\mathrm{H}_{2} \mathrm{O} .
$$




\section{Analytical reactions.}

1. Add to magnesium solution carbonate of potassium or sodium : a white precipitate of magnesium carbonate is produced.

2. Add to magnesium solution carbonate of ammonium (or hydrate of ammonium): part of the magnesium will be precipitated as carbonate (or hydrate). These precipitates, however, are soluble in ammonium chloride and many other ammonium salts; if these latter had been added previously to the magnesium solution, ammonium carbonate (or hydrate) would cause no precipitation. (The dissolving action of the ammonium chloride is due to the tendency of magnesium to form double salts with ammonium salts.)

3. To solution of magnesium add a solution containing sodium phosphate, ammonium chloride, and ammonia : a white crystalline precipitate of magnesium-ammonium phosphate, $\mathrm{MgNH}_{4} \mathrm{PO}_{4}$, is produced.

4. Salts of magnesium are white and soluble, except the carbonate, phosphate, and arseniate; the oxide and hydrate are also insoluble.

\section{Questions.}

211. How is magnesium found in nature?

212. By what process is metallic magnesium obtained?

213. Give the physical and chemical properties of magnesium.

214. State two methods by which magnesium oxide can be obtained.

215. What is calcined magnesia?

216. State the composition and properties of the officinal magnesium carbonate, and how it is made.

217. What is Epsom salt, and how is it obtained?

218. Which compounds of magnesium are insoluble?

219. Give tests for magnesium compounds.

220. How could the presence of magnesium be demonstrated in a mixture of magnesium sulphate and sodium sulphate? 


\section{CALCIUM.}

$$
\mathrm{Ca}^{\mathrm{ii}}=40
$$

General remarks regarding the metals of the alkaline earths. The three metals, calcium, barium, and strontium, form the second group of light metals. Similarly to the alkali-metals, they decompose water at the ordinary temperature with liberation of hydrogen; their separation in the elementary state is even more difficult than that of the alkali-metals.

They differ from the latter by forming insoluble carbonates, and phosphates (those of the alkalies are soluble), from the earths by their soluble hydrates (those of the earths are insoluble), and from all heavy metals by the solubility of their sulphides (those of heavy metals are insoluble). The sulphates are either insoluble (barium) or sparingly soluble (strontium and calcium). The hydrates and carbonates are decomposed by heat, water or carbon dioxide being expelled and the oxides formed. They are bivalent elements.

0ccurrence in nature. Calcium is one of the most abundantly occurring elements. As carbonate $\left(\mathrm{CaCO}_{3}\right)$ it is found in the form of calc-spar, limestone, chalk, marble, shells of eggs and mollusca, etc.; or, as acid carbonate, dissolved in water. The sulphate is found as gypsum or alabaster $\left(\mathrm{CaSO}_{4} 2 \mathrm{H}_{2} \mathrm{O}\right)$; the phosphate $\left(\mathrm{Ca}_{3} 2 \mathrm{PO}_{4}\right)$ in the different phosphatic rocks (apatite, etc.); the fluoride $\left(\mathrm{CaFl}_{2}\right)$ as fluorspar; the chloride $\left(\mathrm{CaCl}_{2}\right)$ in some waters, and the silicate in many rocks. It also enters the vegetable and animal system in various forms of combination, chiefly, however, as phosphate and sulphate.

Calcium oxide, Lime, Calx, $\mathrm{CaO}=56$ (Oxide of calcium, Quicklime, Burned lime), is obtained on a large scale by the common process of lime-burning, which is the heating of limestone or any other calcium carbonate to about $800^{\circ}$, in the so-called limekilns. On a small scale the decomposition may be accomplished in a suitable crucible over a blowpipe flame:

$$
\mathrm{CaCO}_{3}=\mathrm{CaO}+\mathrm{CO}_{2} .
$$

The pieces of oxide thus formed still retain the shape and size of the carbonate used for decomposition. 
Lime is a white, odorless, amorphous, infusible substance, of alkaline taste and reaction; exposed to the air it gradually absorbs moisture and carbon dioxide, the mixture thus formed being known as air-slaked lime.

Lime occupies among bases a position similar to that of sulphuric acid among acids, and is used directly or indirectly in many branches of chemical manufacture.

Calcium hydrate, Ca2HO (Slaked lime). When water is sprinkled upon pieces of calcium oxide, the two substances combine chemically, liberating much heat; the pieces swell up, and are gradually converted into a dry, white powder, which is the slaked lime. When this is mixed with water, the so-called milk of lime. is formed.

Lime-water, Liquor calcis. This is a saturated solution of calcium hydrate in water; 10,000 parts of the latter dissolving about 15 parts of calcium hydrate. In making lime-water, 1 part of calcium oxide is slaked and stirred for about half an hour with 30 parts of water. The mixture is then allowed to settle, and the liquid, containing besides calcium hydrate the salts of the alkali-metals which may have been present in the lime, is decanted and thrown away. To the calcium hydrate left, and thus purified, 300 parts of water are added and occasionally shaken in a well-stoppered bottle, from which the clear liquid may be poured off for use.

Lime-water is a colorless, odorless liquid, having a feebly caustic taste, and an alkaline reaction. When heated to boiling it becomes turbid by precipitation of calcium hydrate (or perhaps dioxide). Carbon dioxide causes a precipitation of calcium carbonate.

Calcium carbonate, Calcii carbonas præcipitatus, $\mathrm{CaCO}_{3}=100$ (Carbonate of calcium). Precipitated carbonate of calcium is obtained as a white, tasteless, neutral, impalpable powder by mixing solutions of calcium chloride and sodium carbonate:

$$
\mathrm{CaCl}_{2}+\mathrm{Na}_{2} \mathrm{CO}_{3}=2 \mathrm{NaCl}+\mathrm{CaCO}_{3} .
$$

Calcium sulphate, $\mathrm{CaSO}_{4}=136$ (Sulphate of calcium, Plaster of Paris). It has been mentioned above, that the mineral gypsum 
is the native calcium sulphate in combination with 2 molecules of water of crystallization. By heating to about $115^{\circ}$ this water is expelled, and the anhydrous sulphate formed. It readily recombines with water, becoming a hard mass, for which reason it is used for making moulds and casts, and in surgery. For the latter purpose plaster is often mixed with some alum and gelatine before adding the water, this mixture being preferred on account of forming a harder, less porous mass, with a smooth surface that can be washed with water containing disinfecting agents.

Tricalcium phosphate, Calcii phosphas præcipitatus, $\mathrm{Ca}_{3} 2 \mathrm{PO}_{4}=\mathbf{3 1 0}$ (Precipitated phosphate of calcium, Phosphate of lime, Bone-phosphate). By dissolving bone-ash (bones from which all organic matter has been expelled by heat) in hydrochloric acid, and precipitating the solution with ammonia water a calcium phosphate is obtained, which contains traces of calcium fluoride and magnesium phosphate.

A pure article is made by precipitating a solution of calcium chloride by sodium phosphate and ammonia:

$$
2 \mathrm{Na}_{2} \mathrm{HPO}_{4}+3 \mathrm{CaCl}_{2}+2 \mathrm{NH}_{4} \mathrm{HO}=\mathrm{Ca}_{3} 2 \mathrm{PO}_{4}+4 \mathrm{NaCl}+2 \mathrm{NH}_{4} \mathrm{Cl}+2 \mathrm{H}_{2} \mathrm{O} \text {. }
$$

It is a white, tasteless, amorphous powder, insoluble in water, soluble in all acids.

Superphosphate, or acid phosphate of lime. Among the inorganic substances which serve as plant-food, calcium phosphate is a highly important one. As this compound is usually found in very small quantities as a constituent of the soil, and as this small quantity is soon removed by the various crops taken from a cultivated soil, it becomes necessary to replace it in order to enable the plant to grow and to form seeds.

For this purpose the various phosphatic rocks (chiefly calcium phosphate) are converted into conmercial fertilizers, which is accomplished by the addition of sulphuric acid to the ground rock. The sulphuric acid removes from the tricalcium phosphate one or two atoms of calcium, forming mono- or dicalcium phosphate and calcium sulphate. The mixture of these substances, containing also more or less of the inpurities originally present 
in the phosphatic rocks, is sold as acid phosphate or superphosphate.

Bone-black and bone-ash. Phosphates enter the animal system in the various kinds of food, and are to be found in every tissue and fluid, but most abundantly in the bones and teeth. Bones contain about 30 per cent. of organic and 70 per cent. of inorganic matter, most of which is tricalcium phosphate. When bones are burned until all the organic matter has been destroyed and volatilized, the resulting product is known as bone-ash. If, however, the bones are subjected to the process of destructive distillation (heating with exclusion of air), the organic matter suffers decomposition, many volatile products escape, and most of the non-volatile carbon remains mixed with the inorganic portion of the bones, which substance is known as boneblack or animal charcoal.

Calcium hypophosphite, Calcii hypophosphis, $\mathrm{Ca} 2\left(\mathrm{PH}_{2} \mathbf{0}_{2}\right)=170$. Obtained by heating pieces of phosphorus with milk of lime until phosphoretted hydrogen ceases to escape. From the filtered liquid the excess of lime is removed by carbon dioxide, and the clear liquid evaporated to dryness. (Great care must be taken during the whole of the operation, which is somewhat dangerous on account of the inflammable and explosive nature of the compounds.)

$$
8 \mathrm{P}+6 \mathrm{H}_{2} \mathrm{O}+3(\mathrm{Ca} 2 \mathrm{HO})=3\left(\mathrm{Ca}_{2} \mathrm{PH}_{2} \mathrm{O}_{2}\right)+2 \mathrm{PH}_{3} .
$$

Chlorinated lime, Calx chlorate (Blecthing-powder, incorrectly called Chloride of lime). This is chiefly a mixture (according to some, a compound) of calcium chloride with calcium hypochlorite, and is manufactured on a very large scale by the action of chlorine upon calcium hydrate:

$$
\begin{aligned}
& 2(\mathrm{Ca} 2 \mathrm{HO})+4 \mathrm{Cl}=2 \mathrm{H}_{2} \mathrm{O}+\underbrace{\mathrm{Ca} 2 \mathrm{ClO}+\mathrm{CaCl}_{2}}_{\text {Chlorinated lime. }} . \\
& \text { Calcium hydrate. }
\end{aligned}
$$

Bleaching-powder is a white powder, having a feeble chlorinelike odor; exposed to the air it becomes damp from absorption of moisture, undergoing decomposition at the same time; with dilute acids it evolves chlorine gas; it is a powerful disinfecting and blcaching agent. 
Sulphuretted lime, Calx sulphurata, is a mixture of calcium sulphide and sulphate, obtained by heating in a crucible a mixture of equal parts of sulphur and calcium oxide.

\section{Analytical reactions.}

1. Add to solution of a calcium salt, the carbonate of either potassium, sodium, or ammonium: a white precipitate of calcium carbonate is produced.

2. Add phosphate of sodium to neutral solution of calcium: a white precipitate of calcium phosphate is produced.

3. Add ammonium (or potassium) oxalate to a calcium solution: a white precipitate of calcium oxalate is produced, which is insoluble in acetic, soluble in hydrochloric acid.

4. Sulphuric acid or soluble sulphates produce a white precipitate in highly concentrated solutions of calcium, but not in dilute solutions.

5. Calcium compounds impart a reddish-yellow color to the flame.

Barium, $\mathrm{Ba}^{\mathrm{ii}}=$ 137. Strontium, $\mathrm{Sr}=87.5$. Both metals are but rarely met with in nature. Barium occurs chiefly as sulphate or heavy-spar, $\mathrm{BaSO}_{4}$, and strontium as sulphate or carbonate.

From the native sulphates the other salts may be made by first converting them into sulphides, by heating with charcoal in crucibles :

$$
\mathrm{BaSO}_{4}+4 \mathrm{C}=\mathrm{BaS}+4 \mathrm{CO} .
$$

When the sulphide is dissolved in hydrochloric acid, barium chloride is formed and sulphuretted hydrogen liberated:

$$
\mathrm{BaS}+2 \mathrm{HCl}=\mathrm{H}_{2} \mathrm{~S}+\mathrm{BaCl}_{2} \text {. }
$$

By precipitating the barium chloride solution with sodium carbonate, barium carbonate is obtained:

$$
\mathrm{BaCl}_{2}+\mathrm{Na}_{2} \mathrm{CO}_{3}=2 \mathrm{NaCl}+\mathrm{BaCO}_{3} .
$$

By dissolving barium carbonate in the various acids the corresponding salts are obtained. For instance:

$$
\mathrm{BaCO}_{3}+2 \mathrm{HNO}_{3}=\mathrm{H}_{2} \mathrm{O}+\mathrm{CO}_{2}+\mathrm{Ba}_{2} \mathrm{NO}_{3} .
$$


Strontium compounds may be made by an analogous process. Neither barium nor strontium enters into any officinal preparation. Strontium is chiefly used in the form of nitrate for pyrotechnical purposes, as it imparts a beautiful red or crimson color to the flame.

Barium is chiefly used as sulphate for adulterating white-lead and other colors, or as chloride or nitrate as reagents for sulphuric acid or soluble sulphates.

Barium salts are poisonous; antidotes are sodium and magnesiun sulphates.

\section{Analytical reactions of barium and strontium.}

1. Like calcium solutions, those of barium and strontium give a white precipitate with soluble carbonates or phosphates.

2. Barium gives with neutral potassium chromate a pale yellow precipitate of barium chromate; calcium and strontium do not.

3. Barium and strontium solutions are precipitated by solution of calcium sulphate; calcium solutions are not.

4. Barium gives with sulphuric acid, or with soluble sulphates, an immediate white precipitate of barium sulphate which is entirely insoluble in all acids; strontium sulpbate is less insoluble, and calcium sulphate may be completely dissolved in water or in dilute hydrochloric acid.

5. Barium colors the flame pale green, strontium crimson, calcium reddish-yellow.

6. Barium, strontium, and calcium compounds are colorless, unless the acid has a coloring effect.

\section{QUESTIONS.}

221. Which metals form the group of the alkaline earths, and in what respect do their compounds differ from those of the alkali-metals?

222. How is calcium found in nature?

223. What is burned lime; from what, and by what process is it made, and how does water act on it?

224. What is lime-water; how is it made, and what are its properties?

225. Mention some varieties of calcium carbonate as found in nature, and how is it obtained by an artificial process from the chloride? 
226. What is plaster of Paris, and what is gypsum; what are they used for?

227. State composition and mode of manufacturing bleaching-powder; what are its properties, and how do acids act upon it?

228. What is bone-black, bone-ash, acid phosphate, and precipitated tricalcium phosphate? How are they made?

229. Give tests for barium, calcium, and strontium; how can they be distinguished from each other?

230. Which compounds of barium and strontium are of interest, and what are they used for?

\section{ALUMINIUM.}

$$
\mathrm{Al} \mathrm{lii}^{\mathrm{ii}}=2 \mathrm{i} .4 \text {. }
$$

Aluminium is the representative of the metals of the earths proper; all other members of this class are found in nature in very small quantities, and are of scientific interest only.

Occurrence in nature. Aluminium is found almost exclusively in the solid mineral portion of the earth; rarely more than traces of aluminium compounds are found dissolved in water, and the occurrence of aluminium in either the vegetable or animal organism seems to be purely accidental.

By far the largest quantity of aluminium is found in combination with silicic acid in the varions silicated roeks forming the greater mass of our earth, such as feldspar, slate, basalt, granite, mica, hornblende, etc., or in the various modifications of clay formed by their decomposition.

The minerals known as corundum, ruby, sapphire, and emery are aluminium oxide in a crystallized state and more or less colored by traces of other substances.

Metallic aluminium is obtained by the decomposition of aluminium chloride by metallic sodiụm :

$$
\mathrm{Al}_{2} \mathrm{Cl}_{6}+6 \mathrm{Na}=6 \mathrm{NaCl}+2 \mathrm{Al} .
$$

Aluminium is an almost silver-white metal of a very low specific gravity (2.6); it is capable of assuming a high polish, and for this reason is used for ornamental articles.

Some of the alloys of aluminium are now used in the arts, 
as, for instance, aluminium-bronze, an alloy resembling gold, and composed of 10 parts of aluminium with 90 of copper.

Aluminium is trivalent, and shows, like a number of other elements (iron, chromium, etc.), the peculiarity that the double atom $\mathrm{Al}_{2}{ }^{\mathrm{vl}}$ acts as a single hexavalent atom.

Alum is the general name for a group of isomorphous salts, composed of one molecule of the sulphate of an univalent metal in combination with one molecule of the sulphate of a trivalent metal, combining in crystallizing with 24 molecules of water. The general formula of an alum is consequently $\mathrm{M}_{2}^{i} \mathrm{SO}_{4}$, $\mathrm{M}_{2}{ }^{11 i} 3 \mathrm{SO}_{4}, 24 \mathrm{H}_{2} \mathrm{O} . \quad \mathrm{M}^{\mathrm{i}}$ represents in this case a univalent, $\mathrm{M}^{\mathrm{iii}}$ a trivalent metal.

Alums known are, for instance:

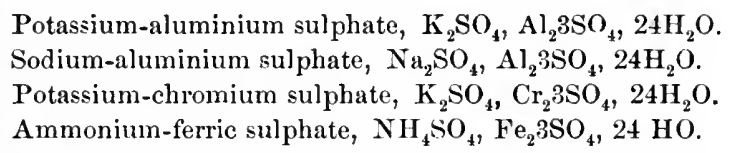

The officinal alum is the potassium-aluminium alum, a white substance crystallizing in large octahedrons, soluble in 10 parts of cold and 0.3 part of boiling water; this solution has an acid reaction and a sweetish, astringent taste.

Alum is manufactured on a large scale by decomposing certain kinds of aluminium silicates by sulphuric acid, when aluminium sulphate is formed, to the solution of which potassium or ammonium sulphate is added, when, on evaporation, potassium or ammonium alum crystallizes.

Dried alum, Alumen exsiccatum, $\mathrm{K}_{2} \mathrm{SO}_{4} \cdot \mathrm{Al}_{2} 3 \mathrm{SO}_{4}=516$. This is common alum, from which the water of crystallization has been expelled by heating it for several days to a temperature of about $80^{\circ} \mathrm{C}$.

Aluminium hydrate, Aluminii hydras, $\mathrm{Al}_{2} 6 \mathrm{HO}=156$. Obtained by adding water of ammonia or solution of sodium carbonate to solution of alum, when aluminium hydrate is precipitated in the form of a highly gelatinous substance, which, after being well.washed, is dried at a temperature not exceeding $40^{\circ}$.

$$
\begin{gathered}
\mathrm{K}_{2} \mathrm{SO}_{4} \cdot \mathrm{Al}_{2} 3 \mathrm{SO}_{4}+6 \mathrm{NH}_{4} \mathrm{HO}=\mathrm{K}_{2} \mathrm{SO}_{4}+3\left[\left(\mathrm{NH}_{4}\right)_{2} \mathrm{SO}_{4}\right]+\mathrm{Al}_{2} 6 \mathrm{HO} \\
\mathrm{K}_{2} \mathrm{SO}_{4} \mathrm{Al}_{2} 3 \mathrm{SO}_{4}+3 \mathrm{Na}_{2} \mathrm{CO}_{3}+3 \mathrm{H}_{2} \mathrm{O}=\mathrm{K}_{2} \mathrm{SO}_{4}+3 \mathrm{Na}_{2} \mathrm{SO}_{4}+3 \mathrm{CO}_{2}+\mathrm{Al}_{2} 6 \mathrm{HO} .
\end{gathered}
$$


The usual decomposition between a soluble carbonate and any other soluble salt (provided decomposition takes place at all) is the formation of an insoluble carbonate; according to this rule, the addition of a soluble carbonate to alum should produce aluminium carbonate. The basic properties of aluminium oxide, however, are so weak that it is not capable of uniting with so weak an acid as carbonic acid, and it is for this reason that the decomposition takes place, as shown by the above formula, with liberation of carbon dioxide, whilst the hydrate is formed. (Other metals, the oxides of which have weak basic properties, show similar reactions, as, for instance, chromium, and iron in the ferric salts.)

The weak basic properties of aluminium are also shown by the fact that aluminium sulphate, chloride, and nitrate, and even alum itself, have an acid reaction, while the corresponding salts of the alkalies or alkaline earths are neutral.

Aluminium hydrate shows considerable surface-attraction towards many substances, which property is made use of in the art of dyeing, where aluminium hydrate is used for retaining coloring matter upon the cotton-fibre. Practically this is accomplished by precipitating aluminium hydrate from solutions containing coloring matter, which latter is carried down and precipitated upon the fibre by the aluminium hydrate, or by impregnating the articles to be dyed with this compound and placing them in the colored solutions.

Aluminium oxide, $\mathrm{Al}_{2} \mathrm{O}_{3}=102$, is obtained as a white, tasteless powder by either burning the metal or by expelling the water from the hydrate by heat:

$$
\mathrm{Al}_{2} 6 \mathrm{HO}=\mathrm{Al}_{2} \mathrm{O}_{3}+3 \mathrm{H}_{2} \mathrm{O} .
$$

Aluminium sulphate, Aluminii sulphas, $\mathrm{Al}_{2} 3 \mathrm{SO}_{4} 18 \mathrm{H}_{2} \mathrm{O}=666$. A white, crystalline powder, soluble in about an equal weight of water; obtained by dissolving the oxide or hydrate in sulphuric acid.

Aluminium chloride, $\mathrm{Al}_{2} \mathrm{Cl}_{6}=267$. This compound is of interest on account of being the salt from which the metal is obtained. Most chlorides may be obtained by dissolving the 
metal, its oxide, hydrate, or carbonate in hydrochloric acid. Accordingly, aluminium chloride may be obtained in solution :

$$
\mathrm{Al}_{2} 6 \mathrm{HO}+6 \mathrm{HCl}=\mathrm{Al}_{2} \mathrm{Cl}_{6}+6 \mathrm{H}_{2} \mathrm{O} .
$$

On evaporating the solution to dryness, however, and heating the dry mass farther with the view of expelling all water, decomposition takes place, hydrochloric acid escapes, and aluminium oxide is left:

$$
\mathrm{Al}_{2} \mathrm{Cl}_{6}+3 \mathrm{H}_{2} \mathrm{O}=\mathrm{Al}_{2} \mathrm{O}_{3}+6 \mathrm{HCl}
$$

Aluminium chloride cannot, consequently, be obtained in a pure state (free from water) by this process, but it may be made by exposing a heated mixture of aluminium oxide and carbon to the action of chlorine. Neither carbon nor chlorine alone causes any decomposition of the aluminium oxide, but by the united efforts of these two substances decomposition is accomplished :

$$
\mathrm{Al}_{2} \mathrm{O}_{3}+3 \mathrm{C}+6 \mathrm{Cl}=3 \mathrm{CO}+\mathrm{Al}_{2} \mathrm{Cl}_{6} \text {. }
$$

Clay is the name applied to a large class of mineral substances, differing considerably in composition, but possessing in common the two characteristic features of plasticity, and the predominance of aluminium silicate in combination with water.

The various kinds of clay have been formed in the course of time from such double silicates as feldspar and others, by a process which is partly of a mechanical, partly of a chemical nature, and consists chiefly in the disintegration of these rocks and a removal of potassium and sodium by the chemical action of carbonic acid, water, and other agents.

The various kinds of clay are used in the manufacture of bricks, earthenware, stoneware, porcelain, etc. The process of burning these substances accomplishes the hardening by expelling the water which is present in the clay. Pure clay is white; the red color of the common varieties is due to the presence of ferric oxide. For china or porcelain clay is used containing silicates of the alkalies which, in burning, melt, causing the production of a more homogeneous mass, while in common earthenware the pores, produced by expelling the moisture, remain unfilled. 
Glass is similar in composition to the better varieties of porcelain. All varieties of glass are mixtures of fusible, insoluble silicates, made by fusing silicic acid (white sand) with different metallic oxides or carbonates, the silicic acid combining chemically with the metals. Sodium and calcium are the chief metals in common glass, though potassium, lead, and others are also frequently used. The color is imparted to the glass by the addition of certain metallic oxides, which have a coloring effect, as, for instance, manganese violet, cobalt blue, chromium green, etc.

Ultramarine is a beautiful blue substance, found in nature as the mineral "lapis lazuli," which was highly valued by artists as a color before the discovery of the artificial process for manufacturing it.

Ultramarine is now manufactured on a very large scale by heating a mixture of clay, sodium sulphate, and carbonate, sulphur and charcoal in large crucibles, when decomposition takes place and the beautiful blue compound is obtained. As neither of the substances used in the manufacture has a tendency to form colored compounds, the formation of this blue ultramarine is rather surprising, and the true chemical constitution of it is yet unknown.

Ultramarine is insoluble in water and is decomposed by acids with liberation of sulphuretted hydrogen, which shows the presence of sulphide of sodium. A green ultramarine is now also manufactured.

\section{Analytical reactions.}

1. To solution of an aluminium salt add potassium or sodium hydrate: a white gelatinous precipitate of aluminium hydrate is produced, which is soluble in excess of the alkali.

2. To aluminium solution add ammonium hydrate: the same precipitate as above is obtained, but it is insoluble in an excess of the reagent.

3. The carbonates of ammonium, sodium, or potassium produce the same precipitate with liberation of carbon dioxide. (See explanation above.)

4. Ammonium sulphide produces the same precipitate with liberation of sulphuretted hydrogen:

$$
\mathrm{Al}_{2} \mathrm{Cl}_{6}+3\left(\mathrm{NH}_{4}\right)_{2} \mathrm{~S}+6 \mathrm{H}_{2} \mathrm{O}=\mathrm{Al}_{2} 6 \mathrm{HO}+6 \mathrm{NH}_{4} \mathrm{Cl}+3 \mathrm{H}_{2} \mathrm{~S} \text {. }
$$


5. Sodium phosphate produces precipitate of aluminium phosphate, soluble in acids.

\section{Questions.}

231. Mention some varieties of crystallized aluminium oxide found in nature and some silicates containing it.

232. Give the general formula of an alum, and mention some alums.

233. Which alum is officinal, how is it made, what are its properties, and what is it used for?

234. What is dried alum, and how does it differ from common alum?

235. How is aluminium chloride made, and how is the metal obtained from it?

236. State the properties of aluminium.

237. What change takes place when ammonium hydrate, and what change when sodium carbonate is added to a solution of alum?

238. What is the composition of earthenware, porcelain, and glass; how and from what materials are they manufactured?

239. What is ultramarine?

240. Give tests for aluminium compounds.

$$
\begin{gathered}
\text { 25. IRON. } \\
\mathrm{Fe}^{\mathrm{ii}}=\text { 55.9. } \quad \mathrm{Fe}_{2}^{\mathrm{vi}}=111.8 .
\end{gathered}
$$

General remarks regarding the metals of the iron group. The six metals ( $\mathrm{Fe}, \mathrm{Co}, \mathrm{Ni}, \mathrm{Mn}, \mathrm{Cr}, \mathrm{Zn}$ ) belonging to this group are distinguished by forming sulphides (chromium excepted) which are insoluble in water, but soluble in diluted mineral acids; they are, consequently, not precipitated from their neutral or acid solutions by hydrosulphuric acid, but by ammonium sulphide as sulphides (chromium as hydrate); their oxides, hydrates, carbonates, phosphates, and sulphides are insoluble; their chlorides, iodides, bromides, sulphates, and nitrates are soluble in water.

With the exception of zinc, these metals are strongly magnetic; they decompose water at a red heat, the oxide being formed and hydrogen liberated; in diluted hydrochloric or sulphuric acid, they dissolve with formation of chlorides or sulphates, respectively, and liberation of hydrogen.

With the exception of zinc, which is bivalent, the metals of the iron group are bivalent in some compounds, trivalent in 
others, and form a number of oxides, the higher of which show, in some cases, decided acid properties, as, for instance, chromic or manganic oxides.

Occurrence in nature. Among all the heavy metals, iron is both the most useful and the most widely and abundantly diffused in nature. It is to be found, though usually in but small quantities, in nearly all forms of rock, clay, sand, and earth; its presence in these being generally indicated by their color (red, reddish-brown, or yellowish-red), as iron is the most common of all natural, inorganic coloring agents. It is also found, though in small quantities, in plants, and in sornewhat larger proportions in the animal system, chiefly in the blood. In the metallic state iron is scarcely ever found, except in the meteorites or metallic masses which occasionally fall upon our earth from the universe.

The chief compounds of iron found in nature are:

Red hematite, ferric oxide, $\mathrm{Fe}_{2} \mathrm{O}_{3}$.

$\mathrm{Magnetic}$ iron ore, ferrous-ferric oxide, $\mathrm{FeO} \cdot \mathrm{Fe}_{2} \mathrm{O}_{3}$.

Spathic iron ore, ferrous carbonate, $\mathrm{FeCo}_{3}$.

Iron pyrites, bisulphide of iron, $\mathrm{FeS}_{2}$.

The carbonate and sulphate are sometimes found in spring waters, which, when containing considerable quantities of iron, are called chalybeate waters. Finally, iron is also a constituent of some organic substances, which are of importance in the animal system.

Manufacture of iron. There is no other metal that is manufactured in such immense quantities as iron, the use of which in thousands of different tools, machines, and appliances is highly characteristic of our present age. Iron is manufactured from the above-named oxides or the carbonate by heating with coal, limestone, and sand in large blast furnaces, which have a somewhat cylindrical shape, and are constantly fed from above with a mixture of the substances named, while hot air is forced into the furnace through suitable apertures from the side below. The chemical change taking place in the upper and less heated part of the furnace is a deoxidation of the ferric oxide or ferrous carbonate by the carbon:

$$
\begin{aligned}
& \mathrm{Fe}_{2} \mathrm{O}_{3}+3 \mathrm{C}=3 \mathrm{CO}+2 \mathrm{Fe} \\
& \mathrm{FeCO}_{3}+\mathrm{C}=\mathrm{CO}+\mathrm{CO}_{2}+\mathrm{Fe} .
\end{aligned}
$$


The heat necessary for this decomposition and fusion of the ore is produced by the combustion of the coal, maintained by the oxygen of the air blown into the furnace. The same air, however, would also burn (oxidize) the iron in the lower and hotter part of the furnace, were it not protected from coming in contact with it by a thin film of a fusible silicate (slag), formed by the combination of the sand and calcium of the limestone, which is added for that purpose. The iron and slag collect at the bottom of the furnace, and are allowed to run off every few hours.

Iron thus obtained is known as cast-iron, and is not pure, but always contains, besides traces of silica (occasionally also sulphur, phosphorus, and various metals), a quantity of carbon varying from 2 to 5 per cent. It is the quantity of this carbon which imparts to the different kinds of iron different properties. Steel contains from 0.5 to 2 per cent., wrought- or bar-iron from 0.03 to 0.3 per cent. of carbon. Wrought-iron is made from cast-iron by the process known as puddling, which is a burning out of the carbon by oxidation. Steel is made either from cast-iron by partially removing the carbon, or from wroughtiron by recombining it with carbon.

Properties. The high position which iron occupies among the useful metals is due to a combination of valuable properties not found in any other metal. Although possessing nearly twice as great a tenacity or strength as any of the other metals commonly used in the metallic state, it is yet one of the lightest, its specific gravity being about 7.7. Though being when cold the least yielding or malleable of the metals in common use, its ductility when heated is such that it admits of being rolled into the thinnest sheets and drawn into the finest wire, the strength of which is so great that a wire of onetenth of an inch in diameter is capable of sustaining 700 pounds. Finally, iron is, with the exception of platinum, the least fusible of all the useful metals.

Iron is little affected by dry air, but is readily acted upon by moist air, when ferric oxide and ferric hydrate (rust) are formed.

Iron forms two series of compounds, distinguished as ferrous and ferric compounds; in the former, iron is bivalent, in the latter trivalent, but it is most likely that the trivalent atom of 
iron is always present as a double atom, and consequently ferric chloride for instance has not the composition $\mathrm{FeCl}_{3}$, but $\mathrm{Fe}_{2} \mathrm{Cl}_{6}$. As the reactions of iron in ferrous and ferric compounds differ considerably, they must be studied separately.

Reduced iron, Ferrum reductum. This is metallic iron, obtained as a very fine, grayish-black powder by passing hydrogen gas (purified and dried by passing it through sulphuric acid) over ferric oxide, heated in a glass tube:

$$
\mathrm{Fe}_{2} \mathrm{O}_{3}+6 \mathrm{H}=3 \mathrm{H}_{2} \mathrm{O}+2 \mathrm{Fe} .
$$

Ferrous oxide, Fe0 (Monoxide or suboxide of iron). This compound is little known in the separate state, as it has (like most ferrous compounds) a great tendency to absorb oxygen from the air. The ferrous hydrate $\mathrm{Fe}_{2} \mathrm{HO}$ may be obtained by the addition of any alkaline hydrate to the solution of any ferrous salt, when a white precipitate is produced which rapidly turns bluishgreen, dark gray, black and finally brown, in consequence of the absorption of oxygen :

$$
\begin{gathered}
\mathrm{FeSO}_{4}+2 \mathrm{NH}_{4} \mathrm{HO}=\left(\mathrm{NH}_{4}\right)_{2} \mathrm{SO}_{4}+\mathrm{Fe} 2 \mathrm{HO} \\
2(\mathrm{Fe} 2 \mathrm{HO})+\mathrm{O}+\mathrm{H}_{2} \mathrm{O}=\mathrm{Fe}_{2} 6 \mathrm{HO} .
\end{gathered}
$$

The precipitation of ferrous hydrate is not complete, some iron always remaining in solution.

Ferrous oxide is a strong base, uniting with acids to form salts, which have usually a pale green color.

Ferric oxide, $\mathrm{Fe}_{2} \mathbf{0}_{3}$. A reddish-brown powder, which may be obtained by heating ferric hydrate to expel water :

$$
\mathrm{Fe}_{2} 6 \mathrm{HO}=\mathrm{Fe}_{2} \mathrm{O}_{3}+3 \mathrm{H}_{2} \mathrm{O} .
$$

It is a feeble base; its salts show usually a brown color.

Ferric hydrate, Ferri oxidum hydratum, $\mathrm{Fe}_{2} 6 \mathrm{HO}=213.8(\mathrm{Hy}$ drated oxide of iron, Per- or sesqui-oxide, Red oxide of iron), is obtained by the precipitation of ferric sulphate or ferric chloride by ammonium or sodium hydrate:

$$
\mathrm{Fe}_{2} 3 \mathrm{SO}_{4}+6 \mathrm{NH}_{4} \mathrm{HO}=3\left[\left(\mathrm{NH}_{4}\right)_{2} \mathrm{SO}_{4}\right]+\mathrm{Fe}_{2} 6 \mathrm{HO} .
$$

The precipitation is complete, no iron remaining in solution as in the case of ferrous salts. 
Ferric hydrate is a reddish-brown powder, frequently used as an antidote in arsenic poisoning; for this purpose it is not used in the dry state, but after having been freshly precipitated and washed, it is mixed with water, and this mixture used. Recently precipitated and consequently highly divided ferric hydrate combines more readily with arsenious acid than the hydrate which has been kept some time, or which has been dried, and thereby assumed a more dense condition.

Hydrated oxide of iron with magnesia, U. S. P., is a mixture made by adding magnesia to a solution of ferric sulphate, when magnesium sulphate and ferric hydrate are formed; the two substances are not separated from each other, the mixture being intended for immediate administration as an antidote in cases of arsenic poisoning.

Ferrous-ferric oxide, $\mathrm{FeO}_{\mathrm{Fe}} \mathrm{O}_{3}$ (Magnetic oxide). This compound, which shows strong magnetic properties, has been mentioned above as one of the iron ores. It has a black color, and is produced in the hydrated state by the addition of ammonium hydrate to a mixture of solutions of ferrous and ferric salts.

Trioxide of iron, $\mathrm{FeO}_{3}$. Not known in a separate state, but in combination with alkalies. In these compounds, called ferrates, $\mathrm{FeO}_{3}$ acts as an acid oxide, analogous to chromic oxide, $\mathrm{CrO}_{3}$, in chromates.

Ferrous chloride, $\mathrm{FeCl}_{2}$ (Protochloride of iron), is obtained as a pale green solution by dissolving iron in hydrochloric acid:

$$
\mathrm{Fe}+2 \mathrm{HCl}=\mathrm{FeCl}_{2}+2 \mathrm{H} .
$$

By evaporation of the solution, the dry salt may be obtained. The solution and salt absorb oxygen very readily:

$$
3 \mathrm{FeCl}_{2}+\mathrm{O}=\mathrm{FeO}+\mathrm{Fe}_{2} \mathrm{Cl}_{6} .
$$

Ferric chloride, ferrous, and afterwards ferric oxide, are formed.

Ferric chloride, Ferri chloridum, $\mathrm{Fe}_{2} \mathrm{Cl}_{6} \cdot 12 \mathrm{H}_{2} \mathrm{O}=\mathbf{5 4 0 . 2}$ (Chloride, sesquichloride, or perchloride of iron), is obtained by adding to the solution of ferrous chloride (obtained as mentioned above) hydrochloric and nitric acids in sufficient quantities, and applying 
heat until complete oxidation has taken place. The nitric acid oxidizes the hydrogen of the hydrochloric acid to water, while the chlorine combines with the ferrous chloride, nitrogen dioxide being also formed :

$$
6 \mathrm{FeCl}_{2}+2 \mathrm{HNO}_{3}+6 \mathrm{HCl}=3 \mathrm{Fe}_{2} \mathrm{Cl}_{6}+4 \mathrm{H}_{2} \mathrm{O}+2 \mathrm{NO} .
$$

By sufficient evaporation of the solution, ferric chloride is obtained as a crystalline mass of an orange-yellow color; it is very deliquescent, has an acid reaction, and a strongly styptic taste. The water of crystallization cannot be expelled by heat, as decomposition of the salt takes place, free hydrochloric acid and ferric oxide being formed.

Solution of chloride of iron, Liquor ferri chloridi, U. S. P. This is a solution in water, containing 37.8 per cent. of the anhydrous ferric chloride. It is a reddish-brown liquid of specific gravity 1.405, having the taste and reaction of the dry salt. This solution, mixed with about 2 parts of alcohol and left standing in a closed vessel for several months, forms the tincture of chloride of iron, Tinctura ferri chloridi. U. S. P.

Dialyzed iron is an aqueous solution of about 5 per cent. of ferric hydrate with some ferric chloride. It is made by slowly adding to a solution of ferric chloride, ammonium hydrate as long as the precipitate of ferric hydrate formed is redissolved in the ferric chloride solution on shaking violently. The clear solution thus obtained is placed in a dialyzer, floating in water, which latter is renewed every day until it shows no reaction with silver nitrate. The ammonium chloride passes through the membrane of the dialyzer into the water, while all irou as hydrate with some chloride is left in solution.

The combination of an oxide or hydrate with a neutral salt is usually called a basic salt or oxy-salt; dialyzed iron is a highly basic oxychloride of iron.

Ferrous iodide, $\mathrm{FeI}_{2}$. When water is poured upon a mixture of metallic iron (fine wire is best) and iodine, the two elements combine directly, forming a pale green solution of the ferrous iodide, from which the salt may be obtained by evaporation. 
As it is easily oxidized and decomposed by the action of the air, an officinal preparation, the succharated iodide of iron, U. S. P., is made by adding about 80 parts of sugar. of milk to 20 parts of the ferrous iodide; the sugar prevents, to some extent, rapid oxidation.

Ferrous bromide, $\mathrm{FeBr}_{2}$. Made analogously to ferrous iodide, by the action of bromine on metallic iron.

Ferrous sulphide, FeS. Easily obtained as a black, brittle mass, by heating iron filings with sulphur, when the elements combine. It is chiefly used for liberating hydrosulphuric acid, by the addition of sulphuric acid. Iron combines with sulphur in several proportions; some of these iron sulphides are found in nature.

Ferrous sulphate, Ferri sulphas, $\mathrm{FeSO}_{4} \cdot 7 \mathrm{H}_{2} \mathbf{0}=277.9$ (Sulphate of iron, Green vitriol, Copperas). Obtained by dissolving iron in sulphuric acid, evaporating, and crystallizing:

$$
\mathrm{Fe}+\mathrm{H}_{2} \mathrm{SO}_{4}=2 \mathrm{H}+\mathrm{FeSO}_{4} .
$$

Also obtained as a by-product in some branches of chemical industry, or by oxidation of the native sulphide of iron:

$$
\mathrm{FeS}_{2}+4 \mathrm{O}=\mathrm{FeSO}_{4}+\mathrm{S} .
$$

Ferrous sulphate crystallizes in large, blnish-green prisms; it is soluble in water, insoluble in alcohol. Exposed to the air, it loses water of crystallization, and absorbs oxygen.

The dried ferrous sulphate, U. S. P., is made by expelling all water by heat; the precipitated ferrous sulphate is made by pouring a strong aqueous solution of ferrous sulphate, slightly acidulated with sulphuric acid, into alcohol, when ferrous sulphate separates as a crystalline powder, which is washed and dried.

Ferric sulphate, $\mathrm{Fe}_{2} 3 \mathrm{SO}_{4}$. The solution of this salt, Liquor ferri tersulphatis, solution of tersulphate of iron, U. S. P., is made by adding sulphuric and nitric acids to a solution of ferrous sulphate, and heating:

$$
6 \mathrm{FeSO}_{4}+3 \mathrm{H}_{2} \mathrm{SO}_{4}+2 \mathrm{HNO}_{3}=3\left(\mathrm{Fe}_{2} 3 \mathrm{SO}_{4}\right)+2 \mathrm{NO}+4 \mathrm{H}_{2} \mathrm{O} .
$$


The action of nitric acid is similar to that described above under ferric chloride. The hydrogen of the sulphuric acid is oxidized, and the liberated radical $\mathrm{SO}_{4}$ unites with the ferrous sulphate, nitrogen dioxide being liberated.

Solution of subsulphate of iron, Liquor ferri subsulphatis (Monsel's solution). This is a solution similar to the preceding, but contains less sulphuric acid, and is therefore looked upon as a basic ferric sulphate, of the doubtful composition $\mathrm{Fe}_{4} \mathrm{O}_{5} \mathrm{SO}_{4}$. The color of the tersulphate of iron solution is reddish-brown; that of Monsel's solution is ruby-red.

Ferric nitrate, $\mathbf{F e}_{2} 6 \mathrm{NO}_{3}$. A 6 per cent. solution of this salt is officinal, under the name solution of nitrate of iron, Liquor ferri nitratis, U.S.P., and is made by dissolving ferric hydrate in nitric acid :

$$
\mathrm{Fe}_{2} 6 \mathrm{HO}+6 \mathrm{HNO}_{3}=6 \mathrm{H}_{2} \mathrm{O}+\mathrm{Fe}_{2} 6 \mathrm{NO}_{3} .
$$

It is an amber-colored or reddish, acid liquid.

Ferrous carbonate, $\mathrm{FeCO}_{3}$. Occurs in nature; may be obtained by mixing solutions of ferrous sulphate and sodium carbonate or bicarbonate:

$$
\mathrm{FeSO}_{4}+\mathrm{Na}_{2} \mathrm{CO}_{3}=\mathrm{Na}_{2} \mathrm{SO}_{4}+\mathrm{FeCO}_{3}
$$

The precipitate is first nearly white, but soon assumes a gray color by oxidation. The saccharated carbonate of iron, U. S. P., is made by mixing the washed precipitate with sugar, and drying. The sugar prevents, to some extent, rapid oxidation.

Ferric carbonate does not exist, the affinity between the feeble ferric oxide and the weak carbonic acid not being sufficient to unite them chemically.

Ferrous phosphate, $\mathrm{Fe}_{3} 2 \mathrm{PO}_{4}$. Obtained as a slate-colored precipitate, when sodium phosphate is added to ferrous sulphate. Like all ferrous salts, it absorbs oxygen from the air, becoming darker in color :

$$
3 \mathrm{FeSO}_{4}+2 \mathrm{Na}_{2} \mathrm{HPO}_{4}=\mathrm{Fe}_{3} 2 \mathrm{PO}_{4}+2 \mathrm{Na}_{2} \mathrm{SO}_{4}+\mathrm{H}_{2} \mathrm{SO}_{4}
$$


As shown by this formula, some sulphuric acid is set free, which has a dissolving action on the ferrous phosphate; to prevent which, sodium acetate may be added forming sodium sulphate and free acetic acid, which latter does not act as a solvent.

Ferric hypophosphite, Ferri hypophosphis, $\mathrm{Fe}_{2} 6 \mathrm{H}_{2} \mathrm{PO}_{2}=501.8$ (Hypophosphite of iron). Made by dissolving ferric hydrate in hypophosphoric acid, and evaporating. It is a grayish-white powder, insoluble in water, soluble in hydrochloric acid.

Scale compounds of iron. Quite a number of officinal preparations of iron are made by mixing solution of ferric citrate (obtained by dissolving ferric hydrate in citric acid) with solutions of other salts, evaporating them to the consistence of thick syrup, spreading this on glass plates, and drying at a low temperature, when the compounds are obtained in the form of thin, translucent scales.

While the Ferri phosphas of the U. S. P. of 1870 was the above-mentioned ferrous phosphate, $\mathrm{Fe}_{3} 2 \mathrm{PO}_{4}$, the Ferri phosphas, phosphate of iron, of the U. S. P. of 1880 is a mixture (or, most likely, double compound) obtained by evaporation of a mixture of ferric citrate and sodium phosphate.

The ferric pyrophosphate of the U. S. P. is a similar scale compound. Of others may be mentioned: Citrate of iron and ammonia, citrate of iron and quinine, citrate of iron and strychnine, etc. In some cases, tartaric acid is used in place of citric acid, as, for instance, in tartrate of iron and ammonium, tartrate of iron and potassium, etc.

Compounds of iron with organic acids will be more fully considered in connection with the acids themselves.

The object of these various organic scale compounds of iron is, doubtless, to present otherwise insuluble iron compounds in a soluble and convenient form for administration. 


\section{Analytical reactions.}

Ferrous salts.

1. Ammonium sulphide.

2. Hydrosulphuric acid.

3. Ammonium, sodium, or potassium hydrate.

4. Ammonium, sodium, or potassium carbonate.

5. Alkaline phosphates or arseniates.

6. Potassium ferrocyanide,

$\mathrm{K}_{4} \mathrm{FeCY}_{6} \cdot{ }^{1}$

7. Potassium ferricyanide,

$\mathrm{K}_{6} \mathrm{Fe}_{2} \mathrm{Cy}_{12}$.

8. Tannic acid.

9. Potassium sulphocyanate.
Black precipitate of ferrous sulphide.

$\mathrm{FeSO}_{4}+\left(\mathrm{NH}_{4}\right)_{2} \mathrm{~S}=$

$\left(\mathrm{NH}_{4}\right)_{2} \mathrm{SO}_{4}+\mathrm{FeS}$.

No change.

White precipitate of ferrous hydrate, soon turning green, black, and brown. Precipitation not complete.

$$
\begin{gathered}
\mathrm{FeCl}_{2}+2 \mathrm{NaHO}= \\
2 \mathrm{NaCl}+\mathrm{Fe} 2 \mathrm{HO} .
\end{gathered}
$$

White precipitate of ferrous carbonate, soon turning darker.

$$
\begin{gathered}
\mathrm{FeCl}_{2}+\mathrm{Na}_{2} \mathrm{CO}_{3}= \\
2 \mathrm{NaCl}+\mathrm{FeCO}_{3} .
\end{gathered}
$$

Almost white precipitate, soon turning darker.

Almost white precipitate, soon turning blue by absorption of oxygen.

Blue precipitate of ferrous ferricyanide, or Turnbull's blue.

$3 \mathrm{FeCl}_{2}+\mathrm{K}_{6} \mathrm{Fe}_{2} \mathrm{Cy}_{12}=$ $6 \mathrm{KCl}+\mathrm{Fe}_{3} \mathrm{Fe}_{2} \mathrm{Cy}_{12}$.

No change, provided oxidation of the ferrous salt has not taken place.

As above.
Ferric salts.

Black precipitate of ferro's sulphide mixed with sulphur.

$$
\begin{gathered}
\mathrm{Fe}_{2} \mathrm{Cl}_{6}+3\left[\left(\mathrm{NH}_{4}\right)_{2} \mathrm{~S}\right]= \\
6 \mathrm{NH}_{4} \mathrm{Cl}+2 \mathrm{FeS}+\mathrm{S} .
\end{gathered}
$$

Ferric salts are converted into ferrous salts with precipitation of sulphur.

$$
\begin{gathered}
\mathrm{Fe}_{2} \mathrm{Cl}_{6}+\mathrm{H}_{2} \mathrm{~S}= \\
2 \mathrm{FeCl}_{2}+2 \mathrm{HCl}+\mathrm{S} .
\end{gathered}
$$

Reddish-brown precipitate of ferric hydrate. Precipitation is complete.

$$
\begin{gathered}
\mathrm{Fe}_{2} \mathrm{Cl}_{6}+6\left(\mathrm{NH}_{4} \mathrm{HO}\right)= \\
6 \mathrm{NH}_{4} \mathrm{Cl}+\mathrm{Fe}_{2} 6 \mathrm{HO} .
\end{gathered}
$$

Reddish-brown precipitate of ferric hydrate, with liberation of carbon dioxide.

$\mathrm{Fe}_{2} \mathrm{Cl}_{6}+3 \mathrm{Na}_{2} \mathrm{CO}_{3}+3 \mathrm{H}_{2} \mathrm{O}=$ $6 \mathrm{NaCl}+\mathrm{Fe}_{2} 6 \mathrm{HO}+3 \mathrm{CO}_{2}$.

A yellowish-white precipitate is produced.

Dark blue precipitate of ferric ferrocyanide, or Prussian blue. Decomposed by alkalies; insoluble in acids.

$$
\begin{gathered}
2 \mathrm{Fe}_{2} \mathrm{Cl}_{6}+3\left(\mathrm{~K}_{4} \mathrm{FeCy}_{6}\right)= \\
12 \mathrm{KCl}+\mathrm{Fe}_{4} 3 \mathrm{FeCy}_{6} .
\end{gathered}
$$

No precipitate is produced, but the liquid is darkened to a greenish or olive hue.

A dark greenish-black precipitate of tannate of iron (ink) is produced.

Deep blood-red precipitate of ferric sulphocyanate. 


\section{Questions.}

241. Which metals belong to the "iron group," and what are their general properties?

242. How is iron found in nature, and what compounds are used in its mannfacture?

243. Describe the process for manufacturing iron on a large scale, and state the difference between cast-iron, wrought-iron, steel, and reduced iron.

244. State the composition and mode of preparation of ferrous and ferric hydrates. What are their properties?

245. Describe in words and chemical symbols the process for making ferric chloride. What is tincture of chloride of iron?

246. How are ferrous iodide and bromide made?

247. State the properties of ferrous sulphate. Under what other names is it known, and how is it made?

248. What change takes place when soluble carbonates are added to soluble ferrous and ferric salts?

249. What is the composition of ferrous phosphate, and what is the phosphate of iron, U. S. P.?

250. Mention tests for ferrous and ferric compounds.

\section{MANGANESE-CHROMIUM-COBALT-NICKEL.}

Manganese, $\mathbf{M n}=54$. Manganese is found either as dioxide (black oxide of manganese, or pyrolusite), $\mathrm{MnO}_{2}$, or as sesquioxide, $\mathrm{Mn}_{2} \mathrm{O}_{3}$. In small quantities it is a constituent of many minerals.

Metallic manganese resembles iron in its physical and chemical properties, and may be obtained by reducing the carbonate with charcoal. Manganese is darker in color than iron, considerably harder, and somewhat more easily oxidized.

0xides of manganese. Four well-defined compounds of manganese with oxygen are known in the separate state, and two others only in combination with other elements.

These oxides are :

Manganese oxide (monoxide or protoxide), MnO.

Manganous-manganic oxide, $\mathrm{MnO} . \mathrm{Mn}_{2} \mathrm{O}_{3}=\mathrm{Mn}_{3} \mathrm{O}_{4}$.

Manganic oxide (sesquioxide), $\mathrm{Mn}_{2}\left(\mathrm{I}_{3}\right.$

Manganese dioxide (binoxide, peroxide, black oxide), $\mathrm{MnO}_{2}$.

Manganic acid, $\left\{\right.$ Normanganic acid, known in a separate state $\left\{\begin{array}{l}\mathrm{H}_{2} \mathrm{O}+\mathrm{MnO}_{3} . \\ \mathrm{H}_{2} \mathrm{O}+\mathrm{Mn}_{2} \mathrm{O}_{7} .\end{array}\right.$ 
Manganous oxidr is a greenish-gray powder, obtainable by heating the carbonate; or as a nearly white hydrate by precipitating a manganous salt by sodium hydrate. It is a strong base, saturating acids completely, and forming salts which hare generally a rose color or a pale reddish tint.

Manganese dioxide, Mangani oxidum nigrum, $\mathrm{MnO}_{2}=86$. This is by far the most important compound of manganese, as it is largely used for generating chlorine:

$$
\mathrm{MnO}_{2}+4 \mathrm{HCl}=\mathrm{MnCl}_{2}+2 \mathrm{H}_{2} \mathrm{O}+2 \mathrm{Cl} .
$$

It is a heavy, grayish-black, crystalline mineral, liberating. oxygen when heated to redness:

$$
3 \mathrm{MnO}_{2}=\mathrm{Mn}_{3} \mathrm{O}_{4}+2 \mathrm{O} .
$$

Manganous sulphate, Mangani sulphas, $\mathrm{MnSO}_{4} \cdot 4 \mathrm{H}_{2} \mathrm{O}=222$, may be obtained by dissolving the oxide or dioxide in sulphuric acid; in the latter case oxygen is evolved:

$$
\mathrm{MnO}_{2}+\mathrm{H}_{2} \mathrm{SO}_{4}=\mathrm{MnSO}_{4}+\mathrm{H}_{2} \mathrm{O}+\mathrm{O} .
$$

It is an almost colorless, or pale, rose-colored substance, isomorphous with the sulphates of magnesium and zinc; it is easily soluble in water.

Potassium permanganate, Potassii permanganas, $\mathbf{K}_{2} \mathbf{M} \mathbf{n}_{2} \mathbf{O}_{8}=\mathbf{3 1 4}$ (Permanganate of potassium). Whenever a compound (any oxide or salt) of manganese is fused with alkaline carbonates (or hydrates) and alkaline nitrates (or chlorates) the manganese is converted into manganic acid, which combines with the alkali, forming potassium (or sodium) manganate:

$$
3 \mathrm{NnO}_{2}+3 \mathrm{~K}_{2} \mathrm{CO}_{3}+\mathrm{KClO}_{3}=3 \mathrm{~K}_{2} \mathrm{MnO}_{4}+3 \mathrm{CO}_{2}+\mathrm{KCl} \text {. }
$$

The fused mass has a dark green color, and when dissolved in water gires a dark emerald-green solution, from which, by evaporation, green crystals of potassium manganate may be obtained.

The green solution is easily decomposed by any acid (or even by water in large quantity) into a red solution of potassium permanganate and a precipitate of dioxide of manganese:

$$
3 \mathrm{~K}_{2} \mathrm{MnO}_{4}+2 \mathrm{H}_{2} \mathrm{SO}_{4}=\mathrm{MnO}_{2}+2 \mathrm{~K}_{2} \mathrm{SO}_{4}+\mathrm{K}_{2} \mathrm{Mn}_{2} \mathrm{O}_{3}+2 \mathrm{H}_{2} \mathrm{O} .
$$


By evaporation and crystallization the potassium permanganate is obtained in slender, prismatic crystals, of a deep, purpleviolet color and a somewhat metallic lustre. The solution in water has a deep purple or, when highly diluted, a pink color. It is a powerful oxidizing agent and an excellent disinfectant, both properties being due to the facility with which a portion of the oxygen is given off to any substance which has affinity for it:

$$
\mathrm{K}_{2} \mathrm{Mn}_{2} \mathrm{O}_{8}+6 \mathrm{HCl}+x=2 \mathrm{KCl}+2 \mathrm{MnCl}_{2}+3 \mathrm{H}_{2} \mathrm{O}+x \mathrm{O}_{5} .
$$

$x$ represents here any substance capable of combining with oxygen while in solution.

\section{Analytical reactions.}

1. Ammonium sulphide produces, with manganous salts, a yellowish-pink or flesh-colored precipitate of manganous sulphide, soluble in acetic and in mineral acids:

$$
\mathrm{MnSO}_{4}+\left(\mathrm{NH}_{4}\right)_{2} \mathrm{~S}=\left(\mathrm{NH}_{4}\right)_{2} \mathrm{SO}_{4}+\mathrm{MnS} .
$$

2. Ammonium hydrate produces a white precipitate of manganous hydrate, which soon darkens by absorption of oxygen. The precipitate is soluble in excess of ammonia.

$$
\mathrm{MnCl}_{2}+2 \mathrm{NH}_{4} \mathrm{HO}=2 \mathrm{NH}_{4} \mathrm{Cl}+\mathrm{Mn} 2 \mathrm{HO} .
$$

3. Potassium and sodium hydrates show the same reaction; the precipitate is insoluble in excess of the alkaline hydrates.

4. Sodium (or potassium) carbonate produces a precipitate of manganous carbonate :

$$
\mathrm{MnSO}_{4}+\mathrm{Na}_{2} \mathrm{CO}_{3}=\mathrm{Na}_{2} \mathrm{SO}_{4}+\mathrm{MnCO}_{3} .
$$

5. Any compound of manganese heated on platinum foil with a mixture of sodium carbonate and nitrate forms a green mass, giving a green solution in water, which turns red on addition of an acid. (See explanation above.)

6. Manganese compounds fused with borax on a platinum wire give a violet coloration to the borax bead.

7. Traces of manganese may be detected by boiling with dilute nitric acid and red lead, when the solution acquires a violet color due to the formation of permanganic acid. 
Chromium, $\mathrm{Cr}=$ 52.4. Found in nature almost exclusively as chrome-iron ore, $\mathrm{FeO} . \mathrm{Cr}_{2} \mathrm{O}_{3}$, a mineral analogous in composition to magnetic iron ore, $\mathrm{FeO} . \mathrm{Fe}_{2} \mathrm{O}_{3}$. The name chromium, from the Greek $\chi \rho \tilde{\omega} \mu a$, (chroma), color, was given to this metal on account of the beautiful colors of its different compounds, none of which is colorless. Chromium forms a basic oxide, $\mathrm{Cr}_{2} \mathrm{O}_{3}$, and an acid oxide, $\mathrm{CrO}_{3}$, the combinations and reactions of which have to be studied separately.

Potassium dichromate, Potassii bichromas, $\mathrm{K}_{2} \mathrm{Cr}_{2} \mathbf{0}_{7^{\circ}}=294.8$ (Bi(chromate or red chromate of potassium). This salt is by far the most important of all combinations of chromium, and is the source from which they are obtained.

Potassium dichromate is manufactured on a large scale by exposing a mixture of the finely ground chrome-iron ore with potassium carbonate and calcium hydrate to the heat of an oxidizing flame in a reverberatory furnace, when both constituents of the ore become oxidized, ferric oxide and chromic acid being formed, the latter combining with the potassium, forming neutral potassium chromate, $\mathrm{K}_{2} \mathrm{CrO}_{4}$ :

$$
2\left(\mathrm{FeOCr}_{2} \mathrm{O}_{3}\right)+4 \mathrm{~K}_{2} \mathrm{CO}_{3}+7 \mathrm{O}=\mathrm{Fe}_{2} \mathrm{O}_{3}+4 \mathrm{CO}_{2}+4\left(\mathrm{~K}_{2} \mathrm{CrO}_{4}\right) .
$$

By treating the heated mass with water, a yellow solution of potassium chromate is obtained, which, upon the addition of sulphuric acid, is decomposed into potassium dichromate and potassium sulphate:

$$
2\left(\mathrm{~K}_{2} \mathrm{CrO}_{4}\right)+\mathrm{H}_{2} \mathrm{SO}_{4}=\mathrm{K}_{2} \mathrm{Cr}_{2} \mathrm{O}_{7}+\mathrm{K}_{2} \mathrm{SO}_{4}+\mathrm{H}_{2} \mathrm{O} .
$$

The two salts may be separated by crystallization. Potassium dichromate forms large, orange-red, transparent crystals, which are easily soluble in water; heated by itself oxygen is evolved, heated with hydrochloric acid chlorine is liberated, heated with organic matter or reducing agents these are oxidized.

To explain the constitution of dichromates we have to assume that chromic anhydride, $\mathrm{CrO}_{3}$, is capable of forming two acids:

$$
\begin{gathered}
\mathrm{CrO}_{3}+\mathrm{H}_{2} \mathrm{O}=\mathrm{H}_{2} \mathrm{CrO}_{4}=\text { Chromic acid. } \\
2 \mathrm{CrO}_{3}+\mathrm{H}_{2} \mathrm{O}=\mathrm{H}_{2} \mathrm{Cr}_{2} \mathrm{O}_{7}=\text { Dichromic acid. }
\end{gathered}
$$

Chromium trioxide, Acidum chromicum, $\mathrm{CrO}_{3}=100.4$ (Chromic acid, Chromic anhydride), is prepared by adding sulphuric acid to 
a saturated solution of potassium dichromate, when chromium trioxide separates in crystals:

$$
\mathrm{K}_{2} \mathrm{Cr}_{2} \mathrm{O}_{7}+\mathrm{H}_{2} \mathrm{SO}_{4}=\mathrm{K}_{2} \mathrm{SO}_{4}+\mathrm{H}_{2} \mathrm{O}+2 \mathrm{CrO}_{3} \text {. }
$$

Thus prepared, it forms crimson, needle-shaped crystals, which are deliquescent, and very soluble in water; it is powerfully corrosive, and one of the strongest oxidizing agents; the solution in water has strong acid properties; it combines with metallic oxides forming chromates and dichromates.

Chromic oxide, $\mathrm{Cr}_{2} \mathrm{O}_{3}$ (Sesquioxide of chromium), is obtained by heating potassium dichromate with sulphur, when potassium sulphate and chromic oxide are formed:

$$
\mathrm{K}_{2} \mathrm{Cr}_{2} \mathrm{O}_{\mathrm{i}}+\mathrm{S}=\mathrm{K}_{2} \mathrm{SO}_{4}+\mathrm{Cr}_{2} \mathrm{O}_{3} .
$$

By washing the heated mass with water, the chromic oxide is left as a green powder, which is insoluble in water and in acids; it is a basic oxide combining with acids to form salts; it is used as a green color, especially in the manufacture of painted glass and porcelain.

Chromic hydrate, $\mathrm{Cr}_{2} 6 \mathrm{H} 0$. A solution of potassium dichromate may be deoxidized by the action of hydrosulphuric acid, sulphurous acid (or any other deoxidizing agent) in the presence of sulphuric or hydrochloric acid:

$$
\mathrm{K}_{2} \mathrm{Cr}_{2} \mathrm{O}_{7}+4 \mathrm{H}_{2} \mathrm{SO}_{4}+3 \mathrm{H}_{2} \mathrm{~S}=\mathrm{K}_{2} \mathrm{SO}_{4}+7 \mathrm{H}_{2} \mathrm{O}+3 \mathrm{~S}+\mathrm{Cr}_{2} 3 \mathrm{SO}_{4} .
$$

As shown by this formula, the sulphates of potassium and chromium are formed and remain in solution, while sulphur is precipitated, the hydrogen of the hydrosulphuric acid having been oxidized and converted into water.

By adding ammonium hydrate to the solution thus obtained, chromic hydrate is precipitated as a bluish-green gelatinous substance:

$$
\mathrm{Cr}_{2} 3 \mathrm{SO}_{4}+6 \mathrm{NH}_{4} \mathrm{HO}=3\left[\left(\mathrm{NH}_{4}\right)_{2} \mathrm{SO}_{4}\right]+\mathrm{Cr}_{2} 6 \mathrm{HO} .
$$

By dissolving this hydrate in the different acids, the various salts, such as chloride, $\mathrm{Cr}_{2} \mathrm{Cl}_{6}$, sulphate, etc., are obtained. Chromic sulphate, similar to aluminium sulphate, combines with potassium or ammonium sulphate and water, forming 
chrome alum, $\mathrm{K}_{2} \mathrm{SO}_{4} \cdot \mathrm{Cr}_{2} 3 \mathrm{SO}_{4} \cdot 24 \mathrm{H}_{2} \mathrm{O}$; it is a purple salt, and is isomorphous with other alums.

\section{Analytical reactions.}

a. Of chromic acid or chromates.

1. Hydrosulphuric acid added to an acidified solution of a chromate, changes the red color into green with precipitation of sulphur. The solution now contaius chromium in the basic form. (See explanation above.)

2. Soluble lead salts produce a yellow precipitate of lead chromate (chrome-yellow), $\mathrm{PbCrO}_{4}$, insoluble in acetic, soluble in hydrochloric acid:

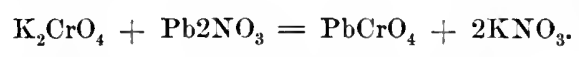

3. Barium chloride produces a pale yellow precipitate of barium chromate, $\mathrm{BaCrO}_{4}$ :

$$
\mathrm{K}_{2} \mathrm{CrO}_{4}+\mathrm{BaCl}_{2}=\mathrm{BaCrO}_{4}+2 \mathrm{KCl} \text {. }
$$

4. Silver nitrate produces a dark red precipitate of silver chromate, $\mathrm{Ag}_{2} \mathrm{CrO}_{4}$ :

$$
2 \mathrm{AgNO}_{3}+\mathrm{K}_{2} \mathrm{CrO}_{4}=2 \mathrm{KNO}_{3}+\mathrm{Ag}_{2} \mathrm{CrO}_{4} .
$$

5. Mercurous nitrate produces a red precipitate of mercurous chromate, $\mathrm{Hg}_{2} \mathrm{CrO}_{4}$.

\section{b. Of salts of chromium.}

6. To chromic chloride oi sulphate add ammonium hydrate or ammonium sulphide: in both cases the green hydrate of chromium is precipitated:

$$
\mathrm{Cr}_{2} \mathrm{Cl}_{6}+3\left[\left(\mathrm{NH}_{4}\right)_{2} \mathrm{~S}\right]+6 \mathrm{H}_{2} \mathrm{O}=6 \mathrm{NH}_{4} \mathrm{Cl}+3 \mathrm{H}_{2} \mathrm{~S}+\mathrm{Cr}_{2} 6 \mathrm{HO} .
$$

7. Potassium or sodium hydrate causes a similar green precipitate of chromic hydrate, which is soluble in an excess of the reagent, but is reprecipitated on boiling for a few minutes.

\section{c. Of chromium in any form.}

8. Compounds of chromium, when mixed with sodium (or potassium) carbonate and nitrate, give, when heated upon platinum foil, a yellow mass of the alkaline chromate. 
9. Compounds of chromium impart a green color to the borax bead.

Cobalt and Nickel, $\mathrm{Co}_{0}=\mathbf{5 9}, \mathrm{Ni}=59$. These two metals have not only the same atomic weight, but show also much resemblance to each other in their chemical and physical properties, and occur in nature associated with each other as sulphides or arsenides.

Both metals are nearly silver-white; the salts of cobalt show generally a red, those of nickel a green color. The solutions of both metals give a black precipitate of the respective sulphides on the addition of ammonium sulphide. Ammonium hydrate produces in solutions of cobalt a blue, in solutions of nickel a green precipitate of the hydrates, both of which are soluble in an excess of the reagent; potassium or sodium hydrate produces similar precipitates, which are insoluble in an excess.

Cobalt is chiefly used when in a state of combination (for coloring glass blue); nickel, when in the metallic state. (German silver is an alloy of nickel, copper, and zinc.)

\section{Questions.}

251. How is manganese found in nature?

252. Mention the different oxides of manganese. What is the binoxide used for?

253. What is the color of manganese salts, of manganates, and of permanganates?

254. How is potassium permanganate made; what are its properties, and what is it used for?

255. Give tests for manganese.

256. State composition and properties of potassium dichromate.

257. How is chromium trioxide made; what are its properties; what is it used for ; and under what other name is it known?

258. By what process may chromium sesquioxide be converted into chromates?

259. What is the composition of the oxide and hydrate of chromium, and how are they made?

260. Mention tests for chromates and chromium salts. 


\section{ZINC.}

$\mathrm{Zn}^{1 \mathrm{i}}=64.9$.

Occurrence in nature. Zinc is chiefly found either as sulphide (zinc-blende), $\mathrm{ZnS}$, or as carbonate (calamine), $\mathrm{ZnCO}_{3}$; it also occurs in combination with silicic acid as silicate.

Metallic zinc is obtained by heating the oxide or carbonate mixed with charcoal in retorts, when decomposition takes place. The liberated metal is vaporized, and distils into suitable receivers, where it solidifies.

Zinc is a bluish-white metal, which slowly tarnishes in the air, becoming coated with a film of oxide; it has a crystalline structure and is, under ordinary circumstances, brittle; when heated to about $130^{\circ}-150^{\circ}$ it is malleable, and may be rolled or hammered without fracture; zinc thus treated retains this malleability when cold; the sheet-zinc of commerce is thus made. When zinc is further heated to about $200^{\circ}$ it loses its malleability and becomes so brittle that it may be powdered; at $410^{\circ}$ it fuses, and at a bright red heat it boils, volatilizes, and, if air be not excluded, burus with a splendid greenish-white light, generating the oxide.

Zinc is frequently used in the metallic state by itself or fused together with other metals (German silver and brass contain it); galvanized iron is iron covered with a coating of metallic zinc.

Zinc is a bivalent metal, forming but one oxide and one series of salts, which have all a white color.

Zinc oxide, Zinci oxidum, $\mathrm{Zn0}=\mathbf{8 0 . 9}$ (Oxide of zinc), may be obtained by burning the metal, but if made for medicinal purposes by heating the carbonate, when carbon dioxide escapes and the oxide is left:

$$
\mathrm{ZnCO}_{3}=\mathrm{ZnO}+\mathrm{CO}_{2} \text {. }
$$

It is a soft, pale yellowish or nearly white, tasteless powder, insoluble in water, soluble in acids; when strongly heated it turns yellow, but reassumes the white color on cooling.

Zinc hydrate, $\mathrm{Zn} 2 \mathrm{HO}$, is obtained by precipitating zinc salts with the hydrate of sodium or ammonium; the precipitate, 
however, is soluble in an excess of either of the alkaline hydrates.

Zinc chloride, Zinci chloridum, $\mathrm{ZnCl}_{2}=135.7$ (Chloride of zinc). Made by dissolving zinc or zinc carbonate in hydrochloric acid and evaporating the solution to dryness:

$$
\mathrm{Zn}+2 \mathrm{HCl}=\mathrm{ZnCl}_{2}+2 \mathrm{H} .
$$

It is met with either as a white, crystalline powder, or in white, opaque pieces; it is very deliquescent and easily soluble in water and alcohol; it combines readily with albuminoid substances; it fuses at about $115^{\circ}$, and is volatilized, with partial decomposition, at a higher temperature.

Zinc bromide, Zinci bromidum, $\mathrm{ZnBr}_{2}=224.5$ (Bromide of zinc). Obtained analogously to the chloride by dissolving zinc in hydrobromic acid:

$$
\mathrm{Zn}+2 \mathrm{HBr}=\mathrm{ZnBr}_{2}+2 \mathrm{H} .
$$

A white powder, resembling the chloride in its properties.

Zinc iodide, Zinci iodidum, $\mathrm{ZnI}_{2}=\mathbf{3 1 8 . 1}$ (Iodide of zinc). The two elements zinc and iodine combine readily when heated with water; the colorless solution when evaporated to dryness, yields a powder whose physical properties resemble those of the chloride.

Zinc carbonate, Zinci carbonas præcipitatus, $2\left(\mathrm{ZnCO}_{3}\right) \cdot 3(\mathrm{Zn} 2 \mathrm{H} 0)$ $=\mathbf{5 4 6 . 5}$ (Precipitated carbonate of zinc, Zinc-white). Solutions of equal quantities of zinc sulphate and sodium carbonate are mixed and boiled, when a white precipitate is formed which is a mixture of the carbonate and hydrate of zinc, while carbon dioxide escapes and sodium sulphate remains in solution:

$$
5 \mathrm{ZnSO}_{4}+5 \mathrm{Na}_{2} \mathrm{CO}_{3}+3 \mathrm{H}_{2} \mathrm{O}=3 \mathrm{CO}_{2}+5 \mathrm{Na}_{2} \mathrm{SO}_{4}+2\left(\mathrm{ZnCO}_{3}\right) \cdot 3(\mathrm{Zn} 2 \mathrm{HO}) \text {. }
$$

Precipitated zinc carbonate is a white, impalpable powder, odorless and tasteless, insoluble in water, soluble in acids.

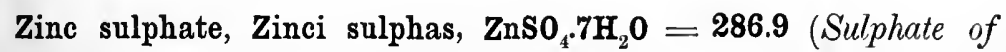
zinc, White vitriol), is obtained by dissolving zinc in diluted sulphuric acid :

$$
\mathrm{H}_{2} \mathrm{SO}_{4}+x \mathrm{H}_{2} \mathrm{O}+\mathrm{Zn}=\mathrm{ZnSO}_{4}+x \mathrm{H}_{2} \mathrm{O}+2 \mathrm{H} .
$$


If zinc be added to strong sulphuric acid, no decomposition takes place; no sufficient explanation has as yet been given for this fact.

Zinc sulphate forms small, colorless crystals, which are isomorphous with magnesium sulphate; it is easily soluble in water.

Zinc phosphide, Zinci phosphidum, $\mathrm{Zn}_{3} \mathrm{P}_{2}=256.7$ (Phosphide of zinc). The two elements zinc and phosphorus combine readily when the latter is thrown upon melted zinc, forming a grayish. black powder, or minutely crystalline, friable fragments, having a metallic lustre on the fractured surface.

Antidotes. Soluble zine salts (sulphate, chloride) have a poisonous effect. If the poison have not produced vomiting, it should be induced. Milk, white of egg, or, still better, some substance containing tannic acid (with which zinc forms an insoluble compound) should be given.

\section{Analytical reactions.}

1. Add to solution of a zinc salt ammonium sulphide: a white precipitate of zinc sulphide is produced. (Zinc sulphide is the only white insoluble sulphide.)

$$
\mathrm{ZnSO}_{4}+\left(\mathrm{NH}_{4}\right)_{2} \mathrm{~S}=\left(\mathrm{NH}_{4}\right)_{2} \mathrm{~S}+\mathrm{ZnS} .
$$

2. Add ammonium, sodium, or potassium hydrate : a white precipitate of zinc hydrate, $\mathrm{Zn} 2 \mathrm{HO}$, is produced, soluble in excess of the reagent.

3. Soluble carbonates and phosphates give white precipitates in neutral solutions of zinc.

4. Potassium ferrocyanide gives a white precipitate of zinc ferrocyanide. (This test may be used to distinguish compounds of zinc from those of magnesium or aluminium.)

5. Zinc is the only heavy metal whose compounds are all colorless. The oxide, carbonate, phosphate, and ferrocyanide are insoluble; the chloride, nitrate, and sulphate soluble.

Cadmium, $\mathbf{C d}=111.8$. Found in nature associated (though in very small quantities) with the various ores of zinc, with which metal it has in common a number of physical and chemi- 
cal properties. Cadmium differs from zinc by forming a yellow sulphide (with hydrosulphuric acid), soluble in diluted acids. Cadmium and its compounds are of little interest; the yellow sulphide is used as a pigment, the sulphate and iodide sometimes for medicinal purposes.

\section{Questions.}

261. How is zinc found in nature, and by what process is it obtained? 262. Mention the properties of metallic zinc, and what is it used for? 263. Mention two processes for making zinc oxide.

264. How does heat act on zinc oxide?

265. Show by chemical symbols the action of hydrochloric and sulphuric acids on zinc.

266. State the properties of chloride and sulphate of zinc.

267. What is white vitriol?

268. Explain the formation of precipitated zinc carbonate, and state its composition.

269. Mention tests for zinc compounds.

270. How many pounds of crystallized zinc sulphate may be obtained from 22.63 pounds of metallic zinc?

\section{LEAD-COPPER-BISMUTH.}

General remarks regarding the metals of the lead group. The six metals belonging to this group $(\mathrm{Pb}, \mathrm{Cu}, \mathrm{Bi}, \mathrm{Ag}, \mathrm{Hg}$, and $\mathrm{Cd})$ are distinguished by forming sulphides which are insoluble in water, insoluble in dilnted mineral acids, insoluble in ammonium sulphide; they are consequently precipitated from neutral, alkaline, or acid solutions by hydrosulphuric acid or ammonium sulphide.

The metals themselves do not decompose water at any temperature, and are not acted upon by diluted sulphuric acid; heated with strong sulphuric acid, most of these metals are converted into sulphates with liberation of sulphur dioxide; nitric acid converts all of them into nitrates with liberation of nitrogen dioxide.

The oxides, iodides, sulphides, carbonates, phosphates, and a few of the chlorides and sulphates of these metals are insoluble; all the nitrates, and most of the chlorides and sulphates are soluble. 
In regard to valence, they show no uniformity whatever, silver being univalent, copper, cadmium, and mercury bivalent, bismuth trivalent, and lead either bivalent or quadrivalent.

Lead, $\mathrm{Pb}^{\text {ii }}=206.5$ (Plumbum). This metal is obtained almost exclusively from the native sulphide of lead, called galena, $\mathrm{PbS}$.

Lead owes its usefulness in the metallic state chiefly to its softness, fusibility, and resistance to acids, which properties are of advantage in using it for tubes or pipes, or in constructing vessels to hold or manufacture sulphuric acid. Lead is also a constituent of many alloys, as, for instance, of type-metal, solder, britannia metal, shot, etc.

Lead oxide, Plumbi oxidum, $\mathrm{Pb0}=222.5$ (Oxide of lead, Litharge). Obtained by exposing melted lead to a current of air, when the metal is gradually oxidized with the formation of a yellow powder, known as massicot; at a higher temperature this fuses, forming reddish-yellow, crystalline scales, and is then known as litharge; by heating still further in contact with air, a portion of the oxide is converted into dioxide (or peroxide), $\mathrm{PbO}_{2}$, and a red powder is formed, known as red lead (or minium), which is probably a mixture (or combination) of oxide and peroxide of lead, $\mathrm{PbO}_{2} 2 \mathrm{PbO}$.

Oxide of lead is used in the manufacture of lead salts, lead plaster, glass, paints, etc.

Nitric acid when heated with red lead combines with the oxide, while the dioxide is left as a dark brown powder, which on heating with hydrochloric acid evolves chlorine (similar to manganese dioxide).

Lead nitrate, Plumbi nitras, $\mathrm{Pb}_{2} \mathrm{NO}_{3}=330.5$ (Nitrate of lead). Obtained by dissolving the oxide in nitric acid:

$$
\mathrm{PbO}+2 \mathrm{HNO}_{3}=\mathrm{H}_{2} \mathrm{O}+\mathrm{Pb}_{2} \mathrm{NO}_{3} .
$$

Lead nitrate is the only salt of lead (with a mineral acid) which is easily soluble in water; it has a white color, and a sweetish, astringent, and afterwards metallic taste.

Lead carbonate, Plumbi carbonas, $2\left(\mathrm{PbCO}_{3}\right) \cdot \mathrm{Pb} 2 \mathrm{HO}=773.5$ (Carbonate of lead, White-lead). This compound may be obtained by 
precipitation of lead. nitrate with sodium carbonate, but is manufactured on a large scale directly from lead, by exposing it to the simultaneous action of air, carbon dioxide, and vapors of acetic acid. The latter combines with the lead, forming a basic acetate which is converted into the carbonate (almost as soon as produced) by the carbon dioxide present.

The action of acetic acid on lead or lead oxide will be considered in connection with acetic acid.

Carbonate of lead is a heavy, white, insoluble, tasteless powder; the white-lead of commerce is frequently found adulterated with barium sulphate.

Lead iodide, Plumbi iodidum, $\mathrm{PbI}_{2}=459.7$ (Iodide of lead). Made by adding solution of potassium iodide to lead nitrate:

$$
\mathrm{Pb}_{2} \mathrm{NO}_{3}+2 \mathrm{KI}=2 \mathrm{KNO}_{3}+\mathrm{PbI}_{2} .
$$

It is a heavy, bright citron-yellow, almost insoluble powder, which may be distinguished from lead chromate by its solubility in ammonium chloride solution on boiling.

Poisonous properties and antidotes. Compounds of lead are directly poisonous, and it happens, not unfrequently, that water passing through leaden pipes or collected in leaden tanks becomes contaminated with lead. Water, free from air and salts, scarcely acts on lead; but if the water contains air, oxide of lead is formed, which is either dissolved by the water or is decomposed by the nitrates or chlorides present in the water, the soluble nitrate or chloride of lead being formed.

If the water contains carbonates and sulphates, however, these will form insoluble compounds, producing a film or coating over the lead, preventing further contact with the water.

Water containing lead will show a dark color on passing hydrosulphuric acid through it; if the quantity present be very small, the water should be evaporated to $\frac{1}{10}$ or even $\frac{1}{100}$ of its original volume before applying the test.

The constant handling of lead compounds is also one of the causes of lead-poisuning (painters' colic).

As an antidote, magnesium sulphate should be used, which forms with lead an insoluble sulphate; the purgative action of magnesia is also useful. 


\section{Analytical reactions.}

1. To solution of a lead salt add hydrosulphuric acid or ammonium sulphide: a black precipitate of lead sulphide is produced:

$$
\mathrm{Pb}_{2} \mathrm{NO}_{3}+\mathrm{H}_{2} \mathrm{~S}=2 \mathrm{HNO}_{3}+\mathrm{PbS} .
$$

2. Add sulphnric acid or soluble sulphate: a white precipitate of lead sulphate is formed:

$$
\mathrm{Pb}_{2} \mathrm{NO}_{3}+\mathrm{Na}_{2} \mathrm{SO}_{4}=2 \mathrm{NaNO}_{3}+\mathrm{PbSO}_{4} .
$$

3. Add hydrochloric acid or soluble chlorides: a white precipitate of lead chloride is produced, which dissolves on the addition of much water, as lead chloride is not entirely insoluble. For the same reason, the precipitate is not formed when the solutions used are highly diluted.

4. Other reagents which give precipitates with lead solutions are:

Potassium chromate, producing yellow lead chromate ;chrome-yellow).

Potassium iodide, producing yellow lead iodide.

Alkaline carbonates, producing white lead carbonate.

Alkaline phosphates, producing white lead phosphate.

Copper, $\mathrm{Cu}^{\mathrm{ii}}=63.2$ (Cupium). Found in nature sometimes in the metallic state, generally, however, combined with sulphur or oxygen. The commonest copper-ore is copper pyrites, a double sulphide of copper and iron, $\mathrm{CuFeS}_{2}$ or $\mathrm{Cu}_{2} \mathrm{~S} . \mathrm{Fe}_{2} \mathrm{~S}_{3}$, having the color and lustre of brass or gold. Other ores are: Copper glance, cuprous sulphide, having a dark gray color and the composition $\mathrm{Cu}_{2} \mathrm{~S}$; malachite, a beautiful green mineral, being a carbonate and hydrate of copper, $\mathrm{CuCO}_{3} \cdot \mathrm{Cu} 2 \mathrm{HO}$. The cupric and cuprous oxide are also occasionally found.

Copper is the only metal showing a distinct red color; it is so malleable that, of the metals in common use, only gold and silver surpass it in that respect; it is one of the best conductors of heat and electricity.

Copper is frequently used in the manufacture of alloys, of which the more important are: 


$\begin{array}{lcccc} & \text { Copper. } & \text { Zine. } & \text { Tin. } & \text { Nickel. } \\ \text { Brass, } & 64 & 36 & \ldots & \ldots \\ \text { German silver, } & 51 & 31 & \ldots & 18 \\ \text { Bell-metal, } & 78 & \ldots & 22 & \ldots \\ \text { Bronze, } & 80 & 4 & 16 & \ldots \\ \text { Gun-metal, } & 90 & \ldots & 10 & \ldots\end{array}$

Copper is also frequently alloyed with gold and silver.

Copper is a bivalent element, forming two oxides and two series of salts, distinguished as cuprous and cupric compounds; the cuprous salts are of but little interest.

Cupric oxide, CuO (Oxide or monoxide of copper). Heated to redness, copper becomes covered with a black scale, which is cupric oxide; it is also obtained by heating cupric nitrate or carbonate, both compounds being decomposed with formation of the oxide; finally, it may be made by adding sodium or potassium hydrate to the solution of a cupric salt, when a bulky, pale blue precipitate of cupric hydrate, $\mathrm{Cu} 2 \mathrm{HO}$, is formed, which, upon boiling, is decomposed into water and cupric oxide, a heavy dark brown powder :

$$
\begin{gathered}
\mathrm{CuSO}_{4}+2 \mathrm{KHO}=\mathrm{K}_{2} \mathrm{SO}_{4}+\mathrm{Cu} 2 \mathrm{HO} \\
\mathrm{Cu} 2 \mathrm{HO}=\mathrm{H}_{2} \mathrm{O}+\mathrm{CuO} .
\end{gathered}
$$

Cuprous oxide, $\mathrm{Cu}_{2} \mathrm{O}$ (Red oxide or suboxide of copper). When cupric oxide is heated with metallic copper, charcoal, or organic matter containing this element, the cupric oxide is decomposed, and cuprous oxide is formed. (Excess of carbon or organic matter. reduces the oxide to metallic copper.)

$$
\begin{gathered}
\mathrm{CuO}+\mathrm{Cu}=\mathrm{Cu}_{2} \mathrm{O} \\
2 \mathrm{CuO}+\mathrm{C}=\mathrm{Cu}_{2} \mathrm{O}+\mathrm{CO} .
\end{gathered}
$$

Some organic substances, especially grape-sugar, decompose strong alkaline solutions of cupric sulphate with precipitation of cuprous oxide, which is a red, insoluble powder.

Cupric sulphate, Cupri sulphas, $\mathrm{CuSO}_{4} \cdot 5 \mathrm{H}_{2} \mathrm{O}=249.2$ (Sulphate of copper, Blue vitriol, Blue-stone). This is the most important compound of copper. It is manufactured on a large scale, either from copper pyrites, or by dissolving copper in sulphuric acid, evaporating and crystallizing the solution:

$$
\mathrm{Cu}+2 \mathrm{H}_{2} \mathrm{SO}_{4}=\mathrm{CuSO}_{4}+2 \mathrm{H}_{2} \mathrm{O}+\mathrm{SO}_{2} .
$$


Cupric sulphate forms large, deep blue crystals, which are easily soluble in water, and have a nauseous, metallic taste. By heating it to about $230^{\circ}$ all water of crystallization is expelled, and the anhydrous cupric sulphate formed, which is a nearly white powder. By further heating this is decomposed, sulphuric and sulphurous oxides are evolved, and cupric oxide is left.

Cupric carbonate is obtained by the addition of sodium carbonate to solution of cupric sulphate, when a bluish-green precipitate is formed, which is cupric carbonate with hydrate; by dissolving this in the various acids, the different cupric salts are obtained.

Ammonio-copper compounds. A number of compounds are known which are either double salts of ammonia and copper, or are derived from ammonium salts and contain copper. Thus, cupric chloride forms with ammonia the compounds: $\mathrm{CuCl}_{2} 2 \mathrm{NH}_{3}$, $\mathrm{CuCl}_{2} 4 \mathrm{NH}_{3}$, and $\mathrm{CuCl}_{2} 6 \mathrm{NH}_{3}$. Cupric sulphate forms, in like manner, cupro-diammonium sulphate, $\mathrm{CuSO}_{4} 2 \mathrm{NH}_{3}$, or $\left(\mathrm{N}_{2} \mathrm{H}_{6} \mathrm{Cu}\right) \mathrm{SO}_{4}$, which is a deep blue compound capable of forming large crystals.

It is this formation of soluble ammonio-copper compounds which prevents ammonium hydrate from precipitating cupric hydrate from its salts.

Poisonous properties and antidotes. The use of copper for culinary vessels is frequently the cause of poisoning by this metal. A perfectly clean surface of metallic copper is not affected by any of the substances used in the preparation of food, but as the metal is very apt to become covered with a film of oxide when exposed to the air, and as the oxide is easily dissolved by the combined action of water, carbonic or other acids, such as are found in vinegar, the juice of fruits, or rancid fats, the use of copper for culinary vessels is always dangerous. Actual adulterations of food with compounds of copper have been detected.

In cases of poisoning by copper the stomach-pump should be used, vomiting induced, and albumen (white of egg) administered, which forms an insoluble compound with copper. Reduced iron or a very diluted solution of potassium ferrocyanide, may also be of use as antidotes. 


\section{Analytical reactions.}

1. Add to solution of copper, hydrosulphuric acid or ammonium sulphide: a black precipitate of cupric sulphide is formed:

$$
\mathrm{CuSO}_{4}+\mathrm{H}_{2} \mathrm{~S}=\mathrm{H}_{2} \mathrm{SO}_{4}+\mathrm{CuS} \text {. }
$$

2. Add sodium or potassium hydrate: a bluish precipitate of cupric hydrate, $\mathrm{Cu} 2 \mathrm{HO}$, is formed, which is converted into dark brown cupric oxide, $\mathrm{CuO}$, by boiling. (See equation above.)

3. Add ammonium hydrate: a dark blue solution is produced, containing an ammonio-copper compound. (See explanation above.)

4. Add potassium ferrocyanide: a reddish-brown precipitate of cupric ferrocyanide falls.

5. Add solution of arsenious acid and carefully neutralize with sodium hydrate: green cupric arsenite is precipitated.

6. Add sodium or potassium carbonate: green cupric carbonate with hydrate is precipitated.

7. Immerse a piece of iron or steel, showing a bright surface, in an acidified solution of copper : the latter is precipitated upon the iron, an equivalent amount of iron passing into solution :

$$
\mathrm{CuSO}_{4}+\mathrm{Fe}=\mathrm{FeSO}_{4}+\mathrm{Cu} .
$$

8. Coinpounds of copper (chloride especially) color the flame green.

9. Cupric compounds give a blue, cuprous compounds a red borax bead.

10. Cupric salts (when not anhydrous) have mostly a blue or green' color; sulphate, nitrate, chloride, and the ammonio-copper compounds are soluble, most other compounds are insoluble.

Bismuth, $\mathrm{Bi}^{\mathrm{iii}}=210$. Found in nature chielly in the metallic state, disseminated, in veins, through various rocks. The extraction of the metal is a mere mechanical process, the earthy matter containing it being heated in iron cylinders, and the melted bismuth collected in suitable receivers.

Bismuth is grayish-white with a pinkish tinge, very brittle, generally showing a distinct crystalline structure. It is occasionally used in alloys and in the manufacture of a few medicinal preparations. 
Bismuth is trivalent, as shown in the chloride, $\mathrm{BiCl}_{3}$, or oxide, $\mathrm{Bi}_{2} \mathrm{O}_{3}$. A characteristic property of this metal is the decomposition of the concentrated solution of any of its normal salts by the addition of much water, with the formation and precipitation of so-called oxy-salts or subsalts of bismuth, while some bismuth with a large quantity of acid remains in solution.

The true constitution of these subsalts is as yet doubtful, but a comparison of them has led to the assumption of a radical Bismuthyl, $\mathrm{BiO}$, which behaves like an atom of a univalent metal.

The relation between the normal or bismuth salts and the subsalts or bismuthyl salts will be shown by the composition of the following compounds:
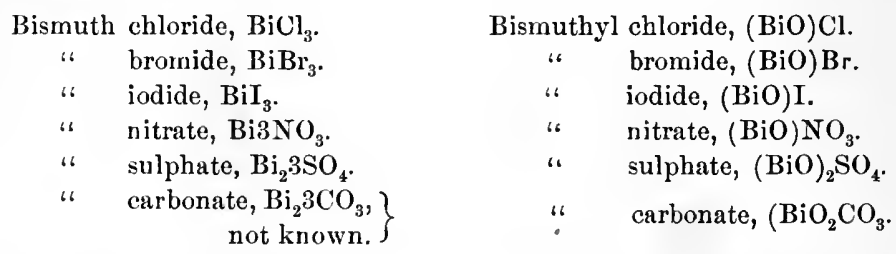

Bismuthyl nitrate, Subnitrate of bismuth, Bismuthi subnitras, $\mathrm{BiONO}_{3} \cdot \mathrm{H}_{2} \mathrm{O}=306$ (Oxynitrate of bismuth). By dissolving metallic bismuth in nitric acid, a solution of bismuth nitrate is obtained, nitrogen dioxide escaping:

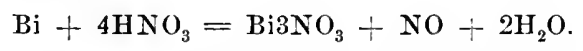

Upon evaporation of the solution, colorless crystals of bismuth nitrate $\left(\mathrm{Bi}_{3} \mathrm{NO}_{3} .5 \mathrm{H}_{2} \mathrm{O}\right)$ are obtained.

If, however, the solution (or the dissolved crystals) be poured into a large quantity of water, the salt is decomposed with the formation of bismuthyl nitrate, some bismuth and the larger quantity of nitric acid remaining in solution :

$$
5\left(\mathrm{Bi}_{3} \mathrm{NO}_{3}\right)+8 \mathrm{H}_{2} \mathrm{O}=4\left(\mathrm{BiONO}_{3} \cdot \mathrm{H}_{2} \mathrm{O}\right)+\mathrm{Bi}_{3} \mathrm{NO}_{3} ; 8 \mathrm{HNO}_{3} .
$$

As bismuth frequently contains arsenic, tests should be applied for this metal before using the bismuth.

Subnitrate of bismuth is a heavy, white, tasteless powder, insoluble in water, soluble in most acids.

Bismuthyl carbonate, Subcarbonate of bismuth, Bismuthi subcarbonas, $(\mathrm{BiO})_{2} \mathrm{CO}_{3} \cdot \mathrm{H}_{2} \mathrm{O}$ (Oxycarbonate of bismuth, Pearl-white). Made 
by adding sodium carbonate to solution of bismuth nitrate, when the subcarbonate is precipitated, some carbou dioxide escaping :

$$
2\left(\mathrm{Bi}_{3} \mathrm{NO}_{3}\right)+3 \mathrm{Na}_{2} \mathrm{CO}_{3}+\mathrm{H}_{2} \mathrm{O}=6 \mathrm{NaNO}_{3}+2 \mathrm{CO}_{2}+(\mathrm{BiO})_{2} \mathrm{CO}_{3} \cdot \mathrm{H}_{2} \mathrm{O} .
$$

A white, or pale yellowish-white powder, resembling the subnitrate. It readily loses water and carbon dioxide on heating, while the yellow oxide, $\mathrm{Bi}_{2} \mathrm{O}_{3}$, is left.

\section{Analytical reactions.}

1. Add to solution of bismuth, hydrosulphuric acid or ammonium sulphide : a dark brown (almost black) precipitate of lismuth sulphide, $\mathrm{Bi}_{2} \mathrm{~S}_{3}$, is produced :

$$
2 \mathrm{BiCl}_{3}+3 \mathrm{H}_{2} \mathrm{~S}=6 \mathrm{HCl}+\mathrm{Bi}_{2} \mathrm{~S}_{3} .
$$

2. Pour a previously concentrated acid solution of bismuth into water: a white precipitate of a bismuthyl salt is formed. (See explanation above.)

3. Add to bismuth solution ammonium or sodium hydrate, or carbonate: a white precipitate of bismuth hydrate, $\mathrm{Bi} 3 \mathrm{HO}$, or of bismuthyl carbonate is produced. (See explanation above.)

\section{Questions.}

271. What are the properties of lead, and from what ore is it obtained?

272. What is litharge, and how does it differ from red lead?

273. Give the composition of nitrate, carbonate, and iodide of lead; how are they made?

274. State the analytical reactions for lead.

275. How is copper found in nature?

276. How many oxides of copper are known; what is their composition and under what circunstances are they formed?

277. What is "blue vitriol ;" how is it made, and what are its properties?

278. How does ammonium hydrate act on cupric solutions?

279. Mention tests for copper.

280. What is the composition of subnitrate and subcarbonate of bismuth; how are they made from metallic bismuth, and what explanation is given in regard to their constitution? 


\section{SILVER-MERCURY.}

Silver, $\mathbf{A g}=107.7$ (Argentum). This metal is found sometimes in the metallic state, but generally as a sulphide, which is nearly always in combination with large quantities of lead sulphide, such ore being known as argentiferous galena. The lead manufactured from this ore contains the silver, and is separated from it by roasting the alloy in a current of air, whereby lead is oxidized and converted into litharge, while pure silver is left.

A second method by which silver is obtained from ores containing it in the metallic state (or in such a form that the compounds are easily decomposed with liberation of metallic silver) is the so-called amalgamation process, which depends upon the solubility of silver in mercury; the ores are treated with this metal, and the silver-amalgam upon being distilled leaves silver behind, the mercury being of course condensed to be used over and over again.

Silver is the whitest of all metals and takes the highest polish; it is the best conductor of heat and electricity, and melts at about $1000^{\circ}$; it is univalent, and forms but one series of salts; it is not affected by the oxygen of the air at any temperature, but is readily acted upon by traces of hydrosulphuric acid, which forms a black film of sulphide upon the surface of metallic silver. Hydrochloric acid scarcely acts on silver, nitric and sulphuric acids dissolve it.

Silver is too soft for use as coin or silverware, and is therefore alloyed with from 5 to 25 per cent. of copper, which causes it to become harder, and consequently gives it more resistance to the wear and tear by friction. .

Pure silver may be obtained by dissolving silver coin in nitric acid, when a blue solution, containing the nitrates of copper and silver, is formed. By the addition of sodium chloride to the solution a white precipitate of silver chloride is formed, while cupric nitrate remains in solution. The silver chloride is washed, dried, mixed with sodium carbonate and heated in a crucible, when sodium chloride is formed, carbon dioxide escapes, and a button of silver is found at the bottom of the crucible:

$$
2 \mathrm{AgCl}+\mathrm{Na}_{2} \mathrm{CO}_{3}=2 \mathrm{NaCl}+\mathrm{CO}_{2}+2 \mathrm{Ag}+0
$$


Silver nitrate, Argenti nitras, $\mathrm{AgNO}_{3}=169.7$ (Nitrate of silver). Pure silver is dissolved in nitric asid:

$$
3 \mathrm{Ag}+4 \mathrm{HNO}_{3}=\mathrm{NO}+2 \mathrm{H}_{2} \mathrm{O}+3 \mathrm{AgNO}_{3} .
$$

The solution is evaporated to dryness with the view of expelling all free acid, the dry mass dissolved in hot water and crystallized. When fused and poured into suitable moulds it yields the white cylindrical sticks which are usually known as caustic, lunar caustic, or lapis infernalis.

When fused with an equal weight of potassium nitrate and formed into similar rods, it represents the diluted nitrate of silver of the U. S. P.

Silver nitrate forms colorless, transparent, tabular, rhombic crystals, or, when fused, a white, hard substance; it is soluble in less than its own weight of water, the solution having a neutral reaction. Exposed to the light, especially in the presence of organic matter, silver nitrate blackens in consequence of decomposition; when brought in contact with animal matter, it is readily decomposed into free nitric acid and metallic silver, which produces the characteristic black stain; it is this decomposition, and the action of the free nitric acid, to which the strongly caustic properties of silver nitrate are due.

Nitrate of silver is used largely in photography, and also in the manufacture of various kinds of indelible inks and hairdyes.

Silver oxide, Argenti oxidum, $\mathrm{Ag}_{2} \mathrm{O}=231.4$ (Oxide of silver). Made by the addition of an alkaline hydrate to silver nitrate:

$$
2 \mathrm{AgNO}_{3}+2 \mathrm{KHO}=2 \mathrm{KNO}_{3}+\mathrm{H}_{2} \mathrm{O}+\mathrm{Ag}_{2} \mathrm{O} .
$$

A dark brown, almost black powder, but very sparingly soluble.in water, imparting to the solution a weak alkaline reaction. It is a strong base, and easily decomposed into silver and oxygen.

Silver iodide, Argenti iodidum, AgI $=234.3$ (Iodide of silver). Made by the addition of potassium iodide to silver nitrate:

$$
\mathrm{AgNO}_{3}+\mathrm{KI}=\mathrm{KNO}_{3}+\mathrm{AgI} .
$$


A heavy, amorphous, light yellowish powder, insoluble in water, and but slightly soluble in ammonium hydrate.

Antidotes. Sodium chloride, white of egg, or milk, followed by an enetic.

\section{Analytical reactions.}

1. Add to solution of a silver salt, hydrosulphuric acid or ammonium sulphide: a black precipitate of silver sulphide is produced:

$$
2 \mathrm{AgNO}_{3}+\mathrm{H}_{2} \mathrm{~S}=2 \mathrm{HNO}_{3}+\mathrm{Ag}_{2} \mathrm{~S} .
$$

2. Add hydrochloric acid, or any soluble chloride: a white, curdy precipitate of silver chloride is produced, which is insoluble in acids, but soluble in ammonium hydrate:

$$
\mathrm{AgNO}_{3}+\mathrm{NaCl}=\mathrm{NaNO}_{3}+\mathrm{AgCl} .
$$

3. Add chromate or bichromate of potassium : a red precipitate of silver chromate or bichromate is formed.

4. Add sodium phosphate: a pale yellow precipitate of silver phosphate, $\mathrm{Ag}_{3} \mathrm{PO}_{4}$, is formed, which is soluble in ammonia and nitric acid.

5. Alkaline hydrates precipitate dark brown silver oxide. (See above.)

6. Potassium iodide or bromide gives a pale yellow precipitate. (See above.)

Mercury, Hydrargyrum, $\mathbf{H g}=199.7$ (Quicksilver). Mercury is sometimes found in small globules in the metallic state, but generally as mercuric sulphide or cinnabar, a dark red mineral. The chief supply was formerly obtained from Spain and Austria; lately, however, large quantities have beeu obtained from California; it is also imported from Peru and Japan.

Mercury is obtained from cinnabar either by roasting it, or by heating it with lime, which combines with the sulphur, while the metal volatilizes, and is condensed by passing the vapors through suitable coolers.

Mercury is the only metal showing the liquid state at the ordinary temperature; it solidifies at $-40^{\circ}$, and boils at $350^{\circ}$, 
but is slightly volatile at all temperatures; it is almost silverwhite, and has a bright metallic lustre.

Mercury is peculiar in that its molecule contains but one atom, at least when in the state of a gas; in the liquid and solid state it may contain two atoms, like most other elements, but we have as yet no means of proving this fact.

Mercury is bivalent, and forms, like copper, two series of compounds, distinguished as mercuric and mercurous compounds; in the former, one atom of mercury exerts its bivalence, as in $\mathrm{HgO}, \mathrm{HgCl}_{2}$, in the mercurous compounds two atoms of mercury exert the same valence, as in $\mathrm{Hg}_{2} \mathrm{O}, \mathrm{Hg}_{2} \mathrm{Cl}_{2}$. We have to explain this fact by assuming that of the four points of attraction, represented by the two atoms of mercury, two are required to hold together or unite these two atoms so as to leave but two for other elements.

Mercury is not affected by the oxygen of the air, nor by hydrochloric acid, while chlorine, bromine, and iodine combine with it directly, and sulphuric and nitric acids dissolve it.

Mercury is used in the metallic state for many physical instruments (thermometer, barometer, etc.); in the silvering of looking-glasses, which is effected by means of an amalgam of tin (amalgams are alloys, in which mercury is one of the constituents); for manufacturing from it all the various mercury compounds, and those officinal preparations in which mercury exists in the metallic state.

These latter preparations are: Mercury with chalk, blue mass or blue pill, mercurial ointment, and mercurial plaster. In these preparations, which are made by intimately mixing (triturating) metallic mercury with the different substances used (viz., chalk, pill-mass, fat, lead-plaster), mercury exists in a metallic, but highly subdivided state. It is most probable that the action of these agents upon the animal system is chiefly due to the conversion of small quantities of mercury into mercurous oxide, which in contact with the acids of the gastric juice or perspiration, are converted into soluble compounds capable of absorption.

Mercurous oxide, $\mathrm{Hg}_{2} \mathrm{O}$ (Black oxide or suboxide of mercury). An almost black, insoluble powder, nade by adding an alkaline hydrate to a solution of mercurous nitrate:

$$
\mathrm{Hg}_{2} 2 \mathrm{NO}_{3}+2 \mathrm{KHO}=2 \mathrm{KNO}_{3}+\mathrm{H}_{2} \mathrm{O}+\mathrm{Hg}_{2} \mathrm{O} .
$$


A similar decomposition takes place when alkaline hydrates are added to the insoluble mercurous chloride. A mixture of lime-water and mercurous chloride (calomel) is known as blackwash; when the two substances are mixed, calomel is converted into mercurous oxide, while calcium chloride is formed:

$$
\mathrm{Hg}_{2} \mathrm{Cl}_{2}+\mathrm{Ca} 2 \mathrm{HO}=\mathrm{CaCl}_{2}+\mathrm{H}_{2} \mathrm{O}+\mathrm{Hg}_{2} \mathrm{O} .
$$

Mercuric oxide, Oxide of mercury, $\mathbf{H g O}=215.7$. There are two mercuric oxides which are officinal; they do not differ in their chemical composition, but in their molecular structure.

The yellow oxide of mercury, Hydrargyrum oxidum flavum, is made by pouring a solution of mercuric chloride into a solution of potassium hydrate, when an orange-yellow, heavy precipitate is produced, which is washed and dried at a temperature not exceeding $40^{\circ}$.

The red oxide of mercury, Hydrargyrum oxidum rubrum, or red precipitate, is made by either heating mercuric nitrate by itself, or after this has been intimately mixed with an amount of metallic mercury equal to the mercury in the nitrate used. In the first case, nitrous fumes and oxygen are given off, rnercuric oxide remaining:

$$
\mathrm{Hg} 2 \mathrm{NO}_{3}=\mathrm{HgO}+2 \mathrm{NO}_{2}+\mathrm{O} .
$$

In the other case, no oxygen is evolved:

$$
\mathrm{Hg}_{2} \mathrm{NO}_{3}+\mathrm{Hg}=2 \mathrm{HgO}+2 \mathrm{NO}_{2} .
$$

The red oxide of mercury differs from the yellow oxide in being more compact, and of a crystalline structure; while yellow oxide is in a more finely divided state, and consequently acts more energetically when used in medicine. Yellow oxide when digested on a water-bath, with a strong solution of oxalic acid, is converted into white mercuric oxalate within 15 minutes, while red oxide is not acted upon by oxalic acid under the same conditions.

When mercuric chloride is added to lime-water, a mixture is formed, holding in suspension a yellow oxychloride of mercury; this mixture is known as yellow-wash.

Mercurous chloride, Hydrargyrum chloridum mite, $\mathbf{H g}_{2} \mathbf{C l}_{2}=$ 470.2 (Calomel, Mild chloride of mercury, Subchloride or protochloride 
of mercury). Mercurous chloride, like mercuric oxide, may be made by two different processes, but the U. S. P. does not state by which method calomel shall be made, though the precipitated salt is in a finer state of subdivision, and consequently more energetic in its action than the sublimed article.

The latter is made by thoroughly mixing with mercuric sulphate a quantity of mercury equal to that contained in the sulphate; by this operation mercurous sulphate is obtained, which is mixed with sodium chloride, and sublimed from a suitable apparatus into a large chamber, so that the sublimate may fall in powder to the floor:

$$
\mathrm{HgSO}_{4}+\mathrm{Hg}+2 \mathrm{NaCl}=\mathrm{Na}_{2} \mathrm{SO}_{4}+\mathrm{Hg}_{2} \mathrm{Cl}_{2} .
$$

The second method for making calomel is the precipitation of a soluble mecurous salt by any soluble chloride:

$$
\mathrm{Hg}_{2} 2 \mathrm{NO}_{3}+2 \mathrm{NaCl}=2 \mathrm{NaNO}_{3}+\mathrm{Hg}_{2} \mathrm{Cl}_{2} \text {. }
$$

Mercurous chloride, made by either process, generally contains traces of mercuric chloride, and should, therefore, be washed with hot water until the washings are no longer acted upon by ammonium sulphide or silver nitrate.

Mercurous chloride is a white, impalpable, tasteless powder, insoluble in water and alcohol; it volatilizes without fusing previously; when given internally, it should not be mixed either with mineral acids, alkaline bromides, iodides, hydrates, or carbonates, all of which have a decomposing action upon this salt.

Mercuric chloride, Hydrargyri chloridum corrosivum, $\mathrm{HgCl}_{2}=$ 270.5 (Corrosive chloride of mercury, Corrosive sublimate, Perchloride or bichloride of mercury). Made by thoroughly mixing mercuric sulphate with sodium chloride, and subliming the mixture, when mercuric chloride is formed, and passes off in white fumes, which are condensed in the cooler part of the apparatus, while sodium sulphate is left:

$$
\mathrm{HgSO}_{4}+2 \mathrm{NaCl}=\mathrm{Na}_{2} \mathrm{SO}_{4}+\mathrm{HgCl}_{2} .
$$

Mercuric chloride is a heavy, white powder, or occurs in heavy, colorless, rhombic crystals or crystalline masses; it is 
soluble in 16 parts of cold and 2 parts of boiling water, in about 2 parts of alcohol, and in 4 parts of ether; when heated, it fuses and is volatilized; it has an acrid, metallic taste, an acid reaction, and strong poisonous properties.

Mercurous iodide, Hydrargyrum iodidum viride, $\mathrm{Hg}_{2} \mathrm{I}_{2}=652.6$ (Green iodide or protiodide of mercury). Both iodides of mercury may be obtained either by rubbing together mercury and iodine in the proportions represented by the respective atomic weights, or by precipitation of soluble mercurous or mercuric salts by potassium iodide.

According to the U. S. P., mercurous iodide is made by triturating 8 parts of mercury with 5 of iodine (and a little alcohol) until all globules of mercury have disappeared and a green powder has been formed, which is washed with alcohol in order to eliminate small quantities of mercuric iodide which may have been formed:

$$
2 \mathrm{Hg}+2 \mathrm{I}=\mathrm{Hg}_{2} \mathrm{I}_{2} .
$$

The powder is finally collected and dried between paper at a low temperature. During the whole operation light should be excluded as much as possible, as it decomposes the compound.

Mercurous iodide is a dull green to greenish-yellow, tasteless powder, insoluble in water, alcohol, and ether. It is easily decomposed into mercuric iodide and mercury.

Mercuric iodide, Hydrargyri iodidum rubrum, $\mathrm{HgI}_{2}=452.9$ (Red iodide or biniodide of mercury). Made by mixing solutions of potassium iodide and mercuric chloride, when a yellow precipitate is forned, turning red immediately :

$$
\mathrm{HgCl}_{2}+2 \mathrm{KI}=2 \mathrm{KCl}+\mathrm{HgI}_{2} .
$$

Mercuric iodide is soluble both in solution of potassium iodide and mercuric chloride, for which reason an excess of either substance will cause a loss of the salt when prepared. It is a scarlet-red, crystalline, tasteless powder, almost insoluble in water and but slightly soluble in alcohol; on heating or subliming it turns yellow in consequence of a molecular change 
which takes place; on cooling, and, more quickly, on pressing or rubbing the yellow powder, it reassumes the original condition and the red color.

Mercuric sulphate, $\mathrm{HgSO}_{4}$. When mercury is heated with strong sulphuric acid (the presence of nitric acid facilitates the formation) chemical action takes place between the two substances, sulphur dioxide being liberated and mercuric sulphate formed, which is obtained as a heavy, white, crystalline powder :

$$
\mathrm{Hg}+2 \mathrm{H}_{2} \mathrm{SO}_{4}=\mathrm{HgSO}_{4}+2 \mathrm{H}_{2} \mathrm{O}+\mathrm{SO}_{2} .
$$

Yellow subsulphate of mercury, Hydrargyri subsulphas flavus, $\mathrm{HgSO}_{4} \cdot \mathbf{2 H g O}=\mathbf{7 2 7 . 1}$ (Basic mercuric sulphate, Turpeth mineral). When mercuric sulphate, prepared as directed above, is thrown into boiling water, it is decomposed into an acid salt which remains in solution, and a basic salt which is precipitated. As shown by its composition, $\mathrm{HgSO}_{4} \cdot 2 \mathrm{HgO}$, it may be looked upon as mercuric sulphate in combination with mercuric oxide. It is a heavy, lemon-yellow, tasteless, insoluble powder.

Mercurous sulphate, $\mathrm{Hg}_{2} \mathrm{SO}_{4}$. When mercuric sulphate is triturated with a sufficient quantity of mercury, direct combination takes place, and the mercurous salt is formed :

$$
\mathrm{HgSO}_{4}+\mathrm{Hg}=\mathrm{Hg}_{2} \mathrm{SO}_{4} \text {. }
$$

Nitrates of mercury. Mercurous nitrate, $\mathrm{Hg}_{2} 2 \mathrm{NO}_{3}$, and Mercuric nitrate, $\mathrm{Hg}_{2} \mathrm{NO}_{3}$, may both be obtained as white salts by dissolving mercury in nitric acid. The relative quantities of the two substances present determine whether mercurous or mercuric nitrate be formed. If mercury is present in excess the mercurous salt, if nitric acid is present in excess the mercuric salt is formed, the latter especially on heating. Both salts are white and soluble in water.

Mercuric sulphide, $\mathbf{H g S}=231.7$. This compound has been mentioned as the chief ore of meleury, occurring crystallized as cinnabar, and has a red color. The same compound may, however, be obtained by passing hydrosulphuric acid gas through mercuric solutions, when at first a white precipitate is formed 
(a double compound of the sulphide of mercury in combination with the mercuric salt), which soon turns black:

$$
\mathrm{HgCl}_{2}+\mathrm{H}_{2} \mathrm{~S}=2 \mathrm{HCl}+\mathrm{HgS} \text {. }
$$

The black, amorphous, mercuric sulphide may be converted into the red, crystallized variety, by sublimation, and is then the officinal preparation known as red sulphide of mercury, Hydrargyrum sulphidum mubrum, cinnabar, or vermilion. It forms brilliant, dark red, crystalline masses, or a tine, bright, scarlet powder, which is insoluble in water, hydrochloric or nitric acid, but soluble in nitrohydrochloric acid.

Mercuric and mercurous sulphides may also be made by triturating the elements mercury and sulphur in the proper proportions, when they combine directly.

Ammoniated mercury, Hydrargyrum ammoniatum, $\mathrm{NH}_{2} \mathrm{HgCl}=$ 251.1 (White precipitate, Mercur ammonium chloride). This compound is made by pouring solution of mercuric chloride into water of ammonia, when a white precipitate falls, which is washed with highly diluted ammonia water and dried at a low temperature :

$$
\mathrm{HgCl}_{2}+2 \mathrm{NH}_{4} \mathrm{HO}=\mathrm{NH}_{2} \mathrm{Hg}^{\mathrm{II}} \mathrm{Cl}+\mathrm{NH}_{4} \mathrm{Cl}+2 \mathrm{H}_{2} \mathrm{O} .
$$

As shown by the composition of this compound, it may be regarded as ammonium chloride, $\mathrm{NH}_{4} \mathrm{Cl}$, in which two atoms of hydrogen have been replaced by one atom of the bivalent mercury. (There are many compounds known in which metallic atoms replace hydrogen in salts of ammonium; the ammonium copper compounds belong to this group of substances.)

Ammoniated mercury is a white, tasteless, insoluble powder.

Antidotes. Albumen (white of egg), of which, however, not too much should be given at one time, lest the precipitate formed by the mercuric salt and albumen be redissolved. The antidote should be followed by an emetic to remove the albuminous mercury compound. 


\section{Analytical reactions.}

Mercurous salts.

Mercuric salts

1. Hydrosulphu- Black precipitate of merric acid, or am- curous sulphide.

monium sulphide.

$$
\begin{gathered}
\mathrm{Hg}_{2} 2 \mathrm{NO}_{3}+\mathrm{H}_{2} \mathrm{~S}= \\
2 \mathrm{HNO}_{3}+\mathrm{Hg}_{2} \mathrm{~S} .
\end{gathered}
$$

2. Potassium iodide.

Green precipitate of mercurous iodide.

$$
\begin{gathered}
\mathrm{Hg} 2 \mathrm{NO}_{3}+2 \mathrm{KI}= \\
2 \mathrm{KNO}_{3}+\mathrm{Hg}_{2} \mathrm{I}_{2} .
\end{gathered}
$$

3. Potassium or Dark-brown precipitate of sodium hydrate. mercurous oxide, $\mathrm{Hg}_{2} \mathrm{O}$.

4. Ammonium hydrate.

Black precipitate of a mercurous ammonium salt is curic ammonium salt is formed. (The insoluble white formed. (See explanation calomel is converted into a above.) black powder.)

5. Potassium or

Yellowish precipitate of Brownish-red precipitate of sodium carbonate. mercurous carbonate, which is mercuric carbonate; unstable. unstable.

6. Hydrochloric acid or soluble

White precipitate of mercurous chloride is produced.

chlorides.

$$
\begin{gathered}
\mathrm{Hg}_{2} 2 \mathrm{NO}_{3}+2 \mathrm{HCl}= \\
2 \mathrm{HNO}_{3}+\mathrm{Hg}_{2} \mathrm{Cl}_{2} .
\end{gathered}
$$

\section{No change.}

Black precipitate of mercuric sulphide. (Precipitate may be white or gray, with an insufficient quantity of the reagent. (See above)

Red precipitate of mercuric iodide. (See above.)

Yellow precipitate of mercuric oxide, $\mathrm{HgO}$. (See above.)

White precipitate of a mer- 
9. A piece of bright metallic copper, when placed in a slightly acid mercury solution (which must not contain any free nitric acid) becomes coated with a dark film of metallic mercury in a fine state of division. The mercury may be separated by placing the copper (after having been washed with water and dried at a very gentle heat) in a narrow test-tube and heating to redness, when the mercury is volatilized and deposited in the cooler part of the tube.

10. All compounds of mercury are completely volatilized by heat, either with or without decomposition.

\section{Questions.}

281. How is silver obtained from the native ores, and how may it be prepared from silver coin?

282. State of silver nitrate: its composition, mode of preparation, properties, and names by which it is known.

283. Give analytical reactions for silver.

284. How is mercury found in nature; how is it obtained from the native ore; what are its physical and chemical properties?

285. Mention the three oxides of mercury; how are they made, what is their composition, what is their color and solubility?

286. State of the two chlorides of mercury : names, composition, mode of preparation, solubility, color, and other properties.

287. Mention the same of the two iodides, as above for the chlorides.

288. State the difference between mercuric sulphate, basic mercuric sulphate, and mercurous sulphate.

289. What is formed when ammonium hydrate, calcium bydrate, potassium or sodium hydrate are added to either mercurous or mercuric chloride?

290. Give tests answering for any mercury compound, and tests by which mercuric compounds may be distinguished from mercurous compounds.

\section{ARSENIC.}

$$
\mathrm{As}=74.9 \text {. }
$$

General remarks regarding the metals of the arsenic group. The metals belonging to either of the five groups considered heretofore, show much resemblance to each other in their chemical properties and consequently in their combinations. This is much less the case among the six metals ( $\mathrm{As}, \mathrm{Sb}, \mathrm{Sn}, \mathrm{Au}$, 
$\mathrm{Pt}, \mathrm{Mo}$ ) which are classed together in this group. In fact, the only resemblance which unites these metals is the insolubility of their sulphides in diluted acids and the solubility of these sulphides in ammonium sulphide (or alkaline hydrates), with which they form soluble double compounds; the oxides have also a tendency to form acids. In all other respects no general resemblance exists between these metals. Arsenic and antimony have many properties in common, and resemble in many respects the non-metallic elements phosphorus and nitrogen, as may be shown by a comparison of their hydrides, oxides, acids, and chlorides:

$\begin{array}{lllll}\mathrm{NH}_{3} & \mathrm{~N}_{2} \mathrm{O}_{3} & \mathrm{~N}_{2} \mathrm{O}_{5} & & \mathrm{NCl}_{3} . \\ \mathrm{PH}_{3} & \mathrm{P}_{2} \mathrm{O}_{3} & \mathrm{P}_{2} \mathrm{O}_{5} & \mathrm{H}_{3} \mathrm{PO}_{4} & \mathrm{PCl}_{3} . \\ \mathrm{AsH}_{3} & \mathrm{As}_{2} \mathrm{O}_{3} & \mathrm{As}_{2} \mathrm{O}_{5} & \mathrm{H}_{3} \mathrm{AsO}_{4} & \mathrm{AsCl}_{3} . \\ \mathrm{SbH}_{3} & \mathrm{Sb}_{2} \mathrm{O}_{3} & \mathrm{Sb}_{2} \mathrm{O}_{5} & & \mathrm{SbCl}_{3} .\end{array}$

Arsenic. Found in nature sometimes in the native state, but generally as sulphide or arsenide. One of the most common arsenic ores is the arsenio-sulphide of iron or mispickel, FeSAs. Realgar is the native red sulphide, $\mathrm{As}_{2} \mathrm{~S}_{2}$, and orpiment or auripigment the native yellow sulphide, $\mathrm{As}_{2} \mathrm{~S}_{3}$. Arsenides of cobalt, nickel, and other metals are not unfrequently met with in nature. Certain mineral waters contain traces of arsenic compounds.

Arsenic may easily be obtained by heating arsenions oxide with charcoal, or by allowing vapors of arsenious oxide to pass over charcoal heated to redness :

$$
\mathrm{As}_{2} \mathrm{O}_{3}+3 \mathrm{C}=3 \mathrm{CO}+2 \mathrm{As} .
$$

In both cases the arsenic, when liberated by the reducing action of the charcoal, exists in the form of vapors, which condense in the cooler part of the apparatus as a steel-gray metallic mass, which, when exposed to the atmospheric air, loses the metallic lustre in consequence of the formation of a film of oxide.

When pure, arsenic is odorless and tasteless; it is very brittle and volatilizes unchanged and without melting when heated to $180^{\circ}$, without access of air. Heated in air, it burns with a bluish-white light, forming arsenious oxide. Although insoluble in water, yet water digested with arsenic soon contains some 
arsenious acid in solution, the oxide of arsenic being formed by oxidation of the metal by the oxygen absorbed in the water.

Arsenic is used in the metallic state as fly poison and in some alloys, chiefly in shot, an alloy of lead and arsenic.

The molecule of arsenic contains four atoms, and not two, like most elements. It is trivalent in some compounds, quinquivalent in others.

Arsenious oxide, Acidum arseniosum, $\mathbf{A s}_{2} \mathbf{0}_{3}=197.8$ (White arsenic, Arsenic trioxide, Arsenious anhydride, improperly Arsenious acid). This compound is frequently obtained as a by-product in metallurgical operations during the manufacture of metals from ores containing arsenic. Such ores are roasted (heated in a current of air), when arsenic is converted into arsenious oxide, which at that temperature is volatilized and condensed in chambers or long flues.

Arsenious oxide is a heavy, white solid, occurring either as an opaque, slightly crystalline powder, or in transparent or semitransparent amorphous masses which frequently show a stratified appearance; recently sublimed, arsenious oxide exists as the semi-transparent glassy mass, known as vitreous arsenious oxide, which gradually becomes opaque and ultimately resembles porcelain.

These two modifications of arsenious oxide differ in their solubility in water, the amorphous or glassy variety dissolving more freely than the crystallized. One part of arsenious oxide is dissolved in from 30 to 100 parts of cold and in 15 parts of boiling water, the solution having at first a faint acrid and metallic, and afterwards a sweetish taste. This solution contains the arsenious oxide not as such, but as arsenious acid, $\mathrm{H}_{3} \mathrm{AsO}_{3}$, which compound, however, cannot be obtained in an isolated condition, but is only known in solution :

$$
\mathrm{As}_{2} \mathrm{O}_{3}+3 \mathrm{H}_{2} \mathrm{O}=2 \mathrm{H}_{2} \mathrm{AsO}_{3} .
$$

The salts of arsenious acid are known as arsenites.

When heated to about $220^{\circ}$, arsenious oxide is volatilized without fusion; the vapors, when condensed, form small, shining, eight-sided crystals; when heated on charcoal, it is deoxidized, giving off at the same time an odor resembling that of garlic. 
Arsenious oxide is frequently used in the arts and for manufacturing purposes, as, for instance, in the manufacture of green colors, of opaque white glass, in calico-printing, as a powerful antiseptic for the preservation of organic objects of natural history, and, finally, as the substance from which all arsenic compounds are obtained.

The officinal solution of arsenious acid, Liquor acidi arseniosi, is a 1 per cent. solution of arsenious acid in water to which 2 per cent. of hydrochloric acid have been added.

The officinal solution of arsenite of potassium, Liquor potassï arsenitis, or Fowler's solution, is made by dissolving 1 part of arsenious oxide and 1 part of potassium bicarbonate in 95 parts of water and adding 3 parts of compound tincture of lavender; the solution contains the arsenic as potassium arsenite.

Arsenic oxide, $\mathbf{A s}_{2} \mathbf{0}_{5}$ (Arsenic pentoxide, Anhydrous arsenic acid). When arsenious oxide is heated with nitric acid, it becomes oxidized and is converted into arsenic acid, $\mathrm{H}_{3} \mathrm{AsO}_{4}$, from which the water may be expelled by further heating, when arsenic oxide is left :

$$
2 \mathrm{H}_{3} \mathrm{AsO}_{4}=\mathrm{As}_{2} \mathrm{O}_{5}+3 \mathrm{H}_{2} \mathrm{O} .
$$

Arsenic oxide is a heavy, white, solid substance which, in contact with water, is converted into arsenic acid. This acid resembles phosphoric acid not only in composition, but also in forming metarsenic and pyroarsenic acids under the same conditions under which the corresponding phosphoric acids are formed. The salts of arsenic acid, the arseniates, also resemble in their constitution the corresponding phosphates.

Arsenic oxide and arsenic acid are largely used as oxidizing agents in the manufacture of aniline colors.

Disodium hydrogen arseniate, Sodii arsenias, $\mathrm{Na}_{2} \mathrm{HAs}_{4} \cdot 7 \mathrm{H}_{2} \mathbf{O}=$ 311.9 (Arseniate of sodium). This salt is made ly fusing arsenious oxide with carbonate and nitrate of sodium:

$$
\mathrm{As}_{2} \mathrm{O}_{3}+2 \mathrm{NaNO}_{3}+\mathrm{Na}_{2} \mathrm{CO}_{3}=\mathrm{Na}_{4} \mathrm{As}_{2} \mathrm{O}_{7}+\mathrm{N}_{2} \mathrm{O}_{3}+\mathrm{CO}_{2} \text {. }
$$

Sodium pyroarseniate is formed, nitrogen trioxide and carbon dioxide escaping. By dissolving in water and crystallizing, the officinal salt is obtained in colorless, transparent crystals :

$$
\mathrm{Na}_{4} \mathrm{As}_{2} \mathrm{O}_{7}+15 \mathrm{H}_{2} \mathrm{O}=2\left(\mathrm{Na}_{2} \mathrm{HAsO}_{4} \cdot 7 \mathrm{H}_{2} \mathrm{O}\right) \text {. }
$$


Arseniuretted hydrogen, $\mathbf{A s H}_{3}$ (Hydrogen arsenide). This compound is always formed when either arsenious or arsenic oxides or acids, or any of their salts, are brought in contact with nascent hydrogen, for instance, with zinc and diluted sulphuric acid, which evolve hydrogen :

$$
\begin{aligned}
& \mathrm{As}_{2} \mathrm{O}_{3}+12 \mathrm{H}=2 \mathrm{AsH}_{3}+3 \mathrm{H}_{2} \mathrm{O} . \\
& \mathrm{As}_{2} \mathrm{O}_{5}+16 \mathrm{H}=2 \mathrm{AsH}_{3}+5 \mathrm{H}_{2} \mathrm{O} . \\
& \mathrm{AsCl}_{3}+6 \mathrm{H}=\mathrm{AsH}_{3}+3 \mathrm{HCl} .
\end{aligned}
$$

Arseniuretted hydrogen is a colorless, highly poisonous gas, having a strong garlic odor. Ignited, it burns with a bluish flame, giving off white clouds of arsenious oxide:

$$
2 \mathrm{AsH}_{3}+6 \mathrm{O}=\mathrm{As}_{2} \mathrm{O}_{3}+3 \mathrm{H}_{2} \mathrm{O} .
$$

When a cold plate (porcelain answers best) is held in the flame of arseniuretted hydrogen, a dark deposit of metallic arsenic (arsenic spots) is produced upon the plate (in a similar manner as a deposit of carbon is produced by a common luminous flame). The formation of this metallic deposit may be explained by the fact that the heat of the flame decomposes the gas, and that, furthermore, of the two liberated elements, arsenic and hydrogen, the latter has the greater affinity for oxygen. In the centre of the flame, to which but a limited amount of oxygen penetrates, the latter is taken up by the hydrogen, arsenic being present in the metallic state until it burns in the outer cone of the flame. It is this liberated arsenic which is deposited upon a cold substance held in the flame.

Arseniuretted hydrogen, when heated to redness, is decomposed into its elements; by passing the gas through a glass tube heated to redness: the liberated arsenic is depositer in the cooler part of the tube, forming a bright metallic ring.

Sulphides of arsenic. Two sulphides of arsenic are known and have been mentioned above as the native disulphide or realgar, $\mathrm{As}_{2} \mathrm{~S}_{2}$, and the trisulphide or orpiment, $\mathrm{As}_{2} \mathrm{~S}_{3}$. Disulphide of arsenic is an orange-red, fusible, and volatile substance, used as a pigment; it may be made by fusing together the elements in the proper proportions. Trisulphide is a golden-yellow, fusible, and volatile substance, which may also be obtained by fusing the elements, or by precipitating an arsenic solution by hydro- 
sulphuric acid. Both sulphides of arsenic are sulphur-acids, uniting with other metallic sulphides to form sulphur-salts, as, for instance, $\mathrm{K}_{2} \mathrm{~S} . \mathrm{As}_{2} \mathrm{~S}_{3}$, or $\left(\mathrm{NH}_{4}\right)_{2} \mathrm{~S} . \mathrm{As}_{2} \mathrm{~S}_{3}$. These compounds are known as sulph-arsenides.

Arsenious iodide, Arsenii iodidum, $\mathbf{A s I}_{3}=\mathbf{4 5 4 . 7}$ (Iodide of arsenic), may be obtained by direct combination of the elements, and forms orange-red crystalline masses, soluble in water and alcohol, but decomposed by boiling with either of these liquids. It is used in the officinal preparation, Solution of iodide of arsenic and mercury, Donovan's solution, which is made by dissolving equal parts of arsenious iodide and mercuric iodide in 100 parts of water.

\section{Analytical reactions.}

1. Add hydrosulphuric acid to a slightly acid solution of arsenic: a yellow precipitate of arsenious sulphide is produced:

$$
\mathrm{As}_{2} \mathrm{O}_{3}+3 \mathrm{H}_{2} \mathrm{~S}=3 \mathrm{H}_{2} \mathrm{O}+\mathrm{As}_{2} \mathrm{~S}_{3} .
$$

If arsenic is present as arsenic acid, this compound is reduced to arsenious acid previous to its precipitation; heating of the liquid facilitates this reaction :

$$
\mathrm{As}_{2} \mathrm{O}_{5}+2 \mathrm{H}_{2} \mathrm{~S}=\mathrm{As}_{2} \mathrm{O}_{3}+2 \mathrm{H}_{2} \mathrm{O}+2 \mathrm{~S} .
$$

2. Add ammonium sulphide or any alkaline hydrate to the yellow precipitate of arsenious sulphide: the latter is readily dissolved but may be reprecipitated by nentralizing with an acid.

3. Ammonio-nitrate of silver (silver nitrate to which enough of water of ammonia has been added to redissolve the precipitate formed at first) produces in neutral solutions of arsenious acid a yellow precipitate of arsenite of silver $\left(\mathrm{Ag}_{3} \mathrm{AsO}_{3}\right)$, in arsenic acid solutions a chocolatc-colored precipitate of arsenite of silver $\left(\mathrm{Ag}_{3} \mathrm{AsO}_{4}\right)$. The two precipitates are soluble, both in alkalies and acids.

4. Ammonio-sulphate of copper (made similarly to ammonio-nitrate of silver from cupric sulphate) added to neutral arsenious solutions produces a green precipitate of cupric arsenite $\left(\mathrm{CuHAsO}_{3}\right)$, known as Scheele's green. (Arsenite of copper mixed with cupric acetate is known as Schweinfurth 
green). The same reagent produces in neutral arsenic acid solutions a similar green precipitate of cupric arseniate.

Instead of using for the above tests the ammonic salts, silver nitrate or cupric sulphate may be added to the acid (or neutral)

Fig. 7 .

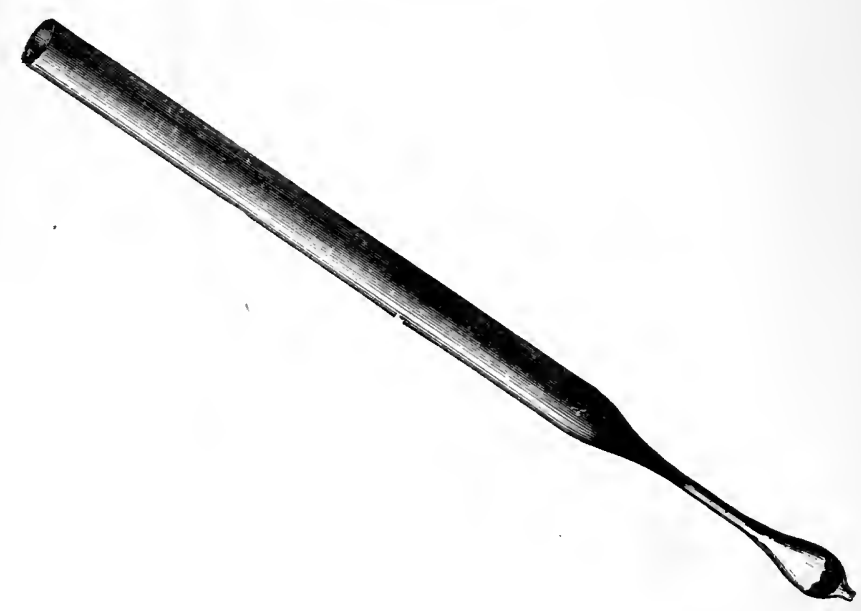

solution of arsenic, then adding water of ammonia carefully in small quantities until a neutral reaction has been obtained, when the precipitate is formed.

5. Soluble arseniates give white precipitates with soluble salts of barium, calcium, magnesium, zinc, and some other metals, soluble arsenites do not.

6. Heat any dry arsenic compound, after being mixed with some charcoal and dry potassium carbonate in a very narrow test-tube (or, better, in a glass tube having a small bulb on one end): the arsenic compound is decomposed and the metallic arsenic deposited as a metallic ring in the upper part of the tube. (Fig. 7.)

7. Heat arsenious or arsenic oxide upon a piece of charcoal by means of a blowpipe: a characteristic odor of garlic is perceptible.

8. Reinsch's test. A thin piece of copper, having a bright metallic surface, placed in a slightly acidified solution of arsenic (nitric acid should not be present) becomes, upon heating the solution, coated with a film of metallic arsenic; the latter may 
be sublimed from the copper by placing it in a narrow test-tube and heating.

9. Fleitmann's test. Zine and solution of sodium hydrate evolve, upon heating, hydrogen gas :

$$
\mathrm{Zn}+2 \mathrm{NaHO}=\mathrm{Na}_{2} \mathrm{ZnO}_{2}+2 \mathrm{H} \text {. }
$$

If arsenious or arsenic acid be present, arseniuretted hydrogen is evolved, which may be recognized by placing over the mouth of the flask in which the reaction takes place a piece of paper, moistened by a drop of silver nitrate solution, which latter is decomposed by the arseniuretted hydrogen with liberation of free silver, which imparts to the paper a purplishblack color. (Antimony compounds do not evolve antimoniuretted lydrogen, when treated with zinc and alkaline hydrates.) (Fig. 8.)

10. Marsh's test. This test is, especially for the detection of traces of arsenic, by far the best and most reliable, and should never be omitted in testing for arsenic in cases of poisoning. The apparatus used for performing this test consists of a glass vessel (flask or Woulf's bottle) provided with a funnel-tube and a delivery-tube (bent at right angles), which is connected with a wider tube, tilled with pieces of calcium chloride or plugs of asbestos; this drying tube is again connected with a piece of hard glass tube, about one foot long, having a diameter of $\frac{1}{4}$ inch, drawn out at intervals of about 3 inches, so as to reduce

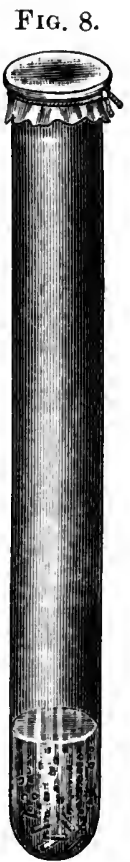
its diameter to $\frac{1}{8}$ inch. Hydrogen is generated in the flask by the action of sulphuric acid on zinc, and examined for its purity by heating the glass tube to redness at one of its wide parts for at least 30 minutes; if no trace of a metallic mirror is formed at the constriction beyond the heated point, the gas and the substances used for its generation may be pronounced to be free from arsenic. (Both zinc and sulphuric acid sometimes contain arsenic.)

After having thus demonstrated the purity of the hydrogen, the suspected liquid, which must contain the arsenic either as 
oxide or chloride (not as sulphide), is poured into the flask through the funnel-tube. If arsenic is present in not too small quantities, the gas ignited at the end of the glass tube shows a flame decidedly different from that of burning hydrogen. The

FIG. 9.

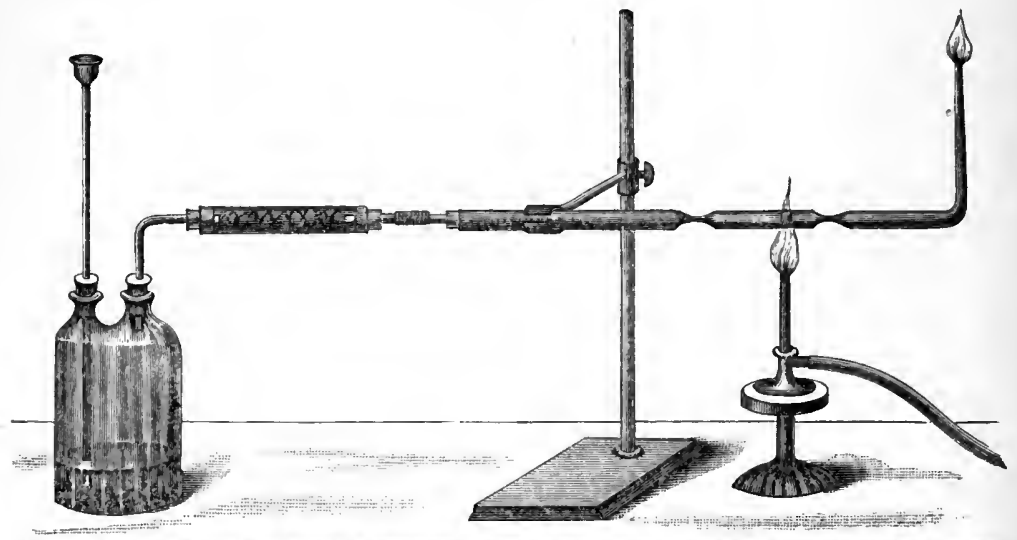

Marsh's apparatus for detection of arsenic.

flame becomes larger, assumes a bluish tint, and emits an odor of garlic, while above it a white cloud appears which is more or less dense; a cold test-tube held inverted over the flame will be covered upon its walls with a white deposit of minute octahedral crystals of arsenious oxide; a cold piece of porcelain held in the flame becomes coated with a brown stain (arsenic spot) of metallic arsenic. (See explanation above in connection with arseniuretted hydrogen.)

The glass tube heated, as above mentioned, at one of its wide parts, will show a bluish-black metallic mirror at the constriction beyond.

The only element which, under the same conditions, forms spots and mirrors similar to arsenic, is antimony; there are, however, sufficiently reliable tests to distinguish arsenic spots from those of antimony.

Arsenic spots treated with solution of hypochlorites (solution of bleaching-powder) dissolve readily; antimony spots are not affected. When nitric acid is added to an arsenic spot, evaporated to dryness and moistened with a drop of silver nitrate, it 
turns brick-red; antimony spots treated in like manner remain white. Arsenic spots dissolved in ammonium sulphide and evaporated to dryness show a bright yellow, antimony spots an orange-red residue.

Fig. 10 represents a simpler form of Marsh's apparatus, which will generally answer for student's tests.

Preparatory treatment of organic matter for arsenic analysis. If organic matter is to be examined for arsenic (or for any other metallic poison), it ought to be treated as follows: The substance, if not liquid, is cut into pieces, well mashed and mixed with water; the liquid or semiliquid substance is heated in a porcelain dish over a steam bath with hydrochloric acid and potassium chlorate until the mass has a uniform light yellow color and has no longer an odor of chlorine. By this operation all poisonous metals are rendered

FIg. 10.

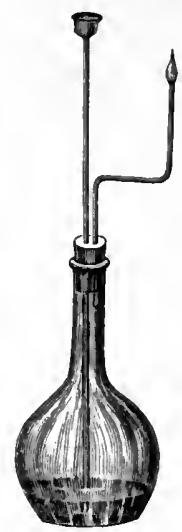

Student's apparatus for making arsenic spots. soluble, even when present as sulphides, and may now be separated from some remaining solid matter by filtration. The clear solution is heated and treated with hydrosulphuric acid gas for several hours, when arsenic and all metals of the arsenic and lead groups are precipitated as sulphides, a little organic matter being also generally precipitated.

The precipitate is collected upon a small filter and treated with warm ammonium sulphide, which dissolves the sulphides of arsenic and antimony, leaving behind the sulphides of the lead group which may be dissolved in nitric, or, if mercury be present, in nitro-muriatic acid and the solution tested by the methods mentioned for the respective metals. The ammonium sulphide solution is evaporated to dryness, this residue mixed with some nitrate and carbonate of sodium and the mixture fused in a small porcelain crucible. By the oxidizing action of the nitrate, both sulphides are converted into the higher oxides, arsenic forming sodium arseniate, antimony forming antimonic oxide. By treating the mass with warm water, sodium arseniate is dissolved and may be filtered off, while antimonic oxide remains undissolved and may be dissolved in hydrochloric acid. 
Both solutions may now be used for making the respective tests for arsenic or antimony.

- Antidotes. Moist, recently prepared ferric hydrate or dialyzed iron are the best antidotes, insoluble ferric arsenite or arseniate being formed. Vomiting should be induced by tickling the fauces or by administering zinc sulphate, but not tartar emetic.

\section{Questions.}

291. Which metals belong to the arsenic group, and what are their characteristics?

292. Which non-metallic elements does arsenic resemble? Mention some of the compounds showing this analogy.

293. How is arsenic obtained in the metallic state; what are its physical and chemical properties; how does heat act upon it?

294. What is white arsenic? State its composition, mode of manufacture, appearance, solubility, and other properties.

295. Which three solutions, containing arsenic, are officinal, and what is their composition?

296. How is arsenic acid obtained from arsenious oxide, and which arseniate is officinal?

297. State composition and properties of arseniuretted hydrogen, and explain its formation. What use is made of it in testing for arsenic?

298. State the composition of realgar, orpiment, Scheele's green, and Schweinfurth green.

299. Give a detailed description of the process by which arsenic can be detected in organic matter.

300. Describe Marsh's test and other tests for arsenic, and state the difference between arsenic and antimony spots.

\section{ANTIMONY-TIN-GOLD-PLATINUM-MOLYBDENUM.}

Antimony, $\mathbf{S b}=120$ (Stibium). This metal is found in nature chiefly as the trisulphide, $\mathrm{Sb}_{2} \mathrm{~S}_{3}$, an ore which is known as black or crude antimony.

The metal is obtained from the sulphide by roasting, when it is converted into oxide, which is reduced by charcoal. Antimony is a brittle, bluish-white metal, having a crystalline structure; it fuses at $450^{\circ}$, and may at a higher temperature be distilled without.change, provided air is excluded; heated in air it burns brilliantly. 
Antimony is used in a number of important alloys, for instance, in type-metal, an alloy of lead, tin, and antimony.

Antimony trisulphide, Antimonii sulphidum, $\mathbf{S b}_{2} \mathbf{S}_{3}=336$ (Antimonious sulphide, Sulphide of antimony). The above-mentioned native sulphide, the black antimony, is purified by fusion; it forms steel-gray masses of a metallic lustre, and a striated, crystalline fracture, forming a grayish-black, lustreless powder, which is insoluble in water, but soluble in hydrochloric acid with liberation of hydrosulphuric acid.

When finely powdered antimonious sulphide is treated with water of ammonia to remove any traces of arsenic (which is frequently found in this ore) and the washed sulphide dried, the purified sulphide of antimony of the U. S. P. is obtained.

Antimonious sulphide found in nature is crystallized and steel-gray, but it may also be obtained in an amorphous condition as an orange-red powder, by passing hydrosulphuric acid gas through an antimonious solution. By heating the orange-red sulphide, it is converted into the black variety.

Sulphurated antimony, Antimonium sulphuratum (Oxysulphide of antimony), chiefly antimonious sulphide with some antimonious oxide. This preparation is made by boiling purified antimonious sulphide with solution of sodium hydrate, and adding to the hot solution sulphuric acid as long as a precipitate is formed, which is collected and dried.

The sulphides and oxides of antimony, like those of arsenic, combine with many metallic sulphides or oxides to form sulphosalts or oxy-salts. Thus, the sodium sulph-antimonite, $\mathrm{Na}_{3} \mathrm{SbS}_{3}$, and the sodium antimonite, $\mathrm{Na}_{3} \mathrm{SbO}_{3}$, are formed, when antimonious sulphide is boiled with sodium hydrate :

$$
\mathrm{Sb}_{2} \mathrm{~S}_{\mathrm{z}}+6 \mathrm{NaHO}=\mathrm{Na}_{3} \mathrm{SbS}_{3}+\mathrm{Na}_{3} \mathrm{SbO}_{3}+3 \mathrm{H}_{2} \mathrm{O} .
$$

By the addition of sulphuric acid, both salts are decomposed, sodium sulphate is formed, and antimonious sulphide and oxide are precipitated:

$$
\begin{aligned}
& 2 \mathrm{Na}_{3} \mathrm{SbS}_{3}+3 \mathrm{H}_{2} \mathrm{SO}_{4}=3 \mathrm{Na}_{2} \mathrm{SO}_{4}+\mathrm{Sb}_{2} \mathrm{~S}_{3}+3 \mathrm{H}_{2} \mathrm{~S} \\
& 2 \mathrm{Na}_{3} \mathrm{SbO}_{3}+3 \mathrm{H}_{2} \mathrm{SO}_{4}=3 \mathrm{Na}_{2} \mathrm{SO}_{4}+\mathrm{Sb}_{2} \mathrm{O}_{3}+3 \mathrm{H}_{2} \mathrm{O}
\end{aligned}
$$


$\mathbf{h}$ is a reddish-brown, amorphous powder, insoluble in water, soluble in hydrochloric acid or. sodium hydrate.

Antimony pentasulphide, $\mathbf{S b}_{2} \mathbf{S}_{5}$ (Golden sulphuret of antimony). A red powder, which, like antimonious sulphide, forms sulphosalts. It may be obtained by precipitation of acid solutions of antimonic acid by hydrosulphuric acid.

Antimonious chloride, $\mathrm{SbCl}_{3}$ (Terchloride of antimony, Butter of antimony). Obtained by boiling the native sulphide with hydrochloric acid :

$$
\mathrm{Sb}_{2} \mathrm{~S}_{3}+6 \mathrm{HCl}=3 \mathrm{H}_{2} \mathrm{~S}+2 \mathrm{SbCl}_{3} .
$$

The clear solution is evaporated and the remaining chloride distilled, when it is obtained as a white, crystalline, semi-transparent mass.

By passing chlorine over antimonious chloride it is converted into antimonic chloride, $\mathrm{SbCl}_{5}$, which is a fuming liquid.

Antimonious oxide, Antimonii oxidum, $\mathrm{Sb}_{2} \mathrm{O}_{3}=288$ (Oxide of antimony). When antimonious chloride is added to water, decomposition takes place, and an oxychloride of antimony, $2 \mathrm{SbCl}_{3} .5 \mathrm{Sb}_{2} \mathrm{O}_{3}$, is precipitated:

$$
12 \mathrm{SbCl}_{3}+15 \mathrm{H}_{2} \mathrm{O}=2 \mathrm{SbCl}_{3} .5 \mathrm{Sb}_{2} \mathrm{O}_{3}+30 \mathrm{HCl} .
$$

This white precipitate was formerly known as powder of Algaroth. It is completely converted into oxide by treating it with sodium carbonate:

$$
2 \mathrm{SbCl}_{3} \cdot 5 \mathrm{Sb}_{2} \mathrm{O}_{3}+3 \mathrm{Na}_{2} \mathrm{CO}_{3}=6 \mathrm{Sb}_{2} \mathrm{O}_{3}+6 \mathrm{NaCl}+3 \mathrm{CO}_{2} \text {. }
$$

The precipitate, when washed and dried, is a heavy, grayishwhite, tasteless powder, insoluble in .water, soluble in acids. Antimonious oxide, while yet moist, dissolves readily in potassium acid tartrate, forming the double tartrate of potassium and antimony, or tartar emetic, which salt will be more fully considered hereafter.

Antidotes. Poisonous doses of any preparation of antimony are generally quickly followed by vomiting; if this, however, 
have not occurred, the stomach-pump must be applied. Tannic acid in any form, or recently precipitated ferric hydrate, should be administered.

\section{Analytical reactions.}

1. Add hydrosulphuric acid to an acidified solution of antimony : an orange-red precipitate of antimonious or antimonic sulphide $\left(\mathrm{Sb}_{2} \mathrm{~S}_{3}\right.$ or $\left.\mathrm{Sb}_{2} \mathrm{~S}_{5}\right)$ is produced.

2. Add ammonium sulphide to the precipitated sulphide of antimony: this is dissolved and may be reprecipitated by neutralizing with an acid.

3. Produce a concentrated solution of antimonious chloride by dissolving the sulphide in hydrochloric acid, and pour it into water: a white precipitate of the oxychloride is formed. (See explanation above.)

4. Boil a piece of metallic copper in the solution of antimonious chloride: a black deposit of antimony is formed upon the copper. By heating the latter in a narrow test-tube, the antimony is volatilized and deposited as a white incrustation of antimonious oxide upon the glass.

5. Use "Marsh's test" as described under analytical reactions for arsenic, and apply tests mentioned there for distinguishing spots of arsenic from those of antimony.

Tin, $\mathbf{S n}=117.7$ (Stannum). This metal is found in nature chiefly as stannic oxide or tin-stone, $\mathrm{SnO}_{2}$, from which the metal is easily obtained by heating with coal:

$$
\mathrm{SnO}_{2}+, 2 \mathrm{C}=\mathrm{Sn}+2 \mathrm{CO} .
$$

Tin is an almost silver-white, very malleable metal, fusing at the comparatively low temperature of $228^{\circ}$. It is used in many alloys, in the silvering of looking-glasses by tin-amalgam, and chiefly in the manufacture of tin-plate, which is sheet-iron covered with a thin layer of tin.

Tin is bivalent in some compounds, quadrivalent in others. These combinations are distinguished as stannous and stannic compounds.

Stannous chloride, $\mathrm{SnCl}_{2}$ (Protochloride of tin). Obtained by dissolving tin in hydrochloric acid by the aid of heat :

$$
\mathrm{Sn}+2 \mathrm{HCl}=\mathrm{SnCl}_{2}+2 \mathrm{H} \text {. }
$$


Sufficiently evaporated, the solution yields crystals of the composition $\mathrm{SnCl}_{2} \cdot 2 \mathrm{H}_{2} \mathrm{O}$. Stannous chloride is a strong deoxidizing agent, frequently used as'a reagent for mercury and gold, which metals are precipitated from their solutions in the metallic state. It is also used in calico-printing.

Stannic chloride, $\mathrm{SnCl}_{4}$ (Perchloride of tin). Stannous chloride may be converted into stannic chloride either by passing chlorine through its solution or by heating with hydrochloric and nitric acids.

\section{Analytical reactions.}

1. Add hydrosulphuric acid to solution of a stannous salt: brown stannous sulphide is precipitated:

$$
\mathrm{SnCl}_{2}+\mathrm{H}_{2} \mathrm{~S}=2 \mathrm{HCl}+\mathrm{SnS} .
$$

The precipitate is soluble in ammonium sulphide.

2. Add hydrosulphuric acid to solution of a stannic salt: yellow stannic sulphide is precipitated:

$$
\mathrm{SnCl}_{4}+2 \mathrm{H}_{2} \mathrm{~S}=4 \mathrm{HCl}+\mathrm{SnS}_{2} .
$$

The precipitate is soluble in ammonium sulphide.

3. Sodium or potassium hydrate added to a stannous salt, produce a white precipitate of stannous hydrate, Sn $2 \mathrm{HO}$. The same reagents added to a stannic salt produce white stannic acid, $\mathrm{H}_{2} \mathrm{SnO}_{3}$. Both precipitates are soluble in excess of the alkali.

Gold, $\mathrm{Au}=196.2($ A 1 (rum). Gold occurs in nature in the free state, and is separated from adhering sand and rock by a mechanical process of washing, in which advantage is taken of the high specific gravity of gold.

Pure gold is too soft for general use, and is therefore alloyed with various proportions of silver and copper. American coin is an alloy of 90 parts of gold and 10 parts of copper; jeweller's gold contains generally 75 per cent. (18 carat) of gold, the other 25 per cent. being copper and silver; the varying proportions are well indicated by the color.

Gold is not affected by either hydrochloric, nitric, or sul- 
phuric acid, but is dissolved by nitrohydrochloric acid, by free chlorine or bromine, and by mercury, with which it forms an amalgam.

Gold is generally trivalent, as in auric chloride, $\mathrm{AuCl}_{3}$, but most likely also univalent in some compounds, as in aurous chloride, AuCl.

Auric chloride, $\mathbf{A u C l}_{3}$. Obtained by dissolving pure gold in nitrohydrochloric acid and evaporating the solution. A mixture of equal parts of auric chloride and sodium chloride is officinal under the name of chloride of gold and sodium. It is an orange-yellow, very soluble powder.

\section{Analytical reactions.}

1. Add hydrosulphuric acid to solution of gold: brown auric sulphide, $\mathrm{Au}_{2} \mathrm{~S}_{3}$, is precipitated, which is soluble in ammonium sulphide.

2. Add ferrous sulphate to solution of gold and set aside for a few hours: metallic gold is precipitated as a dark powder, which, by fusion, is converted into a metallic mass.

Platinum, $\mathrm{Pt}=1$ 194.4. Platinum, like gold, is found in nature in the free state, the chief supply being derived from the Ural mountains, where it is found associated with a number of metals (iridium, ruthenium, osmium, palladium, rhodium) resembling platinum in their properties.

Platinum is of great importance and value on account of its high fusing-point and its resistance to the action of most chemical agents, for which reason it is used in the manufacture of vessels serving in chemical operations.

Platinum, when dissolved in nitrohydrochloric acid, forms platinic chloride, $\mathrm{PtCl}_{4}$, a salt frequently used as a reagent for potassium or ammonium salts, with which it forms insoluble double chlorides of the composition $\mathrm{PtCl}_{4} .2 \mathrm{KCl}$ and $\mathrm{PtCl}_{4}$. $2 \mathrm{NH}_{4} \mathrm{Cl}$. By heating the latter salt sufficiently it is decomposed, and metallic platinum is left as a gray spongy mass.

Molybdenum, Mo $=95.5$. This metal is found in nature chiefly as sulphide, $\mathrm{MoS}_{2}$, from which, by roasting, molybdic oxide, $\mathrm{MoO}_{3}$, is obtained. The oxide, when dissolved in water, 
forms an acid which combines with metals, forming a series of salts termed molybdates. Of interest is ammonium molybdate, a solution of which in nitric acid is an excellent reagent for phosphoric acid, with which it forms a yellow precipitate, insoluble in acids, soluble in ammonium hydrate.

\section{Questions.}

301. How is antimony found in nature, and what are the properties of this metal?

302. State the composition of antimonious sulphide, and its color when crystallized and amorphous.

303. How do hydrochloric acid and alkaline hydrates act upon antimonious sulphide?

304. What is the sulphurated antimony of the U. S. P.?

305. Mention the two chlorides of antimony, and state their properties.

306. How is antimonious oxide made, and what is it used for?

307. Give tests for antimony.

308. State the use made of tin in the metallic state; mention the two chlorides of tin, and what stannous chloride is used for.

309. How are gold and platinum found in nature; by what acid may they be dissolved, and what is the composition of the compounds formed?

310. Which is the most important compound of molybdenum, and what is it used for? 


\section{V.}

\section{ANALYTICAL CHEMISTRY.}

\section{INTRODUCTORY REMARKS AND PRELIMINARY EXAMINATION.}

General remarks. Analytical chemistry is that part of chemistry which treats of the different analytical methods by which substances are recognized and their chemical composition determined. This determination may be either qualitative or quantitative, and, accordingly, a distinction is made between a qualitative analysis, by which simply the nature of the elements (or groups of elements) present in the substance under examination is determined, and a quantitative analysis, by which also the exact amount of these elements is ascertained.

In this book qualitative analysis will chiefly be considered, as the methods for quantitative determinations of the different elements are so numerous and so varied that a detailed description of them would occupy'more space than can be devoted to analytical chemistry in this work. Every one studying analytical chemistry should do it practically, that is, should perform for himself in a laboratory all those reactions which have been mentioned heretofore as characteristic of the different elements and their compounds, and, furthermore, should make himself acquainted with the methods by which substances are recognized when mixed with others, by analyzing various complex substances.

Such a course of practical work in a chemical laboratory is of the greatest advantage to all studying chemistry, and students cannot be too strongly advised to avail themselves of any facilities offered in performing chemical experiments, analytically or otherwise. 


\section{Apparatus needed for qualitative analysis.}

1. Iron stand. (Fig. 11.)

2. Bunsen-lamp with flexible tube (Fig. 11), or (where without gas-supply) spirit-lamp and alcohol.

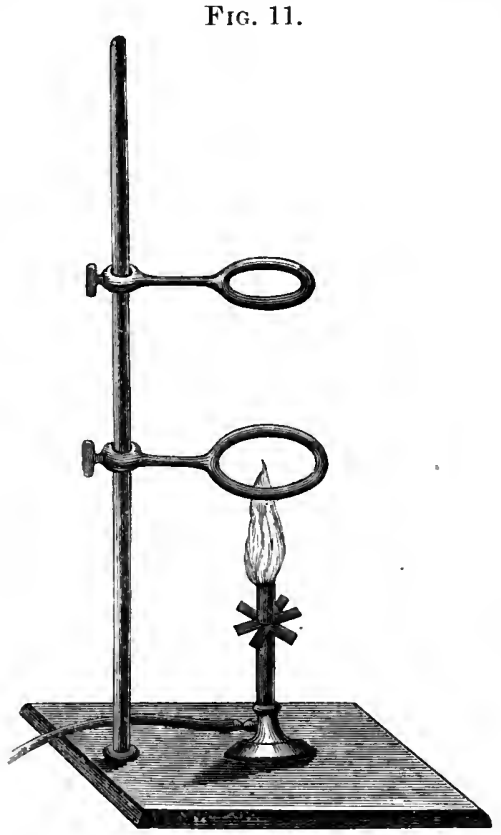

Fig. 12

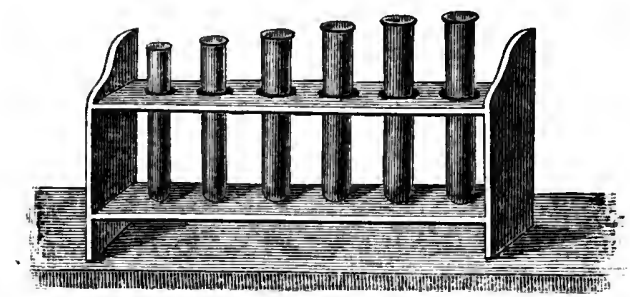

Fig. 13.

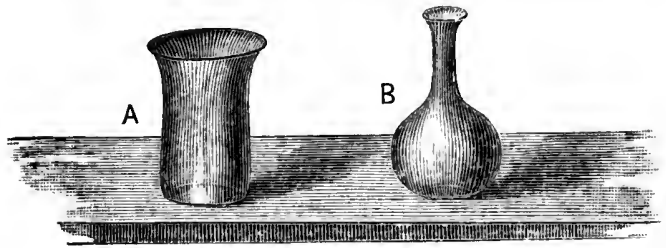

3. Test-tube stand and one dozen assorted test-tubes. (Fig. 12.)

4. Three small beakers from 100 to $150 \mathrm{cc}$. eapacity. (Fig. 13, A.) 
5. Two flasks of 100 to 150 cc. capacity. (Fig. 13, B.)

6. Wash-bottle of about $400 \mathrm{cc}$. capacity. (Fig. 14, A.)

7. Three small glass funnels, about one and a half to two inches in diameter (Fig. 14, B.)

\section{Fig. 14.}

A

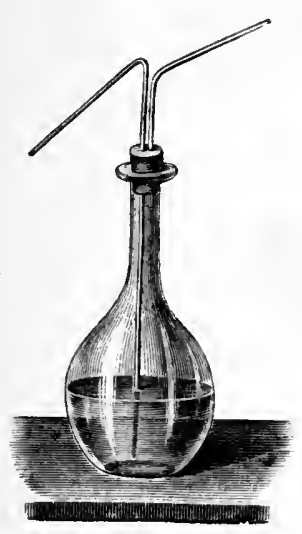

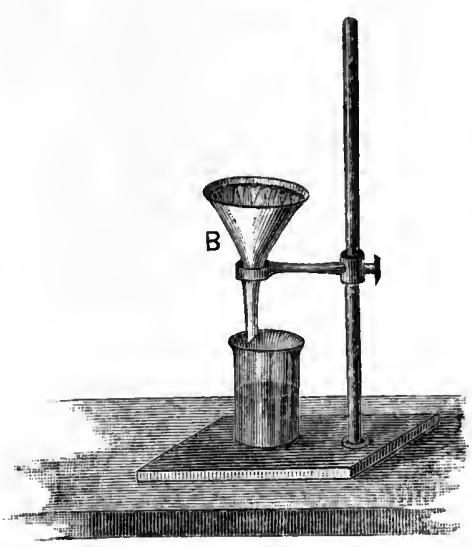

8. A few pieces of glass tubing about ten inches long, and some India-rubber tubing to fit.

9. Three glass rods.

10. Three small porcalain evaporating-dishes, about two inches in diameter. (Fig. 15, A.)

11. Blowpipe. (Fig. 15, B.)

12. Crucible tongs. (Fig. 15, C.)

FIG. 15.

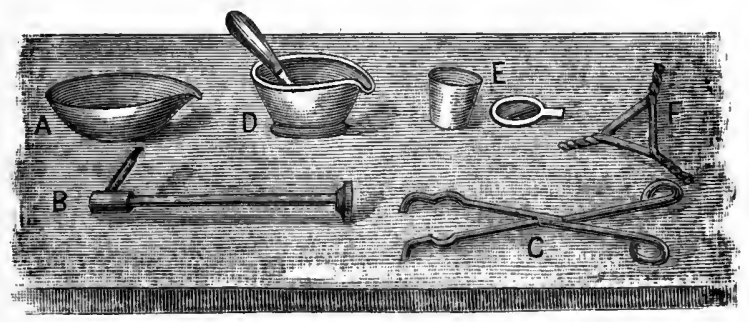

13. Round and triangular file.

14. Wire gauze, about six inches square, or sand-tray.

15. One square inch of platinum foil (not too light), and six inches of platinum wire.

16. Filter-paper.

17. Pair of scissors.

18. One to two dozen assorted corks.

- 19. Sponge and towel.

20. Two watch-glasses. 
21. Small pestle and mortar. (Fig. 15, D.)

22. Small porcelain crucible.

23. Small platinum crucible (Fig. 15, E.)

24. Wire triangle to support the crucible. (Fig. 15, F.)

\section{Reagents needed in qualitative analysis.}

\section{a. Liquids.}

1. Sulphuric acid, sp. gr. $1.84, \mathrm{H}_{2} \mathrm{SO}_{4}$.

2. Sulphuric acid diluted, sp. gr. 1.068 .

3. Hydrochloric acid, sp. gr. 1.16, HCl.

4. Hydrochloric acid diluted, sp. gr., 1.049.

5. Nitric acid, sp. gr. $1.42, \mathrm{HNO}_{3}$.

6. Acetic acid, sp. gr. 1.048, $\mathrm{C}_{2} \mathrm{H}_{4} \mathrm{O}_{2}$.

7. Hydrosulphuric acid, either the gas or its solution in water, $\mathrm{H}_{2} \mathrm{~S}$.

8. Ammonium sulphide, $\left(\mathrm{NH}_{4}\right)_{2} \mathrm{~S}$.

9. Ammonium hydrate (water of ammonia), $\mathrm{NH}_{4} \mathrm{HO}$.

10. Ammonium carbonate, $\left(\mathrm{NH}_{4}\right)_{2} \mathrm{CO}_{3}$. A solution of one part of the salt in a mixture of four parts of water and one part of water of ammonia.

11. Ammonium chloride, $\mathrm{NH}_{4} \mathrm{Cl}$; ten per cent. solution.

12. Ammonium oxalate, $\left(\mathrm{NH}_{4}\right)_{2} \mathrm{C}_{2} \mathrm{O}_{4}$; five per cent. solution.

13. Ammonium molybdate, $\left(\mathrm{NH}_{4}\right)_{2} \mathrm{MoO}_{4}$. A five per cent. solution of the salt in a mixture of equal parts of water and nitric acid.

14. Sodium hydrate, NaHO.

15. Sodium carbonate, $\mathrm{Na}_{2} \mathrm{CO}_{3}$.

16. Sodium phosphate, $\mathrm{Na}_{2} \mathrm{HPO}_{4}$.

17. Sodium acetate, $\mathrm{NaC}_{2} \mathrm{H}_{3} \mathrm{O}_{2}$.

18. Potassium chromate, $\mathrm{K}_{2} \mathrm{CrO}_{4}$.

19. Potassium dichromate, $\mathrm{K}_{2} \mathrm{Cr}_{2} \mathrm{O}_{7}$.

20. Potassium iodide, KI.

21. Potassium ferrocyanide, $\mathrm{K}_{4} \mathrm{FeCy}_{6}$.

22. Potassium ferricyanide, $\mathrm{K}_{6} \mathrm{Fe}_{2} \mathrm{Cy}_{12}$.

23. Potassium sulphocyanate, KCyS.

24. Magnesium sulphate, $\mathrm{MgSO}_{4}$.

25. Barium chloride, $\mathrm{BaCl}_{2}$.

26. Calcium chloride, $\mathrm{CaCl}_{2}$.

27. Calcium hydrate, Ca2HO (lime-water).

28. Calcium sulphate, $\mathrm{CaSO}_{4}$.

29. Ferric chloride, $\mathrm{Fe}_{2} \mathrm{Cl}_{6}$.

30. Lead acetate, $\mathrm{Pb} .2 \mathrm{C}_{2} \mathrm{H}_{3} \mathrm{O}_{2}$.

31. Silver nitrate, $\mathrm{AgNO}_{3}$.

32. Mercuric chloride, $\mathrm{HgCl}_{2}$.

33. Platinic chloride, $\mathrm{PtCl}_{4}$.

Ten per cent. solutions.

Five per cent. solutions.

Ten per cent. solutions.

Saturated solutions.

Five per cent. solutions.

b. Solids.

1. Litmus or blue and red paper.

2. Turmeric paper.

3. Sodium carbonate, dried, $\mathrm{Na}_{2} \mathrm{CO}_{3}$. 
4. Sodium biborate, borax, $\mathrm{Na}_{2} \mathrm{Bo}_{4} \mathrm{O}_{7} \cdot 10 \mathrm{H}_{2} \mathrm{O}$.

5. Sodium-ammonium-hydrogen phosphate (phosphorus salt), $\mathbf{N a}\left(\mathrm{NH}_{4}\right) \mathbf{H} \mathrm{PO}_{4}$. $4 \mathrm{H}_{2} \mathrm{O}$.

6. Potassium carbonate, $\mathrm{K}_{2} \mathrm{CO}_{3}$.

7. Potassium nitrate, $\mathrm{KNO}_{3}$.

8. Potassium chlorate, $\mathrm{KClO}_{3}$.

9. Potassium permanganate, $\mathrm{K}_{2} \mathrm{M} \mathrm{n}_{2} \mathrm{O}_{8}$.

10. Potassium cyanide, $\mathrm{KCy}$.

11. Calcium hydrate, Ca2HO.

12. Ferrous sulphide, FeS.

13. Ferrous sulphate, $\mathrm{FeSO}_{4} \cdot 7 \mathrm{H}_{2} \mathrm{O}$.

14. Manganese dioxide, $\mathrm{MnO}_{2}$.

15. Zinc, granulated, $\mathrm{Zn}$.

16. Copper, $\mathrm{Cu}$.

17. Cupric oxide, CuO.

18. Cupric sulphate, $\mathrm{CuSO}_{4} .5 \mathrm{H}_{2} \mathrm{O}$.

19. Tartaric acid, $\mathrm{H}_{2} \mathrm{C}_{4} \mathrm{H}_{4} \mathrm{O}_{6}$.

20. Starch, $\mathrm{C}_{6} \mathrm{H}_{10} \mathrm{O}_{5}$.

While the apparatus and reagents here enumerated are the more important ones, the analyst will occasionally require others not mentioned in the above lists.

General mode of proceeding in qualitative analysis. Every step taken in analysis should be properly written down in a notebook, and these remarks should be made directly after a reaction has been performed, and not after the nature of the substance has been revealed by perhaps numerous reactions.

Not only the reactions by which positive results have been obtained should be noted, but also those tests and reagents mentioned which have been applied with negative results, that is, which have been applied without revealing the presence of any substance or any group of substances. Such negative results are, however, positive in so far as they prove the absence of a certain substance or certain substances, for which reason they are of direct value and should be noted.

In comparing finally the result obtained by the analysis with the notes taken during the examination, none of them should be contradictory to the conclusions drawn. If, for instance, the preliminary examination showed the substance to have been volatilized by heating upon platinum foil with the exception of a very slight residue, and if, afterwards, other tests show the presence of ammonia and hydrochloric acid and the absence of everything else, and if then the conclusion be drawn that the sub- 
stance is pure ammonium chloride, this conclusion must be incorrect, because pure ammonium chloride is wholly volatile and does not leave a residue. It will then be the task of the operator to find where the mistake occurred, and to correct it.

Use of reagents. A mistake made by most beginners in analyzing is the use of too large quantities both of the substance applied for testing and of the reagents added. This excessive use of material is not only a waste of money, but, what is of greater importance, a waste of time. Some experience in analyzing will soon convince the student of the truth contained in this remark, and will also enable him to select the correct quantities of materials to be used, which rarely exceed $0.5-1$ gram. A smaller amount may frequently answer and a much larger quantity may occasionally be needed, as, for instance, in such cases where highly diluted reagents, such as calcium sulphate solution, lime-water, hydrosulphuric acid-water, etc., are applied.

Preliminary examination. This examination includes the following points :

1. Physical properties. Solid or liquid; crystallized or amorphous; color, odor, hardness, gravity, etc. (On account of possible poisonous properties, the greatest care should be exercised in tasting a substance.)

2. Action on litmus. Examined by holding litmus-paper in the liquid, or by placing the powdered solid upon red and blue litmus-paper, moistened with water. (It should be remembered that many neutral salts, as, for instance, aluminium sulphate, ferrous sulphate, etc., have an acid reaction to litmus-paper, and that such a reaction is consequently not conclusive of the presence of a free acid, nor even of an acid salt.)

3. Heating on platinum foil or in a dry glass tube, open at both ends. (If the substance to be examined be a liquid, it should be evaporated in a small porcelain dish to see whether a solid residue be left or not. If a residue be left, it should be treated like a solid.) The heating of a small quantity of a solid substance upon platinum foil held over the flame of a Bunsen- 
burner or an alcohol lamp, is a test which should never be omitted, as it discloses in most cases the fact whether the substance is of an organic or inorganic nature. Most organic (nonvolatile) substances, when thus heated, will burn with a luminous flame, leaving in many cases a black residue of carbon, which, upon further heating, disappears. In cases where the organic nature of a compound is not clearly demonstrated by heating on platinum foil, the substance is heated with an excess of cupric oxide in a test-tube or other glass tube, provided with a delivery-tube, which passes into lime-water. Upon heating the mixture, the carbon of the organic matter is converted into carbon dioxide, which renders lime-water turbid.

The analytical processes by which the nature of an organic substance is determined, are not considered in this part of the book, but will be mentioned when considering the carbon compounds.

An inorganic substance, heated on platinum foil, may either be volatilized, fused, change color, become oxidized, suffer decomposition, or remain unchanged. (See Table I., page 208.)

Some substances, containing small quantities of water enclosed between the crystals (common salt, for instance), decrepitate when heated, the small fragments being thrown from the foil; such substances should be heated in a test-tube to expel the water and then examined on platinum foil.

4. Heating on charcoal by means of the blowpipe. This test reveals the presence of chlorates and nitrates by the vivid combustion of the charcoal (known as deflagration), which takes place in consequence of the oxidizing action of these substances.

Arsenic is indicated by a characteristic odor of garlic.

5. Heating on charcoal with sodium carbonate and potassium cyanide. A small quantity of the finely powdered substance is mixed with twice its weight of potassium cyanide and dry sodium carbonate. This mixture is placed in a small hole made in a piece of charcoal, and heat applied by means of the blowpipe. Many metallic compounds may be recognized by this test, the metals being liberated and found as metallic globules or shining particles in the fused mass after this has been 
removed from the charcoal and washed with water in a small mortar.

A characteristic incrustation, due to the precipitation of some metallic oxide around the heated spot on the charcoal, is formed by some metals.

If sulphur as such, or in any form of combination, be present in the substance examined by this test, the fused mass contains a sulphide of the alkali (hepar), which may be recognized by placing it on a piece of bright silver (coin) moistened with a drop of water, when the silver will be stained black in consequence of the formation of silver sulphide. The presence of the alkaline sulphide may also be demonstrated by the addition of a few drops of hydrochloric acid to the fused mass, when hydrosulphuric acid is evolved and may be recognized by its odor.

6. Flame tests. Many substances impart a characteristic color to a non-luminous flame. The best mode of performing this test is as follows: A platinum wire is cleaned by washing in hydrochloric acid and water, and heated in the flame until the latter is no longer colored. One end of the wire is fused in a short piece of glass tubing (serving as handle), the other end is bent so as to form a small loop, which is heated, dipped into the substance to be examined, and again held in the lower part of the flame, which then becomes colored.

Some substances show the color-test after being moistened with hydrochloric or sulphuric acid.

A second method of showing flame reactions is to mix the substance with alcohol in a small dish; the alcohol, upon being ignited, shows a colored flame, especially in the dark.

7. Colored borax beads. The compounds of some metals when fused with glass, impart to it characteristic colors. For analytical purposes not the silica-glass, but borax-glass is generally used. This latter is made by dipping the loop of a platinum wire in powdered borax and heating it in the flame (directly, or by means of the blowpipe) until all water has been expelled and a colorless, transparent bead has been formed. To this colorless bead a little of the finely powdered substance is added and strongly heated. The metallic compound is chemically acted upon by the boracic acid, a borate being formed which 
colors the bead more or less intensely, according to the quantity of the metallic compound used.

Some metals (copper, for instance) forming two series of compounds, give different colors to the bead when present in either the higher or lower state of oxidation.

By modifying the blowpipe flame so as either to oxidize (by supplying an excess of atmuspheric oxygen) or deoxidize (by allowing some unburnt carbon to remain in the flame), the metallic compound in the bead may be made to assume the higher or lower state of oxidation. A copper bead may thus be changed from blue to red or red to blue, the blue bead containing the copper in the cupric, the red bead in the cuprous form.

8. Liquefaction of solid substances. Most solid substances have to be dissolved for analysis. The solvents used are water, or for substances insoluble in that liquid the mineral acids, especially diluted, or, if necessary, strong hydrochloric acid. The dissolving action of the acid should be facilitated by the aid of heat. Nitric or even nitrohydrochloric acid may have to be used in some cases.

Three mistakes are frequently made by beginners in dissolving substances in acids, viz.: The substance is not powdered as finely as it should be; sufficient time is not given for the acid to act; too large an excess of the acid is used.

If a substance is partly dissolved by water and partly by one or more other solvents, it may be well to examine the different solutions separately.

Substances insoluble in water and acids have to be rendered soluble by fusion with a mixture of potassium and sodium carbonate, or with potassium acid sulphate, or by the action of hydrofluoric acid.

The insoluble sulphates of the alkaline earths, when fused with the alkaline carbonates, are converted into carbonates, while the sulphates of the alkalies are formed. The latter compounds may be eliminated by washing the fused mass with water and filtering; the solid residue upon the filter contains the carbonates of the alkaline earths, which may be dissolved in hydrochloric acid. 
A NALYTICAL CHEMISTRY.

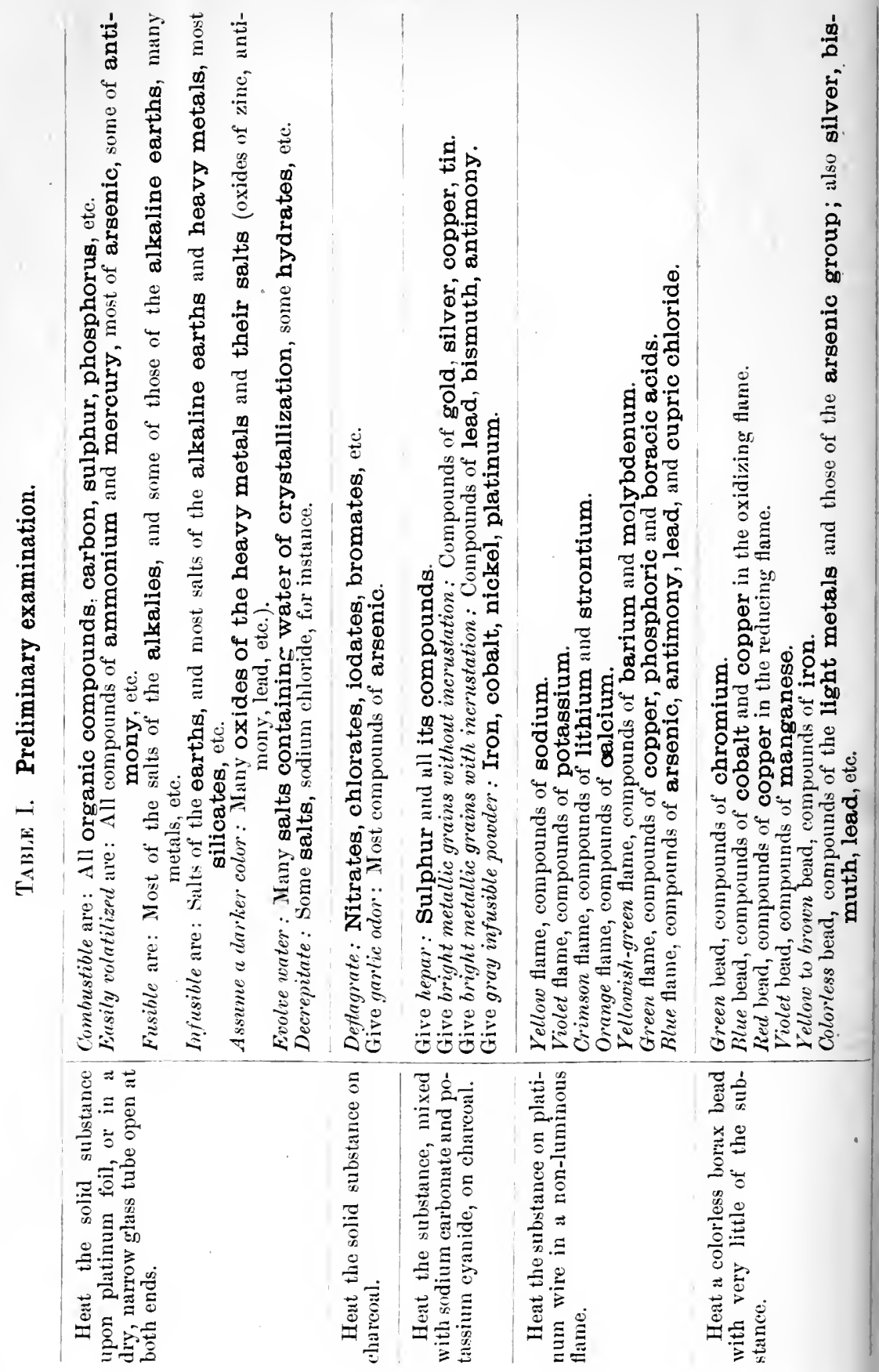




\section{Questions.}

311. What is analytical chemistry, and what, is the object of qualitative and of quantitative analysis?

312. What properties of a substance should first be noticed in making a qualitative analysis?

313. By what tests may organic compounds be distinguished from inorganic compounds?

314. Explain the terms decrepitation and deflagration.

315. Mention some substances which are completely volatilized by heat, some which are fusible, and some which are not changed by heating them.

316. What is meant by "hepar," and which element is indicated by the formation of hepar?

317. Mention some metals which may be liberated from their compounds by heating on charcoal with potassium cyanide and carbonate.

318. Which metallic compounds and which acids are capable of coloring a non-luminous flame? Name the colors imparted.

319. State the metals which impart characteristic colors to a borax bead.

320. Which solvents are used for liquefying solids, and what precautions should be observed in this operation?

\section{SEPARATION OF METALS IN DIFFERENT GROUPS.}

General remarks. The preliminary examination will, in most cases, decide whether or not a metal or metals are present in the substance to be examined. If there be metals, the solution should be treated according to Table II., in order to find the group or groups to which these metals belong, and also to separate them into these groups, the individual nature of the metals themselves being afterwards demonstrated by special methods.

The simplest method of separating the 54 known metals from each other, when all in one solution, would be to add successively 54 different reagents, each of which should form an insoluble compound with but one of the metals. By separating this insoluble compound from the metals remaining in solution (by filtration), and by thus precipitating one metal after the other, they all could be easily separated. We have, however, no such 54 reagents, and are, consequently, compelled to pre- 
cipitate a number of metals together, and the reagents used for this purpose are known as group-reagents.

They are:

1. Hydrosulphuric acid, added to the solution previously acidified by hydrochloric acid. Precipitated are: the metals of the arsenic and lead groups as sulphides.

2. Ammonium sulphide, added after supersaturating with ammonium hydrate. Precipitated are: the metals of the iron group and of the earths as sulphides or hydrates.

3. Ammonium carbonate. Precipitated are: the metals of the alkaline earths as carbonates.

4. In solution are left: the metals of the alkalies and magnesium.

The order in which these group-reagents are added cannot be reversed or changed, because ammonium sulphide added first would precipitate not only the metals of the iron group and the earths, but also the metals of the lead group; ammonium carbonate would also precipitate most of the heavy metals.

For the same reasons, in separating metals of the different groups, the group-reagents must be added in excess, that is, enough of them must be added to precipitate the total quantity of the metals of one group, before it is possible to test for metals of the next group. Suppose, for instance, a solution to contain a salt of bismuth only. Upon the addition of hydrosulphuric acid to the acidified solution, a dark brown precipitate (of bismuth sulphide) is produced, indicating the presence of a metal of the lead group. Suppose, further, that hydrosulphuric acid has not been added in a sufficient quantity to precipitate the whole of the bismuth, then ammonium sulphide, as the next group-reagent, would produce a further precipitation in the filtrate, which fact would lead to the assumption that a metal of the iron group was present, which, however, is not the case.

If the solution contain but one metal, the group-reagents are added successively in small quantities to the same solution, until the reagent is found which causes a precipitation, which reagent is then added in somewhat larger quantity in order to produce a sufficient amount of the precipitate for further examination. 
Acidifying the solution. Hydrosulphuric acid has to be added to the acidified solution for two reasons, viz.: In a neutral or alkaline solution some metals of the arsenic group (which are to be precipitated) would not be precipitated by hydrosulphuric acid; some of the metals of the iron group (which are not to be precipitated) would be thrown down.

The best acid to be used in acidifying is hydrochloric acid; but this acid forms insoluble compounds with a few of the metals of the lead group, causing them to be precipitated. Completely precipitated by hydrochloric acid are mercurous and silver compounds; partially precipitated are compounds of lead, chloride of lead being somewhat soluble in water. The precipitate formed by hydrochloric acid may be exarnined by Table III., page 214.

Hydrochloric acid added to a solution may in a few cases (other than those just mentioned) cause a precipitate, as, for instance, when added to solutions containing certain compounds of antimony or bismuth (the precipitated oxychlorides of these metals are soluble in excess of the acid), to metallic oxides or hydrates which have been dissolved by alkaline hydrates (for instance, hydrate of zinc dissolved in potassium or ammonium hydrate), to solution of alkaline silicates, when silica separates, etc.

Addition of hydrosulphuric acid. This reagent is employed either in the gaseous state (by passing it through the heated solution) or as sulphuretted hydrogen water. The latter reagent answers in those cases where but one metal is present; if, however, metals of the arsenic and lead groups are to be separated from metals of other groups, the gas must be used.

In some cases sulphur is precipitated on the addition of hydrosulphuric acid, while a change in color may take place. This change is due to the deoxidizing action of hydrosulphuric acid, the hydrogen of this reagent becoming oxidized and converted into water, while sulphur is liberated. Thus, brown ferric compounds are converted into pale green ferrous compounds; red solutions of acid chromates become green; and red permanganates or green manganates are decolorized.

The same deoxidizing action of hydrosulphuric acid is the reason why this reagent cannot be employed in a solution con- 
taining free nitric acid, which latter compound oxidizes the hydrosulphuric acid.

Separation of the metals of the arsenic from those of the lead group. The precipitate produced by hydrosulphuric acid in acid solution contains the metals of the arsenic and lead groups. They are separated by means of ammonium sulphide, which dissolves the sulphides of the arsenic group, but does not act on those of the lead group.

Addition of ammonium sulphide. This reagent should never be added to the acid solution, but the solution should be previously supersaturated by ammonium hydrate, as, otherwise, a precipitate of sulphur may be formed. The yellow ammonium sulphide is almost invariably a polysulphide of ammonium, that is, ammonium sulphide which has combined with one or more atoms of sulphur. If an acid be added to this compound, an ammonium salt is formed, hydrosulphuric acid is liberated, and sulphur precipitated:

$$
\left(\mathrm{NH}_{4}\right)_{2} \mathrm{~S}_{2}+2 \mathrm{HCl}=2 \mathrm{NH}_{4} \mathrm{Cl}+\mathrm{H}_{2} \mathrm{~S}+\mathrm{S} .
$$

Ammonium sulphide precipitates the metals of the iron group as sulphides, with the exception of chromium, which is precipitated as hydrate; aluminium is precipitated in the same form of combination.

Ammonium sulphide (or ammonium hydrate) causes also the precipitation of metallic salts which have been dissolved in acids, as, for instance, of the phosphates, borates, silicates, or oxalates of the alkaline earths, magnesium, and others. The processes by which the nature of these precipitates is to be recognized are found in Table VI., page 218.

Addition of ammonium carbonate. The reagent used is the commercial salt, dissolved in water, to which some ammonium hydrate has been added. Heating facilitates complete precipitation of the carbonates of the alkaline earths. 
SEPARATION OF METALS IN DIFFERENT GROUPS. 213

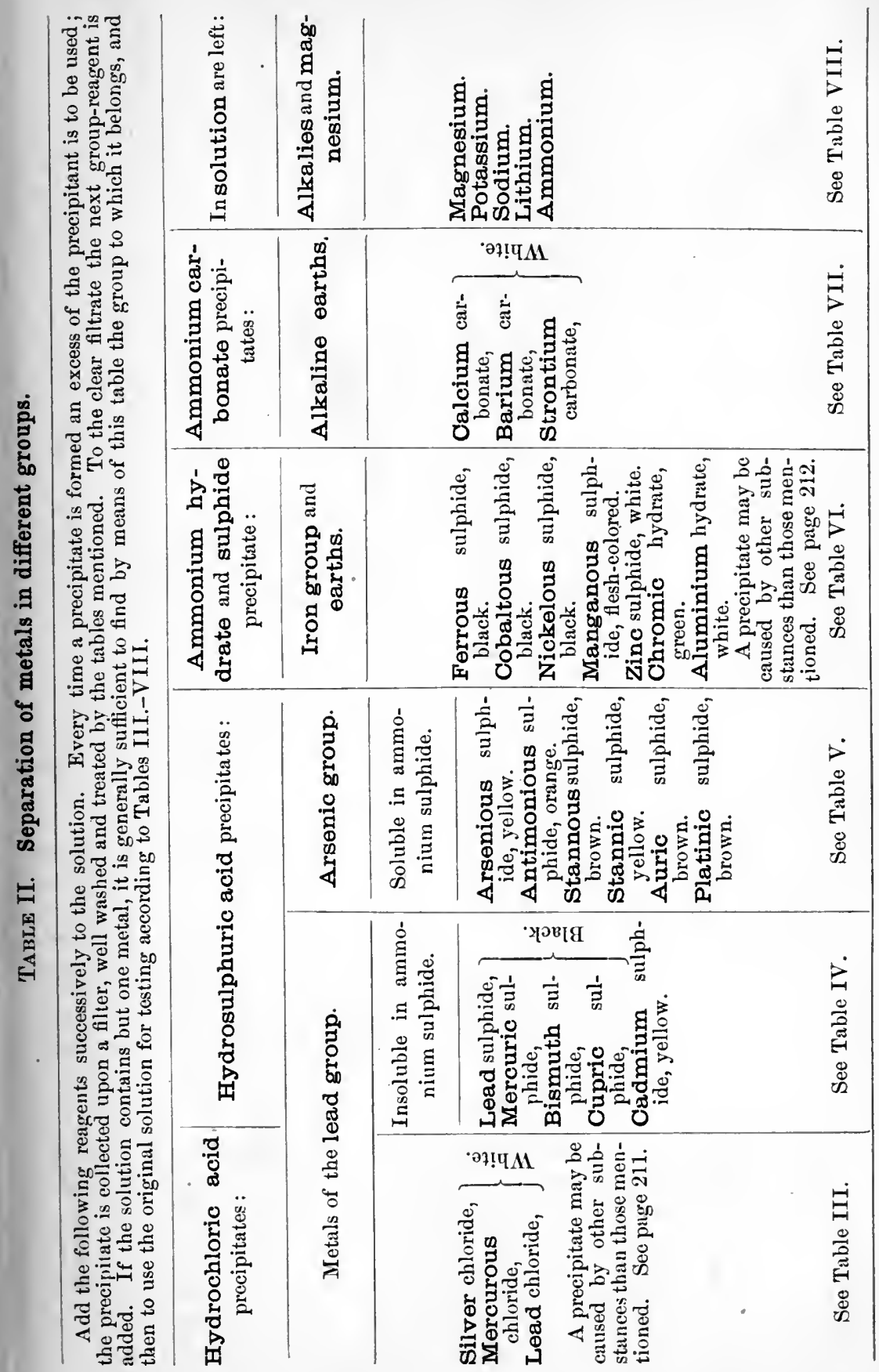




\section{Questions.}

321. State the 3 groups of heavy, and the 3 groups of light metals.

322 . By which two reagents may all heavy metals be precipitated?

323. Why is a solution acidified before the addition of hydrosulphuric acid, when testing for metals?

324. Which metals are precipitated by hydrochloric acid?

325 . Which two groups of metals are precipitated by hydrosulphuric acid in acid solution?

326. How are the sulphides of the arsenic group separated from those of the lead group?

327 . Why is an acid solution neutralized or supersaturated by ammonium hydrate, before adding the ammonium sulphide?

328. Which two groups of metals are precipitated by ammonium sulphide, and in what forms of combination?

329. Name the group-reagent for the alkaline earths.

330. Which metals may be left in solution after hydrosulphuric acil, ammonium sulphide, and ammonium carbonate have been added?

\section{SEPARATION OF THE METALS OF EACH GROUP.}

TABLE III. Treatment of the precipitate formed by hydrochloric acid.

The precipitate may contain silver, mercurous, and lead chlorides. Boil the washed precipitate with much water, and filter.

Filtrate may contain lead chloride. Add sulphuric acid: a white precipitate of lead sulphate is produced.

Residue may consist of mercurous and silver chlorides. Digest residue with ammonium hydrate.

Solution may contain silver. Neutralize with nitric acid, when silver chloride is reprecipitatıd.
A dark gray residue indicates mercury, the white mercurous chloride having been converted into dimercurous ammonium chloride.

Treatment of the precipitate formed by hydrosulphuric acid in warm acid solution. The precipitate is collected upon a small filter, well washed with water, and then examined for its solubility in ammonium sulphide. This is done by placing a portion of the washed precipitate in a test-tube, adding ammonium sulphide, and warming gently. It is either wholly insoluble (metals of the lead group), and treated according to Table IV, 
or fully soluble (metals of the arsenic group), and treated according to Table $V$., or it is partly soluble and partly insoluble (metals of both groups). In the latter case, the total quantity of the washed precipitate is to be treated with warm ammonium sulphide; upon filtering, an insoluble residue is left, which is treated according to Table IV.; to the filtrate, diluted sulphuric acid is added as long as a precipitate is formed, which precipitate contains the metals of the arsenic group as sulphides, generally with some sulphur from the ammonium sulphide.

The precipitation of sulphur in the absence of metals of the arsenic group, frequently leads beginners to the assumption that metals of this group are present. The precipitate consisting of sulphur only is white and milky, but flocculent, and more or less colored in the presence of the metals of the arsenic group.

\section{Questions.}

331. By what tests ean mercurous chloride be distinguished from the chloride of silver or lead?

332. How can it be proved, that a precipitate produced by hydrosulphuric acid in an acid solution contains metals of the arsenic and lead groups?

333. How can mercuric sulphide be separated from the sulphides of copper and bismuth?

334. How does ammonium hydrate act on a solution containing bismuth and copper?

335. State the action of strong, hot hydrochloric acid on the sulphides of arsenic and antimony.

336. Suppose a solution to contain salts of iron, aluminium, zinc, and manganese; by what processes could these four metals be separated and recognized?

337. How can barium, calcium, and strontium be recognized when dissolved together?

338. By what test is magnesium recognized?

339. State a method of separating potassium when mixed with other metallic compounds.

340. How are ammonium compounds recognized when in solution with other metals? 


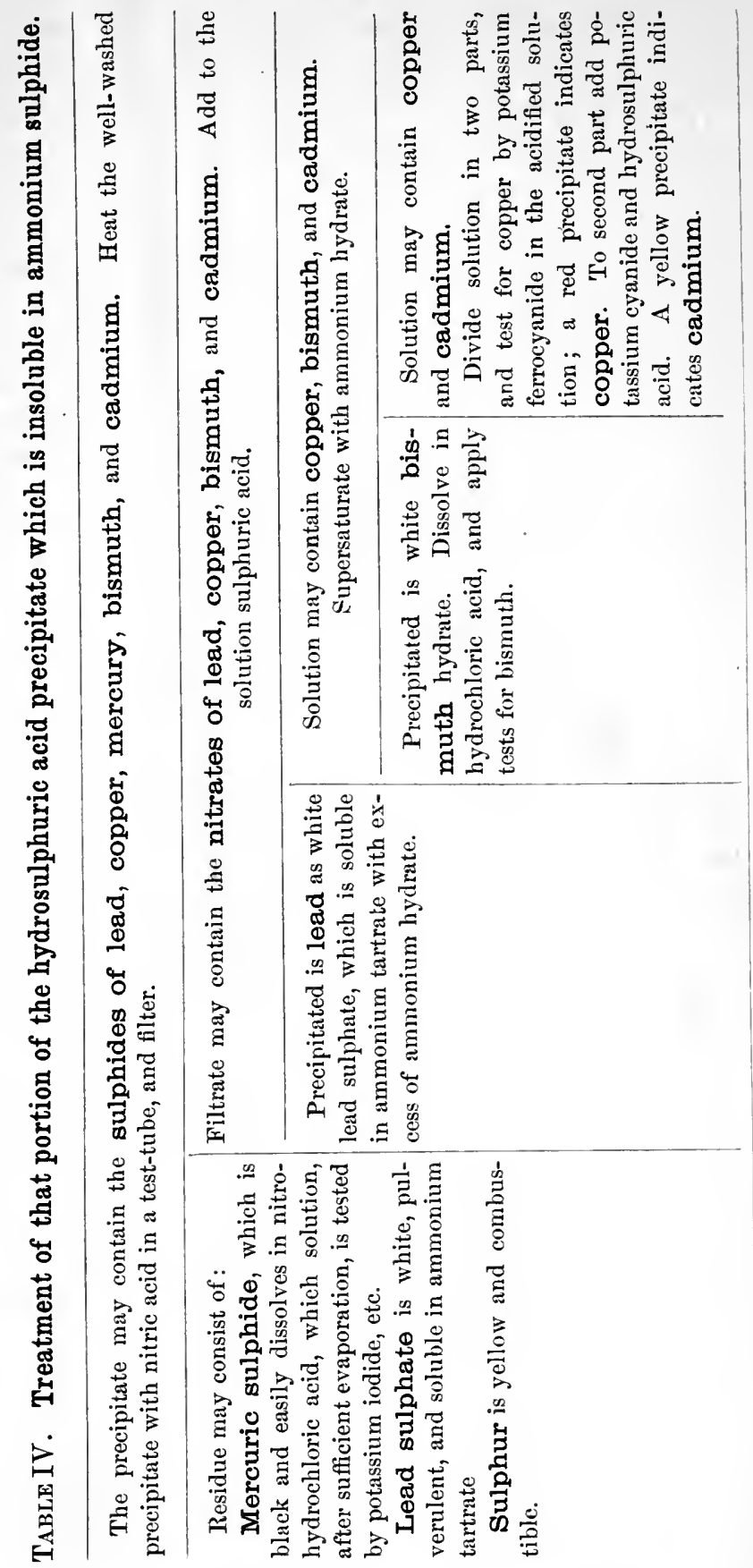


TABLE V. Treatment of the hydrosulphuric acid precipitate which is soluble in ammonium sulphide.

The precipitate may contain the sulphides of arsenic, antimony, tin, and of a few of those metals which are but rarely met with in qualitative analysis, such as gold, platinum, molybdenum, and others, which latter metals, if suspected, may be detected by special tests.

Boil the washed precipitate with strong hydrochloric acid.

\begin{tabular}{l|l}
\hline $\begin{array}{c}\text { An insoluble yellow residue con- } \\
\text { sists of arsenious sulphide. }\end{array}$ & $\begin{array}{l}\text { The solution may contain the chlorides } \\
\text { of antimony and tin. }\end{array}$ \\
The residue is dissolved by boil- & $\begin{array}{l}\text { The solution is introduced into Marsh's } \\
\text { ing with hydrochloric acid and a }\end{array}$ \\
little potassium chlorate, and the & $\begin{array}{l}\text { evolved as antimoniuretted hydrogen, while } \\
\text { solution examined by Marsh's test. } \\
\text { tin remains with the undissolved zinc as a } \\
\text { black metallic powder, which may be col- } \\
\text { lected, washed, dissolved in hydrochloric } \\
\text { acid, and the solution tested by the special } \\
\text { tests for tin. }\end{array}$
\end{tabular}




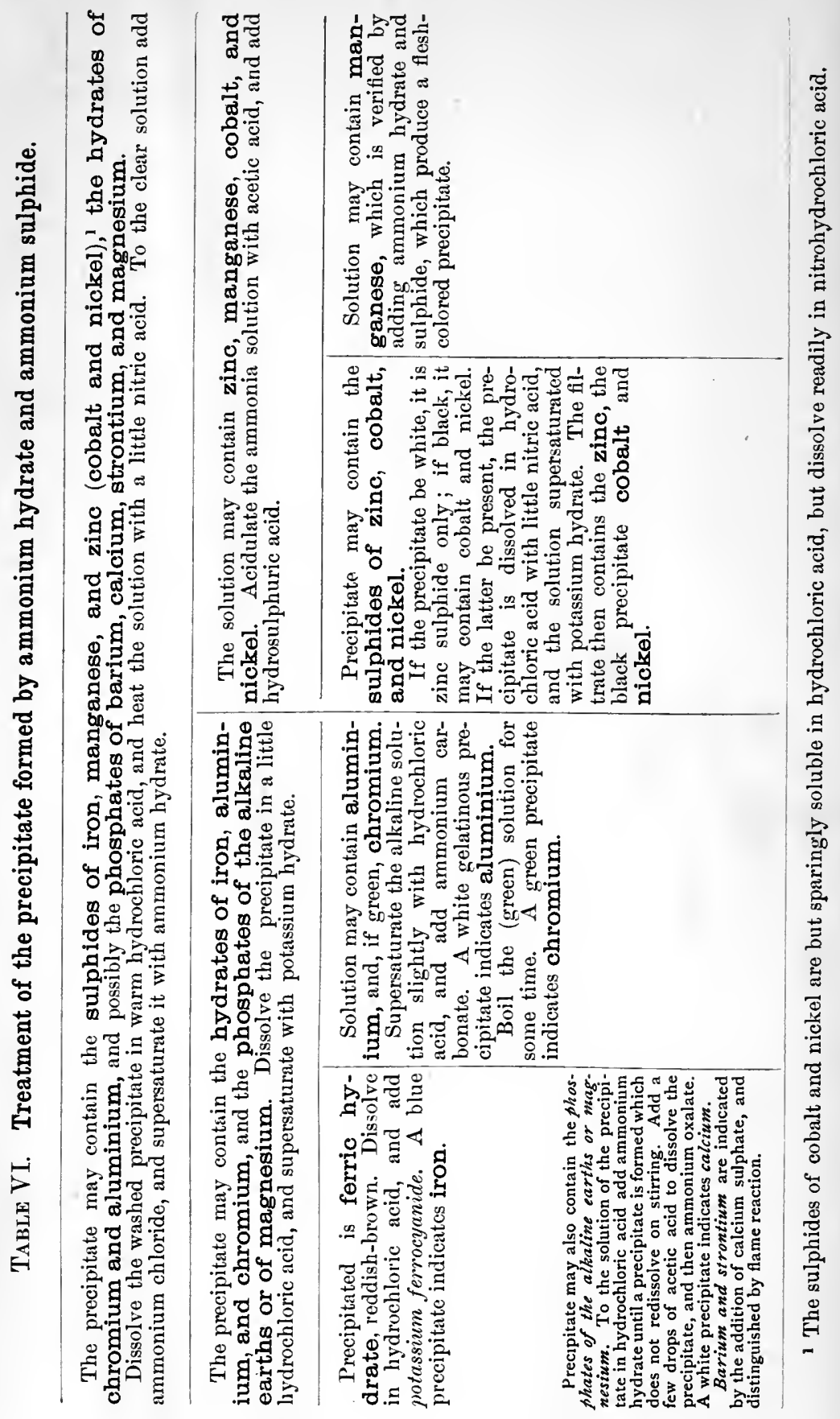




\section{TABLE VII. Treatment of the precipitate formed by ammonium carbonate.}

The precipitate may contain the carbonates of barium, calcium, and strontium.1 Dissolve the precipitate in acetic acid, and add potassium chromate.

Precipitated is ba- Solution may contain calcium and strontium. rium, as pale yellow Add sulphuric acid, and let stand for fifteen minutes. barium chromate.

\begin{tabular}{l|l}
\hline Precipitated is stron- & $\begin{array}{l}\text { Solution may contain } \\
\text { calcium. Add ammo- } \\
\text { tium, as white stron- } \\
\text { tium sulphate. }\end{array}$ \\
nium oxalate. A white \\
precipitate indicates cal- \\
cium.
\end{tabular}

1 If an insufficient quantity of ammonium chloride be present, some magnesia may also be precipitated.

\section{TABLE VIII. Detection of the alkalies and of magnesium.}

The fluid which has been treated with hydrochloric acid, hydrosulphuric acid, ammonium hydrate, sulphide, and carbonate may contain magnesium and the alkalies.

Divide solution into two portions.

To the first portion add sodium phosphate. A white crystalline precipitate indicates magnesium. ${ }^{1}$

The second portion is evaporated to dryness, and all ammonium compounds are expelled by heat. The residue is dissolved in water, and platinic chloride added. A yellow precipitate indicates potassium. The residue is also examined by flame-test: a yellow color indicating sodium; a red color lithium.

Ammonium compounds have to be tested for in the original fluid by treating it with calcium hydrate, when ammonia gas is liberated.

1 If an insufficient quantity of ammonium chloride has been produced in the original solution by the addition of hydrochloric acid and ammonium hydrate, a portion of the magnesia may have been precipitated by the ammonium carbonate. 


\section{DETECTION OF ACIDS.}

General remarks. There are no general methods (similar to those for the separation of metals) by which all acids can be separated, first into different groups, and afterwards into the individual acids. It is, moreover, impossible to render all acids soluble (when in combination with certain metals) without decomposition, as, for instance, in the case of carbonic acid when in combination with calcium; calcium carbonate is insoluble in water, and when the solution by means of acids is attempted, decomposition takes place with liberation of carbon dioxide. Many other acids suffer decomposition, in a similar manner, when attempts are made to render soluble the substances in which they occur.

It is due to these facts that a complete separation of all acids is not so easily accomplished as the separation of metals. There is, however, for each acid a sufficient number of characteristic tests by which it may be recognized; moreover, the preliminary examination, as well as the respective solubility of the substance, and the nature of the metal or metals present, will aid in pointing out the acid or acids which are believed to be present.

If, for instance, a solid substance be completely soluble in water, and if the only metal found were iron, it would become unnecessary to test for carbonic, phosphoric, and hydrosulphuric acids, because the combinations of these acids with iron are insoluble in water; there might, however, be present sulphuric, hydrochloric, nitric, and many other acids, which form soluble salts with iron.

Detection of acids by means of the action of strong sulphuric acid upon the dry substance. The action of sulphuric acid upon a dry powdered substance often furnishes such characteristic indications of the presence or absence of certain acids, that this treatment should never be omitted when a search for acids is made.

When the substance under examination is liquid, a portion should be evaporated to dryness, and, if a solid residue remains, it should be treated in the same manner as solids. 
Most non-volatile, organic substances (including most organic acids) color sulphuric acid dark when heated with it.

Dry inorganic salts when heated with sulphuric acid are either decomposed, with liberation of the acid (which may escape in the gaseous state), or with liberation of volatile products (produced by the decomposition of the acid itself), or no apparent action takes place. See Table IX.

Detection of acids by means of reagents added to their neutral or acid solution. Whenever a substance is soluble in water, there is little difficulty of finding the acid by means of Table X.; but if the substance is insoluble in water, and has to be rendered soluble by the action of acids, this table may, in some cases, be of no use, because the acid originally present in the substance may have been liberated, and escaped in a gaseous state (as, for instance, when dissolving insoluble carbonates in acids), or the tests mentioned in the table may refer to neutral solutions, while it is impossible to render the solution neutral without reprecipitating the dissolved acid. If calcium phosphate, for instance, be dissolved by hydrochloric acid, the magnesium test for phosphoric acid cannot be used, because this test can only be applied to a neutral or alkaline solution; in attempting, however, to neutralize the hydrochloric acid solution, calcium phosphate itself is reprecipitated.

Table XI., showing the solubility or insolubility (in water) of over 300 of the most important inorganic salts, oxides and hydrates, will greatly aid the student in studying this important feature. It will also guide him in the analysis of inorganic substances, as it gives directions for over 300 (positive or negative) tests for metals, and an equal number for acids.

To understand this, it must be remembered that any salt (or oxide or hydrate) which is insoluble in water may be produced and precipitated by mixing two neutral solutions, one containing the metal, the other containing the acid of the insoluble salt to be formed. For instance: Table XI. states that the carbonates of most metals are insoluble in water. To produce, therefore, the carbonate of any of these metals (zinc, for instance) it becomes necessary to add to any solution of zinc (sulphate, chloride, or nitrate of zinc) any soluble carbonate (sodium or potassium carbonate), when the insoluble zinc carbonate is produced. 
Soluble carbonates are consequently reagents for soluble zinc salts, while at the same time soluble zinc salts are reagents for soluble carbonates.

For similar reasons soluble zinc salts are, according to Table XI., reagents for soluble phosphates, arseniates, arsenites, hydrates, and sulphides, but not for iodides, chlorides, sulphates, nitrates, or chlorates.

The insolubility of a compound in water is not an absolute guide for preparing this compound according to the general rule given, there being some exceptions.

For instance: Cupric hydrate is insoluble in water; by adding solution of cupric sulphate to any soluble hydrate, therefore, the insoluble cupric hydrate should be precipitated, and is precipitated by the soluble hydrates of potassium and sodium, but not by the soluble hydrate of ammonium, on account of the formation of the soluble ammonium cupric sulphate.

There are not many such exceptions, and to mention them in the table would have greatly interfered with its simplicity, for which reason they have been omitted.

For the same reason some compounds, which are not known at all, have not been specially mentioned. For instance, according to Table XI., aluminium carbonate and chromium carbonate are insoluble salts; actually, however, these compounds do not exist, the affinity between the weak carbonic acid and the feeble bases not being sufficient to unite them.

It may be finally stated that no well-defined line can be drawn between soluble and insoluble substances. There is scarcely any substance which is not slightly soluble in water, and many of the so-called soluble substances are but very sparingly soluble, as, for instance, the hydrate and sulphate of calcium. 


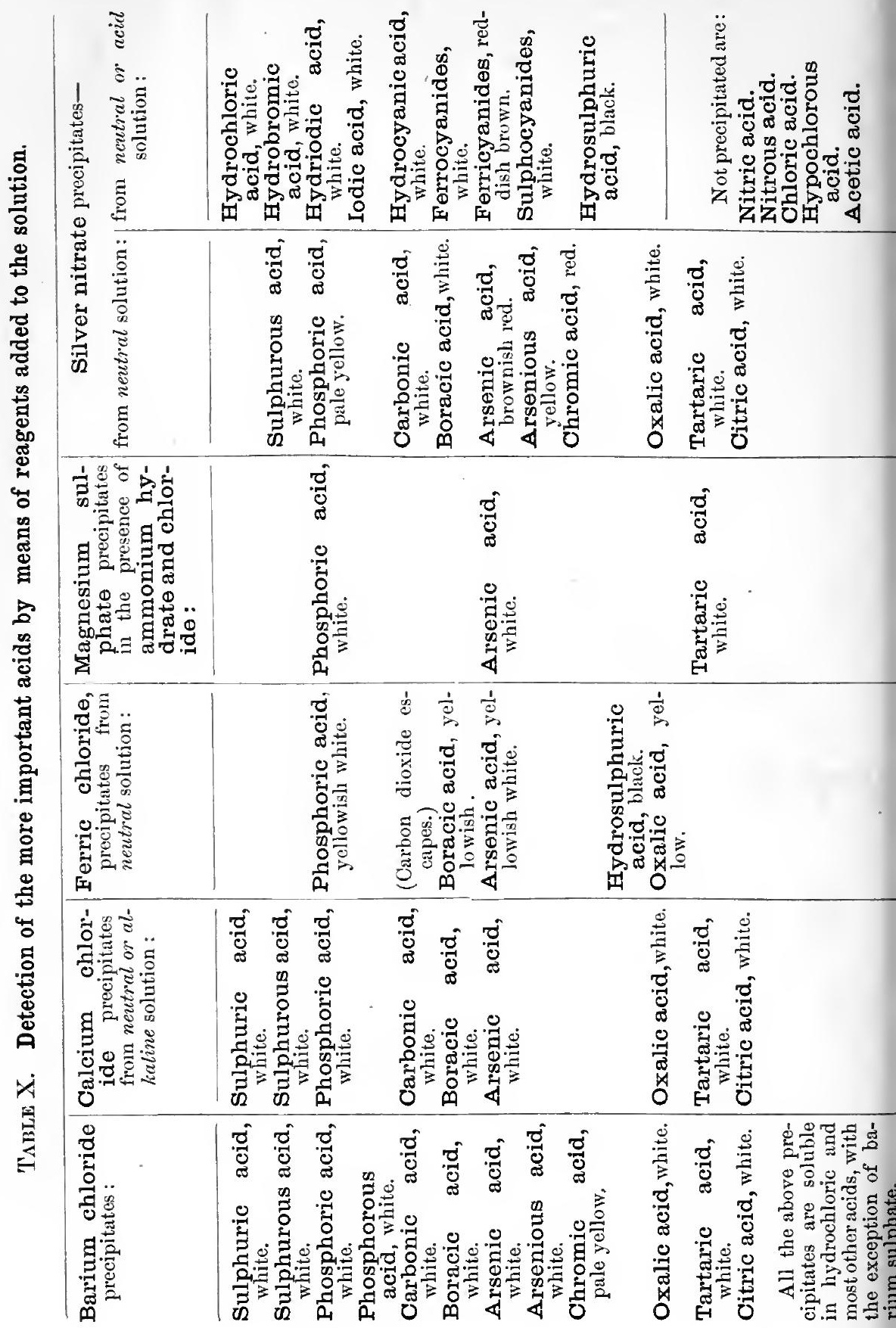


Table XI.

Systematically arranged table showing the solubility and insolubility of inorganie salts and oxides in water.

The dark squares represent insoluble, the white soluble compounds.

\begin{tabular}{|c|c|c|c|c|c|c|c|c|c|c|c|c|c|}
\hline & & 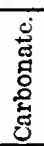 & 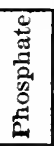 & 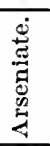 & 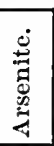 & 递 & 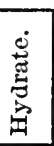 & 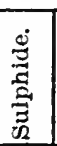 & 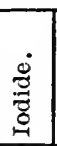 & 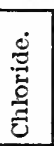 & 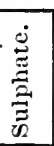 & 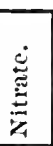 & 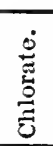 \\
\hline Potassium & \multirow{3}{*}{ 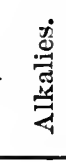 } & & & & & & & & & & & & \\
\hline Sodium & & & & & & & & & & & & & \\
\hline Amnionium & & & & & & & & & & & & & \\
\hline Calcium & \multirow{3}{*}{ 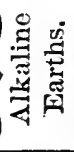 } & & & & 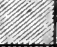 & & & & & & & & \\
\hline Barium & & & & & sena & & & & & & (2) & & \\
\hline Strontium & & & & & - & & & & & & & & \\
\hline \multicolumn{2}{|l|}{ Magnesium } & & & & & & & & & & & & \\
\hline \multicolumn{2}{|l|}{ Aluminium } & & & & & & & & & & & & \\
\hline \multicolumn{14}{|l|}{ Ferric } \\
\hline Ferrous & \multirow{6}{*}{ 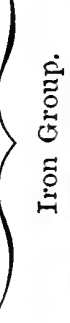 } & & & & III & $y_{1}=2$ & $-1 / 1$ & & & & & & \\
\hline Zine & & & & & 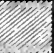 & & & & & & & & \\
\hline Chrominm & & & & & & & & & & & & & \\
\hline Nickel. & & & & V & $y_{2}$ & & I & $4=1$ & & & & & \\
\hline Cobalt & & & & & S & & pe & 1 & & & & & \\
\hline Manganese & & & & & 殓 & & & $=1$ & & & & & \\
\hline \multicolumn{14}{|l|}{ Stannic } \\
\hline Stannous & \multirow{6}{*}{ 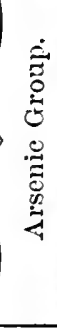 } & & & & & & & $=$ & & & & & \\
\hline Arsenic & & & & L & 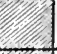 & & & & $=2$ & & & & \\
\hline Arsenious & & II & & (-) & & & & & 两 & & & & \\
\hline Antimony & & & & & & & & & 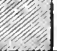 & & & & \\
\hline Gold & & & & 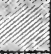 & in & & & & ente & & & & \\
\hline Piatinum & & & & & 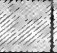 & & & & 2 & & & & \\
\hline \multicolumn{14}{|l|}{ Copper } \\
\hline Bismuth & \multirow{6}{*}{ 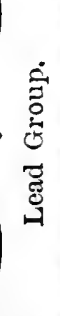 } & & & & & & & & VIn & & & & \\
\hline Cadnifum & & & & & & & & & का & & & & \\
\hline Mercuric & & & & & & & & & VIn & & & & \\
\hline Mercurous & & \multicolumn{11}{|c|}{ Mereurous } & \\
\hline Silver. & & & & & & & 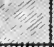 & & 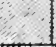 & & & & \\
\hline Leati & & & & & & & 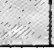 & $=$ & 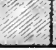 & The & $y x$ & & \\
\hline
\end{tabular}




\section{Questions.}

341. Why is sulphuric acid added to a solid substance when it is to be examined for acids?

342. Mention some acids which cause the liberation of colorless, and some which cause the liberation of colored gases when the salts of these acids are heated with sulphuric acid.

343. Mention an acid which is precipitated by barium chloride in acid solution, and some acids which are precipitated by the same reagent in neutral solution.

344. Which acids may be precipitated by silver nitrate from neutral solutions, and which from either neutral or acid solutions?

345. Mention some acids which form soluble salts only.

346. Mention 3 soluble, and 3 insoluble carbonates, phosphates, arseniates, sulphates, and sulphides respectively.

347. Which oxides or hydrates are soluble, and which are insoluble in water?

348. Mention some metals, the solutions of which are precipitated by soluble chlorides, iodides, and sulphides.

349. State a general rule according to which most insoluble salts may be formed from two other compounds.

350. Why is it sometimes impossible to render a substance soluble in order to test for the acid in the solution obtained?

\section{DETECTION OF IMPURITIES IN OFFICINAL INORGANIC CHEMIICAL PREPARATIONS.}

General remarks. Very little has been said, heretofore, about impurities which may be present in the various chemical preparations, and this omission has been intentional, because it would have increased the bulk of this work beyond the limits considered necessary for the beginner.

Impurities present in chemical preparations are either derived from the materials used in their manufacture, or they have been intentionally added as adulterations. In regard to the last, no general rule for detecting them can be given, the nature of the adulterating article varying with the nature of the substance adulterated; the general properties of the substance to be examined for purity will, in most cases, suggest the nature of those substances which may possibly have been 
added, and for them a search has to be made, or, if necessary, a complete analysis, by which the absence of everything else but the constituents of the pure substance is proved.

Impurities derived from the materials used in the manufacture of a substance (generally through an imperfect or incorrest process of manufacture), or from the vessels used in the manufacture, are generally but few in number (in any one substance), and their nature can, in most cases, be anticipated by one familiar with the process of manufacture itself. For one not acquainted with the mode of preparation, it would be a rather difficult task to study the nature of the impurities which might possibly be present.

The same remarks apply to the methods by which the impurities can be detected. For one familiar with analytical chemistry it is an easy task to suggest, in most cases, the best and quickest method by which the presence or absence of an impurity can be demonstrated; for one unacquainted with these methods, it might be an impossibility to do so, even if a method were given.

For these reasons little stress has been laid upon the occurrence of impurities in the various chemical preparations heretofore considered; moreover, the U. S. P. itself gives, in most cases, directions for the detection of impurities, and, finally, the analyst may avail himself of books specially treating of the examination of chemicals.

In order to give the student, and especially the beginner, a guide in the examination for impurities, the following pages furnish a few directions in regard to the impurities which present in the more important chemical preparations, and also some of the tests used for recognizing them.

Examination of sulphuric, sulphurous, nitric, phosphoric, and hydrochloric acids. The pure acids should be colorless, and upon evaporation leave no residue whatever (phosphoric acid requires red heat for complete evaporation). After being neutralized with an alkali, and then slightly acidulated with hydrochloric acid, they should give no precipitate with hydrosulphuric acid, ammonium hydrate, sulphide, or carbonate.

Sulphuric, phosphoric, and hydrochloric acids sometimes (nitric 
acid very rarely) contain arsenic, and should, therefore, be examined by Marsh's test

Sulphuric acid may contain sulphurous or nitric acid; the former may be recognized by potassium permanganate which is decolorized by sulphurous acid; the latter is detected by carefully pouring solution of ferrous sulphate upon the acid, when, if nitric acid be present, a brownish zone appears at the line of contact.

Sulphurous acid frequently contains sulphuric acid; it should not give more than a very slight turbidity with barium chloride (limit of sulphuric acid).

Nitric acid diluted with 5 parts of water should afford no pre. cipitate with either barium chloride or silver nitrate (absence of sulphuric and hydrochloric acids).

Phosphoric acid should be tested for phosphorous, nitric, sulphuric, hydrochloric, pyrophosphoric, and metaphosphoric acids. Phosphorous acid is indicated by the formation of a dark color when silver nitrate is added to the diluted acid; sulphuric and hydrochloric acids by the formation of white precipitates on the addition of barium chloride or silver nitrate, respectively, to the diluted acid; pyrophosphoric and metaphosphoric acids, by the formation of a precipitate upon adding to the acid an equal volume of tincture of chloride of iron.

Hydrochloric acid, after being diluted, should not give a precipitate with barium chloride (absence of sulphuric acid), and should not liberate iodine from potassium iodide (absence of free chlorine).

The officinal acids should have the strength required by the U. S. P. The amount of actual acid can either be determined by the specific gravity of the liquid acid, or by the quantity of an alkali required to neutralize a certain quantity of the acid.

Exámination of potassium, sodium, and ammonium compounds. The acidulated solution of the hydrates, carbonates, bicarbonates, sulphates, nitrates, and phosphates of potassium, sodium, and ammonium should afford no precipitate with hydrosulphuric acid, or with the hydrate, sulphide, carbonate, or phosphate of ammonium (absence of heavy metals, alkaline earths, and magnesium).

The nitrates, hydrates, carbonates, and bicarbonates (the three 
latter), after being supersaturated with nitric acid, should be tested with solution of barium chloride and silver nitrate for sulphates and chlorides.

Bicarbonates may contain carbonates; the latter are indicated by a white precipitate formed with barium chloride in the cold solution; bicarbonates are not precipitated by this reagent in the cold.

Iodides may contain an iodate, which is indicated by a blue color on the addition of gelatinized starch and some diluted sulphuric acid; chlorides and bromides may be detected by precipitating a solution of the iodide with an excess of silver nitrate, collecting and washing the precipitated iodide (bromide and chloride) of silver, and digesting it with ammonium hydrate, which dissolves the chloride and bromide, but not (or only traces of) the iodide; the filtered ammonium solution, upon being supersaturated with nitric acid, will give a white precipitate if chlorides or bromides are present; a slight turbidity may be due to traces of dissolved iodide.

Potassium salts should impart a violet color to a non-luminous flame, a yellow color indicating sodium.

All compounds of ammonium should be completely volatilized by heat without leaving a residue.

Examination of compounds of calcium. Calcium chloride and calcium carbonate, the latter after having been converted into chloride by neutralizing with hydrochloric acid, should not be precipitated by hydrosulphuric acid or by ammonium hydrate (absence of heavy metals and of aluminium); the solution, after adding ammonium hydrate, ammonium chloride, and an excess of ammonium carbonate, and filtering off the precipitated calcium carbonate, should not be precipitated by sodium phosphate (absence of magnesium). Water digested with calcium carbonate or with tricalcium phosphate, should leave no residue upon evaporation.

Calcium carbonate and phosphate should be tested for sulphates and chlorides by dissolving the salts in diluted nitric acid and adding barium chloride and silver nitrate.

Iron will be indicated in calcium phosphate by saturating its solution in hydrochloric acid with hydrosulphuric acid, and then adding an excess of ammonium hydrate; the precipitate should 
be white, a dark color indicating iron (or possibly other heavy inetals).

Calcium hypochlorite (bleaching-powder) has to be examined by quantitative methods to ascertain the amount of hypochlorous acid present.

Examination of magnesium compounds. Magnesium sulphate, oxide, and carbonate, the two latter after being dissolved in diluted hydrochloric acid, should not be precipitated by hydrosulphuric acid, nor by ammonium hydrate, sulphide, or carbonate after a sufficient amount of ammonium chloride has been added (absence of heavy metals and alkaline earths).

Magnesium sulphate should be tested for chlorides by the addition of silver nitrate.

Water digested with the oxide or carbonate should leave no residue upon evaporation. The same compounds, dissolved in nitric acid, should not be precipitated by either barium chloride or silver nitrate. The oxide should not effervesce with acids (absence of carbonic acid).

Examination of aluminium compounds. Aluminium sulphate and aluminium-potassium sulphate (alum) when dissolved in water, or aluminium hydrate when dissolved in sulphuric acid, should not be precipitated by hydrosulphuric acid and show no blue color on the addition of potassium ferrocyanide (absence of iron). Potassium or sodium hydrate, added to the above solutions, should cause a white, gelatinous precipitate, which is completely dissolved by an excess of the reagent. Water digested with aluminium hydrate should leave no residue upon evaporation.

Examination of compounds of iron. The solution of ferrous salts, acidulated with hydrochloric acid, should not be precipitated by hydrosulphuric acid; solutions of ferric salts give with the same reagent an almost white precipitate of sulphur; a more or less colored precipitate would indicate the presence of metals of the arsenic or lead group.

Solutions of ferric salts (or of ferrous salts, after they have been converted into ferric salts by heating with hydrochloric and nitric acids), after having been precipitated by an excess of 
ammonium hydrate and filtered, should not impart a blue color to the filtrate (absence of copper), and this filtrate should not be precipitated by ammonium carbonate or phosphate (absence of alkaline earths and magnesium) and should not leave a residue on evaporation and gentle ignition in order to expel the ammonium compounds (absence of zinc, manganese, alkalies, etc.).

Solution of potassium hydrate added in excess to ferric solutions and filtered, should not be precipitated by ammonium carbonate after the alkaline solution has been neutralized by hydrochloric acid (absence of aluminium).

Compounds of iron may contain arsenic, and should therefore be examined by Marsh's test.

Ferrous salts (sulphate) should give no immediate blue precipitate with potassium ferrocyanide (absence of ferric compounds). Ferric salts should give no blue color with potassium ferridcyanide (absence of ferrous compounds).

Ferric chloride should also be tested for nitric acid by placing a crystal of ferrous sulphate into the solution to which sulphuric acid has been added (a brown color indicating nitric acid), and for sulphuric acid by barium chloride.

Examination of compounds of zinc. Chloride or sulphate of zinc when dissolved in water, oxide or carbonate of zinc when dissolved in hydrochloric acid, should give no precipitate in the acidulated solution with hydrosulphuric acid (absence of the metals of the arsenic and lead groups).

Ammonium hydrate, added to zinc solutions, should cause a white precipitate, which is completely dissolved by an excess of the reagent (absence of iron, aluminium, etc.).

The filtrate of zinc solutions from which the metal has been precipitated by an excess of ammonium sulphide, should not be precipitated by ammonium carbonate or phosphate (absence of alkaline earths and magnesium), nor should it leave a residue on evaporation and gentle ignition (absence of alkalies).

Water, digested with zinc oxide or carbonate, should leave no residue on evaporation.

Zinc sulphate should be tested for chlorides by silver nitrate, and zinc chloride for sulphates by barium chloride. 
Examination of compounds of manganese. The native manganese dioxide invariably contains other mineral matter; according to the U. S. P., it should contain not less than 66 per cent. of the dioxide, and has, consequently, to be examined by quantitative analysis.

A solution of manganese sulphate, acidulated with hydrochloric acid, should give no precipitate with hydrosulphuric acid (absence of copper, etc.); the aqueous solution of the salt from which the manganese has been precipitated by ammonium sulphide, should leave no residue on evaporation and gentle ignition (absence of magnesium, alkalies, etc.).

Potassium permanganate is completely decolorized (deoxidized) by oxalic acid and sulphuric acid. The colorless solution should be tested with ferrous sulphate for nitric acid, and with silver nitrate for hydrochloric acid. It should also be tested quantitatively.

Examination of compounds of chromium. Solution of potassium dichromate and chromium trioxide, acidulated with nitric acid, should not be precipitated by barium chloride, (absence of sulphuric acid); both substances, when neutralized with potassium hydrate, should give an immediate red precipitate with silver nitrate, a white precipitate indicating chlorides.

Examination of compounds of lead. Solutions of compounds of lead, when completely precipitated with either sulphuric acid or with hydrosulphuric acid, should yield a filtrate which leaves no residue on evaporation and gentle ignition (absence of all other metals).

Lead oxide (litharge) and lead carbonate should dissolve completely in nitric acid.

Lead iodide should be tested for lead chromate by triturating it with 2 parts of ammonium chloride and water, which dissolve the iodide, but not the chromate.

Examination of compounds of copper. Solutions of cupric salts (sulphate), when completely precipitated by hydrosulphuric acid, should yield a filtrate which gives no precipitate with ammonium hydrate, sulphide, or carbonate. Amrnonium hydrate 
added to a cupric solution should form a dark blue solution without leaving a residue.

Examination of compounds of bismuth. Subnitrate and subcarbonate of bismuth should be completely soluble in about 8 parts of a mixture of equal parts of nitric acid and water. When this solution is poured into 50 parts of water a white precipitate falls; the filtrate from this precipitate may be used to test for lead by sulphuric acid, for silver by hydrochloric acid, for sulphates by barium chloride, for chlorides by silver nitrate (all of which reagents give white precipitates if the impurities named are present), and for copper by an excess of ammonium hydrate, which precipitates the bismuth yet in solution, while copper would be indicated by the blue color of the filtrate.

Another portion of the bismuth salt is dissolved in hydrochloric acid and all bismuth precipitated by hydrosulphuric acid; the filtrate should leave no residue (absence of metals of the iron group and of the light metals).

Examination of compounds of silver. A solution of silver nitrate in water, or of silver oxide in nitric acid, should give a white precipitate with hydrochloric acid, which precipitate should be completely dissolved by ammonium hydrate. The filtrate from a solution from which all silver has been precipitated by hydrochloric acid, should leave no residue on evaporation. Silver oxide is readily dissolved by ammonia water.

Examination of compounds of mercury. All compounds of mercury are completely volatilized on heating.

The oxides of mercury are dissolved by heating with about 10 parts of diluted nitric acid; mercuric oxide, when heated in a tube, should evolve no red fumes (absence of nitric acid).

Mercuric chloride should be soluble in water and in alcohol; it should be tested for arsenic by Marsh's test.

Mercurous chloride, when digested with water, should yield a filtrate which, on evaporation, leaves no residue, and which is not changed by either hydrosulphuric acid, silver nitrate, or potassium iodide (absence of mercuric chloride). 
Mercuric iodide should be dissolved by 25 parts of boiling alcohol, or by digesting it with potassium iodide and water.

Mercurous iodide should be tested for mercuric iodide by digesting it with alcohol, which, upon evaporation, should leave no residue.

Ammoniated mercury should be wholly soluble without effervescence in warm hydrochloric, nitric, or acetic acid; when digested with diluted alcohol, the filtrate should not be acted upon by hydrosulphuric acid or potassium iodide (absence of mercuric chloride).

The basic mercuric sulphate is almost insoluble in cold water, but soluble in diluted hydrochloric or nitric acid.

Examination of compounds of arsenic. Arsenious and arsenic oxides, arsenious bromide and iodide are completely volatilized by heating (a residue indicating non-volatile impurities). The four compounds are also soluble in water, the bromide and iodide with decomposition.

Examination of compounds of antimony. Antimonious oxide should be completely soluble in tartaric acid; the solution should be tested for chlorides and sulphates by means of silver nitrate and barium chloride; also for iron, by potassium ferrocyanide.

Antimonious and antimonic sulphides are soluble in concentrated hydrochloric acid, with liberation of hydrosulphuric acid and formation of the trichloride.

Antimonic oxide, as well as antimonions oxychloride, is dissolved by boiling with sodium or potassium hydrate.

\section{QUEsTions.}

351. Give some general methods by which the mineral acids may be examined for metallic impurities and their strength determined.

352. How is sulphuric acid to be tested for sulphurous and nitric acids, and how is nitric acid tested for sulphuric and hydrochloric acids?

353. What impurities are sometimes present in phosphoric acid? How is their presence demonstrated?

354. By what tests is sodium carbonate detected in sodium bicarbonate, and potassium iodate in potassium iodide? 
355. How can the presence of ferrous sulphate be demonstrated in either ammonium chloride, calcium phosphate, magnesium carbonate, aluminium sulphate, or cupric sulphate?

356. State the various methods by which preparations of iron are examined for metallic impurities, and how ferric chloride can also be tested for ferrous chloride, nitric and sulphuric acids.

357. By what methods can zinc oxide be tested for any other metal, and for carbonic, sulphuric, or hydrochloric acid?

358. Give methods for detecting metallic impurities in compounds of lead, copper, bismuth, and silver.

359. What is the action of heat upon mercury compounds, and by what solvents may the various officinal mercury preparations be dissolved?

360. How is mercuric chloride detected in mercurous chloride, and how mercuric iodide in mercurous iodide? 

ORGANIC CHEMISTRY.

\section{INTRODUCTORY REMARKS. ELEMENTARY ANALYSIS.}

Definition of organic chemistry. The term organic chemistry was originally applied to the consideration of compounds formed in plants and in the bodies of animals, and these compounds were believed to be created by a mysterious power, called "vital force," supposed to reside in the living organism.

This assumption was partly justified by the failure of the earlier attempts to produce these compounds by artificial means, and also by the fact that the peculiar character of the compounds, and the numerous changes which they constantly undergo in nature, could not be sufficiently explained by the experimental methods then known, and the laws then established.

It was in accordance with these views that a strict distinction was made between inorganic and organic compounds, and accordingly between inorganic and organic chemistry, the latter branch of the science considering the substances formed in the living organism, and those compounds which were produced by their decomposition.

Since that time, it has been shown that many substances which were formerly believed to be exclusively produced in the living organism, under the influence of the so-called vital force, can be formed artificially from inorganic matter, or by direct combination of the elements. It was in consequence of this fact that the theory of the supposed "vital force," by which organic substances could be formed exclusively, had to be abandoned. 
An organic compound, according to modern views, is simply a compound of curbon generally containing hydrogen, frequently also oxygen and nitrogen, and sometimes other elements.

Organic chemistry may consequently be defined as the chemistry of carbon compounds. The old familiar terms organic compounds and organic chemistry are, however, still in general use.

In a strictly systematically arranged text-book of chemistry organic compounds should be considered in connection with the element carbon itself, but as these carbon compounds are so numerous, their composition often so complicated, and the decompositions which they suffer under the influence of heat or other agents so varied, it has been found best for purposes of instruction to defer the consideration of these compounds until the other elements and their combinations had been studied.

Elements entering into organic compounds. Organic compounds contain generally but a small number of elements. These are, besides carbon, chiefly hydrogen, oxygen, and nitrogen, sometimes sulphur and phosphorus. Other elements, however, enter occasionally into organic compounds, and by artificial means all metallic and non-metallic elements may be made to enter into organic combinations.

Here the question presents itself: Why is it that the four elements carbon, hydrogen, oxygen, and nitrogen are capable of producing such an immense number (in fact, millions) of different combinations? To this question but one answer can be given, which is that these four elements differ more widely from each other, in their chemical and physical properties, than perhaps any other four elements.

Carbon is a black, solid substsnce, which has never yet been fused or volatilized, while hydrogen, oxygen, and nitrogen are colorless gases which can only be converted into liquids with the greatest difficulty. Moreover, hydrogen is highly combustible, oxygen is a supporter of combustion, whilst nitrogen is perfectly indifferent. Finally, hydrogen is univalent, oxygen bivalent, nitrogen trivalent, and carbon quadrivalent. These elements are, therefore, capable of forming a greater number, 
and a greater variety of compounds than would be the case if they were elements of equal valence and of similar properties.

General properties of organic compounds. The substances formed by the union of the four elements just mentioned have properties in some respects intermediate to those of their components. Thus, no organic substance is either permanently solid ${ }^{1}$ like carbon, nor an almost permanent gas like hydrogen, oxygen, and nitrogen.

Some organic substances are solids, others liquids, others gases; they are generally solids when the carbon atoms predominate; they are liquids or gases when the gaseous elements, and especially hydrogen, predominate; likewise, it may also be said that compounds containing a small number of atoms in the molecule are gases or liquids which are easily volatilized; they are liquids of high boiling-points, or solids, when the number of atoms forming the molecules is large.

The combustible property of carbon and hydrogen is transferred to all organic substances, every one of which will burn when sufficiently heated in atmospheric air. (If carbon dioxide, carbonic acid and its salts, be considered organic compounds, we have an exception to the rule, as they are not combustible.)

The properties possessed by organic compounds are many and widely different. There are organic acids, organic bases, and organic neutral substances; there are some organic compounds which are perfectly colorless, tasteless, and odorless, whilst others show every possible variety of color, taste, and odor; many serve as food, whilst others are most poisonous; in short, organic substances show a greater variety of properties than the combinations formed by any four other elements.

And yet, the cause of all the boundless variety of organic matter is that peculiar attraction called chemical aftinity, acting between the atoms of a comparatively small number of elements and uniting them in many thousand different proportions.

1 Non-volatile organic substances are decomposed by heat with generation of volatile products. 
It would, of course, be entirely inconsistent with the object of this book, if all the thousands of organic substances already known (the number of which is continually being increased by new discoveries) were to be considered or even mentioned. It must be sufficient to state the gencral properties of the various groups of organic substances, to show by what processes they are artificially produced or how they are found in nature, how they may be recognized and separated, and finally to point out those members of each group which claim a special attention for one reason or another.

Difference in the analysis of organic and inorganic substances. The analysis of organic substances differs from that of inorganic substances, in so far as the qualitative examination of an organic substance furnishes in many cases but little proof of the true nature of the substance (except that it be organic), whilst the qualitative analysis of an inorganic substance discloses in most cases the true nature of the substance at once.

For instance: If a white, solid substance, upon examination, is found to contain potassium and iodinc, and nothing else, the conclusion may at once be drawn that the compound is potassium iodide, containing 39 parts by weight of potassium, and 126.6 parts by weight of iodine. Or, if another substance is examined, and found to be composed of mercury and chlorine, the conclusion may be drawn that the compound is either mercurous or mercuric chloride, as no other compounds containing these two elements are known, and whether the examined substance be the lower or higher chloride of mercury, or a mixture of both, can easily be determined by a few simple tests.

Whilst thus the qualitative examination discloses the nature of the substance, it is different with organic compounds. Many thousand times the analysis might show carbon, hydrogen, and oxygen to be present, and yet every one of the compounds examined might be entirely different; it is consequently not only the quality of the elements, but chiefly the quantity present which determines the nature of an organic substance, and in order to identify an organic substance with certainty, it frequently becomes necessary to make a quantitative determination of the various elements present, and this quantitative 
analysis by which the elements in organic substances are determined is generally called ultimate or elementary analysis.

There are, however, for many organic substances such characteristic tests that these substances may be recognized by them; these reactions will be mentioned in their proper places.

An analysis by which different organic substances, when mixed together, are separated from each other is frequently termed proximate analysis. Such an analysis includes the separation and determination of essential oils, fats, alcohols, sugars, resins, organic acids, albuminous substances, etc., and is one of the most difficult branches of analytical chemistry.

Qualitative analysis of organic substances, The presence of carbon in a combustible form is decisive in regard to the organic nature of a compound. If, consequently, a substance burns with generation of carbon dioxide (which may be identified by passing the gas through lime-water), the organic nature of this substance is established.

The presence of hydrogen can be proven by allowing the gaseous products of the combustion to pass through a cool glass tube, when drops of water will be deposited.

It is somewhat difficult to show by qualitative analysis the presence or absence of oxygen in an organic compound, and its determination is therefore generally omitted.

The presence of nitrogen is determined by heating the substance with dry soda-lime (a mixture of two parts of calcium hydrate and one part of sodium hydrate), when the nitrogen is converted into ammonia gas, which may be recognized by its odor or by its action on paper moistened with solution of cupric sulphate, a dark blue color indicating ammonia.

Ultimate or elementary analysis. The quantitative determination of carbon and hydrogen is accomplished by the following process: A weighed quantity of the pure and dry substance is mixed with dry cupric oxide, and this mixture introduced into a glass tube, the open end of which is connected by means of a perforated cork and tubing with two glass vessels, one of which (generally a U-shaped tube) is filled with pieces of calcium chloride, the other (usually a tube provided with several bulbs) with solution of potassium hydrate. The two 
glass vessels, containing the substances named, are weighed separately after having been filled. Upon heating the combustion-tube in a suitable furnace, the organic matter is burnt by the oxygen of the cupric oxide, the hydrogen is converted into water (steam), which is absorbed by the calcium chloride, and the carbon is converted into carbon dioxide, which is absorbed by the potassium hydrate. Upon reweighing the two absorbing vessels at the end of the operation, the increase in weight will indicate the quantity of water and carbon dioxide formed during the combustion, and from these figures the amount of carbon and hydrogen present in the organic matter may easily be calculated.

For instance : 0.81 gram of a substance having been analyzed, furnishes, of carbon dioxide $1.32 \mathrm{gram}$, and of water $0.45 \mathrm{gram}$. As every 44 parts by weight of carbon dioxide contain 12 parts by weight of carbon, the above 1.32 gram contains of carbon 0.36 gram, or 44.444 per cent. As every 18 parts of water contain 2 parts of hydrogen, the above 0.45 gram consequently contains 0.05 gram, or 6.172 per cent.

Oxygen is scarcely ever determined directly, but generally indirectly, by determining the quantity of all other elements and deducting their weight, calculated to percentages from 100 . The difference is oxygen.

In the above instance, 44.444 per cent. of carbon and 6.172 per cent. of hydrogen were found to be present, and all other elements, except oxygen, to be absent. The quantity of oxygen is, then, equal to 49.383 per cent. and the composition of the substance as follows:

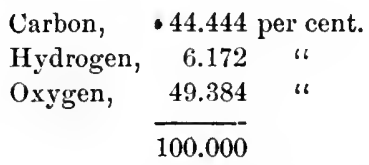

Instead of burning the organic substance by means of cupric oxide, the combustion may be effected by passing dry oxygen gas over the substance heated in a glass tube and collecting the products of combustion as described above.

Determination of nitrogen. Nitrogen is generally determined by heating the substance with soda-lime and passing the gen- 
erated ammonia gas through hydrochloric acid, contained in a suitable glass vessel. Upon evaporation of the acid in a weighed platinum dish over a water bath, ammonium chloride is left, from the weight of which compound the quantity of nitrogen may be calculated.

Determination of sulphur and phosphorus. These elements are determined by mixing the organic substance with sodium carbonate and nitrate, and heating the mixture in a crucible. The oxidizing action of the nitrate converts all carbon into carbon dioxide, hydrogen into water, sulphur into sulphuric acid, phosphorus into phosphoric acid. The two latter acids combine with the sodium of the sodium carbonate, forming sulphate and phosphate of sodium. The fused mass is dissolved in water, and sulphuric acid precipitated by barium chloride, phosphoric acid by magnesium sulphate and ammonium hydrate and chloride. From the weight of barium sulphate and magnesium phosphate, the weight of sulphur and phosphorus is calculated.

Determination of atomic composition from results obtained by elementary analysis. The elementary analysis gives the quantity of the various elements present in percentages, and from these figures the relative number of atoms may be found by dividing the figures by the respective atomic weights. For instance: The analysis above mentioned gave the composition of a compound, as carbon 44.444 per cent., hydrogen 6.172 per cent., and oxygen 49.384 per cent. By dividing each quantity by the atomic weight of the respective element, the following results are obtained :

$$
\begin{aligned}
& \frac{44.444}{12}=3.703 \\
& \frac{6.172}{1}=6.172 \\
& \frac{49.384}{16}=3.087
\end{aligned}
$$

The figures $3.703,6.172$, and 3.087 , represent the relative numbers of atoms present in a molecule of the compound examined. In order to obtain the most simple proportion expressing this relation, the greatest divisor common to the whole has to be found, a task which is sometimes rather difficult on 
account of slight errors made in the quantitative determination itself. In the above case, 0.6172 is the greatest divisor, which gives the following results:

$$
\frac{3.703}{0.6172}=6 ; \quad \frac{6.172}{0.6172}=10 ; \quad \frac{3.087}{0.6172}=5
$$

The simplest numbers of atoms are, accordingly, carbon 6 , hydrogen 10, oxygen 5 , or the composition is $\mathrm{C}_{6} \mathrm{H}_{10} \mathrm{O}_{5}$.

Empirical and molecular formulas. A chenical formula is termed empirical, when it merely gives the simplest possible expression of the composition of a substance. In the above case, the formula $\mathrm{C}_{6} \mathrm{H}_{10} \mathrm{O}_{5}$ would be the empirical formula. It might, however, be possible that this formula does not represent the actual number of atoms in the molecule, which might contain, for instance, twice or three times the number of atoms given, in which case the true composition would be expressed by the formula $\mathrm{C}_{12} \mathrm{H}_{20} \mathrm{O}_{10}$ or $\mathrm{C}_{18} \mathrm{H}_{30} \mathrm{O}_{15}$.

If it could be proven that one of the latter formulas is the correct one, it would be termed the molecular formula, because it expresses not only the numerical relations existing between the atoms, but also the absolute number of atoms of each element contained in the molecule.

The best method to determine the actual number of atoms contained in the molecule is the determination of the specitic weight of the gaseous compound, taking hydrogen as the unit. For instance: The analysis of a liquid substance gave the following result:

$$
\begin{aligned}
& \text { Carbon, } \quad 92308 \text { per cent. } \\
& \text { Hydrogen, } 7.692 \text { " } \\
& \overline{100.000}
\end{aligned}
$$

From this result the empirical formula, $\mathrm{CH}$, is deducted by applying the method stated above. If this formula were also the molecular formula, the density of the vapors of the substance would, when compared with hydrogen (according to the law of Avogadro), be equal to 6.5, because a molecule of hydrogen weighs 2 and a molecule of the compound $\mathrm{CH}$ weighs 13.

Suppose, however, the density of the gaseous substance is 
found to be 39 , then the molecular formula would be expressed by $\mathrm{C}_{6} \mathrm{H}_{6}$, because its molecular weight $(6 \times 12+6 \times 1)$ is equal to 78 , which. weight, when compared with the molecular weight of hydrogen $=2$, gives the proportions $78: 2$, or $39: 1$.

Not all organic compounds can be converted into gases or vapors without undergoing decomposition, and the determination of the molecular formulas of such compounds has to be accomplished by other methods. If the substance, for instance, is an acid or a base, the molecular formula may be determined by the analysis of a salt formed by these substances. For instance: The empirical formula of acetic acid is $\mathrm{CH}_{2} \mathrm{O}$; the analysis of the potassium acetate, however, shows the composition $\mathrm{KC}_{2} \mathrm{H}_{3} \mathrm{O}_{2}$, from which the molecular formula $\mathrm{HC}_{2} \mathrm{H}_{3} \mathrm{O}_{2}$ is deducted for acetic acid.

In many cases, however, it is as yet absolutely impossible to give the molecular formula of a compound with certainty.

Rational, constitutional, structural, or graphic formulas. These formulas are intended to represent the theories which have been formed in regard to the relative arrangement of the atoms within the molecule, or to represent the modes of formation and decomposition of a compound, or the relation which allied compounds bear to one another.

The molecular formula of acetic acid, for instance, is $\mathrm{C}_{2} \mathrm{H}_{4} \mathrm{O}_{2}$, but different constitutional formulas have been used to represent the structure of the acetic acid molecule.

Thus, H. $\mathrm{C}_{2} \mathrm{H}_{3} \mathrm{O}_{2}$ is a formula analogous to $\mathrm{H} . \mathrm{NO}_{3}$, indicating that acetic acid (analogous to nitric acid) is a monobasic acid, containing one atom of hydrogen, which can be replaced by metallic atoms.

$\mathrm{C}_{2} \mathrm{H}_{3} \mathrm{O}^{\mathrm{i}} \cdot \mathrm{HO}^{\mathrm{i}}$ is a formula indicating that acetic acid is composed of two univalent radicals which may be taken out of the molecule and replaced by other atoms or groups of atoms. This fornula also indicates that acetic acid is analogous to hydrates, the radical $\mathrm{C}_{2} \mathrm{H}_{3} \mathrm{O}$ having replaced one atom of hydrogen in $\mathrm{H}_{2} \mathrm{O}$.

$\mathrm{CH}_{3}{ }_{3} \cdot \mathrm{CO}_{2} \mathrm{H}^{2}$ is a formula indicating that acetic acid is composed of two other compound radicals.

It may be finally said, that quite another number of rational formulas has been applied, or, at least, has been proposed by 
different scientists and at different times, to represent the structure of acetic acid, but it should be remembered that these formulas are not intended to represent the actual arrangement of the atoms in space, but only, as it were, their relative mode of combination, showing which atoms are combined directly and which only indirectly, that is, through the medium of others.

\section{QUESTIONS.}

361 . What is organic chemistry, according to modern views?

362. Mention the four chief elements entering into organic compounds, and name the elements which may be made to enter into organic compounds by artificial processes.

363. State the reasons why the four elements carbon, hydrogen, oxygen, and nitrogen, are more apt to form a larger number of compounds than most other elements.

364 . State the general properties of organic compounds.

365. Why does a qualitative analysis of an organic compound, in most cases, not disclose its true nature?

366 . By what tests may the organic nature of a compound be established?

367 . By what tests may the presence of carbon, hydrogen, and nitrogen be demonstrated in organic compounds?

368. State the methods by which the elements carbon, hydrogen, oxygen, sulphur, and phosphorus are determined quantitatively.

369 . By what general method may a formula be deducted from the results of a quantitative analysis?

370. What is meant by an empirical, molecular, and constitutional formula; how are they determined, and what is the difference between them?

\section{CONSTITUTION, DECOMPOSITION, AND CLASSIFICA- TION OF ORGANIC COMPOUNDS.}

Radicals or residues. The nature of a radical or residue has been already stated, but the important part played by residues in organic compounds renders it necessary to consider them more fully.

A residue is an unsaturated group of atoms obtained by removal of one or more atoms from a saturated compound. It is 
not necessary that this removal of atoms should be practically accomplished in order to call a group of atoms a residue, but it is sufficient to prove that the unsaturated group of atoms exists as such in a number of compounds, and that it can be transferred from one compound into another without suffering decomposition.

Residues exist in organic and inorganic compounds; an inorganic residue spoken of heretofore is the water residue or hydroxyl, HO, obtained by removal of one atom of hydrogen from one molecule of water. Hydroxyl does not exist in the separate state, but it exists in hydrogen dioxide, $\mathrm{H}_{2} \mathrm{O}_{2}$, or $\mathrm{HO}-\mathrm{OH}$, and is also a constituent of the various hydrates or hydroxides, as, for instance, of $\mathrm{KHO}, \mathrm{Ca} 2 \mathrm{HO}, \mathrm{Fe}_{2} 6 \mathrm{HO}$, etc.

If one atom of hydrogen be removed from the saturated hydrocarbon methane, $\mathrm{CH}_{4}$, the univalent residue methyl, $\mathrm{CH}_{3}$, is left, which is capable of combining with univalent elements, as in methyl chloride, $\mathrm{CH}_{3} \mathrm{Cl}$, or, with univalent residues, as in methyl hydrate, $\mathrm{CH}_{3} \mathrm{HO}$.

If two atoms of hydrogen be removed from $\mathrm{CH}_{4}$, the bivalent residue methylene, $\mathrm{CH}_{2}$, is left, capable of forming the compounds $\mathrm{CH}_{2} \mathrm{Cl}_{2}, \mathrm{CH}_{2} 2 \mathrm{HO}$, etc.

If three atoms of hydrogen be removed from $\mathrm{CH}_{4}$, the trivalent residue $\mathrm{CH}$ is left, capable of combining with three atoms of univalent elements, as in $\mathrm{CHCl}_{3}$, or with another trivalent residue, etc.

Chains. The expression chain, designates a series of multivalent atoms (generally, but not necessarily of the same element), held together in such a manner that affinities are left unsaturated. For instance :

$$
-\mathrm{O}-\mathrm{O}-,-\mathrm{O}-\mathrm{O}-\mathrm{O}-, \quad-\mathrm{O}-\mathrm{O}-\mathrm{O}-\mathrm{O}-\text {, }
$$

are oxygen chains, each one of which has two free affinities which may be saturated, for instance, with the following results:

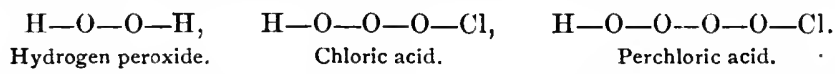

In a similar manner, carbon atoms unite, forming chains, as, for instance :

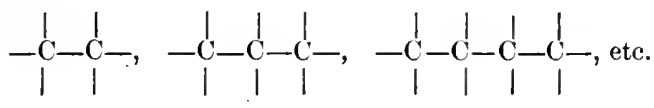


The above carbon chains have 6,8 , and 10 free aftinities, respectively, which may be saturated by the greatest variety of atoms or residues. The chain combination of carbon, above indicated by the first three members of a series, may, as far as it is known, be continued indefinitely. This fact, in connection with the possibility of saturating the free affinities with various atoms or residues, indicates the almost unlimited number of possible combinations to be formed in this way.

In fact, the existence of such an enormous number of carbon compounds is greatly due to the property of carbon to form these chains.

It is not always the case that the atoms when forming a chain are united by one affinity only as above, but they may be united by two or three affinities as indicated by the compounds $\mathrm{C}_{2} \mathrm{H}_{4}$ and $\mathrm{C}_{2} \mathrm{H}_{2}$, the graphic formulas of which may be represented by<smiles>C=CC</smiles>

It is finally assumed that the carbon atoms are united partially by double and partially by single union, as, for instance, in the so-called closed chain of $\mathrm{C}_{6}$, capable of forming the saturated hydrocarbon benzine, $\mathrm{C}_{6} \mathrm{H}_{6}$ :

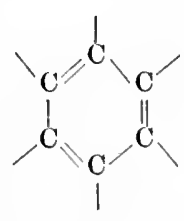<smiles>c1ccccc1</smiles>

A chain has also been termed a skeleton, because it is that part of an organic compound around which the other elements or radicals arrange themselves, filling up, as it were, the unsaturated affinities.

Homologous series. This term is applied to any series of organic compounds the terms or members of which, preceding or following each other, differ by $\mathrm{CH}_{2}$. Moreover, the general character, the constitution, and the general properties of the members of an homologous series are similar. 
The explanation regarding the formation of an homologous series is to be found in the above-described property of carbon to form chains. By saturating, for instance, the affinities in the open carbon chains mentioned above, we obtain the compounds $\mathrm{CH}_{4}, \mathrm{C}_{2} \mathrm{H}_{6}, \mathrm{C}_{3} \mathrm{H}_{8}, \mathrm{C}_{4} \mathrm{H}_{10}$, etc.<smiles>CC</smiles><smiles>CCC</smiles><smiles>CCCCC</smiles>

Many homologous series of various organic compounds are known, as, for instance:

$\begin{array}{ccc}\mathrm{CH}_{3} \mathrm{Cl}, & \mathrm{CH}_{4} \mathrm{O}, & \mathrm{CH}_{2} \mathrm{O}_{2}, \\ \mathrm{C}_{2} \mathrm{H}_{5} \mathrm{Cl}, & \mathrm{C}_{2} \mathrm{H}_{6} \mathrm{O}, & \mathrm{C}_{2} \mathrm{H}_{4} \mathrm{O}_{2}, \\ \mathrm{C}_{3} \mathrm{H}_{7} \mathrm{Cl}, & \mathrm{C}_{3} \mathrm{H}_{8} \mathrm{O}, & \mathrm{C}_{3} \mathrm{H}_{6} \mathrm{O}_{2}, \\ \mathrm{C}_{4} \mathrm{H}_{9} \mathrm{Cl}, & \mathrm{C}_{4} \mathrm{H}_{10} \mathrm{O}, & \mathrm{C}_{4} \mathrm{H}_{8} \mathrm{O}_{2}, \\ \mathrm{C}_{5} \mathrm{H}_{11} \mathrm{Cl}, & \mathrm{C}_{5} \mathrm{H}_{12} \mathrm{O}, & \mathrm{C}_{5} \mathrm{H}_{10} \mathrm{O}_{2}, \\ \text { ete. } & \text { etc. } & \text { etc. }\end{array}$

Types. It has been proposed to select some substances in which the arrangement of atoms in the molecules may be taken as representative of whole classes of other substances, the molecules of which have a similar arrangement, and these normal substances have been termed types. Most substances may be classified under the following five types:

I.

II.

III.

IV.

V.

Hydrogen. Water. Ammonia. Methane. Phosphoric chloride.<smiles>[H][Y3]CO</smiles>

By replacing the constituents of these types by other elements or residues of equal valence, most of the compounds known (both organic and inorganic) may be classed in one of these types.

The following five substances may, for instance, be said to have an atomic arrangement similar to the types above stated: 
I.

Hydrochloric
acid.

$\mathrm{H}-\mathrm{CL}, \mathrm{K}-\mathrm{O}-\mathrm{H}$,
III.

Arsenious

chloride.

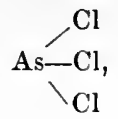

IV.

Ethane. Phosphoric

${ }_{\mathrm{C}}^{\mathrm{CH}_{3} \backslash_{\mathrm{H}}}$,
V. oxychloride.<smiles>O=[Te](Cl)(Cl)Cl</smiles>

The graphic representation of the constitution of compounds according to types has greatly aided in disclosing their structure, and is frequently used to give a picture, as it were, of the theoretical views held regarding the atomic arrangement.

Substitution is a term used for those reactions or chemical changes which depend on the replacement of an atom or a group of atoms by other atoms or groups of atoms. Substitution takes place in organic or inorganic substances, and its nature may be illustrated by the following instances:

$$
\begin{aligned}
& \mathrm{K}+\mathrm{H}_{2} \mathrm{O}=\mathrm{KHO}+\mathrm{H} \text {. } \\
& \text { Potassium. Water. } \begin{array}{c}
\text { Potassium } \\
\text { hydrate. }
\end{array} \text { Hydrogen. } \\
& \underset{\text { Acetic acid. }}{\mathrm{C}_{2} \mathrm{H}_{4} \mathrm{O}_{2}}+\underset{\text { Chlorine. }}{2 \mathrm{Cl}}=\underset{\substack{\text { Monochlor- } \\
\text { acetic acid. }}}{\mathrm{C}_{2} \mathrm{H}_{3} \mathrm{ClO}_{2}}+\underset{\begin{array}{c}
\text { Hydrochloric } \\
\text { acid }
\end{array}}{\mathrm{HCl}} \\
& \underset{\text { Benzine. }}{\mathrm{C}_{6} \mathrm{H}_{6}}+\underset{\text { Nitric acid. }}{\mathrm{HNO}_{3}}=\underset{\text { Nitro-benzine. }}{\mathrm{C}_{6} \mathrm{H}_{5} \mathrm{NO}_{2}}+\underset{\text { Water. }}{\mathrm{H}_{2} \mathrm{O} .}
\end{aligned}
$$

Derivatives. This term is applied to bodies derived from others by some kind of decomposition, generally by substitution. Thus, nitro-benzine is a derivative of benzine; chloroform, $\mathrm{CHCl}_{3}$, is a derivative of methane, $\mathrm{CH}_{4}$, obtained from the latter by replacement of three atoms of hydrogen by the same number of atoms of chlorine.

Isomerism. - Two or more substances may have the same elements in the same proportions by weight (or the same centesimal composition), and yet be different bodies, showing different properties. Such substances are called isomeric bodies. Two kinds of isomerism are distinguished, viz., metamerism and polymerism. 
Metamerism. Substances are metameric when their molecules contain equal numbers of atoms of the same elements. Thus, the oils of juniper, turpentine, lemon, etc., all have the molecular formula $\mathrm{C}_{10} \mathrm{H}_{16}$, and yet they have different physical properties, and may be distinguished by their odor, by their action on polarized light, etc.

The only explanation which can be given regarding this difference of properties is, that the atoms are arranged differently within the molecule. In some cases this arrangement is as yet unknown, in other cases structural or graphic formulas showing this atomic arrangement may be given.

For instance: Acetic acid and methyl formate have both the composition $\mathrm{C}_{2} \mathrm{H}_{4} \mathrm{O}_{2}$, but the arrangement of the atoms (or the structure) is very different, as shown by the formulas:

$$
\begin{array}{rr}
\text { Acetic acid. } & \text { Methyl formate. } \\
\mathrm{C}_{2} \mathrm{H}_{3} \mathrm{O} \backslash \mathrm{H} & \mathrm{CHO} \backslash \mathrm{CHO} \backslash \mathrm{C} .
\end{array}
$$

As another instance, may be mentioned the compound $\mathrm{CN}_{2} \mathrm{H}_{4} \mathrm{O}$, which represents either ammonium cyanate or urea:

$$
\begin{array}{cc}
\text { Ammonium cyanate. } & \text { Urea. } \\
\mathrm{NH}_{4} \backslash \mathrm{ON} & \mathrm{NH}_{2} \succ \mathrm{CO} .
\end{array}
$$

Polymerism. Substances are said to be polymeric when they have the same centesimal composition, but a different molecular weight, or, in other words, when one substance contains some multiple of the number of each of the atoms contained in the molecule of the other.

For instance, some volatile oils have the composition, $\mathrm{C}_{20} \mathrm{H}_{32}$, which is double the number of atoms contained in oil of turpentine, $\mathrm{C}_{10} \mathrm{H}_{16}$; acetylene, $\mathrm{C}_{2} \mathrm{H}_{2}$, is polymeric with benzine, $\mathrm{C}_{6} \mathrm{H}_{6}$; acetic acid, $\mathrm{C}_{2} \mathrm{H}_{4} \mathrm{O}_{2}$, is polymeric with grape-sugar, $\mathrm{C}_{6} \mathrm{H}_{12} \mathrm{O}_{6}$, etc.

: Various modes of decomposition. The principal changes which a molecule may suffer are as follows :

a. The atoms may arrange themselves differently within the molecule. Ammonium cyanate, $\mathrm{NH}_{4} \mathrm{CNO}$, is easily converted into urea, $\mathrm{CO} 2\left(\mathrm{NH}_{2}\right)$. 
b. A molecule may split up into two or more molecules.

For instance :

$$
\underset{\text { Grape-sugar. }}{\mathrm{C}_{6} \mathrm{H}_{12} \mathrm{O}_{6}}=\underset{\text { Alcohol. }}{2 \mathrm{C}_{2} \mathrm{H}_{6} \mathrm{O}}+\underset{\begin{array}{c}
\text { Carbon } \\
\text { dioxide. }
\end{array}}{2 \mathrm{CO}_{2}} \text {. }
$$

c. Two molecules, either of the same kind, or of different substances, may unite together directly:

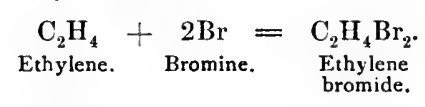

d. Atoms may be removed from a compound without replacing them by other atoms:

$$
\underset{\text { Alcohol. }}{\mathrm{C}_{2} \mathrm{H}_{6} \mathrm{O}}+\underset{\text { Oxygen. }}{\mathrm{O}}=\underset{\text { Aldehyde. }}{\mathrm{C}_{2} \mathrm{H}_{4} \mathrm{O}}+\underset{\text { Water. }}{\mathrm{H}_{2} \mathrm{O}}
$$

e. Atoms may be removed and replaced by others at the same time (substitution):

$$
\underset{\text { Acetic acid. }}{\mathrm{C}_{2} \mathrm{H}_{4} \mathrm{O}_{2}}+\underset{\text { Chlorine. }}{2 \mathrm{Cl}}=\underset{\begin{array}{c}
\text { Monochlor- } \\
\text { acetic acid. }
\end{array}}{\mathrm{C}_{2} \mathrm{H}_{3} \mathrm{ClO}_{2}}+\underset{\begin{array}{c}
\mathrm{HCl} \text { Hydrochloric } \\
\text { acid. }
\end{array}}{\mathrm{H}}
$$

Action of heat upon organic substances. As a general rule, organic bodies are distinguished by the facility with which they decompose under the influence of heat or chemical agents; the more complex the body is, the more easily does it undergo decomposition or transformation.

Heat acts differently upon organic substances, some of which may be volatilized without decomposition, whilst others are decomposed by heat with generation of volatile products. This process of heating non-volatile organic substances in such a manner that the oxygen of the atmospheric air has no access, and to such an extent that decomposition takes place, is called dry or destructive distillation.

The nature of the products formed during this process varies not only with the nature of the substance heated, but also with the temperature applied during the operation. The products formed by destructive distillation are invariably less complex in composition, that is, have a smaller number of atoms in the molecule, than the substance which suffered decomposition; in 
other words, a complex molecule is split up into two.or more molecules less complex in composition.

Otherwise, the products formed show a great variety of properties; some are gases, others volatile liquids or solids, some are neutral, others basic or acid substances. In most eases of destructive distillation a non-volatile residue is left, which is nearly pure carbon.

Action of oxygen upon organic substances. Combustion. Decay. All organic substances are capable of oxidation, which takes place either rapidly with the evolution of heat and light and is called combustion, or it takes place slowly without the emission of light, and is called slow combustion or decay. The heat generated during the decay of a substance is the same as that generated by burning the substance, but as this heat is liberated in the first instance during weeks, months, or perhaps years, so little heat is produced at a time that it can scarcely be noticed.

No organic substance found or formed in nature contains a sufficient quantity of oxygen to cause the complete combustion of the combustible elements (carbon and hydrogen) present; by artificial processes such substances may, however, be produeed, and are then either highly combustible or even explosive.

During common combustion, provided an excess of atmospheric oxygen be present, the total quantity of carbon is converted into carbon dioxide, hydrogen into water, sulphur and phosphorus into sulphuric and phosphoric acids, while nitrogen is generally liberated in the elementary state.

During the process of decay the compounds mentioned above are finally produced, although many intermediate products are also generated. For instance: If a piece of wood be burnt, complete oxidation takes place; intermediate products are also formed chicfly in consequence of the destructive distillation of a portion of the wood, but they are consumed almost as fast as they are produced, as was mentioned in connection with the consideration of flame. Again, when a piece of wood is left exposed to the action of the atmosphere, it slowly burns or decays. The intermediate products formed in this case are entirely different from those producerl during common combustion.

Common alcohol has the composition $\mathrm{C}_{2} \mathrm{H}_{6} \mathrm{O}$; in burning, it 
requires six atoms of oxygen, when it is converted into earbon dioxide and water:

$$
\mathrm{C}_{2} \mathrm{H}_{6} \mathrm{O}+6 \mathrm{O}=2 \mathrm{CO}_{2}+3 \mathrm{H}_{2} \mathrm{O} .
$$

But alcohol may also undergo slow oxidation, in which case oxygen first removes hydrogen, with which it combines to form water, whilst at the same time a compound known as acetic aldehyde, $\mathrm{C}_{2} \mathrm{H}_{4} \mathrm{O}$, is formed:

$$
\mathrm{C}_{2} \mathrm{H}_{6} \mathrm{O}+\mathrm{O}=\mathrm{C}_{2} \mathrm{H}_{4} \mathrm{O}+\mathrm{H}_{2} \mathrm{O} .
$$

This aldehyde, when further acted upon by oxygen, takes up an atom of this element, thereby forming acetic acid:

$$
\mathrm{C}_{2} \mathrm{H}_{4} \mathrm{O}+\mathrm{O}=\mathrm{C}_{2} \mathrm{H}_{4} \mathrm{O}_{2} \text {. }
$$

The three instances given above illustrate the action of oxygen upon organic substances, which action may consist in a mere removal of hydrogen, in a replacement of hydrogen by oxygen, or in an oxidation of both the carbon and hydrogen, and also of sulphur and phosphorus, if they be present.

An organic substance, when perfectly dry and exposed to dry air only, may not suffer decay for a long time (not even for centuries), but in the presence of moisture and air this oxidizing action takes place almost invariably.

Besides the slow oxidation or decay which all dead organic matter undergoes in the presence of moisture, there is another kind of slow oxidation, called respiration, which takes place in the living animal; this process will be more fully considered in the physiological part of this book.

Fermentation and putrefaction. These terms are applied to peculiar kinds of decomposition, by which the molecules of certain organic substances are split up into two or more molecules of a less complicated composition. These decompositions take place when three factors are simultaneously acting upon the organic substance. These factors are : presence of moisture, farorable temperature, and presence of a substance generally termed ferment.

The most favorable temperature for these decompositions lies between $20^{\circ}$ and $40^{\circ}$, but they may take place at lower or higher 
temperatures. No substance, however, will either ferment or putrefy at or below the freezing-point, or at or above the boilingpoint.

The nature of the various ferments differs widely, and their true action cannot, in many cases, be explained; what we do know is, that the presence of comparatively small (often minute) quantities of one substance (the ferment) is sufficient to cause the decomposition of large quantities of certain organic substances, the ferment itself suffering often no apparent change during this decomposition. The ferments are, in a few cases, organic sulstances, but generally living organisms of either vegetable or animal origin.

The nature of the ferment frequently determines the nature of the decomposition which a substance suffers, or, in other words, one and the same substance will under the influence of one ferment decompose with liberation of certain products, while a second ferment causes other products to be evolved. Sugar, for instance, under the influence of yeast, is converted into alcohol and carbon dioxide, while under the influence of certain other ferments it is converted into lactic acid.

The difference between fermentation and putrefaction is, that the first term is used in those cases where the decomposing substance contains carbou, hydrogen, and oxygen only, while substances containing in addition to these three elements either nitrogen or sulphur (or both) undergo putrefaction. The two last-naned elements are generally erolved as ammonia and hydrosulphuric acid, which gases give rise to an offensive odor.

Sugar, having the composition $\mathrm{C}_{6} \mathrm{H}_{12} \mathrm{O}_{6}$, undergoes fermentation, whilst albuminous substances which contain nitrogen and sulphur putrefy.

The oxygen of the air takes no part in either fermentation or putrefaction, but the presence or absence of atmospheric air may cause or prevent decomposition, inasmuch as the atmosphere is filled with millions of minute germs of organic nature, which germs may act as ferments when in contact with organic matter under otherwise favorable conditions.

Whenever organic bodies (a dead animal, for instance) undergo decomposition in nature, the processes of fermentation and putrefaction are generally accompanied by oxidation or decay.

The conditions under which a substance will ferment or 
putrefy have been stated above, and the non-fulfilment of these conditions enables us to prevent deeomposition artificially.

Thus, we freeze substances (meat); or expel all water from or dry them (fruit, etc.), in order to prevent decomposition. The action of the ferments is counteracted either by the so-called antiseptic agents (salt, carbolic or salicylic acid, etc.), which are incompatible with organic life, or by excluding the air, and with it the ferments, by enclosing the substances in air-tight vessels (glass jars, tin cans, etc.), which, when filled, are heated sufficiently to destroy any germs which may have been present.

Action of chlorine and bromine. These two elements act upon organic substances (similarly to oxygen) in three different ways, viz.: they either (rarely, however) combine directly with the organic substance, or remove hydrogen, or replace hydrogen. The following equations illustrate this action :

$$
\begin{aligned}
& \underset{\text { Ethylene. }}{\mathrm{C}_{2} \mathrm{H}_{4}}+\underset{\text { Bromine. }}{2 \mathrm{Br}}=\underset{\substack{\text { Ethylene } \\
\text { bromide. }}}{\mathrm{C}_{2} \mathrm{H}_{4} \mathrm{Br}_{2}} . \\
& \mathrm{C}_{2} \mathrm{H}_{6} \mathrm{O}+2 \mathrm{Cl}=\mathrm{C}_{2} \mathrm{H}_{4} \mathrm{O}+2 \mathrm{HCl} \text {. } \\
& \begin{array}{c}
\text { Ethyl Chlorine. Aldehyde. Hydrochloric } \\
\text { alcohol. }
\end{array} \\
& \mathrm{C}_{2} \mathrm{H}_{4} \mathrm{O}_{2}+2 \mathrm{Cl}=\mathrm{C}_{2} \mathrm{H}_{3} \mathrm{ClO}_{2}+\mathrm{HCl} \text {. } \\
& \text { Acetic acid. Chlorine. } \begin{array}{c}
\text { Monochlor- } \\
\text { acetic acid. }
\end{array}
\end{aligned}
$$

In the presence of water, chlorine and bromine often act as oxidizing agents by combining with the hydrogen of the water and liberating of oxygen; iodine may act in a similar manner as an oxidizing agent, but it rarely acts directly by substitution.

Action of nitric acid. This substance acts either by direct combination with organic bases forming salts, or as an oxidizing agent, or by substitution of nitryl, $\mathrm{NO}_{2}$, for hydrogen. As instances of the latter action, may be mentioned the formation of nitro-benzine and nitro-cellulose:

$$
\begin{aligned}
& \underset{\text { Benzine. }}{\mathrm{C}_{6} \mathrm{H}_{6}}+\underset{\text { Nitric acid. }}{\mathrm{HNO}_{3}}=\underset{\text { Nitro-benzine. }}{\mathrm{C}_{6} \mathrm{H}_{5} \mathrm{NO}_{2}}+\underset{\text { Water. }}{\mathrm{H}_{2} \mathrm{O} .} \\
& \underset{\text { Cellulose. }}{\mathrm{C}_{6} \mathrm{H}_{10} \mathrm{O}_{5}}+\underset{\text { Nitric acid. }}{3 \mathrm{HNO}_{3}}=\underset{\text { Trinitro-cellulose. }}{\mathrm{C}_{6} \mathrm{H}_{7} 3\left(\mathrm{NO}_{2}\right)}+\underset{\text { Water. }}{3 \mathrm{H}_{2} \mathrm{O}} .
\end{aligned}
$$


The additional quantity of oxygen thus introduced into the molecules, renders them highly combustible or even explosive.

Action of dehydrating agents. Substances having a great affinity for water, such as strong sulphuric acid, phosphoric oxide, and others, act upon many organic substances by removing from them the elements of hydrogen and oxygen, and combining with the water formed, while, at the same time, frequently dark or even black compounds are formed, which consist mainly of carbon. The black color imparted to sulphuric acid by organic matter depends on this action.

Action of alkalies. The hydrates of potassium and sodium act in various ways on organic substances.

In some cases direct combination takes place:

$$
\underset{\substack{\text { Carbonic } \\ \text { oxide. }}}{\mathrm{CO}}+\underset{\substack{\text { Potassium } \\ \text { hydrate. }}}{\mathrm{KHO}}=\underset{\substack{\text { Potassium } \\ \text { formate. }}}{\mathrm{KCHO}_{2}}
$$

Salts are formed:

$$
\underset{\substack{\text { Acetic } \\ \text { acid. }}}{\mathrm{C}_{2} \mathrm{H}_{4} \mathrm{O}_{2}}+\underset{\substack{\text { Sodium } \\ \text { hydrate. }}}{\mathrm{NaHO}}=\underset{\substack{\text { Sodium } \\ \text { acetate. }}}{\mathrm{NaC}_{2} \mathrm{H}_{3} \mathrm{O}_{2}}+\underset{\text { Water. }}{\mathrm{H}_{2} \mathrm{O} .}
$$

Fats are decomposed with the formation of soap:

$$
\underset{\text { Oleate of glyceril. }}{\mathrm{C}_{3} \mathrm{H}_{5} 3\left(\mathrm{C}_{18} \mathrm{H}_{33} \mathrm{O}_{2}\right)}+\underset{\substack{\text { Sodium } \\ \text { hydrate. }}}{3 \mathrm{NaHO}}=\underset{\text { Glycerine. }}{\mathrm{C}_{3} \mathrm{H}_{5} 3 \mathrm{HO}}+\underset{\text { Sodium oleate. }}{3\left(\mathrm{NaC}_{18} \mathrm{H}_{33} \mathrm{O}_{2}\right) .}
$$

Oxidation takes place, whilst hydrogen is liberated:

$$
\underset{\substack{\text { Ethyl } \\ \text { alcohol. }}}{\mathrm{C}_{2} \mathrm{H}_{6} \mathrm{O}}+\underset{\substack{\text { Potassium } \\ \text { hydrate. }}}{\mathrm{KHO}}=\underset{\substack{\text { Potassium } \\ \text { acetate. }}}{\mathrm{KC}_{2} \mathrm{H}_{3} \mathrm{O}_{2}}+\underset{\text { Hydrogen. }}{4 \mathrm{H} .}
$$

From compounds containing nitrogen, ammonia is evolved:

$$
\underset{\text { Acetamide. }}{\mathrm{NH}_{2} \mathrm{C}_{2} \mathrm{H}_{3} \mathrm{O}}+\underset{\substack{\text { Potassium } \\ \text { hydrate. }}}{\mathrm{KHO}}=\underset{\substack{\text { Potassium } \\ \text { acetate. }}}{\mathrm{KC}_{2} \mathrm{H}_{3} \mathrm{O}_{2}}+\underset{\text { Ammonia. }}{\mathrm{NH}_{3} .}
$$


Action of reducing agents. Deoxidizing or reducing agents, especially hydrogen in the status nascendi, act upon organic substances either by direct combination:

$$
\underset{\substack{\text { Ethene } \\ \text { oxide. }}}{\mathrm{C}_{2} \mathrm{H}_{4} \mathrm{O}}+2 \mathrm{H}=\underset{\substack{\text { Ethyl } \\ \text { alcohol. }}}{\mathrm{C}_{2} \mathrm{H}_{6} \mathrm{O} .}
$$

or by removing oxygen (and also chlorine or bromine):

$$
\underset{\substack{\text { Benzoic } \\ \text { acid. }}}{\mathrm{C}_{7} \mathrm{H}_{6} \mathrm{O}_{2}}+2 \mathrm{H}=\underset{\substack{\text { Benzoic } \\ \text { aldehyde. }}}{\mathrm{C}_{7} \mathrm{H}_{6} \mathrm{O}}+\mathrm{H}_{2} \mathrm{O} .
$$

In some cases hydrogen replaces oxygen:

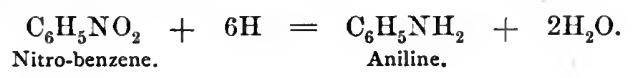

Classification of organic compounds. There are great difficulties in arranging the immense number of organic substances properly, and in such a manner that natural groups are formed the members of which are similar in composition and possess like properties.

Various modes of classification have been proposed, some of which, however, are so complicated that the beginner will find it difficult to make use of them. The grouping of organic substances here adopted, while far from being perfect, has the advantages of being simple, easily understood and remembered.

1. Hydrocarbons. All compounds containing the two elements carbon and hydrogen only. For instance, $\mathrm{CH}_{4}, \mathrm{C}_{6} \mathrm{H}_{6}$, $\mathrm{C}_{10} \mathrm{H}_{16}$, etc.

2. Alcohols. These are unsaturated hydrocarbons or hydrocarbon residues in combination with hydroxyl, $\mathrm{HO}$. For instance, ethyl alcohol, $\mathrm{C}_{2} \mathrm{H}_{5}{ }_{5} \mathrm{HO}$, glycerine, $\mathrm{C}_{3} \mathrm{H}^{\mathrm{iii}}{ }_{5} 3 \mathrm{HO}$, etc.

3. Aldehydes. Compounds intermediate between alcohols and acids, or alcohols from which hydrogen has been removed. For instance :

$$
\begin{gathered}
\mathrm{C}_{2} \mathrm{H}_{6} \mathrm{O}, \\
\text { Elhyl } \\
\text { alcohol. }
\end{gathered} \quad \begin{gathered}
\mathrm{C}_{2} \mathrm{H}_{4} \mathrm{O}, \\
\text { Aldehyde. }
\end{gathered} \quad \begin{gathered}
\mathrm{C}_{2} \mathrm{H}_{4} \mathrm{O}_{2} . \\
\text { Acetic acid. }
\end{gathered}
$$

4. Organic acids. Unsaturated hydrocarbons in combination with carboxyl, a radical having the composition $\mathrm{CO}_{2} \mathrm{H}$, or com- 
pounds formed by replacement of hydrogen in hydrocarbons by carboxyl. Otherwise, organic acids have the general properties of inorganic acids.

5. Ethers. Compounds formed from alcohols by replacement of the hydrogen of the hydroxyl by other unsaturated hydrocarbons, or, what is the same, by other alcohol radicals. For instance :

$$
\begin{array}{ccc}
\mathrm{C}_{2} \mathrm{H}_{5} . \mathrm{HO}, \\
\text { Ethyl } \\
\text { alcohol. }
\end{array} \quad \begin{gathered}
\mathrm{C}_{2} \mathrm{H}_{5} \cdot \mathrm{C}_{2} \mathrm{H}_{5} \mathrm{O}, \\
\text { Ethyl ether. }
\end{gathered} \quad \begin{gathered}
\mathrm{C}_{2} \mathrm{H}_{5} \cdot \mathrm{CH}_{3} \mathrm{O} . \\
\text { Ehyl- } \\
\text { methyl ether. }
\end{gathered}
$$

6. Compound ethers or esters. Formed from alcohols by replacement of the hydrogen of the hydroxyl by acid radicals, or from acids by replacement of the hydrogen of carboxyl by alcohol radicals. For instance :

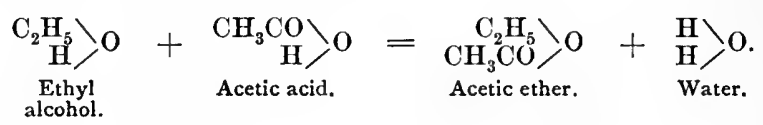

The various fats belong to this group of compound ethers.

7. Carbohydrates. (Sugars, starch, gum, etc.) These compounds contain 6 atoms of carbon (or a multiple of 6 ) in the molecule, and hydrogen and oxygen in the proportion of 2 atoms of hydrogen to 1 atom of oxygen, or in the proportion to form water. Most carbohydrates are capable of fermentation, or of being easily converted into fermentable bodies. Instances: $\mathrm{C}_{6} \mathrm{H}_{12} \mathrm{O}_{6}, \mathrm{C}_{6} \mathrm{H}_{10} \mathrm{O}_{5}$, etc.

Glucosides are substances the molecules of which may be split up in such a manner that several new bodies are formed, one of which is sugar.

8. Amines and amides. Substances formed by replacement of hydrogen in ammonia by alcohol or acid radicals. For instance: ethyl amine, $\mathrm{NH}_{2} \cdot \mathrm{C}_{2} \mathrm{H}_{5}$, urea, $\mathrm{N}_{2} \mathrm{H}_{4} \cdot \mathrm{CO}$, etc. The alkaloids belong to this group.

9. Cyanogen and its compounds. Substances containing the radical cyanogen, CN. For instance : potassium cyanide, KCN.

10. Proteids or albuminous substances. These contain, besides carbon, hydrogen and oxygen, always nitrogen and sulphur, sometimes also other elements. Instances: albumen, casein, fibrin, etc.

In connection with each of these groups have to be considered the derivatives obtained from them directly or indirectly. 


\section{Questions.}

371. Explain the term residue or radical.

372. What is understood by the expression chain, when used in chemistry?

373. What are the characteristics of a homologous series?

374. Give an explanation of the terms isomerism, metamerism, and polymerism.

375. How does heat act upon organic compounds?

376. What is destructive distillation?

377. State the difference between combustion, decay, fermentation, and putrefaction; what is the nature of these processes, and under what conditions do they take place?

378. How do chlorine, nitric acid, and alkalies act upon organic substances?

379. What is the action of hydrogen, and of dehydrating agents upon organic substances?

380. Mention the chief groups of organic compounds.

\section{HYDROCARBONS.}

0ccurrence in nature. Hydrocarbons are seldom derived from animal sources, being generally products of vegetable life; thus, the various essential oils (oil of turpentine and others) of the composition $\mathrm{C}_{10} \mathrm{H}_{16}$ or $\mathrm{C}_{20} \mathrm{H}_{32}$ are frequently found in plants, where they are formed from carbon dioxide and water:

$$
10 \mathrm{CO}_{2}+8 \mathrm{H}_{2} \mathrm{O}=\mathrm{C}_{10} \mathrm{H}_{16}+28 \mathrm{O} .
$$

This equation, whilst showing the possibility of the formation of an essential oil in the plant, must not be taken to mean that 10 molecules of carbon dioxide and 8 molecules of water are simultaneously decomposed, with the production of an essential oil; on the contrary, we know that many intermediate substances are formed, and the formula simply gives the final result, not the intermediate stages of the process.

Other hydrocarbons are found in nature as products of the decomposition of organic matter. Thus methane, $\mathrm{CH}_{4}$, is generally formed during the decay of organic matter in the presence of moisture; the higher members of the methane series are found in crude coal-oil. 
Formation of hydrocarbons. It is difficult to combine the two elements carbon and hydrogen directly; as an instance of such direct combination, may be mentioned acetylene, $\mathrm{C}_{2} \mathrm{H}_{2}$, which is formed when carbon is strongly heated by an electric current in hydrogen gas.

Many hydrocarbons are obtained by destructive distillation of organic matter, and their nature depends on the composition of the material used and upon the degree of heat applied for the decomposition. Hydrocarbons may also be obtained by the decomposition (other than destructive distillation) of numerous organic bodies, such as alcohols, acids, amines, etc., and from derivatives of these substances.

The hydrocarbons found in nature are generally separated from other matter, as well as from each other, by the process known as fructional distillation. As the boiling-points of the various compounds differ more or less, they may be separated by carefully distilling off the compounds of lower boiling-points, while noting the temperature of the boiling liquid by means of an inserted thermometer, and changing the receiver every time an increase of the boiling-point is noticed. 'This separation of volatile liquids, known as tractional distillation, is, however, not absolutely complete, because traces of substances having a higher boiling-point are simultaneously volatilized with the distilling substance.

Properties of hydrocarbons. There are no other two elements which combine together in so many proportions as carbon and hydrogen. Several hundred hydrocarbons are known, many of which form either homologous series or are metameric or polymeric.

Hydrocarbons occur either as gases, liquids, or solids. If the molecule contains not over 4 atoms of carbon, the compound is generally a gas at the ordinary temperature; if it contains from 4 to 10 or 12 atoms of carbon, it is a liquid; and if it contains a yet higher number of carbon atoms, it is generally a solid.

All hydrocarbons may be volatilized without decomposition, all are colorless substances, and many have a peculiar and often characteristic odor; they are generally insoluble in water, but soluble in alcohol, ether, bisulphide of carbon, etc.

In regard to chemical properties, it may be said that hydro- 
carbons are neutral substances, behaving rather indifferently towards most other chemical agents. Most of them are, however, oxidized by the oxygen of the air, by which process liquid hydrocarbons are often converted into solids.

Hydrocarbons of the paraffine or methane series. The hydrocarbons having the general composition $\mathrm{C}_{\mathrm{n}} \mathrm{H}_{2 \mathrm{n}+2}$ are known as paraffines, the name being derived from the higher members of the series which form the paraffine of commerce. The following table gives the composition, boiling-points, etc., of the first 16 members of this series :

\begin{tabular}{|c|c|c|c|}
\hline & & B. P. & Sp.gr. \\
\hline Methyl hydride or methane, & $\mathrm{CH}_{4}$, & & \\
\hline Ethyl hydride or ethane, & $\mathrm{C}_{2} \mathrm{H}_{6}$ & Gases. & \\
\hline Propyl hydride or propane, & $\mathrm{C}_{3} \mathrm{H}_{8}$ & & \\
\hline Butyl hydride or butane, & $\mathrm{C}_{4} \mathrm{H}_{10}$ & $0^{\circ} \mathrm{C}$ & \\
\hline A myl hydride or pentane, & $\mathrm{C}_{5} \mathrm{H}_{12}$ & 30 & 0.628 \\
\hline Hexyl hydride or hexane, & $\mathrm{C}_{6} \mathrm{H}_{14}$ & 68 & 0.669 \\
\hline Heptyl hydride or heptane, & $\mathrm{C}_{7} \mathrm{H}_{16}$ & 93 & 0.690 \\
\hline Octyl hydride or octane, & $\mathrm{C}_{8} \mathrm{H}_{18}$ & 118. & 0.726 \\
\hline Nonyl hydride or nonane, & $\mathrm{C}_{9} \mathrm{H}_{20}$ & 138 & 0.741 \\
\hline Decyl hydride or decane, & $\mathrm{C}_{10} \mathrm{H}_{22}$ & 158 & 0.757 \\
\hline Undecyl hydride or undecane, & $\mathrm{C}_{11} \mathrm{H}_{24}$ & 180 & 0.766 \\
\hline Dodecyl hydride or dodecane, & $\mathrm{C}_{12} \mathrm{H}_{26}$, & 198 & 0.778 \\
\hline Tridecyl hydride or tridecane, & $\mathrm{C}_{13} \mathrm{H}_{28}$ & 218 & 0.796 \\
\hline Tetradecyl hydride or tetradecane, & $\mathrm{C}_{14} \mathrm{H}_{30}$ & 236 & 0.809 \\
\hline Pentadecyl hydride or pentadecane, & $\mathrm{C}_{15} \mathrm{H}_{32}$ & 258 & 0.825 \\
\hline Hexadecyl hydride or paraffine, & $\mathrm{C}_{16} \mathrm{H}_{34}$, & 280 & \\
\hline
\end{tabular}

The above table shows that the paraffines form a homologous series; the first 4 members are gases, most of the others liquids, regularly increasing in specitic gravity, boiling-point, viscidity, and vapor density, as their molecular weight becomes greater.

The paraffines are saturated hydrocarbons, the constitution of which has been already explained; they are incapable of uniting directly with monatomic elements or residues, but they easily yield substitution-derivatives when subjected to the action of chlorine or bromine.

Most of the paraffines are known in two (or even more) modifications; there is therefore another homologous series of hydrocarbons of the same composition as the above normal paraftines, which show some difference from the normal paraftines in boiling- 
points and other properties. In these isomeric paraffines the atoms are arranged differently from those in the normal hydrocarbons, which fact may be proven by the difference in decomposition which these substances suffer when acted upon by chemical agents.

Coal, Coal-oil, Petroleum. The name coal-oil is applied to a mixture of the various liquid paraffines, containing often in solution the gaseous and solid members of the group, and also hydrocarbons belonging to other series. Coal-oil is produced in nature during the formation of coal.

The various substances classed together under the name of coal consist principally of carbon, associated with smaller quantities of hydrogen, oxygen, nitrogen, sulphur, and certain inorganic mineral matters which compose the ash. Coal is formed from buried vegetable matter by a process of decomposition which is partly a fermentation, partly a decay, and chiefly a slow destructive distillation, the heat for this latter process being derived from the interior of the earth or by the decomposition itself.

The principal constituent of the organic matter furnishing coal is wood (or woody fibre, cellulose), and a comparison of the composition of this substance with the various kinds of coal gradually formed will help to illustrate the chemical change taking place:

$\begin{array}{lccc} & \text { Carbon. } & \text { Hydrogen. } & \text { Oxygen. } \\ \text { Wood, } & 100 & 12.18 & 83.07 \\ \text { Peat, } & 100 & 9.85 & 55.67 \\ \text { Lignite, } & 100 & 8.37 & 42.42 \\ \text { Bituminous coal, } & 100 & 6.12 & 21.23 \\ \text { Anthracite coal, } & 100 & 2.84 & 1.74\end{array}$

This table shows a progressive diminution in the proportions of hydrogen and oxygen during the passage from wood to anthracite. These two elements must, therefore, be eliminated in some form of combination which allows them to move, viz., as gases or liquids. The gases formed are chiefly carbon dioxide (which finds its way through the rocks and soils to the surface either in the gaseous state, or after having been absorbed by water in the form of carbonic acid springs) and methane, known to the coal miners as fire-damp, frequently causing the formation 
of explosive gas mixtures in the coal mines, or escaping like carbon dioxide through fissures to the surface of the earth, where it may be ignited.

The liquids formed are water and that mixture of hydrocarbons known as crude coal-oil or petroleurn, which finds its way to the surface either from natural causes, or is lifted up by suitable pumps.

Petroleum, as has been stated, is chiefly a mixture of various hydrocarbons, the boiling-points of which lie between $0^{\circ}$ and $300^{\circ}$, or even higher. The crude oil has to be purified, which is accomplished by a treatment with sulphuric acid, followed by other processes of refining, and finally by fractional distillation, in order to separate the members of low boiling-points from those of higher boiling-points.

The hydrocarbons of low boiling-points, chiefly a mixture of $\mathrm{C}_{5} \mathrm{H}_{12}$ and $\mathrm{C}_{6} \mathrm{H}_{14}$, are officinal, under the name of petrolezm-ether or benzin, which name must not be confounded with benzene or benzol, $\mathrm{C}_{6} \mathrm{H}_{6}$.

Other similar liquids are sold in the market under the name of rhigoline (B. P. about $21^{\circ}$ ) and gasoline (B. P. about $75^{\circ}$ ); they are highly inflammable.

The paraffines distilling above $150^{\circ}$ constitute the common illuminating oil, various kinds of which are sold as kerosene, paraffine oil, astral-oil, mineral sperm-oil, etc. The danger which arises in the use of coal-oil as an illuminating agent is caused by the use of oils which have not been sufticiently freed from the more volatile members of the series, which, when but slightly heated (or even at ordinary temperature), will vaporize, and, upon mixing with atmospheric air, form explosive mixtures. An oil to be safely used for illuminating purposes in common lamps should not give off inflammable vapors (or flash) below $60^{\circ}$, and should not burn at temperatures below $65^{\circ}$.

The residue left from the coal-oil, after the more volatile products have been distilled, is a mixture of substances, some of which are used for lubricating purposes or are officinal under the name of petrolatum, petroleum ointment, or vaseine.

A mixture of the highest and solid members of the paraffine series (and also left in the residue from the coal-oil distillation) is known as paraffine, a white, crystalline, fusible substance used for candles, etc. 
Illuminating gas is a mixture of gases obtained by the destructive distillation of coal (or wood) in iron retorts, with subsequent purification of the gases generated. The constituents of coal have been mentioned above. The products formed from it during its destructive distillation are very numerous; the following are the most important:

$$
\begin{aligned}
& \begin{cases}\text { Hydrogen, } & \mathrm{H} . \\
\text { Methane, } & \mathrm{CH}_{4} . \\
\text { Ethene, } & \mathrm{C}_{2} \mathrm{H}_{4} . \\
\text { Acetylene, } & \mathrm{C}_{2} \mathrm{H}_{2} . \\
\text { Nitrogen, } & \mathrm{N} . \\
\text { Ammonia, } & \mathrm{NH}_{3} . \\
\text { Carbonic oxide, } & \mathrm{CO} . \\
\text { Carbon dioxide, } & \mathrm{CO}_{2} . \\
\text { Hydrosulphuric acid, } & \mathrm{H}_{2} \mathrm{~S} . \\
\text { Hydrocyanic acid, } & \mathrm{HCN} .\end{cases}
\end{aligned}
$$

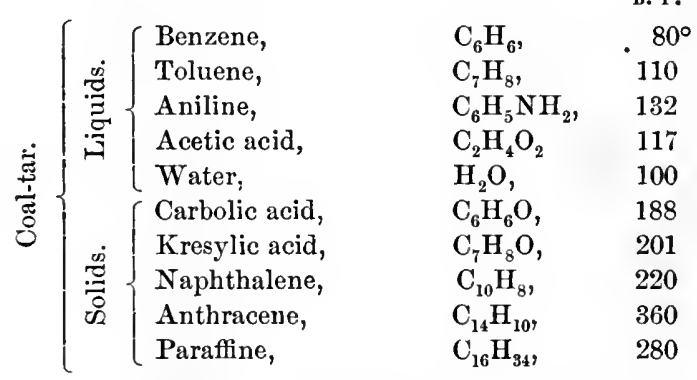

Solid residue: Coke, chiefly carbon and inorganic matter.

The gases are purified by condensing ammonia (and some other gases) by means of water, carbon dioxide and hydrosulphuric acid by calcium hydrate. The following is the composition of a purified illuminating gas obtained from cannel-coal :

$\begin{array}{lrc}\text { Hydrogen, } & 46 \text { volumes. } \\ \text { Methane, } & 41 & \text { " } \\ \text { Ethene, } & 6 & \text { " } \\ \text { Carbonic oxide, } & 4 & \text { " } \\ \text { Carbon dioxide, } & 2 & \text { " } \\ \text { Nitrogen, } & 1 & \text { " }\end{array}$

Coal-tar, obtained as a by-product in the manufacture of illuminating gas contains, as shown by the above table, many valuable substances, such as benzene, aniline, carbolic acid, paraffine, etc., which are separated from each other by making 
use of the difference in their boiling-points and specific gravities, or of their solubility or insolubility in various liquids, or, finally, of their basic, acid, or neutral properties.

Olefines. The hydrocarbons of the general formula $\mathrm{C}_{n} \mathrm{H}_{2^{n} \text {, }}$ are termed olefines. To this series belong:

$$
\begin{array}{ll}
\text { Ethene or ethylene, } & \mathrm{C}_{2} \mathrm{H}_{4} . \\
\text { Propene or propylene, } & \mathrm{C}_{3} \mathrm{H}_{6} . \\
\text { Butene or butylene, } & \mathrm{C}_{4} \mathrm{H}_{8} . \\
\text { Pentene or amylene, } & \mathrm{C}_{5} \mathrm{H}_{10} . \\
\text { Hexene or hexylene, } & \mathrm{C}_{6} \mathrm{H}_{12} .
\end{array}
$$

etc.

Methene, $\mathrm{CH}_{2}$, the lowest term of this series, is not known. The hydrocarbons of this series are not only homologous, but also polymeric with one another.

Of special interest is the first known member of the series, ethene or olefiant gas, on account of its normal occurrence in illuminating gas as well as in most common flames, the luminosity of which depends greatly on the quantity of this compound present in the burning gas.

Benzene series or aromatic hydrocarbons. The members of a series of hydrocarbons having the general composition

\begin{tabular}{|c|c|}
\hline Benzene, & $\mathrm{C}_{6} \mathrm{H}_{6}$, \\
\hline Toluene, & $\mathrm{C}_{7} \mathrm{H}_{8}=\mathrm{C}_{6} \mathrm{H}_{5} \cdot \mathrm{CH}_{3}$, \\
\hline Xylene, & $\mathrm{C}_{8} \mathrm{H}_{10}=\mathrm{C}_{6} \mathrm{H}_{4} \cdot 2 \mathrm{CH}_{3}$, \\
\hline Cumene, & $\mathrm{C}_{9} \mathrm{H}_{12}=\mathrm{C}_{6} \mathrm{H}_{3} 3 \mathrm{CH}_{3}$, \\
\hline Cymene, & $\mathrm{C}_{10} \mathrm{H}_{14}=\mathrm{C}_{6} \mathrm{H}_{2} \cdot 4 \mathrm{CH}_{3}$, \\
\hline Laurene, & $\mathrm{C}_{11} \mathrm{H}_{16}=\mathrm{C}_{6} \mathrm{H} .5 \mathrm{CH}_{3}$ \\
\hline
\end{tabular}
$\mathrm{C}_{\mathrm{n}} \mathrm{H}_{2 \mathrm{n}-6}$ and all the derivatives of this group, including the alcohols, acids, etc., are generally termed aromatic substances on account of the peculiar and fragrant odor possessed by many of them. To the benzene series belong:

The structure of the closed carbon chain of benzene has been referred to, and will be more fully considered in connection with the other aromatic compounds.

The most important member of the above series is benzol or benzene, $\mathrm{C}_{6} \mathrm{H}_{6}$, which is chiefly obtained from coal-tar. It is a colorless, highly volatile and combustible liquid, and an excel- 
lent solvent for many organic substances, as fats, resins, guttapercha, etc., and also for iodine, sulphur, phosphorus, etc.

Volatile or essential oils. The term essential oil is more a pharmaceutical than chemical term, and is used for a large number of liquids obtained from plants, and having in common the properties of being volatile, soluble in ether and alcohol, almost insoluble in water, and having a distinct and in most cases even highly characteristic odor. They stain paper in a similar manner as fats or fat oils, from which they differ, however, by the disappearance of the produced stain after some time, whilst fats leave a permanent stain.

In their chemical composition essential oils differ videly; some are compound ethers, others aldehydes, but most of them are hydrocarbons or oxidized hydrocarbons.

Many essential oils have the composition $\mathrm{C}_{10} \mathrm{H}_{16}$, or the polymeric composition $\mathrm{C}_{20} \mathrm{H}_{32}$. Of essential oils having the composition $\mathrm{C}_{10} \mathrm{H}_{16}$, may be mentioned the oils of turpentine, juniper, lemon, rosemary, bergamot, lavender, etc. The products of oxidation (either found in the plant itself or formed by the subsequent action of the oxygen upon the oils) have often the composition $\mathrm{C}_{10} \mathrm{H}_{16} \mathrm{O}$ or $\mathrm{C}_{10} \mathrm{H}_{14} \mathrm{O}$, but compounds of the composition $\mathrm{C}_{10} \mathrm{H}_{16} \mathrm{H}_{2} \mathrm{O}$ or $\mathrm{C}_{10} \mathrm{H}_{18}$ and $\mathrm{C}_{10} \mathrm{H}_{18} \mathrm{H}_{2} \mathrm{O}$ are also found; the two latter substances, for instance, in the oil of peppermint.

Camphors are substances closely related to the volatile oils, both in chemical and physical properties. Borneo-camphor has the composition $\mathrm{C}_{10} \mathrm{H}_{18} \mathrm{O}$, whilst the camphor found in the camphor-trees of China and Japan has the composition $\mathrm{C}_{10} \mathrm{H}_{16} \mathrm{O}$.

Resins are obtained, together with the essential oils, from plants. Mixtures of a resin and a volatile oil are known as oleo-resins, while mixtures of a resin or oleo-resins and gum are known as gum-resins. The name balsam is also used for a certain group of oleo-resins.

The resins are mostly amorphous, brittle bodies, insoluble in water, but soluble in alcohol, ether, fatty and essential oils; they are fusible, but decompose before being volatilized; they all contain oxygen and exhibit somewhat acid properties.

Turpentine, the olco-resin of the conifers, contains besides the 
oil of turpentine a resin called colophony, rosin, or ordinary resin, consisting chiefly of sylvic acid, $\mathrm{C}_{44} \mathrm{H}_{64} \mathrm{O}_{5}$.

Copaiva balsam consists of a volatile oil and a resin, the latter being principally copaivic acid, $\mathrm{C}_{20} \mathrm{H}_{30} \mathrm{O}_{2}$.

Caoutchouc, $\mathrm{C}_{8} \mathrm{H}_{14}$, and gutta-percha, $\mathrm{C}_{10} \mathrm{H}_{16}$, are intimately related to the essential oils and resins. Caoutchouc heated with sulphur combines with it (becomes "vulcanized"), and is then more elastic and does not become brittle when cold.

Of fossil resins may be mentioned amber and asphalt, the latter having most likely been formed from petroleum.

\section{Questions.}

381. How do hydrocarbons occur in nature, and by what processes are they formed in nature or artificially?

382. State the general physical and chemical properties of hydrocarbons.

383. What is the general composition of the paraffines.

384. State the composition and properties of methane, and also the conditions under which it is formed in nature.

385. What is coal, what are its constituents, from what is it derived, and by what process has it been formed?

386. What is crude coal-oil, what is petroleum ether, and what is petrolatum?

387. How is illuminating gas manufactured, and what are its chief constituents?

388. Mention some of the important substances found in coal-tar.

389. What is understood by aromatic substances; from which series of hydrocarbons are they derived, and what is the name and.composition of the first member of that series?

390. Mention of essential oils: occurrence in nature, physical properties and composition of some of the more important oils.

\section{ALCOHOLS.}

Constitution of alcohols. The old term "alcohol" originally indicated but one substance (ethyl alcohol), but is now applied to a large group of substances which may be looked upon as being derived from hydrocarbons by replacement of one, two, or more hydrogen atoms by hydroxyl, HO.

Any hydrocarbon may be converted into an alcohol radical 
by removal of one or more hydrogen atoms; methane, $\mathrm{CH}_{4}$, for instance, is converted into methyl, $\mathrm{CH}_{3}$, which, upon combining with hydroxyl, forms methyl alcohol, $\mathrm{CH}_{3} \mathrm{HO}$.

Running parallel to the various series of hydrocarbons, we have, therefore, homologous series of alcohols.

If hydroxyl replaces but one atom of hydrogen in a hydrocarbon, the alcohol is termed monatomic; diatomic and triatomic alcohols are formed by replacement of two or three hydrogen atoms respectively. (Diatomic alcohols are also termed glycols.) As an instance of a diatomic alcohol may be mentioned ethylene alcohol, $\mathrm{C}_{2} \mathrm{H}_{4} 2 \mathrm{HO}$, while glycerine, $\mathrm{C}_{3} \mathrm{H}_{5} 3 \mathrm{HO}$, is a triatomic alcohol.

Alcohols correspond in their composition to the hydrates (or hydroxides) of inorganic substances; both classes of compounds containing hydroxyl, $\mathrm{HO}$, which, in the case of alcohols, is in combination with residues containing carbon and hydrogen, in the case of inorganic hydrates with metals, as, for instance, in potassium hydrate, KHO.

If we represent any unsaturated hydrocarbon by Al.R. (alcohol radical), the general formula of the alcohols will be :

Monatomic alcohol. Diatomic alcohol. Triatomic alcohol.

$$
\text { AlRi_-HO AlRii }>\mathrm{HO}
$$

or

AlRiHO AlPii.2HO

$$
\begin{array}{r}
\text { AlRii }-\mathrm{HO} \\
\backslash \mathrm{HO} \\
\mathrm{HO}
\end{array}
$$

AlRiij3HO.

corresponding to

KiHO Caii2HO Biii3HO.

0ccurrence in nature. Alcohols are not found in nature in a free or uncombined state, but generally in combination with acids as compound ethers. Some plants, for instance, contain compound ethers mixed with volatile oils. The triatomic alcohol glycerine is a normal constituent of all fats or fatty oils, and is therefore found in some plants and in most animals.

Formation of alcohols. Alcohols are often produced by fermentation (ethyl alcohol from sugar), sometimes by destructive distillation (methyl alcohol from wood); they are obtained from compound ethers (which are compounds of acids and alcohols) 
by treating them with the alkaline hydrates, when the acid enters into combination with the alkali, whilst the alcohols are liberated according to the general formula :

$$
\stackrel{\mathrm{AlR}}{\mathrm{AcR}}>\mathrm{O}+\mathrm{KHO}=\underset{\mathrm{AcR}}{\mathrm{K}}>\mathrm{O}+\text { AlRHO. }
$$

Alcohols may be obtained artificially by various processes, as, for instance, by treating hydrocarbons with chlorine, when the chloride of a hydrocarbon residue is formed, which may be decomposed by alkaline hydrates in order to replace the chlorine by hydroxyl, when an alcohol is formed. For instance:

$$
\begin{aligned}
& \underset{\text { Ethane. }}{\mathrm{C}_{2} \mathrm{H}_{6}}+2 \mathrm{Cl}=\underset{\substack{\text { Ethyl } \\
\text { chloride. }}}{\mathrm{C}_{2} \mathrm{H}_{5} \mathrm{Cl}}+\mathrm{HCl} \text {. } \\
& \underset{\substack{\text { Ethyl } \\
\text { chloride. }}}{\mathrm{C}_{2} \mathrm{H}_{5} \mathrm{Cl}}+\underset{\substack{\text { Potassium } \\
\text { hydrate. }}}{\mathrm{KHO}}=\underset{\begin{array}{c}
\text { Potassium } \\
\text { chloride. }
\end{array}}{\mathrm{KCl}}+\underset{\substack{\text { Ethyl } \\
\text { alcohol. }}}{\mathrm{C}_{2} \mathrm{H}_{5} \mathrm{HO} .}
\end{aligned}
$$

Properties of alcohols. Alcohols are generally colorless, neutral liquids; some of the higher members are solids, none is gaseous at the ordinary temperature. Most alcohols are specifically lighter than water; the lower members are soluble in or mix with water in all proportions; the higher members are less soluble, and, finally, insoluble. Most alcohols are volatile without decomposition; some of the highest members, however, decompose before being volatilized.

Although alcohols are neutral substances, it is possible to replace the hydrogen of the hydroxyl by metals, as, for instance, $\mathrm{CH}_{3} \mathrm{HO}=$ methyl alcohol $; \mathrm{CH}_{3} \mathrm{NaO}=$ sodium methyl oxide or sodium methylate.

The oxygen of alcohols may be replaced by sulphur, when compounds are formed known as mercaptans; these bodies may be obtained by treating the chlorides of hydrocarbon residues with potassium sulphydrate:

$$
\mathrm{C}_{2} \mathrm{H}_{5} \mathrm{Cl}+\mathrm{KHS}=\mathrm{KCl}+\mathrm{C}_{2} \mathrm{H}_{5} \mathrm{HS} \text {. }
$$

By replacement of the hydrogen of the hydroxyl in alcohols by alcohol radicals ethers are formed; by replacing the same hydrogen with acid radicals compound ethers are produced.

Most alcohols are known in two, or even three, metameric forms, which are distinguished as normal or primary, secondary, and tertiary alcohols. 
Monatomic normal alcohols of the general composition, $\mathrm{C}_{\mathrm{n}} \mathrm{H}_{2^{n+1}} \mathrm{HO}$ or $\mathrm{C}_{\mathrm{n}} \mathrm{H}_{2 \mathrm{n}}+{ }_{2} \mathrm{O}$.

$\left.\begin{array}{llr} & & \text { B. P. } \\ \text { Methyl alcohol, } & \mathrm{CH}_{3} \mathrm{HO}, & 67^{\circ} \\ \text { Ethyl alcohol, } & \mathrm{C}_{2} \mathrm{H}_{5} \mathrm{HO}, & 78 \\ \text { Propyl alcohol, } & \mathrm{C}_{3} \mathrm{H}_{7} \mathrm{HO} & 97 \\ \text { Butyl alcohol, } & \mathrm{C}_{4} \mathrm{H}_{9} \mathrm{HO}, & 115 \\ \text { Amyl alcohol, } & \mathrm{C}_{5} \mathrm{H}_{11} \mathrm{HO}, & 132 \\ \text { Hexyl alcohol, } & \mathrm{C}_{6} \mathrm{H}_{13} \mathrm{HO}, & 150 \\ \text { Heptyl alcohol, } & \mathrm{C}_{7} \mathrm{H}_{15} \mathrm{HO}, & 168 \\ \text { Octyl alcohol, } & \mathrm{C}_{8} \mathrm{H}_{17} \mathrm{HO}, & 186 \\ \text { Nonyl alcohol, } & \mathrm{C}_{9} \mathrm{H}_{19} \mathrm{HO}, & 204 \\ \text { Cetyl alcohol, } & \mathrm{C}_{16} \mathrm{H}_{33} \mathrm{HO}, & 50 \\ \text { Ceryl alcohol, } & \mathrm{C}_{27} \mathrm{H}_{55} \mathrm{HO}, & 79 \\ \text { Melissyl alcohol, } & \mathrm{C}_{30} \mathrm{H}_{61} \mathrm{HO}, & 85\end{array}\right\}$ Fusing-point.

Methyl alcohol, $\mathbf{C H}_{3} \mathrm{HO}$ (Methyl hydrate, Methylic alcohol, Woodspirit, Wood naphtha). Methyl alcohol is one of the many products obtained by the destructive distillation of wood. When pure it is a thin, colorless liquid, similar in smell and taste to ethyl alcohol; crude wood-spirit, which contains many impurities, has an offensive odor and a nauseous, burning taste. Methyl alcohol mixes in all proportions with water; it dissolves resins and volatile oils as freely as ethyl alcohol, and is often substituted for the latter for various purposes in the arts and manufacture.

A mixture of 90 per cent. of ethyl alcohol and 10 per cent. of partially purified wood-spirit is sold in England by licensed dealers under the name of methylated spirit; no internal revenue tax is paid on this mixture, which is unfit for human consumption, but answers well for most purposes for which common alcohol is otherwise used.

Ethyl alcohol, $\mathrm{C}_{2} \mathrm{H}_{4} \mathrm{HO}=46$ (Common alcohol, Ethyl hydrate, Ethylic alcohol), may be obtained from ethene, $\mathrm{C}_{2} \mathrm{H}_{4}$, by addition of the elements of water, which may be accomplished by agitating ethene with strong sulphuric acid, when direct combination takes place and ethyl sulphuric acid is formed:

$$
\underset{\substack{\mathrm{C}_{2} \mathrm{H}_{4} \\ \text { Ethene. }}}{\mathrm{C}_{\text {. }}}+\underset{\substack{\text { Sulphuric } \\ \text { acid. }}}{\mathrm{H}_{2} \mathrm{SO}_{4}}=\underset{\substack{\text { Ethyl sul- } \\ \text { phuric acid. }}}{\mathrm{C}_{2} \mathrm{H}_{5} \mathrm{HSO}_{4}}
$$


Ethyl sulphuric acid mixed with water and distilled yields sulphuric acid and ethyl alcohol:

$$
\mathrm{C}_{2} \mathrm{H}_{5} \mathrm{HSO}_{4}+\mathrm{H}_{2} \mathrm{O}=\mathrm{H}_{2} \mathrm{SO}_{4}+\mathrm{C}_{2} \mathrm{H}_{5} \mathrm{HO} .
$$

Ethyl alcohol may also be obtained, as already mentioned, by treating ethyl chloride with potassium hydrate:

$$
\mathrm{C}_{2} \mathrm{H}_{5} \mathrm{Cl}+\mathrm{KHO}=\mathrm{KCl}+\mathrm{C}_{2} \mathrm{H}_{5} \mathrm{HO} .
$$

While the above methods for obtaining alcohol are of scientific interest, there is but one mode of manufacturing it on a large scale, namely, by the fermentation of certain kinds of sugar, especially grape-sugar or glucose, $\mathrm{C}_{6} \mathrm{H}_{12} \mathrm{O}_{6}$. A diluted solution of grape-sugar under the influence of certain ferments (yeast) suffers decomposition, yielding carbon dioxide and alcohol :

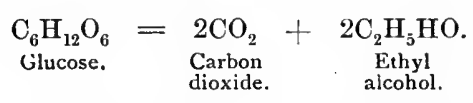

By distilling the fermented liquid an alcohol is obtained containing large quantities of water; on distilling this diluted alcohol a second and third time, collecting the first portions of the distilled liquid separately, an alcohol is obtained containing but little water. These last quantities of water, amounting to about 14 per cent., cannot be removed by simple distillation, but may be separated by mixing the alcohol with half its weight of calcium oxide, which combines with the water to form calcium hydrate, from which the alcohol may now be separated by distillation.

The alcohol thus obtained is known as pure, absolute, or real alcohol. The alcohol of the U.S.P. contains 91 per cent. by weight, or 94 per cent. by volume of real alcohol, and has a specific gravity of 0.820 at $15.6^{\circ}$. The diluted alcohol is made by mixing equal weights of water and alcohol, and has a specific gravity of 0.928. What is generally known as spirit of wine or rectified spirit is an alcohol containing 84 per cent., and proofspirit one containing 49 per cent. by weight of pure alcohol.

Pure alcohol is a transparent, colorless, mobile, and volatile liquid, of a characteristic, pungent, and agreeable odor, and a burning taste; it boils at $78^{\circ}$, has a specitic gravity of 0.794 , is 
of a neutral reaction, has never been solidified, but at $-110^{\circ}$ becomes syrupy; when mixed with water a contraction of volume occurs, and heat is liberated; the attraction of alcohol for water is so great that strong alcohol absorbs moisture from the air or abstracts it from membranes, tissues, and other similar substances immersed in it; to this property are due its coagulating action on albumen, and its preservative action on animal substances. The solvent powers of alcohol are very extensive, both for inorganic and organic substances; of the latter it readily dissolves essential oils, resins, alkaloids, and many other bodies, for which reason it is used in the manufacture of the numerous officinal tinctures, extracts, and fluid extracts.

Alcoholic liquors. Numerous substances containing sugar or starch (which may be converted into sugar) are used in the manufacture of the various alcoholic liquors, all of which contain more or less of ethyl alcohol, besides coloring matter, ethers, compound ethers, and many other substances.

White and red wines are obtained by the fermentation of the grape-juice; the so-called light wines contain from 10 to 12, the strong wines, such as port and sherry, from 19 to 25 per cent. of alcohol; if the grapes contain much sugar, only a portion of it is converted into alcohol, whilst another portion is left undecomposed, such wines are known as sweet wines. Effervescent wines, as champague, are bottled before the fermentation is complete; the carbonic acid is disengaged under pressure and retained in solution in the liquid.

Beer is prepared by fermentation of germinated grain (generally barley) to which much water and some hops have been added; the active principle of hops is lupulin, which confers on the beer a pleasant, bitter flavor, and the property of keeping without injury. Light beers have from 2 to 4 , strong beers, as porter or stout, from 4 to 6 per cent. of alcohol.

Spirits differ from either wines or beers in so far as the latter are not distilled, and therefore contain also non-volatile organic and inorganic substances, as salts, etc., not found in the spirits, which are distilled liquids containing volatile compounds only.

Moreover, the quantity of alcohol in spirits is very much larger, and varies from 45 to 55 per cent. Of distilled spirits may be mentioned: American whiskey, made from fermented rye 
or Indian corn; Irish whiskey, from potatoes; Scotch whiskey, from barley; brandy or cognac, by distilling French wines; rum, by fermenting and distilling molasses; arrack, from fermented rice; gin, from various grains flavored with juniper berries.

Amyl alcohol, $\mathbf{C}_{5} \mathrm{H}_{11} \mathbf{H O}$. This alcohol is frequently formed in small quantities during the fermentation of corn, potatoes, and other substances. When the alcoholic liquids are distilled, amyl alcohol passes over towards the end of the distillation, generally accompanied by propyl, butyl, and other alcohols, and by certain ethers and compound ethers. A mixture of these substances is known as fusel oil, and from this liquid amyl alcohol may be obtained in a pure state. It is an oily, colorless liquid, having a peculiar odor, and a burning, acrid taste; it is soluble in alcohol but not in water. By oxidation of amyl alcohol, valerianic acid is obtained.

Glycerine, Glycerinum, $\mathrm{C}_{3} \mathrm{H}_{5} \mathbf{3 H O}=\mathbf{9 2}$. Glycerine is the triatomic alcohol of the residue glyceryl, $\mathrm{C}_{3} \mathrm{H}_{5}$, formed by removal of three atoms of hydrogen from the saturated hydrocarbon propane, $\mathrm{C}_{3} \mathrm{H}_{8}$, and by combination of the residue with three times HO.

Glycerine is a normal constituent of all fats, which are glycerine in which the three atoms of hydrogen of the hydroxyl have been replaced by residues of fat acids. When fats are treated with alkalies, these latter combine with the fat acids, whilst glycerine is liberated. Upon this decomposition, carried out on a large scale in the manufacture of soap, depends the mode of obtaining glycerine.

Pure glycerine is a clear, colorless, odorless liquid of a syrupy consistence, oily to the touch, hygroscopic, very sweet, and neutral in reaction, soluble in. water and alcohol in all proportions, but insoluble in ether, chloroform, benzol, and fixed oils; its specific gravity is 1.255 ; it cannot be distilled by itself without decomposition, but is volatilized in the presence of water, or when hot steam is allowed to pass through it.

Phenoles. The substances termed phenoles are formed by replacement of hydrogen by hydroxyl in the aromatic hydrocarbons of the benzene series; they have the constitution of alcohols, 
but are not alcohols in the sense in which this term is used. The more importaut substances belonging to this group will be considered later.

\section{Questions.}

391. What is the general constitution of alcohols, and what is the difference between monatomic, diatomic, and triatomic alcohols?

392. How do alcohols occur in nature?

393. By what processes may alcohols be formed artificially, and how may they be separated from their combinations?

394. State the general properties of alcohols.

395. Mention names and composition of the first five members of alcohols of the general composition $\mathrm{C}_{\mathrm{n}} \mathrm{H}_{2 \mathrm{n}}{ }_{1} \mathrm{HO}$.

396. By what process is methyl alcohol obtained, under what other names is it known, and what are its properties?

397. Describe the manufacture of pure alcohol from sugar.

398. Give the alcoholic strength of the alcohol and diluted alcohol of the U. S. P., and also of spirit of wine, proof-spirit, light wines, heavy wines, beers, and spirits.

399. What are the general properties of common alcohol?

400. What is glycerine, how is it found in nature, how is it obtained, and what are its properties?

\section{ALDEHYDES. HALOID DERIVATIVES.}

Aldehydes. The name aldehyde is derived from alcohol dehydrogenatum; referring to its method of formation, viz., by the removal of hydrogen from alcohols, as, for instance:

$$
\underset{\substack{\text { Ethyl } \\ \text { alcohol. }}}{\mathrm{C}_{2} \mathrm{H}_{6} \mathrm{O}}-2 \mathrm{H}=\underset{\substack{\text { Acetic } \\ \text { aldehyde. }}}{\mathrm{C}_{2} \mathrm{H}_{4} \mathrm{O} .}
$$

This removal of hydrogen may be accomplished by various methods, as, for instance, by oxidation of alcohols, when one atom of oxygen combines with two atoms of hydrogen, forming water, whilst an aldehyde is formed at the same time. Aldehydes, when further oxidized, are converted into acids; aldehydes are, consequently, the intermediate products between alcohols and acids, and are frequently looked upon as the hydrides of the acid radicals. Only a few aldehydes are of practical interest, as, for instance, benzoic aldehyde, which substance will be 
more fully considered in connection with the aromatic substances.

Trichloraldehyde, Chloral, $\mathbf{C}_{2} \mathrm{HCl}_{3} \mathbf{0}$ (Trichloracetyl hydride). This substance may be looked upon as acetic aldehyde, $\mathrm{C}_{2} \mathrm{H}_{4} \mathrm{O}$, in which three atoms of hydrogen have been replaced by chlorine. It is made by passing a rapid stream of dry chlorine into pure alcohol to saturation, keeping the alcohol cool during the first few hours, and warming it gradually until the boiling-point is reached. According to the quantity of alcohol operated on, the conversion requires several hours or even days. The crude liquid product separates into two layers; the lower is removed and shaken with three times its volume of strong sulphuric acid and distilled, the distillate is mixed with calcium oxide and again distilled; the portion passing over between $94^{\circ}$ and $99^{\circ}$ is collected.

The decomposition taking place between alcohol and chlorine may be explained by the formation of aldehyde:

$$
\mathrm{C}_{2} \mathrm{H}_{6} \mathrm{O}+2 \mathrm{Cl}=\mathrm{C}_{2} \mathrm{H}_{4} \mathrm{O}+2 \mathrm{HCl},
$$

and by the subsequent replacement of hydrogen by chlorine:

$$
\mathrm{C}_{2} \mathrm{H}_{4} \mathrm{O}+6 \mathrm{Cl}=\mathrm{C}_{2} \mathrm{HCl}_{3} \mathrm{O}+3 \mathrm{HCl} \text {. }
$$

The actual decomposition is, however, somewhat more complicated, numerous other products being formed at the same time. By treatment with sulphuric acid these other substances are renioved.

Chloral is a colorless, oily liquid, having a penetrating odor and an acrid, caustic taste; its specific gravity is 1.5 , and its B. P. $95^{\circ}$.

Chloral hydrate, Chloral, U. S. P., $\mathbf{C}_{2} \mathrm{HCl}_{3} \mathbf{O} \cdot \mathrm{H}_{2} \mathrm{O}=165.2$. When water is added to chloral the two substances combine, heat is disengaged, and the hydrate of chloral is formed, which is a crystalline, colorless substance, having an aromatic, penetrating odor, a bitter, caustic taste, and a neutral reaction; it is freely soluble in water, alcohol, and ether, also soluble in chloroform, carbon disulphide, benzene, fat and essential oils, etc.; it liquefies when mixed with carbolic acid or with camphor; it melts at 
$58^{\circ}$ and boils at $95^{\circ}$, and also volatilizes slowly at ordinary temperature.

Chloral, and its hydrate, are decomposed by weak alkalies into chloroform and a formate of the alkali. metal:

$$
\underset{\text { Chloral. }}{\mathrm{C}_{2} \mathrm{HCl}_{3} \mathrm{O}}+\underset{\substack{\text { Potassium } \\ \text { hydrate. }}}{\mathrm{KHO}}=\underset{\substack{\text { Potassium } \\ \text { formate. }}}{\mathrm{KCHO}_{2}}+\underset{\text { Chloroform. }}{\mathrm{CHCl}_{3}} .
$$

This decomposition was believed to take place in the animal body, and especially in the blood, whenever chloral was given internally, but recent investigations seem to contradict this assumption. There is no chemical antidote which may be used in cases of poisoning by chloral, and the treatment is, therefore, confined to the use of the stomach-pump and to the maintenance of respiration.

Chloroform, Chloroformum, $\mathrm{CHCl}_{3}=119.2$ (Trichlormethane, Dichlormethyl chloride). When chlorine, bromine, or iodine is allowed to act upon methane, $\mathrm{CH}_{4}$, a number of substitution products are formed. Thus, if methane is considered as methyl hydride, $\mathrm{CH}_{3} \mathrm{H}$, the first product of substitution is methyl chloride, $\mathrm{CH}_{3} \mathrm{Cl}$; the second is monochlormethyl chloride, $\mathrm{CH}_{2} \mathrm{ClCl}$; the third is dichlormethyl chloride or chloroforn, $\mathrm{CHCl}_{2} \mathrm{Cl}$; and the fourth is carbon tetrachloride, $\mathrm{CCl}_{4}$. Similar products are formed by the action of iodine or bromine upon methane, or, . in fact, upon any of the paraftines.

Chloroform is, however, not obtained for practical purposes by the above process, but by the action of bleaching-powder and calcium hydrate on alcohol. The three last-named substances, after being mixed with a considerable quantity of water, are heated in a retort until distillation commences; the crude product of distillation is an impure chloroform, which is purified by mixing it with sulphuric acid and allowing the mixture to stand; the upper layer of chloroform is removed and treated with sodium carbonate (to remove any acids) and distilled over calcium oxide (to remove water).

The explanation of the formation of chloroform by the above process has indirectly been given in connection with the consideration of chloral, where it has been shown that alcohol is converted by the action of chlorine first into aldehyde and sub- 
sequently into chloral, which, upon being treated with alkalies, is decomposed into an alkaline formate and chloroform.

The action of the chlorine of the calcium hypochlorite (which is the active principle in bleaching-powder) upon the alcohol is similar to that of free chlorine upon alcohol; in both cases aldehyde, and afterwards chloral, are formed, which latter, in the manufacture of chloroform, is decomposed by the calcium hydrate into calcium formate and chloroform.

If the various intermediate steps of the decomposition are not considered, the process may be represented by the following equation :

$\underset{\text { Alcohol. }}{4 \mathrm{C}_{2} \mathrm{H}_{6} \mathrm{O}}+\underset{\substack{\text { Calcium } \\ \text { hypochlorite. }}}{8 \mathrm{CaCl}_{2} \mathrm{O}_{2}}=\underset{\text { Chloroform. }}{2\left(\mathrm{CHCl}_{3}\right)}+\underset{\substack{\text { Calcium } \\ \text { formate. }}}{2} \underset{\text { Chorom }}{2\left(\mathrm{Ca}_{2}\right)}+\underset{\substack{\text { Calcium } \\ \text { chloride. }}}{5 \mathrm{CaCl}_{2}}+\underset{\text { Water. }}{8 \mathrm{H}_{2} \mathrm{O} .}$

Pure chloroform is a heavy, colorless liquid, of a characteristic, ethereal odor, a burning, sweet taste, and a neutral reaction; it is but very sparingly soluble in water, but miscible with alcohol and ether in all proportions; the specific gravity of pure chloroform is 1.50 , but a small quantity of alcohol (from onehalf to one per cent.), allowed to be present by the U. S. P., causes the specific gravity to be about 1.488 ; boiling-point about $60^{\circ}$, but rapid evaporation takes place at all temperatures.

Chloroform should be tested for excess of alcohol by specific gravity; for hydrochloric acid and chlorine by shaking it with water, which should not give a precipitate with silver nitrate; for aldehyde by heating with solution of potassium hydrate, which should not be colored brown; for empyreumatic and other organic compounds by shaking with an equal volume of pure sulphuric acid, which should remain colorless; or by evaporation, when no residue should be left and no odor should be perceptible after the chloroform has been volatilized.

The only characteristic test for chloroform consists in passing its vapors through a glass tube heated to redness, when chloroform is decomposed, a deposit of carbon being formed, whilst chlorine and hydrochloric acid escape, and may be recognized by their action on silver nitrate (white precipitate of silver chloride) and mucilage of starch, to which potassium iodide has been added (free chlorine liberates iodine, which forms with the starch blue iodized starch). In applying this chloroform test 
to an acid mixture, (possibly containing chlorides) this should be neutralized previous to the expelling of the vapors, as otherwise the acid might decompose chlorides with liberation of bydrochloric acid.

In cases of poisoning, chloroform is generally to be sought for in the lungs and blood, which are placed in a flask connected with a tube of difficultly fusible glass. By heating the flask the chloroform is expelled and decomposed in the heated glass tube, as stated above.

What has been said above regarding antidotes to chloral holds good for chloroform also.

Iodoform, Iodoformum, $\mathrm{CHI}_{3}=392.8$ (Dïodomethyl iodide). This compound is analogous in its constitution to chloroform. It is made by heating together an aqueous solution of potassium carbonate, iodine, and alcohol until the brown color of iodine has disappeared; on cooling, iodoform is deposited in yellow scales, which are well washed with water and dried between filtering paper.

Iodoform occurs in small, lemon-yellow, lustrous crystals, having a peculiar, penetrating odor, and an unpleasant, sweetish taste; it is nearly insoluble in water and acids, soluble in alcohol, ether, fatty, and essential oils.

\section{Questions.}

401. What is an aldehyde, and what are its relations to alcohols and acids?

402. State the composition of acetic aldehyde.

403. Explain the action of chlorine upon alcohol.

404. Give the composition and properties of chloral and chloral hydrate.

405. What decomposition takes place when alkalies act upon chloral?

406. Describe the process of preparing and purifying chloroform.

407. What is the composition of chloroform and what are its properties?

408. How is chloroform tested for impurities?

409. By what test may chloroform be recognized?

410. How is iodoform made, and what are its properties? 


\section{MONOBASIC FATTY ACIDS.}

General constitution of organic acids. When hydroxyl, HO, replaces hydrogen in hydrocarbons, alcohols are formed; when the univalent group $\mathrm{CO}_{2} \mathrm{H}$, known as carboxyl, replaces hydrogen in hydrocarbons, acids are formed. Monatomic, diatomic, and triatomic alcohols are formed by introducing hydroxyl once, twice, or three times respectively into hydrocarbon molecules; monobasic, dibasic, and tribasic acids are formed by substituting one, two, or three hydrogen atoms by carboxyl. For instance:

$\begin{array}{ccc}\text { Hydrocarbons. } & \text { Monobasic acids. } & \text { Bibasic acids. } \\ \mathrm{CH}_{4} & \mathrm{CH}_{3} \cdot \mathrm{CO}_{2} \mathrm{H} & \mathrm{CH}_{2} / \mathrm{CO}_{2} \mathrm{H} \\ \text { Methane. } & \text { Acetic acid. } & \text { Malonic acid. } \\ \mathrm{C}_{2} \mathrm{H}_{6} & \mathrm{C}_{2} \mathrm{H}_{5} \cdot \mathrm{CO}_{2} \mathrm{H} & \mathrm{C}_{2} \mathrm{H}_{4} \backslash \mathrm{CO}_{2} \mathrm{H} \\ \text { Ethane. } & \text { Propionic acid. } & \text { Succinic acid. }\end{array}$

The constitution of carboxyl is represented by $\mathrm{O}=\mathrm{C}-\mathrm{O}-\mathrm{H}$, which shows that of the four affinities of the carbon atom, two are saturated by an atom of oxygen, one by hydroxyl, whilst one is unprovided for ; any univalent hydrocarbon residue may attach itself to this unprovided affinity when an acid is formed. Acids may, therefore, be looked upon as being composed of hydrocarbon residues and hydroxyl, united by the bivalent radical $\mathrm{CO}$. By replacement of the hydrogen of the hydroxyl (or of the carboxyl, which is the same) by metals the various salts are formed.

What is termed the acid radical is the group of the total number of atoms present in the molecule, with the exception of the hydroxyl. In acetic acid, $\mathrm{C}_{2} \mathrm{H}_{4} \mathrm{O}_{2}$, for instance, the radical is $\mathrm{CH}_{3} \mathrm{CO}$ or $\mathrm{C}_{2} \mathrm{H}_{3} \mathrm{O}$, which group of atoms is characteristic of acetic acid and of all acetates, and may often be transferred from one compound into another without decomposition.

The difference between alcohol radicals and acid radicals may also be stated, by saying that the first contain carbon and hydrogen only, whilst acid radicals contain carbon, hydrogen, and. oxygen.

In a similar manner, as there are homologous series of alcohols corresponding to the various series of hydrocarbons, there are 
also homologous series of organic acids running parallel with the corresponding series of hydrocarbons or alcohols.

Occurrence in nature. Organic acids are found and formed both in vegetables and animals, and are present either in the free state, or (and more generally) in combination with bases as salts, or with alcohols as compound ethers. Uncombined or as salts are found, for instance, citric, tartaric, and oxalic acids in plants, formic acid in some insects, uric acid in urine, etc.; as compound ethers, are found many of the fatty acids in the various fats.

Some organic acids are also found as products of the decomposition of organic matter in nature.

Formation of acids. Many acids are produced by oxidation of alcohols. As intermediate products, are formed aldehydes, which may be looked upon (as stated in the last chapter) as alcohols from which two atoms of hydrogen have been removed. For instance:

$$
\begin{aligned}
& \underset{\substack{\text { Ethyl } \\
\text { alcohol. }}}{\mathrm{C}_{2} \mathrm{H}_{5} \mathrm{HO}}+\mathrm{O}=\underset{\substack{\text { Acetic } \\
\text { al.ehyde. }}}{\mathrm{C}_{2} \mathrm{H}_{3} \mathrm{HO}}+\mathrm{H}_{2} \mathrm{O} . \\
& \underset{\substack{\text { Acetic } \\
\text { aldehyde. }}}{\mathrm{C}_{2} \mathrm{H}_{3} \mathrm{HO}}+\mathrm{O}=\underset{\substack{\text { Acetic } \\
\text { acid. }}}{\mathrm{C}_{2} \mathrm{H}_{3} \mathrm{O} . \mathrm{HO} .}
\end{aligned}
$$

Acids are obtained from compound ethers by boiling them with alkalies, when salts are formed, which may be decomposed by sulphuric or other acids. For instance:

$$
\begin{aligned}
& \underset{\text { Ethyl acetate. }}{\mathrm{C}_{2} \mathrm{H}_{5}} \underset{\mathrm{C}}{\mathrm{C}_{2} \mathrm{H}_{3} \mathrm{O}}+\underset{\substack{\text { Potassium } \\
\text { hydrate. }}}{\mathrm{KHO}}=\underset{\substack{\text { Potassium } \\
\text { acetate. }}}{\stackrel{\mathrm{C}_{2} \mathrm{H}_{3} \mathrm{O} \backslash \mathrm{O}}{\mathrm{K}}>\mathrm{O}}+\underset{\text { Ethyl alcohol. }}{\mathrm{C}_{2} \mathrm{H}_{5} \mathrm{HO} .} \\
& \underset{\text { Potassium a etate. }}{2\left(\mathrm{C}_{2} \mathrm{H}_{3} \mathrm{KO}_{2}\right)}+\underset{\substack{\text { Sulphuric } \\
\text { acid. }}}{\mathrm{I}_{2} \mathrm{SO}_{4}}=\underset{\text { Acetic acid. }}{2\left(\mathrm{C}_{2} \mathrm{H}_{4} \mathrm{O}_{2}\right)}+\underset{\substack{\text { Potassium } \\
\text { sulphate. }}}{\mathrm{K}_{2} \mathrm{SO}_{4} .}
\end{aligned}
$$

Acids are also formed by destructive distillation (acetic acid); by fermentation (lactic acid); by putrefaction (butyric acid); by oxidation of many organic substances (formic acid by oxidation of starch), etc. 
Properties. Organic acids show the characteristics mentioned for inorganic acids, viz., when soluble, have an acid or sour taste, redden limus, and contain hydrogen, replaceable by metals with the formation of salts.

Most organic acids, and especially the higher members, show these acid properties in a less marked degree than inorganic acids; in fact, they become so weak that the acid properties can often scarcely be recognized. As stated above, mono-, di-, and tribasic organic acids are known, the two latter being capable of forming neutral, acid, or double salts.

Most organic acids are colorless, some of the lower and volatile acids have a characteristic odor, but most of them are odorless; most organic acids are solids, some liquids, scarcely any gaseous at the ordinary temperature. Any salt formed by the union of an organic acid and a non-volatile metal (especially alkali metal) leaves the carbonate of this metal upon heating the salt sufticiently for combustion. It is for this reason that ashes contain most metals in the form of carbonates.

Whilst the hydrogen of the hydroxyl may be replaced by metals or by other residues, the hydrogen of the acid radical may often be replaced by chlorine, and the oxygen of the hydroxyl by sulphur.

When the hydrogen of the hydroxyl is replaced by a second acid radical (of the same kind as the one forming the acid) the so-called anhydrides are produced, which correspond to the inorganic anhydrides. For instance:

$$
\begin{aligned}
& \mathrm{HNO}_{3} \text { or } \mathrm{NO}_{2} \text {. } \mathrm{HO} \\
& \text { Nitric acid. } \\
& \mathrm{C}_{2} \mathrm{H}_{4} \mathrm{O}_{2} \text { or } \mathrm{C}_{2} \mathrm{H}_{3} \text { O.HO. } \\
& \mathrm{NO}_{2}>\mathrm{O} \\
& \text { Nitric anhydride. } \\
& \text { Acetic acid. } \\
& \begin{array}{l}
\mathrm{C}_{2} \mathrm{H}_{3} \mathrm{O} \\
\mathrm{C}_{2} \mathrm{H}_{3} \mathrm{O}
\end{array} \mathrm{O} . \\
& \text { Acetic anhydride. }
\end{aligned}
$$

Fatty acids of the general composition, $\mathrm{C}_{\mathrm{n}} \mathrm{H}_{2 \mathrm{n}} \mathrm{O}_{2}$ or $\mathrm{C}_{\mathrm{n}} \mathrm{H}_{2 \mathrm{n}}{ }_{1} \mathrm{CO}_{2} \mathrm{H}$.

$\begin{array}{lcc} & \text { Fusing-point. } & \text { Boiling-point. } \\ \text { Formic acid } & +4^{\circ} & 100^{\circ} \\ \text { Acetic acid, } & +17 & 118 \\ \text { Propionic acid, } & -21 & 140 \\ \text { Butyric acid, } & -20 & 162 \\ \text { Valerianic acid, } & -16 & 185 \\ \text { Caproic acid, } & -2 & 205 \\ \text { Enanthylic acid, } & -10 & 224 \\ \text { Caprylic acid, } & +14 & 226\end{array}$

\section{Oecurs in:}

Red ants and some plants, etc.

Vegetable and animal fluids.

Sweat, fluics of the stomach, etc.

Butter.

Valerian root.

Butter.

Castor oil.

Butter; cocoanut oil. 


\begin{tabular}{|c|c|c|c|}
\hline $\begin{array}{l}\text { Pelargonic acid, } \\
\text { Capric acid, }\end{array}$ & $\begin{array}{c}\text { Fusing-point. } \\
18^{\circ} \\
30\end{array}$ & $\begin{array}{l}\text { Boiling-point. } \\
254^{\circ} \\
270\end{array}$ & $\begin{array}{l}\text { Occurs in: } \\
\text { Leaves of geranium. } \\
\text { Butter. }\end{array}$ \\
\hline $\begin{array}{l}\text { Lauric acid, } \\
\text { Myristic acid, }\end{array}$ & $\begin{array}{l}43 \\
54\end{array}$ & & Cocoanut oil. \\
\hline $\begin{array}{l}\text { Palmitic acid, } \\
\text { Margaric acid, } \\
\text { Stearic acid, } \\
\text { Arachidic acid, } \\
\text { Behenic acid, } \\
\text { Hyaenic acid, }\end{array}$ & $\begin{array}{l}62 \\
60 \\
70 \\
75 \\
76 \\
77\end{array}$ & & $\begin{array}{l}\text { Palm oil, butter. } \\
\text { (Obtained artificially.) } \\
\text { Most solid animal futs. } \\
\text { Oils of certain plants. }\end{array}$ \\
\hline $\begin{array}{l}\text { Cerotic acid, } \\
\text { Melissic acid, }\end{array}$ & $\begin{array}{l}80 \\
90\end{array}$ & & Beeswax. \\
\hline
\end{tabular}

The name fatty acids has been given to these acids on account of their frequent occurrence in fats, and also in allusion to the somewhat fatty appearance of the higher members of the series.

The gradual change of properties which the members of a homologous series show, is well marked in the series of fatty acids, thus:

\section{First member}

Is liquid.

Volatilized at $100^{\circ}$.

Strongly acid.

Strongly odoriferous.

Easily soluble in water.

Produces no grease spot.

Forms salts easily soluble without decomposition.

\section{Last member.}

Is solid.

Not volatilized without decomposition.

Scarcely acid.

Odorless.

Insoluble in water.

Produces a grease-spot.

Forms salts which are insoluble or decomposed by water.

The intermediate members of the series show intermediate properties, and this change in properties is in proportion to the gradual change in molecular weight.

Formic acid, $\mathrm{H.CHO}_{2}$ or CHO.HO. This acid is found in the red ant and in other insects, which eject it when irritated. It is also contained in some plants, as, for instance, in the leaves of the stinging-nettle.

It is formed by the oxidation of methyl alcohol :

$$
\underset{\substack{\mathrm{Methyl} \\ \text { alcohol. }}}{\mathrm{CH}_{3} \mathrm{O}}+\mathrm{O}_{2}=\underset{\substack{\text { Formic } \\ \text { acid. }}}{\mathrm{CH}_{2} \mathrm{O}_{2}}+\mathrm{H}_{2} \mathrm{O} \text {, }
$$


by the action of carbonic oxide on potassium hydrate :

$$
\mathrm{KHO}+\mathrm{CO}=\underset{\substack{\text { Potassium } \\ \text { formate. }}}{\mathrm{KCHO}}
$$

by the action of potassium hydrate on chloroform :

$$
\mathrm{CHCl}_{3}+4 \mathrm{KHO}=3 \mathrm{KCl}+2 \mathrm{H}_{2} \mathrm{O}+\mathrm{KCHO}_{2},
$$

by heating equal parts of glycerine and oxalic acid, when the latter is split up into carbon dioxide and formic acid:

$$
\begin{aligned}
& \mathrm{C}_{2} \mathrm{H}_{2} \mathrm{O}_{4}=\mathrm{CO}_{2}+\mathrm{CH}_{2} \mathrm{O}_{2} \text {. } \\
& \text { Oxalic acid. }
\end{aligned}
$$

It is also a product of the decomposition of sugar, starch, etc. Formic acid is a colorless liquid having a penetrating odor, and a strongly acid taste; it produces blisters on the skin; it is a powerful deoxidizer, being then converted into carbon dioxide and water:

$$
\mathrm{CH}_{2} \mathrm{O}_{2}+\mathrm{O}=\mathrm{CO}_{2}+\mathrm{H}_{2} \mathrm{O} \text {. }
$$

Acetic acid, $\mathrm{H} . \mathrm{C}_{2} \mathrm{H}_{3} \mathrm{O}_{2}$, or $\mathrm{C}_{2} \mathrm{H}_{3} \mathrm{O} . \mathrm{HO}$, or $\mathrm{CH}_{3} \cdot \mathrm{CO}_{2} \mathrm{H}=60$. The most important alcohol is ethyl alcohol, and the most important organic acid is acetic acid, obtained from ethyl alcohol by oxidation. Acetic acid is found in combination with alkali metals in the juices of many plants, also in the secretions of the glands, etc.

Acetic acid is chiefly formed either by the oxidation of alcohol (and aldehyde) or by the destructive distillation of wood. It is produced commercially on a large scale as follows: A diluted alcohol (8 to 10 per cent.) is allowed to trickle down slowly through wood-shavings contained in high casks having perforated sides in order to allow a free circulation of the air; the temperature is kept at about $24^{\circ}$ to $30^{\circ}$, and the liquid having passed through the shavings is repeatedly poured back in order to cause complete oxidation. When the latter object has been accomplished the liquid is a diluted acetic acid.

The second process for manufacturing acetic acid is the heating of wood to a red heat in iron retorts, when numerous products (gases, aqueous and tarry substances) are formed. The aqueous products contain, besides other substances, methyl alcohol and acetic acid. The liquid is neutralized with calcium 
hydrate and distilled, when methyl alcohol, water, etc., evaporate and a solid residue is left, which is an inpure calcium acetate. From this latter, acetic acid is obtained by distilling with sulphuric (or hydrochloric) acid, calcium sulphate (or chloride) being formed and left in the retort, whilst acetic acid distils over.

Pure acetic acid is solid at or below $16^{\circ}$, at higher temperatures it is a colorless liquid having a characteristic, penetrating odor, boiling at $118^{\circ}$, and causing blisters on the skin; its specific gravity is 1.056 ; it is miscible with water, alcohol, and ether, is strongly acid, forming salts known as acetates, which are all soluble in water.

Vinegar is a diluted acetic acid (about six per cent.), containing often other substances, such as coloring matter, compound ethers, etc. Vinegar was formerly obtained exclusively by the oxidation of fermented fruit-juices (wine, cider, etc.), the various substances present in them imparting a pleasant taste and odor to the vinegar; to-day vinegar is often made artificially by adding various coloring and odoriferous substances to a diluted acetic acid. Vinegar should be tested for sulphuric and hydrochloric acids, which are sometimes fraudulently added.

Acidum aceticum, Acidum aceticum dilutum, and Acidum aceticum glaciale are the three officinal forms of acetic acid. The firstnamed acid contains 36 per cent., the secoud 6 per cent., the third at least 99 per cent. of pure acetic acid.

Acetic acid shows an exceptional behavior in regard to the specific gravity of its aqueous solutions. The highest specific gravity of 1.0748 belongs to an acid of 77 per cent., which is equal to an acid containing one molecule of water and one of acetic acid, or $\mathrm{C}_{2} \mathrm{H}_{4} \mathrm{O}_{2} . \mathrm{H}_{2} \mathrm{O}$. The addition of either acetic acid or of water causes the liquid to become lighter, so that, for instance, the specific gravity of an acid containing 95 per cent. is equal to that containing 56 per cent. of pure acid, both solutions having a specific gravity of 1.066 .

The specific gravity of a diluted acetic acid cannot therefore be used as a means of determining the amount of pure acid; this is done by exactly neutralizing a weighed portion of the acid with an alkali; from the quantity of the latter used, the quantity of actual acid present may be easily calculated. 


\section{Analytical reactions.}

1. Any acetate heated with sulphuric acid evolves acetic acid, which may be recognized by its odor.

2. Acetic acid or acetates heated with sulphuric acid and alcohol give a characteristic odor of acetic ether.

3. A solution containing acetic acid or an acetate carefully neutralized turns deep red on the addition of solution of ferric chloride, and forms, on boiling, a reddish-brown precipitate of an oxyacetate of iron.

Potassium acetate, Potassii acetas, $\mathrm{KC}_{2} \mathrm{H}_{3} \mathrm{O}_{2}=98$. Sodium acetate, Sodii acetas, $\mathrm{NaC}_{2} \mathrm{H}_{3} \mathrm{O}_{2} \cdot 3 \mathrm{H}_{2} \mathrm{O}=136$. Zinc acetate, Zinci acetas, $\mathrm{Zn} \mathbf{2}\left(\mathrm{C}_{2} \mathrm{H}_{3} \mathbf{O}_{2}\right) \cdot 3 \mathrm{H}_{2} \mathbf{O}=\mathbf{2 3 6 . 9}$. These three salts may be obtained by neutralizing the respective carbonates with acetic acid and evaporating the solution; they are white salts, easily soluble in water.

Ferric acetate, $\mathrm{Fe}_{2} 6\left(\mathrm{C}_{2} \mathrm{H}_{3} \mathrm{O}_{2}\right)$. A 33 per cent. solution of this salt is the Liquor ferri acetatis of the U. S. I'. It is made by dissolving freshly precipitated ferric hydrate in acetic acid; it is a dark, red-brown, transparent liquid of a specific gravity of 1.16 .

Lead acetate, Plumbi acetas, $\mathbf{P b 2}\left(\mathrm{C}_{2} \mathrm{H}_{3} \mathbf{O}_{2}\right) \cdot 3 \mathrm{H}_{2} \mathbf{O}=378.5$ (Sugar of lead), is made by dissolving lead oxide in diluted acetic acid. It forms colorless, shining, transparent crystals, easily soluble in water; on heating, it melts and then loses water of crystallization; at yet higher temperatures it is decomposed; it has a sweetish, astringent, afterwards metallic taste.

When a mixture of lead acetate and lead oxide is digested or boiled with water, the acetate combines with the oxide, forming a basic lead acetate, $\mathrm{Pb} .2\left(\mathrm{C}_{2} \mathrm{H}_{3} \mathrm{O}_{2}\right)+2 \mathrm{PbO}$, a 25 per cent. solution of which is the Liquor plumbi subacetatis, or Goulard's extract, whilst a solution containing about 1 per cent. is the Liquor plumbi subacetatis dilutus, or lead-water.

Cupric acetate, Cupri acetas, $\mathrm{Cu} 2\left(\mathrm{C}_{2} \mathrm{H}_{3} \mathrm{O}_{2}\right) \cdot \mathrm{H}_{2} \mathrm{O}=\mathbf{1 9 9 . 2}$ (Acetate of copper). The commercial verdigris is a basic acetate of copper, $\mathrm{Cu} 2\left(\mathrm{C}_{2} \mathrm{H}_{3} \mathrm{O}_{2}\right) . \mathrm{CuO}$, made by the action of diluted acetic acid and atmospheric air on metallic copper. By adding to this basic 
acetate more acetic acid, the neutral acetate is obtained. It forms deep green, prismatic crystals, which are soluble in water.

Valerianic acid, $\mathrm{HC}_{5} \mathrm{H}_{9} \mathrm{O}_{2}$ (Valeric acid). This acid occurs in valerian root and angelica root, from which it may be separated; it is, however, generally obtained by oxidation of amyl alcohol bp potassium dichromate and sulphuric acid. After oxidation has taken place the mixture is distilled, when valerianic acid with some valerianate of amyl distils over. The change of amyl alcohol into valerianic acid is analogous to the conversion of ethyl alcohol into acetic acid :

$$
\underset{\substack{\mathrm{C}_{5} \mathrm{H}_{11} \mathrm{HO} \\ \text { Amyl } \\ \text { alcohol. }}}{\mathrm{Cl}_{\text {a }}}+2 \mathrm{O}=\underset{\substack{\text { Valerianic } \\ \text { acid. }}}{\mathrm{HC}_{5} \mathrm{H}_{9} \mathrm{O}_{2}}+\mathrm{H}_{2} \mathrm{O} .
$$

Pure valerianic acid is an oily, colorless liquid, having a penetrating, highly characteristic odor; it is slightly soluble in water, but soluble in alcohol; it boils at $185^{\circ}$.

Several of the salts of valerianic acid are officinal; they are the valerianate of iron, of ammonium, of zinc, and of quinine. The three last-named compounds are white salts, whilst the ferric valerianate has a dark-red color; the ammonium salt is easily soluble in water, the three other compounds are insoluble or nearly so.

Oleic acid, Acidum oleicum, $\mathrm{HC}_{18} \mathrm{H}_{33} \mathrm{O}_{2}=282$. As shown by its formula, oleic acid does not belong to the above-described series of fatty acids of the composition $\mathrm{C}_{\mathrm{n}} \mathrm{H}_{2 \mathrm{n}} \mathrm{O}_{2}$, but to a series having the general composition $\mathrm{C}_{\mathrm{n}} \mathrm{H}_{2 \mathrm{n}-2} \mathrm{O}_{2}$.

Oleic acid is a constituent of most fats, especially of fat oils. Thus, olive oil is mainly oleate of glyceril. By boiling olive oil with potassium hydrate, potassium oleate is formed, which may be decomposed by tartaric acid, when oleic acid is liberated.

Oleic acid is a nearly colorless or yellowish, odorless, tasteless, neutral liquid, insoluble in water, soluble in alcohol, chloroform, oil of turpentine, and fat oils, crystallizing near the freezing-point of water; exposed to the air it decomposes and shows then an acid reaction. Lead oleate is soluble in ether, lead palmitate and lead stearate are not.

The officinal oleate of mercury and oleate of veratrine are ob- 
tained by dissolving the yellow mercuric oxide or veratrine in oleic acid.

\section{Questions.}

411. What is the constitution of organic acids, which group of atoms is found in all of them, and how does an alcohol radical differ from an acid radical?

412. Give some processes by which organic acids are formed in nature or artificially?

413. Mention the general properties of organic acids.

414. Which series of acids is known as fatty acids, and why has this name been given to them?

415. Mention names, composition, and occurrence in nature of the first five members of the series of fatty acids.

416. By what processes may formic acid be obtained, and what are its properties?

417. Describe the processes of manufacturing acetic acid from alcohol and from wood.

418. What is vinegar, and what is glacial acetic acid? Give tests for acetic acid and for acetates.

419. Describe the processes for making the acetates of potassium, zinc, iron, lead, and copper, and also of Goulard's extract and leadwater; state their composition and properties.

420. Where and in what form of combination is oleic acid found in nature, and what are its properties?

\section{DIBASIC AND TRIBASIC ORGANIC ACIDS.}

\section{Dibasic acids of the general composition $\mathrm{C}_{n} \mathrm{H}_{2 \mathrm{n}-2} \mathrm{O}_{4}$.}

$\begin{array}{ll}\text { Oxalic acid, } & \mathrm{H}_{2} \mathrm{C}_{2} \mathrm{O}_{4} . \\ \text { Malonic acid, } & \mathrm{H}_{2} \mathrm{C}_{3} \mathrm{H}_{2} \mathrm{O}_{4} \text {. } \\ \text { Succinic acid, } & \mathrm{H}_{2} \mathrm{C}_{4} \mathrm{H}_{4} \mathrm{O}_{4} \text {. } \\ \begin{array}{c}\text { Pyrotartaric acid, } \\ \mathrm{H}_{2} \mathrm{C}_{5} \mathrm{H}_{6} \mathrm{O}_{4}\end{array} \text {. } \\ \begin{array}{c}\text { Adipic acid, } \\ \text { etc. }\end{array} & \mathrm{H}_{2} \mathrm{C}_{6} \mathrm{H}_{8} \mathrm{O}_{4} \text {. }\end{array}$

Of these acids, only the first member is of general interest.

Oxalic acid, $\mathrm{H}_{2} \mathrm{C}_{2} \mathbf{O}_{4}$. This acid may be looked upon as a direct combination of two carboxyl groups, $\mathrm{CO}_{2} \mathrm{H}-\mathrm{CO}_{2} \mathrm{H}$, both atoms of hydrogen being replaceable by metals.

Oxalic acid is largely distributed in the vegretable kingdon in 
the form of potassium, sodium, or calcium salts. It may be obtained from these vegetables, or by the oxidation of many organic substances, chiefly fats, sugars, starch, etc., by nitric acid or other strong oxidizing agents.

Oxalic acid is manufactured on a large scale by heating sawdust with potassium or sodium hydrate, when the oxalate of these metals is formed; by the addition of calcium hydrate to the dissolved alkaline oxalate, insoluble calcium oxalate is formed which is decomposed by sulphuric acid.

Oxalic acid crystallizes in large, transparent, colorless prisms, containing two molecules of water; it is soluble in water and alcohol, and has poisonous properties. When heated slowly it sublimes; when heated rapidly by itself or with sulphuric acid it is decomposed into water, carbonic oxide, and carbon dioxide :

$$
\mathrm{H}_{2} \mathrm{C}_{2} \mathrm{O}_{4}=\mathrm{H}_{2} \mathrm{O}+\mathrm{CO}+\mathrm{CO}_{2} \text {. }
$$

Oxalic acid acts as a reducing agent, decolorizing solutions of the permanganates, and precipitating gold and platinum from their solutions:

$$
\mathrm{PtCl}_{4}+2 \mathrm{H}_{2} \mathrm{C}_{2} \mathrm{O}_{4}=\mathrm{Pt}+4 \mathrm{CO}_{2}+4 \mathrm{HCl} .
$$

\section{Analytical reactions.}

1. Oxalic acid or oxalates when heated with strong sulphuric acid evolve carbonic oxide and carbon dioxide (see above).

2. Neutral solutions of oxalic acid give with calcium chloride a white precipitate of calcium oxalate, $\mathrm{CaC}_{2} \mathrm{O}_{4}$, which is insoluble in acetic, soluble in hydrochloric acid.

3. Silver nitrate produces a white precipitate of silver oxalate, $\mathrm{Ag}_{2} \mathrm{C}_{2} \mathrm{O}_{4}$.

4. A dry oxalate (containing a non-volatile metal) heated in a test-tube evolves carbonic oxide, whilst a carbonate is left which shows effervescence with acids.

Antidotes to oxalic acid. Calcium carbonate or lime-water should be administered, but no alkalies as in cases of poisoning by mineral acids, because the alkaline oxalates are soluble.

0xalates. The acid potassium oxalate, $\mathrm{KHC}_{2} \mathrm{O}_{4}$, or its combination with oxalic acid, is known under the name of salt of sorrel. 
Calcium oxalate, $\mathrm{CaC}_{2} \mathrm{O}_{4}$, is, in small quantities, a normal constituent of urine. Ferrous oxalate, ferri oxalas, $\mathrm{FeC}_{2} \mathrm{O}_{4} \cdot \mathrm{H}_{2} \mathrm{O}$, is made by adding potassium or ammonium oxalate to ferrous sulphate, when double decomposition takes place, and the ferrous oxalate is precipitated as a pale yellow, crystalline, nearly insoluble powder.

Dibasic acids with alcoholic hydroxyl.

$$
\text { Nalic acid }=\mathrm{C}_{4} \mathrm{H}_{6} \mathrm{O}_{5} \text { or } \mathrm{C}_{2} \mathrm{H}_{3}-\mathrm{CO}_{2} \mathrm{H}
$$

In the various acids heretofore considered, the hydrogen is derived either from the unsaturated hydrocarbon residue, or from the hydroxyl in the carboxyl. As shown by the graphic formulas of the above two acids, they contain also hydrogen in the hydroxyl form not in combination with .CO. This hydrogen whilst not replaceable by metals, may be replaced by alcohol radicals; in other words, it behaves like the hydroxyl hydrogen in alcohols. In order to indicate this difference in the function of the hydrogen, malic acid is said to be dibasic, but triatomic ; tartaric acid is dibasic and tetratomic. A few other acids behave in a similar manner, as, for instance, lactic acid.

Malic acid, $\mathrm{H}_{2} \mathrm{C}_{4} \mathrm{H}_{4} \mathrm{O}_{5}$, occurs in the juices of many fruits, as apples, currants, etc.

Tartaric acid, Acidum tartaricum, $\mathrm{H}_{2} \mathrm{C}_{4} \mathrm{H}_{4} \mathbf{0}_{6}=150$. Frequently found in vegetables, and especially in fruits; grapes contain it as potassium acid tartrate, which is obtained in an impure state as a by-product in the manufacture of wine. During the fermentation of grape-juice, the sugar is converted into alcohol; potassium acid tartrate is less soluble in alcoholic fluids than in water, and is, therefore, gradually deposited, forming the crude tartar, or argol of commerce, a substance containing chiefly potassium acid tartrate, but also calcium tartrate, some coloring 
matter, and often traces of other substances. Crude tartar is the source of tartaric acid and its salts.

Tartaric acid is obtained from potassium acid tartrate by neutralizing with calcium carbonate, and decomposing the remaining neutral potassium tartrate by calcium chloride:

$$
\begin{gathered}
\underset{\substack{\text { Potassium } \\
\text { acid tartrate. }}}{2\left(\mathrm{KHC}_{4} \mathrm{H}_{4} \mathrm{O}_{6}\right)}+\underset{\substack{\text { Calcium } \\
\text { carbonate. }}}{\mathrm{CaCO}_{3}}=\underset{\substack{\text { Calcium } \\
\text { tartrate. }}}{\mathrm{CaC}_{4} \mathrm{H}_{4} \mathrm{O}_{6}}+\underset{\substack{\text { Potassium } \\
\text { tartrate. }}}{\mathrm{K}_{2} \mathrm{C}_{4} \mathrm{H}_{4} \mathrm{O}_{6}}+\underset{\text { Water }}{\mathrm{H}_{2} \mathrm{O}}+\underset{\begin{array}{c}
\text { Carbon } \\
\text { dioxide. }
\end{array}}{\mathrm{CO}_{2}} . \\
\underset{\substack{\text { Potassium } \\
\text { tartrate. }}}{\mathrm{K}_{2} \mathrm{C}_{4} \mathrm{H}_{4} \mathrm{O}_{6}}+\underset{\substack{\text { Calcium } \\
\text { chloride. }}}{\mathrm{CaCl}_{2}}=\underset{\substack{\text { Calcium } \\
\text { tartrate. }}}{\mathrm{CaC}_{4} \mathrm{H}_{4} \mathrm{O}_{6}}+\underset{\substack{\text { Potassium } \\
\text { chloride. }}}{2 \mathrm{KCl} .}
\end{gathered}
$$

The whole of the tartaric acid is thus converted into calcium tartrate, which is precipitated as an insoluble powder; this is collected, well washed, and decomposed by boiling with sulphuric acid, when calcium sulphate is formed as an alınost insoluble residue, whilst tartaric acid is left in solution, from which it is obtained by evaporation and crystallization:

$$
\underset{\substack{\text { Calcium } \\ \text { tartrate. }}}{\mathrm{CaC}_{4} \mathrm{H}_{4} \mathrm{O}_{6}}+\underset{\substack{\text { Sulphuric } \\ \text { acid. }}}{\mathrm{H}_{2} \mathrm{SO}_{4}}=\underset{\substack{\text { Tartaric } \\ \text { acid. }}}{\mathrm{H}_{2} \mathrm{C}_{4} \mathrm{H}_{4} \mathrm{O}_{6}}+\underset{\substack{\text { Calcium } \\ \text { sulphate. }}}{\mathrm{CaSO}_{4} .}
$$

Tartaric acid crystallizes in colorless, transparent prisms; it has a strongly acid, but not disagreeable taste; it is readily soluble in water and alcohol.

There are three acids which are isomeric with common tartaric acid, differing from it in physical, but not in chemical properties. These acids are known as inactive tartaric acid, levotartaric acid, and racemic acid, whilst the common tartaric acid is termed dextrotartaric acid. Crude tartar sometimes contains racemic acid.

\section{Analytical reactions.}

1. Neutral solutions of tartaric acid give with calcium chloride a white precipitate of calcium tartrate, which, after being quickly collected on a filter and washed, is soluble in potassium hydrate; from this solution calcium tartrate is reprecipitated on boiling. (Calcium citrate is insoluble in potassium hydrate.)

2. A strong solution of a tartrate, acidulated with acetic acid, gives a white precipitate of potassium acid tartrate on the addition of potassium acetate. (Precipitate forms slowly.) 
3. A neutral solution of a tartrate gives with silver nitrate. a white precipitate of silver tartrate, $\mathrm{Ag}_{2} \mathrm{C}_{4} \mathrm{H}_{4} \mathrm{O}_{6}$, which blackens on boiling, in consequence of the decomposition of the salt with separation of silver. If, before boiling, a drop of ammonia water is added, a mirror of metallic silver will form upon the glass.

4. Sulphuric acid heated with tartrates chars them readily.

5. Tartrates, when heated, are decomposed (blacken), and evolve a somewhat characteristic odor resembling that of burnt sugar.

Potassium acid tartrate, Potassii bitartras, $\mathrm{KHC}_{4} \mathrm{H}_{4} \mathbf{O}_{6}=188(\mathrm{Bi}$ tartrate of potassium, Cream of tartar). The formation of this salt in the crude state (argol) has been explained above. It is purified by dissolving in hot water and crystallizing, when it is obtained in colorless crystals, or as a white, somewhat gritty powder of a pleasant, acidulous taste; it is sparingly soluble in cold, easily soluble in hot water.

Potassium tartrate, Potassii tartras, $2\left(\mathrm{~K}_{2} \mathrm{C}_{4} \mathrm{H}_{4} \mathrm{O}_{6}\right) \mathrm{H}_{2} \mathbf{O}=\mathbf{4 7 0}$ (Tartrate of potassium). Obtained by saturating a solution of potassium acid tartrate with potassium carbonate:

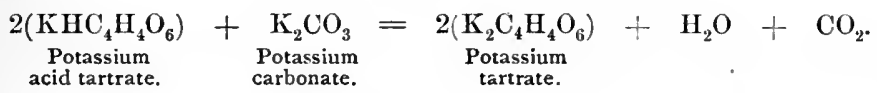

Small, transparent or white crystals, or a white neutral powder, soluble in less than its own weight of water.

Potassium sodium tartrate, Potassii et sodii tartras, $\mathrm{KNaC}_{4} \mathrm{H}_{4} \mathrm{O}_{6}$. $4 \mathrm{H}_{2} \mathrm{O}=282$ (Tartrate of potassium and sodium, Rochelle salt). If in the above-described process for making neutral potassium tartrate, sodium carbonate is substituted for potassium carbonate, the double tartrate of potassium and sodium is formed. It is a white powder, or occurs in colorless, transparent crystals which are easily soluble in water.

Seidlitz powders consist of a mixture of 120 grains of Rochelle salt with 40 grains of sodium bicarbonate (wrapped in blue paper), and 35 grains of tartaric acid (wrapped in white paper). When dissolved in water, the tartaric acid acts upon the sodium 
bicarbonate, causing the formation of sodium tartrate, while the escaping carbon dioxide causes effervescence.

Antimony potassium tartrate, Antimonii et potassii tartras, 2( $\left.\mathrm{KSb0} . \mathrm{C}_{4} \mathrm{H}_{4} \mathbf{O}_{6}\right) \cdot \mathbf{H}_{2} \mathbf{O}=664$ (Tartrate of antimony and potassium, Tartar emetic). This salt is made by dissolving freshly prepared antimonious oxide (while yet moist) in a solution of potassium acid tartrate. From the solution somewhat evaporated, tartar emetic separates in colorless, transparent rhombic crystals:

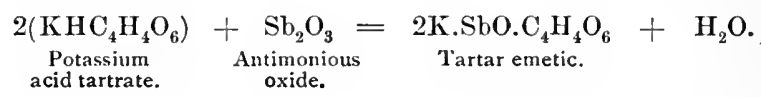

The fact that not antimony itself, but the group $\mathrm{SbO}$ replaces the hydrogen, has led to the assumption of the hypothetical radical $\mathrm{SbO}$, termed antimonyl.

Tartar emetic is soluble in water, insoluble in alcohol; it has a sweet, afterwards disagreeable metallic taste.

Action of certain organic acids upon certain metallic oxides. The solution of a ferric salt (or certain other metallic salts) is precipitated by alkaline hydrates, a salt of the alkali and ferric hydrate being formed. When a sufficient quantity of either tartaric, citric, oxalic, or various other organic acids has been previously added to the iron solution (or to certain other metallic solutions), no such precipitate is produced by the alkaline hydrates, because organic salts or double salts are formed which are soluble, and from which the metallic hydrates are not precipitated by alkaline hydrates. Upon evaporation no crystals (of the organic salt) form, and in order to obtain the compounds in a dry state, the liquid, after being evaporated to the consistence of a syrup, is spread on glass plates which are exposed to a temperature not exceeding $60^{\circ}$, when brown, green, or yellowish-green, amorphous, shining, transparent scales are formed, which are the scale compounds of the U.S.P.

Instead of obtaining these compounds, as stated above, by adding the organic acids (or their salts) to the inorganic salts, they are more generally obtained by dissolving the freshly precipitated metallic hydrate in the organic acid.

The true chemical constitution of many of these scale compounds has as yet not been determined with certainty. 
Of officinal scale compounds containing tartaric acid may be mentioned the tartrate of iron and ammonium, and the tartrate of iron and potassium. The first compound is obtained by dissolving freshly precipitated ferric hydrate in a solution of ammonium acid tartrate, the second by dissolving ferric hydrate in potassium acid tartrate. The clear solutions, after having been sufficiently evaporated, are dried as mentioned above on glass plates.

Citric acid, Acidum citricum, $\mathrm{H}_{3} \mathrm{C}_{6} \mathrm{H}_{5} \mathrm{O}_{7} \cdot \mathrm{H}_{2} \mathrm{O}=210$. Citric acid is a tribasic acid containing three atoms of hydrogen replaceable by metals; its constitution may be expressed by the graphic formula :

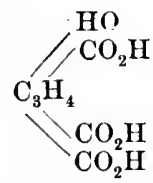

Citric acid is found in the juices of many fruits (strawberry, raspberry, currant, cherry, etc.), and in other parts of plants. It is obtained from the juice of lemons by saturating it with calcium carbonate and decomposing the calcium citrate thus formed by sulphuric acid. It forms colorless crystals, easily soluble in water.

\section{Analytical reactions.}

1. Neutral solutions of citrates yield with calcium chloride on boiling (not in the cold) a white precipitate of calcium citrate, which is insoluble in potassium hydrate, but soluble in cupric chloride.

2. Neutral solutions of citrates are precipitated white by silver nitrate. The precipitate does not blacken on boiling, as in the case of tartrates.

3. A neutral or alkaline solution of a citrate to which a few drops of a solution of potassium permanganate have been added, renders the red solution green or reddish-green, whilst tartrates decolorize it.

Citrates. Potassium citrate, $\mathrm{K}_{3} \mathrm{C}_{6} \mathrm{H}_{5} \mathrm{O}_{7} \cdot \mathrm{H}_{2} \mathrm{O}$, and Lithium citrate, $\mathrm{Li}_{3} \mathrm{C}_{6} \mathrm{H}_{5} \mathrm{O}_{7}$, are both colorless substances, easily soluble in water and obtained by dissolving the carbonates in citric acid. 
Bismuth citrate, $\mathrm{BiC}_{6} \mathrm{H}_{5} \mathrm{O}_{7}$, is obtained by boiling a solution of citric acid with bismuth nitrate, when the latter is gradually converted into citrate whilst nitric acid is set free; the insoluble bismuth citrate is collected, washed and dried; it forms a white, amorphous powder, which is insoluble in water, but soluble in water of ammonia.

Bismuth ammonium citrate is a scale compound obtained by dissolving bismuth citrate in water of ammonia and evaporating the solution at a low temperature.

Ferric citrate, Ferri citras, $\mathrm{Fe}_{2} 2\left(\mathrm{C}_{6} \mathrm{H}_{5} \mathrm{O}_{7}\right) 6 \mathrm{H}_{2} \mathrm{O}$. Obtained in transparent, red scales, by dissolving ferric hydrate in citric acid and evaporating the solution as mentioned heretofore. By mixing solution of ferric citrate with either water of ammonia, or quinine, or strychnine, evaporating to the consistence of syrup and dryiug on glass plates, the following three scale compounds are obtained respectively: Citrate of iron and ammonia, citrate af iron and quinine, citrate of iron and strychnine.

Lactic acid, Acidum lacticum, $\mathrm{HC}_{3} \mathrm{H}_{5} \mathrm{O}_{3}=\mathbf{9 0}$. This acid is the second member of a group of monobasic, diatomic acids which have the general composition $\mathrm{C}_{\mathrm{n}} \mathrm{H}_{2 \mathrm{n}} \mathrm{O}_{3}$, and which contain two hydroxyl groups, the hydrogen of one being capable of replacement by metals, the other by alcohols. The first member of this series is glycollic acid, $\mathrm{HC}_{2} \mathrm{H}_{3} \mathrm{O}_{3}$, a white, deliquescent, crystalline substance.

Lactic acid occurs in many plant-juices, it is formed from sugar by a peculiar fermentation known as "lactic fermentation," which causes the presence of this acid in sour milk and in many sour, fermented substances, as in enselage, sauer-kraut, etc. The formation of lactic acid from sugar may be expressed by the equation :

$$
\underset{\text { Sugar. }}{\mathrm{C}_{6} \mathrm{H}_{12} \mathrm{O}_{6}}=\underset{\text { Lactic acid. }}{2\left(\mathrm{HC}_{3} \mathrm{H}_{5} \mathrm{O}_{3}\right)} \text {. }
$$

For practical purposes lactic acid is made by mixing a solution of sugar with milk, putrid cheese, and chalk, and digesting this mixture for several weeks at a temperature of about $30^{\circ}$. The cheese acts as a ferment, and the chalk neutralizes the acid generated during the fermentation. The calcium lactate thus obtained is purified by crystallization and decomposed by oxalic acid, which forms insoluble calcium oxalate. 
Lactic acid is a colorless, syrupy liquid, of strongly acid properties : it mixes in all proportions with water and alcohol.

Ferrous lactate, Ferri lactas, $\mathrm{Fe} 2\left(\mathrm{C}_{3} \mathrm{H}_{5} \mathrm{O}_{3}\right) 3 \mathrm{H}_{2} \mathrm{O}=287.9$. Made by dissolving iron filings in diluted lactic acid; hydrogen- is liberated and the salt formed. It is a pale, greenish-white, crystalline substance, soluble in water.

\section{QüsTIONS.}

421. Name the more common organic acids found in vegetables and especially in sour fruits.

422. What is the composition of oxalic acid, how is it manufactured, and what are its properties?

423. Explain the formation of crude tartar during the fermentation of grape-juice, and how is tartaric acid obtained from it?

424. Give properties of and tests for tartaric acid.

425. State the composition and formation of cream of tartar, Rochelle salt, and tartar emetic.

426. What are Seidlitz powders, and what change takes place when they are dissolved?

427. Mention some officinal scale compounds of iron, and give a general outline of the mode of preparing them.

428. From what and by what process is citric acid obtained?

429. Mention tests by which citric acid may be distinguished from tartaric acid.

430. From what, and by what process is lactic acid obtained; what are its properties?

\section{AROMATIC ACIDS AND SOME OF THEIR DERIVATIVES.}

Constitution of aromatic compounds. Aromatic compounds are derived from benzene, $\mathrm{C}_{6} \mathrm{H}_{6}$, and the hydrocarbons of the benzene series; they differ considerably in their behavior from the substances derived from the methane series, which latter are generally termed fatty compounds. Aromatic compounds cannot be converted into fatty compounds or the latter into aromatic compounds without suffering the most vital decomposition of the molecule, and in most cases this transformation cannot be accomplished at all. 
On the average, aromatic compounds are richer in carbon than fatty compounds, and yield, when decomposed by various methods, benzene as one of the products; most aromatic substances have antiseptic properties. The following graphic formulas may serve to illustrate the constitution of some aromatic substances:<smiles>c1ccccc1</smiles>

Benzene.<smiles>O=Cc1ccccc1</smiles>

Phenol, or carbolic acid.<smiles>O=[N+]([O-])c1ccc(O)c([N+](=O)[O-])c1[N+](=O)[O-]</smiles>

Trinitro-phenol, or picric acid.<smiles>O=[N+]([O-])c1ccccc1</smiles>

Nitro-benzene.<smiles>Nc1ccccc1</smiles>

Amido-benzene, or aniline.<smiles>O=C(O)c1ccccc1</smiles>

Benzoic acid.<smiles>O=C(O)c1ccccc1</smiles>

Benzaldehyde, or bitter almond oil.<smiles>O=C(O)c1ccccc1</smiles>

Salicylic acid.<smiles>O=C(O)c1ccc(O)c(O)c1O</smiles>

Gallic acid.<smiles>O=C(O)c1ccccc1OCc1c(O)cccc1O</smiles> 
The preceding graphic formulas show that

Benzene, $\quad \mathrm{C}_{6} \mathrm{H}_{6}$, is a saturated hydrocarbon.

Phenol,

$\mathrm{C}_{6} \mathrm{H}_{5} \mathrm{HO}$, is an alcohol obtained from benzene by replacement of one atom of hydrogen by hydroxyl.

Picric acid, $\quad \mathrm{C}_{6} \mathrm{H}_{2}\left(\mathrm{NO}_{2}\right)_{3} \mathrm{HO}$, is phenol in which three hydrogen atoms are replaced by three $\mathrm{NO}_{2}$.

Nitro-benzene, $\quad \mathrm{C}_{6} \mathrm{H}_{5} \mathrm{NO}_{2}$, is benzene in which one hydrogen atom has been replaced by $\mathrm{NO}_{2}$.

Aniline,

Benzoic acid,

$\mathrm{C}_{6} \mathrm{H}_{5} \mathrm{NH}_{2}$, is benzene in which one hydrogen atom has been replaced by $\mathrm{NH}_{2}$.

Bitter almond oil, $\quad \mathrm{C}_{6} \mathrm{H}_{5} \mathrm{COH}$, is the aldehyde of benzoic acid, or benzoic acid from which one atom of oxygen has been removed.

Salicylic acid, $\mathrm{C}_{6} \mathrm{H}_{4} \cdot \mathrm{HO} . \mathrm{CO}_{2} \mathrm{H}$, is benzene in which one hydrogen atom is replaced by hydroxyl and one by carboxyl.

Gallic acid, $\mathrm{C}_{6} \mathrm{H}_{2} \cdot(\mathrm{HO})_{3} \cdot \mathrm{CO}_{2} \mathrm{H}$ is benzene in which three hydrogen atoms are replaced by hydroxyl and one by carboxyl.

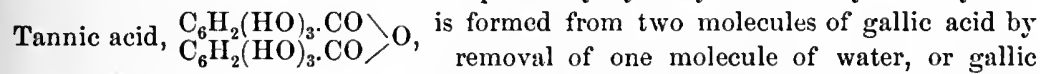
acid in which the hydrogen of the hydroxyl has been replaced by another gallic acid radical.

Carbolic acid, Acidum carbolicum, $\mathrm{C}_{6} \mathrm{H}_{5} \mathrm{HO}=94$ (Phenol). Crude carbolic acid is a liquid obtained during the distillation of coal-tar between the temperatures of $170^{\circ}-190^{\circ}$, and containing chiefly phenol, besides kresylic acid, $\mathrm{C}_{7} \mathrm{H}_{7} \mathrm{HO}$, and other substances. It is a reddish-brown neutral liquid of a strongly empyreumatic and disagreeable odor.

By fractional distillation of the crude carbolic acid, the pure acid is obtained, which forms colorless, interlaced, needle-shaped crystals, sometimes acquiring a pinkish tint; it has a characteristic, slightly aromatic odor, is deliquescent in moist air, soluble in 20 parts of water, and very soluble in alcohol, ether, chloroform, glycerine, fat and volatile oils, etc.; it has, when diluted, a sweetish and afterwards burning, caustic taste; it produces a benumbing and caustic effect, and even blisters on the skin; it is strongly poisonous, and a powerful antiseptic agent, preventing fermentation and putrefaction to a marked degree; fusing-point $42^{\circ}$, boiling-point $182^{\circ}$, specific gravity 1.065 .

Phenol, though generally called carbolic acid, has a neutral reaction, and the constitution of an alcohol, but it readily com- 
bines with strong bases, for instance with sodium, forming sodium phenoxide or sodium phenate:

$$
\mathrm{C}_{6} \mathrm{H}_{5} \mathrm{HO}+\mathrm{NaHO}=\mathrm{C}_{6} \mathrm{H}_{5} \mathrm{NaO}+\mathrm{H}_{2} \mathrm{O} .
$$

Phenol does not yield an aldehyde or acid by oxidation, like fatty alcohols.

As antidotes, may be used olive oil or castor oil, a mixture of both, or a mixture of magnesia and oil.

\section{Tests for carbolic acid.}

1. It coagulates albumen.

2. It colors solutions of neutral ferric chloride intensely and permanently blue or violet.

3. Bromine produces, even in diluted solutions of carbolic acid, a white precipitate of tribrom-phenol.

4. A slip of pinewood moistened with hydrochloric acid, and then dipped into carbolic acid turns blue on exposure to sunlight.

Creasote, Creasotum. This is a product of the distillation of wood-tar, and resembles carbolic acid in many respects, especially in its antiseptic properties and its action on the skin. It is a mixture of substances, but consists chiefly of creasol, $\mathrm{C}_{8} \mathrm{H}_{10} \mathrm{O}_{7}$, and guaiacol, $\mathrm{C}_{7} \mathrm{H}_{8} \mathrm{O}_{2}$.

From carbolic acid creasote may be distinguished by not coagulating albumen, by not being solidified on cooling, by not coloring ferric chloride permanently, and by its lower boilingpoint.

Sulphocarbolic acid, $\mathrm{HC}_{6} \mathrm{H}_{5} \mathrm{SO}_{4}$ (Phenol-sulphonic acid). Formed by dissolving carbolic acid in strong sulphuric acid:

$$
\mathrm{C}_{6} \mathrm{H}_{5} \mathrm{HO}+\mathrm{H}_{2} \mathrm{SO}_{4}=\mathrm{HC}_{6} \mathrm{H}_{5} \mathrm{SO}_{4}+\mathrm{H}_{2} \mathrm{O} .
$$

Sulphocarbolate of sodium, Sodii sulphocarbolas, $\mathrm{NaC}_{6} \mathrm{H}_{5} \mathrm{SO}_{4} \cdot 2 \mathrm{H}_{2} \mathrm{O}$, is obtained as a white soluble salt by dissolving sodium carbonate in the above acid.

Picric acid, $\mathrm{C}_{6} \mathrm{H}_{2}\left(\mathrm{NO}_{2}\right)_{3} \mathrm{HO}$ (Trinitro-phenol, Carbazotic acid). This substance is formed by the action of nitric acid on various 
matters (silk, wool, indigo, Peruvian balsam, etc.), and is manufactured on a large scale by slowly dropping carbolic acid into fuming nitric acid; it is used as a yellow dye for silk and wool, and as a reagent for albumen; most picrates are explosives.

Benzoic acid, Acidum benzoicum, $\mathrm{HC}_{7} \mathrm{H}_{5} \mathrm{O}_{2}$ or $\mathrm{C}_{6} \mathrm{H}_{5} \mathrm{CO}_{2} \mathrm{H}=122$. Found in gum-benzoin and some other gums; it is obtained from benzoin by heating it carefully, when the volatile benzoic acid sublimes. It forms white, lustrous scales or friable needles, having a slight aromatic odor of benzoin, and an acid reaction; it is but slightly soluble in cold water, but easily soluble in alcohol, ether, oils, etc.

Benzoic acid, when neutralized with an alkali, gives a fleshcolored or reddish precipitate of ferric benzoate on the addition of a neutral solution of ferric chloride.

By neutralizing benzoic acid with either ammonium hydrate or sodium hydrate, the two officinal salts ammonium benzoate, $\mathrm{NH}_{4} \mathrm{C}_{7} \mathrm{H}_{5} \mathrm{O}_{2}$, and sodium benzoate, $\mathrm{NaC}_{7} \mathrm{H}_{5} \mathrm{O}_{2} \cdot \mathrm{H}_{2} \mathrm{O}$, are obtained. Both salts are white, soluble in water, and have a slight odor of benzoin.

Oil of bitter almond, Oleum amygdalæ amaræ, $\mathrm{C}_{7} \mathrm{H}_{6} \mathrm{O}$ or $\mathrm{C}_{6} \mathrm{H}_{5} \mathrm{COH}$ (Benzaldehyde). As shown by the formula, oil of bitter almond differs from benzoic acid in containing one atom less of oxygen; in all its reactions it behaves like a true aldehyde, being, for instance, easily converted into benzoic acid by oxidation.

It does not occur in a free state in nature, but is formed by a peculiar fermentation of a glucoside, amygdaline, existing in bitter almonds, in cherry-laurel, and in the kernels of peaches, cherries, etc., but not in sweet almonds. The ferment causing the decomposition of amygdaline is a substance termed emulsine, which is found both in bitter and sweet almonds. As water is required for the decomposition, the emulsine does not act upon the amygdaline contained in the same seed until water is added, when the decomposition takes place as follows:

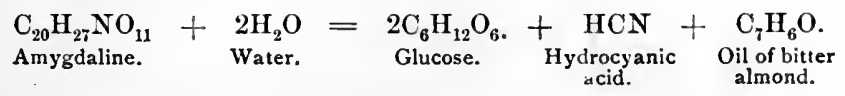

The oil is obtained by distilling bitter almonds with water, . 
when it distils over with hydrocyanic acid and steam, and separates as a heavy oil in the distillate.

It is an almost colorless, thin liquid of a characteristic aromatic odor, a bitter and burning taste, and a neutral reaction. The pure oil is not poisonous, but the crude oil of bitter almond is poisonous on account of its containing hydrocyanic acid.

Bitter almond water, Aqua amygdalce amarae, is made by dissolving 1 part of the oil in 999 parts of water.

Salicylic acid, Acidum salicylicum, $\mathrm{HC}_{7} \mathrm{H}_{5} \mathrm{O}_{3}$ or $\mathrm{C}_{6} \mathrm{H}_{4} \mathrm{HO} 0 . \mathrm{CO}_{2} \mathrm{H}=$ 138. Derived from benzene by introducing one hydroxyl and one carboxyl residue. It is found in several species of violet, and in the form of methyl salicylate in the wintergreen oil (oil of Gaultheria procumbens). May be obtained by fusing potassium hydrate with salicine, a glucoside found in the bark of willow.

Salicylic acid is manufactured from carbolic acid by passing carbon dioxide through sodium acid carbolate (sodium phenoxide), when sodium salicylate remains and carbolic acid distils over:

$$
\begin{aligned}
& \underset{\substack{\text { Carbolic } \\
\text { acid. }}}{\mathrm{C}_{6} \mathrm{H}_{5} \mathrm{HO}}+\underset{\substack{\text { Sodium } \\
\text { hydrate. }}}{\mathrm{HNaO}}=\underset{\substack{\text { Sodium } \\
\text { carbolate. }}}{\mathrm{C}_{6} \mathrm{H}_{5} \mathrm{NaO}^{\circ}}+\mathrm{H}_{2} \mathrm{O} .
\end{aligned}
$$

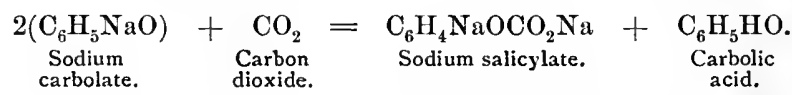

Sodium salicylate, thus obtained, is decomposed by hydrochloric acid:

$$
\underset{\text { Sodium salicylate. }}{\mathrm{C}_{6} \mathrm{H}_{4} \mathrm{NaOCO} \mathrm{Na}}+\underset{\substack{\text { Hydrochloric } \\ \text { acid }}}{2 \mathrm{HCl}}=\underset{6}{\mathrm{C}_{6} \mathrm{H}_{4} \mathrm{HOCO}_{2} \mathrm{H}}+\underset{\substack{\text { Salicylic acid } \\ \text { chloride. }}}{2 \mathrm{NaCl}}
$$

Salicylic acid is a white, solid substance, odorless, or of a slight aromatic odor, having a sweetish, slightly acrid taste, and an acid reaction; it is but sparingly soluble in cold water, but readily. soluble in alcohol, ether, etc.; it fuses at about $175^{\circ}$, and sublimes at $200^{\circ}$; it is a valuable disinfectant and antiseptic.

Salicylic acid assumes a fine violet color with ferric chloride. By dissolving the alkaline hydrates in salicylic acid, the various salts may be obtained, as, for instance, sodium salicylate, 
$2\left(\mathrm{NaC}_{7} \mathrm{H}_{5} \mathrm{O}_{3}\right) \cdot \mathrm{H}_{2} \mathrm{O}$, and lithium salicylate, $2\left(\mathrm{LiC}_{7} \mathrm{H}_{5} \mathrm{O}_{3}\right) \cdot \mathrm{H}_{2} \mathrm{O}$, both of which are white, soluble salts.

Gallic acid, Acidum gallicum, $\mathrm{HC}_{7} \mathrm{H}_{5} \mathrm{O}_{5}$ or $\mathrm{C}_{6} \mathrm{H}_{2}(\mathrm{HO})_{3}, \mathrm{CO}_{2} \mathrm{H}=188$. Obtained by exposing moistened gall-nuts to the air for about six weeks, when a peculiar fermentation takes place, during which tannic acid is converted into gallic acid, which is purified by crystallization. The crystals contain one molecule of water, which may be expelled at $100^{\circ}$. It is a white, solid substance, forming long, silky needles; it has an astringent and slightly acidulous taste, and an acid reaction; it gives a bluish-black color with ferric salts, and does not coagulate albumen; by heating, it is decomposed into carbon dioxide and pyrogallic acid, $\mathrm{C}_{6} \mathrm{H}_{6} \mathrm{O}_{3}$.

Tannic acid, Acidum tannicum, $\mathrm{C}_{14} \mathrm{H}_{10} \mathbf{O}_{9}=322$. There are a number of tannic acids, or tannins, found in various parts of different plants (oak-bark, gall-nuts, cinchona, coffee, tea, etc.), the composition of which is not identical with the above formula. All tannins, however, are amorphous, soluble substances, having a faint acid reaction and astringent properties; they all precipitate albumen, alkaloids, ferric salts (bluish-black), and form with animal substances compounds which do not putrefy.

The officinal tannic acid is obtained by extracting nut-galls with ether and alcohol, and evaporating the solution.

Tannic acid forms light-yellowish, amorphous scales, having a faint and characteristic odor, a strongly astringent taste, and an acid reaction; it is easily soluble in water and diluted alcohol.

Analytical reactions. Tannic acid forms with ferric salts a bluish-black precipitate (ink), and also precipitates gelatine, alkaloids, albumen, gelatinized starch, and solution of tartar emetic.

\section{Questions.}

431. What is the difference between aromatic and fatty compounds, and from which hydrocarbons are aromatic compounds derived?

432. State the composition of carbolic acid. In what respects does it differ from true acids?

433. Mention properties of and tests for carbolic acid.

434. What is the difference between creasote and carbolic acid; from what are the two substances obtained? 
435. What is picric acid, and what is it used for?

436. What relation exists between benzoic acid and oil of bitter almond?

437. What is the source of amygdaline, and into what substances is it decomposed by emulsine in the presence of water?

438. Explain the process for the manufacture of salicylic acid from carbolic acid, and state its properties.

-439. State the general properties of tannins.

440. From what, and by what processes are gallic and tannic acids obtained?

\section{ETHERS.}

Constitution. It has been shown that alcohols are hydrocarbon residues in combination with hydroxyl, $\mathrm{HO}$, and that acids are hydrocarbon residues in combination with carboxyl, $\mathrm{CO} . \mathrm{OH}$; it has further been shown that carboxyl may be considered as being composed of $\mathrm{CO}$, and hydroxyl $\mathrm{HO}$, and that the term acid radical is applied to that group of atoms in acids which embraces the hydrocarbon residue $+\mathrm{CO}$. If we represent an alcohol radical by $\mathrm{AlR}$, and an acid radical by $\mathrm{AcR}$, the general formula of an alcohol is AlR.HO or $\stackrel{A}{\mathrm{H}} \mathrm{H} \backslash \mathrm{O}$, and of an acid, AcR.HO or $\stackrel{\mathrm{AcR}}{\mathrm{H}}>\mathrm{O}$.

Ethers are formed by replacement of the hydrogen of the hydroxyl in alcohols by hydrocarbon residues(or alcohol radicals), and compound ethers or esters are formed by replacement of the hydrogen of the hydroxyl (or carboxyl) in acids by hydrocarbon residues. While alcohols correspond in their constitution to hydrates, ethers correspond to oxides, and compound ethers to salts. For instance :

\begin{tabular}{|c|c|c|c|}
\hline Hydrates. & Oxides. & Acids. & Salts. \\
\hline $\mathrm{KHO}=\stackrel{\mathrm{K}}{\mathrm{H}}>\mathrm{O}$ & $\mathrm{K}_{2} \mathrm{O}=\underset{\mathrm{K}}{\mathrm{K}} \succ \mathrm{O}$ & $\mathrm{HNO}_{3}=\underset{\mathrm{H}}{\mathrm{NO}}>\mathrm{O}$ & $\left.\mathrm{KNO}_{3}=\underset{\mathrm{K}}{\mathrm{NO}_{2}}\right\rangle \mathrm{C}$ \\
\hline Potassium hydrate. & Potassium oxide. & Nitric acid. & Potassium nitrate. \\
\hline $\begin{array}{c}\mathrm{C}_{2} \mathrm{H}_{5} \\
\mathrm{H}^{\prime}\end{array}$ & $\begin{array}{l}\mathrm{C}_{2} \mathrm{H}_{5} \\
\mathrm{C}_{2} \mathrm{H}_{5}\end{array} \mathrm{O}$ & $\begin{array}{r}\mathrm{C}_{2} \mathrm{H}_{3} \mathrm{O} \\
\mathrm{H}\end{array}>\mathrm{O}$ & $\begin{array}{c}\mathrm{C}_{2} \mathrm{H}_{3} \mathrm{C} \\
\mathrm{C}_{2} \mathrm{H}_{3}\end{array}$ \\
\hline Ethyl alcohol. & Ethyl ether. & Acetic acid. & $\begin{array}{l}\text { Ethyl acetate, or } \\
\text { acetic ether. }\end{array}$ \\
\hline$\stackrel{\mathrm{AlR}}{\mathrm{H}} \backslash \mathrm{O}$ & $\begin{array}{l}\mathrm{AlR} \backslash \mathrm{O} \\
\mathrm{AlR}\end{array}>\mathrm{O}$ & $\begin{array}{r}\mathrm{AcR} \\
\mathrm{H}\end{array}>\mathrm{O}$ & $\begin{array}{c}\mathrm{AcR} \\
\mathrm{AlR}\end{array}>0$ \\
\hline Alcohol. & Ether. & Acid. & $\begin{array}{l}\text { Compound } \\
\text { ether. }\end{array}$ \\
\hline
\end{tabular}


It is not necessary that the two hydrocarbon residues in an ether should be alike, as in the above ethyl ether, but they may be different, in which case the ethers are termed mixed ethers. For instance :

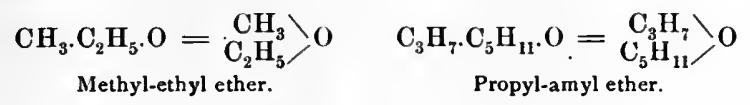

In diatomic or triatomic alcohols, or in dibasic or tribasic acids, containing more than one atom of hydrogen derived from hydroxyl or carboxyl, these hydrogen atoms may be replaced by various other univalent, bivalent, or trivalent residues. This fact shows that the number of ethers or compound ethers which are capable of being formed is very large.

Formation of ethers. Ethers may be formed by the action of the chloride or iodide of a hydrocarbon residue upon an alcohol in which the hydroxyl hydrogen has been replaced by a metal. For instance :

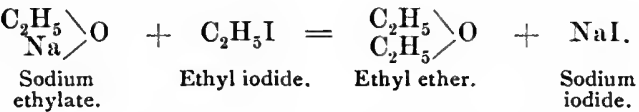

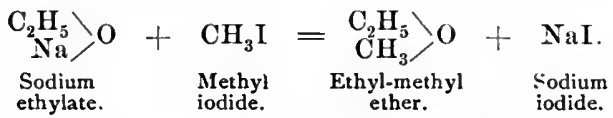

Ethers are also formed by the action of sulphuric acid upon alcohols; the sulphuric acid removing water in this case, thus :

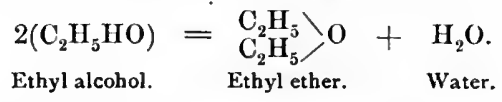

Compound ethers are formed by the combination of acids with alcohols and elimination of water. (Presence of sulphuric acid facilitates this action.)

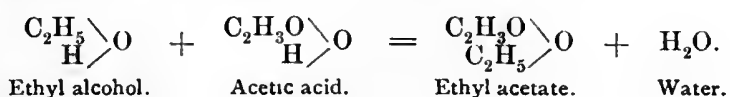

They are also formed by the action of hydrocarbon chlorides (or iodides) on salts. For instance :

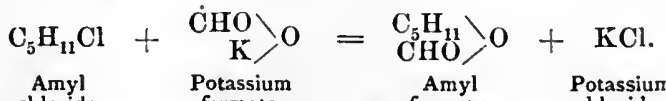

$$
\begin{aligned}
& \text { chloride. formate. formate. chloride. }
\end{aligned}
$$


0ccurrence in nature. Many ethers are products of vegetable life and occur in some essential oils; wax contains the compound ether palmitate of melissyl, $\mathrm{C}_{30} \mathrm{H}_{61} . \mathrm{C}_{16} \mathrm{H}_{31} \mathrm{O}$.O, and spermaceti, a solid substance found in the head of the whale, is the palmitate of cethyl, $\mathrm{C}_{16} \mathrm{H}_{33} \cdot \mathrm{C}_{16} \mathrm{H}_{31} \mathrm{O}$.O. The most important group of compound ethers are the fats and fatty oils, which are widely distributed in the vegetable, but even more so in the animal kingdom.

General properties. The ethers and compound ethers of the lower members of the monatomic alcohols and fatty acids have generally a characteristic and pleasant odor. Fruit essences consist mainly of such compound ethers, and what is generally known as the "bouquet" or "flavor" of wines and other alcoholic liquors is chiefly due to ethers or compound ethers, which are formed during (and after) the fermentation by the action of the acids present on the alcohol or the alcohols formed. The improvement which such alcoholic liquids undergo "by age" is caused by a continued chemical action between the substances named.

All ethers are neutral substances; those formed by the lower alcohols and acids are generally volatile liquids, those of the higher members are non-volatile solids. When compound ethers are heated with alkalies, the acid combines with the latter, whilst the alcohol is liberated. (The properties of the compound ethers, termed fats, will be considered further on.)

Ethyl ether, Ether $\left(\mathbf{C}_{2} \mathrm{H}_{5}\right)_{2} \mathbf{O}=\mathbf{7 4}$ (Ether, Sulphuric ether, Ethyl oxide). The name of the whole group of ethers is derived from this (ethyl-) ether, in a similar manner as common (ethyl-) alcohol has given its name to the group of alcohols. The name sulphuric ether was given at a time when its true composition was yet unknown, and for the reason that sulphuric acid was used in its manufacture.

Ether is manufactured by heating to about $140^{\circ}$ a mixture of about equal parts of alcohol and sulphuric acid in a retort, which is so arranged that additional quantities of alcohol may be allowed to flow into it, while the open end is connected with a tube, leading through a suitable cooler in order to condense the highly volatile product of the distillation. 
The action of sulphuric acid upon alcohol is not quite so simple as described above, in connection with the general methods for obtaining ethers, where the final result only was given. An intermediate product, known as ethyl-sulphuric acid or sulphovinic acid, is formed, which, by acting upon another molecule of alcohol, forms sulphuric acid and ether, which latter is volatilized as soon as formed. The decomposition is shown by the equations:

$$
\begin{aligned}
& \underset{\text { Alcohol. }}{\mathrm{C}_{2} \mathrm{H}_{5} \mathrm{HO}}+\underset{\substack{\text { Sulphuric } \\
\text { acid. }}}{\mathrm{H}_{2} \mathrm{SO}_{4}}=\underset{\substack{\text { Ethyl-sulphuric } \\
\text { acid. }}}{\mathrm{C}_{2} \mathrm{H}_{5} \mathrm{HSO}_{4}}+\underset{\text { Water. }}{\mathrm{H}_{2} \mathrm{O} .} \\
& \underset{\substack{\text { Ethyl. sulphuric } \\
\text { acid. }}}{\mathrm{C}_{2} \mathrm{H}_{5} \mathrm{HSO}_{4}}+\underset{\text { Alcohol. }}{\mathrm{C}_{2} \mathrm{H}_{5} \mathrm{HO}}=\underset{\substack{\text { Sulphuric } \\
\text { acid. }}}{\mathrm{H}_{2} \mathrm{SO}_{4}}+\underset{\text { Ether. }}{\left(\mathrm{C}_{2} \mathrm{H}_{5}\right)_{2} \mathrm{O}} .
\end{aligned}
$$

The liberated sulphuric acid at once attacks another molecule of alcohol, again forming ethyl-sulphuric acid, which is again decomposed, etc. Theoretically, a given quantity of sulphuric acid should therefore be capable of converting any quantity of alcohol into ether; practically, however, this is not the case, because secondary reactions take place sinultaneously, and because the water which is constantly formed does not all distil with the ether, and therefore dilutes the acid to such an extent that it no longer acts upon the alcohol.

Ether thus obtained is not pure, but contains water, alcohol, sulphurous and sulphuric acids, etc.; it is purified by mixing it with chloride and oxide of calcium, pouring off the clear liquid and distilling it.

Pure ether is a very mobile, colorless, highly volatile liquid, of a refreshing, characteristic odor, a burning and sweetish taste, and a neutral reaction; it is soluble in alcohol, chloroform, liquid hydrocarbons, fixed and volatile oils, and dissolves in eight volumes of water. Specific gravity at $0^{\circ}$ is 0.720 ; boiling-point $35^{\circ}$. It is easily combustible and burns with a luminous flame. When inhaled, it causes intoxication and then loss of consciousness and sensation. The great volatility and combustibility of ether necessitate special care in the handling of this substance near fire or light.

Stronger ether, Ether fortior of the U. S. P., contains about 94 per cent. of pure ether and 6 per cent. of alcohol, with a little water, whilé ether, wether of the U. S. P., contains 74 per cent. of pure ether and 26 per cent. of alcohol, with some water. 
Spiritus cetheris and Spiritus cetheris compositus are mixtures of about one part of ether and two parts of alcohol, three per cent. of certain ethereal oils being added to the second preparation.

Acetic ether, Ether aceticus, $\mathbf{C}_{2} \mathrm{H}_{5} \mathrm{C}_{2} \mathrm{H}_{3} \mathbf{O}_{2}=\mathbf{8 8}$ (Ethyl acetate). Made by mixing dried sodium acetate with alcohol and sulphuric acid, distilling and purifying the crude product by shaking with calcium chloride and rectifying:

$$
\underset{\substack{\text { Ethyl } \\ \text { alcohol. }}}{\mathrm{C}_{2} \mathrm{H}_{5} \mathrm{HO}}+\underset{\text { Sodium acetate. }}{\mathrm{NaC}_{2} \mathrm{H}_{3} \mathrm{O}_{2}}+\mathrm{H}_{2} \mathrm{SO}_{4}=\underset{\text { Acetic ether. }}{\mathrm{C}_{2} \mathrm{H}_{5} \mathrm{C}_{2} \mathrm{H}_{3} \mathrm{O}_{2}}+\underset{\substack{\text { Sodium acid } \\ \text { sulphate }}}{\mathrm{NaHSO}_{4}}
$$

It is a colorless, neutral, and mobile liquid, of a strong ethereal and somewhat acetous odor, soluble in alcohol, ether, chloroform, etc., in all proportions, and in 17 parts of water. Specific gravity 0.889 . Boiling-point $76^{\circ} \mathrm{C}$.

Ethyl nitrite, $\mathbf{C}_{2} \mathrm{H}_{5} \mathrm{NO}_{2}$ (Nitrous ether). Made by distilling a mixture of alcohol, sulphuric and nitric acids at a temperature of $80^{\circ}$.

By the deoxidizing action of alcohol on nitric acid, $\mathrm{HNO}_{3}$, the latter is converted into nitrous acid, $\mathrm{HNO}_{2}$, which in its turn acts on alcohol, the two substances combining with elimination of water, which is absorbed by the sulphuric acid:

$$
\mathrm{C}_{2} \mathrm{H}_{5} \mathrm{HO}+\mathrm{HNO}_{2}=\mathrm{C}_{2} \mathrm{H}_{5} \cdot \mathrm{NO}_{2}+\mathrm{H}_{2} \mathrm{O} \text {. }
$$

Spirit of nitrous ether, Spiritus atheris nitrosi, Sweet spirit of nitre. This is a mixture of 5 parts of the crude ethyl nitrite with 95 parts of alcohol. It is a clear, mobile, volatile, and inflammable liquid, of a pale straw color, inclining slightly to green, a fragrant, ethereal odor, and a sharp, burning taste. It slightly reddens litmus paper, but evolves no carbon dioxide with carbonate.

Amyl nitrite, Amyli nitris, $\mathrm{C}_{5} \mathrm{H}_{11} \mathrm{NO}_{2}=117$ (Nitrite of amyl). Made by distilling equal volumes of pure amyl alcohol and nitric acid from a retort until an inserted thermometer shows a temperature of $100^{\circ}$. The crude distillate is purified by agitating it with a solution of potassium carbonate and hydrate, separating the upper layer of the liquid and redistilling it; the liquid passing over between $96^{\circ}$ and $100^{\circ}$ is the amyl nitrite. 
It is a clear, pale yellowish liquid, of an ethereal, fruity odor, an aromatic taste, and a neutral or slightly acid reaction. Specific gravity 0.872 . Boiling-point $96^{\circ}$.

Fats and fat oils. All true fats are compound ethers of the triatomic alcohol glycerine, in which the three replaceable hydrogen atoms of the hydroxyl are replaced by three univalent radicals of the higher members of the fatty acids. For instance:

$$
\begin{aligned}
& \text { Glycerine }=\mathrm{C}_{3} \mathrm{H}_{5} \cdot 3(\mathrm{HO}) \text { or } \mathrm{C}_{3} \mathrm{H}_{5} \underset{-\mathrm{HO}}{\stackrel{\mathrm{HO}}{\mathrm{HO}}} \\
& \text { Stearic acid, }=\mathrm{C}_{18} \mathrm{H}_{35} \mathrm{O} . \mathrm{HO} \text { or } \mathrm{C}_{18} \mathrm{H}_{35} \mathrm{O} \text { 的. } \\
& \text { Stearine or tristearine }=\mathrm{C}_{3} \mathrm{H}_{5} \cdot 3\left(\mathrm{C}_{18} \mathrm{H}_{35} \mathrm{O}\right) \cdot \mathrm{O}_{3} \text { or } \mathrm{C}_{3} \mathrm{H}_{5} \underset{\sim}{\sim} \underset{\left(\mathrm{C}_{18} \mathrm{H}_{35} \mathrm{O}\right) . \mathrm{O}}{\left(\mathrm{C}_{18} \mathrm{H}_{35} \mathrm{O}\right) . \mathrm{O}}
\end{aligned}
$$

Whilst all natural fats are glycerine in which the three hydrogen atoms are replaced, we may by artificial means introduce but one or two acid radicals, thus forming:

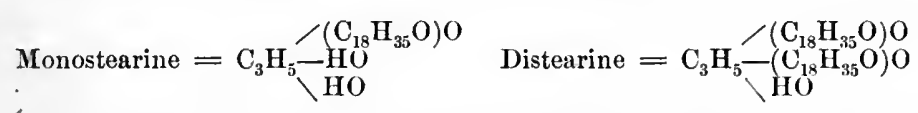

Fats are often termed glycerides; stearine being, for instance, the glyceride of stearic acid.

The principal fats consist of mixtures of palmitine, $\mathrm{C}_{3} \mathrm{H}_{5}$. $3\left(\mathrm{C}_{16} \mathrm{H}_{31} \mathrm{O}\right) . \mathrm{O}_{3}$, stearine, $\mathrm{C}_{3} \mathrm{H}_{5} 3\left(\mathrm{C}_{18} \mathrm{H}_{35} \mathrm{O}\right) . \mathrm{O}_{3}$, and oleine, $\mathrm{C}_{3} \mathrm{H}_{5}$. $3\left(\mathrm{C}_{18} \mathrm{H}_{33} \mathrm{O}\right) \cdot \mathrm{O}_{3}$. Stearine and palmitine are solids, oleine is a liquid at ordinary temperature; the relative quantity of the three fats mentioned determines its solid or liquid condition. The liquid fats, containing generally oleine as their chief constituent, are called fatty oils or fixed oils in contradistinction to volatile or essential oils.

All fats, when in a pure state, are colorless, odorless, and tasteless substances, which stain paper permanently; they are insoluble in water, difficultly soluble in cold alcohol, easily soluble in ether, bisulphide of carbon, benzene, etc. The taste and color of fats are due to foreign substances, often produced by a slight decomposition which has taken place in some of the fat. All fats are lighter than water, and all solid fats fuse below $100^{\circ}$; fats can be distilled without change at about $300^{\circ}$, but are 
decomposed at a higher temperature with the formation of numerous products, some of which have an extremely disagreeable odor, as, for instance, acroleine, $\mathrm{C}_{3} \mathrm{H}_{4} \mathrm{O}$, an aldehyde which in composition is equal to glycerine minus two molecules of water :

$$
\mathrm{C}_{3} \mathrm{H}_{5} 3 \mathrm{HO}-2 \mathrm{H}_{2} \mathrm{O}=\mathrm{C}_{3} \mathrm{H}_{4} \mathrm{O} \text {. }
$$

Some fats keep without change when pure; as they, however, generally contain impurities, such as albuminous matter, etc., they suffer decomposition (a kind of fermentation aided by oxidation), which results in a liberation of the fatty acids which impart their odor and taste to the fats, causing them to become what is generally termed rancid.

Some fats, especially some oils, suffer oxidation which renders them hard. These drying oils differ from other oils in being mixtures of oleine with another class of glycerides, containing unsaturated acids with less hydrogen in relation to carbon than oleic acid. Drying oils are prevented from drying by albuminous impurities, which may be removed by treating the oils with 4 per cent. of concentrated sulphuric acid; the acid does not act on the fat, but quickly destroys the albuminous matters, which, with the sulphuric acid, sink to the bottom, whilst the "refined" oil may be removed by decantation.

Fats are largely distributed in the animal and vegetable kingdoms. They exist in plants chiefly in the seeds, while in animals they are generally found under the skin, around the intestines, and on the muscles.

Human fat, beef tallow, mutton tallow, and lard are mixtures of palmitine and stearine with some oleine. Butter consists of the glycerides of butyric acid, caproic acid, caprylic acid, and capric acid.

The principal non-drying vegetable oils (consisting chiefly of oleine) are olive oil, cottonseed oil, cocoanut oil, palm oil, almond oil.

Among the drying oils are of importance: linseed oil, castor oil, croton oil, hemp oil, cod-liver oil.

Whenever fats are treated with alkaline hydrates, or with a number of other metallic oxides, decomposition takes place, the fatty acids combining with the metals, whilst glycerine is set free. Some of the substances thus formed are of great importance, as, for instance, the various kinds of suap. 
Soap. Any fat boiled with sodium or potassium hydrate will form soap. Soft soap is potassium soap, hard soap is sodium soap. The better kinds of hard soap are made by boiling olive oil with sodium hydrate :

$$
\begin{aligned}
& \mathrm{C}_{3} \mathrm{H}_{5} 3\left(\mathrm{C}_{18} \mathrm{H}_{33} \mathrm{O}_{2}\right)+3 \mathrm{NaHO}=3\left(\mathrm{NaC}_{18} \mathrm{H}_{33} \mathrm{O}_{2}\right)+\mathrm{C}_{3} \mathrm{H}_{5} 3 \mathrm{HO} . \\
& \text { Oleate of glyceryl Sodium Sodium oleate Glycerine. } \\
& \text { (olive oil). hydrate. (hard soap). }
\end{aligned}
$$

Soaps are soluble in water and alcohol; they contain rarely less than 30 per cent., but sometimes as much as $70-80$ per cent. of water.

Ammonia liniment, Linimentum ammonice, and lime liniment, Linimentum calcis, are obtained by mixing cottonseed oil with water of ammonia and lime-water, respectively. The oleate of ammonium or calcium is formed, and remains mixed with the liberated glycerine.

Lead plaster, Emplastrum plumbi. Chiefly lead oleate, $\mathrm{Pb}_{2} \mathrm{C}_{18} \mathrm{H}_{33} \mathrm{O}_{2}$. Obtained by boiling lead oxide with olive oil and some water for several hours, until a homogeneous mass is formed. Lead oleate differs from the oleates of the alkalies by its complete insolubility in water.

\section{Questions.}

441. Explain the constitution of simple, mixed, and compound ethers. To what inorganic compounds are they analogous?

442. State the general processes for the formation of ethers and compound ethers.

443. What is the composition of ethyl ether? Explain the process of its manufacture in words and symbols, and state its properties.

444. How is acetic ether made, and what are its properties?

445. What is sweet spirit of nitre, and how is it made?

446. State the general composition of fats.

447. What is the solubility of fats in water, alcohol, and ether; how do heat and oxygen act upon them; what is the cause of their becoming rancid?

448. Mention the chief constituents of tallow, butter, and olive oil.

449. Explain the composition and manufacture of soap, and state the difference between hard and soft soap.

450. How are ammonia liniment, lime liniment, and lead plaster made, and what is their composition? 


\section{CARBOHYDRATES.}

Constitution. The term carbohydrates or carbhydrates is not well chosen, because it implies that these substances are carbon in combination with water. Carbohydrates do contain hydrogen and oxygen in the proportion of two atoms of hydrogen to one atom of oxygen, or in the proportion to form water, but this does not exist as such in the carbohydrates.

The true atomic structure of carbohydrates is as yet but little known. The compounds of the composition $\mathrm{C}_{6} \mathrm{H}_{12} \mathrm{O}_{6}$ are now looked upon as the aldehyde of the hexatomic alcohol mannite, $\mathrm{C}_{6} \mathrm{H}_{14} \mathrm{O}_{6}$, the chief constituent of manna:

$$
\mathrm{C}_{6} \mathrm{H}_{14} \mathrm{O}_{6}-2 \mathrm{H}=\mathrm{C}_{6} \mathrm{H}_{12} \mathrm{O}_{6} .
$$

Mannite itself is formed from the saturated hydrocarbon $\mathrm{C}_{6} \mathrm{H}_{14}$, by replacement of 6 atoms of hydrogen by $6 \mathrm{HO}$; its constitutional formula is, therefore, $\left(\mathrm{C}_{6} \mathrm{H}_{8}\right)^{\text {vi }} \cdot 6(\mathrm{HO})$.

Carbohydrates generally contain 6 atoms of carbon or a multiple of 6 .

Properties. Carbohydrates are either fermentable or can be converted into substances which are capable of fermentation. They are not volatile, but suffer decomposition when sufficiently heated; they have neither acid nor basic properties, but are of a neutral reaction. Oxidizing agents convert them into saccharic and mucic acids and finally into oxalic acid. (Soluble carbohydrates have the property of bending the plane of polarized light.)

Most carbohydrates are white, solid substances, and, with the exception of a few, soluble in water. The members of the first two groups (glucoses and saccharoses) have a more or less sweet taste.

0ccurrence in nature. No other organic substances are found in such immense quantities in the vegetable kingdom as the members of this group, cellulose being a chief constituent of all, starch and various kinds of sugar of most plants. Carbohydrates are also found as products of animal life, as, for instance, the sugar in milk, in bees' honey, etc. 
Groups of carbohydrates.

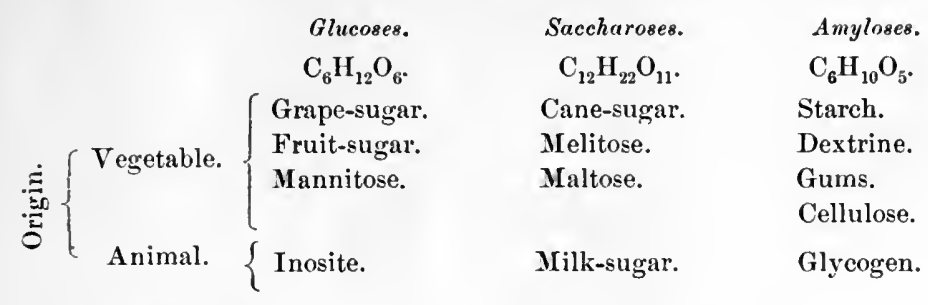

Grape-sugar, $\mathbf{C}_{6} \mathbf{H}_{12} \mathbf{0}_{6}$ (Ordinary glucose, Dextrose). This substance is very abundantly diffused throughout the vegetable kingdom, and is often accompanied by fruit-sugar. It is contained in large quantity in the juice of many fruits; the percentage of grape-sugar in the dried fig is about 65 , in grape $10-20$, in cherry 11 , in mulberry 9 , in strawberry 6 , etc.

Grape-sugar is also found in honey and in minute quantities in the normal blood ( 0.1 per cent. or less), and traces occur, perhaps, in normal urine, the quantity in both liquids rising, however, during certain diseases as high as 5 per cent.

Grape-sugar is produced in the plant from starch by the action of the vegetable acids present; it may be obtained artificially from starch (or from many other carbohydrates) by heating with diluted mineral (sulphuric) acids. Starch is also converted into glucose by the action of diastase, a substance formed during the fermentation of various seeds (grain, etc.); it is for this reason that grain, used for alcoholic liquids, is allowed to germinate, during which process diastase is formed, which, acting upon the starch, converts it into fermentable glucose.

Glucose is generally met with as a thick syrup which crystallizes with difficulty, combining during erystallization with one molecule of water. Glucose is very soluble in water and less sweet than cane-sugar; when heated to $170^{\circ}$ it loses water, and is converted into glucosan, $\mathrm{C}_{6} \mathrm{H}_{10} \mathrm{O}_{5}$; by stronger heating it loses more water and forms caramel, a mixture of various substances.

Grape-sugar combines with various metallic oxides (alkalies, alkaline earths, etc.), and also with organic acids, forming a series of compounds known as glucosides.

Grape-sugar may be recognized analytically:

1. By causing a bright red precipitate of cuprons oxide when 
boiled with a solution of cupric sulphate in sodium hydrate, to which some tartaric acid has been added.

2. By precipitating metallic silver, bismuth, and mercury, when compounds of these metals are heated with it in the presence of alkalies.

3. By easily fermenting when yeast is added to the solution, alcohol and carbon dioxide being formed :

$$
\mathrm{C}_{6} \mathrm{H}_{12} \mathrm{O}_{6}=2 \mathrm{C}_{2} \mathrm{H}_{5} \mathrm{HO}+2 \mathrm{CO}_{2} \text {. }
$$

Fruit-sugar, $\mathbf{C}_{6} \mathbf{H}_{12} \mathbf{0}_{6}$ (Levulose). Occurs with glucose in sweet fruits and honey; it resembles glucose in most chemical and physical properties, but does not crystallize from an aqueous solution; it may, however, be obtained in white, silky needles from an alcoholic solution.

Mannitose, $\mathrm{C}_{6} \mathrm{H}_{12} \mathrm{O}_{6}$. Obtained by the oxidation of mannite; it does not crystallize, and resembles grape-sugar.

Inosite, $\mathbf{C}_{6} \mathrm{H}_{12} \mathbf{0}_{6}$ (Muscle-sugar). Occurs in various muscular tissues, in the lungs, kidneys, liver, spleen, brain, and blood. Although identical in composition with grape-sugar, inosite differs from the latter in not being fermentable and by not precipitating cuprous oxide from alkaline copper-solutions.

Cane-sugar, Saccharum, $\mathbf{C}_{12} \mathbf{H}_{22} \mathbf{0}_{11}=342$ (Ordinary saccharose, Common sugar, Beet-sugar). Cane-sugar is found in the juices of many plants, especially in that of the different grasses (sugarcane), and also in the sap of several forest trees (maple), in the roots, stems, and other parts of various plants (sugar-beet), etc. All plants containing cane-sugar do not contain free organic acids, which latter would convert it into grape-sugar.

Cane-sugar is manufactured from various plants containing it by crushing them between rollers, expressing the juice, heating and adding to it milk of lime, which precipitates vegetable albuminous matter. The clear liquid is evaporated to the consistence of a syrup, which is further purified (refined) by filtering it through bone-black and evaporating the solution in "vacuum pans" to the crystallizing point.

Cane-sugar forms white, hard, distinctly crystalline granules; it dissolves in 0.2 part of boiling, in 0.5 part of cold water, 
and in 175 parts of alcohol; when heated to $160^{\circ}$ it fuses, and the liquid, on cooling, forms an amorphous, transparent mass, known as barley-sugar; at a higher temperature cane-sugar is decomposed, water is evolved, and a brown, almost tasteless substance is formed, which is known as caramel or burnt sugar. Oxidizing agents act energetically upon cane-sugar, which is a strong reducing agent. A mixture of cane-sugar and potassium chlorate will deflagrate when moistened with sulphuric acid; potassium permanganate is readily deoxidized in acid solution; cane-sugar, however, does not affect an alkaline copper-solution.

Melitose, $\mathrm{C}_{12} \mathrm{H}_{22} \mathrm{O}_{11}$, is the chief constituent of Australian manna.

Maltose, $\mathrm{C}_{12} \mathrm{H}_{22} \mathrm{O}_{11}$, is a sugar resembling cane-sugar in some, glucose in other properties; it is formed along with dextrine during the conversion of starch into sugar.

Milk-sugar, Saccharum lactis, $\mathbf{C}_{12} \mathbf{H}_{22} \mathbf{0}_{11}+\mathbf{H}_{2} \mathbf{O}=\mathbf{3 6 0}$ (Lactose). Found almost exclusively in the milk of the mammalia. Obtained by freeing milk of casein and fat and evaporating the remaining liquid (whey) to a small bulk, when the milk-sugar crystallizes on cooling.

It forms white, hard, crystalline masses; it is soluble in 7 parts of water (at $15^{\circ}$ ) and in one part of boiling water, insoluble in alcohol and ether; it is much harder than cane-sugar, and but faintly sweet; it is not easily brought into alcoholic fermentation by the action of yeast, but easily undergoes "lactic fermentation," when cheese is added. During this process milk-sugar is converted into lactic acid.

Milk-sugar resembles grape-sugar in its action on alkaline solution of copper, from which it precipitates cuprous oxide.

Starch, Amylum, $\mathbf{C}_{6} \mathbf{H}_{10} \mathbf{O}_{5}=162$. Starch is very widely distributed in the vegetable kingdom, and found chiefly in the seed of cereais and leguminosæ, but also in the roots, stems, and seeds of nearly all plants.

It is prepared from wheat, potatoes, rice, beans, sago, arrowroot, etc., by a mechanical operation. The vegetable matter containing the starch is comminuted by rasping or grinding in order to open the cells in which it is deposited, and then steeped in water; the softened mass is then rubbed on a sieve under a 
current of water which washes out the starch while cellular fibrous matter remains on the sieve; the starch slowly deposits from the washings, and is further purified by treating it with water.

Starch forms white, amorphous, tasteless masses, which are peculiarly slippery to the touch, and easily converted into a powder; it is insoluble in cold water, alcohol, and ether; when boiled with water, it yields a white jelly (mucilage of starch) which cannot be looked upon as a true solution, but is a suspension of the swollen starch particles in water; by continued boiling with much water some starch passes into solution.

Starch, when examined under the microscope, is seen to consist of granules differing in size, shape, and appearance, according to the plant from which the starch was obtained. Concentric layers, which are more or less characteristic of starchgranules, show that they are formed in the plant by a gradual deposition of starch matter.

The most characteristic test for starch is the dark-blue color which iodine imparts to it (or better to the mucilage). This color is due to the formation of iodized starch, Amylum iodatum, U. S. P., an unstable dark-blue compound of the doubtful composition $\mathrm{C}_{6} \mathrm{H}_{9} \mathrm{IO}_{5} \mathrm{I}$.

Starch is an important article of food, especially when associated, as in ordinary flour, with albuminous substances.

Dextrine, $\mathbf{C}_{6} \mathbf{H}_{10} \mathbf{O}_{5}$ (British gum). Obtained by boiling starch with diluted acids, or by subjecting starch to a dry heat of $175^{\circ}$, or by the action of diastase (infusion of malt) upon hydrated starch.

Dextrine is a colorless or slightly yellowish, amorphous powder, resembling gum-arabic in some respects; it is soluble in water, reduces alkaline copper solutions, and is colored light wine-red by iodine.

Gums. These are amorphous substances of vegetable origin, soluble in water or swelling up in it, forming thick, sticky masses; they are insoluble in alcohol, and are converted into glucose by boiling with diluted sulphuric acid.

Gum-arabic consists chiefly of the calcium salt of arabic acid, 
$\mathrm{C}_{6} \mathrm{H}_{10} \mathrm{O}_{5} \cdot \mathrm{H}_{2} \mathrm{O}$. Other gums oceur in the cherry tree, in linseed or flaxseed, in Irish moss, in marsh-mallow root, etc.

Cellulose, $\mathbf{C}_{6} \mathbf{H}_{10} \mathbf{0}_{5}$, perhaps $\mathbf{C}_{18} \mathbf{H}_{30} \mathbf{0}_{15}$ (Plant-fibre, Lignine). Cellulose constitutes the fundamental material of which all cells and vessels of vegetables are built up, and forms, therefore, the largest portion of the solid parts of every plant; it is well adapted for this purpose on account of its insolubility in water and most other solvents, its resistance to either alkaline or acid liquids, and its tough and flexible nature. Some parts of vegetables (cotton, for instance) are nearly pure cellulose.

Pure cellulose is a white, translucent mass, insoluble in all the common solvents, but soluble in an ammoniacal solution of basic cupric carbonate; it is not colored blue by iodine.

Treated with concentrated sulphuric acid it swells up, and gradually dissolves; water precipitates from such solutions a substance known as amyloid, which is an altered cellulose giving a blue color with iodine.

Unsized paper (which is chiefly cellulose) dipped into a mixture of two volumes of sulphuric acid and one volume of water, forms, after being washed and dried, the so-called "parchment paper," which possesses all the valuable properties of parchment.

Pyroxyline, Pyroxylinum, $\mathbf{C}_{6} \mathrm{H}_{8}\left(\mathbf{N O}_{2}\right)_{2} \mathbf{0}_{5}$ (Dinitro-cellulose, Soluble gun-cotton). By the action of nitric acid of various strengths on cellulose, three different substitution products may be obtained, which are distinguished as mono-, di-, and trinitro-cellulose:

$$
\begin{aligned}
& \mathrm{C}_{6} \mathrm{H}_{10} \mathrm{O}_{5}+\mathrm{HNO}_{3}=\mathrm{C}_{6} \mathrm{H}_{9}\left(\mathrm{NO}_{2}\right) \mathrm{O}_{5}+\mathrm{H}_{2} \mathrm{O} . \\
& \mathrm{C}_{6} \mathrm{H}_{10} \mathrm{O}_{5}+2 \mathrm{HNO}_{3}=\mathrm{C}_{6} \mathrm{H}_{8}\left(\mathrm{NO}_{2} \mathrm{O}_{2} \mathrm{O}_{5}+2 \mathrm{H}_{2} \mathrm{O} .\right. \\
& \mathrm{C}_{6} \mathrm{H}_{10} \mathrm{O}_{5}+3 \mathrm{HNO}_{3}=\mathrm{C}_{6} \mathrm{H}_{7}\left(\mathrm{NO}_{2}\right)_{3} \mathrm{O}_{5}+3 \mathrm{H}_{2} \mathrm{O} .
\end{aligned}
$$

The trinitro-cellulose is the highly explosive gun-cotton; the dinitro-cellulose or pyroxyline is soluble in a mixture of ether and alcohol; this solution is known as collodion.

Pyroxyline is best made by immersing cotton for 10 hours in a cooled mixture of 6 parts of sulphuric acid and 5 parts of nitric acid, and washing it with cold water until the washings no longer show an acid reaction. Neither the mono- nor trinitro-cellulose is soluble in a mixture of ether and alcohol. 
Glycogen, $\mathbf{C}_{6} \mathbf{H}_{10} \mathbf{0}_{5}$. Found exclusively in animals; it occurs in the liver, the white blood-corpuscles, in many embryonic tissues, and in muscular tissue. Pure glycogen is a white, starch-like, amorphous substance, soluble in water, insoluble in alcohol; by the action of diluted acids it is converted into glucose.

Glucosides. This term is applied to a group of substances (chiefly of vegetable origin), which by the action of acids, alkalies, or ferments sufter decomposition in such a manner that one. of the products formed is grape-sugar. Glucosides may, therefore, be looked upon as compound sugars, or sugar in combination with various other substances. The following is a list of the more important glucosides, giving also the composition and the source whence they are obtained:

\begin{tabular}{|c|c|c|}
\hline $\begin{array}{l}\text { Amygdalin, } \\
\text { Arbutin, } \\
\text { Cathartic acid, } \\
\text { Carminic acid, } \\
\text { Colocynthin, } \\
\text { Digitalin, } \\
\text { Elaterin, } \\
\text { Gentiopicrin, } \\
\text { Glycyrrhizin, } \\
\text { Helleborin, } \\
\text { Indican, } \\
\text { Jalapin, } \\
\text { Myronic acid, } \\
\text { Picrotoxin, } \\
\text { Salicin, } \\
\text { Santonin, } \\
\text { Scammonin, } \\
\text { Solanin, } \\
\text { Tannins, }\end{array}$ & $\begin{array}{l}\mathrm{C}_{20} \mathrm{H}_{27} \mathrm{NO}_{11}, \\
\mathrm{C}_{25} \mathrm{H}_{34} \mathrm{O}_{14}, \\
\mathrm{C}_{180} \mathrm{H}_{192} \mathrm{~N}_{4} \mathrm{SO}_{82} ? \\
? \\
\mathrm{C}_{58} \mathrm{H}_{84} \mathrm{O}_{23} ? \\
\mathrm{C}_{27} \mathrm{H}_{45} \mathrm{O}_{15} ? \\
\mathrm{C}_{26} \mathrm{H}_{28} \mathrm{O}_{5}, \\
\mathrm{C}_{20} \mathrm{H}_{30} \mathrm{O}_{12}, \\
\mathrm{C}_{24} \mathrm{H}_{36} \mathrm{O}_{9}, \\
\mathrm{C}_{36} \mathrm{H}_{42} \mathrm{O}_{6}, \\
? \\
\mathrm{C}_{31} \mathrm{H}_{50} \mathrm{O}_{16}, \\
\mathrm{C}_{10} \mathrm{H}_{19} \mathrm{NS}_{2} \mathrm{O}_{10}, \\
\mathrm{C}_{9} \mathrm{H}_{10} \mathrm{O}_{4}, \\
\mathrm{C}_{13} \mathrm{H}_{18} \mathrm{O}_{7}, \\
\mathrm{C}_{15} \mathrm{H}_{18} \mathrm{O}_{3}, \\
\mathrm{C}_{34} \mathrm{H}_{56} \mathrm{O}_{16}, \\
?\end{array}$ & $\begin{array}{l}\text { Bitter almonds, etc. } \\
\text { Arbutus uva ursa } \\
\text { Senna. } \\
\text { Cochineal. } \\
\text { Colocynthis. } \\
\text { Digitalis. } \\
\text { Cucumber fruit. } \\
\text { Root of gentiana. } \\
\text { Liquorice root. } \\
\text { Root of hellebore. } \\
\text { Indigo plant. } \\
\text { Jalap resin. } \\
\text { Seeds of black mustard. } \\
\text { Cocculus indicus. } \\
\text { Bark of willow. } \\
\text { Wormseed. } \\
\text { Resin scammony. }\end{array}$ \\
\hline
\end{tabular}

Some of the above glucosides, such as amygdalin, salicin, tannin, have been mentioned before.

Santonin, Santoninum, $\mathbf{C}_{15} \mathbf{H}_{81} \mathbf{O}_{3}=246$. Santonin is the active principle of wormseed, the unexpanded flower-heads of Artemisia. It crystallizes in colorless prisms, which turn yellow on exposure to light; it is but sparingly soluble in water, more soluble in alcohol and ether; it has distinct acid properties, combining with strong bases, as, for instance, with sodium hydrate, forming the officinal santoninate of sodium, $2\left(\mathrm{NaC}_{15} \mathrm{H}_{19} \mathrm{O}_{4}\right) \cdot 7 \mathrm{H}_{2} \mathrm{O}$. 
Santonin solutions give a white precipitate with silver, zinc, and mercurous salts; with an alcoholic solution of potassium hydrate they yield a scarlet red liquid, which gradually becomes colorless. Santonin taken internally confers upon the urine a dark color resembling the color of urine containing bile; upon heating such urine it turns cherry-red or crimson, the color disappearing on the addition of an acid, and reappearing on neutralization.

\section{Questions.}

451. To which group of substances is the term "carbohydrates" applied?

452. State the general properties of carbohydrates.

453. Mention the three groups of carbohydrates, and the composition and characteristics of the members of each group.

454. Mention some fruits in which grape-sugar, and some plants in which cane-sugar is found.

455. What is the difference between grape-sugar and cane-sugar, and by what tests can they be distinguished?

456. From what source, and by what process is milk-sugar obtained?

457. What is starch, what are its properties, by what tests can it be recognized, and what substance is formed when diastase or diluted acids act upon it?

458. Where is cellulose found in nature, and what are its properties?

459. What three compounds may be obtained by the action of nitric acid upon cellulose, and what are they used for?

460. Which substances are termed glucosides? Mention some of the more important glucosides.

\section{AMINES AND AMIDES. ORGANIC BASES. ALKALOIDS.}

Forms of nitrogen in organic compounds. Nitrogen may be present in organic compounds in three forms, viz., ammonia, cyanogen, nitric acid, or derivatives of these compounds. Substances containing nitrogen in the nitric acid form may either be organic salts of this acid (nitrates), or may have been formed by replacement of hydrogen atoms by the nitric acid radical $\mathrm{NO}_{2}$. These latter compounds, termed nitro-compounds, such as nitro-cellulose, nitro-benzene, nitro-glycerine, etc., do not occur in nature, but are obtained exclusively by artificial means, 
generally by treatment of the organic substance with concentrated nitric acid; all these nitro-compounds are more or less explosive.

Cyanogen compounds contain nitrogen in the form of cyanogen, $\mathrm{CN}$, a radical the compounds of which will be considered hereafter.

Organic compounds containing nitrogen in the ammonia form are known as amines or amides, organic bases or alkaloids. (Albuminous substances also contain nitrogen in the ammonia form.)

Amines. Whenever the hydrogen of ammonia is replaced by alcoholic radicals (or hydrocarbon residues) compounds are formed which are termed amines. For instance :

\begin{tabular}{|c|c|c|c|c|}
\hline $\mathrm{Or}$ & $\underset{\mathrm{N}-\mathrm{H}}{\angle \mathrm{C}_{2} \mathrm{H}_{5}}$ & 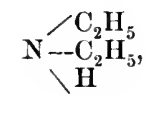 & $\stackrel{\mathrm{N}-\mathrm{C}_{2} \mathrm{H}_{5}}{-\mathrm{C}_{2} \mathrm{H}_{5},}$ & $\begin{array}{c}\angle \mathrm{CH}_{3} \\
\mathrm{~N}-\mathrm{C}_{2} \mathrm{H}_{5}, \\
\underset{\mathrm{C}_{4} \mathrm{H}_{9}}{ },\end{array}$ \\
\hline $\begin{array}{c}\mathrm{NH}_{3} \text {, } \\
\text { Ammonia. }\end{array}$ & $\begin{array}{c}\mathrm{N}\left(\mathrm{C}_{2} \mathrm{H}_{5}\right) \mathrm{H}_{2}, \\
\text { Ethylamine. }\end{array}$ & $\begin{array}{l}\mathrm{N}\left(\mathrm{C}_{2} \mathrm{H}_{5}\right)_{2} \mathrm{H}, \\
\text { Diethylamine. }\end{array}$ & $\begin{array}{c}\mathrm{N}\left(\mathrm{C}_{2} \mathrm{H}_{5}\right)_{3}, \\
\text { Triethylamine. }\end{array}$ & $\begin{array}{r}\mathrm{NCH}_{3} \cdot \mathrm{C}_{2} \mathrm{H} \\
\text { Methyl-ethyl-b }\end{array}$ \\
\hline
\end{tabular}

Amines resemble ammonia in their chemical properties; they are, like ammonia, basic substances; they combine with acids directly and without elimination of water, thus:

$$
\underset{\substack{\mathrm{NH}_{4}+\mathrm{HCl} \\ \text { Triethylamine. }}}{\mathrm{N}\left(\mathrm{C}_{2} \mathrm{H}_{5}\right)_{3}+\mathrm{HCl}}=\underset{\substack{\text { Triethylammonium } \\ \text { chloride. }}}{\mathrm{NH}(\mathrm{CH}) \mathrm{HCl}}
$$

Amides are substances derived from ammonia by replacement of hydrogen atoms by acid radicals. Thus:

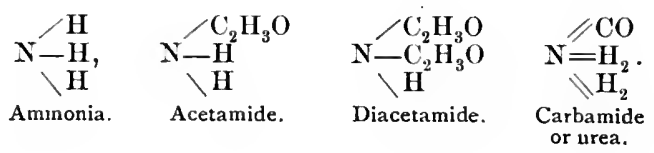

Amides also resemble ammonia in their chemical properties; to a less extent, however, than amines, because the acid radicals have a tendency to neutralize the basic properties of ammonia. 
Formation of amines and amides. Amines and amides may be formed by the action of ammonia upon the chloride or iodide of an alcohol or acid radical:

$$
\begin{aligned}
& \underset{\text { Ethyl iodide. }}{\mathrm{C}_{2} \mathrm{H}_{5} \cdot \mathrm{I}}+\underset{\text { Ammonia. }}{\mathrm{NH}_{3}}=\underset{\substack{\text { Hydriodic } \\
\text { acid. }}}{\mathrm{HI}}+\underset{\substack{\mathrm{NH}_{2} \\
\mathrm{NH}_{2} \cdot \mathrm{C}_{2} \mathrm{H}_{5}}}{\mathrm{H}_{3}} . \\
& \underset{\begin{array}{c}
\text { Acetyl } \\
\text { chloride. }
\end{array}}{\mathrm{C}_{2} \mathrm{H}_{3} \mathrm{O} . \mathrm{Cl}}+\underset{\text { Ammonia. }}{2 \mathrm{NH}_{3}}=\underset{\begin{array}{c}
\text { Ammonium } \\
\text { chloride. }
\end{array}}{\mathrm{NH}_{4} \mathrm{Cl}}+\underset{\text { Acetamide. }}{\mathrm{NH}_{2} \cdot \mathrm{C}_{2} \mathrm{H}_{3} \mathrm{O}} .
\end{aligned}
$$

Amines may also be formed by the action of nascent hydrogen upon the cyanides of the alcoholic radicals:

$$
\underset{\text { Methyl cyanide. }}{\mathrm{CH}_{3} \mathrm{CN}}+4 \mathrm{H}=\underset{\text { Ethylamine. }}{\mathrm{NH}_{2} \cdot \mathrm{C}_{2} \mathrm{H}_{5}} .
$$

Aniline, Phenylamine, $\mathbf{N H}_{2} \cdot \mathbf{C}_{6} \mathrm{H}_{5}$. Amines may in some cases be formed by the action of nascent hydrogen upon nitro-compounds; the manufacture of aniline depends on this decomposition :

$$
\underset{\text { Nitro-benzene. }}{\mathrm{C}_{6} \mathrm{H}_{5} \mathrm{NO}_{2}}+\underset{\text { Hydrogen. }}{6 \mathrm{H}}=\underset{\text { Water. }}{2 \mathrm{H}_{2} \mathrm{O}}+\underset{\text { Phenylamine. }}{\mathrm{NH}_{2} \cdot \mathrm{C}_{6} \mathrm{H}_{5}} .
$$

Pure aniline is a colorless, neutral liquid, having a peculiar, aromatic odor. Oxidizing agents, such as arsenious and arsenic oxide, chromic oxide, chlorides, nitrates, etc., convert it into blue, red, violet, green, or black derivatives; the substances thus formed are the various aniline colors, which are now manufactured on a large scale.

Occurrence of organic bases in nature. (Alkaloids.) The various organic basic substances found in nature are either amines (compounds containing carbon, hydrogen, and nitrogen only), or amides (compounds containing, besides the three elements named, also oxygen). But a small number of organic bases is found in the animal system, urea being the most important one. In plants organic bases are more frequently met with, and are grouped together under the name of alkaloids, this term signifying alkali-like in allusion to the alkaline or basic properties of these substances.

Alkaloids are found in the leaves, stems, roots, barks, and seeds of various plants; it often happens that a certain alkaloid 
is found in the different species of one family, and it is also often the case that various alkaloids of a similar composition are found in the same plant.

\section{General properties of alkaloids.}

1. They combine with acids (without elimination of water) to form well-defined salts, and are set free from the solutions of these salts by alkalies and alkaline carbonates.

2. Those containing no oxygen (amines) are volatile liquids, those containing oxygen (amides) are non-volatile solids.

3. The volatile alkaloids have a peculiar, disagreeable odor reminding of ammonia, the non-volatile alkaloids are odorless.

4. Most solid alkaloids fuse at a temperature above $100^{\circ}$ without decomposition, but are decomposed when the heat is raised much beyond the fusing-point.

5. Alkaloids are insoluble, or nearly so, in water, but soluble in alcohol, chloroform, benzene, and many also in ether.

6. The chlorides, sulphates, nitrates, acetates (and most other salts) of alkaloids are either soluble in water, or in water which has been slightly acidulated, and also in alcohol; but they are insoluble, or nearly so, in ether, chloroform, benzene, benzin, and amyl alcohol.

7. The solid alkaloids, as well as their salts, may be obtained in a crystallized state.

8. Most alkaloids are white.

9. Most alkaloids have a very strong, generally bitter taste.

10. Most alkaloids act very energetically upon the animal system.

11. Most alkaloids give precipitates with tannic acid, picric acid, phospho-molybdic acid, potassium-mercuric iodide, and the higher chlorides of platinum, gold, and mercury.

12. Most alkaloids give beautiful color reactions when treated with oxidizing agents, such as nitric acid, chloric acid, chromic acid, ferric chloride, chlorine water, etc.

General mode of obtaining alkaloids. The disintegrated vegetable substance (bark, seeds, etc.) is extracted with acidified water, which dissolves the alkaloids. When the alkaloid is volatile, it is obtained from this solution by distillation, after having been liberated by an alkali. 
Non-volatile alkaloids are precipitated from the acid solution by the addition of an alkali, and the impure alkaloid thus obtained is purified by again dissolving in an acid and reprecipitating, or by dissolving in alcohol and evaporating the solution.

Antidotes. In cases of poisoning by alkaloids the stomachpump and emetics (zinc sulphate) should be applied as soon as possible; astringent liquids may be given, because tannic acid forms insoluble compounds with most of the alkaloids. In some cases suecial physiological antidotes exist, and should be used. .

Detection of alkaloids in cases of poisoning. The separation and detection of poisonous alkaloids in organic matter (food, contents of stomach, etc.), especially when present, as is generally the case, in very small quantities, is one of the most difficult tasks of the toxicologist, and none but an expert, who has made himself thoroughly familiar with the methods of discovering minute quantities of organic poisons in the animal system, should undertake to make such an analysis in case legal proceedings depend on the result of the chemist's report.

Of the various methods applied for the separation of alkaloids from organic matter, the following may be mentioned.

The substance to be examined is properly comminuted (if this be necessary) and repeatedly digested at $40^{\circ}$ to $50^{\circ}$ with water, slightly acidulated with sulphuric acid. The filtered liquids (containing the sulphates of the alkaloids) are evaporated over a water-bath to a thin syrup, which is mixed with three or four times its own volume of alcohol; this mixture is digested at about $35^{\circ}$ for several hours, cooled, filtered, and again evaporated nearly to dryness. (By this treatment with alcohol many substances soluble in the acidified water, but insoluble in diluted alcohol, are eliminated and left on the filter, whilst the alkaloids remain in solution as sulphates.)

A little water is now added to the residue and the liquid supersaturated with sodium carbonate, which decomposes the salts of the alkaloids, liberating the latter. The mixture is now well shaken with five times its volume of pure ether, which dissolves the poisonous alkaloids, except morphine and strych- 
nine, which are almost insoluble in ether. The ethereal liquid, which forms a distinct layer over the aqueous solution, is removed and allowed to evaporate in watch-glasses; the slight residues left are used for the various tests characteristic of the different alkaloids.

The aqueous alkaline solution separated from the ethereal liquid is well shaken with chloroform, which dissolves strychnine; after having subsided, the chloroform is removed by a pipette and evaporated to dryness over a water-bath; if the residue upon being slightly heated with a drop of sulphuric acid does not darken, the tests for strychnine may be applied; if, however, a brown or dark color is produced, the residue has to be further purified by heating with a few drops of sulphuric acid, which destroys most organic matter, but does not affect strychnine, which is extracted from the charred material by slightly acidulated water.

The aqueous alkaline solution (previously treated with ether and chloroform) is evaporated to a small bulk over a water-bath and exhausted with alcohol, which dissolves morphine; the alcoholic solution may leave the morphine sufficiently pure for further examination.

It is not claimed for the above method of detecting the alkaloids in presence of much organic matter, that it is the best one, but I have found that students of even limited experience in analytical operations obtained better results by using this than any other method. The practical toxicologist has in most cases of poisoning some data (deducted from the symptoms before death, or from the results of the post-mortem examination) pointing to a certain poison, which, of course, facilitate his work considerably.

\section{Important alkaloids.}

a. Liquid and volatile alkaloids.

Coniine, $\quad \mathrm{C}_{8} \mathrm{H}_{15} \mathrm{~N}, \quad$ Conium maculatum.

Nicotine, $\mathrm{C}_{10} \mathrm{H}_{14} \mathrm{~N}_{2}$, Tobacco plant.

b. Solid and fixed alkaloids.

\begin{tabular}{llcc|} 
Morphine, & $\mathrm{C}_{17} \mathrm{H}_{19} \mathrm{NO}_{3}$, & 10.00 per cent. \\
Codeine, & $\mathrm{C}_{18} \mathrm{H}_{21} \mathrm{NO}_{3}$, & 0.25 " \\
Thebaine, & $\mathrm{C}_{19} \mathrm{H}_{21} \mathrm{NO}_{3}$, & 0.15 " \\
Papaverine, & $\mathrm{C}_{21} \mathrm{H}_{21} \mathrm{NO}_{4}$ & 1.00 " & \multicolumn{2}{|c}{ In opium. } \\
The percentages \\
Narcotine, & $\mathrm{C}_{22} \mathrm{H}_{23} \mathrm{NO}_{7}$ & 1.30 & given are an aver- \\
Narceine, & $\mathrm{C}_{25} \mathrm{H}_{29} \mathrm{NO}_{9}$ & 0.70 & age, but vary \\
widely.
\end{tabular}




\begin{tabular}{|c|c|c|}
\hline $\begin{array}{l}\text { Quinine, } \\
\text { Cinchonine, } \\
\text { Quinidine, } \\
\text { Cinchonidine, } \\
\text { Strychnine, } \\
\text { Brucine, } \\
\text { Solanine, } \\
\text { Atropine, } \\
\text { Hyoscyamine, } \\
\text { Veratrine, } \\
\text { Aconitine, } \\
\text { Colchicine, } \\
\text { Berberine, } \\
\text { Piperine, } \\
\text { Emetine, } \\
\text { Sinapine, } \\
\text { Physostigmine, } \\
\text { Pilocarpine, } \\
\text { Caffeine, } \\
\text { Theobromine, }\end{array}$ & $\begin{array}{l}\mathrm{C}_{20} \mathrm{H}_{24} \mathrm{~N}_{2} \mathrm{O}_{2}+3 \mathrm{H}_{2} \mathrm{O}, \\
\mathrm{C}_{19} \mathrm{H}_{22} \mathrm{~N}_{2} \mathrm{O} \text {. } \\
\text { isomere to quinine, } \\
\text { isomere to cinchonine, } \\
\mathrm{C}_{21} \mathrm{H}_{22} \mathrm{~N}_{2} \mathrm{O}_{2}, \\
\mathrm{C}_{23} \mathrm{H}_{26} \mathrm{~N}_{2} \mathrm{O}_{4}+4 \mathrm{H}_{2} \mathrm{O}, \\
\mathrm{C}_{43} \mathrm{H}_{71} \mathrm{NO}_{16}, \\
\mathrm{C}_{17} \mathrm{H}_{23} \mathrm{NO}_{3}, \\
\mathrm{C}_{15} \mathrm{H}_{23} \mathrm{NO}_{3}, \\
\mathrm{C}_{32} \mathrm{H}_{50} \mathrm{NO}_{9} \text { ? } \\
\mathrm{C}_{30} \mathrm{H}_{47} \mathrm{NO}_{7} \text { ? } \\
\mathrm{C}_{17} \mathrm{H}_{23} \mathrm{NO}_{6}, \\
\mathrm{C}_{20} \mathrm{H}_{17} \mathrm{NO}_{4}, \\
\mathrm{C}_{17} \mathrm{H}_{19} \mathrm{NO}_{3}, \\
\mathrm{C}_{30} \mathrm{H}_{46} \mathrm{~N}_{2} \mathrm{O}_{7}, \\
\mathrm{C}_{16} \mathrm{H}_{23} \mathrm{NO}_{5}, \\
\mathrm{C}_{30} \mathrm{H}_{21} \mathrm{~N}_{3} \mathrm{O}_{4}, \\
\mathrm{C}_{11} \mathrm{H}_{16} \mathrm{~N}_{2} \mathrm{O}_{2}, \\
\mathrm{C}_{8} \mathrm{H}_{10} \mathrm{~N}_{4} \mathrm{O}_{2}+\mathrm{H}_{2} \mathrm{O}, \\
\mathrm{C}_{7} \mathrm{H}_{8} \mathrm{~N}_{4} \mathrm{O}_{2},\end{array}$ & $\begin{array}{l}\text { In solanaceæ. } \\
\text { Veratrum album and viride. } \\
\text { Aconitum napellas. } \\
\text { Colchicum autumnale. } \\
\text { Berberis vulgaris. } \\
\text { Pepper. } \\
\text { Ipecacuanha root. } \\
\text { White mustard seed. } \\
\text { Calabar bean. } \\
\text { Pilocarpus. } \\
\text { Coffee, tea. } \\
\text { Seeds of theobroma cacao. }\end{array}$ \\
\hline
\end{tabular}

Coniine, $\mathrm{C}_{8} \mathrm{H}_{15} \mathrm{~N}$. Occurs in conium maculatum (hemlock), accompanied by two other alkaloids. It is a colorless, oily liquid, having a disagreeable, penetrating odor.

Nicotine, $\mathrm{C}_{10} \mathrm{H}_{14} \mathrm{~N}_{2}$. Tobacco leaves contain from 2 to 8 per cent. of nicotine, which is a colorless, oily liquid, having a caustic taste and a disagreeable, penetrating odor. It gives with hydrochloric acid a violet, with nitric acid an orange color.

Opium is the concrete, milky exudation obtained, in Asia Minor, by incising the unripe capsules of papaver somniferum, poppy. Chemically, opium is a mixture of a large number of substances containing besides gum, albumen, wax, volatile and coloring matter, meconic acid, meconin, etc.,-not less than $\mathbf{1 6}$ or 18 different alkaloids, many of which are, however, present in minute quantities.

Ordinary opium should contain not less than 9 per cent., and when dried at $85^{\circ}$ from 12 to 16 per cent. of morphine.

Dried and powdered opium, after having been exhausted with ten times its weight of stronger ether (which dissolves chiefly the narcotine, but not the morphine salts), the ethereal solution 
filtered off, and the weight of the opium restored by sugar of milk, forms the denarcotized opium of the U. S. P.

Quantitative estimation of morphine in opium. The U.S.P. proposes the following method for the determination of morphine in opium.

Seven grams of opium are triturated with 3 grams of calcium hydrate and 20 c.c. of water, until a uniform mixture results, to which 50 c.c. of water are added. The mixture is filtered, and to 50 c.c. of the filtrate (representing 5 grams of opiurn) are added 5 c.c. of alcohol and 25 c.c. of stronger ether. After the mixture is well shaken, 3 grams of ammonium chloride are added, again shaken, and then set aside for 12 hours, when morphine separates in small crystals, which are collected upon a tilter, dried at $60^{\circ}$, and weighed.

The explanation of the above process is as follows: Calcium hydrate liberates the alkaloids, most of which are precipitated, while morphine is redissolved by the excess of calcium hydrate; by the addition of ammonium chloride, calcium chloride and ammonia are formed, which latter causes the dissolved alkaloids to be precipitated; ether is added in order to dissolve and eliminate traces of other alkaloids, especially narcotine, which it dissolves, whilst it does not act as a solvent upon the morphine.

Morphine, Morphina, $\mathbf{C}_{17} \mathrm{H}_{19} \mathrm{NO}_{3} \cdot \mathrm{H}_{2} \mathbf{O}=\mathbf{3 0 3}$ (Morphia). A white, crystalline powder, or colorless, shining, prismatic crystals, odorless, of a bitter taste, and an alkaline reaction; almust insoluble in ether and chloroform, very slightly soluble in cold water, soluble in 100 parts of cold and 36 parts of boiling alcohol; it fuses at $120^{\circ}$, losing its water; heated with excess of hydrochloric acid for some hours, under pressure, to $150^{\circ}$, it loses water, and is converted into apomorphine, $\mathrm{C}_{17} \mathrm{H}_{17} \mathrm{NO}_{2}$, a crystalline, solid alkaloid, valuable as an emetic. The hydrochlorate of apomorphine, $\mathrm{C}_{17} \mathrm{H}_{17} \mathrm{NO}_{2} \cdot \mathrm{HCl}$, is officinal.

The above-mentioned process for the quantitative estimation of morphine in opium may be used for its manufacture, or it may be made by adding to infusion of opium an equal bulk of alcohol, then slight excess of ammonia, and setting aside for crystallization. The crude morphine thus obtained is purified by crystallization. 
Morphine combines with acids, and of the salts are officinal :

Acetate of morphine, morphinæ acetas, $\mathrm{C}_{17} \mathrm{H}_{19} \mathrm{NO}_{3} \cdot \mathrm{HC}_{2} \mathrm{H}_{3} \mathrm{O}_{2} \cdot 3 \mathrm{H}_{2} \mathrm{O}$.

Hydrochlorate of morphine, morphinæ hydrochloras, $\mathrm{C}_{17} \mathrm{H}_{19} \mathrm{NO}_{3} . \mathrm{HCl} .3 \mathrm{H}_{2} \mathrm{O}$.

Sulphate of morphine, morphinæ sulphas, $\left(\mathrm{C}_{17} \mathrm{H}_{19} \mathrm{NO}_{3}\right)_{2} \mathrm{H}_{2} \mathrm{SO}_{4} .5 \mathrm{H}_{2} \mathrm{O}$.

The above three salts are white and soluble in water.

\section{Analytical reactions for morphine.}

1. Nitric acid first reddens morphine, and then renders it yellow.

2. Strong neutral solution of ferric chloride causes a blue color with morphine or with neutral solutions of morphine salts; the color is changed to green by an excess of the reagent, and is destroyed by free acids or alcohol, but not by alkalies.

3. A fragment of iodic acid added to a strong solution of a morphine salt is decomposed, with liberation of iodine, which imparts a violet color to chloroform upon shaking the latter with the mixture.

4. A mixture of 1 part of morphine and 4 parts of cane-sugar added to concentrated sulphuric acid gives a dark-red color, which is intensified by a drop of bromine water.

5. Morphine dissolves in cold, concentrated sulphuric acid, forming a colorless solution, which, after standing for several hours, turns pink on the addition of a trace of nitric acid.

6. Aqueous or acid solutions of morphine salts are precipitated by alkaline hydrates; the precipitated morphine is soluble in potassium or sodium hydrate, but not in ammonium hydrate.

7. Neutral solutions of morphine afford yellow precipitates with the chloride of gold or platinum, with potassium chromate or dichromate, and with picric acid, but not with mercuric chloride.

Codeine, Codeina, $\mathrm{C}_{18} \mathrm{H}_{21} \mathrm{NO}_{3} \cdot \mathrm{H}_{2} \mathrm{O}=317$. A white or yellowishwhite, crystalline powder, sparingly soluble in cold water, easily soluble in alcohol and chloroform.

\section{Analytical reactions :}

1. Codeine, dissolved in sulphuric acid containing 1 per cent. of sodium molybdate, forms at first a dirty-green solution, which after a while becomes pure blue, and gradually fades within a few hours to pale yellow. 
2. Codeine, dissolved in sulphuric acid, forms a colorless liquid, which, upon being warmed with a trace of ferric chloride, becomes deep blue.

3. Codeine forms with chlorine water a colorless solution, which turns yellowish-red with ammonium hydrate.

Narcotine, $\mathrm{C}_{22} \mathrm{H}_{23} \mathrm{NO}_{7}$, and Narceine, $\mathrm{C}_{23} \mathrm{H}_{29} \mathrm{NO}_{9} \cdot 2 \mathrm{H}_{2} \mathrm{O}$, are white, crystalline opium alkaloids, which are almost insoluble in water, soluble in alcohol. Concentrated sulphuric acid forms with narcotine a solution which is at first colorless, but turns yellow in a few minutes, and purple on heating. Narceine dissolves in concentrated sulphuric acid with a gray-brown color, which changes to red when heated.

Meconic acid, $\mathrm{C}_{7} \mathrm{H}_{4} \mathrm{O}_{7} \cdot 3 \mathrm{H}_{2} \mathbf{0}$. A tribasic acid, characteristic of opium, in which.it exists chiefly combined with the alkaloids. It is a white crystalline substance, soluble in water and alcohol.

Meconic acid forms with ferric chloride a blood-red color, which is not affected by diluted acids or by mercuric chloride (different from ferric sulphocyanate), but disappears on the addition of stannous chloride and of the alkaline hypochlorites. This test may be used in cases of poisoning to decide whether opium or morphine is present.

Cinchona alkaloids. The bark of various species of cinchona contains a number of alkaloids, of which the most important are quinine, cinchonine, quinidine, and cinchonidine. These alkaloids exist in the bark in combination with a peculiar acid, termed kinic acid. The quantity and relative proportion of the alkaloids vary widely in different barks, but the officinal bark should not contain less than 3 per cent. of alkaloids.

Determination of the total alkaloids and of quinine in cinchona. The U. S. P. has adopted the following method for the quantitative estimation of the cinchona alkaloids:

Powdered cinchona bark is thoroughly mixed with milk of lime, containing of calcium oxide about one-fourth of the weight of cinchona used. This mixture, which now contains the alkaloids in an uncombined state, is dried at a temperature not above $80^{\circ}$, and then exhausted by hot alcohol, which dis- 
solves the alkaloids. The filtered alcoholic sulution is slightly acidulated with sulphuric acid, and then evaporated to expel the alcohol. The residue is mixed with a little water, and the clear solution, containing the alkaloids as sulphates, is precipitated by sodium hydrate. The precipitated alkaloids are collected on a small filter, washed and dried at $100^{\circ}$.

From the total alkaloids the quinine is separated by dissolving them in water acidulated with sulphuric acid, and adding to this solution (which should be just distinctly acid to test-paper, and should weigh seventy times as much as the alkaloids used) diluted solution of sodium hydrate until exactly neutral, when the "effloresced sulphate of quinine," $\left(\mathrm{C}_{20} \mathrm{H}_{24} \mathrm{~N}_{2} \mathrm{O}_{2}\right)_{2} \cdot \mathrm{H}_{2} \mathrm{SO}_{4} \cdot 2 \mathrm{H}_{2} \mathrm{O}$, separates; this is collected on a small filter, dried at $60^{\circ}$, and weighed.

The precipitation of the salt is facilitated by heating the mixture to about $60^{\circ}$ for five minutes, and subsequent cooling to $15^{\circ}$ for half an hour.

As sulphate of quinine is soluble in water of $15^{\circ}$ to the extent of 0.12 per cent., the filtered liquid has to be weighed, and for every 100 grams of the filtrate 0.12 gram has to be added to the weight of the quinine sulphate collected on the filter. If the quantity of quinine in the alkaloids be too small, or the quantity of the filtered liquid too large, no separation of the salt may take place. In order to calculate from the precipitated "effloresced sulphate of quinine" the quantity of "crystallized sulphate of quinine," 11.5 per cent. of its weight have to be added for water of crystallization.

Quinine, Quinina, $\mathrm{C}_{20} \mathrm{H}_{24} \mathrm{~N}_{2} \mathbf{O}_{2} \cdot 3 \mathrm{H}_{2} \mathbf{O}=378$. This formula represents the crystallized alkaloid, but it is also known as anhydrous, and in combination with either one or two molecules of water. The anhydrous quinine is a resinous substance, whilst the crystallized quinine is a white powder, having a bitter taste and an alkaline reaction. It is nearly insoluble in water, but soluble in alcohol, ether, and chloroform.

Quinine sulphate, Quininæ sulphas, $\left(\mathrm{C}_{20} \mathrm{H}_{24} \mathrm{~N}_{2} \mathrm{O}_{2}\right)_{2} \mathrm{H}_{2} \mathrm{SO}_{4} \cdot 7 \mathrm{H}_{2} \mathrm{O}=872$ (Sulphate of quinine). This salt, containing two molecules of the alkaloid in combination with one of sulphuric acid and seven of water, is the common form of sulphate of quinine. It forms 
snow-white, loose, filiform crystals, 'fragile and somewhat flexible, making a very light and easily compressible mass; it has a very bitter taste and a neutral reaction; it is soluble in 740 parts of cold and in 30 parts of boiling water, soluble in 65 parts of alcohol, but nearly insoluble in ether and chloroform; it readily dissolves in diluted sulphuric or hydrochloric acid.

Quinine acid sulphate, Qúininæ bisulphas, $\left(\mathrm{C}_{20} \mathrm{H}_{24} \mathrm{~N}_{2} \mathbf{O}_{2}\right) \mathrm{H}_{2} \mathrm{SO}_{4} \cdot \mathbf{7} \mathrm{H}_{2} \mathbf{O}$ $=548$ (Bisulphate of quinine). This salt is formed when the common sulphate is dissolved in an excess of diluted sulphuric acid. It crystallizes in colorless, silky needles, has a strongly acid reaction and is soluble in 10 parts of water.

Hydrochlorate of quinine, $\mathrm{C}_{20} \mathrm{H}_{24} \mathrm{~N}_{2} \mathrm{O}_{2} . \mathrm{HCl} .2 \mathrm{H}_{2} \mathrm{O}=396.4$. '

Hydrobromate of quinine, $\mathrm{C}_{20} \mathrm{H}_{24} \mathrm{~N}_{2} \mathrm{O}_{2} \cdot \mathrm{HBr} \cdot 2 \mathrm{H}_{2} \mathrm{O}=440.8$.

Valerianate of quinine, $\mathrm{C}_{20} \mathrm{H}_{24} \mathrm{~N}_{2} \mathrm{O}_{2} \cdot \mathrm{C}_{5} \mathrm{H}_{10} \mathrm{O}_{2} \cdot \mathrm{H}_{2} \mathrm{O}=444$.

The above three salts are obtained by dissolving quinine in the respective acids; they are white, crystalline substances; the first two are easily, the valerianate is sparingly soluble in water.

Citrate of iron and quinine is a scale compound obtained by dissolving ferric hydrate and quinine in citric acid, evaporating, etc.

\section{Analytical reactions for quinine.}

1. Quinine or its salts, dissolved in water or in diluted acids, give, after having been shaken with fresh chlorine water, an emerald-green color on the addition of ammonium hydrate.

2. Solutions of quinine, treated with chlorine water, then with fragments of potassium ferrocyanide, turn pink, then red on the addition of ammonium hydrate not in excess.

3. Solutions of quinine give with water of ammonia a white precipitate of quinine, which is readily dissolved in an excess of ammonia. The precipitate is also soluble in about twenty times its own weight of ether (the other cinchona alkaloids requiring larger proportions of ether for solution).

4. Most solutions of quinine, especially when acidulated with sulphuric acid, show a vivid, blue fluorescence.

5. Chlorine, passed through water holding quinine in suspension, forms a red solution. 
6. Neutral solutions of quinine are precipitated by alkaline oxalates.

7. Quinine and its salts, when heated on platinum foil, burn entirely away.

8. Quinine and its salts form colorless solutions with concentrated sulphuric acid. A dark or red color indicates the presence of other organic substances.

Cinchonine, Cinchonina, $\mathrm{C}_{20} \mathbf{H}_{24} \mathbf{N}_{2} \mathbf{O}=308$. This alkaloid is found in cinchona bark in quantities varying from 0.5 to 3 per cent. It crystallizes without water, forming white. needles; it is almost insoluble in water, soluble in $\mathbf{1 1 0}$ parts of alcohol or in 370 parts of ether, readily soluble in diluted acids.

By dissolving the alkaloid in sulphuric acid is obtained:

Cinchonine sulphate, Cinchonince sulphas, $\left(\mathrm{C}_{20} \mathrm{H}_{24} \mathrm{~N}_{2} \mathrm{O}\right)_{2} \mathrm{H}_{2} \mathrm{SO}_{4} \cdot 2 \mathrm{H}_{2} \mathrm{O}$ $=750$. It is a white, crystalline substance. Cinchonine differs from quinine by its greater insolubility in ether, by its insolubility in ammonia water, by not forming fluorescent solutions, and by not giving a green color with chlorine water and ammonia.

Cinchonidine, $\mathrm{C}_{20} \mathrm{H}_{24} \mathbf{N}_{2}$. An alkaloid isomeric with cinchonine; soluble in 75 times its weight of ether. The sulphate is officinal.

Quinidine, $\mathbf{C}_{24} \mathbf{H}_{24} \mathbf{N}_{2} \mathbf{O}_{2}$. Isomeric with quinine; it gives, like the latter, a green color with chlorine water and ammonia, and forms fluorescent solutions. Unlike quinine, it is not precipitated from neutral solutions by alkaline oxalates.

Strychnine, Strychnina, $\mathbf{C}_{21} \mathbf{H}_{22} \mathbf{N}_{2} \mathbf{O}_{2}=334$. This alkaloid is found, together with brucine, in the seeds and bark of different varieties of strychnos, and is generally obtained from nux vomica. Strychnine is a white, crystalline powder, having an intensely bitter taste, which is still perceptible in solutions containing 1 in 700,000. It is nearly insoluble in water and in ether, soluble in chloroform and in diluted acids.

By dissolving it in sulphuric acid the officinal strychnine sulphate, Strychnince sulphas, $\left(\mathrm{C}_{21} \mathrm{H}_{22} \mathrm{~N}_{2} \mathrm{O}_{2}\right)_{2} \cdot \mathrm{H}_{2} \mathrm{SO}_{4} \cdot 7 \mathrm{H}_{2} \mathrm{O}=892$, is obtained. 
Strychnine has strong basic properties and is one of the most powerful poisons known, one-quarter of a grain having caused death within a few hours.

\section{Analytical reactions of strychnine.}

1. Strychnine dissolves in sulphuric acid and in nitric acid without color.

2. A fragment of potassium dichromate, drawn through a solution of strychnine in concentrated sulphuric acid, produces momentarily a blue, then brilliant violet color, which slowly passes to cherry-red, then to rose-pink, and finally to yellow. This reaction may yet be noticed with $\frac{1}{50000}$ grain of strychnine.

3. Strychnine, when moistened with a solution of iodic acid in sulphuric acid, produces a yellow color, changing to brickred and then to violet-red.

4. Solutions of strychnine give with diluted solution of potassium dichromate a yellow, crystalline precipitate, which, when collected, washed, and heated with concentrated sulphuric acid, shows the play of colors described in test 2 .

5. Neutral solutions of strychnine give yellow precipitates with the chlorides of gold and platinum and with picric acid, a white precipitate with mercuric chloride.

Bracine, $\mathrm{C}_{23} \mathrm{H}_{26} \mathrm{~N}_{2} \mathbf{O}_{4} 4 \mathrm{H}_{2} \mathbf{0}$. This alkaloid acts similarly to strychnine, but less energetically, upon the animal system. It is distinguished from strychnine by giving a bright red color with concentrated nitric acid, soon changing into yellow, whilst stannous chloride changes the red color to violet; chlorine water colors brucine bright red, changed to yellowish brown by ammonium hydrate.

Atropine, Atropina, $\mathrm{C}_{17} \mathrm{H}_{23} \mathrm{NO}_{3}=289$ (Daturine). Occurs in belladonna; it is a white, crystalline powder, having a bitter and acrid taste, and an alkaline reaction; it is nearly insoluble in water, but very soluble in alcohol and chloroform. The sulphate of atropine, Atropince sulphas, $\left(\mathrm{C}_{17} \mathrm{H}_{23} \mathrm{NO}_{3}\right)_{2} \cdot \mathrm{H}_{2} \mathrm{SO}_{4}$, is a white, crystalline powder, easily soluble in water.

Analytical reactions :

1. Atropine dissolves in concentrated sulphuric acid without color. 
2. The above solution is not colored by nitric acid (difference from morphine), and not at once by potassium dichromate (difference from strychnine). Prolonged contact with potassium dichromate causes the solution to turn green.

3. The green solution obtained by the action of potassium dichromate upon atropine which has been dissolved in sulphuric acid, evolves, on the addition of a few drops of water and warming, a pleasant odor reminding of roses and orange flowers.

4. Solutions of atropine dilate the pupil of the eye to a marked extent.

Veratrine, Veratrina, $\mathrm{C}_{32} \mathrm{H}_{50} \mathrm{NO}_{9}$ ? An alkaloid, or a mixture of alkaloids, obtained from veratrum officinale. It is a white, amorphous, rarely crystalline powder, highly irritating to the nostrils; nearly insoluble in water, readily soluble in alcohol.

Analytical reactions :

1. Concentrated sulphuric acid causes with veratrine first a yellow, then reddish-yellow, intense scarlet, and finally violetred color.

2. Veratrine when heated with concentrated hydrochloric acid, dissolves with a blood-red color.

3. Bromine water colors veratrine violet.

4. Veratrine forms with nitric acid a yellow solution.

Caffeine, Caffeina, $\mathbf{C}_{8} \mathrm{H}_{10} \mathbf{N}_{4} \mathbf{0}_{2} \cdot \mathrm{H}_{2} \mathbf{O}=212$ (Theine). Occurs in coffee, tea, Paraguay tea, and a few other plants. It forms long, silky needles, which are soluble in 75 parts of water and in 35 parts of alcohol; it has a slightly bitter taste and a neutral reaction.

Caffeine is dissolved by sulphuric acid without color; when treated with strong nitric acid it forms a yellow liquid which, after evaporation, assumes a purplish color when moistened with water of ammonia.

\section{Questions.}

461. What are the three chief forms in which nitrogen enters into organic compounds?

462. What are amines and amides?

463. State the composition of aniline, how it is obtained from benzene, and by what processes it is converted into aniline colors. 
464. State the general physical and chemical properties of alkaloids. 465. Give a general method for the extraction and separation of alkaloids from vegetables.

466. Mention the chief constituents of opium, and explain the process for determining the percentage of morphine in opium.

467. Mention the properties of morphine and its salts; give tests for them.

468. Mention the principal alkaloids found in cinchona bark, and give a process by which the total quantity of these alkaloids and of quinine may be determined.

469. State the physical and chemical properties of quinine and cinchonine. Which of their salts are officinal, and by what tests may these alkaloids be recognized and distinguished from each other?

470. Give tests for strychnine, brucine, atropine, and veratrine.

\section{CYANOGEN AND ITS COMPOUNDS.}

Occurrence in nature. Cyanogen itself does not occur in nature, but compounds of it are found in a few plants (amygdalin), and also in some animal fluids (saliva contains sodium sulphocyanate). Gases issuing from volcanoes (or from iron furnaces) sometimes contain cyanogen compounds.

Constitution of cyanogen compounds. The univalent residue cyanogen, $-\mathrm{C} \equiv \mathrm{N}$, or $\mathrm{CN}$, or $\mathrm{Cy}$, was the first compound radical distinctly proved to exist, and isolated by Gay-Lussac, in 1814. The name cyanogen signifies "generating blue," in allusion to the various blue culors (Prussian and Turnbull's blue) containing it. The symbol $\mathrm{Cy}$, frequently used in place of $\mathrm{CN}$, has been adopted merely to simplify the writing of. formulas of cyanogen compounds.

Cyanogen and its compounds show much resemblance to the halogens and their compounds, as indicated by the composition of the following substances:

$\begin{array}{cccc}\begin{array}{c}\mathrm{ClCl}, \\ \text { Chlorine. }\end{array} & \begin{array}{c}\mathrm{HCl}_{1} \\ \text { Hydrochloric } \\ \text { acid. }\end{array} & \begin{array}{c}\mathrm{KI}, \\ \text { Potassium } \\ \text { iodide. }\end{array} & \begin{array}{c}\mathrm{HClO}, \\ \text { Hypochlorons } \\ \text { acid. }\end{array} \\ \begin{array}{c}\mathrm{CNCN}, \\ \text { Cyanogen. }\end{array} & \begin{array}{c}\mathrm{HBr}, \\ \text { Hydrobromic } \\ \text { acid. }\end{array} & \begin{array}{c}\mathrm{KCN}, \\ \text { Potassium } \\ \text { cyanide. }\end{array} & \begin{array}{c}\mathrm{HCNO}, \\ \text { Cyanic acid. }\end{array} \\ \begin{array}{c}\text { CNCl, } \\ \text { Cyanogen } \\ \text { chloride. }\end{array} & \begin{array}{c}\mathrm{HCN}, \\ \text { Hydrocyanic } \\ \text { acid. }\end{array} & \begin{array}{c}\mathrm{AgCN}, \\ \text { Silver cyanide. }\end{array} & \begin{array}{c}\text { HCNS, } \\ \text { acid. }\end{array}\end{array}$


Dicyanogen, $(\mathbf{C N})_{2}$ or $\mathbf{C y}_{2}$. The unsaturated radical $\mathrm{CN}$ does not exist as such in a free state, but combines whenever liberated with another $\mathrm{CN}$, forming dicyanogen. It may be obtained by heating mercuric cyanide:

$$
\mathrm{HgCy}_{2}=\mathrm{Hg}+2 \mathrm{Cy} .
$$

It is a colorless gas, having an odor of bitter almonds, and burning with a purple flane, forming carbon dioxide and nitrogen; it is soluble in water, and may be converted into a colorless liquid by pressure; it acts as a poison, both to animal and vegetable life, even when present in but small proportions in the air.

Hydrocyanic acid, HCN or HCy $=27$ (Cyanhydric acid, Hydrogen cyanide, Prussic acid). This compound is found in the water distilled from the disintegrated seeds or leaves of amygdalus, prunus, laurus, etc. It is also found among the products of the destructive distillation of coal, and is formed by a great number of chemical decompositions. For instance :

By passing ammonia over red-hot charcoal:

$$
\underset{\text { Ammonia. }}{4 \mathrm{NH}_{3}}+\underset{\text { Carbon. }}{3 \mathrm{C}}=\underset{\substack{\text { Ammonium } \\ \text { cyanide. }}}{2\left(\mathrm{NH}_{4} \mathrm{CN}\right)}+\underset{\text { Methane. }}{\mathrm{CH}_{4}} .
$$

By the action of ammonia on chloroform:

$$
\underset{\text { Chloroform. }}{\mathrm{CHCl}_{3}}+\mathrm{NH}_{3}=\underset{\begin{array}{c}
\text { Hydrocyanic } \\
\text { acid. }
\end{array}}{\mathrm{HCN}}+\underset{\begin{array}{c}
\text { Hydrochloric } \\
\text { acid. }
\end{array}}{3 \mathrm{HCl} .}
$$

By heating ammonium formate to $200^{\circ}$ :

$$
\underset{\substack{\text { Ammonium } \\ \text { formate. }}}{\mathrm{NH}_{4} \mathrm{CHO}_{2}}=\underset{\substack{\text { Hydrocyanic } \\ \text { acid. }}}{\mathrm{HCN}}+\underset{\text { Water. }}{2 \mathrm{H}_{2} \mathrm{O} .}
$$

By the action of hydrosulphuric acid upon mercuric cyanide:

$$
\mathrm{HgCy}_{2}+\mathrm{H}_{2} \mathrm{~S}=\mathrm{HgS}+2 \mathrm{HCy} \text {. }
$$

By the decomposition of alkaline cyanides by diluted acids:

$$
\mathrm{KCy}+\mathrm{HCl}=\mathrm{KCl}+\mathrm{HCy} .
$$


By the action of hydrochloric acid upon silver cyanide:

$$
\mathrm{AgCy}+\mathrm{HCl}=\mathrm{AgCl}+\mathrm{HCy} .
$$

By distilling potassium ferrocyanide with diluted sulphuric acid :

$$
\underset{\substack{\text { Potassium } \\ \text { ferrocyanide. }}}{2\left(\mathrm{~K}_{4} \mathrm{FeCy}_{6}\right)}+\underset{\substack{\text { Sulphuric } \\ \text { acid. }}}{6\left(\mathrm{H}_{2} \mathrm{SO}_{4}\right)}=\underset{\substack{\text { Potassium } \\ \text { ferrous ferrocyanide. }}}{\mathrm{K}_{2} \mathrm{Fe}_{2} \mathrm{Cy}_{6}}+\underset{\substack{\text { Potassium } \\ \text { acid sulphate. }}}{6\left(\mathrm{KHSO}_{4}\right)}+\underset{\substack{\text { Hydrocyanic } \\ \text { acid. }}}{6 \mathrm{HCy} .}
$$

Pure hydrocyanic acid is (at a temperature below $26^{\circ}$ ) a colorless, mobile liquid, of a penetrating, characteristic odor resembling that of bitter almonds; it boils at $26.5^{\circ}$, and crystallizes at $-15^{\circ}$. It is readily soluble in water, and a 2 per cent. solution is the diluted hydrocyanic acid, Acidum hydrocyanicum dilutum.

According to the U. S. P., this diluted acid is made either by the decomposition of potassium ferrocyanide by diluted sulphuric acid in a retort, the delivery tube of which passes into a receiver containing a mixture of water and alcohol by which the liberated gas is absorbed, this liquid being afterwards diluted with a sufficient quantity of water to make a 2 per cent. solution, or it is made extemporaneously by the decomposition of 6 parts of silver cyanide by 5 parts of hydrochloric acid, diluted with 55 parts of water, allowing the silver chloride to subside and pouring off the clear liquid.

The diluted acid has the characteristic odor of bitter almonds, a slightly acid reaction, and is completely volatilized by heating. Whilst the pure acid is very readily decomposed by exposure to light, etc., the diluted acid is fairly stable, and is rendered more so by a trace of mineral acids.

Potassium cyanide, Potassii cyanidum, $\mathrm{KCN}=65$ (Cyanide of potassizm). The pure salt may be obtained by passing hydrocyanic acid into an alcoholic solution of potassium hydrate. The commercial article, however, is a mixture of potassium cyanicle with potassium cyanate. It is obtained by fusing potassium ferrocyanide with potassium carbonate in a crucible, when potassium cyanide and cyanate are formed, whilst carbon di- 
oxide escapes, and metallic iron is set free and collects on the bottom of the crucible. The decomposition is as follows:

$$
\underset{\substack{\text { Potassium } \\ \text { cyanide. }}}{2\left(\mathrm{~K}_{4} \mathrm{FeCy}_{6}\right)}+\underset{\substack{\text { Potassium } \\ \text { carbonate. }}}{2\left(\mathrm{~K}_{2} \mathrm{CO}_{3}\right)}=\underset{\substack{\text { Potassium } \\ \text { cyanide. }}}{10 \mathrm{KCy}}+\underset{\substack{\text { Potassium } \\ \text { cyanate. }}}{2 \mathrm{KCyO}}+\underset{\text { Iron. }}{2 \mathrm{Fe}}+\underset{\substack{\text { Carbon } \\ \text { dioxide. }}}{2 \mathrm{CO}_{2}}
$$

Potassium cyanide is a white, deliquescent salt, odorless when perfectly dry, but emitting the odor of hydrocyanic acid when moist. Potassium cyanides and other alkaline cyanides show a tendency to combine with the cyanides of heavy metals, forming a number of double cyanides, such as the cyanide of sodium and silver, $\mathrm{NaCy} . \mathrm{AgCy}$, ctc.

Silver cyanide, Argenti cyanidum, AgCN $=133.7$ (Cyanide of silver). A white powder, obtained by precipitating solution of potassium cyanide with silver nitrate. It is insoluble in water, slightly soluble in water of ammonia; evolves cyanogen when heated.

Mercuric cyanide, Hydrargyri cyanidum, $\mathbf{H g}(\mathbf{C N})_{2}$ (Cyanide of mercury). A white, soluble, crystalline salt obtained by dissolving mercuric oxide in hydrocyanic acid; it evolves cyanogen when heated.

\section{Analytical reactions for hydrocyanic acid.}

1. Hydrocyanic acid or soluble cyanides give with silver nitrate a white precipitate of silver cyanide, which is sparingly soluble in ammonia, soluble in alkaline cyanides or hyposulphites, but insoluble in diluted nitric acid. Concentrated nitric acid dissolves it with decomposition:

$$
\mathrm{HCy}+\mathrm{AgNO}_{3}=\mathrm{AgCy}+\mathrm{HNO}_{3} \text {. }
$$

2. Hydrocyanic acid mixed with ammonium hydric sulphide and evaporated to dryness forms sulphocyanic acid, which upon being slightly acidulated with hydrochloric acid gives with ferric chloride a blood-red color of sulphocyanate of iron. (Excess of ammonium sulphide must be avoided.)

3. Hydrocyanic acid, or soluble cyanides, give, when mixed 
with ferrous and ferric salts and potassium hydrate, a greenish precipitate, which, upon being dissolved in hydrochloric acid, forms a blue precipitate of Prussian blue, $\mathrm{Fe}_{4} 3 \mathrm{FeCy}_{6}$. This reaction depends on the formation of potassium ferrocyanide by the action of the cyanogen upon both the potassium of the potassium hydrate and the iron of the ferrous salt. In alkaline solutions, the blue precipitate does not form, for which reason hydrochloric acid is added.

4. Hydrocyanic acid heated with diluted solution of picric acid gives a deep-red color on cooling.

5. In cases of poisoning, the matter under examination is distilled (if neessary after the addition of water) from a retort connected with a cooler. To the distilled liquid the above tests are applied. If the substance under examination should have an alkaline or neutral reaction, the addition of some sulphuric acid may be necessary in order to liberate the hydrocyanic acid. The objectionable feature to this aeidifying is the fact that nonpoisonous potassium ferrocyanide might be present, which upon the addition of sulphuric acid would liberate hydrocyanic acid. In cases where the addition of an acid becomes neessary, a preliminary examination should, therefore, decide whether or not ferro- or ferricyanides are present.

Antidotes. Hydroeyanic acid is a powerful poison both when inhaled or swallowed in the form of the acid or of soluble eyanides. As an antidote, is recommended a mixture of ferrous sulphate and ferric ehloride with either sodium carbonate or magnesia. The action of this mixture is explained in the above reaction 3 , the object being to convert the soluble cyanide into an insoluble ferrocyanide of iron. In most cases of poisoning by hydroeyanic acid, there is, however, no time for the action of such an antidote, in consequence of the rapidity of the action of the poison, and the treatment is chiefly directed to the maintenance of respiration by artificial means.

Cyanic acid, HCNO, and Sulphocyanic acid, HCNS, are both colorless aeid liquids, the salts of which are known as cyanates and sulphocyanates. These salts are oltained from alkaline cyanides by treating them with oxidizing agents or by boiling their solu- 
tions with sulphur, when either oxygen or sulphur is taken up by the alkaline cyanide:

$$
\begin{aligned}
& \mathrm{KCy}+\mathrm{O}=\mathrm{KCy} \mathrm{O}=\text { Potassium cyanate. } \\
& \mathrm{KCy}+\mathrm{S}=\mathrm{KCyS}=\text { Potassium sulphocyanate. }
\end{aligned}
$$

The acids themselves may be liberated from their salts by diluted mineral acids. Sulphocyanates give with ferric salts a deep red color, which is not affected by hydrochloric acid, but disappears on the addition of mercuric chloride.

Metallocyanides. Cyanogen not only combines with metals to form the true cyanides, which may be looked upon as derivatives of hydrocyanic acid, but cyanogen also enters into combination with certain metals (chiefly iron), forming a number of complex radicals, which upon combining with hydrogen form acids or with basic elements form salts. It is a characteristic feature of the compound cyanogen radicals thus formed that the analytical characters of the metals (iron, etc.) entering into the radical are completely hidden. Thus, the iron in ferroor ferricyanides is not precipitated by either alkalies, soluble carbonates, ammonium sulphide, or any of the common reagents for iron, and its presence can only be demonstrated by these reagents after the organic nature of the compound has been destroyed by burning it (or otherwise), when ferric oxide is left, which may be dissolved in hydrochloric acid and tested for in the usual manner.

The principal iron-cyanogen radicals are ferrocyanogen $\left[\mathrm{Fe}^{\mathrm{ii}}(\mathrm{CN})_{6}{ }^{\mathrm{i}}\right]^{\mathrm{iv}}$ or $\mathrm{FeCy_{6 }}{ }^{\mathrm{iv}}$, and ferricyanogen $\left[\mathrm{Fe}_{2}{ }^{\mathrm{vi}}(\mathrm{CN})_{12}{ }^{\mathrm{i}}\right]^{\mathrm{vi}}$ or $\mathrm{Fe}_{2} \mathrm{Cy}_{12}{ }_{12}$. These two radicals contain iron in the ferrous and ferric state respectively, and form, upon combining with hydrogen, acids which are known as hydroferrocyanic acid, $\mathrm{H}_{4} \mathrm{FeCy}_{6}$ (tetrabasic), and hydroferricyanic acid, $\mathrm{H}_{6} \mathrm{Fe}_{2} \mathrm{CN}_{12}$, or $\mathrm{H}_{6} \mathrm{Fe}_{2} \mathrm{O}_{12}$ (hexabasic); the salts of these acids are termed ferrocyanides and ferricyanides.

Potassium ferrocyanide, Potassii ferrocyanidum, $\mathrm{K}_{4} \mathrm{Fe}(\mathrm{CN})_{6}, \mathbf{H}_{2} \mathbf{0}=$ 421.9 (Ferrocyanide of potassium, Yellow prussiate of potash). This salt is manufactured on a large scale by heating refuse animal matter (scrapings of horns, hoofs, hides, etc.) with potassium carbonate and waste iron (filings, etc.). The fused mass is 
boiled with water, and from the solution thus formed the crystals separate on cooling.

The nitrogen and carbon of the organic matter (heated as above stated) combine, forming cyanogen, which enters into combination first with potassium and afterwards with iron.

Potassium ferrocyanide forms large, translucent, pale, lemonyellow, soft, odorless, non-poisonous, neutral crystals, easily dissolving in water.

Analytical reactions:

1. Ferrocyanides heated on platinum foil burn and leave a residue of (or containing) ferric oxide.

2. Ferrocyanides heated with concentrated sulphuric acid evolve carbonic oxide; with diluted sulphuric acid liberate hydrocyanic acid; with concentrated hydrochloric acid liberate hydroferrocyanic acid.

3. Soluble ferrocyanides give a blue precipitate with ferric salts :

$$
\underset{\substack{\text { Potassium } \\
\text { ferrocyanide. }}}{3\left(\mathrm{~K}_{4} \mathrm{FeCy}_{6}\right)}+\underset{\substack{\mathrm{Ferric} \\
\text { chloride. }}}{2\left(\mathrm{Fe}_{2} \mathrm{Cl}_{6}\right)}=\underset{\substack{\text { Polassiun } \\
\text { chloride. }}}{12 \mathrm{KCl}}+\underset{\begin{array}{c}
\text { Ferric fer- } \\
\text { rocyanide. }
\end{array}}{\mathrm{Fe}_{4} \cdot 3 \mathrm{FeCy}_{6} .}
$$

The blue precipitate of ferric ferrocyanide, or Prussian blue, is insoluble in water and diluted acids, soluble in oxalic acid (blue ink), and is decomposed by alkalies with separation of brown ferric hydrate and formation of potassium ferrocyanide. The addition of an acid restores the blue precipitate.

4. Soluble ferrocyanides give with eupric solutions a brownishred precipitate of cupric ferrocyanide.

5. Soluble ferrocyanides produce, with solutions of silver, lead, and zinc, white precipitates of the respective ferrocyanides.

6. Ferrocyanides give with ferrous salts a white precipitate of ferrous ferrocyanide, soon turning blue by absorption of oxygen.

Potassium ferricyanide, $\mathbf{K}_{6} \mathbf{F e}_{2}(\mathbf{C N})_{12}$ (Red prussiate of potash). Obtained by passing chlorine through solution of potassium ferrocyanide :

$$
\underset{\substack{\text { Potassium } \\ \text { ferrocyanide. }}}{2\left(\mathrm{~K}_{4} \mathrm{FeCy}_{6}\right)}+\underset{\text { Chlorine. }}{2 \mathrm{Cl}}=\underset{\substack{\text { Potassium } \\ \text { chluride. }}}{2 \mathrm{KCl}}+\underset{\substack{\text { Potassium } \\ \text { ferricyanide. }}}{\mathrm{K}_{6} \overline{\mathrm{Fe}}_{2} \mathrm{Cy}_{12}}
$$

While apparently this decomposition consists merely in a removal of two atoms of potassium from two molecules of 
potassium ferrocyanide, the change is actually more complete, as the atoms arrange themselves differently, the iron passing also from the ferrous to the ferric state.

Potassium ferricyanide crystallizes in red prisms, soluble in water. It forms, with ferrous solutions, a blue precipitate of ferricyanide of iron or Turnbull's blue:

$$
\mathrm{K}_{6} \mathrm{Fe}_{2} \mathrm{Cy}_{12}+3\left(\mathrm{FeSO}_{4}\right)=3\left(\mathrm{~K}_{2} \mathrm{SO}_{4}\right)+\mathrm{Fe}_{3} \mathrm{Fe}_{2} \mathrm{Cy}_{12} .
$$

With ferric solutions no precipitate is produced by potassium ferricyanide, but the color is changed to a dark olive green.

Nitrocyanmethane, $\mathbf{C H}_{2} \cdot \mathbf{C N} \cdot \mathrm{NO}_{2}$ (Fulminic acid). This substance may be looked upon as a derivative of methane, $\mathrm{CH}_{4}$, in which two atoms of bydrogen are replaced by cyanogen and $\mathrm{NO}_{2}$ respectively. It is not known in the separate state, but its combinations with metals are well known, especially mercuric fulminate, which is manufactured and used as an explosive in percussion caps, etc. It is made by adding alcohol to a solution of mercury in nitric acid. Silver fulminate can be obtained by a similar process.

\section{- Questrions.}

471. What is cyanogen, what is dicyanogen, and how is the latter obtained?

472. How does cyanogen occur in nature, and which non-metallic elements does it resemble in the constitution of various compounds?

473. State the composition and properties of hydrocyanic acid.

474. Mention some reactions by which hydrocyanic acid is formed, and state the two processes by which the officinal diluted acid is obtained.

475. What strength and what properties has the diluted hydrocyanic acid?

476. State the composition of pure potassium cyanide and of the commercial article. How is the latter made?

477. Give reactions for hydrocyanic acid and cyanides.

478. Explain the constitution and give the composition of ferro- and ferricyanides.

479. Give composition, mode of manufacture, and tests of potassium ferrocyanide.

480. What is red prussiate of potash, how is it obtained, and by what reaction can it be distinguished from the yellow prussiate? 


\section{ALBUMINOUS SUBSTANCES OR PROTEIDS.}

Occurrence in nature. Albuminous substances form the chief part of the solid and liquid constituents of the animal body; they occur in blood, tissues, muscles, nerves, glands, and all other organs; they are also found in small quantities in nearly every part of plants, and in larger quantities in many seeds. They have never yet been formed by artificial means, but are almost exclusively products of vegetable life, and undergo but little change when consumed as food and assimilated by animals.

General properties. The various proteids resemble one another closely in their properties. Their composition is so complex that, as yet, no chemical formula has been assigned to them with any certainty. The percentage composition and other reasons have led to a formula represented by $\mathrm{C}_{144} \mathrm{H}_{224} \mathrm{~N}_{36} \mathrm{O}_{44} \mathrm{~S}_{2}$, which represents about the average composition of the proteids. The percentage composition is shown in the following figures:

$\begin{array}{lrlrl}\text { Carbon, } & 51.5 & \text { per cent. to } 53.6 \text { per cent. } \\ \text { Hydrogen, } & 6.7 & \text { " } & 7.1 & \text { " } \\ \text { Nitrogen, } & 15.6 & \text { " } & 17.4 & \text { " } \\ \text { Oxygen, } & 21.8 & \text { " } & 24.0 & \text { " } \\ \text { Sulphur, } & 0.8 & \text { " } & 1.5 & \text { " }\end{array}$

Of other properties may be mentioned:

1. They are amorphous, colorless, odorless, and nearly tasteless substances, incapable of dialysis.

2. They easily undergo that decomposition known as putrefaction.

3. Some are soluble in water, others only in water containing alkalies, acids, or other substances, whilst some are insoluble.

4. They are not volatile without decomposition.

5. The soluble proteids are converted into insoluble modifications either by heating to $60^{\circ}$ or $70^{\circ}$, or by the addition of mineral acids, alcohol, or certain metallic salts. This process of converting soluble into insoluble proteids is called coagulation; and proteids when once coagulated will not return to their original soluble form without suffering some alteration.

6. They are converted into peptones by the action of gastric juice. (See later.) 


\section{Analytical reactions.}

1. Proteids are colored yellow by warm nitric acid, the color changing to orange with ammonia.

2. Millon's reagent colors them purple-red on heating. This reagent is a solution of mercuric nitrate, containing some excess of nitric acid; it is best made by dissolving 1 part of mercury in 2 parts of nitric acid of a specific gravity of 1.42 , and diluted with 2 volumes of water.

3. A few drops of enpric sulphate solution and then potassium hydrate added, give a violet color.

4. A few drops of solution of 1 part of cane-sugar in 4 parts of water and then strong sulphuric acid added, produce a purple color.

5. They are often precipitated by highly diluted acids, but redissolved by boiling with strong hydrochloric acid, forming a violet-red solution. The precipitated proteids are also generally dissolved by caustic alkalies.

Classification. Our present unsatisfactory state of knowledge regarding proteids, the close resemblance which they show in properties, and the difficulties which are met with in separating them in a pure state, make it difficult to arrange these bodies properly. Three principal groups may, however, be distiuguished; they are:

\section{True albumins.}

Serum-albumin.

Egg-albumin.

Plant-albumin.

Soluble in water and precipitated (coagulated) by warming their solution to $60^{\circ}$ or $70^{\circ}$.

\section{Fibrins}

Blood-fibrin.

Muscle fibrin.

Plant-fibrin or gluten.

Insoluble in water; soluble in water containing certain neutral salts, alkalies, or acids. They become generally insoluble as soon as they leave the organism.
III. Caseins.

Milk-casein.

Serum-casein.

Plant-casein or legumin.

Not coagulated by heat, but by diluted acids, and also by the mucous membrane of the stomach of the calf (rennet).

True albumins occur in the whites of birds' eggs, in milk, in the plasma of the bluod, chyle, lymph, in the juices of muscles, in serous and nutritive liquids; they are also found in the juices of plants. Solutions of albumin become turbid at $60^{\circ}$, and are coagulated at or below $75^{\circ}$; the presence of a little free acid 
facilitates precipitation, whilst strongly alkaline solutions are not precipitated by heating. (The presence of too much acid may also prevent precipitation.) During the coagulation some sulphuretted hydrogen is generally liberated, indicating a decomposition.

Coagulated albumin is dissolved by strong solutions of alkaline hydrates; compounds of albumin with an alkali, so-called alkali-albumins, are found in vegetable and animal fluids.

Albumin is also precipitated by picric acid, metaphosphoric acid, and by mercuric chloride.

Serum-albumin is the most abundant albuminous substance in animal bodies, and may be obtained from blood (after having separated the fibrin) by heating to $70^{\circ}$, when it coagulates. It is an almost white, or pale yellow, elastic substance. Pathologically it occurs in urine.

Egg-albumin differs but little from the former, but may be distinguished from it by being coagulated by ether, which does not affect blood-albumin. It exists in solution in the white of eggs, where it is contained in a network of delicate membrane.

The yolk of egg contains a substance in solution called vitelin, an albuminous substance containing also phosphorus; it is probably a mixture of albumin and casein; it is precipitated by heating to $75^{\circ}-80^{\circ}$, or by alcohol, which latter decomposes it into albumin and lecithir, a peculiar glyceride containing phosphorus, but no sulphur.

Vegetable albumin exists in nearly all vegetable juices, and is the most valuable constituent of vegetables used as food. It is coagulated at $61^{\circ}-63^{\circ}$, and by nearly all acids.

Blood-fibrin is found in the blood, chyle, lymph, and in many serous exudations; it coagulates (clots) as soon as the blood leaves the organism, and may be obtained from coagulated blood by whipping with a bundle of twigs, and washing the separated fibrin with water. It is, when recently obtained, a white, gelatinous, tenacious mass, consisting of numerous minute fibrils; when dried it becomes hard and brittle. It is in- 
soluble in water, alcohol, and ether, but swells up and dissolves slowly in diluted acids.

Whether or not fibrin exists as such in the blood, or is produced by the decomposition of another substance when blood leaves the organism, is a question not yet decided; it is, however, highly probable that there exist in the blood two substances, known as paraglobulin and tibrinogen, which combine together (forming tibrin) as soon as the blood leaves the organism.

Muscle-fibrin or muscle-protoplasm is a liquid contained in the muscles; after death this liquid coagulates, becomes solid (rigor mortis), and this insoluble body is termed myosin or flesh-fibrin.

Vegetable fibrin or gluten exists in many parts of vegetables, and is best obtained from wheat-flour by kneading it on a sieve with water, when the starch passes through and gluten remains as a soft, elastic mass, insoluble in water, alcohol, and ether. It is probably a mixture of several proteids.

Milk-casein, most likely an alkali-albumin, is the principal albuminous substance of milk, in which it occurs in solution.

Casein is precipitated by acids, alcohol, many metallic salts, and by rennet; it is soluble in alkalies.

Vegetable casein or legumin occurs in considerable quantities in the seeds of leguminosæ, such as peas and bcans, which contain nearly 25 per cent.; it is also found in almonds, various nuts, etc. It shows reactions very similar to milkcascin.

Peptone is a term assigned to the products formed by the action of gastric and pancreatic juices upon proteids during the process of digestion. Peptones are soluble in water, insoluble in alcohol; the neutral aqueous solution is precipitated by alcohol, but not by heat, acids, or alkalies. Peptones are capable of dialysis, or of diffusion through membranes, whilst proteids are not. Whilst peptones are looked upon as but slightly altered albumins by some scientists, they are considered by 
others as mixtures of leucine, $\mathrm{C}_{6} \mathrm{H}_{13} \mathrm{NO}_{2}$, tyrosine, $\mathrm{C}_{9} \mathrm{H}_{11} \mathrm{NO}_{3}$, asparaginic acid, etc.

Hæmoglobin (Hematocrystalline). This substance is the coloring agent of the blood; it resembles the proteids in many respects, but differs from them in being crystallizable and in containing iron. Its composition has been found to correspond to the formula $\mathrm{C}_{600} \mathrm{H}_{960} \mathrm{~N}_{154} \mathrm{O}_{179} \mathrm{FeS}_{3}$.

The most characteristic feature of a solution of hæmoglobin is its power of absorbing various gases; it absorbs oxygen in considerable quantities, thereby assuming a bright red color, but gives up the oxygen again when another gas, carbon dioxide for instance, is passed through it, the latter gas causing the bright red color to change into a dark red. Upon this absorption and exchange of the two gases in the hæmogloblin depend its action in the blood, where it exists in the red blood-corpuscles. Arterial blood is bright red, and contains oxyhemoglobin, hæmoglobin loaded with oxygen; this latter element is exchanged by the blood during its passage through the system for carbon dioxide, produced by the oxidation of the various organic substances, and the dark venous blood, now loaded with carbon dioxide, returns to the lungs, where the carbon dioxide is again exchanged for oxygen.

Hæmoglobin absorbs certain other gases, for instance, carbonic oxide, nitrogen dioxide, and hydrocyanic acid more readily than either oxygen or carbon dioxide, and the poisonous properties of these gases are due to their rendering hæmoglobin incapable of taking up oxygen.

Hæmoglobin may be obtained in beautiful red crystals, which differ somewhat in shape and solubility in water according to the species of animal from whose blood they were obtained.

Hæmoglobin may be decomposed by boiling with alcohol (or by other agents) into albumin and a substance called homatin, $\mathrm{C}_{34} \mathrm{H}_{34} \mathrm{~N}_{4} \mathrm{O}_{5} \mathrm{Fe}$, which is soluble in acidified alcohol. Hæmatin is a bluish-black powder, which forms with hydrochloric acid a crystalline compound, which fact is made use of as a characteristic microscopical test for the presence of blood.

Animal cryptolites. This term is applied to a number of animal substances of an unknown composition, but resembling 
somewhat the albuminoids in their properties. Their characteristic feature is the power of effecting changes in other organic substances, but the true cause of this action is unknown. The most important cryptolites are pepsin, ptyalin, and pancreatin.

Pepsin is the active principle of gastric juice, capable of converting albumin readily into soluble peptones. Saccharated pepsin, Pepsinum saccharatum, is officinal. Pepsin, obtained from the mucous membrane of the stomach of the hog, is mixed with powdered sugar of milk; this mixture is a white powder, not completely soluble in water, but readily and completely dissolves on the addition of a small quantity of hydrochloric acid. One part of saccharated pepsin dissolved in 500 parts of water, acidulated with 7.5 parts of hydrochloric acid, should dissolve at least 50 parts of hard-boiled egg-albumin, in 5 or 6 hours, at a temperature of $38^{\circ}-40^{\circ}$.

Ptyalin is a compound found in saliva, and having the property of converting starch into dextrine.

Pancreatin, the active principle or principles of the pancreatic secretion, is capable of converting starch into sugar, albumins into peptones, neutral fats into emulsions, and also into glycerine and fatty acids.

Gelatinoids. To this group belongs a number of substances occurring in bones, skins, horns, hair, nails, feathers, etc., and having generally the property of forming a jelly with water. The organic matter in bones, usually called ossein, contains, bcsides an albuminous substance, the two gelatinoids collagen and gelatin, an impure mixture of which forms common glue.

\section{Questions.}

481. To which class of substances is the term proteids applied, and which elements enter into their composition?

482. By what processes are albuminous substances formed in nature, and where do they occur?

483. State the general properties of albuminous substances.

484. How are proteids acted upon by heat, nitric acid, and Millon's reagent?

485. Into which three groups may proteids be classified, and how do they differ from each other? 
486. Mention some true albumins; state where they are found, and by what tests they are characterized.

487. Which substance is the cause of the clotting of blood, and how may that substance be obtained?

488. Where is animal, and where vegetable casein found?

489. What elements are found in hæmoglobin, where does it exist, and what are its characteristic properties?

490. What is pepsin, and how does it act upon albuminous substances? 


\section{VII. \\ PHYSIOLOGICAL CHEMISTRY.}

\section{CHEMICAL CHANGES IN PLANTS AND ANIMALS.}

General remarks. Physiological chemistry is that part of chemistry which has more especially for its object the various chemical changes which take place in the living organism of either plants or animals. It considers the chemical nature of the different substances used as "food," follows up the changes which this food undergoes during its absorption and assimilation in the organism, and treats finally of the products which are eliminated by it. The chemical changes taking place in the organism are either normal (in health) or abnormal (in disease). The abnormal products formed under abnormal conditions are generally termed "pathological" products.

Difference between vegetable and animal life. As a general rule, it may be stated that the chemical changes in a plant are progressive or constructive, in an animal regressive or destructive. That is to say, plants take up as food a small number of inorganic substances of a comparatively simple composition, convert them into organic substances of a more and more complicated composition with the simultaneous liberation of oxygen, whilst animals take up as food those organic vegetable substances of a complex composition, assimilate them in their system, where they are gradually used (burned up) and finally discharged as waste products, which are identical (or nearly so) with those substances serving as plant food. 
Plant food.

Carbon dioxide.

Water.

Ammonia, $\mathrm{NH}_{3}$.

Nitrates, $\mathrm{M}_{x} \mathrm{NO}_{3}$.

$\left.\begin{array}{l}\text { Phosphates } \\ \text { Sulphates } \\ \text { Chlorides }\end{array}\right\}$ of $\left\{\begin{array}{l}\text { Calcium. } \\ \text { Magnesium. } \\ \text { Sodium. } \\ \text { Potassium. }\end{array}\right.$
Waste products of animal life.

Carbon dioxide.

Water.

Urea, $\mathrm{CO}\left(\mathrm{NH}_{2}\right)_{2}$.

Urates, $\mathrm{M}_{x} \mathrm{C}_{5} \mathrm{H}_{2} \mathrm{~N}_{4} \mathrm{O}_{3}$.

$\left.\begin{array}{l}\text { Phosphates } \\ \text { Sulphutes } \\ \text { Chlorides }\end{array}\right\}$ of $\left\{\begin{array}{l}\text { Calcium. } \\ \text { Magnesium. } \\ \text { Sodi } \lambda \text { m. } \\ \text { Potassium. }\end{array}\right.$

Formation of organic substances by the plant. As shown in the above table, plants take up the necessary elements for organic matter from a comparatively small number of compounds. All carbon is derived from carbon dioxide; hydrogen chiefly from water; oxygen from either of the two substances named, as well as from the various salts; nitrogen either from ammonia, nitrates or nitrites; while sulphur and phosphorus are derived from sulphates and phosphates respectively. These substances are taken into the plant chiefly by the roots, the assimilation of the necessary mineral constituents being facilitated by an acid secretion (discharged from the roots) which has a tendency to render these salts, present in the soil and surrounding the roots, soluble.

Water having absorbed more or less of carbon dioxide, of ammonia or ammonium salts, and of nitrates, phosphates, and sulphates of potassium, calcium, etc., enters the plant through the roots by a simple process of diffusion, and is carried to the various green parts of the plant (chiefly to the leaves), where, under the influence of sunlight, a chemical decomposition and the formation of new compounds take place, the liberated oxygen being discharged directly through the leaves into the atmosphere.

It is difficult to explain fully the process of the formation of highly complex organic compounds in the plant, because we know so little in regard to the intermediate products which are formed. It is, however, fair to assume that the various compounds above mentioned as plant food, are first decomposed (with liberation of oxygen) in such a manner that residues or unsaturated radicals are formed, which combine together. From these compounds, produced at first, nore complicated ones will 
be gradually formed by replacement of more hydrogen, oxygen, or other atoms by other residues.

The following equations, while not showing the various radicals and intermediate compounds formed, may illustrate some of the results obtained by the plant in forming organic compounds :

$$
\begin{aligned}
\mathrm{CO}_{2}+\mathrm{H}_{2} \mathrm{O}= & \mathrm{H}_{2} \mathrm{CO}_{3} \\
& \mathrm{H}_{2} \mathrm{CO}_{3}-\mathrm{O}=\mathrm{H}_{22} \mathrm{CO}_{2}=\text { Formic acid. } \\
2 \mathrm{CO}_{2}+\mathrm{H}_{2} \mathrm{O}= & \mathrm{H}_{2} \mathrm{C}_{2} \mathrm{O}_{5} \\
& \mathrm{H}_{2} \mathrm{C}_{2} \mathrm{O}_{5}-\mathrm{O}=\mathrm{H}_{2} \mathrm{C}_{2} \mathrm{O}_{4}=\text { Oxalic acid. } \\
6 \mathrm{CO}_{2}+6 \mathrm{H}_{2} \mathrm{O}= & \mathrm{C}_{6} \mathrm{H}_{12} \mathrm{O}_{18} \\
& \mathrm{C}_{6}^{\prime} \mathrm{H}_{12} \mathrm{O}_{18}-12 \mathrm{O}=\mathrm{C}_{6} \mathrm{H}_{12} \mathrm{O}_{6}=\text { Glucose. } \\
10 \mathrm{CO}_{2}+8 \mathrm{H}_{2} \mathrm{O}= & \mathrm{C}_{10} \mathrm{H}_{16} \mathrm{O}_{28} \\
& \mathrm{C}_{10} \mathrm{H}_{16} \mathrm{O}_{28}-28 \mathrm{O}=\mathrm{C}_{10} \mathrm{H}_{16}=\text { Oil of turpentine. } \\
10 \mathrm{CO}_{2}+4 \mathrm{H}_{2} \mathrm{O}+2 \mathrm{NH}_{3}= & \mathrm{C}_{10} \mathrm{H}_{14} \mathrm{O}_{24} \mathrm{~N}_{2} \\
& \mathrm{C}_{10} \mathrm{H}_{14} \mathrm{O}_{24} \mathrm{~N}_{2}-24 \mathrm{O}=\mathrm{C}_{10} \mathrm{H}_{14} \mathrm{~N}_{2}=\text { Nicotine. }
\end{aligned}
$$

The above formulas show that the formation of organic compounds in the plant is always accompanied by the liberation of oxygen, and it may be stated as a general rule, that no organic substance (produced in nature) contains a quantity of oxygen sufficient to convert all carbon into carbon dioxide and all hydrogen into water, which fact also explains the combustibility of all organic substances.

Why it is that the living plant has the power of forming organic substances in the manner above indicated, we know not, and we know very little even in regard to the means by which the living eell accomplishes this formation, but we do know that sunlight is that agent, the action of which is indispensable for the plant in the formation of more complicated organic substances from simpler ones.

Decomposition of vegetable matter in the animal system. It has been stated above, that the process of chemical decomposition taking place in the animal system is chiefly regressive or destructive, that is to say: The substances formed in the plant are taken into the animal system, where they are assimilated, and then gradually oxidized by the inhaled atmospheric oxygen, 
thereby being converted into simpler forms of combination which are finally eliminated as waste products.

It has been shown above how a molecule of glucose which is formed in the plant requires not less than 6 molecules of carbon dioxide, and the same number of molecules of water for its formation, 6 molecules of oxygen being eliminated. A moleeule of gluense taken into the animal system undergoes the reverse process; by combining there with 6 molecules of oxygen it is converted into 6 molecules of carbon dioxide and the same number of molecules of water, thus :

$$
\mathrm{C}_{6} \mathrm{H}_{12} \mathrm{O}_{6}+12 \mathrm{O}=6 \mathrm{CO}_{2}+6 \mathrm{H}_{2} \mathrm{O}
$$

Animal food. The food taken by animals is (besides water and a few of its mineral constituents) all derived from vegetables, but it is taken from them either directly or indirectly; in the latter ease it has been previously taken into and assimilated by other animals, as in case of food taken in the form of meat, milk, eggs, etc. While some animals (herbivora) feed upon vegetable, and some (carnivora) upon animal food exclusively, others are capable of taking and assimilating either.

The fact that animal food is derived from vegetable matter, renders it superfluous to state that the elements taking an active part in the formation of either vegetable or animal matter, are identical. Of the total number of 66 clements, only 14 are found as necessary constituents of the animal body. These elements are carbon, hydrogen, oxygen, nitrogen, sulphur, phosphorus, chlorine, fluorine, silicium, calcium, magnesium, sodium, potassium, and iron. A fer other elements, such as aluminium, manganese, copper, etc., are sometimes found in the animal system, but they eannot be looked upon as normal or necessary constituents.

The various kinds of animal food are derived chiefly from three groups of organic substances, viz., carbohydrates (sugars, stareh, ete.), fats, and albuminous or nitrogenous substances. The inorganic substances, such as phosphates, chlorides, etc., required by the animal in the construction of bones, for the liberation of hydrochloric acid in the gastric juice, ete., are generally found as constituents of various kinds of food or are derived from drinking water. Milk contains all the necessary 
organic or inorganic constituents; bread is rich in phosphates, which latter are also found in smaller or larger quantities in nearly all kinds of vegetable and animal food.

Food has a twofold office, viz., to build up or nourish the whole body, and to maintain the animal heat. The latter action is chiefly accomplished by the oxidation of carbohydrates and fats, which are therefore also spoken of as heat-giving or respiratory food, whilst nitrogenous or albuminous substances are those which serve to build up and nourish the animal frame, and are termed plastic or nutritive food.

Of the heat produced by the oxidation of fats and carbohydrates, a certain amount escapes, whilst another quantity is employed in working the involuntary machinery of the body (motion of the heart, lungs, intestinal canal, etc.), and the rest is available for conversion into voluntary muscular actions. Whilst the nitrogenous substances have primarily the task of continuously replacing the wear and tear of the nitrogenous tissues, any excess of them may serve to keep up the animal heat and consequently also the involuntary or voluntary motion.

The relative proportions in which the two kinds of food are taken by animals depend upon the nature of the animal and upon its particular condition of existence. A young, growing animal, for instance, requires a relatively larger quantity of nitrogenous food than an animal whose growth has ceased, and an animal exposed to a low temperatare requires more of heatgiving food than one living in a warmer locality.

The relative proportions of nitrogenous and non-nitrogenous matter in various kinds of food are shown in the following table :

\begin{tabular}{lcc|lcc} 
Sweet potatoes, & $\begin{array}{r}\text { Nitro- } \\
\text { genous. }\end{array}$ & $\begin{array}{c}\text { Non-ni- } \\
\text { trogenons. }\end{array}$ & & $\begin{array}{r}\text { Nitro- } \\
\text { genous. }\end{array}$ & $\begin{array}{c}\text { Non-ni- } \\
\text { trogenous. }\end{array}$ \\
Rice, & 1 & 17 & Pork, & 1 & 3 \\
Carrots, & 1 & 12 & Fat mutton, & 1 & 2.7 \\
Potatoes, & 1 & 11 & Peas (dried), & 1 & 2.5 \\
Bread, & 1 & 10 & White beans, & 1 & 2.3 \\
Flour, & 1 & $5.0-6.8$ & Milk, & 1 & 2.2 \\
Turnips, & 1 & 6 & Beef, & 1 & 1.7 \\
Onions, & 1 & 6 & Cheese, & 1 & 0.7 \\
Oatmeal, & 1 & 5.5 & Veal, & 1 & $0.5-1.5$ \\
Cocoa, & 1 & 5 & White of egg, & 1 & 0.1 \\
& & & & & 0
\end{tabular}


Nutrition of animals. Before the food can be utilized for the construction and sustenance of the animal body it has to undergo digestion, which means that it has to be dissolved or otherwise converted into such a form that it can be absorbed by the blood, which carries it first to the lungs and from there to all the various parts and organs of the body, which have to be continually repaired or reconstructed by the constituents of the blood.

The first step towards the digestion of the food is its disintegration, which is accomplished by the teeth with the aid of the saliva, an alkaline fluid acting chemically upon starch, converting it into dextrine and sugar and also upon the fatty portions of the food by enulsifying them at least partly.

The masticated food thus prepared passes down into the stomach, where it undergoes another change, consisting chiefly in the conversion of insoluble albuminous matter into soluble peptones. The agent producing this change is the gastric juice, secreted by the lining membrane of the stomach.

The action in the stomach lasts for several hours; the partially digested food resulting from this action, and known as chyme, is then propelled by peristaltic motion into the commencement of the intestines (the duodenum), where it meets with and is acted upon by two other agents, the bile and pancreatic juice. The special function of bile in the digestion of food has not been sufficiently explained, but it appears that, in coöperating with the pancreatic juice, it acts upon the fats, causing them either to form intimate mixtures (emulsions) with water, or saponifying them partly. Pancreatic juice, moreover, acts powerfully upon those starchy portions of the food which nay have escaped the previous action of the saliva, and it also acts upon proteids, converting then into peptones.

From the duodenum the almost digested food now passes into other intestines, from which again juices are liberated further acting upon the semiliquid mass, which, upon its arrival in the small intestines, contains the soluble nutritious matters in the form of a thin, milky fluid called chyle. This chyle is a mixture of liquefied and chemically slightly altered albuminous, starchy, and fatty matters, which, during their passage through the intestines, are gradually absorbed (by a simple process of diffusion), 
whilst the undissolved and undigested portions of the food are further propelled until they leave the organism as feces.

The chyle, after having passed through the walls of the intestines, is absorbed and carried in the blood to the lungs, where it is acted upon by the inhaled oxygen, absorbing a certain quantity of this element, whilst about an equal volume of carbon dioxide is given off. The blood, thus regenerated by the absorption of chyle and oxygen, returns to the system as arterial (oxidized) blool, which in its course through the body comes in contact with all the various parts of the system, nourishing and regenerating them by leaving behind albuminous (and other) matter, whilst, at the same time, it oxidizes another quantity of matter which had been previously left behind, had assumed cellular structure, and had been rendered useless during the performance of its functions.

Waste products of animal life. The changes which the food suffers after having been absorbed by the animal system are extremely complicated, and far from being thoroughly understood. Numerous products and organs are formed and nourished from and by the blood; among them muscular-, nerve-, and brainsubstance, excretions and secretions, such as milk, saliva, bile, gastric and pancreatic juice, etc., also bones, teeth, hair, and many others.

Most of these substances (some excretions, such as milk and others, excepted) suffer a constant oxidation in the system, and are finally eliminated as waste products. There are three channels through which the waste products are given off; they are the lungs, the skin, and the kidneys. By the lungs are eliminated chiefly carbon dioxide and some water, by the kidneys urine (which is a weak aqueous solution of urea, uric acid, urates, phosphates, chlorides, and sulphates of calcium, magnesium, sodium, potassium, etc.), and by the skin are constantly eliminated carbon dioxide and water, and during the process of sweating also more or less of the constituents of urine.

Chemical changes after death. After the death of either a plant or animal, a chemical decomposition cornmences which finally results in the formation of those inorganic compounds from which the plant originally derived its food, viz., carbon 
dioxide, water, ammonia, sulphates, phosphates, etc. This decomposition of a dead body is generally a simultaneous fermentation or putrefaction, aided by decay or slow combustion.

There are numerous intermediate products formed, which differ according to the nature of the decomposing substance, or according to the conditions (degree of temperature, amount of moisture and air present, etc.) under which the decomposition takes place.

During the decomposition of dead vegetable matter (especially of moist wood) the internediate products are frequently called humus, which substance (or better mixture of substances) forms the chief part of the organic matter in the soil.

During the decomposition of dead animals, the sulphur is first eliminated as hydrosulphuric acid, and a number of other intermediate products have been shown to be formed; among them certain organic bases often called ptomaines or cadaveric alkaloids, substances which have poisonous properties. The decomposition of organic matter may be prevented under conditions which have been mentioned heretofore in connection with putrefaction.

\section{Questions.}

491. What is the difference between vegetable and animal life in a chemical point of view?

492. Mention the chief substances serving as plant food.

493. Explain the formation of organic substances in the plant.

494. What elements enter into the animal system as necessary constituents?

495. The members of which three groups of organic substances are chiefly used as food by animals?

496. Which substances are generally termed "nutritive," and which "heat-giving?"

497. Explain the chenical change which food suffers during digestion.

498. Mention the four principal fluids which are secreted by various organs of the animal body, in order to facilitate or cause digestion.

499. What are the waste products of animal life, and through which channels are they eliminated:

500 . What is the final result of the decomposition of dead plants or animals? 


\section{¡1. ANIMAL FLUIDS AND TISSUES.}

Constituents of the animal body. The auimal body cousists mainly of three kinds of matter, viz., water, organic and inorganic matter. It contains of water, about 60 per cent., of organic matter 35 per cent., and of inorganic matter about 5 per cent. The water may be determined by drying a weighed. quantity in an air-bath at a temperature of $100^{\circ}$ to $105^{\circ}$; the organic matter is estimated by burning the dried substance, and the inorganic matter (ash) by weighing the residue. Some of the elements which are left in the inorganic residue have, however, been actually constituents of organic compounds; iron, for instance, which is left in the ash, has been chiefly a constituent of hæmoglobin; sulphur, left as a sulphate, may have been a constituent of albumin, etc.

The relative quantities of the three constituents in some of the animal fluids and tissues is shown in the following table:

\begin{tabular}{lccc} 
& Water. & $\begin{array}{c}\text { Organic and } \\
\text { volatile matter. }\end{array}$ & $\begin{array}{c}\text { Inorganic resi- } \\
\text { due }(a, h) .\end{array}$ \\
Saliva, & 99.50 & 0.32 & 0.18 \\
Gastric juice, & 99.43 & 0.33 & 0.24 \\
Pancreatic juice, & $94 . ?$ & $4 . ?$ & $2 . ?$ \\
Bile, & 8592 & 13.30 & $0.78^{1}$ \\
Chyle, & 91.80 & 7.40 & 0.80 \\
Lymph, & \multicolumn{1}{c}{ Similar to chyle. } & \\
Pus, & 87.00 & 12.20 & 0.80 \\
Cows' milk, & 87.00 & 12.25 & 0.75 \\
Human milk, & 86.80 & 12.85 & 0.35 \\
Blood, & 79.50 & 19.70 & 0.80 \\
Blood-corpuscles, & 54.60 & 44.68 & 0.72 \\
Blood-serum, & 90.50 & 8.68 & 0.82 \\
Urine, & 95.70 & 3.00 & 1.32 \\
Bone (varies widely), & 22.00 & 26.00 & 52.00 \\
Dentine, & 10.00 & 25.00 & 65.00 \\
Enamel, & 0.4 & 3.60 & 96.00
\end{tabular}

The complex nature of the various organic matters has been referred to in the preceding chapter, and will be more fully considered below; but it may be mentioned here, that some of these organic substances (or groups of substances) may be separated by a successive treatment of the animal matter with various solvents. Thus, by treating with ether or carbon disul-

1 The metals in combination with the biliary acids not included. 
phide, all fats may be extracted; by treating then with alcohol and water successively other substances (generally termed extractive matter or extractives) are dissolved, which may be obtained by evaporating the solution.

Among the extractives are found kreatin and kreatinin, urea, uric acids, organic salts, etc. After the fatty matter and the extractives have been removed, there remains an elastic and somewhat horny mass, which consists chiefly of proteids (albumin, fibrin, globulin, etc.).

The complete separation of all substances is extremely difficult on account of the great similarity in properties of many of these substances, and the rapid changes which they suffer when acted upon by solvents or chemical agents.

As the nature or composition of many of the inorganic salts present in the animal tissues is changed during the burning off of the organic matter, it is necessary to determine them either in the aqueous solution (extract) or by subjecting the animal matter to dialysis, by which process they may be more or less completely separated from the organic matter, which is left in the dialyzer, whilst the salts pass through the membrane.

Blood. Two kinds of blood are distinguished, the arterial or oxidized and the venous or deoxidized blood. Arterial blood as it is present in the system, or immediately after it has been drawn from the body, is a red liquid of an alkaline reaction and a specific gravity of about 1.060. Upon examination under the microscope, blood is seen to consist of a colorless fluid, called plasma or serum, in which float small globules'or corpuscles. These corpuscles are capable of transmitting red light only, thus imparting to the blood its red color; their shape is that of a biconcave disk, and their size about $\frac{1}{3500}$ of an inch in diameter. Besides the red corpuscles, there are found some of a white color, but their number is very much smaller, the proportion of white to red corpuscles being about 1 to 350 .

The composition of normal human blood is about:

$\begin{array}{lrl}\text { Water, } & 79.50 \text { per cent. } \\ \text { Serum-albumin, } & 7.34 \text { “ } \\ \text { Fibrin, } & 0.21 & \text { "6 } \\ \text { Hæmoglobin, } & 11.64 & \text { “ } \\ \text { Fatty matters, } & 0.18 & \text { " } \\ \text { Extractives, } & 0.32 & \text { " } \\ \text { Ash, } & 0.81 \text { " }\end{array}$


Blood serum contains of water 90.5 per cent.; of solids 9.5 per cent., the latter being chiefly proteids 8 to 9 per cent., fats, extractives, and salts from 1 to 2 per cent. Wet blood-corpuscles contain of water 54.63 per cent., hæmoglobin 41.1 per cent., other proteids 3.9 per cent., fats (chiefly cholesterin and lecithin) 0.37 per cent. The quantity of water in corpuscles varies widely, and most likely ranges in healthy blood from 76 to 80 per cent.

The alkaline reaction of blood is due to the presence of acid sodium carbonate, $\mathrm{NaHCO}_{3}$, and sodium phosphate, $\mathrm{Na}_{2} \mathrm{HPO}_{4}$, both of which have a weak alkaline reaction. Besides these alkaline salts, blood also contains others, among them chiefly sodium chloride, and also the chlorides, phosphates, and sulphates of calcium, magnesium, sodium, potassium, etc.

When blood leaves the body and is allowed to stand a while (or, quicker, on shaking or agitating it violently) it separates into a semisolid mass termed clot, and a pale, yellow liquid termed serum, which latter, however, also solidifies after a time in consequence of the coagulation of the serum-albumin. Clot consists of fibrin, holding in its meshes the blood-corpuscles; the latter may be removed by washing the clot in a stream of water. Another method for obtaining the corpuscles is to dilute mammalian blood with 10 volumes of a 2 per cent. sodium chloride solution, which prevents coagulation, but allows the corpuscles to settle at the bottom of the fluid.

Fibrin exists most likely not as such in the blood, but forms after it leaves the body from two kinds of albumin, termed fibrinoplastic matter (identical with paraglobulin) and fibrinogen.

Hæmoglobin is the chief constituent of the red corpuscles and the substance which carries oxygen to the various tissues, as described in connection with the consideration of hæmoglobin itself.

Examination of blood stains. Blood stains may be recognized after having been washed off with as little water as possible, by the following methods:

1. Examine the reddish fluid under the microscope for bloodcorpuscles.

2. Evaporate a drop of the fluid on a microscopic slide with a fragment of sodium chloride, cover with a cover-glass, allow 
a drop of glacial acetic acid to enter from the side and warm gently: abundant crops of hæmin crystals are seen under the microscope.

3. Add a drop of the fluid to some tincture of guaiacum in a test-tube and float on surface ethereal solution of hydrogen dioxide: a blue ring forms at junction of the ethereal solution and the guaiacum. (Blood is, however, not the only substance showing this reaction.)

4. The spectroscope shows bands characteristic of hæmoglobin.

Chyle is a white, creamy liquid, of a strongly alkaline reaction, having in common with blood the property of coagulating (upon leaving the organism) into white fibrin and turbid serum. The composition of chyle differs according to the state of digestion; it contains :

\begin{tabular}{lcccc} 
& During full digestion. & \multicolumn{2}{c}{ During fasting. } \\
Water, & 91.8 per cent. & \multicolumn{2}{c}{96.8 per cent. } \\
Fibrin, & 0.2 “ & 0.09 & " \\
Proteids, & 3.5 & " & 2.30 & " \\
Fats, & 3.3 & " & 0.04 & " \\
Extractives, & 0.4 & " & 0.28 & " \\
Salts, & 0.8 " & 0.49 "
\end{tabular}

Lymph is a clear, colorless, or slightly yellow liquid, of a faint alkaline reaction; in composition it closely resembles chyle but differs from it in containing smaller quantities of fibrin and fatty matters.

Saliva is secreted by several glands situated in the mouth, and represents in its mixed condition a viscid, generally slightly alkaline liquid of a specific gravity 1.008. It contains of

$\begin{array}{lcc}\text { Water, } & 99.49 & \text { per cent. } \\ \text { Ptyalin, } & 0.12 \text { “ } \\ \text { Mucin, } & 0.13 \text { " } \\ \text { Fatty matters, } & 0.11 \text { " } \\ \text { Salts, } & 0.15 \text { " }\end{array}$

Plyalin, the active principle of saliva, is a ferment which has the power of converting starch into glucose; its composition is doubtful. Among the various salts of saliva is found potassium 
sulphocyanate, as may be shown by the addition of a drop of ferric chloride solution, which produces a deep red color, disappearing on the addition of mercuric chloride (difference from meconic acid).

Gastric juice is a liquid secreted by the follicles of the stomach; it has always a decidedly acid reaction, due to free hydrochloric acid, which is most likely formed by the action of sodium phosphate on calcium chloride :

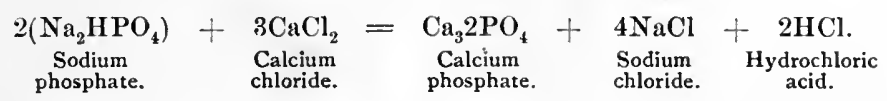

According to others the hydrochloric acid is liberated by the action of acid sodium carbonate on sodium chloride:

$$
\underset{\substack{\text { Sodium } \\ \text { acid carbonate. }}}{\mathrm{NaHCO}_{3}}+\underset{\substack{\text { Sodium } \\ \text { chloride. }}}{\mathrm{NaCl}}=\underset{\substack{\text { Sodium } \\ \text { carbonate. }}}{\mathrm{Na}_{2} \mathrm{CO}_{3}}+\underset{\text { Hydrochloric }}{\text { acid. }} \text { HCl. }
$$

The above formulas show the reverse action of that which these substances exert upon each other under common couditions, but it must be remembered that the living cell is capable of decomposing matter generally in a manner different from that which it suffers ordinarily.

Gastric juice contains of :

$\begin{array}{lr}\text { Water, } & 99.460 \text { per cent. } \\ \text { Pepsin, } & 0.302 \\ \text { Free hydrochloric acid, } & 0.022 \text { " } \\ \left.\begin{array}{c}\text { Alkaline chlorides, } \\ \text { Phosphates of calcium, } \\ \text { magnesium, and iron, }\end{array}\right\} & 0.200 \text { " }\end{array}$

Pepsin, the most important constituent of gastric juice, has been spoken of heretofore; it has, in the presence of free hydrochloric acid the power of converting proteids into peptones; it does not act on starch, but does not prevent the continued action of saliva on it; acting on fats, it dissolves the albuminous envelopes of the fat cells, but does not act chemically upon the fats themselves.

Bile, secreted by the liver, is a thin, transparent liquid of a golden-yellow color, and a specific gravity of 1.020 ; it has a 
very bitter taste and an alkaline reaction; it varies widely in composition, the total solids ranging from 9 to 17 per cent., being always highest after a meal ; its composition, moreover, is highly complex; the following is an average of five analyses of bile from subjects with healthy liver :

$\begin{array}{lcc}\text { Water, } & 91.68 \text { per cent. } \\ \text { Mucus pigment, } & 1.29 \text { " } \\ \text { Taurocholate of sodium, } & 0.87 & \text { " } \\ \text { Glycocholate of sodium, } & 3.03 & \text { " } \\ \text { Fat, } & 0.73 \text { " } \\ \text { Soaps, } & 1.39 \text { " } \\ \text { Cholesterin, } & 035 \text { " } \\ \text { Lecithin, } & 053 \text { " }\end{array}$

The true physiological action of bile is yet doubtful; it is, however, known that it acts on fats, forming with them an emulsion which renders their passage through animal membranes more easy; it also precipitates peptones, and it most likely serves as a disinfectant, preventing putrefaction of the organic matters during their passage through the intestines; it finally acts as a natural purgative by irritation of the muscular tissue of the intestines.

Bile obtained after death is of a brownish-yellow color; freed from mucus it will remain undecomposed for an almost indefinite period. The mucus may be separated by the addition of diluted alcohol and subsequent filtration.

The quantity of bile discharged daily by a grown person may be put at forty ounces, but a considerable quantity of this discharged bile is reabsorbed in a changed form by the intestines; only a small amount of bile matters (in a decomposed state, however) is discharged by the feces.

Biliary pigments. Of these four are known, but it is probable that more exist. Bilirubin, $\mathrm{C}_{32} \mathrm{II}_{36} \mathrm{~N}_{4} \mathrm{O}_{6}$, is, when amorphous, an orange-yellow powder; when crystalline, it forms red prisms. It is sparingly soluble in water, alcohol, and ether, readily soluble in hot chloroform and carbon disulphide. When treated with a mixture of concentrated nitric acid and sulphuric acid it turns first green, then blue, violet, red, and finally yellow. This reaction, known as Gmelin's test, is used for the detection of bile-pigments in urine and otler fluids. 
Biliverdin, $\mathrm{C}_{32} \mathrm{H}_{36} \mathrm{~N}_{4} \mathrm{O}_{8}$, is a green powder existing in green biles; it responds to Gmelin's test.

Biliary acids. Glycocholic acid, $\mathrm{C}_{26} \mathrm{H}_{43} \mathrm{NO}_{6}$, and taurocholic acid, $\mathrm{C}_{26} \mathrm{H}_{45} \mathrm{NO}_{7} \mathrm{~S}$, exist as sodium salts in the bile of man and most animals. Both salts may be obtained as colorless crystals, which dissolve in water, forming solutions of an acid reaction, and an intensely bitter taste. Both acids are easily decomposed by heating with alkalies or with diluted acids, also by the action of putrefying material or by the chemical changes taking place in the intestines. In all these cases is formed cholic acid, $\mathrm{C}_{24} \mathrm{H}_{40} \mathrm{O}_{5}$, and a second product, which in the case of glycocholic acid is glycocol, $\mathrm{C}_{2} \mathrm{H}_{5} \mathrm{NO}_{2}$, and in the case of taurocholic acid taurine, $\mathrm{C}_{2} \mathrm{H}_{7} \mathrm{NO}_{3} \mathrm{~S}$.

Pettenliofer's test. The biliary acids and their salts show a characteristic reaction known as Pettenkofer's test. This reaction is shown by adding some cane-sugar to the liquid substance under examination, and adding concentrated sulphuric acid in such a manner that the temperature does not rise above $70^{\circ}$. In the presence of biliary acids a beautiful cherry-red color is developed, which gradually changes to dark reddish-purple.

The bile acids are, however, not the only substances which show this reaction, and it, therefore, becomes in many cases necessary to separate the bile acids from other organic matter. This separation is accomplished by evaporating the substance under examination (urine, for instance), after having been mixed with a small quantity of coarse animal charcoal, to dryness at $100^{\circ}$. The residue is extracted with absolute alcohol, the filtered alcoholic solution is again partially evaporated, and mixed with 10 volumes of absolute ether. The biliary acids are soluble in alcohol, but not in ether, or in ether containing one-tenth of alcohol. After standing an hour or two, the biliary acids will form a deposit, which is collected on a small filter, dissolved in a little water, and mixed with a few drops of a strong solution of sugar. Upon the addition of sulphuric acid, with the precaution above mentioned, the characteristic colors will indicate the presence of the bile acids.

Cholesterin, $\mathbf{C}_{26} \mathbf{H}_{43} \cdot \mathbf{H O}$. This substance is classed by physiologists among the fats, because it is greasy and soluble in ether, 
but its chemical constitution is that of an alcohol. It is found chiefly in bile, but also in blood, nerve-tissue, brain, contents of the intestines, feces, etc.; its presence in certain vegetables, as peas, beans, etc., has also been demonstrated.

Cholesterin crystallizes in colorless, silky needles, which are insoluble in water, alkalies, and diluted acids, but soluble in ether. It sometimes forms in the organism solid masses, known as biliary calculi or gallstones, some of which are almost pure cholesterin.

\section{Tests for cholesterin:}

1. Evaporated with nitric acid it gives a yellow mass, which turns brick-red on addition of ammonia.

2. Mixed in the dry state with strong sulphuric acid, it produces a blue-red or violet color on the addition of chloroform, the color changing to green on exposure to air.

3. Evaporated with a mixture of 2 volumes of sulphuric acid and 1 volume of ferric chloride solution, it turns violet.

Lecithins. Lecithin, one of the constituents of bile, is the member of a group of substances generally termed phosphorized fats or lecithins. These bodies are highly complex in composition, and may be looked upon as fats formed from glycerine, in which hydrogen atoms are replaced by the radicals of phosphoric and fatty acids.

Pancreatic juice. There is no thoroughly reliable analysis of this highly complex liquid on record. It contains from 3 to 6 per cent. of solids, two-thirds of which are of organic, one-third of inorganic nature. Among the organic constituents are a number (certainly two, probably more) of cryptolites or animal ferments, which manifest themselves: 1 , by converting starch into sugar (this action is more energetic than that of ptyalin); 2 , by converting albuminoids into peptones (this action takes place in alkaline, but not in acid solution, as in case of pepsin); 3 , by emulsifying neutral fats; 4 , by decomposing fats into gly- . cerine and fatty acids.

Feces consist of that portion of the food which has not been taken into the system by absorption, and is discharged from the body mixed with some of the products of the biliary and intes- 
tinal secretions. The quantity passed depends on the nature of the food taken, and on the energy of the digestive powers. A grown person, in normal condition, discharges from 7 to 9 ounces daily. An approximate analysis of the feces of a healthy adult shows:

\begin{tabular}{|c|c|c|}
\hline Water, & \multicolumn{2}{|c|}{77.3 per cent } \\
\hline Mucin, & 2.3 & " \\
\hline Proteids, & 5.4 & $\because$ \\
\hline Extractives, & 1.8 & “ \\
\hline Fats, & 1.5 & " \\
\hline Salts, & 1.8 & " \\
\hline $\left.\begin{array}{c}\text { Resinous, biliary, and } \\
\text { coloring matters, }\end{array}\right\}$ & 5.2 & "6 \\
\hline Insoluble residue of food, & 4.7 & " \\
\hline
\end{tabular}

Bone is chemically distinguished from other tissues by the large quantity of inorganic salts which it contains. Bones contain about 35 per cent. of organic matter combined with 65 per cent. of mineral matter. Different bones (and even different parts of the same bone) of the same person differ somewhat in composition; moreover, the bones of a child contain somewhat more of organic matter than those of a grown person, as may be shown by the following analyses of the corresponding bone in children and a grown person:

Child one year. Child five years. 'Man twenty-five years.

Organic matter,

Tricalcium phosphate

Magnesium phosphate,

Calcium carbonate,

Soluble salts,

\subsection{2 per cent.}

48.55 "

1.00 "

5.79 "

1.24 "
32.29 per cent.

59.74 "

1.34 "

6.00 "

0.63 "
31.17 per cent.

58.95 " "

1.30 "

7.08 "

1.50 "

Frequently human bones contain calcium fluoride, which substance, to the amount of 1 to 2 per cent., is a normal constituent of the bones of many animals. The organic matter of bone is called ossein, and is a mixture of collagen, elastin, and an albuminoid existing in the bone-cells. Collagen is a nitrogenous substance, insoluble in water, but forming when treated with it under the influence of heat and pressure, gelatine, an amorphous, tasteless, translucent substance, which swells up in boiling water, forming on cooling a soft jelly; an impure form of gelatine is common glue. 
Teeth are similar in composition to bone, but contain even less of organic matter; the enamel containing as much as 96 to 98 per cent. of inorganic matter; traces of fluorides and silicium are found in the teeth.

Hair, nails, horns, hoofs, feathers, epithelium, are nearly identical in composition. They all contain a nitrogenous substance, termed keratin, which is probably not a distinct chemical compound, but a mixture of several substances similar in composition and properties.

Mucus is secreted by the various mucous membranes, and is found in saliva, bile, connective tissues, feces, urine, etc. When pure it forms a clear, translucent or viscid mass; it contains a substance termed mucin, which swells up in water, and readily dissolves in water containing an alkali; from these solutions it is precipitated by acetic acid.

Muscles contain fibrin, albumin, myosin, kreatin, $\mathrm{C}_{4} \mathrm{H}_{9} \mathrm{~N}_{3} \mathrm{O}_{2}$, sarkin, $\mathrm{C}_{5} \mathrm{H}_{4} \mathrm{~N}_{4} \mathrm{O}$, xanthin, $\mathrm{C}_{5} \mathrm{II}_{4} \mathrm{~N}_{4} \mathrm{O}_{2}$, uric acid, glucose, inosite, lactates, and salts.

Kreatin, sarkin, and xanthin are substances formed in the organism by oxidation of proteids, and may be looked upon as compound ureas or substances formed as intermediate products of the tinal conversion of proteids into urea, carbon dioxide, water, etc. These substances may indeed be decomposed artificially, in such a manner that urea is produced as one of the produrcts of decomposition.

Brain consists of so many individual parts that the analysis of it as a whole is of little value, and to separate these parts successfully is a task not jet accomplished. Brain, as a whole, contains cercbrin, lecithin, neurin, cholesterin, and many other substances, some of which are distinguished by the large quantity of phosphorus they contain.

\section{Questions.}

501. What three kinds of matter are found as constituents of the animal body, and how can they be determined quantitatively?

502. Mention the chief constituents of blood, and state those which predominate in serum and in the corpuscles respectively. 
503. What substances cause the clotting of blood, how are they separated, and what are their properties?

504. How may blood stains be recognized?

505. What is the active principle of saliva, and how does it act on starch?

506. State the composition of gastric juice, and explain its physiological action.

507. State the general properties of bile, and mention its chief constituents.

508. Give Gmelin's test for biliary pigments, and Pettenkofer's test for biliary acids. What precautions are necessary in "using the latter test?

509. What is cholesterin? State its properties and reactions.

510. Mention the principal constituents of muscles, bone, teeth, and hair.

\section{MILK.}

Properties and composition. Milk is the secretion of the mammary glands, the presence of which is characteristic of the class of animals known as mammalia. The milk of different animals differs somewhat in composition, but it always contains all the constituents necessary for a normal development of the various tissues, liquids, organs, etc., of the young mammal, which generally feeds exclusively upon milk for a shorter or longer period of its early life.

Milk is a slightly alkaline, aqueous solution of casein, albumin, lactose, and-inorganic salts, holding in suspension small globules of fat. Its specific gravity ranges from 1.029 to 1.033 .

The average composition of various kinds of milk is given below, but, it must be remembered that milk not only differs in certain species, but also in the same animal at different times; for instance, the quality and quantity of food taken, as also various physiological changes, have a decided influence upon the milk secreted.

\begin{tabular}{lrcrcc} 
& \multicolumn{3}{c}{ Human milk. } & \multicolumn{2}{c}{ Cow's milk. } \\
Wariations. & Average. & Variations. & Average. \\
Water, & 90.4 to 85.7 & 88.05 & 90.2 to 83.7 & 86.95 \\
Casein and albumin, & 1.8 to 3.1 & 2.45 & 33 to 5.5 & 4.40 \\
Fut (butter), & 3.0 to 3.8 & 3.40 & 2.8 to 4.5 & 3.65 \\
Lactose, & 4.5 to 7.0 & 5.75 & 3.0 to 5.5 & 4.25 \\
Inorganic salts, & 0.3 to 0.4 & 0.35 & 0.7 to 0.8 & 0.75
\end{tabular}




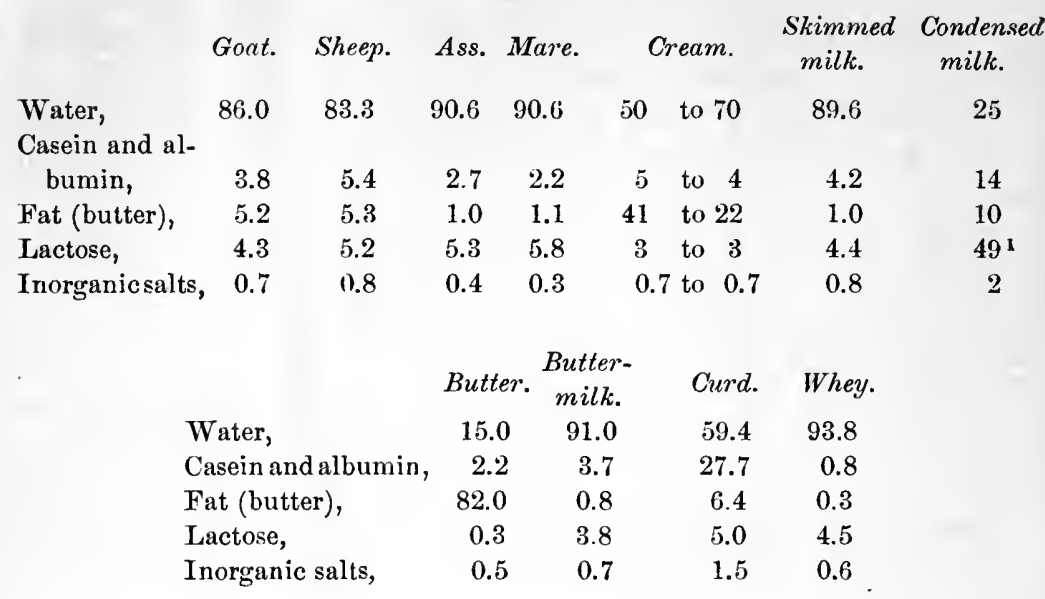

The inorganic salts consist chiefly of calcium or sodium phosphate and sodium and potassium chloride, but contain also some magnesium and iron.

The proteids consist mainly of casein with some albumin, the proportion being about as 9 to 1 .

Beside the constituents mentioned in the above analyses, milk also contains a very small quantity of extractives, among which are found peptone, kreatin, leucin, etc. The principles which give to milk its peculiar odor have not yet been conclusively pointed out.

Changes in milk. Soon after milk leaves the animal system changes take place which are either of a physical or chemical nature. The first change in milk, when allowed to stand for a few hours, is a separation of the suspended fat globules towards. the upper part of the liquid, which gradually becomes loaded with fat, forming a distinct layer over the liquid. This upper layer having a slightly yellowish color (cream-color) is cream, the watery liquid below having a bluish-white color is skimmed milk.

Another change taking place in milk (rarely after a few hours, but generally after a day or a few days) is the coagulation of casein, which takes place both in the cream and in the skimmed milk, converting the whole into a thick, semi-liquid mass,

1 Including cane-sugar added by the manufacturer. 
which gradually separates into a solid white curd, and a thin, transparent milk-serum or whey.

The coagulation of the casein is caused by lactic acid, produced by the so-called lactic fermentation of lactose. The ferments causing this fermentation are undoubtedly floating in the air, as it is possible to prevent the decomposition of milk-sugar for a considerable length of time by taking proper precautions for destroying and excluding them. Simultaneously with the coagulation of milk the alkaline reaction becomes acid and the sweet taste gradually more and more sour.

These changes in milk can to some extent be artificially produced, hindered, and controlled. Thus, the casein may be precipitated by the addition of rennet or acetic acid (or any mineral acid) and heating. The decomposition of the milksugar and with it the "curdling" may be prevented-1, by chemical treatment with alkaline salts or antiseptics; 2 , by physical treatment, such as cooling or icing, boiling and aeration; 3 , by condensation or evaporation, with or without the addition of a preservative agent. All these systems of preservation, however, are subject to serious disadvantages because they either interfere with the natural constitution and properties of the milk, or because they serve their purpose for too limited a time.

The addition of alkalies such as lime-water, sodium carbonate or bicarbonate, does not prevent the lactic fermentation, but prevents the action of the liberated acid on the casein by forming a lactate of calcium or of sodium.

Of antiseptics, salicylic acid has been used with good results (2 grains to a pint).

Of all preservatives, cold is the most efficient and least objectionable, and milk when cooled to within a few degrees of the freezing-point may be kept for 8 to 12 days sweet and without change.

The condensation of milk is effected either simply by evaporating (generally in vacuum pans) a portion of the water, or by first dissolving in it a certain quantity of sugar (generally canesugar) and then evaporating to the consistence of a thick syrup, which is placed in suitable air-tight jars. The sugar which is added serves as an additional preventive of decomposition.

The following gives the constituents of milk which may be 
obtained from it by mechanical processes after it has been changed as described above:

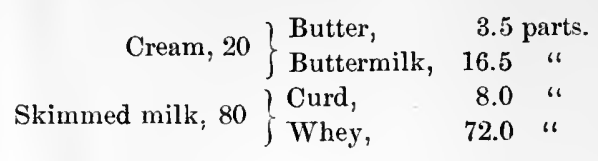

Butter. Even in the thickest varieties of cream there is no cohesion between the fat globules, whilst in butter the fat has actually cohered. This change is accomplished by violently agitating (churning) the crean, when the fat particles gradually combine with each other, whilst the liquid (buttermilk) separates.

Chemically, butter is milk-fat, a mixture of different fats or glycerides of the fatty acids, chiefly palmitic and butyroleic acid, with butyric, capronic, and caprylic, and occasionally stearic acid; it always contains a certain proportion, 15 to $\mathbf{1 6}$ per cent., of water, besides traces of casein, salts, coloring matter, etc.

For curing butter, common salt is often used; the quantity added should not exceed 5 per cent.

The composition of buttermilk has been given above; when freshly obtained from sweet cream it is a pleasant drink and a wholesome food.

Cheese consists mainly of casein, milk-fat, water, and inorganic salts; these constituents vary as follows:

Water, 61 to 28 parts.
Casein, 15 to 35 "
Fat, 20 to 30 "
Salts, 4 to 7 "

Cheese is made either from pure milk, from skimmed milk, or from a mixture of milk and cream, and accordingly varies highly in composition. Practically, cheese is made by causing milk to coagulate (either by allowing it to stand or by the addition of rennet, acids, or other substances), and separating the curd (casein and fat) from the whey by mechanical means, such as filtering and pressing. The curd is placed in suitable moulds and afterwards allowed to stand or "ripen" for a shorter or 
longer period. The process of ripening is a partial decomposition (decay and putrefaction) of the casein, and the value of cheese depends mainly upon the nature of the products formed during this decomposition.

Adulterations of milk. Of these, the most commonly practised are removal of cream, addition of water, or both. Sometimes sodium carbonate, sugar, and even chalk are added, but these latter adulterations are fortunately but rarely practised by milkdealers. The question whether or not milk has been tampered with is generally decided by ascertaining whether cream has been removed or water added. It is, therefore, chiefly the quantity of total solids which has to be determined in order to decide the purity of milk. But it has been shown by the above tables of milk analysis that the quantity of these solids varies considerably, and a minimum of total solids-should therefore be adopted legally. While no such minimum quantity is officially recognized in most States of this country, it is safe to say that milk containing less than $\mathbf{1 1}$ per cent. of total solids may be looked upon as adulterated. (The above given lowest quantity of 9.8 per cent. of total solids in cow's milk is very abnormal.) The methods for detecting such fraud will be considered below.

Testing milk. There is, unfortunately, no instrument which will indicate the purity or quality of milk directly. An instrument heretofore used for that purpose (especially in France), and known as the lactometer, is simply a hydrometer which indicates the specific gravity of milk. There are, however, in milk substances which have a tendency to increase the specific gravity, such as lactose, salts, and casein, whilst there is at the same time one substance, the fat, which is specifically lighter than water. The specific gravity of milk ranges from 1027 to 1034, the average being about 1030. If water be added to milk, the specific gravity will become lower, but the same effect may be obtained by adding fat or cream. Again, if cream be removed, the specific gravity will be higher, and in order to bring the milk back to the standard of 1030 , water may be added. In other words, cream may be removed from and water added to the same milk and the specific gravity will be unchanged; or a 
natural milk containing large quantities of fat has the same specific gravity of a poorer milk to which water has been added.

These facts show that the lactometer alone is of no value whatever in milk analysis, although it is useful in determining the quantity of eream present. This is generally accomplished by the so-called creamometer, a glass tube or glass eylinder about one foot long, half' an inch in diameter, and graduated into 100 parts by volume, the 0 being about an inch from the top. The tube is filled with milk to the 0 and set aside for 12 or 18 hours, when the line of demarcation between the cream and the liquid below is well defined and may be easily read off.

The quantity of cream varies from 8 to 20 per cent., but should not fall below 10 per eent. Milk which shows a large quantity of cream (15 to 18 per cent.) may fall considerably below 1030 in specific gravity, but if there is little cream (8 to 10 per cent.) and the milk shows a low specific gravity, there ean be little doubt that it has been tampered with.

There are a number" of other instruments, the so-called "lactoscopes," used for the determination of cream, the operations of which are based on the fact that milk rich in cream is a much more opaque (or more white) Huid than that from which eream has been taken or to which water has been added.

One of the lactoscopes has been introduced under the name of pioskop, and consists of a round disk about two and a half inches in diameter, with a shallow disk-like depression in the centre, in which a few drops of milk are placed. This is then covered by a glass disk, transparent in the middle where it rests. on the milk, the rim being covered by six radial strips of oilpaint, varying from white to dark gray, and marked with the quality corresponding to it, from "cream" to "very poor milk." The color of the thin layer of milk, as seen through the transparent part of the glass plate, corresponds with one of the six color strips, and its quality is thus readily, though not very accurately, deternined.

The above methods of determining the purity of milk, although answering for ordinary purposes, are absolutely insufficient for scientific purposes or as evidence upon which to base legal proceedings. In such cases a complete analysis, including the 
exact determination of total solids and of the various constituents, is required.

Analysis of milk. The total solids are determined by placing a weighed quantity (from 5 to 10 c.c.) of the well-mixed milk in a weighed platinum dish and heating for several hours in a water-bath until no more weight is lost. The loss in weight represents the water, the weight of the residue the total solids. The fat is determined by extracting the solid residue repeatedly with warm ether, filtering this solution through a small filter, which is to be well washed with ether, and evaporating the ethereal solution in a weighed platinum dish.

Milk-sugar is next determined by treating the residue (from which fat has been extracted) with hot diluted alcohol; lactose and a few soluble salts enter into solution; the liquid evaporated to dryness in a weighed dish, gives the quantity of sugar plus some salts. Upon igniting the milk-sugar a residue of salts is left, which is also weighed and this weight deducted from the first one.

Casein. The residue now left (after treatment with ether and alcohol) contains chiefly casein with some albumin and salts. If any casein should have been washed upon the filters accidentally, it has to be transferred back to the dish, the contents of which are dried and weighed. By burning off the casein and reweighing the dish plus the salts, the quantity of the casein is determined.

The remaining salts added to those previously obtained from the alcoholic solution form the total ash or inorganic solids, an analysis of which may be made according to the methods given heretofore.

Casein may also be determined directly by precipitating it from milk, by the addition of acetic acid and boiling. The precipitated casein is filtered off, and has to be well washed, first with water, and then with ether, as it contains most of the fat.

\section{Questions.}

511. Mention the five principal coustituents of milk.

512. Give the average composition of human and of cow's milk.

513. What compounds constitute milk-ashes? 
514. What physical and what chemical changes does milk suffer on standing?

515. What acid is formed in milk on standing, and how does this acid act on the casein?

516. Describe the processes used for preventing the decomposition of milk. What are their advantages and their disadvantages?

517. Give the quantities of the chief components of cream, skimmed milk, butter, buttermilk, curd, whey, and cheese, and state how these substances are obtained.

518. Why does the specific gravity of milk not indicate its purity and richness?

519. Describe the advantages of the combined use of the lactometer and creamometer in testing milk.

520. Give a process for the complete quantitative analysis of milk.

\section{URINE AND ITS NORMAL CONSTITUENTS.}

Secretion of urine. It has been explained in a former chapter how blood absorbs the digested food as chyle, how this is acted upon by the atmospheric oxygen in the lungs, and how this arterial blood, whilst passing through the system, deposits albuminous and other substances, receiving in exchange the products formed by the oxidation of the various tissues. These products are either gases (chiefly carbon dioxide), liquids (chiefly water), and solids held in solution by the water. These waste solids must necessarily be eliminated from the system, and the organs which accomplish this result are the kidneys.

The process of separating the waste materials from the blood is chiefly of a physical nature, partly a transudation or filtration, and partly a diffusion or osmosis. The conditions essential for such an exchange are given in the kidneys. Blood is separated by delicate membranes from a thin, aqueous, saline solution; the interchange taking place is chiefly a passage of the waste crystalline products of the blood into the aqueous solution, which is thereby gradually converted into urine, that liquid, which is finally discharged, carrying off nearly the total quantity of all the nitrogen taken into the system in the form of nitrogenous food.

General properties. Normal human urine, when in a fresh state, is a clear and transparent aqueous liquid, of a lighter or 
deeper amber color, having a peculiar, faintly aromatic odor, a bitter, saline taste, a distinct acid reaction on blue litmus-paper, and a specific gravity heavier than water (average about 1020). When urine is kept in a clean vessel it may remain unchanged for several days, provided the temperature be not too high, and the amount of total solid constituents not too small.

In urine, shortly after cooling, especially if it be concentrated, a light, cloudy film of mucus is formed, which slowly sinks to the bottom; the acid rcaction gradually increases, small yellowish-red crystals of acid urates, or uric acid, are deposited. In this condition, the urine may often continue unchanged for several weeks, provided the temperature be low. If, however, the urine be very dilute, and the temperature above the mean, a decomposition speedily takes place. The urine is then found to be covered with a thin, shining, and frequently iridescent membrane, fragments of which sink gradually to the bottom. The urine then becomes turbid, acquires a pale color, its reaction becomes alkaline, and it begins to develop a nauseous ammoniacal odor, due to the products formed during the decomposition of urea and other substances.

Composition. Urine is chiefly an aqueous solution of urea and inorganic salts, containing, however, always some uric acid, mucus, coloring and other organic matters. The average composition of normal human urine may be stated thus :

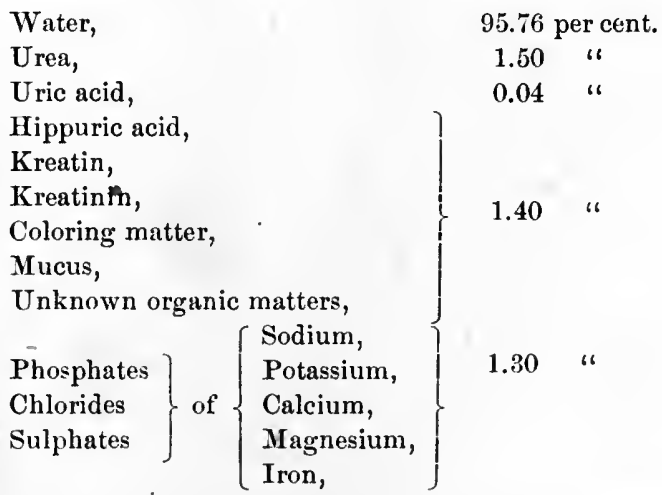

The above average composition of human urine varies considerably, and is influenced by the water and food taken, amount of work done, time of day, temperature of air, age, sex, etc. 
The quantity of urine passed in a day also varies widely, an adult discharging from 500 to 2300 e.c. in twenty-four hours. The quantity of total solids contained in this urine varies from 60 to 120 grams, and of urea from 20 to 40 grams.

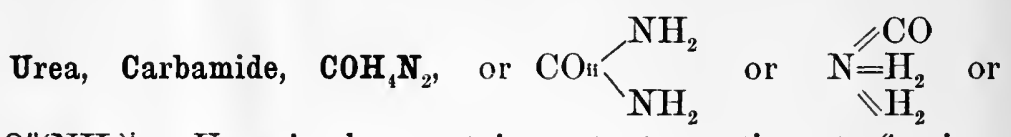
$\mathrm{CO}^{\prime \prime}\left(\mathrm{NH}_{2}\right)_{2}$. Urea is the most important constituent of urine, and is the substance which carries off by far the largest quantity of all nitrogen taken in the food. Urea has never yet been found as a product of regetable life, but is found as a normal constituent of the urine of the mammalia, and in smaller quantity in the excrement of birds, fishes, and some reptiles. It occurs also in the blood, muscular tissue, chyle, lymph, bile, perspiration, and many other animal fluids.

When pure, urea crystallizes from an aqueous solution in colorless prisms; it is odorless, and has a cooling, bitter taste; it easily dissolves in water, the solution having a neutral reaction; it fuses when heated to $130^{\circ}$, but decomposes at a higher temperature, giving off ammonia gas and water, whilst a nunıber of other substances are formed at the same time. A pure solution of urea does not decompose at ordinary temperature, but on boiling, and especially under pressure, it takes up water, and is decomposed into ammonia or carbon dioxide, or into ammonium carbonate :

$$
\mathrm{CO}\left(\mathrm{NH}_{2}\right)_{2}+\mathrm{H}_{2} \mathrm{O}=\mathrm{CO}_{2}+2 \mathrm{NH}_{3} \text {. }
$$

The same decomposition takes place in urine under the influence of a ferment (most likely present in urine, or perhaps derived from the air), if the temperature be not too low.

A solution of urea is decomposed by the action of chlorine or bromine with generation of hydrochloric (or hydrobromic) acid, carbon dioxide, and nitrogen :

$$
\mathrm{CO}\left(\mathrm{NH}_{2}\right)_{2}+6 \mathrm{Cl}+\mathrm{H}_{2} \mathrm{O}=6 \mathrm{HCl}+\mathrm{CO}_{2}+2 \mathrm{~N} \text {. }
$$

Alkaline hypochlorites or hypobromites cause the similar decomposition, upon which is based the quantitative estimation of urea. 
Urea forms with acids definite salts and with certain oxides and salts definite compounds.

Urea is formed artiticially by numerous decompositions, as, for instance :

$a$. By a process similar to the one taking place in the animal system, viz., by limited oxidation of albuminous substances by potassium permanganate.

$b$. By oxidation of uric acid in the presence of water:

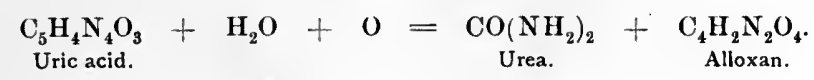

c. By the action of caustic alkalies upon kreatin :

$$
\underset{\text { Kreatin. }}{\mathrm{C}_{4} \mathrm{H}_{9} \mathrm{~N}_{3} \mathrm{O}_{2}}+\mathrm{H}_{2} \mathrm{O}=\underset{\text { Urea. }}{\mathrm{CO}\left(\mathrm{NH}_{2}\right)_{2}}+\underset{\text { Sarcosine. }}{\mathrm{C}_{3} \mathrm{H}_{7} \mathrm{NO}_{2}}
$$

d. By the molecular transformation of ammonium cyanate, which takes place when its solution is evaporated and allowed to crystallize:

$$
\underset{\substack{\text { Ammonium } \\ \text { cyanate. }}}{\mathrm{NH}_{4} \mathrm{CNO}}=\underset{\text { Urea. }}{\mathrm{CO}\left(\mathrm{NH}_{2}\right)_{2} .}
$$

e. By the action of ammonia on carbonyl chloride :

$$
\underset{\substack{\text { Carbonyl } \\
\text { chloride. }}}{\mathrm{COCl}_{2}}+\underset{\text { Ammonia }}{2 \mathrm{NH}_{3}}=\underset{\begin{array}{c}
\text { Hydrochloric } \\
\text { acid. }
\end{array}}{2 \mathrm{HCl}}+\underset{\text { Urea. }}{\mathrm{CO}\left(\mathrm{NH}_{2}\right)_{2} .}
$$

$f$. By the action of ammonia on ethyl carbonate:

$$
\underset{\substack{\text { Ethyl } \\ \text { carbonate. }}}{\left(\mathrm{C}_{2} \mathrm{H}_{5}\right)_{2} \mathrm{CO}_{3}}+\underset{\text { Ammonia. }}{2 \mathrm{NH}_{3}}=\underset{\text { Ethyl alcohol. }}{2\left(\mathrm{C}_{2} \mathrm{H}_{5} \mathrm{HO}\right)}+\underset{\text { Urea. }}{\mathrm{CO}\left(\mathrm{NH}_{2}\right)_{2} .}
$$

Urea may be obtained from urine by evaporating it to the consistency of a syrup and mixing the cooled residue with an equal volume of nitric acid, when crystals of urea nitrate, $\mathrm{CO}\left(\mathrm{NH}_{2}\right)_{2} \cdot \mathrm{HNO}_{3}$, form. These crystals are washed with a little cold water, dissolved in hot water, and the solution decomposed by potassium carbonate, in which case potassium nitrate, free urea, carbon dioxide, and water are formed. Upon evaporating the solution crystals of potassium nitrate form, which are separated from the mother liquor, which is evaporated to dryness and exhausted by alcohol, which dissolves the urea; the some- 
what evaporated alcoholic solution yields crystals of urea, which are generally more or less colored by urinary pigments.

Determination of urea. There are no very characteristic reactions by which urea can be well recognized. From organic mixtures it is separated by digesting them with from 3 to 4 volumes of alcohol in the cold; the filtered liquid is evaporated to dryness and extracted with alcohol, which again is evaporated. The dry residue may be tested for urea as follows :

1. Dissolved in a few drops of water, the addition of an equal quantity of colorless nitric acid causes the formation of white, shining, crystalline plates or prisms of urea nitrate.

2 . If a strong solution of oxalic acid is added, instead of nitric acid, rhombic plates of urea oxalate form.

3. The residue (or urea) heated in a test-tube to about $160^{\circ}$, until no more vapors of ammonia are evolved, leaves a substance termed biuret, $\mathrm{C}_{2} \mathrm{H}_{6} \mathrm{~N}_{3} \mathrm{O}_{2}$, which upon the addition of a few drops of potassium hydrate solution and a drop of cupric sulphate solution, causes the solution of the cupric hydrate with a reddishviolet color.

The quantitative estimation of urea in urine may be effected by various methods, of which but one will be mentioned, because it requires less time and less skill in manipulation than most other methods. This determination is based upon the fact that urea is decomposed by alkaline hypobromites into carbon dioxide, water, and nitrogen:

$$
\underset{\text { Urea. }}{\mathrm{CO}\left(\mathrm{NH}_{2}\right)_{2}}+\underset{\substack{\text { Sodium } \\
\text { hypobromite. }}}{3(\mathrm{NaBrO})}=\underset{\substack{\text { Sodium } \\
\text { bromide. }}}{3 \mathrm{NaBr}}+\underset{\begin{array}{c}
\text { Carbon } \\
\text { dioxide. }
\end{array}}{\mathrm{CO}_{2}}+\underset{\text { Water. }}{2 \mathrm{H}_{2} \mathrm{O}}+\underset{\text { Nitrogen. }}{2 \mathrm{~N} .}
$$

The liberated nitrogen is collected, and from its volume its weight and that of the urea are calculated.

Practically the operation is conducted as follows: 100 grams of sodium hydrate are dissolved in 250 c.c. of water, and to this cooled solution are added 25 c.c. of pure bromine, when sodium hypobromite is formed, leaving, however, an excess (over one-half) of the sodium hydrate in an unaltered condition. (The solution easily decomposes, and should, therefore, be freshly prepared for analysis.)

The apparatus required (Fig. 16) consists, in its most simple 
form, of a wide-mouth bottle $\mathrm{A}$; a small test-tube $\mathrm{B}$, of about 10 c.c. capacity; a glass cylinder $\mathrm{C}$, and a graduated burette $\mathrm{D}$.

Into the bottle is fitted a perforated cork, which is connected by means of tubing with the burette. 5 c.c. of urine are introduced into the test-tube and 20 c.c. of the alkaline hypobromite solution into the bottle, care being taken not to bring the

FIG. 16.

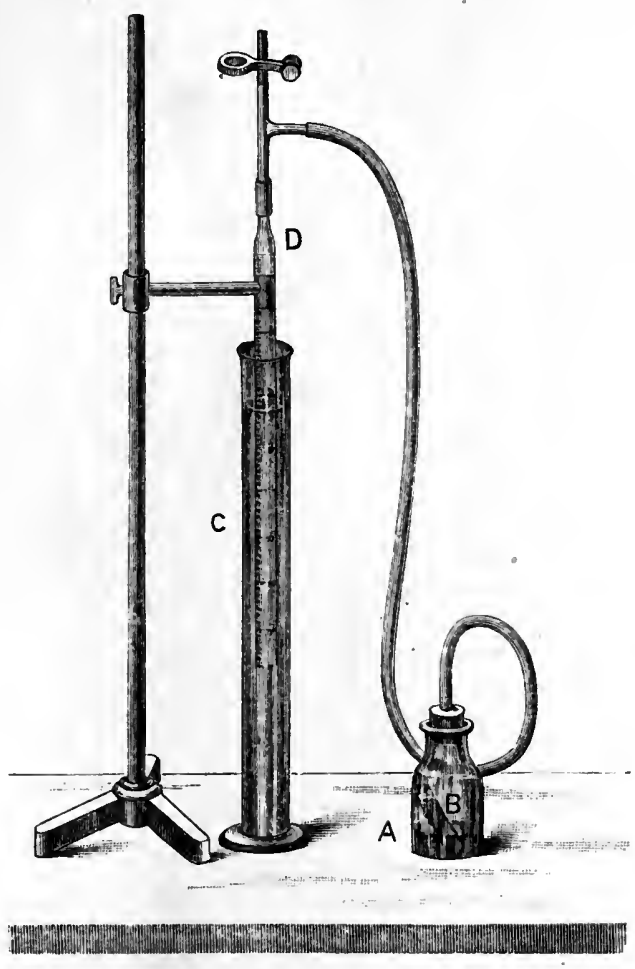

Apparatus for the volumetric estimation of urea.

liquids in contact with each other. The graduated burette is lowered in the cylinder, until the zero mark is on a level with the surface of the water in the cylinder and the connection between the burette and the bottle made. By now inclining the bottle so that the urine comes in contact with the hypobromite, decomposition of urea takes place energetically. The liberated carbon dioxide is absorbed by the sodium hydrate while the 
nitrogen increases the volume of air present in the apparatus. The burette is gradually raised as the nitrogen is evolved and the whole allowed to stand for half an hour. The cubic centimeters of nitrogen gas are read off (whilst the water in the burette and cylinder are on a level), and give, multiplied by 0.0027 , the grams of urea in 5 c.c. of urine.

As the volume of a gas depends upon temperature and pressure, corrections for these have to be made by using the following formula:

$$
p=\frac{100 . v b}{760.370 . a(1+0.003665 t)}
$$

$p=$ Weight of urea for 100 c.c. urine.

$a=$ Volume of urine used, expressed in c.c.

$v=$ Volume of nitrogen read off.

$b=$ Barometric pressure in $\mathrm{mm}$.

$t=$ Temperature during the measurement of nitrogen.

370 represents the c.c. of nitrogen (at $0^{\circ}$ and $760 \mathrm{~mm}$. pressure) obtained from one gram of urea.

The above-described process for estimation of urea is for various reasons far from being perfect (uric acid and kreatinin, for instance, are also decomposed with liberation of nitrogen), but it has been found that the results obtained are quite sufficient for clinical purposes.

Uric acid, $\mathrm{H}_{2} \mathrm{C}_{5} \mathrm{H}_{2} \mathrm{~N}_{4} \mathbf{O}_{3}$. Uric acid is found in small quantities in human urine, chiefly in combination with sodium, potassium, and ammonium, but also with calcium and magnesium. In larger proportions, uric acid is found in the excrement of birds, mollusks, insects, and chiefly of serpents, the solid urine of the latter consisting almost entirely of uric acid and urates. It is also found in Peruvian guano.

Pure uric acid is a white, crystalline, tasteless, and odorless substance, almost insoluble in water, requiring 1900 parts of boiling and 15,000 parts of cold water for its solution; it is also insoluble in alcohol and ether. The great insolubility of uric acid causes to its separation in the solid state, both in the bladder and in the tissues.

Determination of uric acid. Uric acid may be recognized by its crystalline form, and by the murexid test, which is made by 
placing a framment of uric acid in a porcelain dish, adding a drop of nitric acid, and carefully evaporating over a flame. To the dry residue a drop of ammonia water is added, which produces a beautiful purplish-red color. This reaction occurs, however, also with a number of substances which are similar to, but more complex in composition than, uric acid.

The quantitative estimation of uric acid in urine is best accomplished by adding 10 c.c. of hydrochloric acid to 250 c.c. of urine, setting aside for 24 hours in a cool place, aud collecting the crystals of uric acid on a small filter, which has been previously weighed. The crystals are washed with a little water, and dried at $100^{\circ}$.

If the urine (to be tested for uric acid) be very dilute, it should be evaporated to about one-half its bulk before adding hydrochloric acid; if it contain albumin, this should be removed by adding a drop of acetic acid, boiling, and filtering.

Hippuric acid, $\mathrm{C}_{9} \mathrm{H}_{9} \mathrm{NO}_{3}$ (Benzyl-glycocol, Benzyl-amido-acetic acid), is a normal constituent of human urine, but is found in much larger quantities in the urine of herbivora. Its constitution may be considered as ammonia in which two hydrogen atoms are replaced by the radicals of benzoic and acetic acid respectively, thus, $\underset{-\mathrm{H}}{\mathrm{N}} \underset{-\mathrm{C}_{2}}{-\mathrm{C}_{3} \mathrm{H}_{3} \mathrm{O}} \mathrm{O}_{2}$. Hay, and especially aromatic herbs, contain benzoic acid, or compounds having a similar composition, and a portion of these compounds is eliminated in hippuric acid. Administration of benzoic acid also increases the amount of hippuric acid in urine.

When pure, hippuric acid crystallizes in transparent, colorless, odorless prisms, which have a bitter taste, and are sparingly soluble in water.

Analytically hippuric acid is characterized:

1. By giving a sublimate of benzoic acid, and an odor of hydrocyanic acid, when heated in a dry test-tube.

2. By giving a brown precipitate with ferric chloride.

3. By giving off benzene and ammonia, when heated with calcium hydrate.

Other organic substances, such as kreatin, kreatinin, xanthin, lactic acid, mucus, coloring matters, etc., occur in such small 
quantities in normal urine that their detection, separation, and quantitative estimation are very difficult, and almost exclusively attempted during scientific investigations.

\section{Questions.}

521. What is urine, where and by what process is it formed in the animal body, and what is its function?

522. Mention the general physical and chemical properties of urine.

523. Give the average composition of human urine, and state by what conditions the composition is influenced.

524. State the composition and properties of urea.

525. By what process is urea formed in the animal body, and how can it be obtained artificially?

526. Describe a process by which urea may be estimated quantitatively in urine.

527. In what forms is uric acid found in urine, and what are its properties?

528. Describe the murexid test.

529 . How can uric acid be determined quantitatively in urine?

530 . What is hippuric acid, and by what tests may it be recognized ?

\section{EXAMINATION OF NORMAL AND ABNORMAL URINE.}

Points to be considered in the analysis of urine. They are:

1. Color, odor, general appearance, whether clear, smoky, clondy, turbid, etc.

2. Reaction: whether acid, neutral, or alkaline to test-paper.

3. Specific gravity.

4. Total amount of organic and inorganic solids.

5. Total amount of inorganic matter (ash).

6. Determination of urea.

7. Determination of uric acid.

8. Determination of inorganic acids and bases. (Hydrochloric, sulphuric, and phosphoric acids; sodium, potassium, calcium, magnesium, and iron.)

9. Determination of albumin.

10. Determination of sugar.

11. Examination for bile. 
12. Examination of any organic or inorganic sediment, either by chemical means or by the microscope.

Samples of urine should always be drawn from the wellmixed and exactly measured quantity of the total urine discharged in 24 hours.

Color. Normal urine is generally pale yellow or reddish-yellow, but it may be as colorless as water, or as dark brownishblack as porter; a smoky tint generally indicates the presence of blood, and a brownish-green suggests the presence of the coloring matter of bile.

The true nature of the normal coloring matters of urine is as yet doubtful; the existence of at least two has, however, been demonstrated; they have been named urobilin and urine-indican or uroxanthin, and are, most likely, products of the decomposition of biliary matters.

Abnormal coloring matters are chiefly those of blood, bile, and of certain vegetables; thus, rhubarb and senna leaves cause a reddish-yellow to deep red color, especially in alkaline urine; santonin produces a bright yellow color, changing to red or crimson on the addition of an alkali. Carbolic acid introduced into the system causes a dark, or even black discoloration of urine.

The coloring matters of blood may be recognized by adding to a few drops of urine a drop of tincture of guaiacum, and agitating with a solution of ozonized ether (ethereal solution of hydrogen dioxide): the latter is colored blue in case hæmoglobin is present.

Detection of biliary coloring matter will be considered below.

0dor. The normal odor of fresh urine is characteristic, and is sometimes spoken of as aromatic; it is not known by what substance or substances this odor is cansed. The ammoniacal and putrescent odor which urine acquires on standing, is due to the products of decomposition formed, chiefly to ammonia.

A number of substances taken internally and separated by the kidneys from the blood, cause the urine to assume a characteristic odor; aromatic substances especially impart such odors; oil of turpentine gives an odor reminding of violets, and the odor of cubebs, copaiba, and other similar substances is promptly 
transferred to the urine of persons using these drugs internally. A sweetish smell sometimes attends the presence of large quantities of sugar in urine.

Reaction. This is generally acid in healthy uripe which has been recently passed, but may become neutral or alkaline within a short period, by decomposition of urea and formation of ammonium carbonate.

Specific gravity. The normal specitic gravity of an average amount of 1500 c.c. of urine passed in twenty-four hours is about 1020, but it varies, even in health, from 1012 to 1025 or more. A specific gravity above 1028 generally indicates the presence of sugar, larger quantities of which may cause the specific gravity to rise to 1050 .

The determination of the specific gravity of urine is generally accomplished by the urinometer, which is a small hydrometer indicating specific gravities from zero (or 1000) to 60 (or 1060). As the temperature influences the density of liquids, a urinometer can only give correct results at a certain degree of temperature, which is generally marked upon the instrument. Many of the urinometers manufactured and sold, even at the normal (or stated) temperature, show incorrect gravities, and for this reason a urinometer should always be thoroughly tested before placing full confidence in the results obtained by it.

Determination of total solids. An approximate determination of total solids may be deducted from the specific gravity of the urine, as it has been found that the last two figures of the specific gravity of urine, multiplied by 2.2 , correspond to the number of grams in 1000 c.c. of urine. If, for instance, 1750 c.c. of urine, of a specific gravity of 1018 , have been discharged in twenty-four hours, then the quantity of total solids in 1000 c.c. will be $18 \times 2.2$, or 39.6 grams; and in 1750 c.c., 69.3 grams.

A more exact method of determining the total solids in urine is the evaporation of about 50 c.c. in a weighed platinum dish over a water-bath (or, better, under the receiver of an air-pump 
over sulphuric acid), until it is found that no more loss in weight ensues on continued exposure of the dish in the drying apparatus. By now reweighing the dish, plus contents, and deducting from the weight that of the empty dish, the weight of total solids is found.

Determination of inorganic constituents. The platinum dish containing the known quantity of total solids is exposed to the action of a non-luminous flame, and the heat continued until all organic matter has been destroyed and expelled. By reweighing now, and deducting the weight of the platinum dish, plus ash from the weight of the dish, plus total solids, the quantity of total organic matter is determined; and by deducting weight of dish from weight of dish plus ash, the total quantity of inorganic matter is found.

The analysis of this ash is effected by the methods given in connection with the consideration of the various acid and basic constituents themselves. Chlorine is determined by precipitating the solution of the ash in nitric acid with silver nitrate, sulphuric acid by barium chloride, phosphoric acid by ammonium molybdate, calcium by ammonium oxalate, potassium by platinic chloride, iron by potassium ferrocyanide, etc.

For the determination of many of the inorganic constituents, it is not necessary to destroy the organic matter as described above, but this determination can be effected directly. Thus, chlorine may be precipitated directly from urine (slightly acidulated with nitric acid) by silver nitrate; the precipitated silver chloride is collected upon a small filter, well washed, dried, and weighed in a porcelain crucible, after the filter (to which particles of silver chloride adhere) has been burned separately and its ash added to the contents of the crucible, which is moderately heated before weighing.

The so-called earthy phosphates (phosphates of calcium and magnesium) may be approximately determined by adding a few drops of an alkaline hydrate to about 50 c.c. of urine, heating to the boiling-point, collecting on a filter, washing, igniting, and weighing in a platinum crucible.

The methods for estimating urca and uric acid have been described in the preceding chapter. 
Detection of albumin. There are chiefly three methods by which the presence of albumin in urine nay be demonstrated; they are based upon the coagulation of albumin by heat, by nitric acid, or by picric acid.

The urine used for any of these tests must be perfectly clear; if it be not clear, it must be rendered so by processes which vary according to the nature of the substance causing the turbidity. In most cases filtration through good filter-paper may be sufficient; but if this does not accomplish the desired result, it may become necessary to use other means. Thus, if earthy, amorphous phosphates be present (which, especially in alkaline urine, are apt to pass through the best filter-paper), they may be removed by adding to the urine about a fourth part of potassium hydrate solution, warming the mixture, and filtering. If the turbidity be caused by urates, the urine will generally become clear by passing the test-tube once or twice through a flame.

The clear urine is then tested by either (or all) of the following methods:

a. Coagulation by heat. A test-tube is filled about one-half with the urine, to which, if not distinctly acid to test-paper, a few drops of acetic acid are added. (In case potassium hydrate has been added in order to precipitate the phosphates, enough of acetic acid must be added to cause a distinct acid reaction.) The test-tube is then held over the flame in such a manner that the heat acts upon the upper half of the urine only, heating this portion gradually to the boiling-point. By thus operating, two strata of fluid are obtained for comparison, and by holding the test-tube against the light, or against a black background, any difference in the appearance of the upper and lower strata may easily be noticed. Any cloudiness or opacity seen may be due to albumin, but may also be caused by phosphates.

To decide this question, a few drops (10 to 15) of nitric acid are allowed to flow gently down the side of the tube into the urine. The precipitate will readily disappear when caused by phosphates, but will be permanent when albumin is present.

Instead of heating, as above described, merely the upper half of the urine, the total quantity of the urine (acidulated by a few drops of acetic acid) may be heated, and the test-tube set aside for several hours (after having added 10 to 15 drops of nitric 
acid), in order to allow the albumin to subside, when it can be more distinctly seen and its quantity noticed.

b. Nitric acid test. A test-tube is filled to the deptl of about half an inch with colorless nitric acid, and an equal quantity of urine is allowed to flow down the side of the test-tube in such a manner that the specifically lighter urine forms a distinct and separate layer over the nitric acid. (If the urine be allowed to flow from a pipette, the formation of the two strata is easily accomplished.) In case albumin is present, a white band or zone of varying thickness (according to the quantity of albumin present) appears at the point of contact.

If the urine be highly concentrated, a similar white zone is formed between the acid and urine, due to the separation of insoluble acid urates; the difference between the separated urates and albumin is that the latter forms a sharply defined zone, whilst the urates diffuse into the urine above. Moreover, the urates dissolve on the application of heat. Finally, the separation of acid urates may be avoided by diluting the urine with an equal volume of water and placing this diluted urine upon the nitric acid.

c. Picric acid test. This test has the advantage that neither phosphates nor urates can be mistaken for albumin. It consists in slowly dropping urine into a test-tube filled to about onefourth with a highly colored solution of picric acid in water. In the presence of albumin a white cloud or sharply defined white turbidity is formed, and on warming the liquid the albumin collects into balls which rise to the surface of the liquid.

In the above methods the manipulations and precautions are so minutely described, in order to detect small quantities or even traces of albumin. When albumin is abundantly present, there is no difficulty whatever in its determination, as heat will precipitate it from an acid, neutral, or sometimes even alkaline urine; the precipitate should, however, always be tested by the addition of a few drops of nitric acid, and the previous addition of a few drops of acetic acid is also advisable.

A neutral urine should never be acidified by nitric acid (instead of acetic acid), because a drop or two of nitric acid may in some cases prevent the coagulation of albumin by heat, though a larger quantity (10 to 20 drops) has no such effect. 
Quantitative estimation of albumin. An approximate method for the comparative estimation of albumin is to precipitate it (with the precautions above given) in a graduated test-tube by heat and setting aside for 11 (or better for 24) hours. At the end of that time the proportion of the coagulated albumin which has collected at the bottom of the fluid is noticed. If the albumin occupy one-fourth, one-sixth, one-tenth of the height of the liquid, there is said to be one-fourth, one-sixth, or one-tenth of albumin in the urine. If, however, at the end of 12 or 24 hours scarcely any albumin has collected at the bottom, there is said to be a trace.

A better method of exactly estimating the amount of albumin is to collect the precipitate on a small filter, previously dried at $100^{\circ}$ and weighed. After having been well washed, the filter and contents are dried and weighed. As it may happen that the precipitated albumin encloses earthy phosphates, it is well to burn filter with contents in a platinum crucible, and to deduct the weight of the remaining inorganic residue from that of the albumin.

Detection of sugar. Traces of sugar, or as much as 0.01 per cent., are said to occur normally in urine, and are of no significance; moreover, it is as yet doubtful whether these traces of sugar are actually present in normal urine. Large quantities of sugar are often indicated by a high specitic gravity of the urine, which then varies from 1030 to 1050 .

Of the many tests by which sugar may be detected in urine three only will be mentioned; they are generally known as the copper tests (also termed Trommer's or Fehling's test, according to the manner in which the reaction is conducted), the bismuth test, and the fermentation test. The copper tests as well as the bismuth test are based upon the deoxidizing or reducing power which grape-sugar possesses for many metallic oxides such as cupric, bismuthic, and silver oxide, which, in the presence of alkalies, are converted into the lower state of oxidation, or are reduced to metals.

The tests for sugar should always be preceded by tests for albumin, which latter, if present, should be remored by coagulation and filtration. 
Trommer's test. A few drops (2-4) of a 5 per cent. solution of cupric sulphate are added to about 5 to 8 c.c. of urine in a test-tube and then an equal volume of potassium (or sodium) hydrate solution is added. The alkaline hydrate precipitates both earthy phosphates and cupric hydrate, the latter however dissolving (especially if sugar be present) in the excess of the alkali, producing a beautiful blue transparent liquid. (If no sugar is present, the color is less blue, but more of a greenish hue.) The liquid is now boiled for a few seconds, when, if sugar be present, a yellow precipitate of cuprous hydrate is formed which subsequently loses its water and becomes the red cuprous oxide, which falls to the bottom or adheres to the sides of the test-tube.

As various organic substances (other than sugar) have a tendency to reduce cupric oxide at a temperature of $100^{\circ}$, it is well to set aside a test-tube prepared as above (without heating it) for from 6 to 24 hours. If sugar be present, the formation of cuprous hydrate will gradually take place, whilst most other organic matters do not act upon cupric oxide at ordinary temperature.

In drawing conclusions from the above test, it should be remembered that a change of color does not indicate sugar; that a precipitate of earthy phosphates must not be mistaken for cuprous oxide; and that substances other than sugar may deoxidize cupric oxide at the temperature of $100^{\circ}$.

Fehling's test differs from Trommer's test merely in using a previously mixed reagent instead of producing this reagent, as it were, in the urine by adding to it cupric sulphate and an alkaline hydrate successively. This reagent, known as Fehling's solution, is made as follows:

Crystallized cupric sulphate, .

34.65 grams.

Dissolved in

Pure water, 200

This solution is poured gradually into a solution of

Crystallized sodium potassium tartrate, . 173 Dissolved in

Solution of sodium hydrate of sp. gr. 1.12, The clear, well-mixed fluid is diluted to 1000 c.c.

The addition of sodium-potassium tartrate in Fehling's solution prevents the precipitation of cupric hydrate by the alka- 
line hydrate. This action is analogous to the formation of the soluble scale compounds of iron, where the precipitation of ferric hydrate is also prevented by tartaric or other organic acids.

Fehling's solution is very apt to decompose, and if not recently made should be tested by boiling some of it alone and some of it mixed with about three volumes of water in testtubes; if no precipitate occurs in either case, the fluid may safely be used.

This is done by heating about 10 c.c. of Fehling's solution in a test-tube, and adding drop by drop the suspected urine; if the latter contains larger quantities of sugar a yellow or red precipitate of cuprous hydrate and oxide will be produced very readily; if but small quantities are present, an equal volume of urine may be added to the solution, and the boiling repeated several times before the reaction takes place.

Bötger's bismuth test consists in adding to a mixture of equal volumes of urine and potassium (or sodium) hydrate solution a few grains of subnitrate of bismuth and boiling for half a minute. If sugar be present, a gray or dark brown, finally black, precipitate of metallic bismuth is formed. If but very little sugar is present, the undecomposed excess of bismuthic nitrate mixes with the metallic bismuth, imparting to it a gray color; the test should then be repeated with a smaller amount of the bismuth salt.

If the urine contains hydrogen disulphide (sometimes produced by decomposition of certain urinary constituents), black bismuthic sulphide will be formed, which may be mistaken for metallic bismuth; albumin itself may be the cause of the formation of alkaline sulphides; the previous complete separation of albumin is therefore indispensable.

The fermentation test is based upon the decomposition of sugar by the action of yeast with generation of carbon dioxide. The test is made by adding to about 50 or 75 c.c. of urine (contained in a large test-tube or small flask) a few c.c. of ordinary baker's or brewer's yeast. The vessel containing the urine is provided with a perforated cork, through which is passed one limb of a bent glass tube, long enough to reach nearly to the bottom of the vessel, which should be completely filled with urine. If the urine be acid, it should be rendered slightly alkaline by a little 
sodium carbonate. Under the second limb of the bent glass tube is placed a beaker.

The apparatus, thus prepared, is placed in a room having a temperature of about $18-28^{\circ}$. If sugar be present, fermentation will commence within 12 hours, and will manifest itself by the formation of carbon dioxide, which will force a portion of the fluid through the bent tube into the beaker placed there for its reception.

The disadvantages of this process are the length of time required for its performance, the unreliability of the ferment, and the fact that small quantities of sugar (less than 0.5 per cent.) evolve so little carbon dioxide that a doubt may be felt as to the presence of sugar at all.

Quantitative estimation of sugar. Various methods for the determination of sugar in urine have been suggested, some of which depend on the loss in weight caused by the escape of carbon dioxide during fermentation. The disadvantages of the fermentation test for qualitative determination have been pointed out above, and apply also to quantitative determinations.

By far the best method is the decomposition of a copper solution of a known strength, and Fehling's solution, prepared as stated above, answers this purpose well.

1000 c.c. of Fehling's solution, containing 34.65 grams of crystallized cupric sulphate, $\mathrm{CuSO}_{4} .5 \mathrm{H}_{2} \mathrm{O}$, are exactly decomposed by 5 grams of grape-sugar, or 1 c.c. solution by 0.005 of grape-sugar.

To make the quantitative determination, operate as follows: 10 c.c. of Fehling's solution are poured into a porcelain dish of about 200 c.c. capacity, placed over a flame. The copper solution is diluted with about 40 c.c. of water, and heated to boiling; to the boiling liquid, urine (which has been previously diluted with 9 parts of water) is added from a burette very gradually, until the blue color of the solution has disappeared, and there remains, upon subsidence of the cuprous oxide, an almost colorless, clear liquid. A filtered portion of this liquid, acidified with hydrochloric acid, should not give a reddish-brown precipitate with potassium ferrocyanide (a precipitate would show that all copper had not been precipitated, and that more 
urine was needed), whilst a second portion of the filtered fluid should not produce a red precipitate on boiling with a few drops of Fehling's solution (a precipitate would indicate that too much urine has been added, in which case the operation has to be repeated).

The calculation of the amount of sugar present is easily made. 10 c.c. of Fehling's solution are decomposed by 0.05 gram of sugar; this quantity must, therefore, be contained in the number of c.c. of urine used. Suppose 30 c.c. of urine, diluted with 9 parts of water, or 3 c.c. of pure urine have been required to decompose the 10 c.c. of Fehling's solution, then 3 c.c. of urine contain of grape-sugar 0.05 gram, or 100 c.c. of urine 1.666 gram, according to the equation :

$$
\begin{array}{r}
3: 0.05:: 100: x \\
x=1.666 .
\end{array}
$$

If the urine contains but very little sugar, it may be used directly without diluting it, or instead of diluting it with 9 parts of water, it may be diluted with 4 volumes or with an equal volume of water.

Detection of bile. The presence of bile in urine is generally indicated by a decided color, which varies from a deep brownishred to a dark brown; the foam of such urine (produced by shaking) has a distinct yellow color, and a piece of filtering paper or a piece of linen dipped into the urine assumes a yellow color, which does not disappear on drying.

The further detection of bile depends upon the reactions of the biliary coloring matters or biliary acids; it frequently happens, however, that the pigments are present, whilst the acids are not.

Gmelin's test for biliary coloring matters has been already considered, and may be applied to urine either by allowing a small quantity of nitric acid, containing some nitrous acid, to flow down the sides of a test-tube (containing the urine) in such a manner that the two fluids do not mix, or by placing upon a porcelain plate a few drops of the urine, near it a few drops of nitric acid, to which one drop of sulphuric acid has been added, and allowing the two liquids to approach gradually. In both cases (if bile pigment is present) a play of color is seen at the point of union between the two fluids, the colors changing 
from green to blue, violet-red, and yellow or yellowish-green; while the appearance of the green at the beginning is indispensable to prove the presence of bile, the presence of all the other colors is not essential.

The above test may be made in a somewhat modified form by mixing the urine with a concentrated solution of sodium nitrate, and pouring down the sides of the test-tube concentrated sulphuric acid, in such a manner as to form two distinct layers; the colors are seen at the point of contact as above.

If the urine be very dark in color, it should be diluted with water before applying the above tests.

Ultzmann's test for bile pigment is made by mixing 10 c.c. of urine with 3 or 4 c.c. of potassium hydrate solution $(1$ in 3 of water), and supersaturating with hydrochloric acid; the mixture assumes a beautiful emerald-green color.

Pettenkofer's test for biliary acids is made by dissolving a few grains of cane-sugar in urine contained in a test-tube, and adding to it some concentrated sulphuric acid; the liquid is at first turbid, then becomes clear and almost simultaneously turns yellow, then pale cherry-red, dark carmine-red, and finally a beautiful purple violet.

As many substances (other than biliary acids) show a similar reaction, it is often necessary to separate the bile acids by the process described in connection with the consideration of bile itself.

Urinary deposits (sediments). When urine is allowed to stand for a few hours, a deposit is frequently formed, consisting either of solids which have been suspended in the urine, or of substances which are less soluble in cold urine (and are, therefore, separated on cooling), or which are formed by chemical decomposition.

When such a deposit is to be examined, a few ounces of the urine should be set aside for several hours in a tall, narrow, cylindrical glass; when the sediment has collected at the bottom, the supernatant urine may be removed by a siphon, or the sediment may be taken out by means of a pipette for examination.

Sediments are either organized or unorganized. To the first belong: mucus, blood, pus, urinary casts, epithelium, spermatozoids, fungi, infusoriæ, etc.; to the second belong: uric acid, 
urates, calcium oxalate, phosphate, or carbonate, magnesiumammonium phosphate, cystin, hippuric acid, etc.

The chemical examination of any urinary sediment should always be preceded by a microscopical examination, which latter is in many cases the only way of determining the nature of the sediment, especially of the organized substances. Most of the unorganized and either crystalline or amorphous sediments may be easily recognized by chemical means.

Urates of ammonium, calcium, and sodium dissolve on heating the urine, and are reprecipitated on cooling. The murexid test is used in addition.

Phosphates of calcium or ammonium-magnesium dissolve in acetic acid, and ammonium molybdate dissolved in nitric acid produces a yellow precipitate on heating.

Calcium oxalate is insoluble in acetic, but soluble in hydrochloric acid, from which solution it is reprecipitated on neutralizing with ammonia.

Uric acid is not dissolved by heat, nor by acetic or hydrochloric acid, but burns on platinum foil without leaving a residue; it is recognized by the murexid test.

Urinary calculi are solid deposits of larger or smaller size. formed from the urine within the tracts (kidneys, ureter, bladder, and urethra). The chenical composition of the calculi is generally that of either of the above-named unorganized sediments, and their nature can easily be determined by using the tests mentioned for them.

Most common are calculi of uric acid; often met with are those of urates, phosphates, and oxalates; rarely, however, those of xanthin and cystin.

\section{Questions.}

531. What points are to be considered, and what substances determined in the analysis of normal and abnormal urine?

532. What is the color of urine, and what are the chief causes influencing the color?

533. What is the specific gravity of healthy urine, how is it determined, and how is the total amount of solids approximately calculated from the specific gravity? 
EXAMINATION OF NORMAL AND ABNORMAL URINE. 393

534. Describe three tests by which albumin may be recognized, and state the precautions necessary in making these tests.

535. How may the quantity of albumin in urine approximately and how accurately be determined?

536. Describe 'Trommer's, Fehling's, bismuth, and fermentation test for sugar. On what principles are they based?

537. How is sugar determined quantitatively?

538. By what tests are biliary pigments and acids recognized in urine?

539. What is the nature of urinary sediments, and by what means are they recognized?

540. What are urinary calculi generally composed of, and by what simple tests can their nature be determined? 



\section{A P P E N I) I X.}

TABLE OF WEIGHTS AND MEASURES.

Measures of length.

1 millimeter $=0.001$ meter $=0.0394$ inch.

1 centimeter $=\quad 0.01$ meter $=0.3937$ inch.

1 decimeter $=0.1$ meter $=3.9371$ inches.

1 meter $\quad=39.3708$ inches.

1 decameter $=10 \quad$ meters $=32.8089$ feet.

1 hectometer $=100 \quad$ meters $=328.089$ feet.

1 kilometer $=1000 \quad$ meters $=0.6214$ mile.

Measures of capacity.

\begin{tabular}{|c|c|c|c|c|c|c|c|}
\hline 1 milliliter & $=$ & 1 c.c. & $=$ & 0.00 & liter & $=$ & 0.0021 U. S. pint. \\
\hline 1 centiliter & $=$ & 10 c.c. & $=$ & 0.01 & liter & $=$ & 0.0211 U. S. pint. \\
\hline 1 deciliter & $=$ & 100 c.c. & $=$ & 0.1 & liter & $=$ & 0.2113 U. S. pint. \\
\hline 1 liter & $=$ & 1000 c.c. & $=$ & & & $=$ & 1.0567 U. S. quart. \\
\hline 1 decaliter & & & $=$ & 10 & litres & $=$ & 2.6418 U. S. gallons. \\
\hline 1 hectoliter & & & $=$ & 100 & litres & $=$ & 26.418 U. S. gallons. \\
\hline 1 kiloliter & & & $=$ & 1000 & litres & $=$ & U. S. gallons. \\
\hline
\end{tabular}

Weights.

1 milligram $=0.001$ gram $=0.015$ grain Troy.

1 centigram $=0.01$ gram $=0.154$ grain Troy.

1 decigram $=0.1$ gram $=1.543$ grain Troy.

$1 \mathrm{gram}=15.432$ grains Troy.

1 decagram $=10 \quad$ grams $=154.324$ grains Troy.

1 hectogram $=100 \quad$ grams $=0.268$ pound Troy.

1 kilogram $=1000 \quad$ grams $=2.679$ pounds Troy. 
Commercial weights and measures of the US.A.

1 pound avoirdupois $=16$ ounces.

1 ounce $=437.5$ grains.

1 gallon $=231$ cubic inches

1 gallon $=4$ quarts $=8$ pints.

1 pint of water weighs 7291.2 grains at a temperature of $15.6^{\circ}$.

\section{Troy weight.}

$1 \mathrm{drachm}=60$ grains.

1 ounce $=8$ drachms $=480$ grains. 
TABLE OF ELEMENTS.

\begin{tabular}{|c|c|c|c|c|c|c|c|}
\hline & & Symbol. & $\begin{array}{l}\text { A tomic } \\
\text { weight. }\end{array}$ & & & Symbol. & $\begin{array}{l}\text { Atomic } \\
\text { weight. }\end{array}$ \\
\hline Aluminium, & & . Al & 27 & Molybdenum, & & Mo & 95.5 \\
\hline Antimony, & & . $\mathrm{Sb}$ & 120 & Nickel, & 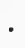 & $\mathrm{Ni}$ & 58 \\
\hline Arsenic, & $\cdot$ & . As & 74.9 & Niobium, & & $\mathrm{Nb}$ & 94 \\
\hline Barium, & • & - $\mathrm{Ba}$ & 136.8 & Nitrogen, & " & . $\mathrm{N}$ & 14 \\
\hline Beryllium, & . & - $\mathrm{Be}$ & 9 & Osmium, & . & Os & 198.5 \\
\hline Bismuth, & & . $\mathrm{Bi}$ & 210 & Oxygen, & - & . $\mathrm{O}$ & 16 \\
\hline Boron, . & & - $\mathrm{Bo}$ & 11 & Palladium, & . & $\mathrm{Pd}$ & 105.7 \\
\hline Bromine, & & . $\mathrm{Br}$ & 79.8 & Phosphorus, & . & . $\mathrm{P}$ & 31 \\
\hline Cadmium, & & . $\mathrm{Cd}$ & 111.8 & Platinum, & & - $\mathrm{Pt}$ & 194.4 \\
\hline Cresium, & • & . $\mathrm{Cs}$ & 132.6 & Potassium, & & . $\mathbf{K}$ & 39 \\
\hline Calcium, & & - $\mathrm{Ca}$ & 40 & Rhodium, & & . $\mathrm{Rh}$ & 104.1 \\
\hline Carbon, . & & . $\mathrm{C}$ & 12 & Rubidium, & & . $\mathrm{Rb}$ & 85.3 \\
\hline Cerium, & • & . $\mathrm{Ce}$ & 141 & Ruthenium, & & . $\mathrm{Ru}$ & 104.2 \\
\hline Chlo & & . $\mathrm{Cl}$ & 35.4 & Scandium, & & - $\mathrm{Sc}$ & 44 \\
\hline Chromium, & & . $\mathrm{Cr}$ & 52.4 & Selenium, & & . Se & 78.8 \\
\hline Cob & & . $\mathrm{Co}_{0}$ & 58.9 & Silicon, . & & . $\quad \mathbf{S i}$ & 28 \\
\hline Copper, . & & - $\mathrm{Cu}$ & 63.2 & Silver, . & & . Ag & 107.7 \\
\hline Didymium, & & . $\mathrm{Di}$ & 144.6 & Sodium, & & . $\mathrm{Na}$ & 23 \\
\hline Erbium, & & . $\mathrm{E}$ & 165.9 & Strontium, & & . $\mathrm{Sr}$ & 87.4 \\
\hline Fluorine, & & . $\mathrm{Fl}$ & 19 & Sulphur, & & & 32 \\
\hline um, & $\cdot$ & . $\mathrm{G}$ & 68.8 & Tantalum, & & . $\mathrm{Ta}$ & 182 \\
\hline Gold, & 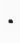 & - $\mathrm{Au}$ & 196.2 & Tell & & - Te & 128 \\
\hline Hydrogen, & $\cdot$ & . $\mathrm{H}$ & 1 & Thallium, & & . $\mathrm{TI}$ & 203.7 \\
\hline Indium, & & . In & 113.4 & Thorium, & & . $\mathrm{Th}$ & 233 \\
\hline Iodine, & . & . $\mathrm{I}$ & 126.6 & Tin, & & . Sn & 117.7 \\
\hline Iridium, & . & . $\mathbf{I r}$ & 192.7 & Titanium, & & . $\mathrm{Ti}$ & 48 \\
\hline Iron, . & . & . $\mathrm{Fe}$ & 55.9 & Tungsten, & & . W & 183.6 \\
\hline Lanthanum, & - & - La & 138.5 & Uranium, & & . $\mathrm{U}$ & $238 . \overline{5}$ \\
\hline Lead, . & & . $\mathrm{Pb}$ & 206.5 & Vanadium, & & V & 51.3 \\
\hline Lithium, & ? & . $\mathrm{Li}$ & 7 & Ytterbium, & & . $\mathbf{Y b}$ & 172.7 \\
\hline Magnesium, & - & . $\quad \mathbf{M g}$ & 24 & Yttrium, & & . $\mathbf{Y}$ & 89.8 \\
\hline Ianganese, & • & . $\mathbf{M n}$ & 54 & Zinc, & & . $\quad Z \mathbf{n}$ & 64.9 \\
\hline Aercury, & ' & . $\quad \mathbf{H g}$ & 199.7 & Zirconium, & , & . $\mathrm{Zr}$ & 90 \\
\hline
\end{tabular}





\section{N D E X.}

BSORPTION, 33

A Acetic acid, 283 analy tical reactions of, 285

Acetic ether, 306

\section{Acetylene, 260}

Acid, acetic, 283

adipic, 287

arachidic, 282

arsenic, 185

arsenious, 184

behenic, $28 \%$

benzoic, 299

boracic, 84

bromic, 106

butyric, 281

cupric, 282

caproic, 281

caprylic, 281

carbazotic, 298

carbolic, 297

carbonic, 79

cerotic, 282

chloric, 104

cholic, 361

chromic, 156

citric, 29 ?

cyanic, 336

fluoric, 108

formic, 282

fulminic, 339

gallic, 301

glacial acetic, 284

glycocholic, 361

hippuric, 379

hyænic, 282

hydriodic, 107

hydrobromic, 106

hydrochloric, 102

hydrocyanic. 333

hydroferricyanic, 337

hydroferrocyanic, 337

hydrofluoric, 108

hydrosulphuric, 90

hypochlorous, 104

hypophosphorous, 9 R

hyposulphurous, 90

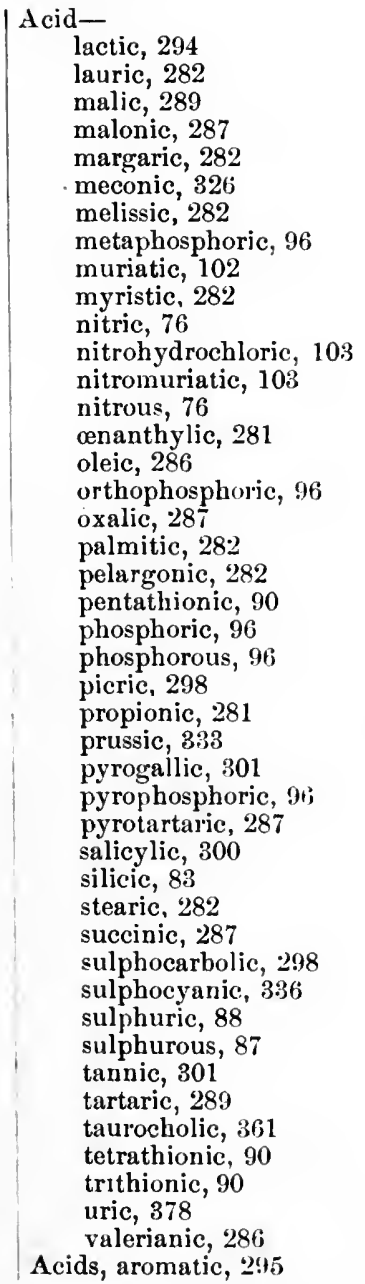


Acsds-

biliary, 361

definition of, 55

detection of, 220

fatty, 281

organic, 279

Aconitine, 322

Adhesion, 31

Ather, 304

Affinity, chemical, 38

Agate, 83

Air, composition of, 74

Alabaster, 132

Albumin, 341

in urine, 384

Albuminous substances, $3 \mathbf{4 0}$

Alcohol, 270 analytical reactions of, 341

absolute, 271

amyl, 273

butyl, 270

dilute, 271

ethyl, 270

methyl, 270

real, 271

Alcoholic liquors, 272

Alcohols, 267

monatomic, 270

Aldehydes, 274

Alkali metals, 115

Alkaline earths, 132

Alkaloids, 322

antidotes to the, 321

detection of, 321

Allotropic modification, 60

Alloy, definition of, 114

Alum, 139

Aluminium, 138

and ammonium sulphate, 139

analytical reactions, 142

chloride, 140

hydrate, 139

oxide, 140

sulphate, 140

A malgam, 175 ammonium, 125

Amber, 267

Amides, 317

Amines, 317

Ammonia, 75 water, 75

Ammoniated mercury, 180

Ammonio-copper compounds, 168

Ammonium, 125

amalgam, 125

analytical reactions of, 128

benzoate, 299

bromide, 127

carbamate, 126

carbonate, 126

chloride, 126

hydrate, 126

\author{
Ammonium- \\ iodide, 127 \\ molybdute, 198 \\ nitrate, 127 \\ phosphate, 127 \\ sulphate, 127 \\ sulphide, 127 \\ sulphydrate, 127 \\ valerianate, 286 \\ Amorphism, 18 \\ Amorphous phosphorus, 94 \\ Amygdalin, 299 \\ Amyl alcohol, 273 \\ nitrite, 306 \\ Analysis, definition of, 55 \\ organic, 240 \\ qualitative, 199 \\ quantitative, 199 \\ ultimate, 240
}

Analytical chemistry, 199

Analytical reactions of acetates, 285

albuminous substances, 341

aluminium, 142

ammonium, 128

antimony, 195

arsenic, 187

atropine, 330

barium, 137

benzoates, 299

bile, 360,390

bismuth, 171

borates, 84

bromides, 106

calcium, 136

carbolic acid, 298

carbonates, 81 .

chlorates, 104

chlorides, 102

cholesterin, 362

chromates, 158

chromium, 158

citrates, 293

codeine, 325

copper, 169

cyanides, 335

ferric salts, 152

ferricyanides, 339

ferrocyanides, 338

ferrous salts, 152

hippuric acid, 379

hydrocyanic acid, 335

hy pochlorites, 103

hypophosphites, 98

iodides, 107

iron, 152

lead, 166

magnesium, 131

manganese, 155

mercury, 181

morphine, 325

nitrates, 77

oxalates, 288 
Analytical reactions ofphosphates, 97 phosphites, 96 potassium, 120 quinine, 328 silicates, 84 silver, 174 sodium, 124 starch, 314 strontium, 137 strychnine, 330 sugar, 311 sulphates, 90 sulphides, 91 sulphites, 87 tannic acid, 301 tartrates, 290 tin, 196 urates, 378 urea, 376 veratrine, 331 zinc, 163

Aniline, 297

Animal charcoal, 135 fluids and tissues, 355

Antidotes to acids, 77, 90 alkalies, 116 alkaloids, 321 antimony, 194 arsenic, 192 barium, 137 carbolic acid, 298 copper, 169 cyanides, 336 hydrocyanic acid, 386 lead, 165 mercury, 180 nitric acid, 77 oxalic acid, 288 phosphorus, 95 silver, 174 sulphuric acid, 90 zinc, 162

Antimonious chloride, 194 oxide, 194

Antimony, 192 analytical reactions of, 195 and potassium tartrate, 292 antidotes to, 194

black, 192

butter, 194

chloride, 194

crude, 192

oxide, 194

pentasulphide, 194

potassio-tartrate, 194

sulphide, 193

sulphurated, 193

trisulphide, 193

Apomorphine, 324

Argentum, 172

Argol, 289
Aromatic compounds, 296

Arrack, 273

Arsenic, 182

acid, 185

analytical reactions of, 187

antidotes to, 192

detection of, in case of poisoning, 191

Fleitmann's test for, 189

Marsh's test for, 189

oxide, 185

red native sulphide, 183

Reinsch's test for, 188

sulphides, 186

yellow native sulphide, 183

Arsenious acid, 184

anhydride, 184

iodide, 187

oxide, 184

Arseniuretted hydrogen, 186

Ash, black-, 122

bone-, 135

soda-, 122

Asphalt, 267

Atmosphere, composition of, 74

Atmospheric pressure, 29

A tom, definition of, 39

Atomic theory, 39

Atomic weights, determination of, 47

Atoms, 39 relation of specific heat to, 49 quantivalence of, 44

Atropine, 330 analytical reactions of, 330 sulphate, 330

Auric chloride, 197 sulphide, 197

Aurum, 196

A uripigment, 183

Avogadro's law, 23

BALSAM, copaiva, 267

B Balsams, 266

Barium, 136

analytical reactions of, 137

antidotes to, 137

curbonate, 136

chloride, 136

chromate, 158

sulphate, 136

sulphide, 136

Barometer, 28

Basalt, 84

Bases, definition of, 55

Beer, 272

Benzaldehrde, 299

Benzene, 265

Benzin, 363

Benzol, 265

Benzoic acid, 299

Berberine, 32.2

Beryllium, 58 
Bicarbonate of potassium, 117 sodium, 123

Bichloride of mercury, 177

Bichromate of potassium, 156

Bile, 359 detection of, in urine, 390

Biliary acids, 361 calculi, 362 pigments, 360

Bilirubin, 360

Biliverdin, 361

Bismuth, 169

analytical reactions of, 171

carbonate, 170

hydrate, 171

nitrate, 170

oxide, 171

oxy-salts, 170

subcarbonate, 170

subnitrate, 170

sulplate, 170

sulphide, 171

Bismuthyl, 170

carbonate, 170

nitrate, 170

Bisulphide of carbon, 91

Biuret, 376

Black antimony, 192

-ash, 122

-lead, 78

oxide of copper, 167 manganese, 153

mercury, 175

Bleaching-powder, 135

Blood, 356

corpuscles, 357

detection of, 357

-fibrin, 342

-serum, 357

-stains, 357

Blue mass, 175

pill, 175

Prussian, 152

-stone, 167

Turnbull's, 152

vitriol, 167

Bone, 363

- -ash, 135

i- -black, 135

Boracic acid, 84

Borax, 124 analytical reactions of, 84 bead, 206

Boron, 83

Bötger's bismuth-test, 388

Brain, 364

Brandy, 273

Bromides, analytical reactions of, 106

Bromine, 105

Brucine, 330

Butter, 368 of antimony, 194
CADAVERIC alkaloids, 354

$\bigcup$ Cadmium, 162 iodide, 163

sulphate, 163

sulphide, 163

Cæsium, 58

Caffein, 331

Calamine, 160

Calcined magnesia, 130

Calcium, $132^{\circ}$

analytical reactions of, 136

carbonate, 133

fluor:de, 108

hydrate, 133

hypophosphite, 135

oxalate, 289

oxide, 132

phosphate, 134

sulphate, 133

superphosphate, 134

tartrate, 290

Calc-spar, 132

Calomel, 176

Camphors, 266

Cane-sugar, 312

Canutchouc, 267

Capillary attraction, 32

Carbamide, 374

Carbazotic acid, 298

Carbohydrates, 310

Carbolic acid, 297

analytical reactions of, 298 antidotes to, 298

Carbou, 78

bisulphide, 91

dioxide, 79

disulphide, 91

monoxide, 81

Carbonates, analytical reactions of, 81

Carbonic acid, 79 oxide, 81

Carboxyl, 279

Casein, 341 vegetable, 343

Cast-iron, 145

Caustic, 173 lunar, 173 potash, 116

Cellulose, 315

Centigrade thermometer, 25

Cerebrin, 364

Cerium, 58

Chains, 246

Chalk, 132

Charcoal, animal, 135

Cheese, 368

Chemical action, definition of, 38 affinity, 38

formulas, 40

reactions, 54

symbol, definition of, 40

Chemism, 38 
Chemistry, definition of, 39 how to study it, 62

Chili saltpetre, 124

Chloral, 275 hydrate, 275

Chlorates, analytical reactions of, 104

Chloric acid, 104 oxides, 103

Chlorides, analytical reactions of, 102

Chlorinated lime, 135

Chlorine, 100 acids, 104 oxides, 103 -water, 101

Chloroform, 276

Chlorous oxide, 103 tetroxide, 103

Cholic acid, 361

Cholesterin, 362

Chromates, analytical reactions of, 158

Chrome-alum, 139 -iron ore, 156 -yellow, 158

Chromic acid, 156 hydrate, 157 oxide, 157

Chromiun, 156 chloride, 157 sulphate, 157

Chyle, 358

Cinchona alkaloids, 326

Cinchonidine, 329

Cinchonine, 329 sulphate, 329

Cinnabar, 174, 179

Citrates, analytical reactions of, 293

Citric acid, 293

Clay, 141

Clot, 357

Coagulation, 340

Coal, 262

Coal-uil, 262

Cobalt, 159

Codeine, 325

Cognac, 273

Cohesion, 18

Colchicine, 322

Collagen, 345,363

Collodion, 315

Colloids, 35

Columbium, 58

Combustion, 68, 252

Compound radicals, 56

Compounds, decomposition of, 51 definition of, 37

Coniine, 323

Copaiva balsam, 267

Copper, 166 acetate, 285 ammonio-sulphate, 168 analytical reactions of, 169 antidotes to, 168
Copper-

arsenite, 169

black oxide, 167

glance, 166 .

hydrate, 167

oxide, 167

pyrites, 166

sulphate, 167

sulphide, 166, 169

Corrosive chloride of mercury, 177 sublimate, 177

Corundum, 138

Cream, 370 of tartar, 291

Creamometer, 370

Creasol, 298

Creasote, 298

Crude antimony, 192

Cryptolites, 344

Crystallization, 18

Crystalloids, 35

Cubic nitre, 124

Cumene, 265

Cupric acetate, 285

arsenite, 169

ferrocyanide, 169

hydrate, 167

oxide, 167

sulphate, 167

sulphide, 169

Cuprous oxide, 167

Cuprum, 166

Curd, 367

Cyanates, 336

Cyanhydric acid, 333

Cyanic acid, 336

Cyanides, analytical reactions of, 335 antidotes to, 336

Cyanogen, 332

Cymene, 265

ALTON'S atomic theory, 42

Decay, 252

Decomposition by electricity, 52 heat, 52

light, 52

various modes of, 250

Decrepitation, 205

Deflagration, 205

Detection of impurities in officinal preparations, 226

Deposits, urinary, 391

Derivatives, 249

Destructive distillation, 251

Dextrine, 314

Dialysis, 34

Dialyzed iron, 148

Diamond, 78

Diastase, 311

Dibasic acids, 55

Dicyanogen, 333 
Didymium, 58

Diffusion, 34

Digitalin, 316

Digestion, 352

Distillation, 25

destructive, 251

dry, 251

fractional, 260

Disulphide of carbon, 91

Divisibility, 20 chemical, 36

Donovan's solution, 187

Double salts, 56

Drinking water, 71

Dried alum, 139

Dry distillation, 251

GARTHS, alkaline, 132

E Elasticity, 19

Electricity, 52

Elementary analysis, 240

Element, definition of, 37

Element, 58

derivation of names of, 64,109

natural groups of, 59

non-metallic, 64

metallic, 109

relative importance of, 57

time of discovery, 65,111

valence, 66,112

Emetine, 322

Emery, 138

Empirical formulas, 243

Endosmosis, 34

Epithelium, 364

Epsom salt, 130

Equivalence, 44

Erbium, 58

Essential oils, 266

Ethane, 261

Ethene, 265

Ether, 304

acetic, 306

nitrous, 306

sulphuric, 304

Ethers, 302

Ethyl, 270

acetate, 306

alcohol, 270

ether, 204

hydrate, 270

hydride, 261

nitrite, 306

oxide, 304

Ethylene. 265

Ethylic alcohol, 270

TAHRENHEIT'S thermometer, 25 Fatty acids, 281

Fats, 307

Feathers, 364
Feces, 362

Fehling's solution, 387

Feldspar, 84

Fermentation, 253

Ferric acetate, 285

chloride, 147

citrate, 294

hydrute, 146

hypophosphite, 151

iodide, 148

nitrate, 150

oxide, 146

salts, analytical reactions of, 152

sulphate, 149

sulphocyanate, 152

tartrate, 293

valerianate, 286

Ferricyanogen, 337

Ferricyanides, analytical reactions of, 339

Ferrocyanides, analy tical reactions of, 338

Ferrocyanogen, 337

Ferrous bromide, 149

carbonate, 150

chloride, 147

hydrate, 146

iodide, 148

oxide, 146

phosphate, 150

salts, analytical reactions of, 152

sulphnte, 149

sulphide, 149

Ferrum, 109

Fibrin, 341

vegetable, 343

Flame, structure of, 83 tests, 206

Fleitmann's test, 189

Flesh-fibrin, 343

Flowers of sulphur, 86

Fluorine, 108

Fluorspar, 108

Food, animal, 350

heat-giving, 351

nutritive, 351

plant, 348

plastic, 351

respiratory, 351

Formic acid, 282

Formulas, chemical, 40

empirical, 243

graphic, 243

molecular, 243

rational, 244

Fowler's solution, 185

Fractional distillation, 260

Fusel oil, 273

Fusibility of metals, 110

CALENA, 164

G argentiferous, 172

Gallic acid, 301 
Gallium, 58

Gall-stones, 362

Galvanized iron, 160

Gas, definition of, 19 illuminating, 264

Gasoline, 263

Gastric juice, 359

Gelatine, 345, 363

Gelatinized starch, 314

Gelatinoids, 345

German silver, 159

Gin, 273

Glacial acetic acid, 284 phosphoric acid, 96

Glass, 14:

Glauber's salt, 123

Glucinum, 58

Glucose, 311

Glucosides, 316

Glue, 345,363

Gluten, 343

Glycerin, 273

Glycholic acid, 361

Glycocol, 361

Glycols, 268

Gmelin's test, 360, 390

Gold, 196 and sodium chloride, 197 analytical reactions of, 197 chloride of, 197 coin, 196 sulphide, 197

Golden sulphuret of antimony, 194

Goulard's extract, 285

Granite, 84

Grape-sugar, 311

Graphic formulas, 243

Graphite, 78

Gravitation, 27

Green vitriol, 149

Gum, 314

-arabic, 314

British, 314

-resins, 266

Gun-cotton, 315

-powder, 118

Gutta-percha, 267

Gypsum, 132

IAIR, 364

11 Hæmatin; 344

Hæmatocrystalline, 344

Hromaglobin, 344

Haloids, 99

Halogens, 99

Heat, 23

action upon compounds, 51

action upon organic substances, 251

decomposition by, 36

latent, 24

specific, 26
Heavy magnesia, 130

-spar, 136

Hippuric acid, 379

Homologous series, 247

Hoofs, 364

Horns, 364

Humus, 357

Hydrargyrum, 174

Hydriodic acid, 107

Hydrobromic acid, 106

Hydrocarbons, 259

Hydrochloric acid, 102

analytical reactions of, 102

Hydrocyanic acid, 333

analytical reactions of, 33.5 antidotes to, 336

IIrdroferricyanic acid, 337

Hydroferrocyanic acid, 337

Hydrofluoric acid, 108

Hydrogen, 69

arseniuretted, 186

dioxide, 72

peroxide, 72

sulphuretted, 90

Hydrometers, 28

Hydrosulphuric acid, 90

Hydroxyl, 246

Hyoscyamine, 322

Hypochlorites, tests for, 103

Hypochlorous acid, 104 oxide, 103

Hypophosphites, tests for, 98

Hypophosphoric acid, 98

Hy posulphurous acid, 90

ILLUMINATING gas, 264 oil, 26 ?

Indium, 58

Iodides, analytical reactions of, 107

Indine, 105

tests for it, 107

tincture of, 107

Iodized starch, 314

Iodoform, 278

Iridium, $\tilde{58}$

Iron, 143 acetate, 285

analytical reactions of, 152

bromide, 149

carbonate, 150

cast-, 145

chlorides, 147

dialyzed, 148

galvanized, 160

hydrates, 146

iodide, 148

nitrate, 150

ores, 144

oxides, 146

phosphates. 150

pyrites, 144 


\section{Iron-}

reduced, 146

scale compounds of, 151

sulphates, 149

wrought-, 145

Isomerism, 249

Isomorphism, 19
TCTIC acid, 294

L Lactometer, 369

Lactoscope, 370

Lactose, 313

Lanthanum, 58

Lapis infernalis, 173

Latent heat, 24

Laughing-gas, 76

Laurene, 265

Law, Avogadro's, 23

of chemical combination by weight, 41

by volume, 42

of constancy of composition, 41

of diffusion of gases, 35

Dulong and Petit's, 50

of equivalents, 44

Mariotte's, 19

of multiple proportions, 42

Laws of chemical combination, 41

Lead, 164

acetate, 285

analytical reactions of, 166

antidotes to, 165

carbonate, 164

chloride, 166

chromate, 166

iodide, 165

nitrate, 164

oxide, 164

plaster, 309

phosphate, 166

-water, 285

white, 164

Lecithin, 342, 362

Legumin, 343

Le dcine, 344

Light, decomposition by, 52

Light magnesia, 130

Lime, acid phosphate of, 134

chloride of, 135

chlorinated, 135

-kiln, 132

quick-, 132

slaked, 133

superphosphate of, 134
Lime-water, 133

Limestone, 132

Liquefaction of solids, 207

Liquids, definition of, 19

Litharge, 164

Lithium, 124

citrate, 293

salicylate, 301

Litmus, 55

Lunar caustic, 173.

Lymph, 358

MAGNESIA, 130

II calcined, 130

Magnesium, 129

analytical reactions of, 131

carbonate, 129

oxide, 130

sulphate, 130

sulphite, 130

Malachite, 166

Malic acid, 289

Manganates, 154

Manganese, 153

analytical reactions of, 155

black oxide of, 154

dioxide, 154

oxides of, 153

Manganous carbonate, 155

chloride, 155

hydrate, 155

oxide, 154

sulphate, 154

Marble, 132

Mariotte's law, 19

Marsh-gas, 82

Marsh's test for arsenic, 189

Matter, definition of, 17

Mass, 17

Meconic acid, 326

Meerschaum, 129

Melissic acid, 282

Melting-points of metals, 110

Mercurial ointment, 175 plaster, 175

Mercuric chloride, 177

cyanide, 335

fulminate, 339

iodide, 178

nitrate, 179

oxide, 176

salts, analytical reactions of, 181

sulphate, 179

sulphide, 179

Mercurous chloride, 176

chromate, 158

iodide, 178

nitrate, 179

oxide, 175

salts, analytical reactions of, 181 
Mercurous-

sulphate, 179

sulphide, 179

Mercury, 174

ammoniated, 180

analytical reactions of, 181

antidotes to, 180

basic sulphate, 179

black oxide, 175

carbonates, 181

chlorides, 17 i

iodides, 178

nitrates, 179

oleate, 286

oxides, 176

sulphates, 179

sulphides, 179

with chalk, $17 j$

Metallic elements, 109

Metallocyunides, 337

Metalloids, 64

Metals, 109

classification of, 113

derivation of names, 10 !

melting-points, 110

specific gravity, 111

valence, 112

Metamerism, 250

Metaphosphoric acid, 96

Methane, 261 series, 261

Methyl alcohol, 270

hydrate, 270

hydride, 261

Methylated spirit, 270

Mica, 84

Milk, 365

adulterations of, 369

analysis of, 371

-casein, 343

-sugar. 313

Mineral waters, 71

Minium, 164

Mispickel, 183

Molecular theory, 21

Molecule, definition of, 21

Molybdenum, 197

Molybdic acid, 198 oxide, 197

Monobasic acids, 55

Monsel's solution, 150

Morphine, 324 acetate, 325

analytical reactions of, 325

hydrochlorate, 325

sulphate, 325

Muscle-fibrin, 343

Mucilage of starch, 314

Mucus, 364

Murexid test, 378

Muscles, 364

Myosin, 343
N A IIS, 364

1 Narceine, 326

Narcotine, $326^{\circ}$

Natrium, 110

Neutral substances, 56

Neurin, $3 \dot{6} 4$

Nickel, 159

Nicotine, 323

Nitrates, analytical reactions of, 77

Nitre, 117

Nitric acid, 76

Nitro-benzene, 297

Nitro-cellulose, 315

Nitrocyanmethane, 339

Nitrogen, 73

determination in organic compounds, 241

oxides, 76

Nitrohydrochloric acid, 103

Nitromuriatic acid, 103

Nitrous acid, 76 oxide, 76

Nomenclature, 60

Non-metallic elements, 64

Nordhausen sulphuric acid, 88

Nutrition of animals, 352

ILS, bergamot, 266

bitter almond, 299

castor, 308

cod-liver, 308

cotton-seed, 308

juniper, 266

lavender, 266

lemon, 266

linseed, 308

olive, 308

peppermint, 266

rosemary, 266

turpentine, 266

vitriol, 88

Oils, essential, 266 fat, 308

Olefiant gas, 265

Olefines, 265

Oleic acid, 286

Oleo-resins, 266

Olive oil, 308

Opium, 323 denarcotized, 324

Organic analysis, 240

chemistry, 236

substances, classification of, 257

decomposition of, 250

decomposition of, in the animal system, 349

formation of, in plants, 348

Orpiment, 183

Orthophosphoric acid, 97

Osmium, 58

Ossein, 345, 363 
Oxalates, analytical reactions of, 288

Oxalic acid, 287 antidotes, 288

Oxide, definition of, 68

Oxygen, 66

Ozone, 68

DA LLA UIU M, 58

Palmitic acid, 282

Pancreatic juice, $362^{\circ}$

Pancreatin, 345

Papaverine, 322

Puraffine, 263

Pelargonic acid, 282

Pentathionic acid, 90

Pepsin, 345 saccharated, 345

Peptone, 343

Perchloric acid, 103

Permanganates, 154

Petrolatum, 263

Petroleum, 262 -ether, 263 ointment, 263

Pettenkofer's test, 361,391

Phenol, 273, 297

Phosphates, analytical reactions of, 97

Phosphites, analytical reactions of, 96

Phosphoretted hydrogen, 99

Phosphoric acid, 96

Phosphorous acid, 96

Phosphorus, 92 antidotes to, 95 detection of, 95 determination in organic compounds, 242 red or amorphous, 94

Physiological chemistry, 347

Physostigmine, 322

Picric acid, 298

Pilocarpine, 322

Pioskop, 370

Piperin, 322

Plant-food, 348

Plaster of Paris, 133

Platinic chloride, 197

Platinum, 197 and ammonium chloride, 197 and potassium chloride, 197

Plumbago, 78

Plumbum, 110

Polymerism, 250

Polymorphism, 19

Porcelain, 141

Porosity, 31

Potush, 116 caustic, 116

Potassium, 115 acetate, 285
Potassium-

acid carbonate, 117

acid oxalate, 288

acid tartrate, 291

analytical reactions of, 120

bicarbonate, 117

bichromate, 156

bitartrate, 291

bromide, 120

carbonate, 117

chlorate, 118

chromate, 156

citrate, 293

cyanate, 337

cyanide, 334

dichromate, 156

ferricyanide, 338

ferrocyanide, 337

hydrate, 116

hypophosphite, 119

iodide, 119

manganate, 154

nitrate, $1: 7$

oxalate, 288

permanganate, 154

prussiate, 337,338

sodium tartrate, 291

sulphate, 118

sulphite, 119

sulphocyanate, 337

sulphurated, 119

tartrate, 291

Preliminary examination, 204

Proof-spirit, 271 table for, 208

Propionic acid, 281

Propyl alcohol, 270

Proteids, 340

Prussian blue, 338

Prussiate of potash, red, 338 yellow, 337

Prussic acid, 333

Ptomaines, 354

Ptyalin, 345, 358

Putrefaction, 253

Pyrites, copper, 166 iron, 144

Pyrophosphoric acid, 97

Pyroxylin, 315

() UANTIVALENCE, 44

Quicksilver, 174

Quinine, 327

acid sulphate, 328

analytical reactions of, 328

citrate of iron and, 328

sulphate, 327

valerianate, 286

Quinidine, 326 
RADICAL, definition of, 56,245

R Reactions, 54 analytical, 55 synthetical, 55

Reagents, list of, 202 use of, 204

Real alcohol, 271

Realgar, 183

Reinsch's test, 188

Residue, definition of 56,245

Rhodium, 58

Rochelle salt, 291

Rock-crystal, 83

Rubidium, 58

Ruby, 138

Rum, 273

Ruthenium, 58

SALICIN, 300

S Salicylic acid, 300

Saliva, 358

Sal sodr, 122

Salt-cake, 122 common, 121

Saltpetre, 117

$$
\text { Chili, } 124
$$

Salts, definition of, 56 table of the solubility or insolubility of, 225

Sand, 83

Santonin, 316

Sapphire, 138

Sarkin, 364

Scale compounds of iron, 151

Scheele's green, 187

Schweinfurth green, 187

Seidlitz powder, 291

Selenium, 58

Serum, 357

-albumin, 342

Sherry wine, 272

Silica, 83

Silicates, 83

Silicic acid, 83

Silicon, 83

Silicium, 83

Silver, 172

analytical reactions of, 174

antidotes to, 174

chloride, 172

chromate, 158,174

cyanide, 335

fulminate, 339

German, 159

iodide, 178

nitrate, 173

oxide, 173

sulphide, 174

Sinapin, 322

Slaked lime, 133

Soap, 309
Soda, 122

-ash, 122

-lime, 240

Sodium, 121

acetate, 285

analytical reactions of, 124

arseniate, 185

benzoate, 299

bicarbonate, 123

carbonate, 122

chloride, 121

hydrate, 121

hyposulphite, 123

nitrate, 124

phosphate, 123

salicylate, 300

sulphate, 123

sulphite, 123

sulphocarbolate, 298

Solanin, 322

Solids, definition of, 18

Specific heat, 26 weight, 27

Spirit of hartshorn, 75

proof, 271

rectified, 271

of wine, 271

wood-, 270

Stannic acid, 196

chloride, 196

sulphide, 196

Stannous chloride, 195

hydrate, 196

sulphide, 196

Stannum, 195

Starch, 313

iodized, 314

Stearic acid, 282

Stearine, 307

Steel, 145

Strontium, 136 analytical reactions of, 137

carbonate, 137

nitrate, 137

sulphate, 137

Structure of flame, 83

Strychnine, 329

analytical reactions of, 330

sulphate, 329

Sublimation, 25

Sublimed sulphur, 86

Substitution, 249

Succinic acid, 287

Sugar, 311

cane-, 312

detection of, in urine, 386

fruit-, 312

grape-, 311

of lead, 285

milk, 313

Sulphates, analytical reactions of, 90

Sulphides, anulytical reactions of, 91 
Sulphites, analytical reactions of, 87

Sulphocarbolates, 298

Sulphocyanic acid, 336

Sulphur, 85

dioxide, 86

determination in organic compounds, 24:2

flowers of, 86

milk of, 86

precipitated, 86

sublimed, 86

trioxide, 88

Sulphurated antimony, 193

Sulphuretted hydrogen, 90

Sulphuric acid, 88

antidotes to, 90

dilute, 90

fuming, 88

Nordhausen, 88

Sulphuric ether, 304

Sulphurous acid, 87

Superphosphate of lime, 134

Surface-action, 31

Sweet spirit of nitre, 306

Symbols, function of, 40

Synthesis, 55

TANNIC acid, 301

Tamin, 301

Tantalum, 58

Tartar, cream of, 291 crude, 289 emetic, 292

Tartaric acid, 289

Tartrates, analytical reactions of, 290

Taurine, 361

Taurocholic acid, 361

Teeth, 364

Tellurium, 58

Thallium, 58

Thebaine, 322

Theine, 381

Theobromine, 322

Thermometers, 25

Thorium, 58

Tin, 195

amalgam, 195

analytical reactions of, 196

cblorides of, 196

perchloride, 196

-stone, 195

Titanium, 58

Toluene, 265

Trichloraldehyde, 275

Trichlormethane, 276

Trinitro-cellulose, 315 -phenol, 298

'Trommer's test, 387

Tungsten, 58

Turpentine, 266

Turpeth mineral, 179
Tyrosine, 344

Type-metal, 193

Types, chemical, 248

JTTIMATE analysis, 240

Ultramarine, 142

Ultzmann's test, 391

Uranium, 58

Urates, 392

Urea, 374

determination of, 376

nitrate, 375

Uric acid, 378

Urinary calculi, 391

dejosits, 391

sediments, 3!1

Urine, 372

analysis, 380

color, 381

composition, 373

reaction, 382

secretion, 372

specific gravity, 382

Urinometer, 382

Urobilin, 381

Uroxanthin, 381

VALENCE, 45

Valerianates, 286

Valerianic acid, 286

Vanadium, 58

Vaseline, 263

Veratrine, 331 analytical reactions of, 331 oleate, 2863

Verdigris, 285

Vinegar, 284

Vitelin, 342

Vitriol, blue, 167 green, 149 oil of, 88

white, 161

Volatile oils, 266

WATER, 70

distilled, 72

drinking, 71

lime-, 133

mineral, 71

Weight, 27

of ammonia, 75

specific, 27

Whey, 367

Whiskey, 273

White arsenic, 184 precipitate, 180 vitriol, 161

Wine, 273 
INDEX.

Wood-naphtha, 270

-spirit, 270

Wrought-iron, 145

Y ANTHIN, 364

$\lambda$ Xylene, 265

VTTERBIUM, 58

1 Yttrium, 58

\section{T/ $\begin{aligned} & \text { INC, } 160 \\ & \text { acetate, } 285\end{aligned}$}

analytical reactions of, 163
Zinc-
antidotes to, 162
-blende, 160
bromide, 161
carbonate, 161
chloride, 161
ferrocyanide, 162
hydrate, 160
iodide, 161
oxide, 160
phosphide, 162
sulphate, 161
valerianate, 286
Zirconium, 58





\section{HENRY C. LEA'S SON \& CO.'S}

(LATE HENRY C. LEA)

\section{CLASSIFIEDCATALOGUE}

O F

\section{MEDICALA ND SURGICAL PUBLICATIONS.}

In asking the attention of the profession to the works advertised in the following pages, the publishers would state that no pains are spared to secure a continuance of the confidence earned for the publications of the house by their careful selection and accuracy and finish of execution.

The large number of inquiries received from the profession for a finer class of bindings than is usually placed on medical books has induced us to put certain of our standard publications in half Russia; and, that the growing taste may be encouraged, the prices have been fixed at so small an advance over the cost of sheep as to place it within the means of all to possess a library that shall have attractions as well for the eye as for the mind of the reading practitioner.

The printed prices are those at which books can generally be supplied by booksellers throughout the United States, who can readily procure for their customers any works not kept in stock. Where access to bookstores is not convenient, books will be sent by mail postpaid on receipt of the price, and as the limit of mailable weight has been removed, no difficulty will be experienced in obtaining through the post-office any work in this catalogue. No risks, however, are assumed either on the money or on the books, and no publications but our own are supplied, so that gentlemen will in most cases find it more convenient to deal with the nearest bookseller.

A handsomely illustrated catalogue will be sent to any address on receipt of a two-cent stamp.

$$
\text { HENRY C. LEA'S SON \& CO. }
$$

Nos. 706 and 708 Sansom St, Philadelphia, September, 1884.

PROSPECTUS FOR 1884.

\section{A WEEKLY MEDICAL JOURNAL.}

SUBSCRIPTION RATES.

The Medical News . . . . . . . Five Dollars.

The American Journal of the Medical Sciences . Five Dollars.

COMMUTATION RATES.

The Medical News $\}$ Nine Dollars per

The American Jounnal of the Medical Sciences $\}$ annum, in advance.

\section{THE MEDICAL NEWS.}

\section{A National Weekly Medical Periodical, containing 28 to 32 Quarto Pages of Reading Matter in Each Issue.}

In making the change, nearly three years since, from a monthly to a weekly, those in charge of The Medicar News, proposed to furnish the profession what it had never before 
enjoyed-a journal national in the widest sense of the word, devoted to the highest ideals of professional morals and honor, an unsparing enemy of quackery and fraud, a scientific magazine in elaboration, and a newspaper in energy and vitality. They believe that in every respect it has fulfilled its promises. Its readers and contributors are found in every State and Territory; its Editorial Staff includes some of the brightest minds in the profession, and in every issue living topics are editorially discussed in a scliolarly and practical manner; its corps of qualified reporters and correspondents covers all the medical centres of both hemispheres, and secures for its columns the earliest information on matters of medical interest, and its reports of Medical Progress are culled from all the important professional journals published on both continents.

The determination of THE NEws to maintain its position as the leading medical weekly of America, is evidenced by the special cable dispatches from its own representative at the recent International Medical Congress in Copenhagen. Its special articles on Koch's discovery of the Bacillus of Cholera have likewise commanded the attention of the profession, press and laity in all parts of the country. In short, its unrivalled organization enables THE NEws each week to lay upon the table of its readers an epitome of a week's advance of the whole medical world.

THE News, always endeavoring to enhance its usefulness, has pleasure in announcing to the profession that arrangements have been perfected for the publication during this year of a highly valuable series of practical articles by eminent men on the more important diseases met with by every practitioner in his daily duties.

Original articles from foreign authorities may also be expected, the first of which appeared in the issue of January 5th, 1884, on Digital Exploration of the Bladder in Obscure Vesical Diseases, with its results, with 7 original illustrations, by Sir Henrx Triompson, of London, Surgeon Extraordinary to the King of the Belgians, etc. From the high character of the articles already published, a fair conception may be formed of the value of the series to every professional man in active practice.

In typographical appearance, THE NEws of 1884 shows an advance even upon the issues of 1882-83, and nothing has been left undone to economize the time and promote the comfort of its readers. It appears in a double-columned quarto form, printed by the latest improved Hoe speed presses, on handsome paper, from a clear, easily read type, specially cast for its use.

The Medicar News employs all the approved methods of modern journalism with the intention of rendering itself indispensable to the profession; and, in the anticipation of an unprecedented circulation, its subscription has been placed at the exceedingly low rate of $\$ 5$ per annum, in advance. At this price it ranks as the cheapest medical periodical in this country, and when taken in connection with The American Journal at NINE DOLLARS per annum, it is confidently asserted that a larger amount of material of the highest class is offered than can be obtained elsewhere, even at a much higher price. 


\title{
THE AMERICAN JOURNAL of the MEDICAL SCIENCES,
}

\author{
Edited by I. MINIS HAYS, A. M., M. D.,
}

\section{Is published Quarterly, on the first days of Jaunary, April, July and October, each Number containing over 'Three Hundred Octavo Pages, fully Illustrated.}

Founded in 1820, The American Jourval is now completing its sixty-fifth consecutive year of faithful and honorable service to the profession. I3eing the only periodical in the English language capable of presenting elaborate articles-the form in which the most important discoveries have always been communicated to the professionThe Americax Journal cannot fail to be of the utmost value to physicians who would keep themselves au courant with the nedical thought of the day. It may justly claim that it numbers amoug its contributors all the most distinguished members of the profession, that its history is identified with the advances of medical knowledge, and that its circulation is co-extensive with the use of the English language.

During 1885 The JourNal will continue to present those features which have long proved so attractive to its readers.

The Original Department will consist of elaborate and richly illustrated articles from the pens of the most eminent members of the profession in all parts of the country.

The Review Department will maintain its well-earned reputation for discernment and impartiality, and will contain elaborate reviews of new works and topics of the day, and numerous analytical and bibliographical notices by competent writers.

Following these comes the Quarterly Summary of Improvements and Discoveries in the Medical Sciences, which, being a classified and arranged condensation of important articles appearing in the chief medical journals of the world, furnishes a compact digest of medical progress abroal and at lome.

The subscription price of The American Journal of the Medical Sciences has never been raised during its long career. It is still sent free of postage for Five Dollars per annum in advance.

Taken together, the Jounsal and Nwws combine the advantages of the elaborate preparation that can be devoted to a quarterly with the prompt conveyance of intelligence by the weekly; while, by special management, duplication of matter is rendered impossible.

It will thus be seen that for the very moderate sum of NINE DOLLARS in advance the subscriber will receive free of postage a weekly and a quarterly journal, both reflecting the latest advances of the medical sciences, and containing an equivalent of more than 4000 octavo pages, stored with the choicest material, original and selected, that can be furnished by the best medical minds of both hemispheres. It would be impossible to find elsewhere so large an amount of matter of the same value offered at so low a price.

The safest mode of renittance is by bank check or postal money orler, drawn to the order of the undersigned; where these are not accessible, remillances for subscriptions may be made at the risk of the publishers by forwirding in registered letters. Address,

Henry C. Lea's Son \& Co., Nos. 706 and 708 Sansom St., Philadelphia.

* * Communications to both these periodicals are invited from gentlemen in all parts of the country. Original articles contributed exclusively to either periodical are liberally paid for upon publication. When necessary to elucidate the text, illustrations will be furnished without cost to the author.

All letters pertaining to the Editorial Department of The Mrdical Nrws and The Americax Journat of tife Medical Sciexces should be addressed to the Editorial OfFices, 1004 Walnut Street, Philadelphia.

All letters yertaining to the Business Department of these journals should be addressed exclusively to Hexry C. Les's Sox \& Co., 706 and 708 Sansom Street, Philadelphia. 
DUNGLISON, ROBLEY, M. D.,

Late Professor of Instututes of Medicine in the Jefferson Medical College of Philadelphia.

MEDICAL LEXICON; A Dictionary of Medical Science: Containing a concise explanation of the various Subjects and Terms of Anatomy, Physiology, Pathology, Hygiene, Therapeutics, Pharmacology, Pharmacy, Surgery, Obstetrics, Medical Jurisprudence and Dentistry, Notices of Climate and of Mineral Waters, Formulæ for Officinal, Empirical and Dietetic Preparations, with the Accentuation and Etymology of the Terms, and the French and other Synonymes, so as to constitute a French as well as an English Medical Lexicon. A new edition, thoroughly revised, and very greatly modified and augmented. By Richard J. DUNGLison, M. D. In one very large and handsome royal octavo volume of 1139 pages. Cloth, $\$ 6.50$; leather, raised bands, $\$ 7.50$; very handsome half Russia, raised bands, $\$ 8$.

The object of the autlior, from the outset, has not been to make the work a mere lexicon or dictionary of terms, but to afford under each word a condensed view of its various medical relations, and thus to render the work an epitome of the existing condition of medical science. Starting with this view, the immense demand which has existed for the work has enabled him, in repeated revisions, to augment its completeness and usefulness, until at length it has attained the position of a recognized and standard authority wherever the language is spoken. Special pains have been taken in the preparation of the present edition to maintain this enviable reputation. The additions to the vocabulary are more numerous than in any previous revision, and particular attention has been bestowed on the accentuation, which will be found marked on every word. The typographical arrangement has been greatly improved, rendering reference mucl more easy, and every care has been taken with the mechanical execution. The volume now contains the matter of at least four ordinary octavos.

A book of which every American ought to be prond. When the learned author of the work passed away, probably all of us feared lest the book should not maintain its place in the advaneing science whose terms it defines. Fortunately, Dr. Richard J. Dunglison, having assisted his father in the revision of several editions of the work, and having been, therefore, trained in the methods and imbued with the spirit of the book, has been able to edit it as a work of the kind should be edited-to carry it on steadily, witlıout jar or interruption, along the grooves of thought it has travelled during its lifetime. To show the magnitude of the task which Dr. Dunglison has assumed and carried through, it is only necessary to state that more than six thousand new subjects lave been added in the present edition.-Philadelphia Medical Times, Jan. 3, 1874.

About the first book purchased by the medical student is the Medical Dictionary. The lexicon explanatory of technical terms is simply a sine qua non. In a seience so extensive and with such collaterals as medicine, it is as much a necessity also to the practising physician. To meet the wants of students and most physicians the dictionary must be condensed while comprehensive, and practical while perspicacious. It was becalse Dunglison's met these indications that it became at once the dictionary of general use wherever medicine was studied in the English language. In no former revision have the alterations and additions been so great. The chief terms have been set in black letter, while the derivatives follow in small caps; an arrangement which greatly facilitates reference. - Cincinnati Lancet and Clinic, Jan. 10, 1874.

As a standard work of reference Dunglison's

work has been well known for about torty years and needs no words of praise on our part to recom mend it to the members of the medical, and likewise of the pharmacentical, profession. The latter especially are in need of a work which gives ready and reliable information on thousands of subjects and terms which they are liable to encounter in pursuing their daily vocations, but with which they cannot be expected to be familiar. The work before as fully supplies this want.-American Journal of Pharmacy, Feb. 1874.

Particular care has been devoted to derivation and accentuation of terms. With regard to the latter, indeed, the present edition may be considered a complete "Pronouncing Dictionary of Medical Science." It is perhaps the most reliable work published for the busy practitioner, as it contains information upon every medical subject, in a form for ready access, and with a brevity as admirable as it is practical.-Southern Medical Record, Feb. 1874.

A raluable dictionary of the terms employed in medicine and the allied sciences, and of the rels tions of the subjects treated under each head. It well deserves the authority and popularity it has obtained.-British Mad. Jour., Oct. 31, 1874.

Few works of this class exhibit a yrander monument of patient research and of scientific lore.London Lancet, May 13, 1875 .

Dunglison's Dictionary is incalculably valuable, and indispensable to every practitioner of medicine, pharmacist and dentist.-Western Lancet, March, 1874.

It has the rare merit that it certainly has no rival in the English language for accuracy and extent of references.-London Medical Gazette.

\section{HOBLYN, RICHARD D., M. D.}

A Dictionary of the Terms Used in IMedicine and the Collateral Sciences. Revised, with numerous additions, by IsAAC HAYs, M. D., late editor of The American Journal of the Medical Sciences. In one large royal $12 \mathrm{mo}$. volume of 520 double-columned pages. Clotl, $\$ 1.50$; leather, $\$ 2.00$.

It is the best book of definitions we have, and ought always to be upon the student's table.-Southern Medical and Surgical Journal.

RODWELL, G. F., F. R. A.S., F. C. S.,

Lecturer on Natural Science at Clifton College, England.

A Dictionary of Science: Comprising Astronomy, Chemistry, Dynamics, Electricity, Heat, Hydrodynamics, Hydrostaties, Light, Magnetism, Mechanics, Meteorology, Pneumatics, Sound and Statics. Contributed by J. T. Bottomley, M. A., F. C.S., William Crookes, F.R.S., F.C.S., Frederick Guthrie, B.A., Ph. D., R. A. Proctor, B.A., F.R.A.S., G. F. Rodwell, Editor, Charles Tomlinson, F.R.S., F.C.S., and Richard Wornell, M.A., B.Sc. Preceded by an Essay on the History of the Plysical Sciences. In one handsome octavo volume of 702 pages, with 143 illustrations. Clotl, $\$ 5.00$. 
HARTSHORNE, HENRY, A. M., M. D.,

Lately Professor of Hygiene in the University of Pcnnsylvania.

A Conspectus of the Medical Sciences; Containing Handbooks on Anatomy, Physiology, Chemistry, Materia Medica, Practice of Medicine, Surgery and Obstetrics. Second edition, thoroughly revised and greatly improved. In one large royal $12 \mathrm{mo}$. volume of 1028 pages, with 477 illustrations. Cloth, $\$ 4.25$; leather, $\$ 5.00$.

The object of this manual is to afford a conven- industry and energy of its able editor.-Boston ient work of reference to students during the brief $\mid$ Medical and Surgical Journal, Sept. 3, 1874.

moments at their command while in attendance We can say, with the strictest truth, that it is the upon medical lectures. It is a favorable sign that best work of the kind with which we are acquaintit has beeu found necessary, in a short space of ed. It embodies in a condensed form all recent time, to issue a new and carefully revised edition. contribntions to practical medicine, and is thereThe illustrations are very numerous and unusu- fore useful to every busy practitioner throughout ally clear, and each part seems to have received our country, besides being admirably adapted to its due share of attention. We can conceive such the nse of students of medicine. The book is a work to be useful, not only to students, but to faithfully and ably executed.-Charleston Medical practitioners as well. It reflects credit upon the Journal, April, 1875.

\section{STUDENTS' SERIES OF MANUALS.}

A Series of Fifteen Manuals, for the use of Students and Practitioners of Medicine and Surgery. They will be written by eminent Teachers or Examiners, and will be issued in pocket-size $12 \mathrm{mo}$. volumes of $300-540$ pages, richly illustrated and at a low price. The following volumes may now be announced: KLEIN's Elements of Histology, PePPER's Surgical Pathology, Treves' Surgical Applied Anatomy, Ralfe's Clinical Chemistry, Clarke and Lock woon's Dissectors' Manual, Power's Human Physiology, and Bruce's Materia Medica and Therapeutics, (Just ready); Ronertson's Physiological Physics, Bentamy's Operative Surgery, BenL's Comparative Physiology and Anatomy, Gould's Surgical Diagnosis, PEPPER's Forensic Medicine, and CuRnow's Medical Applied Anatomy, (In active preparation for early publication.) For separate notices see index on last page.

\section{SERIES OF CLINICAL MANUALS.}

In arranging for this Series it has been the design of the publishers to provide the profession with a collection of authoritative monographs on important clinical subjects in a cheap and portable form. The appended list of authors and titles will give an idea of the general plan, and details regarding size and price may be expected at an early day: Hutchinson on Syphilis; Savage on Insanity, including Hysteria; Bryant on the Breast; Treves on Intestinal Obstruction; Morris on Surgical Diseases of the Kidney; Broadbent on the Pulse; Butlin on the Tongue; Owen on Surgical Diseases of Children; LuCAS on Diseases of the Urethra; MARSH on Diseases of the Joints; PICK on Fractures and Dislocations. For separate notices see index on last page.

\section{NEILL, JOHN, M. D., and SMTTH, F. G., M. D.,}

Late Surgeon to the Penna. Hospital. Prof. of the Institutes of Med. in the Univ. of Penna.

An Analytical Compendium of the Various Branches of Medical Science, for the use and examination of Students. A new edition, revised and improved. In one very large royal $12 \mathrm{mo}$. volume of 974 pages, with 374 woodcuts. Cloth, $\$ 4$; strongly bound in leather, raised bands, $\$ 4.75$.

\section{LUDLOW, J.L., M.D.,}

Consulting Physician to the Philadelphia Hospital, etc.

A Manual of Examinations upon Anatomy, Physiology, Surgery, Practice of Medicine, Obstetrics, Materia Medica, Chemistry, Pharmacy and Therapeutics. To which is arlded a Medical Formulary. Third edition, thoroughly revised, and greatly extended and enlarged. In one handsome royal 12mo. volume of 816 large pages, with 370 illustrations. Cloth, $\$ 3.25$; leather, $\$ 3.75$.

The arrangement of this volume in the form of question and answer renders it especially suitable for the office examination of students, and for those preparing for graduation.

\section{WILSON, ERASMUS, F. R. S.}

A System of Human Anatomy, General and Special. Edited by W. H. Gobrecht, M. D., Professor of General and Surgical Anatomy in the Medical College of Ohio. In one large and handsome octavo volume of 616 pages, with 397 illustrations. Cloth, $\$ 4.00$; leatlier, $\$ 5.00$.

\section{SMITH, H. H., M. D., and HORNER, WM. E., M. D.,}

Emeritus Prof. of Surgery in the Univ. of Penna., etc. Late Prof. of Anat. in the Univ. of Penna.

An Anatomical Atlas, Illustrative of the Structure of the Human Body. In one large imperial octavo volume of 200 pages, with 634 beautiful figures. Cloth, $\$ 4.50$.

\section{CLELAND, JOHN, M. D., F. R. S.,}

Professor of Anatomy and Physiology in Queen's College, Galvay.

A Directory for the Dissection of the Human Body. In one 12mo. volume of 178 pages. Cloth, $\$ 1.25$. 


\section{ALLEN, HARRISON, M. D.,}

Professor of Physiology in the University of Pennsylvania.

A System of Human Anatomy, Including Its Medical and Surgical Relations. For the use of Practitioners and Students of Medicine. With an Introductory Section on Histology. By E. O. Shakespeare, M. D., Ophthalmologist to the Philadelphia Hospital. Comprising about 825 double-columned quarto pages, with 380 illustrations on 109 full page lithographic plates, many of which are in colors, and 241 engravings in the text. In six Sections, each in a portiolio. Section I. Histology. Section II. Boxfs axd Jolnts. Section III. Muscles and Fasciz. Section IV. Arteries, Veiss axd Lymphatics. Section V. Nervods System. Section VI. Organs of SENse, of Digestion and Gexito-URinary ORgans, Embryology, Deveropment, Teratology, Superficial Anatomy, Post-Mortem Examinations, AND Genfray AND Cuinical Indexfs. Iust ready. Price per Section, each in a handsome portfolio, $\$ 3.50$; also bound in cloth $\$ 23.00$; very handsome half Russia, raised bands and open back, \$25.00. For sale by subscription only. Apply to the Publishers.

\section{Extract from Introduction.}

It is the design of this book to present the facts of human anatomy in the manner best suited to the requirements of the student and the practitioner of medicine. The author believes that such a book is needed, inasmuch as no treatise, as far as he knows, contains, in addition to the text descriptive of the subject, a systematic presentation of such anatomical facts as can be applied to practice.

A book which will be at once accurate in statement and concise in terms; which will be an acceptable expression of the present state of the science of anatomy; which will exclude nothing that can be made applicable to the medical art, and which will thus embrace all of surgical importance, wlile onitting nothing of valne to clinical medicine,-would appear to have an excuse for existence in a country where most surgeons are general practitioners, and where there are few general practitioners who have no interest in surgery.

It is to be considered a study of applied anatomy |care, and are simply superb. There is as much in its widest sense-a systematic presentation of of practical application of anatomical points to such anatomical facts as can be applied to the the every-day wants of the medical clinician as practice of medicine as well as of surgery. Our to those of the operating surgean. In fact, few author is concise, accurate and practical in his general practitioners will read the work without a statements, and succeeds admirably in infusing feeling of surprised glatification that so many an interest into the study of what is generally con. points concerning which they may never have sidered a dry subject. The department of Histol- thought before are so well presented ior their conogy is treated in a masterly manner, and the sideration. It is a work which is destined to be ground is travelled over by one thoroughly famil- the best of its $\mathrm{kind}$ in any language.-Medical iar with it. The illustrations are made with great Rccord, Nov. 25,1882 .

\section{CLARKE,W.B., F.R.C.S. \& LOCKWOOD,C.B., F.R.C.S.} Demonstrators of Anatomyat St. Bartholomew's Hospital Medical School, London.

The Dissector's Manual. In one pocket-size 12mo. volume of 396 pages, with 49 illustrations. Limp cloth, red edges, \$1.50. Just ready. See Students' Series of Manuals, page 5 .

This is a very excellent manual for the use of the part, are good and instructive. The book is neat student who desires to learn anatomy. The meth- and convenient. We are glad to recommend it.ods of demonstration seem to us very satisfactory. Boston Medical and Surgical Journal, Jan. 17, 1884. There are many woodcuts which, for the most

\section{TKEVES, FIEDERICK, F.R.C.S.,}

Senior Demonstrator of Anatomy and Assistant Surgcon at the London Hospital.

Surgical Applied Anatomy. In one pocket-size $12 \mathrm{mo}$. volume of 540 pages, with 61 illustrations. Limp cloth, red edges, \$2.00. Just ready. See Students' Series of Manuals, page 5 .

He has produced a work which will command a quickened by daily use as a teacher and practilarger circle of reader's than the class for which it tioner, has enabled our author to prepare a work was written. This union of a thorough, practical which it would be a most difficult task to excel.acçnaintance with these fundamental branches, The American Practitioner, Feb., 1884.

\section{CURNOW, JOHN, M. D., H. R. C. P.,}

Professor of Anatomy at King's College, Plysician at King's College IIospital.

Medical Applied Anatomy. In one pocket-size 12mo. volume. Preparing. See Students' Series of Manuals, page 5.

\section{BELLAMI, EDWARD, F. R. C.S., \\ Senior Assistant-Surgeon to the Charing-Cross Hospital, London.}

The Student's Guide to Surgical Anatomy: Being a Description of the most Important Surgical Regions of the Human Body, and intended as an Introduction to Operative Surgery. In one 12mo. volume of 300 pages, with 50 illustrations. Cloth, \$2.25.

HARTSHORNE'S HANDBOOK OF ANATOMY AND PHISIOLOGY. Second edition, revised. In one royal $12 \mathrm{mo}$. volume of 310 pages, with 220 woodcuts. Cloth, $\$ 1.75$.
HORNER'S SPECIAL ANATOMY AND HISTOLOGY Eirhth edition, extensively revised and modified. In two octavo volumes of 1007 pages, with 320 woodcuts. Cleth, $\$ 6.00$. 


\section{GRAY, HENRY, F. R. S.,}

Leclurer on Analomy at St. George's Hospital, London.

Anatomy, Descriptive and Surgical. The Drawings by H. V. CARTER, M. D., and Dr. Westmacotr. The dissections jointly by the AUtror and Dr. CArTer. With an Introduction on General Anatomy and Development by T. Hormes, M. A., Surgeon to St. George's Hospital. Edited by T. Pickering Pick, F. R. C. S., Surgeon to and Lecturer on Anatomy at St. George's Hospital, London, Examiner in Anatomy, Royal College of Surgeons of England. A new American from the tenth enlarged and improved London edition. To which is added the second American from the latest English edition of Landmarks, Medical and Surgical, by Lutiner Holden, F.R.C.S., author of "Human Osteology," "A Manual of Dissections," etc. In one imperial octavo volume of 1023 pages, with 564 large and elaborate engravings on wood. Cloth, $\$ 6.00$; leather, $\$ 7.00$; very handsome lialf Russia, raised bands, $\$ 7.50$.

This work covers a more extended range of subjects than is customary in the ordinary text-books, giving not only the details necessary for the student, but also the application to those details to the practice of medicine and surgery. It thus forms both a guide for the learner and an admirable work of reference for the active practitioner. The engravings form a special feature in the work, many of them being the size of nature, nearly all original, and having the names of the various parts printed on the body of the cut, in place of figures of reference with descriptions at the foot. They thus form a complete and splendid series, which will greatly assist the student in forming a clear idea of Anatomy, and will also serve to refresh the memory of those who may find in the exigencies of practice the necessity of recalling the details of the dissecting-room. Combining, as it does, a complete Atlas of Anatomy with a thorough treatise on systematic, descriptive and applied Anatomy, the work will be found of great service to all physicians who receive students in their offices, relieving both preceptor and pupil of much labor in laying the groundwork of a thorough medical education.

Landmarks, Medical and Surgical, by the distinguished Anatomist, Mr. Luther Holden, has been appended to the present edition as it was to the previous one. This work gives in a clear, condensed and systematic way all the information by which the practitioner can determine from the external surface of the body the position of internal parts. Thus complete, the work, it is believed, will furnish all the assistance that can be rendered by type and illustration in anatomical study.

This well-known work comes to us as the latest There is probably nowork nsed so universally American from the tenth English edition. As its by physicians and medical students as this one. title indicates, it has passed through many hands It is deserving of the confidence that they repose and has received many additions and revisions. in it. If the present edition is compared with that The work is not susceptible of more improvement. issued two years ago, one will readily see how Taking it all in all, its size, manner of make-up, much it has been improved in that time. Many its character and illustrations, its general accur: pages have been added to the text, especially in acy of description, its praetieal aim, and its per- those parts that treat of histology, and many new spicuity of style, it is the Anatomy best adapted to cuts have been introduced and old ones modified. the wauts of the student and practitioner.-Medical $\frac{- \text { Journal of the American Medical Association, Sept. }}{1,1883 .}$
Record, Sept. $15,1883$. Record, Sept. 15, 1883.

\section{A ISO FOR SAIE SEPARATE-}

\section{HOLDEN, LUTHER, F. R. C.S.,}

Surgeon to St. Bartholomew's and the Foundling Hospitals, London.

Landmarks, Medical and Surgical. Second American from the latest revised English edition, with additions by W. W. KEEx, M. D., Professor of Artistic Anatomy in the Pennsylvania Academy of the Fine Arts, formerly Lecturer on Anatomy in the Philadelphia School of Anatomy. In one handsome $12 \mathrm{mo}$. volume of 148 pages. Cloth, $\$ 1.00$.

This little book is all that can be desired within almost to learn it by heart. It teaehes diagnosis by its scope, and its contents will be found simply in- external examination, ocular and palpable, of the valuable to the young surgeon or physician, since body, with such anatomical and physiological facts they bring before him such data as he requires at as directly bear on the subject. It is eminently every examination of a patient. It is written in the student's and young practitioner's book.-Phylanguage so clear and concise that one ought sician and Surgeon, Nov. 1881.

\section{DALTON, JOHN C., M. D.,}

Professor of Physiology in the College of Physicians and Surgeons, Neto York.

The Topographical Anatomy of the Brain. In one very handsome quarto volume of about 200 pages of descriptive text. Illustrated with forty-nine life-size photographic illustrations of Brain Sections, with a like number of outline explanatory plates, as well as many carefully-executed woodcuts through the text. In press.

\section{ELLIS, GEORGE VINER,}

Emeritus Professor of Anatomy in University College, London.

Demonstrations of Anatomy. Being a Guide to the Knowledge of the Human Body by Dissection. From the eighth and revised London edition. In one very handsome octavo volune of 716 pages, with 249 illustrations. Cloth, \$4.25; leather, \$5.25.

Ellis' Demonstrations is the favorite text-book speeial line. The descriptions are clear, and the of the English student of anatomy. In passing rnethods of pursting anatomieal investigations are through eight editions it has been so reviserl anil given with such detail that the book is honestly adapted to the needs of the student that it would entitled to its name.-St. Louis Clinical Record, aeem that it had almost reached perfeetion in this June, 1879. 


\section{DALTON, JOHN C., M. D.,}

Professor of Physiology in the College of Physicians and Surgeons, New York, etc.

A Treatise on Human Physiology. Designed for the use of Students and Practitioners of Medicine. Seventh edition, thoroughly revised and rewritten. In one very handsome octavo volume of 722 pages, with 252 beautiful engravings on wood. Cloth, $\$ 5.00$; leather, $\$ 6.00$; very handsome half Russia, raised bands, $\$ 6.50$.

The merits of Professor Dalton's text-book, his | more compact form, yet its delightful charm is resmooth and pleasing style, the remarkable clear- tained, and no subject is thrown into obscurity. ness of his descriptions, which leave not a chapter Altogether this edition is far in advance of any obscure, his cautious judgment and the general previous one, and will tend to keep the profession correctness of his facts, are perfectly known. They posted as to the most recent additions to our have made his text-book the one most familiar physiological knowledge--Michigan Medical News, to American students.-Med. Record, March 4, 1882. A A pril, 1882.

Certainly no physiological work has ever issued One can scarcely open a college catalogue that from the press that presented its subject-matter in does not have mention of Dalton's Physiology as a clearer and more attractive light. Almost every the recommended text or consultation-book. For puge bears evidence of the exhaustive revision American students we would unreservedly recomthat has taken place. The material is placed in a mend Dr. Dalton's work.-Va.Med. Monthly, July,'s2.

\section{FOSTER, MICHALL, M. D., F. R. S.,}

Professor of Physiology in Cambridge University, England.

Text-Book of Physiology. Third American from the fourth English edition In one handsome royal $12 \mathrm{mo}$. volume of over 1000 pages, with about 300 illust. Preparing.

A notice of the previous edition is appended.

A more compact and scientific work on physiol- | eration the late discoveries in physiological chemogy has never been published, and we believe our- istry and the experiments in localization of Ferrier selves not to be mistalien in aserting that it las now been introduced into every medical college in which the English language is spoken. This work conforms to the latest researches into zoology and comparative anatomy, and takes into considand others. Tlue arrangement followed is such as to render the whole subject lucid and well connected in its various parts. - Chicago Medical Journal and Examiner, August, 1882.

\section{POWER, HENTY, M. B., F. R. C. S.,}

Examiner in Physiology, Royal College of Surgeons of England.

Human Physiology. In one handsome pocket-size 12mo, volume of 396 pages. with 47 illustrations. Cloth, $\$ 1.50$. Just ready. See Students' Series of Manuals, page 5.

This little work is deserving of the highest / as to place it within the reach of all, while the expraise, and we can hardly conceive how the main cellence of its text will certainly secure for it most facts of this science could have been more clearly favorable commendation._Cincinnati Lancet and or concisely stated. The price of the work is such C'tinic, Feb. 16, 1884 .

\section{DRAPER, JOHN C., M. D., LL. D.,}

Professor of Chemistry in the University of the City of New York.

Medical Physics. A Text-book for Students and Practitioners of Medicine. In one handsome octavo rolume of about 600 pages, with about 250 woodcuts. Preparing.

The object of the author has been to present in a clear and concise manner, without undue technicalities, the most modern views of physics in their special bearing on medical science. Familiarity with the laws and principles which govern the relations of force and matter is necessary, not only to a clear comprehension of plysiology, but is an inestimable aid to the physician and surgeon in their daily practice; yet the subject is strangely neglected in professional education and is one for which the medical student has no special text-book. This want Professor Draper has endeavored to supply, and his distinguisled reputation guarantees such a presentation of the subject that the work will be one, not only essential to the student, but of interest and importance to the intelligent practitioner.

ROBERTSON, J. McGREGOR, M. A., M. B.,

Muirhead Demonstrator of Physiology, University of Glasgow.

Physiological Physics. See Students' Series of Manuals, page 5. Preparing.

\section{BELL, F. JEFFTEY, M. A.,}

Professor of Comparative Anatomy at King's College, London.

Comparative Physiology and Anatomy. In active preparation for early publication. See Students' Series of Manuals, page 5.

\section{CARPENTER, WM. B., M. D., F. R. S., E. G. S., F. L. S.,}

Registrar to the University of London, etc.

Principles of Human Physiology. Edited by Hexry Power, M. B., Lond., F.R.C.S., Examiner in Natural Sciences, University of Oxford. A new American from the eiglith revised and enlarged edition, with notes and additions by Fraxcis G. SiITH, M. D., late Professor of the Institutes of Medicine in the University of Pennsylvania. In one very large and handsome octavo volume of 1083 pages, with two plates and 373 illus. trations Cloth, $\$ 5.50$; leather, $\$ 6.50$; half Russia, $\$ 7$. 
ATTEIELD, JOHN, Ph. D.,

Professor of Practical Chentistry to the Pharmaceutical Society of Great Britain, etc.

Chemistry, General, Medical and Pharmaceutical; Including the Chemistry of the U. S. Pharmacopœia. A Manual of the General Principles of the Science, and their Application to Medicine and Pharmacy. A new American, from the tenth English edition, specially revised by the Author. In one handsome royal $12 \mathrm{mo}$. volume of 728 pages, with 87 illustrations. Cloth, $\$ 2.50$; leatler, $\$ 3.00$.

It is a book on which too much praise cannot be indispensable to all persons engaged in those debestowed. As a text book for medical schools it partments of science. It includes the whole is unsurpassable in the present state of chemical clemistry of the last Pharmacopoia.-Pacific Mediscience, and having been prepared with a special cal and Surgical Journal, Jan. 1884.

view towards medicine and pharmacy, it is alike

\section{BLOXAM, CHARLES L.,}

Professor of Chemistry in K'ing's College, London.

Chemistry, Inorganic and Organic. New American from the fifth London edition, thoroughly revised and much improved. In one very handsome octavo volume of 727 pages, with 292 illustrations. Cloth, $\$ 3.75$; leather, $\$ 4.75$. Just ready.

The general plan of this work remains the laws and principles involved. We gladly repeat same as in previous editions, the evident object now the opinion we expressed about a form er being to give clear and concise descriptions of all edition, that we regard Bloxam's Chemistry as known elements and of their most important one of the best treatises on general and applied compounds, with explanations of the chemical chemistry.-American Jour. of Pharmacy, Dec. 1883.

\section{FTANKLAND, E., D.C.L., F.R.S., d JAPP, E. R., F.C.S.}

Inorganic Chemistry. In one handsome volume, with illustrations. Preparing.

\section{SIMON, W., Ph. D., M. D.,}

Professor of Chemistry and Toxicology in the College of Physicians and Surgeons, Baltimore, and Professor of Chemistry in the Maryland Collcge of Pharmacy.

Manual of Chemistry. A Guide to Lectures and Laboratory work for Beginners in Chemistry. A Text-book, specially adapted to Students of Pharmacy and Medicine. In one $12 \mathrm{mo}$. vol. of $400 \mathrm{pp}$, with 17 woodcuts and 7 plates with colors illustrating over 50 of the most important chemical reactions. Shortly.

\section{REMSEN, IRA, M. D., Ph. D.,}

Professor of Chemistry in the Johns Hopkins University, Baltimore.

Principles of Theoretical Chemistry, with special reference to the Constitution of Chemical Compounds. Second and revised edition. In one handsome royal $12 \mathrm{mo}$. volume of 240 pages. Cloth, $\$ 1.75$. Just ready.

The book is a valuable contribution to the chemical literature of instruction. That in so few years a second edition has been called for indicates that many chemical teachers have been folnd ready to eudorse its plan and to adopt its methods. In this edition a considerable proportion of the book has been rewritien, much new matter has been \begin{tabular}{l|l} 
added and the whole has been brought up to date. & to the average text books of $t$ \\
Wo earnestly commend this book to every student & Journal of Science, March, 1884.
\end{tabular}

of chemistry. The high reputation of the author assures its accuracy in all matters of fact, and its judicious conservatism in matters of theory, combined with the fulness with which, in a small compass, the present attitude of chemical science towards the constitution of compounds is considered, gives it a value much beyond that accorded to the average text books of the day.-American

\section{FOWNES, GEORGE, Ph. D.}

A Manual of Elementaly Chemistry; Theoretical and Practical. Revised and corrected by Henry WATts, B.A., F. R.S., Editor of A Dictionary of Chemistry, etc. A new American from the twelfth and enlarged London edition. Edited by RosERT Bridges, M. D. In one large royal 12mo. volume of 1031 pages, witl 177 illustrations on wood and a colored plate. Cloth, $\$ 2.75$; leather, $\$ 3.25$.

The book opens with a treatise on Chemical Physics, including Heat, Light, Magnetism and Electricity. 'These subjects are treated clearly and briefly, but enongh is given to enable the student to comprehend the facts and laws of Chemistry proper. It is the fashion of late years to omit these topics from works on chemistry, but their om the great advance in the science of Cliemistry of late years, the chapter on the General Principles of Chemical Philosophy has been entirely rewritten. The latest views on Equivalents, Quantivalence, etc., are clearly and fully set forth. This last edition is a great improvement upon its prede. cessors, which is saving not a little of a book that has reached its twelfth edition.-Ohio Medical Recorder, Oct., 1878.

Wöhler's Outlines of Organic Chemistry. Edited by FrrtiG. Translated by Ira REmsen, M. D., Ph. D. In one $12 \mathrm{mo}$. volume of 550 pages. Cloth, $\$ 3$.

GALLOWAY'S QUALITATIVE ANALYSIS. New edition. Preparing.

LEHMANN'S MANUAL OF CHEMICAL PHYSIOLOGY. In one octaro rolume of 327 pages, with 41 illustrations. Cloth, $\$ 2.25$.
CARPENTER'S PRIZE ESSAY ON THE USE AND AbUse of Alcollolic Liquons in Health aNd DigEASE. With explanations of scientific words. Small 12mo. 178 pages. Cloth, 60 cents. 
HOFWMANN, F., A.M., Ph.D., \& POWIR F.B., Ph.D., Public Analyst to the State of New York.

Prof. of Anal. Chem. in the Phill. Coll. of Pharmacy.

A Manual of Chemical Analysis, as applied to the Examination of Medicinal Chemicals and their Preparations. Being a Guide for the Determination of their Identity and Quality, and for tle Detection of Impurities and Adulterations. For the use of Pharmacists, Plıysicians, Druggists and Manufacturing Chemists, and Pharmaceutical and Medical Students. Third edition, entirely rewritten and much enlarged. In one very handsome octavo volume of 621 pages, with 179 illustrations. Cloth, $\$ 4.25$.

We congratulate the author on the appearance tion of them singularly explicit. Moreover, it is of the third edition of this work, published for the exceptionally free from typographical errors. We first time in this country also. It is admirable and have no hesitation in recommending it to those the information it undertakes to supply is both who are engaged either in the manufacture or the extensive and trustworthy. The selection of pro- testing of medicinal chemicals.-London Pharmacesses for determining the purity of the substances of which it treats is excellent and the descrip-

\section{WATTS, HENRY, B. A., F. R. S.}

Author of "A Dictionary of Chemistry," etc.

A Manual of Physical and Inorganic Chemistry. In one 12mo. volume of 500 pages with 150 illustrations. Preparing.

\section{CLOWES, FRANK, D. Sc., London,}

Senior Science-Master at the High School, Newcastle-under-Lyme, etc.

An Elementary Treatise on Practical Chemistry and Qualitative Inorganic Analysis. Specially adapted for use in the Laboratories of Schools and Colleges and by Beginners. Second American from the third and revised English edition. In one very haudsome royal $12 \mathrm{mo}$. volume of 372 pages, with 47 illustrations. Cloth, \$2.50.

The chief object of the author of the present work renders it unintelligible to the primary stndent was to furnish one which was sufficiently elemen. tary in the description of apparatuses, chemicals, modes of experimentation, etc., so as to "reduce to a minimum the amount of assistance required from a teacher." It is a generally recognized fact that one of the most serious hindrances to the utility of many of the smaller text-books is the too great conciseness of the language employed, which unless supplenented by copious verbal explana tions from the teacher. The Elementary Treatise of Dr. Clowes, examined with reference to the above claims, is found to be a great improvement on other elementary works. A student who carefully reads this text will scarcely need the assistance of a tutor in following ont any of the experiments described.-Va. Med. Monthly, Ap., 1881.

\section{RALFE, CHARLES H., M. D., F. R. C.P.,}

Assistant Physician at the London Hospital.

Clinical Chemistry. In one pocket-size 12 mo. volume of 314 pages, with 16 illustrations. Limp cloth, red edges, \$1.50. See Students' Series of Manuals, page 5.

This is one of the most instructive little works | cine. Dr. Ralfe is thoroughly acquainted with the that we have met with in a long time. The author latest contributions to his seience, and it is quite is a physician and physiologist, as well as a chem- refreshing to find the subject dealt with so clearly ist, consequently the book is unqualifiedly prac- and simply, yet in snch evident harmony with the tical, telling the physician just what he ought to modern scientific methods and spirit.-MIedical know, of the applications of chemistry in medi- Record, February 2, 1884.

\section{CHARLES, T. CRANSTOUN, M. D., F. C. S., M. S.,}

Formerly Asst. Prof. and Demonst. of Chemistry and Chemical Physics, Queen's College, Belfast.

The Elements of Physiological and Pathological Chemistry. A Handbook for Medical Students and Practitioners. Containing a general account or Nutrition, Foods and Digestion, and the Chemistry of the Tissues, Organs, Secretions and Excretions of the Body in Health and in Disease. Together with the methods for preparing or separating their clief constituents, as also for their examination in detail, and an outline syllabus of a practical course of instruction for students. In one octavo volume, with 38 woodcuts and 1 colored plate. Shortly.

\section{CLASSEN, ALEXANDER,}

Professor in the Royal Polytechnic School, Aix-la-Chapelle.

Elementary Quantitative Analysis. Translated, with notes and additions, by Edgar F.Smiri. Ph. D., Assistant Professor of Chemistry in the Towne Scientific School, University of Peına. In one 12mo. volume of 324 pages, with 36 illust. Cloth, $\$ 2.00$.

It is probably the best manual of an elementary $\mid$ and then advancing to the analysis of minerals and nature extant, insomuch as its methods are the such products as are met with in applied chemisbest. It reaches by examples, commeneing with try. It is an indispensable book for students in single determinations, followed by separations, ehemistry.-Boston Journal of Chemistry, Oct. 1878.

\section{GREENE, WILLIAM H., MI. D.,}

Demonstrator of Chemistry in the Medical Department of the University of Pennsylvania.

A Manual of Medical Chemistry. For the use of Students. Based upon Bowman's Medical Chemistry. In one $12 \mathrm{mo}$. volume of 310 pages, witl 74 illus. Cloth, \$1.75.

It is a coucise manual of three hundred pages, the recognition of compounds due to pathologica giving an excellent summary of the best methods of analyzing the liquids and solids of the body, both for the estimation of their normal constituents and conditions. The detection of poisons is treated with sufficient fulness for the purpose of thestudent or practitioner.-Boston $J$. of Chem., June, ' 80 


\section{PARTISH, EDWARD,}

Late Professor of the Theory and Practice of Pharmacy in the Philadelphia College of Pharmacy.

A Treatise on Pharmacy: designed as a Text-book for the Student, and as a Guide for the Physician and Pharmacentist. With many Formulæ and Prescriptions. Fifth edition, thoroughly revised, by Thomas S. Wiegand, Ph. G. In one handsome octaro volume of 1093 pages, with 256 illustrations. Cloth, \$5; leather, \$6. Just ready.

A new edition of this masterly work, with nu- ' a valuable guide and compend for the physician merous additions, and so revised as to be in accord and medical student.--The Physician and Surgeon, with the new Pharmacopoia and the latest teach- $A$ pril, 1884.

ings in chemistry, is a publication of the first im. It would be difficult to find a more complete portance. No thoroughgoing pharmacist will fail work, for it contains minute information upon to possess himself of so useful a guide to practice, every subject pertaining to pharmacy,-Cincinnati and no physician who properly estimates the value of an accurate knowledge of the remedial agents employed by him in daily practice, so far as their miscibility, compatibility and most effective methods of combination are concerned, can afford to leave this work out of the list of their works of reference. The country practitioner, who must always be in a measure his own pharmacist, will find it indispensable.-Louisville Mcdical Nexs, March $29,1884$.

This treatise on Pharmacy is as indispensable to the dispensing or manufacturing druggist, and student of pharmacy, as Dunglison's Medical Dictionary is to the doctor and student of medicine. It has ceased being a literary luxury and has become a necessity. The work is not merely a textbook for pharmacy students and druggists, but is Icdical Neus, Jan. 1884,

This well-known work presents itself now based upon the recently revised new Pharmacopceia. Several important modifications of the internal arrangement have been made, and we believe they will be found to increase the practical usefulness of the book. Each page bears evidence of the care bestowed upon it, and conveys valuable information from the rich store of the edjtor's experience. In fact, all that relates to practical pharmacy-apparatus, processes and dispensinghas been arranged and described with clearness in its various aspects, so as to afford aid and advice alike to the student and to the practical pharmacist. The work is judiciously illustrated with good woodcuts-American Journal of Plarmacy, January, 1884

\section{HERMANN, Dr. L.,}

Professor of Physiology in the University of Zurich.

Fxperimental Pharmacology. A Handbook of Methods for Determining the Physiological Actions of Drugs. Translated, with the Author's permission, and with extensive additions, by RobERT MEAde Sirth, M. D., Demonstrator of Pliysiology in the University of Pennsylvania. In one handsome 12mo. volume of 199 pages, with 32 illustrations. Cloth, $\$ 1.50$.

Prof. Hermann's handbook, which Dr. Smith has translated and enriched with many valuable additions, will be gladly welcomed by those engaged in this department of physiology. It is an excellent little book, full of concise information, and it should find a place in every laboratory. It explains the various methods and instruments used, and points out what lines of investigation are to be pursued for studying different phenomena, and also how and what particularly to observe.American Journal of the Medical Sciences, Jan. 1884. The selection of animals and their management, the paths of elimination and clianges of poisons in the body, the explanation of the symptoms produced by poisons, alterations in tissue, in the reproductive function and in temperature, action on muscles and in nerves, anatomical and chemical

changes produced by poisons, all are successirely passed in review in a practical instructive fashion, which speaks well for both the author and the translator. The book is deserving of an encomium as a correct exponent of the spirit and tendencies of modern pharnacological research. After closely perusing the pages, all laden to overflowing with the richest facts of physiological inrestigation, and after following the astounding progress of toxic pharmacology as revealed by the author, we feel that we are fast approaching the realization of that utopian dream in which we behold experimental and clinical experience firmly and inseparably united. It is a reliable, concise and practical vade mecum for the timepressed worker in the laboratory.-New Orleans Medical and Surgical Journal, May, i883.

\section{MAISCI, JOHN M., Phar. D.,}

Professor of Materia Medica and Botany in the Philadelphia College of Pharmacy.

A Manual of Organic Materia Medica; Being a Guide to Materia Medica of the Vegetable and Animal Kingdoms. For the use of Students, Drnggists, Plarmacists and Physicians. New edition. In one handsome royal 12mo. volume. Preparing.

\section{BRUNTON, T. LAUDER, M. D.,}

Lecturer on Materia Medica and Therapeutics at St. Bartholomew's Hospital, etc.

A Manual of Materia Medica and Therapeutics, including the Pharmacy, the Physiological Action and the Therapentical Uses of Drugs. In one landsome octavo volume. In press.

\section{BRUCE, J. MITCHELL, M. D., F. R. C.P.}

Materia Medica and Therapeutics. An Introdnction to Rational Treatment. In one pocket-size $12 \mathrm{mo}$. volume of 555 pages. Limp cloth, $\$ 1.50$. Just ready. See Students' Series of Manuals, page 5.

\section{GRIFFITH, ROBERT EGLESFIELD, M. D.}

A Universal Formulary, containing the Methods of Preparing and Administering Officinal and other Medicines. The whole adapted to Physicians and Pharmaceutists. Third edition, thoroughly revised, with numerons additions, by JoHs M. MAISCH, Phar.D., Professor of Materia Medica and Botany in the Philadelphia College of Pharmacy. In one octavo volume of 775 pages, with 38 illustrations. Cloth, $\$ 4.50$; leather, $\$ 5.50$. 
STILLÉ, A., M. D., LL. D., \& MAISCH, J. M., Phar. D.,

Professor Emeritus of the Theory and Practice of Medicine and of Clinical Medicne

in the University of Pennsylvania.

Prof. of Mat. Med. and Botany in Phila. Callege of Pharmacy, Sec'y to the American Pharmaceutical Association.

The National Dispensatory: Containing the Natural History, Chemistry, Pharmacy, Actions and Uses of Medicines, including those recognized in the Pharmacopœias of the United States, Great Britain and Germany, with numerous references to the French Codex. Third edition, thoroughly revised and greatly enlarged. In one magnificent imperial octavo volume of 1776 pages, with 311 fine engravings. Cloth, $\$ 7.25$; leather, $\$ 8.00$; half Russia, open back, $\$ 9.00$. With Dennison's "Ready Reference Index" $\$ 1.00$ in addition to price in any of above styles of binding. Just ready.

When The National Dispensa tory first appeared in 1879 it was hailed as supplying a want that had long been felt in both the Medical and Pharmaceutical Professions. Its accuracy, its fulness, its conciseness, the happy manner in which, while omitting all that was obsolete or merely curious, it gave all the information that the practitioner or druggist could desire, not only with regard to the selection, preparation and compounding of drugs, but their physiological effects, their therapeutical use and their clinical value, gave it at once an unapproached position as a standard work and an indispensable book of reference.

In the present revision the authors have labored incessantly with the view of making the third edition an even more complete representative of the science of 1884 than its first edition was of that of 1879 . For this, ample material has been afforded not only by the new United States Pharmacopoia, but by those of Germany and France, which have recently appeared and been incorporated in it, besides a large number of new non-officinal remedies. It is thus rendered the representative of the most advanced state of American, English, French and German pharmacology and therapeutics. The vast amount of new and important material thus introduced may be gathered from the fact that the additions to this edition amount in themselves to the matter of an ordinary full-sized octavo volume, rendering the work larger by twenty-five per cent. than the last edition. The Therapentic Index, so suggestive and convenient to the practitioner, contains 1600 more references than the last edition-the general index 3700 more, while the list of illustrations has been increased by 80 .

Yet these facts inadequately represent the amount of labor bestowed on the revision, for it has not simply consisted in making additions. The effort has been to prevent undue increase of the volume in bulk by having in it nothing that could be regarded as superfluous, yet care has been taken that nothing should be omitted which a member of either profession could expect to find in it.

The appearance of the work has been delayed by nearly a year in consequence of the determination of the authors that it should attain as near an approach to absolute accuracy as is humanly possible. With this view an elaborate and laborions series of examinations and tests have been made to verify or correct the statements of the Pharmacopœia, and very numerous corrections have been found necessary. It has thus been rendered indispensable to all who consult the Pharmacopœia.

The work is therefore presented in the full expectation that it will maintain the position universally accorded to it as the standard authority in all matters pertaining to its subject, as registering the furthest advance of the science of the day, and as embodying in a shape for convenient reference the recorded results of human experience in the laboratory, in the dispensing room, and at the bed-side.

\section{FARQUHARSON, ROBERT, M. D.,}

Lecturer on Materia Medica at St. Mary's Hospital Medical School.

A Guide to Therapeutics and Materia Medica. Third American edition, specially revised by the Author. Enlarged and adapted to the U.S. Pharmacopoeia by Frank Woodbury, M. D. In one handsome $12 \mathrm{mo}$. volume of 524 pages. Cloth, $\$ 2.25$.

Dr. Farquharson's Therapeutics is constructed / nmned pages-one side containing the recognized upon a plan which brings before the reader all the physiological action of the medicine, and the other essential points with reference to the properties of the disease in which observers (who are nearly aldrugs. It impresses these upon him in such a way ways mentioned) have obtained from it good reas to enable him to take a clear view of the actions sults-make a very good arrangement. The early of medicines and the disordered conditions in chapter containing rules for prescribing is excelwhich they must prove useful. The double-col-| lent.-Canada Med. and Surg. Journal, Dec. i882.

\section{STILLÉ, ALFRED, M. D., LL. D.,}

Professor of Theory and Practice of Med, and of Clinical Med. in the Univ. of Penna.

Therapeutics and Materia Medica. A Systematic Treatise on the Action and Uses of Medicinal Agents, including their Description and History. Fourth edition, revised and enlarged. In two large and handsome octavo volumes, containing 1936 pages. Cloth, $\$ 10.00$; leather, $\$ 12.00$; very handsome half Russia, raised bands, $\$ 13.00$.

We can hardly admit that it has a rival in the in pharmacodynamies, but as by far the most commultitude of its citations and the fulness of its plete treatise upon the clinical and practical side research into clinical histories, and we mustassign of the question.-Boston Medical and Surgical Jourit a place in the physician's library; not, indeed, nal, Nov. $5,1874$. as fully representing the present state of knowledge 


\title{
COATS, JOSEPH, M. D., F. F. P. S.,
}

Pathologist to the Glasgow Western Infirmary.

A Treatise on Pathology. In one very handsome octavo volume of 829 pages, with 339 beautiful illustrations. Cloth, $\$ 5.50$; leather, $\$ 6.50$. Just ready.

The work before us treats the subject of Patl- / condition effected in strnetures by disease, and ology more extensively than it is usually treated points out the characteristics of varions morbid in similar works. Medical students as well as agencies, so that they can be easily recognized. But, physicians, who desire a work for study or refer- not limited to morbid anatomy, it explains fully how ence, that treats the subjects in the various de- the functions of organs are disturbed by abnormal partments in a very thorough manner, but without eonditions. There is nothing belonging to its deprolixity, will certainly give this one the prefer- partment of medicine that is not as fully elucidated ence to any with which we are acquainted. It sets as our present knowledge will admit.-Cincinnati forth the most recent discoveries, exhibits, in an Medical News, Oct. 1883.

interesting manner, the changes from a normal

\section{GREEN, T. HENTY, M. D.,}

Lecturer on Pathology nnd Morbid Anatomy at Charing-Cross Hospital Medical School, London.

Pathology and Morbid Anatomy. Fifth American from the sixth revised and enlarged English edition. In one very handsome octavo volume of 482 pages, with 150 fine engravings. Cloth, $\$ 2.50$. Just ready.

The issue of the sixth edition of this work indi- and that the anthor's ardor remains unabated. We catesits deservedly sustained popularity and valne. may confidently recommend it to the medical stuIt $w i l l$ be a double pleasure to those who have not dent and practitioner as al together the best in our forgotten their early debts, to find that the demand language.-Lancet, July 19, 1884.

for Dr. Green's manual continues as great as ever,

\section{WOODHEAD, G. SIMS, M. D., F. R. C.P. E.,}

Demonstrator of Pathology in the University of Edinburgh.

Practical Pathology. A Manual for Students and Practitioners. In one very beautiful octavo volume of 497 pages, with 136 exquisitely colored illustrations. Cloth, \$6.00. Just ready.

It cannot often be said in these days of literary activity, that a book meets a distinet want, that it opens up new ground, and that it is sure to be largely in request. All these things are perfectly true of the admirable and handsome volume before us. It is literally the first thorough attempt to deal fully with the subject of practical pathology, especially in its histological aspect, and in manner and scope it stands alone. The vast majority of the figures interpolated in the text are colored, and colored so as to reproduce with tolerable exaetitude the appearances of sections stained with varions

\section{CORNIL, V., and \\ Prof. in the Faculty of Med. of Paris. \\ RANVIER, L., \\ Prof. in the College of France.}

A Manual of Pathological Histology. Translated, with notes and additions by E. O. Shakespeare, M. D., Pathologist and Ophthalmic Surgeon to Philadelphia Hospital, and by J. Henry C. Simes, M. D., Demonstrator of Pathological Histology in the University of Penusylvania. In one very handsome octavo volume of 800 pages, with 360 illustrations. Cloth, $\$ 5.50$; leather, $\$ 6.50$; half Russia, raised bands, $\$ 7$.

One of the most complete volumes on patholog- Thus side by side physiological and pathological ical histology we have ever seen. The plan of study anatomy go hand in hand, affording that best of embraced within its pages is essentially practical. all processes in demonstrations, comparison. The Normal tissues are discussed, and after their thor- admirable arrangement of the work affords facility ough demonstration we are able to compare any in the study of any part of the human economy.pathological clange which has occnrred in them. New Orleans Medical and Surgical Journal, June,1882.

\section{KLEIN, E., M. D., F. R. S.,}

Joint Leeturer on General Anat. and Phys., in the Med. School of St. Bartholomew's Hosp. London.

Elements of Histology. In one pocket size 12mo. volume of 360 pages, with 181 illus. Limp cloth, red edges, $\$ 1.50$. (See Students' Series of Manuals, page 5.)

Althongh an elementary work, it is by no means | The illustrations are numerous and excellent. We superficial or incomplete, for the author presents eommend Dr. Flein's Elements inost heartily to In concise language nearly all the fundamental faets the student.-Medical Record, Dec. 1, 1883.

regarding the microscopic structure of tissues.

\section{PEPPER, A. J., M. B., M. S., F. R. C. S.,}

\author{
Surgeon and Lecturer at St. Mary's Hospital, London.
}

Surgical Pathology. In one pocket-size 12mo. volume of 511 pages, with 81 illustrations. Limp cloth, red edges, \$2.00. See Students' Series of Manuals, page 5 .

It is prepared especially to meet the requirements | illustrations are numerous and well selected. The of the student, but contains much of interest for the arrangement is easy and natural. We would espe. general practitioner. The author has succeeded cially recommend it not only to students, but to all admirably in priting the work forward in the most who wish a conclse and clear exposition of some of practical form, and he deserves great praise for the the intricate problems of surgical pathology - Nashlucidity of style and brevity of descriptions. The ville Journal of Medicine and Surgery, Jan. 1884.

SCHÄFER'S PRACTICAL HISTOLOGY. In one OGY. Translated by Josepr Lmidv, M. D. In one handsome royal $12 \mathrm{mo}$. volume of 308 pages, with volume, very large imperial quarto, with 320

\begin{tabular}{c|l} 
40 illustrations. & $\begin{array}{l}\text { copper-plate figures, plain and co } \\
\text { GLUGE'S ATLAS OF PATHOLOGICAL HISTOL }\end{array}$ \\
eriptive letter-press. & Cloth, \$4.00.
\end{tabular} 


\section{FLINT, AUSTIN, M. D.,}

Prof. of the Principles and Practice of Med. and of Clin. Med. in Bellevue Hospital Medical College, N. Y.

A Treatise on the Principles and Practice of Medicine. Designed for the use of Students and Practitioners of Medicine. With an Appendix on the Researches of Koch, and their bearing on the Etiology, Pathology, Diagnosis and Treatment of Phthisis. Fifth edition, revised and largely rewritten In one large and closely-printed octavo volume of 1160 pages. Cloth, $\$ 5.50$; leather, $\$ 6.50$; half Russia, $\$ 7$.

Koch's discovery of the bacillus of tubercle gives promise of being the greatest boon ever conferred by science on humanity, surpassing even vaccination in its benefits to mankind. In the appendix to his work, Professor Flint deals with the subject from a practical standpoint, discussing its bearings on the etiology, pathology, diagnosis, prognosis and treatment of pulmonary phthisis. Thus enlarged and completed, this standard work will be more than ever a necessity to the physician who duly appreciates the responsibility of his calling.

A well-known writer and lecturer on medicine recently expressed an opinion, in the highest degree complimentary of the admirable treatise of Dr. Flint, and in enlogizing it, he described it accurately as "readable and reliable." No text-book is more calculated to enchain the interest of the student, and none better classifies the naltitudinous subjects ineluded in it. It has already so far won its way in Engtand, that no ineonsiderable number of men use it alone in the study of pure medicine; and we ean say of it that it is in every way adapted to serve, not only as a com plete guide. but also as an ample instruetor in the science and practice of medicine. The style of Dr. Flint is always polished and engaging. The work abounds in perspicuous explanation, and is a most valuable text-book of medicine.-London Medical News.

\section{HARTSHORNE, HENRY, M. D., \\ Lately Professor of Hygiene in the University of Pennsylvania.}

Essentials of the Principles and Practice of Medicine. A Handbook fur Students and Practitioners. Fifth edition, thoroughly revised and rewritten. In one royal $12 \mathrm{mo}$. volume of 669 pages, with 144 illustrations. Cloth, $\$ 2.75$; half bound, $\$ 3.00$.

Within the compass of 600 pages it treats of the this one; and probably not one writer in our day history of medieine, general pathology, general had a better opportunity than Dr. Hartshorne for symptomatology, and physical diagnosis (including condensing all the views of eminent practitioners laryngoscope, ophthalmoseope, ete.), general ther- into a $12 \mathrm{mo}$. The numerous illustrations will be apeuties, nosology, all special pathology and prac- rery nseful to students especially. These essentiee. Thele is a wonderful amount of information tials, as the name suggests, are not intended to contained in this work, and it is one of the best supersede the text-books of Flint and Bartholow, of its kind that we have seen.-Glasgow Medical but they are the most valuable in affording the Journal, Noy. 1882.

An indispensable book. No work ever exhibited a better average of actual practical treatment than means to see at a glanee the whole literature of any disease, and the most valuable treatment.-Chicago Medical Journal and Examiner, April, 1882.

\section{BRISTOWE, JOHN SYER, M. D., F. R. C. P.,}

Physician and Joint Lecturer on Medicine at St. Thomas' Hospital.

A Treatise on the Practice of Medicine. Second American edition, revised by the Author. Edited, with addlitions, by James H. Hutchrssos, M.D., physician to the Pennsylvania Hospital. In one handsome octavo volume of 1085 pages, with illustrations. Cloth, $\$ 5.00$; leather, $\$ 6.00$; very handsome half Russia, raised bands, $\$ 6.50$.

The reader will find every conceivable subject | are appropriate and practical, and greatly add to conneeted with the practice of medicine ably pre- its usefulness to Ameriean readers. - Buffalo $M \epsilon d-$ sented, in a style at onee clear, interesting and ical and Surgical Journal, March, 1880. concise. The additions made by Dr. Hutchinson

\section{WATSON, SIR THOMAS, M. D.,}

Late Physician in Ordinary to the Queen.

Lectures on the Principles and Practice of Physic. A new American from the fifth English edition. Edited, with additions, and 190 illustrations, by HENRY Hartshorne, A. M., M. D., late Professor of Hygiene in the University of Pennsylvania. In two large octavo volumes of 1840 pages. Cloth, $\$ 9.00$; leather, $\$ 11.00$.

LECTURES ON THE STUDY OF FEVER. By A. Hudsox, M. D., M. R. I. A. In one octaro volume of 308 pages. Cloth, $\$ 2.50$.

STOKES' LECTURES ON FEVER. Edited by John William Moore, M. D., F. K. Q. C. P. In one octavo volume of 280 pages. Cloth, $\$ 2.00$.

A TREATISE ON FEVER. By Ronert D. LyoNs, K. C. C. In one 8ro. vol, of $354 \mathrm{pp}$. Cloth, $\$ 2.25$.
LA ROCHE ON YELLOW FEVER, considered in its Historieal, Pathological, Etiological and Therapeutical Relations. In two large and handsome oetavo volumes of $1468 \mathrm{pp}$. Cloth, 87.00 .

A HANDBOOK OF THE PRINCIPLES AND PRACTICE OF MEDICINE. For the use of Students and Practitioners. By Frask WoodEUkr, M. D. In one royal $12 \mathrm{mo}$. volume, with illustrations. Preparing.

A CENTURY OF AMERICAN MEDICINE, 17\%6-1876. By Drs. E. H. ClaRKe, H. J.

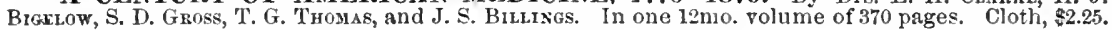


For Sale by Subscription Only.

T H E

\title{
AMERICAN SYSTEM OF PRACTICAL MEDICINE.
}

\author{
Edited by WILLTAM PEPPER, M. D., LL. D., \\ PROVOST AND PROFESSOR OF THE THEORY AND PRACTICE OF MEDICINE AND OF \\ CLINICAL MEDICINE IN THE UNIVERSTY OF PENNSYLVANIA.
}

In five imperial octavo volumcs, containing about 1000 pages each, with illustrations.

Volume I., now in press.

The publishers fecl pardonable pride in amnouncing this magnificent work. For three years it has been in active preparation, and it is now in a sufficient state of forwardness to justify them in calling the attention of the profession to it as the work in which for the first time American medicine will be thoroughly represented by its worthiest teachers, and presented in the full development of the practical utility which is its preëminent characteristic. The most able men-from the East and the West, from the North and the Sonth, from all the prominent centres of education, and from all the hospitals which afford special opportunities of study and pratice-have united in generons rivalry to bring together this vast aggregate of specialized experience.

The distinguished editor has so apportioned the work that each author has had assigned to him the subject which he is peculiarly fitted to cliscuss, and in which his views will be accepted as the latest expression of scientific and practical knowledge. The practitioner will therefore find these volumes a complete and unfailing work of reference, to which he may at all times turn with full certainty of finding what he needs in its most recent aspect, whether he seeks information on the general principles of medicine, or minute guidance in the treatment of special disease. So wide is the scope of the work that, with the exception of midwifery and matters strictly surgical, it embraces the whole domain of medicine, including the deptrtments for which the physician is accustomed to rely on special treatises, such as diseases of women and children, of the genito-nrinary organs, of the skin, of the nerves, hygiene and sanitary science, and medical ophthalmology and otology. Moreover, authors have inserted the formulas which they have found most efficient in the treatment of the varions affections. It may thus be truly regarded as a Complete Limrary of Practical Medicine, and the general practitioner possessing it may feel secure that he will require little else in the daily round of professional duties.

Although every effort has been made to avoid the introduction of matters purely speculative, and to condense, as far as possible, the vast amount of practical information furnished, yet the accumulation of indispensable material hats been such that it lias not been practicable to present it in less than five splendid imperial octavo volumes, containing about 5000 beautifully printed pages, and embodying the matter of about fifteen ordinary octavos. Such illustrations as serve really to elucidate the subject lave been introduced, but the editor has done this with a sparing hand, feeling that space might be occupied more usefully and worthily than by superfluous pictures.

As a work of which every American physician may reasonably feel prond, and in which every practitioner will find a safe and trustworthy counsellor in the daily responsibilities of practice, the publishers confidently anticipate a circulation unexampled in the annals of medical literature.

The material for the work is substantially complete in the hands of the editor, and as the printing is progressing as rapidly as is consistent with the accuracy indispensable in a work of this nature, the profession may look for the first volume in the fall, and for the subsequent volumes at reasonable intervals thereafter. publishers.

A detailed prcspectus of the work will be mailed to any address on application to the

\section{REYNOLDS, J. RUSSELL, M. D.,}

Professor of the Principles and Practice of Medicine in University College, London.

A System of Medicine. With notes and additions by Henry Hartshorne, A. M., M. D., late Professor of Hygiene in the University of Pennsylvania. In three large and handsome octavo volumes, containing 3056 double-columned pages, with 317 illustrations. Price per volume, cloth, $\$ 5.00$; sheep, $\$ 6.00$; very handsome half Russia, raised bands, $\$ 6.50$. Per set, cloth, $\$ 15$; leather, $\$ 18$; half Russia, $\$ 19.50$. Sold only by subscription.

There is no medical work which we have in I may be supplied, the publishers have committed times past more frequently and fully consulted the preparation of the book for the press to Dr. when perplexed by doubts as to treatment, or by Henly Hartshorne, whose judicious notes distribhaving unusual or apparently incxplicable symp- uted throughout the volume afford abundant evitoms presented to us, than "Reynolds' System of dence of the thoroughness of the revision to which Medicine." It contains just that kind of informa- he has subjected it. -American Journal of the Medtion which the busy practitioner frequently inds himself in need of. In order that any deficiencies 


\section{FOTHERGILL, J. M., M. D., Edin., M. R. C. P., Lond.,}

Physician to the City of London Hospital for Diseases of the Chest.

The Practitioner's Handbook of Treatment; Or, The Principles of Therapeutics. Second edition, revised and enlarged. In one very handsome octavo volume of 651 pages. Cloth, $\$ 4.00$; very handsome half Russia, raised bands, $\$ 5.50$.

The junior members of the profession will find of physiology. Every chapter, every line, has the it a work that should not only be read but care- impress of a master-hand; and while the work is fully studied. It will assist them in the proper thoroughly scientific in every particular, it presents selection and combination of therapeutical agents to the thoughtful realer all tle charms and beaubest adapted to each case and condition, and ties of a well-written novel. No physician can enable them to prescribe intelligently and success- well afford to be without this valuhle work, for its fully.-St. Louis Courier of Medicine, Nov, 1880. originality makes it fill a niche in medical litera-

The author merits the thanks of every well-edu- ture hitherto vacant.-Nashville Journ, of Med, and cated plyysician for his efforts toward rationalizing $\mid$ Surg., Oct. 1880.

the treatment of diseases upon the scientific basis

\section{FLINT, AUSTIN, M. D.}

Clinical Medicine. A Systematic Treatise on the Diagnosis and Treatment of Diseases. Designed for Students and Practitioners of Medicine. In one large and liandsome octavo volume of 799 pages. Cloth, $\$ 4.50$; leather, $\$ 5.50$; half Russia, $\$ 6.00$.

It is here that the skill and learning of the great sistently with orevity and clearness, the different clinician are displayed. He has given us a store- subjects and their several parts receiving the house of medical knowledge, excellent for thestu- attention which, relatively to their imıortance, dent, convenient for the practitioner, the result of medical opinion claims for them, is still more diffia long life of the most faithful clinical work, col- cult. 'J'his task, we feel bound to say, has been lected by an energy as vigilant and systematic as executed with more than partial success by Dr. untiring, and weighed by a judgment noless clear Flint, whose name is already familiar to students than his observation is close.-Archives of Medicine, of advanced medicine in this country as that of Dec. 1879.

To give an adequate and nseful conspectus of the subjects, and of numerous papers exhibiting much extensive field of modern elinical medicine is a task originality and extensive research.-The Dublin of no ordinary difficulty; but to acromplish this con- Journal, Dec. 1879.

\section{By the Same Author.}

Essays on Conservative Medicine and Kindred Topics. In one very handsome royal $12 \mathrm{mo}$. volume of 210 pages. Cloth, $\$ 1.38$.

\section{BROADBENT, W. H., M. D., F.R.C.P.,}

Physician to and Lecturer on Medicine at St. Mary's Hospital.

The Pulse. In one $12 \mathrm{mo}$. volume. See Series of Clinical Manuals, page 5 .

\section{SCHREIBER, DR. JOSEPH.}

A Manual of Treatment by Massage and Methodical Muscle Exercise. Translated by Walter Mendelson, M. D., of New York. In one handsome octavo volume of about 300 pages, with about 125 fine engravings. Preparing.

\section{FINLAYSON, JAMES, M. D., Editor,}

Physician and Lecturer on Clinical. Medicine in the Glasgow Western Infirmary, etc.

Clinical Diagnosis. A Handbook for Students and Practitioners of Medicine. With Chapters by Prof. Gairdner on the Physiognomy of Disease; Prof. Stephens on Diseases of the Female Organs; Dr. Robertson on Insanity; Dr. Gemmell on Physical Diagnosis; Dr. Coats on Laryngoscopy and Post-Mortem Examinations, and by the Editor on Case-taking, Family History and Symptoms of Disorder in the Various Systems. In one handsome $12 \mathrm{mo}$. volume of 546 pages, with 85 illustrations. Cloth, $\$ 2.63$.

This is one of the really useful books. It is at- bulkier volumes; and because of its arrangement tractive from prefacc to the final page, and ought and complete index it is unusually convenient for to be given a place on every office table, because it quick reference in anv emergency that may come contains in a condensed form all that is valuable upon the busy practitioner.-N. C. Med. Journ., in semeiology and diagnosties to be found in Jan. 1879.

\section{FENWICK, SAMUUEL, M. D.,}

Assistant Physician to the London Hospital.

The Student's Guide to Medical Diagnosis. From the third revised and enlarged English edition. In one very handsome royal $12 \mathrm{mo}$, volume of 328 pages, with 87 illustrations on wood. Cloth, $\$ 2.25$.

\section{TANNER, THONAS HAWKES, M. D.}

A Manual of Clinical Medicine and Physical Diagnosis. Third American from the second London edition. Revised and enlarged by Tilbury Fox, M. D., Physician to the Skin Department in University College Hospital, London, etc. In one small $12 \mathrm{mo}$. volume of 362 pages, with illustrations. Cloth, $\$ 1.50$. 
RICFARDSON, B. W., M.A., M.D., LL. D., F.R.S., F.S.A. Fellow of the Royal College of Physicians, London.

Preventive Medicine. In one octavo volume of 729 pages. Cloth, \$4; leather, $\$ 5$; very handsome half Russia, raised bands, $\$ 5.50$. Just ready.

Excerpt from Contents.

I.-Disease as a Unity, with a variety of Phenomena. The Preventive Scheme of Medicine. General Diseases of Mankind. 1. Constitutional Diseases. 2. Local Diseases. 3. Diseases from Natural Accidents,-Lightning-Sunstroke-Starvation-PoisonsVenoms-Poisonous Food-Pregnancy. II. Acquired Diseases of Artificial Origin; Phenomena and Course. 1. Aequired Diseases from Inorganic and Organic Poisons,Tea-Coffee-Alcohol-Tobacco-Soot-Gases. 2. Acquired Diseases from Physical Agencies, Mechanical and General,-Dusts-Pressure on Lungs-Concussions and Shocks - Muscular Overwork and Strain-Acquired Deformities-Physical Injuries-Surgical Operations. 3. Acquired Diseases from Mental Agencies,-Moral, Emotional and Habitual. Diseases from Mental Shock, from Moral Contagion,-Tarantism-Suicide, from Hysterical Emotion, from Passion, from Habits of Life-Insomnia-DementiaSloth-Luxury - Secret Immorality. III.-1. Origins and Causes of Discase,-Congenital, Hereditary or Constitutional Causes; Atmospheric and Climatic Causes; Parasitic Causes, -Bacteria-Bacilli-Spirila-Trichine; Zymotic Causes; Industrial and Accidental Causes; Social and Psychical Cuuses; Senile Degenerative Causes. 2. Preventions of Disease. Prevention of Hereditary or Constitutional Diseases,-Personal Rules for Pregnancy, Infancy, Adolescence, Maturity ; Prevention of Atmospheric and Climatic Diseases; of Parasitic Diseases,-Personal Rules; of Zymotic Diseases,-Contagion-DrainageIsolation of Sick-Water and Milk Supply-Hospitals-Registration-VaccinationOther Inoculations-Legislation; Prevention of Industrial Diseases-Lead PoisoningDusts - Gases, etc.; Prevention of Social and Psychical Diseases,-Warming and Ventilation-Light-Water-the Bed-room-Bread-Äbattoirs-Schools-Sepulture-Drunkenness; Prevention of Sellile Disease.

Dr. Richardson has succeded in producing a the question of disease is comprehensive, masterly work which is elevated in conception, comprehen- and fully abreast with the latest and best knowlsive in scope, scientific in character, systematic in edge on the subject, and the preventive measures arrangement, and which is written in a clear, con- advised are accurate, explicit and reliable.-The cise and pleasant manner. He evinces the happy American Journal of the Medical Sciences, April, 1884. faculty of extracting the pith of what is known on This is a book that will surely find a place on the the subject, and of presenting it in a nost simple, table of every progressive physician. To the intelligent and practical form. There is perhaps medical profession, whose duty is quite as much to no similar work written for the general public prevent as to cure disease, the book will be a boon. that contains such acomplete, reliable and instruc- -Boston Medical and Surgical Journal, Mar. 6, 1884. tive collection of data upon the diseases common The treatise contains a vast amount of solid, valuto the race, their origins, canses, and the measures able hygienic information.-Medical and Surgical for their prevention. The descriptions of diseases Reporter, Feb. 23, 1884.

are clear, chaste and scholarly; the discussion of

\section{BARTHOLOW, ROBERTS, A. M., M. D., LL. D.,}

Prof. of Materia Medica and General Therapeutics in the Jefferson Med. Coll. of Phila., etc.

Medical Flectricity. A Practical Treatise on the Applications of Electricity to Medicine and Surgery. Second edition. In one very handsome octavo volume of 292 pages, with 109 illustrations. Cloth, $\$ 2.50$.

The second edition of this work following so A most excellent work, addressed by a practisoon upon the first would in itself appear to be a tioner to his fellow-practitioners, and therefore sufficient announcement; nevertheless, the text thoroughly practical. The work now before us has been so considerably revised and condensed, has the exceptional merit of clearly pointing out and so much enlarged by the addition of new mat- where the benefits to be derived from electricity and so much enlarged by the addition of new mat-
ter, that we cannot fial to recognize a vast improve-
must come. It contains all and everything that ment upon the former work. The author has pre- the practitioner needs in order to understand inpared his work for students and practitioners-for telligently the nature and laws of the agent he is those who lave never acquainted themselves with making use of, and for its proper application in the subject, or, having done so, find that after a practice. In a condensed, practical form, it pretime their knowledge needs refreshing. We think sents to the physician all that he would wish to he has accomplished this object. The book is not remember after perusing a whole library on medical too voluminous, but is thoroughly practical, sim. electrieity, including the results of the latest inple, complete and comprehensible. It is, mole- vestigations. It is the book for the practitioner, over, replete with numerous illustrations of instru- and the necessity for a second edition proves that ments, appliances, ctc.-Medical Record, November it has been appreciated by the profossion.-Physi15,1882 . cian and Surgeon, Dec. 1882.

\section{HABERSHON, S. O., M. D.,}

Senior Physician to and late Lect. on Principles and Practice of Med. at Guy's Hospital, London.

On the Diseases of the Abdomen; Comprising those of the Stomach, and other parts of the Alimentary Canal, Csophagus, Cacum, Intestines and Peritoneum. Second American from third enlarged and revised English edition. In one handsome octavo volume of 554 pages, with illustrations. Cloth, $\$ 3.50$.

PAVY'S TREATISE ON THE FUNCTION OF DIGES'ION; its Disorders and their Treatment. From the second J.ondon edition. In one octavo volume of 238 pages. Cloth, $\$ 2.00$.

CHAMBERS' MANUAL OF DIET AND REGIMEN IN HEALTH AND SICKNESS. In one handsome octavo volume of $302 \mathrm{pp}$. Cloth, $\$ 2.75$.
BARLOW'S MANUAL OF THE PRACTICE OF MEDICINE. With additions by D. F. CoNDle, M. D. 1 vol. 8vo., pp. 603. Cloth, $\$ 2.50$.

TODD'S CLINICAL LECTURES ON CERTAIN ACUTE DISEASES. In one octavo volume of 320 pages. Cloth, $\$ 2.50$.

HOLLAND'S MEDICAL NOTES AND REFLECTIONS. i vol. 8vo., pp. 493. Cloth, $\$ 3.50$. 
COHEN, J. SOLIS, M. D.,

Lecturer on Laryngoscopy and Discases of the Throat and Chest in the Jefferson Medical College.

Diseases of the Throat and Nasal Passages. A Guide to the Diagnosis and Treatment of Affections of the Pharynx, Esophagus, Tracheal, Larynx and Nares. Third edition, thoronghly revised and rewritten, with a large number of new illustrations. In one very handsome octavo volume. Preparing.

\section{SEILER, CARL, M. D.,}

Lecturer on Laryngoscopy in the University of Pennsylvania.

A Handbook of Diagnosis and Treatment of Diseases of the Throat, Nose and Naso-Pharynx. Second edition. In one handsome royal 12no. rolume of 294 pages, with 77 illustrations. Cloth, $\$ 1.75$.

Dr. Seiler's book is a clear, concise, practical Dr. Seiler's treatise contains all the essentials of exposition of the subject, such as only a master of the knowledge of the important localities comit could have written. It is better suited to the / pressed into a small space and put together by wants of advanced students and young plysicians one of the ablest of American specialists. To stuthan any other at present in the hands of the pro- dents and others this book ean be recommended fession. - Anericrn Prartitioner, Aug, 1883. Canada Medical and Surgical Journal, July, 1883.
:

\section{BROWNL, LENNOX, F. R. C. S., Edin.,}

Senior Surgeon to the Central London Throat and Ear Hospital, etc.

The Throat and its Diseases. Second American from the second English edition, thoroughly revised. With 100 typical illustrations in colors and 50 wood engravings, designed and executed by the Author. In one very handsome imperial octavo volume of about 350 pages. Preparing.

\section{WLINT, AUSTIN, M. D.,}

Professor of the Principles and Practice of Medicine in Bcllevue Hospital Merlical College, N. Y.

A Manual of Auscultation and Percussion; Of the Physical Diagnosis of Diseases of the Lungs and Heart, and of Thoracic Aneurism. Third edition. In one handsome royal 12mo. volume of 240 pages. Cloth, \$1.03. Just ready.

This practical and jnstly popular manual is con- the author's plan is to simplify the subject as reniently divided into eight chapters, and the mach as possible; to impress the fact that student is gradually led up from a general con- close study of the physieal conditions in health sideration of physical signs in health and disease and disease is a sine qua non of success in both to the differential diagnosis of diseased conditions diagnosis and treatment.-The Medical News, by a knowledge of these physical signs. As in his April 28, 1883. courses of practical instruction, so in this book

\section{BY THE SAUE AUTHOR.}

Physical Exploration of the Lungs by Means of Auscultation and Percussion. Three lectures delivered before the Philadelphia County Medical Society, 1882-83. In one handsome small 12mo. volume of 83 pages. Cloth, $\$ 1.00$.

A Practical Treatise on the Physical Exploration of the Chest and the Diagnosis of Diseases Affecting the Respiratory Organs. Second and rerised edition. In one handsome octavo volume of 591 pages. Cloth, $\$ 4.50$.

Phthisis: Its Morbid Anatomy, Etiology, Symptumatic Events and Complications, Fatality and Prognosis, Treatment and Physical Diagnosis; In a series of Clinical Studies. In one handsome octaro volume of 442 pages. Cloth, $\$ 3.50$.

A Practical Treatise on the Diagnosis, Pathology and Treatment of Diseases of the Heart. Second revised and enlarged edition. In one octaro volume of 550 pages, with a plate. Cloth, \$4.

\section{DALTON, JOHN C., M. D.,}

Professor of Physiology and Hygiene in the College of Physicians and Surgeons, New York.

Doctrines of the Circulation of the Blood. In one handsome $12 \mathrm{mo}$. volume of 150 pages. In press.

\section{GROSS, S. D., M.D., LL.D., D.C.L. Oxon., LL.D. Cantab.}

A Practical Treatise on Foreign Bodies in the Air-passages. In one octavo volume of 452 pages, with 59 illustrations. Cloth, $\$ 2.75$.
FULLER ON DISEASES OF THE IUNGS ANU AIR-PASSAGES. Their Pathology, Physical Di-
agnosis, Symptoms and 'Treatment. From the second and revised English edition. In one octavo volume of 475 pages. Cloth, $\$ 3.50$.
SLADE ON DIPHTHERIA; its Nature and Treat- ment, with an account of the History of its Pre- valence in various Conntries. Second and revised edition. In one 12mo. vol., pp. 158. Cloth, $\$ 1.25$. WALSHE ON THE DISEASES OF THE HEART AND GREAT VESSELS. 'Third American edi- tion. In 1 vol. 8 ro., $416 \mathrm{pp}$. Cloth, $\$ 3.00$.
SMITH ON CONSUMPTION; its Early and Reme- diable Stages. 1 vol. 8 ro., pp. 253 . Cloth, \$2.25.
LA ROCHE ON PNEUMONIA. I vol. 8vo. of 490 pages. Cloth, $\$ 3.00$.
WILLIAMS ON PULMONARY CONSUMPTION; its Nature, Varieties and Trealment. With an analysis of one thousand cases to exemplify its duration. In one 8 vo. vol. of $303 \mathrm{pp}$. Cloth, $\$ 2.50$. CLINICAL OBSERVATIONS ON FUNCTIONAL NERVOUS DISORDERS, by C. HANDFIELD JONES, M. D. Second American edition. In one hand- some octavo volume of 340 pages. Cloth, $\$ 3.25$. 


\section{HAMLLTON, ALLAN MCLANE, M. D.,}

Attending Physician at the IIospital for Epileptics and Paralytics, Btackwetl's Istand, N. Y.

Nervous Diseases; Their Description and Treatment. Second edition, thoroughly revised and rewritten. In one octavo volume of 598 pages, with 72 illustrations. Cloth, $\$ 4$.

When the firstedition of this good book appeared / characterized this book as the best of its kind in we gave it our emplatic endorsement, and the any language, which is a handsome endorsement present edition enhances our appreciation of the from an exalted source. The improvements in the book and its author as a safe guide to students or new edition, and the additions to it, will justify its clinical neurology. One of the best and most purchase even by those who possess the old.critical of English neurological journals, Brain, has $\mid$ Alienist and Neurologist, April, 1882.

\section{TUKE, DA NTEL HACK, M. D.,}

Joint Author of The Manut of Pyychotogical Hedicine, etc.

Illustrations of the Influence of the Mind upon the Body in Health and Disease. Designed to elucirlate the Action of the Imagination. New edition. Thoroughly revised and rewritten. In one handsome octavo volume of 467 pages, with two colored plates. Cloth, $\$ 3.00$. Just ready.

*** These few extracts from Dr. Tuke's book The bibliography and general index at the end of give an idea in a limited manner of the interesting the book are excellent.-Boston Medical and Surgical matters there discussed. 'They fail, howerer, to Journal, May 1, 1884.

give even a faint idea of the strength of treatment, It is really one of those books that nerer grow the rich coloring and clearncss and skill of style old. The more it is read, the more it will be apwith which every subject is elaborated. As physi- preciated. Difficult and arduous as is the handcians we should constantly bear in mind the im- ling of so wide and important a subject in its portance of the expectant treatment of disease. varied aspects, the distinguished authol has most We probably all suppose that we know almost etfectually succeeded in placing upon a firm and everything about this trentment now, but Dr. rational basis, the complex phenomena resulting Tuke's book, which we all should own and read, from the influence of the mind upon the body.will make the modest among us hang our heads. Southern Practitioner, May, 1884.

CLOUSTON, THOMAS S., M. D., F. R. C.P., L.R. C. S., Lccturer on Mcnlal Diseases in the University of Eainburgh.

Clinical Lectures on Mental Diseases. With an $\Lambda$ ppendix, containing an Abstract of the Statutes of the United States and of the Sereral States and Territories relating to the Custody of the Insane. By Charles F. Forsom, M. D., Assistant Professor of Mental Diseases, Medical Department of Harvard University. In one handsome octavo volume of $5 \pm 1$ pages, illustrated with eight lithographic plates, four of which are beautifully colored. Cloth, \$4. Just ready.

Dr. Clonston's book is one long record of accu- disposal in this interesting volume. Repeatedly rate and laborious observation. From cover to at frequent intervals the results of Dr. Clonston's cover the book is positively bursting with facts. ripe experience are put before 118 in the form It presents to the student of alienism an almost of empirical generalizations, which, representing embarrassing profusion of data. The number of multitudinons observations, are equivalent to large cases which are recorded in full is considerable, groups of facts. Nor is this all, for in addition to and the skill with whicl the salient points of each the excellent therapenties witli which the book is case have been chosen for presentation, and the saturated, we meet with many shrewd remarks, graphic terseness with which they are described, and occasionally with a flash of true philosophic will be fully appreciated. The records of eases insight.-Brin, April, 1854 .

are far from being the only materials placed at our

\section{Also in separate form-}

\section{FOLSOM, CHARLES F., M. D.,}

Assistant Professor of Mental Diseases in Medical Department of Harvard Univcrsity.

An Abstract of the Statutes of the United States, and of the Several States and Territories relating to the Custody of the Insane. In one 8ro. volume of 108 pages. Cloth, \$1.50. Just ready.

\section{SAVAGE, GEORGE H., M. D.,}

Lecturer on Mental Diseases at Guy's Hospitat, London.

Insanity, including Hysteria. In one 12mo. volume. Preparing. See Series of Clinical Manuals, page 5.

\section{PLAYFATR, W. S., M. D., E. R. C. P.,}

The Systematic Treatment of Nerve Prostration and Hysteria. In one handsome small $12 \mathrm{mo}$, volume of 97 pages. Cloth, $\$ 1.00$. Just ready.

The book is well worth perusal, and will repay | to which are added the histories of a number of anyone for the time spent in its careful study, in- cases illustrative of the method and its results. asmuch as it will lead to a better understanding of An appendix contains a description of the method the management of those bêtes noirs of the physi- of performing massage, which is clear and concian, nerve prostration and hysteria. Details are cise.-New Orleans Medical and Surgicat Journal, given of the manner of carrying out the treatment, [ May, 1883.

\section{MITCHELL, S. WEIR, M. D.,}

Physician to Orthopodic Hospital and the Infirmary for Diseases of the Nervous System, Phila., etc.

Lectures on Diseases of the Nervous System; Especially in Women. Second edition. In one very handsome $12 \mathrm{mo}$. volume of about 250 pages. Preparing.

Blandford on Insanity and its Treatment: Lectures on the Treatment, Medical and Legal, of Insaue Patients. In one very handsome octavo volume. 


\section{GROSS, S. D., M. D., LL. D., D. C. L. Oxom., LL. D. Cantab., \\ Emeritus Professor of Surgery in the Jefferson Medical College of Philadelphia.}

A System of Surgery: Pathological, Diagnostic, Therapeutic and Operative. Sixth edition, thoroughly revised and greatly improved. In two large and beautifullyprinted imperial octavo volumes containing 2382 pages, illustrated by 1623 engravings. Strongly bound in leather, raised bands, $\$ 15$; half Russia, raised bands, $\$ 16$.

His System of Surgery, which, since its first edi- and, above all, has conscientiously adhered to tion in 1859 , has been a standard work in this truth and fact, weighing the evidence, pro and country as well as in America, in "the whole con, accordingly. A considerable amonnt of new domain of surgery," tells how ealnest and labori- material has been introduced, and altogether the domain of surgery," tells how eal west and labori- material has been introduced, and altogether the he appreciated the work done by men in other that he has placed the work fully abreast of the countries, and how much he contributed to pro- state of our knowledge.-Med. Record, Nov. 18, 1882. mote the science and practice of surgery in his We regard Gross' System of Surgery not only as own. There has been no man to whom America a singularly rich storehouse of scientific informais so much indebted in this respect as the Nestor tion, but as marking an epoch in the literary hisof surgery.-British Mcdical Journal, May 10, 1884. tory of surgery. 'The present edition has received

Dr. Gross' System of Surgery has long been the the most careful revision at the hands of the emistandard work on that snbject for students and practitioners.-London Lancet, May 10, 1884,

The work as a whole needs no commendation. Many years ago it earned for itself the enviable reputation of the leading American work on surgery, and it is still capable of maintaining that standard. The reason for this need only be mentioned to be appreciated. The author has always been calm and judicious in his statements, has based his conclusions on much study and personal experience, has been able to grasp his subject in its entirety, nent author himself, assisted in various instances by able specialists in various branches. All departments of the vast and ever-increasing literature of the science have been drawn upon for their most recent expressions. The late advances made in surgical practice have been carefully noted, such as the recent developments of Listerism and the improvements in gynæcological operations. In every respect the work reflects lasting credit on American medical literature.-Medical and Surgical

\section{ASHHURST, JOHN, Jr., M. D.,}

Professor of Clinical Surgery, Univ. of Pcnna., Surgeon to the Episcopal Hospital, Philadelphia.

The Principles and Practice of Surgery. Third edition, enlarged and revised. In one large and handsome octavo volume of 1060 pages, with 555 illustrations. Cloth, $\$ 6$; leather, $\$ 7$; very handsome half Russia, raised bands, $\$ 7.50$.

Dr. Ashhurst's Surgery is a condensed treatise covering the whole domain of the science in one manageable volume. 'The present edition has had a thorough revision. The novelties in surgical practice and the recent observations in surgical science have been incorporated, but the size of the volume has not been materially increased. The author's arrangement is perspicnous, and his language correct and clear. An excellent index closes the work.-Med. and Surg. Reporter, Oct. 28,'82. The author, long known as a thorougli student of surgery, and one of the most accomplished scholars in the country, aims to give in this work "a condensed but comprehensive description of the modes of practice now generally employed in the treatment of surgical affections, with a plain exposition of the principles upon which these modes of practice are based." In this he has so well succeeded that it will be a surprise to the reader to know how much practical knowledge extending over such a wide range of research is com-

\begin{abstract}
pressed in a volume of this size. This feature of the work must be its best claim for continued popularity with students and practitioners. In fact, in this respect it is withont an equal in any language. In the present edition many novelties in surgical practice are introduced, many modifications of previons statements made, and several new illustrations added.-Med. Rec., Nov. 18, 1882

It treats in a very thorough and satisfactory manner all the subjects in the various departments of surgery. The medical student and general practitioner of medicine will find it admirably adapted to their wants, the former as a text-book, and the latter as a most valuable work of reference when lie wishes to refresh his mind and obtain the latest information on any subject of surgery. In revising his work for a third edition, the author has spared no pains to render it worthy of a continuance of the favor with. which it has heretofore been received. We predict an increasing demand for the work. Cincinnati Medical News, Nov., 1882.
\end{abstract}

\section{GIBNEY, V.P., M. D.}

Surgeon to the Orthopcedic Hospital, New York, etc.

Orthopædic Surgery. For the use of Practitioners and Students. In one handsome octavo volume, profusely illustrated. Preparing.

\section{ROBERTS, JOHN B., A. M., M. D.,}

Lecturer on Anatomy and on Operative Surgery at the Philadelphia School of Anatony.

The Principles and Practice of Surgery. For the use of Students and Practitioners of Medicine and Surgery. In one very handsome octavo volume of about 500 pages, with many illustrations. Preparing.

\section{BELLAMY, EDWARD, F. R. C. S.}

Operative Surgery. In activepreparation. See Students' Series of Mranuals, page 5.

\section{STIMSON, LEWIS A., B. A., M. D.,}

Prof. of Pathol. Anat. at the Univ. of the City of New York, Surgeon and Curator to Bellevue Hosp.

A Manual of Operative Surgery. In one very handsome royal 12mo. volume of 477 pages, with 332 illustrations. Cloth, $\$ 2.50$.

This volume is devoted entirely to operative sur- $\mid$ every student shonld possess one. This work gery, and is intended to familiarize the student does away with the necessity of pondering over with the details of operations and the different larger works on surgery for descriptions of operamodes of performing them. The work is hand. tions, as it presents in a nutshell what is wanted somely illustrated, and the descriptions are clear by the surgeon without an elaborate search to and well-drawn. It is a clever and useful volume; find it.-Maryland Medical Journal, August, 1878. 
BRYANT, THOMAS, F. R. C. S.,

Surgeon and Lecturer on Surgery to Guy's Hospital, London.

The Practice of Surgery. Fourth American from the fourth and revised English edition. In one large and very handsome imperial octavo volume of over 1000 pages, with about 750 illustrations. In press.

A notice of the previous edition is appended.

It is a work especially adapted to the wants of | sent the student with practical information, and students and practitioners. It affords instruction that alone, than to burden his memory with the in sufficient detail for a full understanding of sur- views of different writers, however distinguished gical principles and the treatment of surgical dis- they might have been. In this edition the whole eases. It embraces in its scope all the diseases work has been carefully revised much of it has that are recognized as belonging to surgery, and been rewritten, and important additions have been all traumatic injuries. In discussing these it has made to almosi every chapter.-Cincinnati Medical seemed to be the aim of the author rather to pre- News, Jan. 1881.

\section{ERICHSEN, JOHN E., F. R. S., F. R. C. S., \\ Professor of Surgery in Unversity College, London, etc.}

The Science and Art of Surgery; Being a Treatise on Surgical Injuries, Diseases and Operations. From the eighth and enlarged English edition. In two large and beautiful octavo volumes of about 2000 pages, illustrated with about 900 engravings on wood. In press.

\section{ESMARCH, Dr. FRIEDRICH,}

Professor of Surgery at the University of Kiel, etc.

Farly Aid in Injuries and Accidents. Five Ambulance Lectures. Translated by H. R. H. Princess Christian. In one handsome small 12mo. volume of 109 pages, with 24 illustrations. Cloth, 75 cents.

The excellent little handbook by Dr. Esmarch /organization of the human body, illustrated by may be referred to by all for clear, safe and practi- clear, suitable diagrams. The second teaches how cal directions and instructions for rendering the to give judicious help in ordinary injuries-conturight kind of aid until the doctor arrives, in the sions, wounds, hæmorrhage and poisoned wounds. event of the numerous injuries that are liable to The third treats of first aid in cases of fracture happen in a family or neighborhood in the circum- and of dislocations, in sprains and in burns. Next, stances of daily life. The manual is earnestly the methods of affording first treatment in cases and justly commended for its excellence and clear- of frost-bite, of drowning, of suffocation of loss of ness, and especially for the minuteness and extent consciousness and of poisoning are described; of its practical details.-Harpers' Magazine, Aug., and the fifth lecture teaches how injured persons 1883.

The course of instruction is divided into five homes, to a medical man, or to a hospital. The sections or lectures. The first, or introductory illustrations in the book are clear and good.-Medilecture, gives a brief account of the structure and cal Times and Gazette, Nov, 4, 1882.

\section{BRYANT, THOMAS, F. R. C.S.,}

Surgeon to and Lecturer on Surgery at Guy's Hospital, London.

Diseases of the Breast. In one 12mo. volume. Preparing. See Series of Clinical Manuals, page 5.

\section{TREVES, FREDERICK, F. R. C. S.,}

Assistant Surgeon to and Lecturer on Surgery at the London Hospital.

Intestinal Obstruction. In one 12mo. volume. Preparing. See Series of Clinical Manuals, page 5.

\section{BUTLIN, HENTY T., F. R. C.S.,}

Assistant Surgeon to St. Bartholomew's Hospital, London.

Diseases of the Tongue. In one 12mo. volume. See Series of Clinical Manuals, page 5 .

GOULD, A. PEARCE, M. S., M. B., F. R. C. S., Assistant Surgeon to Middlesex Hospital.

Surgical Diagnosis. A Manual for the Wards. In one pocket-size 12mo. volume. Preparing. See Students' Series of Manuals, page 5.

\section{DRUITT, ROBERT, M. R. C. S., etc.}

The Principles and Practice of Modern Surgery. From the eighth London edition. In one 8vo. volume of 687 pages, with 432 illus. Cloth, $\$ 4$; leather, $\$ 5$.

SARGENT ON BANDAGING AND OTHER OPERATIONS OF MINOR SURGERY. New edition, with a Chapter on military surgery. One $12 \mathrm{mo}$. volume of 383 pages, with 187 cuts. Cloth, $\$ 1.75$.

MILLER'S PRINCIPLES OF SURGERY. Fourth American from the third Edinburgh edition. In one 8vo. vol. of 688 pages, with 340 illustrations. Cloth, \$3.75.

MILLER'S PRACTICE OF SURGERY. Fourth and revised American from the last Edinburgh edition. In one large 8vo. vol. of 682 pages, with 364 illustratlons. Cloth, $\$ 3.75$.
PIRRIE'S PRINCIPLES AND PRACTICE OF SURGERY. Edited by Joln NELL, M. D. In one 8vo. vol. of $784 \mathrm{pp}$. with 316 illus. Cloth, $\$ 3.75$ COOPER'S LECTURES ON THE PRINCIPLES AND PRAC'TICE OF SURGERY. In one 8ro. vol. of 767 pages. Cloth, $\$ 2.00$.

SKEY'S OPERATIVE SURGERY. In one vol.8vo of 661 pages, with 81 woodcuts. Cloth, \$3.25.

GIBSON'S INSTITUTES AND PRACTICE OF SURGERY. Eighth edition. In two octavo rols. of 965 pages, with 34 plates. Leather $\$ 6.50$. 


\section{HOLMES, TIMOTHY, M. A.,}

Surgeon and Lecturer on Surgery at St. George's Hospital, London.

A System of Surgery; Theoretical and Practical. IN TREATISES BY VARIOUS AUTHIORS. AMERICAN EDITION, THOROUGHLY REVISED AND RE-EDITED by Jorn H. PAckard, M. D., Surgeon to the Episcopal and St. Joseph's Hospitals, Philadelphia, assisted by a corps of thirty-three of the most eminent Americau surgeons. In three large and very handsome imperial octavo volumes containing 3137 doublecolumned pages, with 979 illustrations on wood and 13 lithographic plates, beautifully colored. Price per volume, cloth, $\$ 6.00$; leather, $\$ 7.00$; half Russia, $\$ 7.50$. Per set, cloth, $\$ 18.00$; leather, $\$ 21.00$; half Russia, $\$ 22.50$. Sold only by subscription.

Volune I. contains General Pathology, Morbid Processes, Injuries in GenERAL, CoMplications of INJURIES AND INJURIES OF Regions.

Tolune II. contains Diseases of Organs of Special Sense, Circulatory System, Digestive Tract and Gentro-Urinary Organs.

Volude III. contains Diseases of the Respiratory Organs, Bones, Joints and Muscles, Drseases of the Nervous System, Gunshot Wounds, Operative axd Minor Surgery, and Miscella nous Subjects (including an essay on Hospitals).

This great work, issued some years since in England, has won such universal confidence wherever the language is spoken that its republication liere, in a form more tlioroughly adapted to the wants of the American practitioner, lias seemed to be a duty owing to the profession. To accomplish this, each article las been placed in the hands of a gentleman specially competent to treat its subject, and no labor has been spared to bring each one up to the foremost level of the times, and to adapt it thoroughly to the practice of the country. In certain cases this has rendered necessary the substitution of an entirely new essay for the original, as in the case of the articles on Skin Diseases, on Diseases of the Absorbent System, and on Anæsthetics, in the use of which American practice differs from that of England. The same careful and conscientious revision lias been pursued throughout, leading to an increase of nearly one-fourth in matter, while the series of illustrations has been nearly trebled, and the whole is presented as a complete exponent of Britisl and American Surgery, adapted to the daily neels of the working practitioner.

In order to bring it within the reach of every member of the profession, the five volumes of the original have been compressed into three by employing a double-columned royal octavo page, and in this improved form it is offered at less than one-half the price of the original. It is printed and bound to match in every detail with Reynolds' System of Medicine. The work will be sold by subscription only, and in due time every member of the profession will be called upon and offered an opportunity to subscribe.

The authors of the original English edition are men of the front rank in England, and Dr. Packard has been fortunate in securing as his American coadjutors such men as Bartholow, Hyde, Hunt, Conner, Stimson, Morton, Hodgen, Jewell and their colleagues. As a whole, the work will be solid and substantial, and a valuable addition to the library of any medical man. It is more wieldky and more useful than the English edition, and with its companion work-"Reynolds' System of Medicine" -will well represent the present state of our science. One who is familiar with those two works will be fairly well furnished head-wise and handwise.-The Hedical Tews, Jan. 7, 1882.

Great credit is due to the American editor and his co-labolel's for revising and bringing within easy reach of American surgeons a work which has been received with such universal favor on the other side of the Atlantic as Holmes' System of Surgery. With regard to the mechanical execution of the work, neither pains nor money seem to have been spared by the publishers. - Mted. and Surg. Reporter, Sept. 14, 1881.

This work is cyclopedic in character, and every subject is treated in an exhaustive mauner. It is especially designed for a reference book, which every practising surgeon should have under hand in cases which require more than ordinary knowledge.-Chieago Med. Journ. and Exam., Feb. 1882.

\section{HAMTLTON, FRANK H., M. D., LL.D.,}

Surgeon to Bellevue Hospital, New York.

A Practical Treatise on Fractures and Dislocations. Seventh edition, thoroughly revised and much improved. In one very handsome octavo volume of about 1000 pages, with about 375 illustrations. Cloth, $\$ 5.50$; leather, $\$ 6.50$; very handsome half Russia, open back, $\$ 7.00$. Shortly.

So exalted is the position universally accorded to this now classic work that during its twenty-five years' existence, no attempt has been made to occupy the ground it so ably covers. It therefore continues to hold its place as the only complete work in any language on Fractures and Dislocations, and its translation into French and German is a well-deserved recognition of its merit. This edition has been subjected to a thorough revision both in text and illustrations, and will doubtless maintain its position as the standard authority on its subject.

\section{MARSH, HOWARD, F. R. C. S.,}

Senior Assistant Surgeon to and Lecturer on Anatomy at St. Bartholomew's Hospilal, London.

Diseases of the Joints. In one $12 \mathrm{mo}$. volume. Preparing. See Series of Clinical Manuals, page 5.

PICK, T. PICKERING, E. R. C. S.,

Surgeon to and Lecturer on Surgery at St. George's Hospital, London.

Fractures and Dislocations. In one 12mo. volume. Preparing. See Series of Clinical Manuals, page 5 . 


\section{STIMSON, LEWIS A., B. A., M. D.,}

Professor of Pathological Anatomy at the University of the City of New York, Surgeon and Curator to Bellevue Hospital, Surgcon to the Presbyterian Mospital, New York, etc.

A Practical Treatise on Fractures. In one very handsome octavo volume of 598 pages, with 360 beautiful illustrations. Cloth, $\$ 4.75$; leather, $\$ 5.75$.

The author gives in clear language all that the practical surgeon nced know of the science of fractures, their etiology, symptoms, processes of union, and treatment, according to the latest developments. On the basis of mechanical analysis the author accurately and clearly explains the clinical features of fractures, and by the same method arrires at the proper diagnosis snd rational treatment. A thorough explanation of the pathological anatomy and a careful description of the valious methods of procedure make the book full of value for every practitioner. The diction is simple, clear and vivid. Wherever desirable, brief clinical histories are introducted, which, being skillfully chosen to illustrate particular points, attest the rich experience of the anthor. The numerons beautifully-executed illustrations form an especial attraction of the book.-Ccntralbiat für Chirurgie, May 19, 1883.

\section{WELLS, J. SOELBERG, F. R. C. S.,}

Professor of Ophthalmology in King's College Hospital, London, etc.

A Treatise on Diseases of the Fye. Fourth American from the third London edition. Thoronghly revised, with copious additions, by CHARLES S. BuLL, M. D., Surgeon and Pathologist to the New York Eye and Ear Infirmary. In one large octavo volmue of 822 pages, with 257 illustrations on wood, six colored plates, and selections from the Testtypes of Jaeger and Snellen. Cloth, $\$ 5.00$; leather, $\$ 6.00$; half Russia, $\$ 6.50$.

The present edition appears in less than three for the physician to have an acquaintance with the years since the publication of the last American edition, and yet, from the numerous recent investigations that have been made in this branch of medicine, many changes and additions have been required to meet the present scope of knowledge upon this subject. A eritical examination at once shows the fidelity and thoroughness with which the editor has accomplished his part of the work. The illnstrations throughout are good. This edition can be recommended to all as a complete treatise on diseases of the eye, than which probably none better exists.-Medical Record, Aug. 18,'83.

This magnificent work is par excellence the standard work of the times on the important subjects of which it treats. It is absolutely necessary pathology and therapeuties of the eye. From no source can he more accurately derive this needed knowledge than from the volume before us.Mcdical and Surgical Reporter, August 4, 188 .

Anyone desirous of obtaining the most contplete work on diseases of the eye in the English language, will find in this treatise the fulfiment of that desire. Dr. Bull's additions to the volume may be taken as a brief but very excellent résume of the progress made in ophthialmology during the past ten years. It is no cxaggeration to say that there are few more readalyle books in medi. cine than this; certainly no medical library can be considered complete withont it.-Crnada Medical and Surgical Journal, November, 1883.

\section{NETTLESHIP, EDWARD, F. R. C. S.,}

Ophthalmic Surg. and Lect. on Ophth. Surg. at St. Thonas' Hospital, London.

The Student's Guide to Diseases of the Eye. New edition. With a chapter on the Detection of Color-Blindness, by Wirliam Tromson, M. D., Ophthalmologist to the Jefferson Medical College. In one royal $12 \mathrm{mo}$. volume of 416 pages, with 138 illustrations. Cloth, $\$ 2.00$.

This admirable guide bids fair to become the been received slows its real value and tlie apprefavorite text-book on ophthalmic surgery with stu- ciation by the profession of its intrinsic merits. dents and general practitioners. It bears throngh- Dr. Thomson has added a Chapter on Color-Blindout the imprint of sound judgment combined with
vast experience. The illustrations are numerous
are well known. Wich subjert his extensive investigations
Wh th is valuable addition the and well chosen. This book, within the short com- book becomes the most valualle guide to diseases pass of about 400 pages, contains a lucid exposition of the eye yet published. We commend it to the of the modern aspect of ophthalmic science.- notice of students of medicine and to such pracMedical Record, June 23, 1883.

This work is essentially a student's manual of of diseases which are frequently met with in daily ophthalmology, and the favor with which it has practice-Buffalo Med. and Surg. Journ., May, 1883.

\section{JULER, HENRY, F.R. C. S.,}

Scnior Ass't Surgeon, Royal Westminster Ophthalmic IIosp.; late Cïnical Ass't, Moorfields, London.

A Handbook of Ophthalmic Science and Practice. In one handsome octavo volume, with miny woodcuts and chromo-lithographs. In press.

\section{BROWNE, EDGARA.,}

Surgcon to the Liverpool Eye and Ear Infirmary and to the Dispensary for Skin Diseases.

How to Use the Ophthalmoscope. Being Elementary Instructions in Opllthalmoscopy, arranged for the use of Students. In one small royal 12mo. volume of 116 pages, with 35 illustrations. Cloth, \$1.00.

LAWSON ON INJURIES TO THE EYE, ORBIT AND EYELIDS: Their Immediate and Remote Effects. 8 vo., 404 pp., 92 illus. Cloth, $\$ 3.50$.

LAURENCE AND MOON'S HANDY BOOK OF OPHTHAIMIC SURGERY, for the use of Prac- titioners. Second edition. In one octavo volume of 227 pages, with 65 illust. Cloth, \$2.75.

CAR'IER'S PRACTICAL TREATISE ON DISEAS. ES OF THE EYE. Edited by JoHs Green, M. D. In one handsome octavo rolume. 


\section{POLITZER, ADAM,}

Imperial-Royal Prof. of Aural Therap. in the Univ. of Vienna.

A Text-Book of the Ear and its Diseases. Trauslated, at the Author's request, by James PatTerson Casselts, M. D., M. R. C. S. In one handsome octavo volume of 800 pages, with 257 original illustrations. Cloth, $\$ 5.50$.

The anatomy, physiology, pathology, therapen- | of each separate division of the ear are admirable, tics and bibliography of the ear are so ably and and profusely illustrated by woodents. They are thoroughly presented, that he who has carefully followed immediately by the physiology of the read this imposing volume can feel sure that very section, and this again by the pathological physilittle of interest or value in the past or present of ology, an arrangement which serves to keep up the aural surgery has escaped him.-Am. Jour. of the interest of the student by showing the direet apMed. Sciences, July, 1883.

The work itself we do not hesitate to pronounce the best upon the subject of aural diseases which has ever appeared, systematic withont being ton diffuse on obsolete subjects, and eminently practical in every sense. The anatomical descriptions

plication of what has preceded to the study of disease. 'The whole work can be recommended as a reliable guide to the student, and an efficient aid to the practitioner in his treatment.-Boston Med ical and Surgical Journal, June 7, 1883.

\section{BURNETT, CHARLES H., A. M., M. D.,}

Professor of Otology in the Philadelphic Potyclinic; President of the American Otological Society.

The Far, Its Anatomy, Physiology and Diseases. A Practical Treatise ror the use of Medical Students and Practitioners. New edition. In one handsome octavo volume of 580 pages, with 107 illustrations. Cloth, \$4.00; leather, \$5.00. Just ready.

In preparing a second cdition of his book, the author has been fully aware of the entlusiastic and meritorious work done in this specialty in late years. A thorough search has been made into the literature of Otology, and the work is now put forth as a complete and satisfactory guide for students and practitioners in mastering this confessedly difficult branch of medical science.

\section{COLEMIN, A., L. R. C. P., F. R. C. S., Exam. L. D. S.,}

Senior Dent. Surg. and Lect. on Dent. Surg. at St. Bartholomew's Hosp. and the Dent. Hosp., London.

A Manual of Dental Surgery and Pathology. Thoroughly revised and adapted to the use of American Students, by Thomas C. STellwagex, M. A., M. D., D. D. S., Prof. of Physiology at the Pliladelphia Dental College. In one handsome octavo volume of 412 pages, with 331 illustrations. Cloth, \$3.25.

This volume deserves to rank among the most $\mid$ of practice for the instruction of those commenc important of recent contributions to dental litera- ing a professional career, and he has faithfully enture. Mr. Coleman has presented his methods of deavored to teach to others all that he has acquired practice, for the most part, in a plain and concise by his own observation and experience. The book manner, and the work of the American editor has deserves a place in the library of every dentist. been conscientiously performed. He has evidently - Dental Cosmos, May, 1882. labored to nresent his convictions of the best modes

\section{GROSS, S. D., M. D., LL. D., D. C. L., etc.}

A Practical Treatise on the Diseases, Injuries and Malformations of the Urinar'y Bladder, the Prostate Gland and the Urethra. Tlird edition, thoroughly revised by SAMuEL W. Grose, M. D., Professor of the Pr:nciples of Surgery and of Clinical Surgery in the Jefferson Medical College, Philadelphia. $n$ one octavo volume of 574 pages, with 170 illustrations. Cloth, $\$ 4.50$.

\section{ROBERTS, WILLIAI, M. D.,}

Lecturer on Medicine in the Manchester S'chool of Hedicine, etc.

A Practical Treatise on Urinary and Renal Diseases, including Urinary Deposits. Fourth American from the fourth London edition. Illustrated by numerous engravings. In one large and handsome octavo volume. Preparing.

\section{MORIIS, HENRT, M. B., F.R.C.S.,}

Surgeon to and Lecturcr on Surgery at Middlesex Hospital, London.

Surgical Diseases of the Kidney. In one 12mo. volume. Preparing. See Series of Clinical Manuals, page 5 .

\section{LUCAS, $\underset{\text { Senior Assistant Surgeon to Giuy's Hospital, London. }}{C L}$ I. R. C. S.,}

Diseases of the Urethra. In one 12mo. volume. Preparing. See Series of Clinical Manuals, page 5 .

\section{THOMPSON, SIR HENRY,}

Surgeon and Professor of Clinical Surgery to University College Hospital, London.

Lectures on Diseases of the Urinary Organs. Second American from the third Englisl edition. In one 8vo. volume of 203 pp., with 25 illustrations. Cloth, \$2.25.

\section{By the Same Author.}

On the Pathology and Treatment of Stricture of the Urethra and Urinary Fistulæ. From the third English edition. In one octavo volume of 359 pages, with 47 cuts and 3 plates. Cloth, $\$ 3.50$. 


\section{BUMSTEAD, F. J., M. D., LL. D., \\ and \\ Late Professor of Venereal Diseases at the College of Physicians and Surgeons, New York, etc.}

\section{TAYLOR, R. W. A. $\boldsymbol{M}_{\text {., }} \boldsymbol{M}$. D.,}

Surgeon to Charily Fospital, New York, Prof, of Venereat and Skin Diseases in the University of Vermont, Pres. of the Am. Dermatological Ass'n.

The Pathology and Treatment of Venereal Diseases. Including the results of recent investigations upon the subject. Fifth edition, revised and largely rewritten, by Dr. Taylor. In one large and handsome octavo rolume of 898 pages with 139 illustrations, and thirteen chromo-lithographic figures. Cloth, $\$ 4.75$; leather, $\$ 5.75$; very handsome half Russia, $\$ 6.25$.

It is a splendid record of honest labor, wide research, just comparison, careful scrutiny and original experience, which will always be ticld as a high credit to American medical literature. This is not only the best work in the English langnage upon the subjects of which it treats, but also one which has no equal in other tongues for its clear, comprehensive and practical handing of its themes.-American Journal of the Medical Sciences, Jan, 1884.

It is certainly the best single treatise on venereal in our own, and probably the best in any language.-Boston Medical and Surgical Journal, April 3,1884 .

The character of this standard work is so well known that it would be superfiuons here to pass in review its general or special points of excellence. The verdict of the profession lias been passed; it has been accepted as the most thorough and complete exposition of the pathology and treatment of venereal diseases in the language. Admirable as a model of clear description, an exponent of sound pathological doctrine, and a guide for rational and successful treatment, it is an ornament to the medical literature of this country. The additions made to tlie present edition are eminently jndicious, from the standpoint of practical utility._Journal of Cutaneous and Venereal Diseases, Jan. 1884.

\section{HUTCHINSON, JONATHAN, F. R. S., F. R. C. S.,}

Consulting Surgeon to the Landon Hospital.

Syphilis. In one $12 \mathrm{mo}$. volume. Preparing. See Series of Clinical Manuals, page 5.

\section{CORNIL, V.,}

Professor to the Faculty of Medicine of Paris, and Physician to the Lourcine Hospital.

Syphilis, its Morbid Anatomy, Diagnosis and Treatment. Specially revised by the Author, and translated with notes and additions by J. Henry C. Srmes, M. D., Demonstrator of Pathological Histology in the University of Pennsylvania, and J. William White, M. D., Lecturer on Venereal Diseases and Demonstrator of Surgery in the University of Pennsylvania. In one handsome octavo volume of 461 pages, witl 84 very beautiful illustrations. Cloth, $\$ 3.75$.

The anatomical and histological characters of the the whole volume is the clinical experience of the hard and soft sore are admirably described. The author or the wide acquaintance of the translators multiform cutaneous manifestations of the disease, with medical literature morc evident. The anatare dealt with histologically in a masterly way, as omy, the histology, the pathology and the clinical we should indeed expect them to be, and the features of syphilis are represented in this work in accompanying illustrations are executed carefully their best, most practical and most instructive and well. The various nervous lesions which are form, and no one will rise from its perusal without the recognized outcome of the syphilitic dyserasia the feeling that his grasp of the wide and imporare treated with carc and consideration. Syphilitic tant subject on which it treats is a stronger and epilepsy, paralysis, cerebral syphilis and locomotor surer one.-The London Practitioner, Jan. 1882. ataxia are subjects full of interest; and nowhere in

\section{GROSS, SAMUEL W., A. M., M. D.,}

Professor of the Principles of Surgery and of Clinical Surgery in the Jefferson Medical College.

A Practical Treatise on Impotence, Sterility, and Allied Disorders of the Male Sexual Organs. Second edition, thorouglily revised. In one very handsome octavo volume of 168 pages, with 16 illustrations. Cloth, $\$ 1.50$.

The author of this monograph is a man of posi- This work will derive value from the high stand. tive convictions and vigorous style. This is justi- ing of its author, aside from the fact of its passing fied by his experience and by hisstudy, which has so rapidly into its second edition. This is, indeed, gone hand in hand with his experience. In regard a book that cvery physician will be glad to place to the various organic and functional disorders of in his library, to be read with profit to himself, the male generative apparatus, he has had ex. and with incalculable benefit to his patient. Beceptional opportunities for observation, and his sides the subjects embraced in the title, which are book shows that he has not neglected to compare treated of in their various forms and degrees, his own views with those of other authors. The spermatorrhoe and prostatorrlicea are also fully result is a work whleh can be safely recommended considered. The work is thoroughly practical in to both physicians and surgeons as a guide in the character, and will be especially useful to the treatment of the disturbances it refers to. It is general practitioner.-Medical Record, Aug. 18, the best treatise on the subject with which we are 1883. acquainted.-The Medical News, Sopt. 1, 1883.

\section{CULLERIER, A., d BUMLTEAD, F. J., M.D., LL.D.,} Surgeon to the Hopital du Midi.

Late Professor of Venereal Diseases in the Collegc of Physicians and Surgeons, New Fork.

An Atlas of Venereal Diseases. Translated and edited by Freman J. BumSTEAD, M. D. In one imperial 4to. volume of 328 pages, double-columns, with 26 plates, containing about 150 figures, beautifully colored, many of them the size of life. Strongly bound in clotl, $\$ 17.00$. A specimen of the plates and text sent by mail, on receipt of 25 cts.

HILL ON SYPHILIS AND LOCAL CONTAGIOUS FORMS OF LOCAL DISFASE AFFECTIXG DISORDERS. In one 8vo vol. of $479 \mathrm{p}$. Cloth, \$3.25. PRINCIPALLY THE ORGANS OF GENERA. LEE'S LECTURES ON SYPHILIS AND SOME TION. In one 8vo. vol. of 246 pages. Cloth, $\$ 2.25$. 


\section{HYDE, J. NEVINS, A. M., M. D.,}

Professor of Dermatology and Venereal Diseases in Rush Medical College, Chicago.

A Practical Treatise on Diseases of the Skin. For the use of Students and Practitioners. In one handsome octavo volume of 570 pages, with 66 beautiful and elaborate illustrations. Cloth, $\$ 4.25$; leather, $\$ 5.25$.

The author has given the student and practi- / cian in active practice. In dealing witl these tioner a work admirably adapted to the wants of questions the author leaves nothing to the preeach We can heartily commend the book as a sumed knowledge of the reader, but enters thorvalnable addition to our literature and a reliable oughly into the most minute description, so that \begin{tabular}{l|l} 
valnable addition to our literature and a retiable & oughily into the most matents and practitioners in their studies \\
one is not only told what should be done under
\end{tabular} and practice.-Am. Journ. of Med. Sci., July, 1883.

Especially to be praised are the practical sug. gestions as to what may be called the commonsense treatment of eczema. It is quite impossible to exaggerate the judiciousness with which the formulæ for the external treatment of eczema are selected, and what is of equal importance, the full and clear instructions for their nse.-London Medicat Times and Gazette, July 28, 1883.

The work of Dr. Hyde will be awarded a high position. The student of medicine will find it peculiarly adapted to his wants. Notwithstanding the extent of the subject to which it is devoted, yet it is limited to a single and not very large volume, without omitting a proper discussion of the topics. The conciseness of the volume, and the setting forth of only what can be held as facts will also make it acceptable to general practitioners. - Cincinnati Medrcal News, Feb. 1883.

The aim of the author has been to present to his readers a work not only expounding the most modern conceptions of his subject, but presenting what is of standard value. He has more especially devoted its pages to the treatment of disease, and by his detailed descriptions of therapeutic measures has adapted them to the needs of the physi-

\section{FOX, T., M.D., F.R.C.P., and FOX, T.C., B.A., M.R.C.S.,}

Physician to the Department for Skin Diseases, Univcrsity College Hospital, London.

Physician for Diseases of the Skin to the Westminster Hospital, London.

An Epitome of Skin Diseases. With Formulæ. For Students and Practitioners. Third edition, revised and enlarged. In one very handsome $12 \mathrm{mo}$, volume of 238 pages. Cloth, $\$ 1.25$.

The third edition of this convenient handbook calls for notice owing to the revision and expansion which it has undergone. The arrangement of skin diseases in alphabetical order, which is the method of classification adopted in this work, becomes a positive advantage to the student. The book is one which we can strongly recommend, not only to students but also to practitioners who require a compendions summary of the present state of dermatology.-British Mcdical Journal, July 2, 1883.

We cordially recommend Fox's Epitome to those whose time is limited and who wish a handy

manual to lie upon the table for instant reference. Its al phabetical arrangement is suited to this use, for all one has to know is the name of the disease, and here are its description and the appropriate treatment at hand and ready for instant applico tion. The present edition has been very carefully revised and a number of new diseases are described, while most of the recent additions to dermal therapentics find mention, and the formulary at the end of the book has been considerably augmented.-The Medical News, December, 1883.

\section{MORRIS, MALCOLM, M. D.,}

Joint Lecturer on Dermatology at St. Mary's Hospital Mcdical School, Lonilon.

Skin Diseases; Including their Definitions, Symptoms, Diagnosis, Prognosis, Morbid Anatomy and Treatment. A Manual for Students and Practitioners. In one $12 \mathrm{mo}$. volume of 316 pages, with illustrations. Cloth, $\$ 1.75$.

To physicians who would like to know something about skin diseases, so that when a patient presents himself for relief they can make a correct diagnosis and prescribe a rational treatment, we unhesitatingly recommend this little book of Dr. Morris. The affections of the skin are described in a terse, lucid mauner, and their several characteristics so plainly set forth that diagnosis will be easy. The treatment in each case is such as the experience of the most eminent dermatologists advises.-Cincinnati Medicat N'ews, April, 1880.

This is emphatically a learner's book; for we can safely say, that in the whole range of medical literature there is no book" of a like scope which

for clearness of expression and methodical arrangement is better adapted to promote a rational conception of dermatology $\rightarrow$ a branch confessedly difficult and perplexing to the beginner. -St. Louts Courier of Melicine, A pril, 1880.

The writer has certainly given in a small compass a large amount of well-compiled information, and his little book compares favorably with any other which has emanated from England, while in many points lie has emancipated himself from the stubbornly adhered to errors of others of his countrymen. There is certainly excellent material in the book which will well repay perusal.-Boston Med and Surg. Journ., March, 1880.

\section{WILSON, ERASMIUS, E.R.S.}

The Student's Book of Cutaneous Medicine and Diseases of the Skin. In one handsome small octavo volume of 535 pages. Cloth, $\$ 3.50$.

\section{HILLIER, THOHAS, M. D.,}

Physician to the Skin Dcpartment of University College, London.

Handbook of Skin Dissases; for Students and Practitioners. Second American edition. In one $12 \mathrm{mo}$. volume of 353 pages, with plates. Cloth, $\$ 2.25$. 


\section{AN AMTRICAN SYSTEM OF GYNAECOLOGY.}

A System of Gynæcology, in Treatises by Various Authors. In two handsome octavo volumes, richly illustrated. In active preparation.

\section{LIST OF CONTRIBUTORS.}

FORDYCE BARKER, M. D.,

ROBERT BATTEY, M. D.,

SAMUEL C. BUSEY, M. D.,

HENRY F. CAMPBELL, M. D.,

BENJAMIN F. DAWSON, M. D.,

WILLIAM GOODELL, M. D.,

II ENRY F. GARRIGUES, M. D., SAMUEL W. GROSS, M. D., JAMES B. HUNTER, M. D., WILLIAM T. HOWARD, M. D., A. REEVES JAClisON, M. D., EDWARD W. JENKS, M. D.
CIIARLES CARROLL LFE, M. D.,

WILLIAM T. LUSK, M. D., MATTHEW D. MANN, M. D., ROBERT B. MAURY, M. D., C. D. PALMER, M. D.,

WILLIAM M. POLK, M. D., THADUEUS A. REAMY, M. D., A. D. ROCKWELL, M. D.,

ALBERT H. SMITH, M. D. R. STANSBURY SUTTON, A. M., M. D., T. GAILLARD THOMAS, M. D., CHARLES S. WARD, M. D.,

WILLIAM H. WELCH, M. D.

\section{THOMAS, T. GATLLARD, M. D.,}

Professor of Diseases of Women in the College of Physicians and Surgeons, N. Y.

A Practical Treatise on the Diseases of Women. Fiftl edition, thoroughly revised and rewritten. In one large and handsome octavo volume of 810 pages, with 266 illustrations. Cloth, $\$ 5.00$; leather, $\$ 6.00$; very handsome half Russia, raised bands, $\$ 6.50$. The words which follow "fifth edition" are in ' vious one. As a book of referenco for the busy this case no mere formal announcement. The practitioner it is unequalled.-Boston Metical and alterations and additions which have been made are Surgical Journal, A pril 7, 1880.

both numerous and important. The attraction It has been enlarged and carefully revised. It is and the permanent character of this book lie in a condensed encyclopiedia of gynzecological medithe clearness and truth of the elinical descriptions eine. The style of arrangement, the masterly of diseases; the fertility of the author in thera- manner in which cach subject is treated, and the peutic resources and the fulness with which the honest convictions derived from probably the details of treatment are described; the definite largest clinical experience in that specialty of any character of the teaching; and last, but not least, in this country, all serve to commend it in the the evident candor which pervades it. We wotld highest terms to the practitioner.-Nashville Jour. also partieularize the fulness with which the hise 0 tory of the subject is gone into, which makes the book additionally interesting and gives it value as a work of reference.-London Medrcal Thmes and Gazelte, July $30,1881$.

The determination of the author to keep his book foremost in the rank of works on gyuæcology is most gratifying. Recognizing the fact that this can only be accomplished by frequent and thorongh revision, he has spared no pains to make the present edition nore desirable even than the pre-

\section{of Mca. and Surg., Jan. 1881.}

That the previous editions of the treatise of $\mathrm{Dr}$. Thomas were thought worthy of translation into German, French, Italian and Spantsh, is enough to give it the stamp of genuine merit. At home it has made its way into the library of every obstetrician and gynecologist as a safe guide to practice. No small number of additions have been made to the present edition to make it correspond to recent improvements in treatment.-Pacific Medical and Surgical Journal, Jan. 1881.

\section{EDIS, ARTHUR W., M. D., Lond., F.R.C.P., M.R.C.S.,} Assist. Obstetric Physician to Middlesex Hospital, lale Physician to British Lying-in Hospilal.

The Diseases of Women. Including their Pathology, Causation, Symptoms, Diagnosis and Treatment. A Manual for Students and Practitioners. In one handsome octavo volume of 576 pages, with 148 illustrations. Cloth, $\$ 3.00$; leather, $\$ 4.00$.

It is a pleasure to read a book so thoronghly good as this one. The special qualities which are conspicuous are thoroughness in covering the whole ground, clearness of description and conciseness of statement. Anotlier marked feature of the book is the attention jaid to the details of many minor surgical operations and procedures, as, for instance, the use of tents, application of leeches, and use of hot water injections. These are among the more common methods of treatment, and yet very little is said about them in many of the text-books. The book is one to be warmly recommended especially to students and general practitioners, who need a concise but complete résumé of the whole subject. Specialists, too, will find many useful hints in its pages.-Boston Med. and Surg. Journ., March 2, 1882.

\section{BARNES, ROBERT, M. D., F. R. C.P., \\ Obsletric Physician to St. Thomas' Hospital, London, etc.}

The greatest pains hare been taken with the selating to treatment. A liberal selection of remedies is given for each morbid condition, the strength, mode of application and other details being fully explained. The descriptions of gyniecological manipulations and operations aro full, clear and practical. Nuch care has also been bestowed on the parts of the book which deal with diagnosis-we note especially the pages dealing with the differentiation, one from another, of the different kinds of abdominal tumors. The practitioner will therefore find in this book the kind of knowledge he most needs in his daily work and he will be pleased with the clearness and fulness of the information there given. - The Practitioner, Feb. 1882 .

A Clinical Exposition of the Medical and Surgical Diseases of Women. In one handsome octavo volume, with numerous illustrations. New edition. Preparing.

CHADWICK, JAMES R., A. M., M. D.

A Manual of the Diseases Peculiar to Women. In one 12mo. vol. Prepg.

\section{WEST, CIIARLES, M. D.}

Lectures on the Diseases of Women. Third American from the third Iondon edition. In one octavo volume of 543 pages. Cloth, $\$ 3.75$; leather, $\$ 4.75$. 


\section{EMMET, THOMAS ADDIS, M. D., LL. D.,}

Surgeon to the Woman's Hospital, New York, etc.

The Principles and Practice of Gynæcology ; For the use of Students and Practitioners of Medicine. New (third) edition, thoroughly revised. In one large and very handsome octavo volume of about 900 pages, with about 150 illustrations. Shortly.

A few notices of the previous edition are appended:

No gynæcological treatise has appeared which $/$ ceived more attention than in America. It is, contains an equal amount of original and useful then, with a feeling of pleasure that we welcome a matter; nor does the medical and surgical history work on diseases of women from so eminent a of America include a book more nove and useful. gynæcologist as Dr. Emmet. The work is essenThe tabular and statistical information which it tially clinical, and leaves a strong impress of the contains is marvellous, both in quantity and accu- author's individuality. 'To criticise, with the care racy, and cannot be otherwise than invaluable to it merits, the book throughout, would demand far future investigators. It is a work which demands more space than is at our command. In parting not careless reading but profound study. Its value we can say that the work teems with original as a contribution to gynæcology is, perhaps, jdeas, fresh and raluable methods of practice, and greater than that of all previons literature ol the is written in a clear and elegant style, worthy of subject combined.-Chicago Med. Gaz., A pril 5, '80. the literary reputation of the country of Longfellow

In no country of the world has gynæcology re- ana O. W. Holnes.-British Med. jour., Feb. 21 , '80.

DUNCAN, J. MATTHEWS, M.D., LL. D., F. R. S. E., etc.

Clinical Lectures on the Diseases of Women; Delivered in Saint Bartholomew's Hospital. In one handsome octaro volume of 175 pages. Cloth, $\$ 1.50$.

They are in every way worthy of their author; stamp of individuality that, if widely read, as they indeed, we look upon them as among the most certainly deserve to be, they cannot fail to exert a valuable of his contributions. They are all npon | wholesome restraint upon the undue eagerness matters of great interest to the general practitioner. With which many young physicians seem bent Some of them deal with subjects that are not, as a upon following the wild teachings which so infest rule, adequately handled in the text-books; others the gynæcology of the present day. $-N$. $Y$. Medical of them, while bearing upon topics that are usually Journal, March, 1880 .

treated of at length in such works, yet bear such a

\section{GUSSEROW, A.,}

Professor of Midwifery and the Diseases of Children at the University of Berlin.

A Practical Treatise on Uterine Tumors. Specially revised by the Author, and translated with notes and additions by EDMUND C. WENDr, M. D., Pathologist to the St. Francis Hospital, N. Y., etc., and revised by NATHAN BozEMAN, M. D., Surgeon to the Woman's Hospital of the State of New York. In one handsome octavo volume, with about 40 illustrations. Preparing.

\section{HODGE, HOGH L., M. D.,}

Emeritus Professor of Obstetrics, etc., in the University of Pennsylvania.

On Diseases Peculiar to Women; Including Displacements of the Uterus. Second edition, revised and enlarged. In one beautifully printed octavo volume of 519 pages, with original illustrations. Cloth, $\$ 4.50$.

\section{By the Same Author.}

The Principles and Practice of Obstetrics. Illustrated with large lithographic plates containing 159 figures from original photographs, and with numerous woodcuts. In one large quarto volume of 542 double-columned pages. Strongly bound in cloth, $\$ 14.00$.

* * Specimens of the plates and letter-press will be forwarded to any address, free by mail, on receipt of six cents in postage stamps.

\section{TARNIER, S., and CHANTREUIL, G.}

A Treatise on the Art of Obstetrics. Translated from the French. In two large octavo volumes, richly illustrated.

\section{RAMSBOTHAM, FRANCIS H., M. D.}

The Principles and Practice of Obstetric Medicine and Surgery; In reference to tlie Process of Parturition. A new and enlarged edition, thoroughly revised by the Author. With additions by W. V. KeAting, M. D., Professor of Obstetrics, etc., in the Jefferson Medical College of Philadelphia. In one large and handsome imperial octavo volume of 640 pages, with 64 full-page plates and 43 woodcuts in the text, containing in all nearly 200 beautiful figures. Strongly bound in leather, with raised bands, $\$ 7$.

ASHWELL'S PRACTICAL TREATISE ON THE DISEASES PECULIAR TO WOMEN. Third American from the third and revised London edition. In one 8vo. vol., .pp. 520 . Cloth, \$3.50.

CHURCHILL ON THE PUERPERAL FEVER
AND OTHER DISEASES PECULIAR TO WO MEN. In one 8vo. vol. of 464 pages. Cloth, $\$ 2.50$. MEIGS ON THE NATURE, SIGNS AND TREATMENT OF CHILDBED FEVER. In one 87o. volume of 346 pages. Cloth, $\$ 2.00$. 


\section{PLAYFATR, W. S., M. D., F. R. C. P., \\ Professor of Obstetric Medicune in King's College, London, etc.}

A Treatise on the Science and Practice of Midwifery. Third American edition, revised by the Author. Edited, with additions, by RoBRRT P. HARRIS, M. D. In one handsome octavo volume of 659 pages, with 183 illustrations. Cloth, \$4; leather, $\$ 5$; half Russia, \$5̃.50.

The medical profession has now the opportunity | all details not necessary for a full understanding of adding to their stock of standard medical work one of the best volumes on midwifery ever published. The subject is taken up with a master hand. The part devoted to labor in all its various presentations, the management and results, is admirably arranged, and the views entertained will be found essentially modern, and the opinions expressed trustworthy. The work abounds with plates, illustrating various obstetrical positions; they are admirably wrought, and afford great assistance to the stiudent. $-N$. O. Medical and Surgical Journal, March, 1880.

If inquired of by a medical student what worls on obstetrics we should recommend for him, par excellence, we would undoubtedly advise him to choose Playfair's. It is of convenient size, but what is of chief importance, its treatment of the various subjects is concise and plain. While the discussions and descriptions are sufficiently elaborate to render a very intelligible idea of them, yet News, Jan., 1880.

It certainly is an admirable exposition of the science and practice of midwifery. Of course the additions made by the American editor, Dr. R. P. Harris, who never utters an idle word, and whose studious researches in some special departments of obstetrics ale so well known to the profession, are of great value.-The American Practitioner, April, 1880.

The third edition-so soon following the secondshows that the good qualities of the book have been recognized by the profession. 'The second American has been exhausted before the second English edition, and this is therefore especially prepared and revised by the author for this country; a fact which ought to be satisfactory as to the profession here being furnished with the latest work upen all subjects pertaining to obstetrics.-Am. Journal of Med. Sciences, April, 1880.

\section{KING, A. F. A., M. D.,}

Professor of Obstetrics and Diseases of Women in the Medical Department of the Columbian University, Washington, D. C., and in the University of Vermont, etc.

A Manual of Obstetrics. New edition. In one very handsome 12mo. volume of 331 pages, with 59 illustrations. Clotl, $\$ 2.00$. Just ready.

In a series of short paragraphs and by a con- that it will be in great demand by them, so suited densed style of composition, the writer has pre- is it to their wants. Of a size that it can be easily sented a great deal of what it is well that every carried, yet it contains all of the main points in obstetrician should know and be ready to practice obstetrics sufficiently elaborated to give a full and or prescribe. The fact that the demand for the correct idea of them. The general practitioner volume has been such as to exhaust the first will also find it very useful for reference, for the edition in a little over a year and a half speals purpose of refreshing the mind. We can confiwell for its popularity.-American Journal of the dently assert that it will be found to be the best Merical Sciences, April, 1884.

This little work upon obstetrics will be highly issued from the press.-Cincinnati Medical News, valued by medical students. We feel quite sure March, $\mathbf{1 8 8 4}$

\section{PARVIN, THEOPHILUS, M. D., LL. D.,}

Professor of Obstetrics and the Diseases of Women and Children in the Jefferson Medical College.

A Treatise on Midwifery. In one very handsome octavo volume of about 550 pages, with numerous illustrations. In press.

\section{BARNES, ROBERT, M. D., and FANCOURT, M. D.,} Phys. to the General Lying-in Hosp., Lond.

Obstetric Phys. to St. Thomas' Hosp., Lond.

A System of Obstetric Medicine and Surgery, Theoretical and Clinical. For the Student and the Practitioner. The Section on Embryology contributed by Prof. Milnes Marshall. In two handsome octavo volumes, profusely illustrated. In press.

\section{BARNES, FANCOURT, M. D.,}

Obstetric Physician to St. Thomas' Hospital, London.

A Manual of Midwifery for Midwives and Medical Students. In one royal $12 \mathrm{mo}$. volume of 197 pages, with 50 illustrations. Cloth, $\$ 1.25$.

\section{PARRY, JOHN S., M. D.,}

Obstetrician to the Philadelphia Hospital, Vice-President of the Obstet. Society of Philadelphia.

Extra-Uterine Pregnancy : Its Clinical History, Diagnosis, Prognosis and Treatment. In one handsome octavo volume of 272 pages. Clotl, $\$ 2.50$.

\section{TA NNER, THOMAS HAIWKES, M. D.}

On the Signs and Diseases of Pregnancy. First American from the second English edition. In one handsome octavo volume of 490 pages, with 4 colored plates and 16 woodcuts. Cloth, $\$ 4.25$.

\section{WINCKEL, $\mathrm{F}$.}

A Complete Treatise on the Pathology and Treatment of Childbed. For Students and Practitioners. Translated, with the consent of the Author, from the second German edition, by J AMes READ CHADwick, M. D. In one octavo volume of 484 pages. Cloth, $\$ 4.00$. 


\section{LEISHMAN, WILLIAM, M. D.,}

Regius Professor of Midwifery in the University of Glasgow, etc.

A System of Midwifery, Including the Diseases of Pregnancy and the Puerperal State. Third American edition, revised by the Author, with additions by JoHN S. PARry, M. D., Obstetrician to the Philadelphia Hospital, etc. In one large and very handsome octavo volume of 740 pages, with 205 illustrations. Cloth, $\$ 4.50$; leather, $\$ 5.50$; very handsome half Russia, raised bands, $\$ 6.00$.

The author is broad in his teachings, and discusses briefly the comparative anatomy of the pelvis and the mobility of the pelvic articulations. The second chapter is devoted especially to the study of the pelvis, while in the third the female organs of generation are introduced. The structire and development of the ovum are admirably described. Then follow chapters upon the varions subjects embraced in the stully of midwifery. The descriptions thronghout the work are plain and pleasing. It is sufficient to state that in this, the last edition of this well-known work, every recent advancement. in this field has been brought forward-Physician and Surgeon, Jas. 1830.

We gladly welcome the new edition of this cxcellent text-book of midwifery. 'The former editions have heen most favorably received by the profession on both sides of the Atlantic. In the

preparation of the present edition the anthor has made such alterations as the progress of obstetrical science seems to require, and we cannot but admile the ability with which the task has been performed. We consider it an admirable textbook for students during their attendance upon lectures, and have great pleasure in recommending it. As an exponent of the midwifery of the present day it has no superior in the English languagc.-Canata Lancet, Jan. 1880.

To the American student the work before us must prove admirably adapted. Complete in all jts parts, essentially modern in its teachings, and with demonstrations noted for clearuess and precision, it will gain in favor and be recognized as a work of staudard merit. The work cannot fail to be popular and is cordially recommended. $-N$. $O$. Med. and Siurg. Journ., Warch, 1880.

\section{SMITII, J. IEWIS, IL. D.,}

Clinical Professor of Discases of Children in the Bellevue Hospital Medical Collcge, N. Y.

A. Complete Practical Treatise on the Diseases of Children. Fifth edition, thoroughly revised and rewritten. In one handsome octaro volume of 836 pages, with illustrations. Cloth, $\$ 4.50$; leather, $\$ 5.50$; very handsome half Russia, rised bands, $\$ 6$.

This is one of the best books on the subject with which we venture to say will be a favorable one.which we have met and one that has giren us Dublin Journal of Medical Scienre, March, 1883.

satisfaction on every occasion on which we have There is no book published on the subjects of consulted it either as to diagnosis or treatment. which this one treats that is its equal in value to It is now in its fith edition and in its present form the physician. While he has said just enough to is a very adequate representation of the subject it impart the information desired by general practitreats of as at present understood. The important tioners on such questions as etiology, pathology, subject of intant hygiene is fully dealt with in the prognosis, etc., he bas deroted more attention to early portion of tho book. The great bulk of the the diagnosis and treatment of the ailments which work is appropriately devoted to the diseases of he so accurately describes; and such information infancy and childhood. We would recommend is exactly what is wanted by the vast majority of any one in need of information on the subject to "family physicians."-Va. Med. Monthly, Feb. 1882. procure the work and form his own opinion on it,

\section{KEATING, JOHN M., M. D.,}

Lecturer on the Diseases of Children at the University of Pennsylvania, etc.

The Mother's Guide in the Management and Feeding of Infants. In one handsome $12 \mathrm{mo}$. volume of 118 pages. Cloth, $\$ 1.00$.

Works like this one will aid the physician im-' the employment of a wet-nurse, about the proper mensely, for it saves the time he is constantly giv- food for a nursing mother, about the tonic effects ing his patients in instructing them on the sub- of a bath, about the perambulator versus the nurses' jects here dwelt upon so thoroughly and prac- arms, and on many other subjects concerning tically. Lr. Keating has written a practical book, which the eritic might say, "surely this is obvihas carefully avoided unnecessary repetition, and ons," but which experience teaches us are exactly successfully instructed the mother in such details the things needed to be insisted upon, with the rich of the treatment of her child as devolve upon her. as well as the ponr.-London Lancet, January, 281882. He has studiously omitted gising prescriptions, and instructs the mother when to call upon the doctor, as his duties are totally distinct from hers. -American Journal of Obstetrics, October, 1881.

Dr. Keating has kept clear of the common fanlt of worlss of tnis sort, viz., mixing the duties of the mother with those proper to the doctor. There is the ring of common sense in the remarks abont

A book small in size, written in pleasant style, in anguare which can be readily nnderstood by any mother and eminently practical and safe; in fact a book for which we liave been waiting a long time, and which we can most heartily recominend to mothers as the book ou this subject-New Iork Medical Journal and Obstelrical Review, Feb. 1882.

\section{OWEN, EDMUND, TR. B., F. R. C. S.,}

Surgeon to the Children's ILospital, Grcat Ormorid St, London.

Surgical Diseases of Children. In one 12mo. volume. Preparing. See Series of Clinical Manuats, page j.

\section{WEST, CHARLES, M. D.,}

Physician to the Hospital for Sick Children, London, etc.

Leetures on the Diseases of Infaney and Childhood. Fifth American from 6th English edition. In one octavo volume of 686 pages. Cloth, $\$ 4.50$; leather, $\$ 5.50$.

By the Same Author.

On Some Disorders of the Nervous System in Childhood. In one small $12 \mathrm{mo}$. volume of 127 pages. Cloth, $\$ 1.00$. 


\section{TIDY, CHARLES MEYMOTT, M. B., I. C. S.,}

Professor of Chemistry and of Forensic Medicine and Public Health at the London Hospital, etc.

Legal Medicine. Volume II. Legitimacy and Paternity, Pregnancy, Abortion, Rape, Indecent Exposure, Sodomy, Bestiality, Live Birtl, Infanticide, Aspliyxia, Drowning, Hanging, Strangulation, Suffocation. Making a very handsome imperial octavo volume of 529 pages. Cloth, $\$ 6.00$; leather, $\$ 7.00$. Just ready.

Volume I. Containing 664 imperial octavo pages, with two beautiful colored plates. Cloth, $\$ 6.00$; leatlier, $\$ 7.00$. Recently issued.

He whose inclinations or necessities lead him to The fact that the very numerous illustrative cases assume the functions of a medical jurist wants a are drawn from many sources, and are not limited, book encyclopæedic in character, in which he may as in Casper's Handbook, to the author's own exbe reasonably sure of finding medico-legal topies perience, and the additional fact that they are discussed wih judicial fairness, with sufficient brought down to a very recent date, give them, completeness, and with due attention to the most for purposes of reference, a very obvious value.recent advances in medical seience. Mr. 'Tidy's $\mid$ Boston Medical and Surgical Journal, Feb. 8, 1883.

work bids fair to meet this need satisfactorily.

\section{TAYLOR, ALFRED S., M. D.,}

Lecturer on Medical Jurisprudence and Chemistry in Guy's Hospital, London.

A Manual of Medical Jurisprudence. Eightl American from the tenth London edition, thoroughly revised and rewritten. Edited by JoHN J. REese, M. D., Professor of Medical Jurisprudence and Toxicology in the University of Pennsylvania. In one large octavo volume of 937 pages, with 70 illustrations. Cloth, $\$ 5.00$; leather, $\$ 6.00$; half Russia, raised bands, $\$ 6.50$.

The American editions of this standard manual | only have to seek for laudatory terms.- American have for a long time laid claim to the attention of the profession in this country; and the eighth comes before us as embodying the latest thoughts and emendations of Dr. Taylor upon the subject to which he devoted his life with an assiduity and success which made him facile princeps among English writers on medical jurisprudence. Both the author and the book have made a mark too deep to be affected by criticism, whether it be censure or praise. In this case, however, we should Journal of the Medical Sciences, Jan. 1881 .

This celebrated work has been the standard authority in its department for thirty-seven years, both in England and America, in both the professions which it concerns, and it is improbable that it will be superseded in many years. The work is simply indispensable to every physician, and nearly so to every liberally-educated lawyer, and we heartily commend the present edition to both professions.-Albany Law Journal, March 26, 1881.

\section{By the Same Author.}

The Principles and Practice of Medical Jurisprudence. Third edition. In two handsome octavo volumes, containing 1416 pages, with 188 illustrations. Cloth, $\$ 10$; leather, \$12. Just ready.

Taylor's Treatise at the hands of Dr. Stevenson books of its class. Including within its purview, has undergone a diminution of bulk with an in - as the subject does, something from every divi. crease of mass. This edition only asserts with sion of medical science, this exhaustive treatise stronger reason the allowed claims of the late Dr. will ever remain an invaluable collection of data. Taylor's work to the first position among English - New York Medical Journal, Dec. $1,1883$.

\section{By the Same Author.}

Poisons in Relation to Medical Jurisprudence and Medicine. Third American, from the third and revised English edition. In one large octavo volume of 788 pages. Cloth, $\$ 5.50$; leather, $\$ 6.50$.

\section{PEPPER, AUGUSTUS J., M. S., M. B., F. R. C. S.,}

Examiner in Forensic Medicine at the Unversity of London.

Forensic Medicine. In one pocket-size 12mo. volume. Preparing. See Students' Series of Manuals, page 5 .

\section{LEA, HENRY C.}

Superstition and Force: Essays on The Wager of Law, The Wager of Battle, The Ordeal and Torture. Third revised and enlarged edition. In one handsome royal $12 \mathrm{mo}$, volume of 552 pages. Cloth, $\$ 2.50$.

This valuable work is in reality a history of civ- should not be most carefully studied; and however llzation as interpreted by the progress of jurispru- well versed the reader may be in the science of dence. . In "Superstition and Force" we have a jurisprudence, he will find much in Mr. Lea's volphilosophic survey of the long period intervening ime of which he was previously ignorant. The between primitive barbarity and civilized enlight- book is a valuable addition to the literature of soenment. There is not a chapter in the work that cial science.-Westminster Review, Jan. 1880.

\section{By the Same Author.}

Studies in Church History. The Rise of the Temporal Power-Benefit of Clergy-Excommunication. New edition. In one very handsome royal octavo volume of 605 pages. Cloth, $\$ 2.50$.

The anthor is pre-eminently a scholar. He takes up every topic allied with the leading theine, and traces it ont to the minutest letail with a wealth of knowledge and impartiality of treatment that compel admiration. The amount of information compressed into the book is extracrilinary. In mo other single volume is the development of the

Just ready.

primitive church traced with so much clearness, and with so definite a perception of complex or conflicting sources. The fifty pages on the growth of the papacy, for instance, are admirable for concisenew and freedom from prejudice.-Boston Traveller, May 3, 1883. 
Allen's Anatomy

Amerlcan Journal of the Medical Siclences American Sy'stem of Gynæcology

American System of Practical Medicine

*Ashhurst's Surgery

Ashwell on Diseases of Women

Attfield's C'hemistry

Barlow's Practice of Medicine

Barnes' Midwifer

* Barnes on Diseases of Vomen

Barnes' System of Obstetric Medicine

Bartholow on Electricity

Bartholow on Electricity

Bell's Comparative Physiology and Anatomy

Bellamy's Operative Surgery

Blandford on Insanity

Bownan's Practical Cliemistr

* Bristowe's Practice of Medicine

Broadbent on the Pulse

Browne on the Ophthalmoscope

Browne on the Throat

Bruce's Materia Medica and 'Therapeutics

Brunton's Materia Medica and Therapeutic

Bryant on the Breast

* Bryant's Practice of Surgery

* Bumstead on Venereal Diseases

* Burnett on the Ear

Butlin on the Tongue.

Carpenter on the Use and Abuse of Alcobol

*Carpenter's Human Physiology

Carter on the Fre

Century of American Medicine

Chadwick on Diseases of Women

Chambers on Diet and Regimen

Charles' Phy'siological and Pathological Chem.

Churchill on Puerperal Fever

Clarke and Lockwood's Dissectors' Mannal

Classen's Quantitative Analy'sis

Cleland's Dissector

Clowston on Insanity

Coats' Pathology

Colsen on the Throat

Coleman's Dental Surgery

Condie on Diseases of Children

Cooper's Lectures on Surgery

*Cornil on syphilis and Jauvier's Pathologicai Histolagy

Cullerier's Atlas of Venereal Diseases

Curnow's Medical Anatomy

Dalton on the Circulation

*Dalton's Human Pliysiologs :

Dalton's Topograplical Anatomy of the Brain

Davis' Climical Lectures

Drapel"s Medical Physic

Duncan on Diseases of Vomen

*Dunglison's Medical Dictionary

Edis on Iiseases of W omen

Ellis' Demonstrations of Anatomy

*Emmet's Gynæcology

*Erichsen's Sy'stem of Surgery

Esmarch's Early Aid in Injuries and Accid'ts

Farquharson's Therapeuties and Mat. Med.

Fenwick's Medical Diagnosis

Finlayson's C'linical Diagnosis

Flint on Auscultation and Percussion

Flint on Phthisis

Flint on Physical Exploration of the Lungs

Flint on Respiratory Organs

Flint on the Heart

* Flint's Clinical Medicine

Flint's Essay's

*Flint's Praetice of Medicine

Folsom's Laws of U. S. on Custody of Insane Foster's Phvsiolog

*Fothergill's Handbook of 'Treatment

Fownes' Elementary Chemistry

Fox on Diseases of the skin

Frankland and Japp's Inorganic Chemistry

Fuller on the Lungs and Air Passages

Galloway's Analysis

Gibney's Orthoptedic Surgery

Glbson's Surgery

Gluge's Pathological Histology, by Leidy

Gould's Surgical Diagnosis

*Gray's Anatomy

Greene's Medical Chemistry.

Green's Pathology and Morbid Anatomy

Grith th's Universal Formulary

Grithith's Universal Formulary .

Gross on Foreign Bodies in Air-Passages

Gross on Impotence and

Gross on Urimary Organs

*Gross' System of Surgery .

Gusserow on Uterine Tumors

Habershon on the Abdomen .

* Hamilt on on Fractures and Disiocations

Hamilton on Nervons Diseases

Hartshorne's Anatomy and Physiology :

Hartshorne's Conspectus of the Med. Seiences

Hartshorne's Essentials of Medicine

Hermann's Experimental Pharmacology

Hill on Syphilis

Hillier's Handbook of $\dot{S} k$ in Diseases

Hoblyn's Medical Dictionary
6 Hodge on Women

3 Hodge's Obstetrics

Oofmann and Power's Chemical Analysis

Holland's Medical Notes and Reflections

*Holmes' System of Surgery

Horner's Anatomy and Histoiogy

Hudson on Fever

Hutchinson on Syphilis

es of the Skin

Jones (C. Handfield) on Nervous Disorder

Juler's Ophthalmic Science and Practice

ing's Manual of Obstetrics

Klen's Histology

19 Calaria, etc.

Laurence and Moon's Ophthämic Surgery

Lawson on the Eye, Orbit and Eselid

Lea's superstition and Force

Pary on Digestion and its Disorders

18 Pepper's Forensic Medicine

24 Pick on Fractures and Dislocations

30 Pirrie's System of Surgery

21 Playfair on Nerve Prostration and Hysteria

5 * Playfair's Midwifery

13 Politzer on the Ear and its Diseases

25 Power's Human Phy'siology.

Pamsbotham on Parturition

Remsen's 'Theoretical (henistry

'System of Mfedicine

- Richardson's Preventìve Medicine

Roberts on Urinary Diseases

21 Roberts' Irinciples and Practice of Surgery

Robertson's Physiological Physics

Rodwell's Dictionary of Sclence

27 Sargent's Minor and Military Surgery.

Savare on Insanity, including Hysteria .

Schider's Histology

Schreiber on Massage.

21 Seiker on the Throat, Nose and Naso-Pharynx

Series of Clinical Manuals

Simon's Mannal of Chemistry

6 Skey's Operatıve Surgery

8 Slade on Diphtheria

18 Smith (Edward) on Consumpition -

15 Smith (H. H.) and IIorner's Anatomical Allas

*Smith (J I ewis) on Children

8 *Stille \& Maisch's National Dispensatory

*Stillé's Therapeutics and I I ateria Medica

Stimson on Fractures

4 : Stimson's Operative Surgery

Stokes on Feyer

Students' Series of Mariuals

6 Sturges' Clinical Medicine

9 Tanner on Signs and Iiseases of Pregnancy

6 Tanner's Manual of Clinical Medicine

9 Tarnier and Chantreuil's Obstetrics

Taylor on Poisons

9 \#ruylor's Medical Jurisprudence

20 Tavior's Prin. and Prac. of Med. Jurisprudence

* Thomas on Diseases of Women

13 Thompson on Stricture

, 21 Thompson on Urinary Organs

Tidy's Lega] Medicine .

Todd on Acute Diseases

13 'Treves' Applied Anatomy

1 Treves on Jntestinal Obstructio

1 Tike on the influence of Mind on the Body

Wratsine

Watson's Practice of Physic

Watts Physical and Inorganic Chemistry

8 *trels on the Eye

West on Diseases of Childhood

22 VYest on Diseases of Women

19 West on Nervous J)isorders in Childhood

6 Williams on Consumption

5 Wilson's Handbook of Cutaneous iredicine

14 Wilson's Human Anatomy 'Treatment of Childbed

25 VVöller's Orouic Cluemistry

26 Woodhury's Pract ice of Medicine

4 Woodhead's Practical Pathology

also bound in half Russia. 






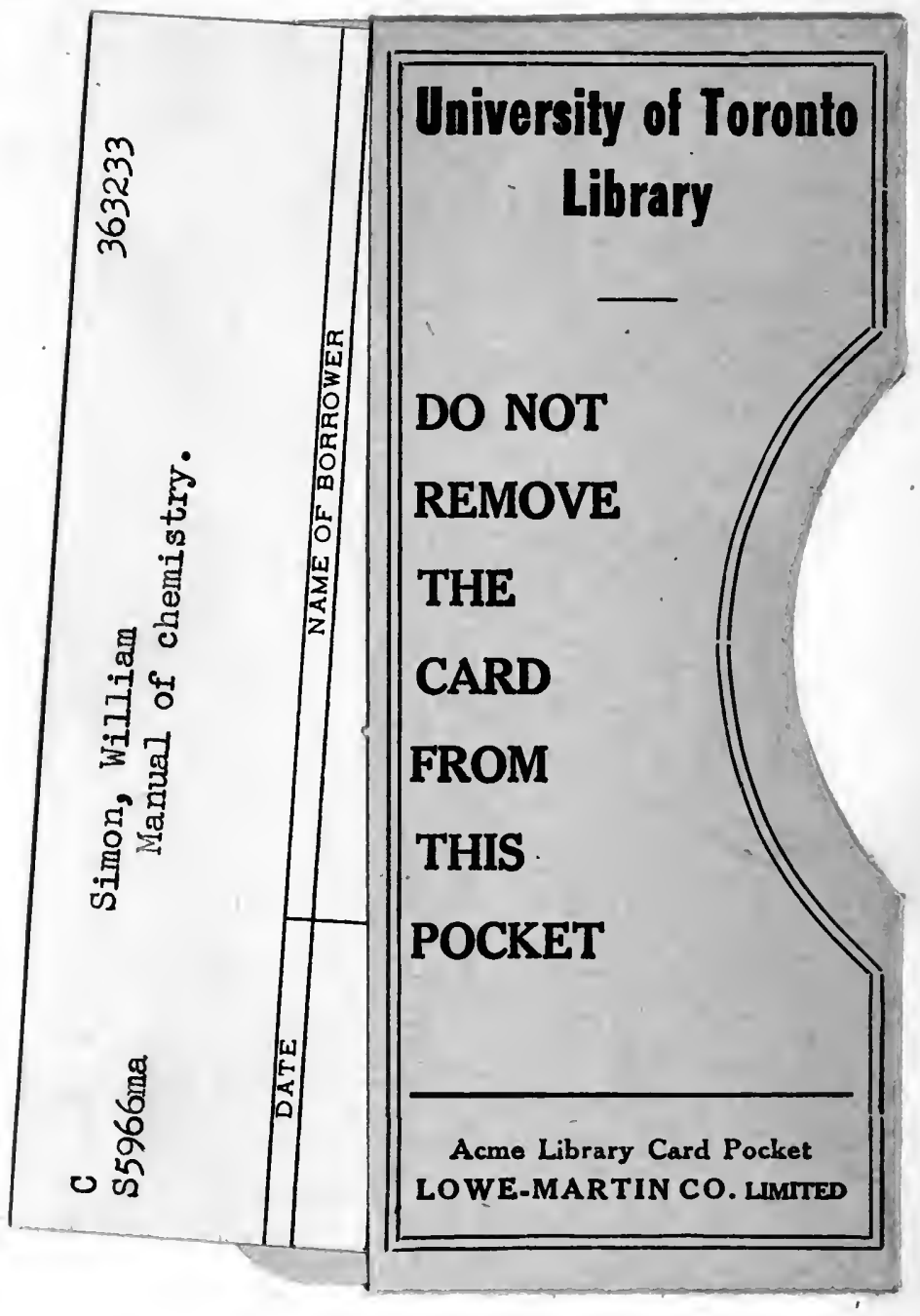


- 